
(5)

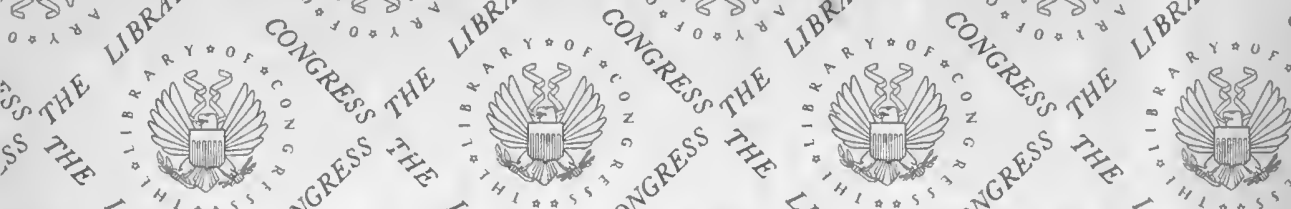

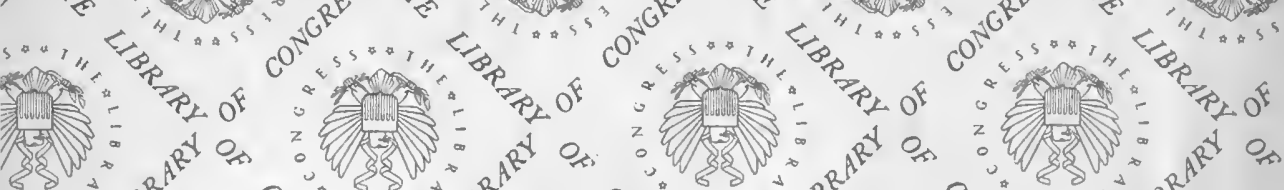
So $n^{2}$

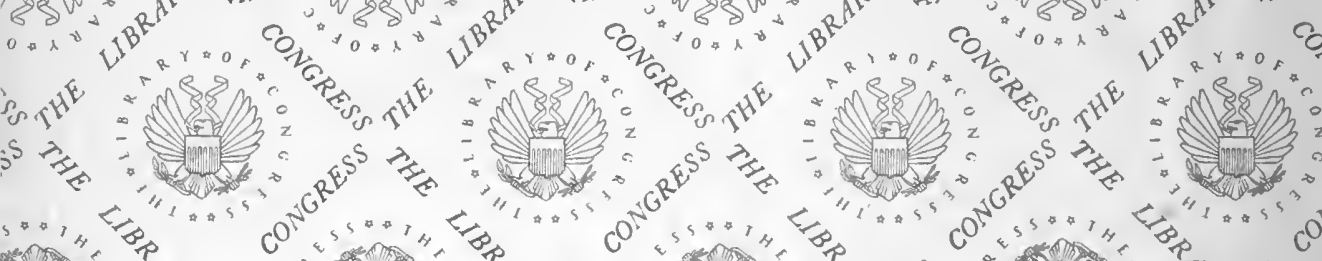

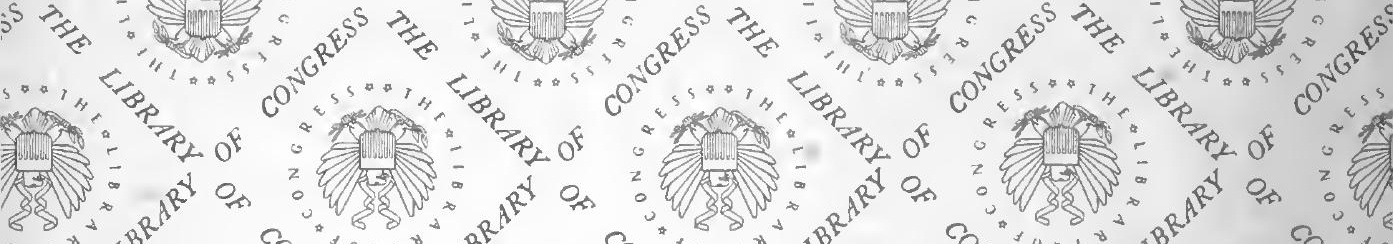

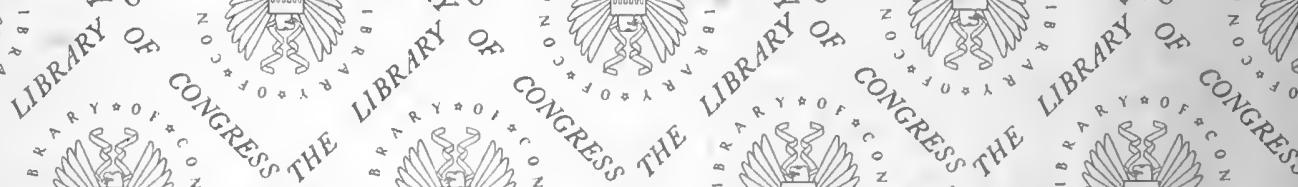

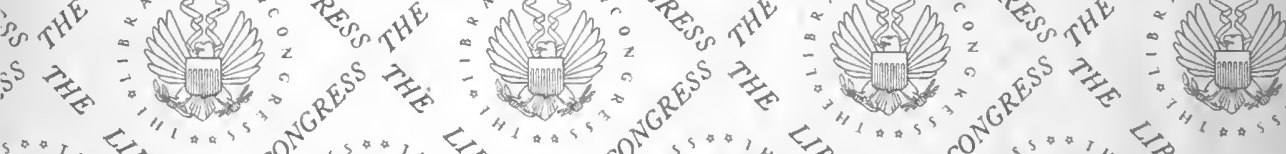

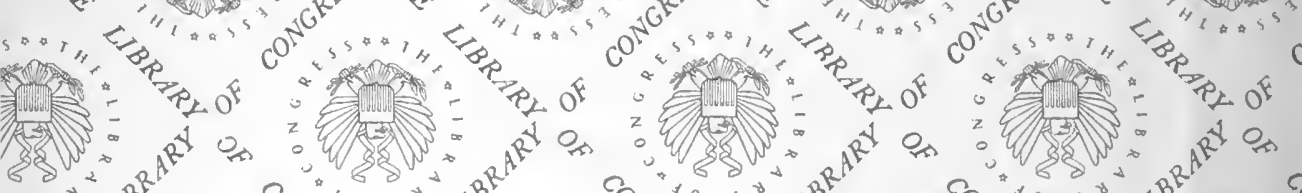

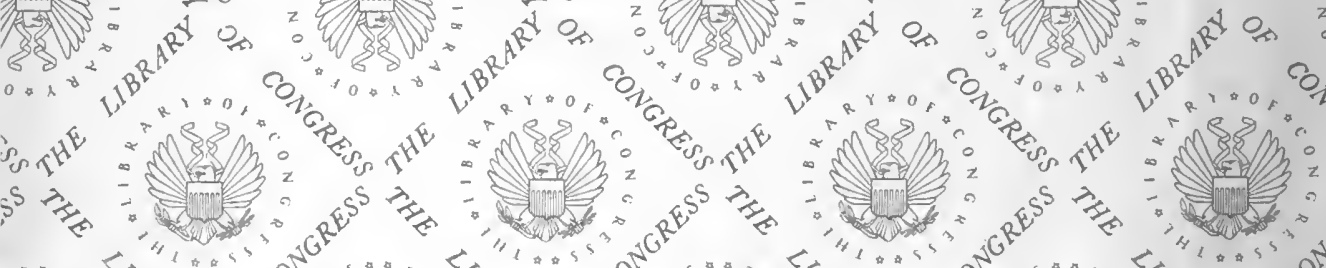

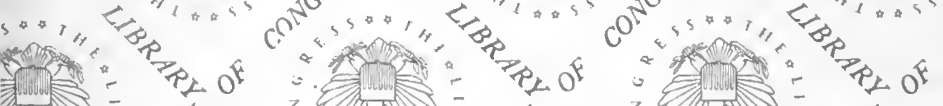

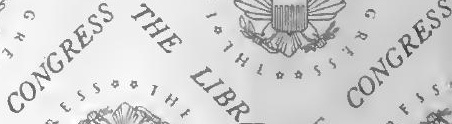
0 



\section{EDUCATION AND WORLD CITIZENSHIP}


CAMBRIDGE UNIVERSITY PRESS

C. F. CLAY, MANAGER

LONDON : FETTER LANE, E.C. 4

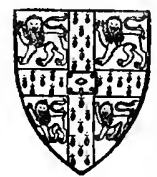

NEWYORK : THE MACMILLAN CO. BOMBAY

CALCUTTA MACMILLAN AND CO., LtD. MADRAS

TORONTO : THE MACMILLAN CO. OF CANADA, LTD.

TOKYO : MARUZEN-KABUSHIKI-KAISHA

ALL RIGHTS RESERVED 


\title{
EDUCATION
}

AND

\section{WORLD CITIZENSHIP}

\section{AN ESSAY TOWARDS A SCIENCE OF EDUCATION}

\author{
BY \\ JAMES CLERK MAXWELL GARNETT . \\ C.B.E., M.A. \\ Late Fellow of Trinity College, Cambridge; Barrister-at-law; \\ General Secretary of the League of Nations Union; Late \\ Principal of the Manchester Municipal College of Techno- \\ logy, and Dean of the Faculty of Technology in the Victoria \\ University of Manchester
}

\section{CAMBRIDGE}

AT THE UNIVERSITY PRESS

I 92 I 


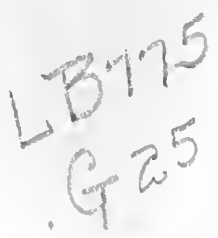

If any man think that he knoweth anything he knoweth nothing yet as he ought to know.

S. Paul.

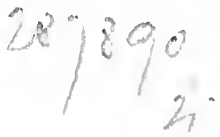




\section{PREFACE}

THE object of the enquiry described in this book is stated on

It is to attempt, in the light of modern knowledge of physiological psychology, to formulate, however tentatively, a few simple and general principles of education, and so to take a further step towards a science of education. The need, and even the duty, of making this attempt became more and more impressed upon me during eight years of work for the Board of Education and eight following years of work for education in the North of England....The enquiry has been in progress for most of this latter period, and many of the conclusions reached have been applied in practice by the university and the college of which I was then a member. Some of these results have formed the subject of lectures, addresses and papers that have already been printed.

The present volume, of which the first draft was finished in July, I9I9, is divided into three 'Books.'

Book I is introductory and historical.

Book II is concerned with the aim of education. It is summarised in Chapter I9 which the reader is advised to look at before beginning this section of the work. There should then be no difficulty in following the argument; for, although mathematical analysis has not been wholly dispensed with, its use has been confined to footnotes and appendices which the non-mathematical reader may neglect. In the course of Book II, I was brought face to face with several philosophical issues. These I deliberately abstained from avoiding. I am consequently prepared to find that, here especially, many of the positions I have taken up may prove to be provisional only.... The investigations described in Book II, and especially in Chapter I7, first made me realise that a perfect system of education must be world-wide; or, at least, that, in the interests of human progress, the ultimate aim of education should be the same the world over. So it is that 'world citizenship' appears upon the title-page.

Book III treats of a system of education designed to achieve the aim set forth in Book II. But, if such a system of education was to be described within reasonable limits, it became necessary to 
focus attention upon a smaller and more homogeneouscommunity than the whole 'Great Society' of mankind. Accordinglyalthough Book III, particularly in Chapter 2r, deals with the manner in which the principles formulated in Book II may be applied to any system of education designed to achieve the aim therein defined-most of Book III, summarised by the coloured plate facing p. 3I9, is specially concerned with a national system of education that might be established in England within the next decade, if only the League of Nations is sufficiently supported by public opinion to make large-scale war impossible, and to set free for productive expenditure most of the money that is still being dissipated upon armaments.

To William James, I owe far more than I can express or even estimate; and to Dr William McDougall, who now occupies William James' chair of psychology, I am deeply indebted, not only for the stimulus of his writings, but also for his great kindness in reading and commenting upon my typoscript while he was busily preparing to migrate from Oxford to Harvard. I am also most grateful to my former colleague in Manchester, Professor Alexander, and to two other friends-Professor John Adams and Professor Percy Nunn-who also read my typoscript during the spring of 1920 and sent me valuable suggestions. I cannot omit a word of thanks to Miss Eira Davies who prepared the typoscript and willingly gave me much other effective help during more than a year. And I should like, in a very special degree; to thank my father, who has twice read my proofs, and to whom I have always been able to look for advice, assistance, and encouragement.

But to my wife's interest and cooperation, the possibility of writing the book at all has been due. Since the work began, she has allowed it to have the first claim upon my vacations as well as upon my spare moments during term. To her I dedicate the book.

J. C. M. G.

HAMPSTEAD,

January, 1921 


\section{Table of Contents}

\section{BOOK I}

\section{INTRODUCTION}

Chapter i. The Object and Method of the Present En-

PAGE

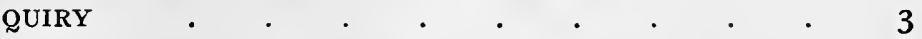

Chapter 2. The aims of Education in the Past . . . I2

Chapter 3. The Present Position . . . . . . . I9

\section{BOOK II}

\section{THE AIM OF EDUCATION}

Chapter 4. Education and Neurology . . . $\quad 27$

Chapter 5. Neurograms . . . . . . . 42

$\S$ I. Neurograms or Neural Dispositions . . . . . 42

$\S 2$. Psychoses and Neuroses . . . . . . . . 50

$\S 3$. Instincts and Emotions . . . . . . $5 \mathrm{I}$

$\S 4$. Sentiments . . . . . . . . . . 58

$\S 5$ 5. Interest-Systems $\quad . \quad$. $\quad . \quad$. $\quad . \quad$. $\quad$. 62

Chapter 6. Involuntary Thought . . . . . 65

$\S$ I. The First Law: Psycho-neural Parallelism . . . 65

$\S 2$. The Second Law: Diffusion $\quad . \quad$. $\quad . \quad$. $\quad .66$

$\S 3$. The Third Law: Inhibition by Drainage . . . 70

Chapter 7. Will and General Ability . . . . 95

$\S$ I. Psycho-physical Interaction . . . . . . 95

$\S 2$. Will-power, the single general factor in human qualities $\quad 98$

$\S 3$. Cleverness, a group factor in intellectual qualities . II9

§ 4. The Fourth Law: Free Will . . . . . 127

$\S 5$. Educability of Will . . . . . . . . . $\quad$ I35

$\S 6$. Will in Everyday Life . . . . . . . . $\quad$ r 38

Chapter 8. Purpose . . . . . . . . . . $\mathrm{I} 43$

$\S$ I. Purpose-Neurograms . . . . . . . . . $\quad$ I43

$\S 2$. Formation of Purpose-Neurograms . . . . . . 149

$\S 3$. Influence of Purposes on the Stream of Thought . $\quad{ }_{152}$

$\S$ 4. Dr Webb's Group Factor in Character Qualities . $\quad$ I55 
Chapter 9. Conflict

$\S$ I. Opposing Interest-Systems

$\S 2$. Resoluticn of Conflicts

$\S 3$. Two Types of Conflict

Chapter io. Some Characteristics of Reasoning .

Chapter it. The Organisation of Thought . . . . $\quad$ I 89

$\S$ I. The World of Experience . . . . . . . . . . . $\quad$ ' 189

$\S$ 2. The Endarchy of Science . . . . . . . . . $\quad$ r 94

$\S 3$. Growth of the Endarchy of Science . . . . . . $\quad 197$

$\S$ 4. Maximal Endarchies . . . . . . . 206

\$ 5. Efficiency and Utility in Thought Organisation . . 2II

$\S 6$. Subdivisions of Knowledge . . . . . . . 213

$\S 7$. The Value of an Essence . . . . . . . . $2 \mathrm{r6}$

Chapter I2. Neurographies . . . . . . . 220

$\S$ I. The Nature of Individual Neurographies . . . . 220

$\S 2$. Individual Neurographies and the Endarchy of Science 226

§ 3. Personal Endarchies . . . . . . . . . 232

$\S 4$. The Central Elements in Personal Endarchies . . $\quad 237$

Chapter r3. Curiosity; and the Involuntary Growth of SiNGLE Wide INTERESTS . . . . . . . . 245

Chapter I4. Reasoning; and the Voluntary Development of Single Wide Interests ․ . . . . . $\quad 255$

$\S$ I. Will and Curiosity $\quad . \quad$. $\quad . \quad . \quad . \quad . \quad . \quad . \quad 255$

$\S 2$. The First Period of Conscious Work . . . . $\quad 256$

$\S 3$. The Interval of Unconscious Work . . . . $\quad 257$

$\S 4$. The Second Period of Conscious Work . . . 259

$\S 5$. An Illustration and a Digression . . . . . 264

$\S 6$. A Personal Endarchy facilitates Reasoning . $\quad 266$

$\S 7$. Will, the principal factor in developing Single Wide Interests . . . . . . . . 268

Chapter i5. The Fifth Law . . . . . . . 272

$\S$ I. The End of a Train of Thought . . . . . 272

$\S 2$. The Fifth Law fits Experience . . . . . 273

$\S 3$. Pedagogic Corollaries: Practical Work in Schools $\quad 278$

Chapter i6. Conduct . . . . . . . . 28I

$\S$ I. Conduct as affected by Neurography . . . . 28I

$\S 2$. Conduct as affected by Will . . . . . 287

$\S 3$. The Five Laws of Thought. $\quad . \quad$. $\quad . \quad$. $\quad . \quad 289$ 
PAGE

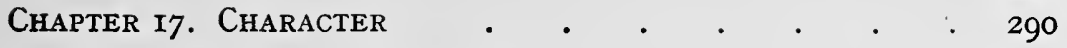

$\S$ I. Character and Conduct.$\quad$. $\quad . \quad$. 290

$\S 2$. Character, Neurography and Will . . . . 293

$\S 3$. Character in the perfect Commonwealth . . . 295

$\S 4$. Character and Progress . . . . . . 303

$\S 5$. The Christian Hypothesis . . . . . . 305

Chapter i8. The Aim of Education . . . . . 3II

Chapter i9. The Aim of Education-Summary . . . 3I2

\section{BOOK III}

\section{A SYSTEM OF EDUCATION}

Chapter 20. Types of Social Service . . . . . 3ig

$\S$ I. Some Principles of Selection . . . . . . 3I9

$\S 2$. Classification of Services . . . . . . . 325

Chapter 21. Applications of Principles . . . . 33 I

$\S$ I. The Power of Education . . . . . . . . 33 I

$\S 2$. Training the Will . . . . . . . 332

$\S 3$. The Central Purpose . . . . . . . . 334

$\S 4$. The Scientific Endarchy and Organised Thought . 335

$\S 5$. A Single Wide Interest: not Separate Subjects . . 336

$\S 6$. The Growth of a Single Wide Interest . . . . 340

$\S 7$. Continuity in Education . . . . . . . . . 349

$\S 8$. Contrasted Types of Study . . . . . . . . 35I

§. Handwork . . . . . . . . . $36 \mathrm{I}$

Chapter 22. A Flow-Diagram of Education . . . 363

$\S$ I. Introduction . . . . . . . . . . 363

$\S$ 2. The Province as a Unit . . . . . . . 365

§ 3. The Horizontal Scale . . . . . . . 367

$\S$ 4. The Vertical Scale . . . . . . . . . 368

$\S 5$. Types of Education . . . . . . . . . . . $\quad . \quad 372$

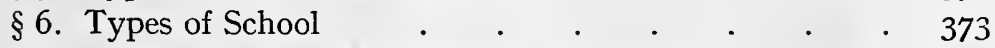

$\S 7$. Scholarships and Maintenance Allowances . . . 374

Chapter 23. Types of Education . . . . . . . 376

$\S$ I. Nursery Education . . . . . . . . . . $\quad .376$

$\S$ 2. Elementary and Preparatory Education . . . 377

$\S 3$. Secondary Education: Junior, Intermediate, and Advanced . . . . . . . . 383 
§ 4. University Entrance .

$\S 5$. Undergraduate Studies . . . . . . . $\quad$. 397

$\S 6$. Graduate Study and Research . . . . . . 402

$\S 7$. University Part-time Studies . . . . . . . 406

$\S 8$. Senior Technical Education _ . . . . . . . 409

$\S$ 9. Advanced and Miscellaneous Part-time Studies . . 4 4 3

$\S$ Io. Senior Secondary Education . . . . . . 4I5

$\S$ II. Intermediate Part-time Studies . . . . . 420

$\S$ I2. Senior Elementary Education . . . . . . . 422

§ 13. Junior and Senior Part-time (Secondary) Education . 424

$\S$ I4. Byways of Education . . . . . . . . . . 429

Chapter 24. Types of School and College . . . . 432

$\S$ I. Public Spirit . . . . . . . . . . . . 432

$\S$ 2. Universities . $\quad . \quad . \quad . \quad . \quad . \quad . \quad . \quad 435$

$\S 3$. Senior Technical Schools . . . . . . . . . 440

$\S$ 4. Secondary Schools . . . . . . . . . . . . 443

$\S 5$. Junior Technical Schools . . . . . . . . . 445

$\S 6$. Part-time Secondary Schools . . . . . . . 448

$\S 7$. Elementary Schools . . . . . . . . . 45I

$\S 8$. Summary . . . . . . . . . . . . . 452

Chapter 25. A National Scholarship System . . . 453

$\S$ I. Maintenance Allowances . . . . . . . 453

$\S 2$. Provincial Authorities . . . . . . . . 454

$\S$ 3. Multiplicity of Scholarships . . . . . . 459

$\S 4$. Methods of Selection . . . . . . . 460

Chapter 26. Teachers . . . . . . . . . . 464

Appendix A. The 'Broad Foundation' Metaphor . 469

„ B. Mathematical Appendix to Chapters 7 and 8 . 475

" C. Note on Maximal Endarchies . . . . 492

" D. Note on Hobbies and Holidays . . . . . 495

" E. Provinces of England and Wales. . . . 497

" F. A Higher School Certificate examination . . 498

" G. The cost of a National Scholarship System . 499

" H. University Education and Local Rates . . 502

INDEX $\cdot \quad \cdot \quad \cdot \quad \cdot \quad \cdot \quad \cdot \quad \cdot \quad \cdot \quad \cdot \quad \cdot \quad 505$

\section{DIAGRA M}

A National.System of Education _ . . . . to face p. 3I9 
BOOK I

INTRODUCTION

G. E. 



\section{CHAPTER 1}

\section{THE OBJECT AND METHOD OF THE PRESENT ENQUIRY}

'I'M not an educationist and I hope I never shall be' said one who, years ago, ranked high in the administration of English education: 'the greatest man who was ever in this Office,' according to his colleague who told me the story. Small wonder that he was reputed great, even greatest! In holding that his wide general knowledge of education was unsystematic, inexact, amateurish, he must have been unique. Rare indeed is the man who does not think himself expert in education. 'Even if he has had none, he is ready to say how much (or how little) he has missed it, or what it ought to have been if he had had it.'*

So our friend was great in disclaiming expert knowledge. But was he great in refusing to hope for it, even to strive for it, although its acquisition might well have seemed hopeless? Hopeless enough it was in all appearance; for our friend, had he lived until our own day, might still be excused for denying the existence of any generally accepted principles of education. 'Plato disagrees with you' was the crushing rejoinder made quite recently by an ex-President of his University Union, to whom the writer had suggested that education might become a science!

We recognise expert or systematic knowledge in any branch of natural science when we find a body of closely connected propositions which, with their logical consequences, all the recognised experts in that science agree to accept as corresponding with experience-not merely their own experience, but also so much of other people's as they are able to disentangle from the interpretations which those other people have put upon it $\uparrow$. Thus any two expert mathematicians or physicists or chemists or engineers, discussing matters relating to their science, will be able to assume from the outset a number of wide generalisations upon which both are agreed: generalisations which

* Professor Arthur Smithells, F.R.S., Presidential Address to the Society of British Gas Industries. (3rd March, I9I I.)

$\dagger$ The systematic or expert character of this knowledge still remains, when subsequent discoveries have disproved its truth: the Ptolemaic astronomers were not less expert in their science, after Copernicus had shewn that the facts might be more simply (and therefore more credibly) accounted for by a different hypothesis. 
connect together, and thus 'explain,' a much larger number of facts. So nearly universal is this agreement that when two expert chemists disagree before a court of law, not upon the validity of one of the generalisations which form the principles of their science, but merely upon the question whether a particular fact belongs to one generalisation or to another, their disagreement is sufficiently remarkable to excite comment.

But, when the discussion is of education, disagreement concerning first principles is the rule rather than the exception. There is little agreement concerning the end of education, and still less concerning the means. Even the agreement that does appear to exist is often fictitious and due either to the misuse of metaphor or to the absence of any esoteric or symbolic language in which ideas concerning education can be unequivocally expressed. And yet the need for established principles of education and for the general recognition of such principles is beyond dispute. It is only by means of organised systems of ideas that our thinking, whether of education or of any other matter, can make permanent progress; and it is only by the wide acceptance (which need not be other than provisional) of a single set of principles that a coherent and effective system of public education may be built up. For such a system implies a number of schools and colleges cooperating with each other and with the industries, commerce and other.departments of the life of the people so that the effect which each school or college in turn produces upon the development of the person being educated shall be continuous with that which precedes as well as with that which follows it. Not until such a system of education has been established will there be any assurance that, as the pupil passes from school to school or from form to form, each teacher in turn will not aim at undoing what his predecessor has done. Meanwhile, in England and Wales alone, $£ 36,000,000$ of public money was, before 19r4, being spent yearly on elementary education, and many other millions on higher education in addition. And, since the purpose of this expenditure must be either to modify or to facilitate the development of individuals so as to produce the several types of men who shall not only render the multifarious service required by the community, but also realise their best selves in that service, the need for a larger measure of agreement among educators is evidently urgent.

Our friend who, in spite of his responsibility for the administration of national expenditure on education in England, hoped that he never would be an 'educationist' ought then rather to have striven to 
understand, and even to construct, principles of education. The hopelessness of such a task was no sufficient excuse for failing to attempt it. Mr Bergson has reminded us that tasks which seemed impossible to sedentary thinkers may nevertheless be accomplished by acts of faith. For example, the impossibility of swimming might be demonstrated by one who should shew that unless a man could float he could not swim and unless he could first swim he could not float; and yet this arm-chair logic is stultified by the action of the man of faith who, leaping in and struggling, finds that he can swim.

If the apparent hopelessness of the task which our friend refused to attempt was no sufficient excuse for his refusal, such an excuse is still less valid to-day. Much fine work has been accomplished in recent years by physiologists and psychologists. It is, more than ever it was, the duty of those who are concerned with the administration of education to formulate the principles* upon which they are acting and to endeavour to coordinate them with the principles that underlie the practice not only of their colleagues in the service of education, but also of those who control the training of young people who have passed from their last school or college into the employment by which they mean to serve their fellows and to earn their daily bread. The present essay is an attempt to perform this duty; and its publication is intended to stimulate other educators to make similar but more successful attempts.

The first step in the direction of securing more general agreement concerning the principles of education should be to give up the useor, at least, the misuse-of metaphor. The prevalence of metaphors in educational literature, in its prosaic official reports $\dagger$ as well as in the poetic writings of distinguished ex-officials, is responsible for much of the obscurity which now envelopes educational thought. The plain man readily adopts and employs a metaphor, even a mixed metaphor like that of the 'broad foundation of general culture,' $\ddagger$ when he would never agree to the psychological theories it implies. He supposes that the metaphor by which he has been misled expresses in plain language a general principle capable of universal application, its limitations having been concealed from him by a change of metaphor whenever his author sees that absurdities are about to appear£. Mr Stelling

* Such principles must, of course, be consistent with all the facts known to those who formulate them.

$\dagger$ See Appendix A.

f See Appendix A.

$\S$ "For many minds, to say "as the twig is bent so is the tree inclined" not only illustrates the aphorism "train up a child in the way he should go, and when he is old he will not depart from it," but actually proves it ' (Adams, Evolution of Educational Theory, p. 286.) 
never realised that his whole system of education would have to be changed were he to regard Tom Tulliver's mind as an intellectual stomach with a delicate digestion instead of as a field whose culture demanded ploughing and harrowing by such potent implements as etymology and grammar (to which poor Tom's mind was peculiarly impervious) in order to prepare it for the receipt of any subsequent crop! 'O Aristotle,' adds George Eliot, 'had you been the freshest modern instead of the wisest ancient, would you not have mingled with your praise of metaphor a complaint that to-day we are seldom able to say what a thing is except by saying that it is something else!'

When metaphors have been banned, at least for a while in order to enable the literature of education to recover a more healthy tone, the next step is to permit a more general use of esoteric or symbolic language*. So long as writers and speakers continue to use, without defining, such words as character, culture, imagination, or interest, words which are used every day with a variety of different meanings, so long will each of their readers or hearers put his own interpretation upon the word in question, if indeed he trouble to give it any clear interpretation at all. So long therefore will it remain impossible to convey, or to secure agreement concerning, precise conceptions of the facts or principles of education. So long, too, will any close connexion between education and most branches of social service-education and industry, for example-appear incongruous to many people. Is there not a great gulf fixed, say they, between hazy views of education high in the clouds above and the hard facts of science or technology down in the depths beneath? And yet the intimate relation of education to industry is obvious enough; for of all that goes to make industry possible, let alone prosperous, the human element is by far the most important.

After seizing upon the facts themselves, instead of upon quite different facts contained in a metaphor, and after expressing the true facts in unequivocal even if technical language, the third step towards the formulation of principles is to select from among the vast mass of available material a limited number of facts to be associated together by means of a generalisation. The selection of the simplest facts and their relation to one another by means of wide generalisa-

* Cf. W. McDougall: "When we come to describe the facts of consciousness we find that the notions and the words in popular use are very inadequate to the work of analytic description...the greatest authorities have not yet learnt to use the same descriptive terms, or to apply the same terms in exactly the same senses.' (Psychology, Pp. 42 and 47.) 
tions is, according to one of the greatest of mathematicians*, the method by which all systematic knowledge of natural science has been organised; and the same method is that by which we instinctively acquire such organised knowledge as we possess of the world in which we live.

The selection of certain facts in order to make our main lines of association, our first generalisations, our principles, will involve ignoring other facts, to some of which however we may afterwards return and relate them to our organised body of knowledge by means of other secondary generalisations; further sets of facts may then be related to these; and so on. Moreover, as Poincaré has pointed out, it is of the first importance that the facts selected for linking up by the earliest generalisations shall be simple† facts, simpler perhaps than can be directly perceived by our senses. A direction and position in space, represented by a line having length without breadth or thickness, is an example of such a simple fact. It is just the kind of fact that would be included in our first generalisations were we to begin the systematic study of any material object, whether the leg of a table, the continent of Europe, or the Dresden Madonna. And this remains true, notwithstanding the entire absence of a line as we have defined it from any of these objects as apprehended by our senses In short, we are accustomed to think-and this is the more true the more precise we want our thinking to be-not of things as they really are, but of simplified and organised representations or abstracts which for the purpose in hand correspond in all essential respects with more complex sense impressions of those things. Such abstracts are more easily and more accurately reasoned about than the more complex sense impressions. Thus, if we wish to determine the area of a triangular field, we do not think of the field with its crop of wheat, its boundary hedges, its sandy soil, its flatness, its view of the sea, its summer heat and its winter cold; but we think quite simply of an imaginary triangle having more in common with the plan of the field included in the title deeds than with the field itself as it appeals to our senses. And the reason is given by Bergson: 'Whatever is geometrical in things is entirely accessible to human intelligence.' $\S$

* Henri Poincaré, Science and Method, pp. 17, 18. † Science and Method, p. I8.

$\ddagger$ Cf. Bernard Hart: 'Atoms are merely constructions of the scientific imagination.... The ether and its waves have never been observed in nature, they have been invented by the scientist in order to explain the facts of light and heat. But their actual non-existence does not in the least vitiate the value which they have for science. They enable us to resume and predict a vast number of facts, and this is the sole justification which a scientific law is ever required to possess.' (The Psychology of Insanity, p. 15.)

$\S$ Creative Evolution, p. 200. 
For accurate and easy thinking about education it is necessary to make use of the same method: to select the facts about which to think and, above all, to choose facts which are simple, even if imaginary like the line which represents the direction of a hedge. Thus, in formulating principles of education, we shall for the most part focus our attention upon the comparatively simple material aspects of the brain, rather than upon the mind or soul, of the person being educated. This procedure implies no low material view of education. It does not suggest that education is concerned with the central nervous system rather than with the soul, although it recognises that the soul can only be reached by human educators through the brain of the pupil. Nor does it assume that we know more of the brain than we do of the soul: the contrary is more probably the case. But, as Huxley pointed out, " there can be little doubt that the further science advances, the more extensively and consistently will all the phenomena of Nature be represented by materialistic formulae and symbols.'* Bergson states the same truth more fully. 'The intellect,' he writes 'is characterised by a natural inability to comprehend life.' $\dagger$ And again, 'The intellect is at home in the presence of unorganised matter.... Now, when the intellect undertakes the study of life, it necessarily treats the living like the inert, applying the same forms to this new object, carrying over into this new field the same habits that have succeeded so well in the old; and it is right to do so, for only on such terms does the living offer to our action the same hold as inert matter. But the truth we thus arrive at...is no more than a symbolic verity. It cannot have the same value as the physical verity, being only an extension of physics to an object which we are a priori agreed to look at only in its external aspect. The duty of philosophy should be to intervene here actively....' $\ddagger$

Moreover in reasoning about material things, there is less danger of going astray 'between the moment when we meet a proposition for the first time as the conclusion of one syllogism, and the moment when we find it once more as the premise of another syllogism,' $\S$ by forgetting the meaning of the proposition in the meanwhile. Our reasoning is therefore more likely to be accurate if, without by any means asserting that the brain is merely a material thing, we concentrate our attention upon its material aspects. And then, not only will our conclusions more certainly follow from our premises, but a

* Collected Essays, Vol. I, p. 164 quoted by W. Temple: 'The Nature of Personality' (p. 2).

$\dagger$ Loc. cit. p. 174. Bergson's italics.

$\ddagger$ Loc. cit. p. 206.

$\S$ H. Poincaré, loc. cit. p. $4^{8}$. 
comparison of these conclusions with observed facts will therefore also shew us more certainly if our premises or hypotheses are incorrect*. And if from certain premises we deduce conclusions which accord with all the observed facts, we shall feel the more sure of the truth of the premises themselves and of other unverifiable conclusions drawn from them.

For these reasons we shall, as far as possible, employ 'materialistic formulae and symbols' in this enquiry. While, however, we recognise and make use of the fact that 'all mental phenomena are accompanied by a physiological phenomenon,' $\dagger$ and while we choose to imagine the latter, rather than the former, in our discussions, we need not suppose that, if we could completely describe the structure of the nervous system of any man or animal and had a complete knowledge of the laws of the physical and chemical processes that occur in it, we should be able to account completely for all the conduct of that individual. In fact, we shall not follow Huxley in regarding consciousness as no more than an 'epiphenomenon' caused by the play of nervous processes in the brain but neither modifying those processes nor reacting upon them. Nor shall we accept the view that the processes of mind and brain run parallel to each other but never meet or interact, the doctrine of psycho-physical parallelism. On the contrary, we shall assume what Dr McDougall calls 'the old commonsense view' that psycho-physical interaction does in fact take place, 'that soul and body, or psychical and physical processes, interact or react upon one another, so that psychical processes play a part in determining conduct.' +

Having pointed out that the first step towards a more coherent and effective system of public education is to secure some measure of agreement, however provisional, upon the aim of education as a whole, and having indicated a starting-point and a method, we are ready to proceed with our enquiry concerning principles of education with a view to discovering and formulating, however tentatively and provisionally, such an aim of education as will be consistent with these principles.

We begin in the next chapter-the second chapter of this first

* In other words, we shall be more likely to discover our mistake if we attribute imaginary properties to the brain than if we erroneously imagine properties of the mind or soul.

$\dagger$ Alfred Binet, The Psychology of Reasoning, p. I2.

† W. McDougall, Physiological Psychology, p. 8 , to which reference may be made for a summary of the argument for 'psycho-physical interaction' as against 'epiphenomenalism ' and 'psycho-physical parallelism.' A fuller statement is given in Body and Mind by the same author. 
book-with a summary of Professor Adams' account of the principles of education as they have been accepted in the past.

In our second book we shall briefly describe a few leading features of the human nervous system, and, using the results of some recent researches in neurology and experimental psychology, we shall formulate five laws of thought consistent with each other and with all available facts. While eagerly awaiting new discoveries and generalisations that will confirm or modify the accepted facts or assumptions upon which these five laws are based, we shall use the laws in question as a base for further advance, deducing from these laws results that may be compared with experience and, if they fit, be accepted along with the laws from which they are derived as forming a nucleus around which a science of education may continue to grow. Our second book concludes by pointing out that the aim of education cannot be determined until the aim of life has first been agreed upon; but that, if we assume continuous progress towards some far-off goal, whatever that goal may be, to be the aim of human life, then we can deduce the aim of education from our five laws of thought. The deduction is accordingly made, and a single aim for education ultimately formulated.

The third and final book is concerned with the effect upon a system of education-and especially upon the system, if system it can be called, at present in operation in England-of adopting and pursuing in practice the aim of education defined in our second book. A reformed system of education, consistent with this aim and based upon the principles that led us to its definition, is next described and illustrated by a diagram. This reformed system is especially adapted to English conditions so as to involve the smallest changes in the present public provision of education in this country. It is discussed in some considerable detail, for it is intended to be capable of being brought into operation in England within the next ten years, a decade which in any case bids fair to be more critical than any in English history. Indeed the future, not of England only but of Christendom, largely depends upon harmony of purpose and community of effort among the English-speaking peoples. Education is the most powerful, if not the only effective, instrument for securing this harmony of purpose, that is bound to result in practical cooperation. It is, however, lamentably true that England lags far behind the greater part of the English-speaking world in its public provision for education. The reform of English public education, so as to provide this country with a coherent and effective system that will bring every kind of 


\section{1 OBJECT AND METHOD OF PRESENT ENQUIRY II}

education within the reach of all who are of sufficient educational promise, in whatever part of the land they may happen to live and however poor they or their parents may happen to be, is one of the most urgent needs of the present critical time. It is for this reason that our third book, altogether inadequate though it be for a complete account of a system of education designed to realise the aim set forth in the central portion of the present enquiry, is longer than would be needed to indicate only a few of the principal changes that would follow if this aim, in all its diverse applications to different schools and colleges and to different courses of study, were consistently pursued by every English educator. 


\section{CHAPTER 2}

\section{THE AIMS OF EDUCATION IN THE PAST}

John Stuart Mill, in his rectorial address to the University of St Andrews (I867), defined education as including 'whatever we do for ourselves, and whatever is done for us by others, for the express purpose of bringing us somewhat nearer to the perfection of our nature; it does more: in its largest acceptation it comprehends even the indirect effects produced on character, and on the human faculties by things of which the direct purposes are different; by laws, by forms of government, by the industrial arts, by modes of social life; nay, even by physical facts not dependent on human will, by climate, soil, and local position.' *

According to this definition education includes all those influences that operate upon the person being educated from outside himself and so modify his nature. But for practical purposes the definition is too wide, as Mill was well aware. What we commonly call education is that education in which man intervenes $\uparrow$ : 'It is a bi-polar process in which one personality acts upon another in order to modify the development of that other. The process is not only a conscious but a deliberate one. The educator has the clearly realised intention of modifying the development of the educand.' $\ddagger$

In prehistoric times, and among the primitive communities with which history begins, individual habits and social customs differed but little from one generation to another. Evolution was a slow process in those days. As was the father, so was the son. Every individual's walk in life could be foreseen with small risk of error. It was the function of such education as he received to prepare him

* Quoted by John Adams, The Evolution of Educational Theory, p. 1o. To Professor Adams' work I am indebted for much that appears in this chapter and the next.

$\dagger$ Huxley, Collected Essays, Vol. III, p. 85. Quoted by Adams (loc. cit. p. 30) who distinguishes between this human education and that cosmic education which consists of wider processes wherein no human educator takes part.

$\ddagger$ Adams, loc. cit. p. 39. Educand is Professor Adams' term for the person being educated. We shall follow Professor Adams in its use so as to avoid calling the same person by several different names-child, pupil, student-as he or she goes from one school to another and so on to college. 
for his particular walk in life. 'All early education is a direct preparation for the life work of the individual concerned. It is an education ad hoc.... Education begins by being specific.' * The idea of a general education which should fit the educand for any walk in life to which he might happen to be called arose comparatively late in the evolution of educational theory.

We must distinguish here between specific education-preparing the educand for his whole life-work, social as well as vocational-and what is technically known in schools as specialisation. 'Specific education historically precedes while school specialisation historically succeeds the development of the theory of a liberal education. Specific education is the form that naturally appeared as soon as humanity rose to the possibility of passing on its gains from generation to generation. The child was brought up to do what its parents found it necessary for them to do in order to carry on life successfully. With the coming of the conception of a liberal education there was a tendency to a certain rigidity of curriculum because of a natural desire on the part of every one to insist on having only those elements that were generally recognised as constituting the really free training.' $\dagger$ On the other hand, school specialisation consists in the manipulation of the elements of this rigid curriculum, often without reference to the lifework of the educand. Thus a boy who intends to study science or medicine at the university may specialise in classics at school on the ground that 'the classics develop the power of sustained and orderly thinking ' $\div$; or another may specialise in mathematics at school and at college in order to win a place in the Indian Civil Service and so administer laws in India.

The specific education of early times was passed on from parent to child or was acquired by the educand for himself in the mere process of living. It is only in modern states and in very recent times that the education of every inhabitant has been deliberately planned. But even among primitive peoples it soon became usual for the education of the rulers to be given them deliberately, while other people were left to make shift with what education they could find for themselves. The education given to future rulers aimed specifically at preparing them for the whole of their life-work. And, since whoever was well educated as prophet, priest, or king must have had his whole nature concerned in the process, no distinction was apparent at the

* Adams, loc. cit. pp. I62, I63.

$\dagger$ Adams, loc. cit. pp. I78, I 79 .

$\ddagger$ Quoted from Dr Alex Hill (formerly Master of Downing College, Cambridge) by Adams, loc. cit. p. 2 II. 
earliest stage between the vocational and social aspects of specific education*.

The education of the 20,000 cultured Athenian citizens of the time of Socrates was still within the region of the specific. They were, in fact, specifically prepared for their life-work of cultured leisure $\uparrow$. Theirs it was to govern and defend their city-state, as well as to understand and direct the work of the swarming slaves among whom were some of the finest craftsmen the world has ever seen. The 'liberal' education of the free citizen of Athens was wide enough to fit him for the performance of these manifold duties. But it did not include subjects-Sanskrit, for example, or Egyptian art-which, while having no bearing upon the work he would be called upon to $d o$, he nevertheless required to know about in order to be recognised as 'cultured.' $\ddagger$ At this stage the specific education that best prepared the free man for his life-work as a citizen was not distinguished from the training that best developed him as a man. The vocational and social aims continued coalesced. But we must not forget that the educational theory here in question was concerned only with the education of the citizens who counted. The slaves were specifically trained for their several occupations. Relatively to that of the slaves, the education of the free citizens was a general education. The specific education of the free man was, in fact, the most general education practised or conceived.

In mediaeval Europe, as in ancient Athens, the training of the masses of the people for the practice of industrial or artistic crafts had little relation to the education of those who were to rule in church or state. Indeed, the noble and the learned clerk would probably have been unwilling to concede that the apprentice who could not read or write had received any education whatever. Education was still the preserve of the rulers of the people who were prepared for a wide kind of life. But the growing complexity of social organisation broke up the ancient unity that marked the life-work for which old time rulers had been educated. The specific education provided in the

* Cf. Adams, loc. cit. pp. I82, 183 .

$\dagger$ Adams, loc. cit. p. 183 .

$\ddagger$ Cf. Professor A. N. Whitehead, F.R.S.: 'Classical learning has had its moments of triumph. It triumphed with radiant genius in Athens during the age of Pericles, a genius so splendid as to beget the theory that all subsequent ages should bend their intellectual activities to the parasitic existence of contemplation of this radiance. But this was a triumph of modern learning, exactly what I am contending for. Happily for mankind there were at Athens no schoolmasters to impress upon them that the only wisdom was to be found among the Egyptians. Herodotus was a modern of the moderns.' (Address to the Education Section (L) of the British Association, Bournemouth, 1919.) 
grammar schools and universities for those who intended to take Holy Orders or to follow the profession of law vas in the hands of the clergy. This was a bookish education, Latin being the basis of the curriculum. 'For the children of the aristocracy...a more elaborate form of social training was in vogue. This included much training of the body and of manual aptitude in those arts and exercises which were practised in the daily life, in the duties and in the amusements of the nobility and courtly families. At the time of the Italian Renaissance, this type of knightly or courtly education became highly developed and elaborate.'*

The cleric from the university and the courtier from the palace school might both claim to have received a general education. Each had, it is true, been specifically educated for a different walk in life; but their training had not been specialised. The former had studied the seven liberal arts; while the 'sons of the nobility and higher gentry were able to study whatever subjects their intelligent parents chose to select for them, and thus secure a clear advantage for their future life by this specific education.' $\dagger$ And yet 'the finished courtier that Castiglione describes was no doubt free of all the great courts of Europe, but he would have cut a less creditable figure in the circle of scholars at a University. It was the increase in the number of spheres in which a man could claim to be generally educated...that led to the development of [the idea of a still more general education which should be] a new opposite to specific education, an education that was not intended to prepare a man for this, that or the other sphere, however wide, but just to enable him to become a complete all round man, a man who was not educated for this or that, but who was just educated, and nothing more.' $\ddagger$

The growth of this theory of a general education that was to fit a man, not for this or that sphere of life, however wide and satisfying, but for any possible sphere that might claim him-not even for actual life at all, but for a sort of potential life§-was fostered by several cooperating causes.

In the first place it appealed to those writers who dealt definitely with the theoretical aspects of education. "The tendency of the educational theorist is to erect for himself a typical educand, and then set about finding the best way to educate him as a mere human being, apart altogether from any consideration of the particular rôle that

* Report of the Consultative Committee of the Board of Education on Practical Work in Secondary Schools, Appendix B, p. I32.

+ Adams, loc. cit. pp. I65, I66.

+ Adams, loc. cit. p. 184 .

$\S$ Cf. Adams, loc. cit. p. $\mathrm{r} 83$. 
the educand may have to play in life.... The theorist sets up before him an ideal of pure cultural education, something that will fit a man to fill any post to which he may be called.'* And yet, when we examine the writings of the theorists with some care, we find that 'each of them has in view some more or less definite type of man, and that their theories are directed towards the moulding of the educands on this model.' $\dagger$

Another reason for the wide acceptance of the new theory of general education was its consistence, if not its confusion, with the evident fact that there are certain instrumental subjects, such as was Latin in the middle ages, that every educand must study as a preparation for those further studies that shall specifically prepare him for his walk in life. It was not that Latin was supposed to produce an effect on the soul that prepared it for the reception of cultured ideas; but, quite simply, that Latin was the medium in which all higher education was then conducted. In Latin the books were written; university lectures were delivered in Latin; and Latin was the language of the church.

Then again the idea of a general education, quite independent of the lives which the educands might expect to lead, simplified the school curricula at a time when it tended to become intolerably complex. For while specific education was still practically universal the only way to adapt school education to the growing variety of careers that were becoming open to the educands was to add to the curriculum every subject which might help to prepare for any one of these careers. The increase of knowledge, brought about by the revival of classical learning and the beginnings of natural science, added further to the volume of school studies. Even in Milton's ideal school which still aimed at specific preparation for a particular walk in lifethat of a sturdy and cultured squire of the commonwealth + -the school course extended over nine strenuous years, and had to be followed by further specialised courses for those who wished to qualify for the professions of law or medicine or theology! At this rate, 'encyclopaedic instruction soon became impossible.' $\$$ The necessary relief was afforded by the theory of a general education, an education in vacuo. And this relief was the more welcome when it became a question of educating the masses of the people for their

* Adams, loc. cit. pp. I76, I77.

$\dagger$ Professor Adams cites the cases of Milton, Locke, Chesterfield, Montaigne, Rousseau, Pestalozzi and Herbert Spencer in support of this statement. Loc. cit. pp. 177, r 78 .

$\ddagger$ Cf. Adams, loc. cit. p. $177 . \quad$ § Adams, loc. cit. p. 208. 
almost infinite variety of different occupations as well as for their common life as citizens.

So the schools came to offer a general education more or less divorced from any attempt to prepare the educands for their future activities, whether vocational or social. There could be no valid objection to this in so far as it meant only that schools which prepared the educands not directly for life itself but for specific courses in the universities confined themselves to those instrumental subjects without which the further study was impossible. The general preparatory courses provided in the Arts Faculties of the universities themselves were equally defensible.

In fact, however, the universities gave up specific education no less readily than did the schools. But, as the professions which university men entered were already controlled by university men who readily accepted university standards in appraising qualifications, questions concerning the suitability of university education as a preparation for these walks of life did not easily arise.

The case of the schools was different. Even those schools which professed to prepare for the universities must have been attended by many pupils who did not afterwards receive a university education. There were also many schools which made no such profession, and all of whose pupils passed directly into practical life. The parents or employers of these pupils, brought up perhaps in a different tradition, naturally enquired what the schools had done to prepare their pupils for the life which followed school. So abstract a conception as the 'general education' that we have been discussing would not satisfy such critics who 'wanted to know why obsolete and, for practical purposes at any rate, useless subjects were taught to the exclusion of what the world regarded as essential. A defence had to be found, and it was ready to the hands of the school-master in this theory of formal training'*: namely, that the soul possessed a number of faculties (of memory, of observation, of invention, and the like) each of which could only be developed, or at least could be most effectively trained, by the study of certain appropriate subjects at school; so that the omission of any one of these subjects would stunt the development of the soul and maim the personality. Formal training was thus a simple and by no means abstract conception which critics of what appeared impractical in school curricula were ready enough to accept: the more so since the truth of the doctrine could not be easily tested. Indeed it is only in recent years that experimental psychologists have 
succeeded in shewing that this doctrine does not fit the facts, so that Professor Adams is able to write: 'The balance of expert opinion is now so solidly against the general dogma of formal training that as an educational force it must be regarded as moribund.'*

What then? As the dogma of formal training becomes discredited, its opposite-specific education-comes back to take its place as the most widely accepted theory of education. According to Professor Adams, 'the whole evolution of educational theory may be said to be a great sweep from specific education back again to specific education, through a long period during which formal training held the field.' $\dagger$

* Adams, loc. cit. p. 222, where references are given to some of the literature. Further references appear below.

$\dagger$ Loc. cit. p. 225. 


\section{CHAPTER 3}

\section{THE PRESENT POSITION}

WE have thus briefly traced the swing of the pendulum from specific education to formal training and part of the way back again. But the swing back is not yet complete; for there is as yet no general agreement that education should have a specific aim. In the sequel we shall look more closely into the causes of the modern movement towards specific education. Meanwhile, we have to estimate the present position as nearly as we may.

The most easily observed characteristic of English education at the present time is perhaps its aimlessness*. It is not merely that the theorists have been unable to agree among themselves what should be the aim of the national system of education as a whole, and what should be the aims of each particular school or college in order to achieve the aim of the whole. But in actual practice also there is dissimilarity and even inconsistency between such individual or partial aims as appear to be pursued, and are sometimes confessed, by the educational institutions of this country. "There is no longer a universally recognised circle of knowledge constituting a liberal education preparatory to specialist studies, as there was in the middle ages. Nor is there general agreement...as to the end that should be sought by education as a whole. Nor can agreement on such points be expected while men differ widely as to the meaning and purpose of life.' $\dagger$

But wide differences concerning the meaning or purpose of life are not related to differences concerning the aim of education simply as cause is related to effect: the relation is also that of effect to cause. The one kind of difference acts and reacts upon the other. We need not, therefore, despair of securing such a larger measure of agreement among English educators as shall make for the higher development

* Cf. Dr Lyttelton, when Headmaster of Eton: 'If Louvain, Rheims, etc. are the outcome of falsehoods thoroughly taught, what might be the power of truth if taught with equal thoroughness? So we turn to look within [at education in England], and find instead of thoroughness divergence of aim; instead of concentration a vast unwillingness to make clear to ourselves what we are trying to do.' (Times Educational Supplement, Ist August, 1916.)

+ Professor J. Welton, Encyclopaedia Britannica, I Ith edition, article on 'Education.' Cf. conclusion of Book II below. 
of individual citizens and for their more effective cooperation in the service of the society to which they belong. Even though at first the rate of change towards agreement may be very slow, this rate of change may grow quickly and soon result in finite progress.

And, in fact, most writers do not despair of agreement concerning the aim of education. Some of those whose opinions carry most weight even venture to state what the aim should be. Indeed, it is difficult to see how anyone can think clearly or effectively concerning the means of education until he has provisionally made up his mind concerning its end. Among very recent* pronouncements upon the aim of education two may be cited by way of illustration of the diversity of view that characterises the age in which we live.

Sir William Ramsay, in the course of an article on English Education, refers to 'the aim of training, namely, the power of concentration, the exercise of judgment, and, most of all, the development of the inventive faculties.' $\dagger$

Dr C. A. Mercier also regards education as having a threefold aim. He writes 'The aims of education are, I take it, these three: It should inculcate first, character; second, a habit of clear thinking; and third, a knowledge of facts...it is better to be good than to be wise: it is better to be wise than to be learned.' $\ddagger$

Except that both these statements assign an inferior place in education to the inere imparting of knowledge of facts, they appear, at first sight, to have little in common. There is a real difference between the two aims as stated, in that Dr Mercier's statement recognises, while Sir William Ramsay's statement does not recognise, the paramount importance of those instinctive (emotional) processes which play so important a part in the constitution of character. But, as our enquiry proceeds we shall see that the other apparent differences between the two statements almost disappear.

The important points for us to note at this stage are: firstly, that the need for more agreement concerning the aim of education is becoming increasingly recognised; and secondly, that the statements of this aim made from time to time by public men differ widely from one another.

But the most astonishing aspect of the aimlessness of English education is not that would-be reformers differently define the objects of their efforts. Rather is it that the authorities responsible for

* This was written in April, 1916.

$\uparrow$ Sir William Ramsay, K.C.B., F.R.S., in the Daily Dispatch of 21 st January I915.

$\ddagger$ Times, 17th January, 1915. 
spending more than thirty million pounds sterling of public money every year on education in England and Wales have given so little indication of the aim they have in view, whether for English education as a whole, or for that large part of it which they are able to control. Even the teachers, on whose hearty and intelligent cooperation the achieving of this aim depends, are no better informed than other people what the aim may be! Parliament has, wisely enough no doubt, abstained from putting any definition of education on the statute book. And in vain do we search official literature for guidance. It is true that the introduction to the Public Elementary School Code tells us that 'the purpose of the Public Elementary School is to form and strengthen the character...'; but we are not told what is here to be understood by 'character,' a word that means different things to different people and to most people perhaps conveys no clear meaning at all; nor are we told in which direction the character is to be trained. To these questions we shall return later*.

Meanwhile, if we are right in describing aimlessness as characteristic of the education of the English nation to-day, defects in our national system of education cannot be remedied until our legislators, administrators and educators are sufficiently agreed upon the aim of the whole to enable them to test particular proposals for reform by asking whether, and to what extent, each suggested change of means would help to secure the end in view. Every one agrees that such defects exist and are serious. It is only concerning the nature of the defects and the appropriate remedies that people differ.

The first business of all who are interested in education reform should therefore be to make up their minds, in the light of the available evidence, what the aim of education ought to be, being ready to modify the aim on which they have fixed when further evidence is forthcoming.

Professor Adams has enumerated some fifteen statements of the aim of education and concludes that 'there are two...that stand out from the others as embodying all the essentials, and as between them covering the whole field.... The first is self-realisation, the second many-

* In the absence of any general concensus of opinion concerning the aim of education, in the absence even of any esoteric language in which to express such an opinion without equivocation or risk of misunderstanding, most men fall back on metaphor. According to Professor Adams (loc.cit. p. 285), no branch of study is perhaps so metaphor-ridden as education and psychology. That is why we began in our first chapter by condemning the misuse of metaphor. The most popular metaphor to-day is that of the 'broad foundation of liberal education,' which is sometimes combined with Pestalozzi's favourite plant metaphor so as to produce the mixed metaphor of the 'broad foundation of general culture.' See Appendix A. 
sided interest. These have been frequently treated as antagonistic ideals, and each has its enthusiastic supporters. But...far from opposing each other they are really complementary. Neither can be attained apart from the other.'*

The aim of Herbart and his followers was to build up in each educand a many-sided interest. In order to emphasise its essential unity and to avoid asking how many sides the interest is to have, we shall describe it as a single wide interest. According to the Herbartian theory, education can modify the natural development of the educand: indeed the core of Herbart's teaching is that instruction supplies the only force that can modify character $\dagger$. By building up different interests in different individuals, the educator is thus able to produce men who shall be fitted to serve each other and the community in a variety of different occupations.

Those who advocate self-realisation as the aim of education generally maintain that the educand should be protected as long as possible from a cramping environment so that he may experience a free and full development, which is self-realisation. From Froebel's point of view, the educator's function is not to produce men to serve the state in this, that or the other capacity; but is rather to watch over and protect while the educand freely becomes what his own nature makes him. It is true that, as the educand grows older, he should gradually become his own chief educator. In the university, at least, he should be allowed considerable freedom to choose his own courses. Even in the university, however, he will do well to receive advice from his tutor or director of studies. And the master or mistress in a secondary school not only must in practice, but also should in theory, prescribe the greater part of the pupil's studies. Rousseau himself, after giving Emile a tutor all to himself (and so avoiding the administrative difficulty in the way of allowing each pupil in a school to select his own curriculum) made the tutor suggest the questions which Emile should ask. He thus interfered with Emile's natural development so as to make him become a particular type of man: in fact an eighteenth century dandy $\ddagger$.

Some active interference with the natural development of the educand is, indeed, demanded from every educator. Too much freedom in early youth leads to the formation of bad habits which leave the grown man anything but free. No man can realise his best self through selfishness. Whoever aims first at saving his life, loses it. To achieve a complete self-realisation it is necessary to aim, in the

\footnotetext{
* Loc. cit. pp. 39, 40. † Adams, loc. cit. p. 326. $\quad \ddagger$ Adams, loc. cit. p. 178 .
} 
first instance, at becoming fit for the service of others*. If then we assume that (except in the case of quite young children, under say twelve years of age, whose interests are normally multifarious $\dagger$ ) the two aims, self-realisation and single wide interest, between them cover the whole field, it follows that, so far as the facts we have yet considered enable us to determine it, the first aim of education during adolescence and maturity must be to build up a single wide interest.

In Book II we shall reconsider, explain and confirm this provisional conclusion in the light of further facts.

* Cf. the Master of Marlborough on character-building in the public schools: 'In season and out of season we teach that the liberty we largely grant to our boys is to be used in the service of the common weal: that a boy must live for his society.' (Times, 5 th June, I9r 5. .)

$\dagger$ Cf. W. James' remark that 'Childhood...has few organised interests.' Principles of Psychology, Vol. I, p. 4I 7 



\section{BOOK II}

\section{THE AIM OF EDUCATION}





\section{CHAPTER 4}

\section{EDUCATION AND NEUROLOGY}

OUR summary of the account given by Professor Adams of The Evolution of Educational Theory has been little concerned with the new science of Physiological Psychology. And in truth the work of the physiologists and anatomists has hitherto but little affected educational theory. Neurology is seldom discussed at educational conferences or at the meetings of associations of teachers. Neurograms and synapses do not figure in educational literature.

But, as we have indicated in our first chapter, we do not propose thus to restrict our discussion. Two reasons for departing from the practice of most writers on education we have already given*. We may now add a third reason, namely that 'many recent advances in psychology are directly due to physiological observations' $\dagger$; in fact, as Dr McDougall adds, 'our mental life is intimately bound up with, and to a great extent conditioned by, the processes of the nervous system, and therefore cannot be understood without the aid of knowledge of those processes....and it may be confidently predicted that psychology will cease to be regarded as a purely academic study, and will be recognised as providing the only sound theoretical basis for the art of the teacher...in proportion as it becomes a truly physiological psychology.' $\ddagger$

We shall therefore employ some of the conceptions of physiological psychology in the following attempt to analyse the foundations of character and their effect upon behaviour.

To every thought which passes through the mind, to every feeling on the fringe of consciousness, and to every reflex movement of which we are unconscious, there corresponds a 'nervous impulse' travelling swiftly along a series of nerve elements and comparatively slowly traversing the junctions between the consecutive elements in the series; and each different thought or feeling or movement involves a different series of nerve elements-different, at least, as regards some of its units.

* See above, pp. $8,9$.

$\dagger$ W. McDougall, Physiological Psychology, p. I2.

$\$$ Loc. cit. p. I2. 
These nerve elements*, or neurones, consist for the most part of relatively long axis cylinders projecting from a central nucleus or cell-body and, after giving off collaterals at various points, subdivided into a number of branches at their further extremities. The cell-bodies also have other projections or processes consisting of many fibres that are called 'dendrons' on account of their tree-like formation $\dagger$. Each neurone is separately nourished by its own cell-body; it is seldom if ever continuous with its neighbours; but, on the other hand, it is no more independent of them than a human individual is independent of the society in which he lives: if his neighbours degenerate and die, he is likely to do the same. While each neurone is thus a separate trophic unit, it is not a separate functional unit; for the functional unit of the nervous system is a 'nervous arc': a series of two or more neurones placed end on to one another and capable of conducting a 'nervous impulse' from one to the other across the junction, or synapse, between them. The neurones, bound together and supported by connective tissue and finely branching neuroglia cells, constitute the nervous system.

The nature of the nervous impulse which it is the function of the neurones to conduct is still uncertain. It probably consists in a progressive 'partial decomposition of some very complex and unstable molecules' $\ddagger$ of which the neurones are composed. The chemical activity stimulated in a neurone by the incoming impulse we shall call the 'activity' of the neurone. The activity is to be distinguished

* This chapter's brief account of some of the principal features of the nervous system will, it is hoped, enable the general reader sufficiently to understand the physiological terms - 'the materialistic formulae and symbols'-which we shall employ. But every reader who has not made a special study of the nervous system would do well to acquire at least so much information as is given in Dr McDougall's attractive primer of Physiological Psychology (published in 1905 by J. M. Dent $\&$ Co.), and to have at hand some general description and figures of the gross anatomy of the nervous system, such as are included in many text-books of anatomy and physiology.

$\dagger$ Cf. W. McDougall, 'The neurones are of various shapes and sizes, but each one consists of a nucleus, which with a certain amount of protoplasm surrounding it constitutes the cell-body, and one or more delicate protoplasmic processes [or projections] continuous with the cell-body. In many neurones one of these processes is much longer than the rest and is called the axis-cylinder process or axon. All the nerve-fibres of the peripheral nerves are axons of neurones. The axon, in most cases, has for its protection a delicate sheath of fatty matter known as the medulla, and in many cases it is of great length, those, for example, which run from the spinal cord to the ends of the toes of a tall man are several feet in length. Some axons give off at short intervals a number of branches or collatevals, which connect them to numerous other neurones. The other processes of the body of the neurone are generally shorter and much branched; they are known as dendrons because in many cases a single large process of this kind divides, like the stem of a tree, into many branches ending in a great number of twigs.' (Loc. cit. pp. 24, 25.)

$\ddagger$ McDougall, loc. cit. p. 28. 
both from incoming impulses and from outgoing impulses. Of the latter some may be due to the activity of the neurone itself and some to incoming impulses conducted through the neurone with or without loss en route. The 'activity' of a nervous arc is the sum of the activities of the neurones that compose the arc. An arc, or a system of arcs, that is being traversed by a nervous impulse we shall speak of as 'excited.'* The 'intensity of the excitement' of-or the 'flow' through-an arc are terms we shall use to denote the measure of the impulses traversing the arc in unit time.

In many cases the passage of a nervous impulse is accompanied by a current of negative electricity travelling along the neurone in the same direction as the impulse, and there appears no reason to doubt that all nervous impulses are similarly associated with the transfer of negative electricity. If this supposition is well founded, it follows that to every thought that flits across the mind there corresponds not only a nervous impulse traversing the neurones of the brain, but also an electro-magnetic wave which is started as the electric current rises and falls and which gradually diffuses itself throughout all space. It is conceivable that such a wave, traversing the brain of another individual, might give rise to nervous impulses there and so affect that other individual's consciousness.

The nervous impulse normally originates in the stimulation of a sense-organ and is normally discharged in producing movement. The normal path of the impulse is thus from a sense-organ to a muscle. In the ideally simple case we may imagine that only two neurones are concerned: one sensory or 'afferent,' the other motor or 'efferent'; and these two together form a 'sensori-motor arc,' the functional unit of the nervous system. 'In the higher animals and in man we must distinguish two systems of muscles and two corresponding systems of sense-organs. The one system of muscles is attached for the most part to the bones of the skeleton, and the contractions of the muscles of this skeletal system produce all those movements of the limbs, trunk, head, and organs of speech by means of which relations with the outer world are maintained. In man these movements are under the control of the will.' $\dagger \ldots$ The muscles of the other system are embedded in the viscera, or internal organs of the body, and cannot for the most part be controlled by the will. The corresponding sense-organs of the visceral system are also embedded in the viscera and are stimulated

* An arc or a system of arcs which is 'excited' will therefore also be active. But, as we shall see, it may continue to be active after it has ceased to be excited, i.e. after impulses have ceased to traverse it and to pass on. See below, p. 66.

$\dagger$ McDougall, loc. cit. p. I6. 
by movements, changes of pressure, and chemical changes occurring in their neighbourhood. The nervous impulse caused by the stimulation of one of these sense-organs traverses a sensory neurone of the visceral system, crosses one or more synapses, and finally travels back by a motor neurone of the same system until it reaches the visceral muscle, the consequent movement of which adjusts the viscus to meet the changes which gave rise to the nervous impulse. In this way are brought about the involuntary movements of the heart, stomach, and other viscera. It was formerly supposed that this system of nerves was quite separate from, and independent of, the other larger system. But we know now that 'the two systems of sensori-motor arcs, the skeletal or voluntary and the visceral or involuntary, are intimately connected. The skeletal muscles are controlled not only by impulses from sense-organs on the surface of the body, but also by impulses initiated in sense-organs that are embedded in the muscles themselves and in the surrounding tissues, the sheaths of the muscles and their tendons and the joint-surfaces of the bones; these sense-organs, which are known as the organs of the "muscular [or kinaesthetic] sense," are stimulated by the contractions of the muscles and the movements of the parts resulting from those contractions. The nerves from these sense-organs join the sensori-motor arcs that connect the skeletal muscles with the sense-organs of the surface, and so cooperate with them in determining the sequence and the force of the contractions of these muscles, which are thus under a double sensory control as well as the control of the will. These are the functional units which with their complicated interconnexions constitute the nervous system. They are represented schematically in the diagram.'*

The sensory or afferent nerves of the skeletal system, including those of the kinaesthetic sense as well as those conveying impulses from the sense-organs of the body's surface, communicate with the motor or efferent nerves of the skeletal system only in the spinal cord and in the brain. Many of the sensory neurones, after entering a segment of the cord and giving off collaterals which communicate immediately-in the same segment--with motor nerves leaving the cord from that segment (and which thus provide the paths used in the case of spinal reflex movements $\dagger$ ), are continued up the cord giving

* Loc.cit. pp. 16, 17. The diagram (Fig. I on p. 31 ) is reproduced with modifications from p. I 7 of Dr McDougall's work.

$\dagger$ Sometimes the impulse arising from a stimulus applied to a sense-organ of the surface of the body or of the muscular sense, will, on reaching the spinal cord, give rise to an impulse in the efferent motor neurone alone. No impulse 
off further collaterals in various segments and finally communicating in the upper part of the cord with other long neurones which prolong the afferent or sensory path until it reaches the cerebrum* or great

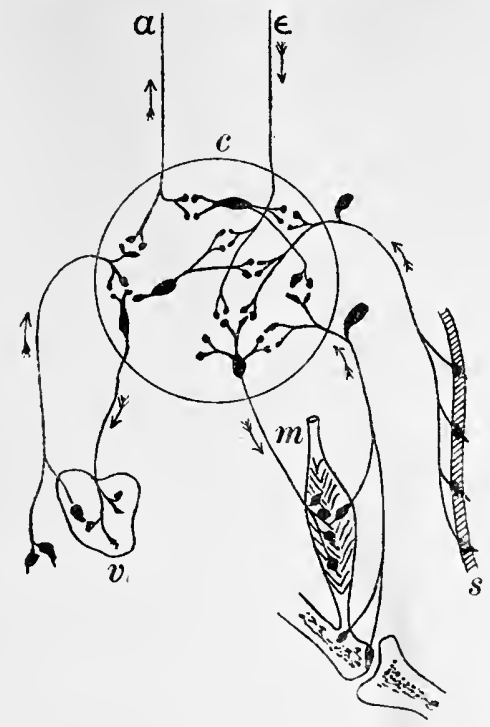

Fig. I. Diagram illustrating the functional units, the skeletal and visceral sensorimotor arcs of each segment of the nervous system of a vertebrate animal: $c$, the central nervous system; $m$, a skeletal muscle attached to the bone; $s$, the skin covering it; $v$, a muscular viscus. On the right is shewn a skeletal arc with double afferent path from sense-organs of the skin and from sense-organs of the 'muscular sense' in tendon, joint-surface, and muscle; on the left an arc of the visceral system with afferent path from sense-organs in and about the viscus.

[Descending (efferent) fibres, $\epsilon$, connect the brain through synapses in the spinal cord, $c$, with the other neurones represented in the diagram and enable skeletal movements to be produced not only reflexly from below, but also voluntarily from above. Ascending (afferent) fibres, $a$, also connecting with the other neurones through synapses in the spinal cord, $c$, enable consciousness to be affected by skeletal movements whether of voluntary or reflex origin. There are also other ascending (afferent) fibres linking the brain with sense-organs in various parts of the body and especially upon its surface. The long neurones, $\epsilon$ and $a$, running lengthwise in the spinal cord are not represented in Dr McDougall's original diagram.]

will then travel up the spinal cord. In such cases the resulting movement is described as a spinal reflex movement. In the case of dogs it is possible to sever all the afferent and efferent paths that connect the brain with the remainder of the central nervous system, and yet to obtain these spinal reflex movements. Examples are quoted from Professor Sherrington on p. 35.

* Until recently it was generally believed that every sensation was accompanied by activity in certain sensory regions of the cortex or outer layer of the cerebrum. But the researches of Dr Henry Head and Dr Gordon Holmes, beginning with those described in the Croonian Lectures of I9I I (see Brain, Vol. 34, Nov. I9II, pp. Io2 et seq.), have shewn that two bodies known as the optic thalami and 
brain on the side of the body opposite to that in which the impulse originated by the stimulation of a sense-organ. The sense-organs of all parts of one side of the body are thus connected with the cerebrum on the opposite side*. Not until it reaches the cerebrum does a nervous impulse originating in a physical stimulus to a sense-organ give rise to sensation. But sensations do not always result from a physical stimulus acting on the peripheral nervous system even though the stimulus may be adequate in quantity and quality to produce sensation under more favourable conditions. Indeed, as we have just seen, 'many afferent impulses remain on the physiological level and never form the basis of a sensation; they are destined to control reflex activity or to coordinate movements of the body and limbs.' $\uparrow$

We have said that the sense-organs of all parts of one side of the body are connected with the cerebrum on the opposite side $\neq$. The nerves of the special senses-for example, sight, hearing or smellare also connected with the opposite side of the cerebral cortex $\S$. In this way the sensory neurones of all parts of the body are connected with the cerebrum, and those of each of the principal senses-tactile, kinaesthetic, visual, auditory, olfactory, etc.-are connected with a certain 'sensory area' of the cortex or outer layer of the cerebrum.

When the fibres, by which the various sensory paths are thus continued to the cerebrum, enter the layer of grey matter which constitutes the cortex, we can no longer trace their connexions anatomically in the dense tangle of neurones which are the principal constituents of this grey matter. But we know that from these sensory areas fibres pass down to join the various motor neurones which convey the nervous impulses to the skeletal or 'voluntary' muscles. Thus in the kinaesthetic or 'Rolandic' area are the cellbodies of large neurones, known from their shape as the 'pyramidal'

situated one on either side of the base of the cerebrum are not merely intermediate receiving stations or junctions between successive neurones in sensory paths from the surface of the body to the cortex of the cerebrum, but are themselves the seats of certain primitive elements in sensation. 'Thus, reactions of the human optic thalamus, freed from the restricting influence of the cortex, are an almost perfect expression of the non-discriminative aspects of sensation.' (Henry Head, on 'Sensation and the Cerebral Cortex,' Brain, Vol. 4I, Sept. I9I8, p. 200.) In particular those components of sensation which underlie feeling-tone tend to excite thalamic but not cortical centres. (See Head and Holmes, Brain, Vol. 34, p. I90.)

* This is not strictly true of the eyes, where there is partial crossing only. For particulars of the bilateral representation of each retina in the brain, see any treatise on Physiology or Anatomy. Cf. the diagram given by Dr Purves Stewart in The Diagnosis of Nervous Diseases, $4^{\text {th }}$ edition, p. 46.

$\dagger$ Henry Head, Brain, Vol. 4I (I918), p. 201.

$\ddagger$ But see the last footnote but one. $\S$ See the first two footnotes above. 
cells, whose axons, forming the 'pyramidal tract,' pass down to connect directly with the motor neurones whose cell-bodies lie in the various segments of the spinal cord and whose axons thence conduct impulses to the skeletal muscles of the trunk and limbs. And from physiological data we can conclude that the afferent neurones, which enter each of the sensory areas, are connected by shorter or longer chains of small neurones in the grey matter of the area with the efferent neurones of the same area; we are able to infer also that in the main the afferent neurones of any one sensory field or region of the body are most intimately connected, in the sensory areas of the cortex, with the efferent neurones that make connexions with the motor systems of the same region, the motor systems that have the most intimate functional relations to that sensory field. Similar connexions between the afferent neurones of any one sensory field and the motor neurones of that field are made, as we have seen, in that segment of the spinal cord to which enter and from which emerge the sensory and motor nerves of the field in question. But we may assume that the connexions effected by means of loop lines (or, as Dr McDougall calls them, 'arcs of the intermediate level') that connect, through a sensory area of the cerebrum, the sensory and motor neurones of the same field-neurones that are already connected in the corresponding segment of the spinal cord-are far more complex than those of the spinal level and so render possible a far more complicated series of coordinated movements*.

A still higher degree of coordination, adapted to still more complex situations, is effected by means of the neurones of the large cerebral 'association areas' which surround various sensory areas of the cerebrum and connect them with one another. Series of these neurones form arcs among which we may distinguish two principal kinds.

In the first place there are those which connect the sensori-motor arcs of the Rolandic or kinaesthetic area with sensori-motor arcs in the other sensory areas of the cortex. They converge upon the Rolandic area from all the other sensory areas, and the impulses that they bring find, in the large pyramidal neurones already mentioned $\dagger$, their efferent paths to the motor neurones of the skeletal muscles. Hence this system of pyramidal neurones with the cortical paths converging upon it from all parts of the cortex has been aptly likened by Professor William James $\ddagger$ to a funnel, the mouth and conical body

* This account of the sensori-motor arcs of the intermediate level is abridged from that given by $\mathrm{Dr}$ McDougall, pp. 43-45.
t P. 32 .
$\ddagger$ Loc. cit. Vol. II, p. $58 \mathrm{r}$.

G. E. 
of the funnel being represented by the converging cortical paths, its neck by the Rolandic cortex, and its stem, through which the outflow takes place, by the neurones of the pyramidal tract.

A second important class of association-area or 'higher level' arcs consists of arcs that connect with one another the various sensory areas, other than the kinaesthetic area, of each of the two hemispheres, formed, one on the right side and one on the left, by the cortex and adjoining parts of the cerebrum. Moreover, transversely running fibres connect each part of one hemisphere with the corresponding part, as well as with other parts, of the other hemisphere.

The superior function of the arcs of these association areas is indicated by the fact, clearly demonstrated by Professor Flechsig, that the neurones of which they are composed attain structural perfection in each individual at a much later age than those of other parts of the nervous system. While the latter are fully developed at birth, most of the former are not perfected until a much later age, some not until adult life is reached*.

A clearer idea of the functions of the nervous arcs of the different levels-spinal, intermediate and higher-may be obtained by considering a simple case of the conduction of a nervous impulse from its origin in a sense-organ to its discharge in the production of movement. I imagine myself walking barefoot by the sea shore. Suppose, further, that I am, at the same time, engaged in an interesting conversation with a friend. Not noticing where I tread, I step-with my right foot, let us say-on a sharp pebble. Immediately and involuntarily my right knee and hip joints are bent so as to lift the injured foot, while at the same time my left leg is more rigidly extended to take the extra weight thus thrown upon it.

If the hurt is slight, and if my conversation is sufficiently engrossing, this coordinated series of movements may take place without attracting my attention and without the intervention of my will. In that case the impulses, which the sharp stone has originated in the sensory nerve endings in the sole of my right foot, travel up the sensory neurones at the rate of several yards a second. Where these neurones enter a segment $\uparrow$ of the spinal cord in the lumbar region they give off collaterals or branches which connect, through small neurones in the grey matter of the segment, with motor neurones leading to the muscles of both legs. Parts of the impulses probably

* This account of the higher level arcs is slightly abridged from that given by Dr McDougall, loc. cit. pp. 90, 9I.

$\dagger$ This segment is represented schematically by the circle in the diagram on p. $3 \mathrm{r}$. 
leave the sensory neurones by way of those collaterals; and, after crossing the synapse or synapses in the grey matter of the segment, leave it by way of motor neurones whose roots lie in the same segment, and whose function it is to conduct the impulse to the flexor muscles of the right leg and the extensor muscles of the left*.

On reaching the muscles, the nervous impulse gives rise to chemical changes which result in the contractions of the muscles and the motion of the limbs. These movements stimulate kinaesthetic sense-organs from which impulses return to the same segment of the cord. As in the case of the impulses from the sense-organs of the sole of my foot, parts of these impulses probably pass out from the cord immediately along the motor neurones, thus serving to maintain and regulate the contractions of the extensor and flexor muscles in question.

Experiments, conducted by Professor C. S. Sherrington, upon 'spinal' dogs-dogs the neural connexions of whose brains with their spinal cords had been severed-prove that in these 'spinal' animals the stimulation of sense-organs on the sole of one hind foot will result in the withdrawal $\dagger$ of that foot and the extension $\ddagger$ of the other hind leg. In such a case we have to suppose that the whole of the impulses arising both from the original stimulus and afterwards from the kinaesthetic sense-organs are discharged directly through the spinal nerves; for they cannot traverse the higher portion of the afferent (sensory) paths which lead to the brain. We have then an example of a simple spinal reflex, in which the brain takes no part. And more complicated spinal reflexes, involving several segments of the cord, may also be evolved in spinal animals; for if a chemical irritant be applied to the flank of such an animal the hind foot of the same side will make scratching movements§ which are almost as well aimed as if the animal were entire; and if that foot be held, then the other hind leg will make similar movements.

But it is probable that in man even so simple an automatic movement as the withdrawal of one leg and the extension of the other cannot take place without affecting the 'fringe of consciousness'the vague background of thought. We must therefore suppose that,

* The impulse which, crossing a synapse and entering another neurone, acts upon it as a stimulus will excite in it katabolic changes. The intensity of the resulting impulse in the second neurone may considerably exceed that of the impulse which reached it from outside. Cf. Dr McDougall, loc. cit. p. 3o. Cf. also Dr Keith Lucas who found that in every case which he examined 'the intensity of the impulse set up in a nerve is independent of the strength of the stimulus which evokes it.' (The Conduction of Nervous Impulse, I917, p. 92.)

t The Integrative Action of the Nervous System, p. Io.

Loc. cit. p. 30.

§ Sherrington, loc. cit. p. Io. 
in the case we have imagined, part of the impulses from the sole of my right foot (and from the kinaesthetic sense-organs so soon as they have been stimulated by the beginning of movement) pursue afferent paths as far as the cortex of the cerebrum and thence return, by the neurones of the pyramidal tract, to be discharged along the motor neurones which produce the movements we have described. They thus traverse a long loop through sensory regions of the brain-in the thalamus as well as in the cortex-where their passage may, as we have seen, make me conscious of pain in my foot and of the movements of my limbs; and they probably also traverse a shorter circuit wholly on the spinal level. In fact the spinal reflex of the spinal dog becomes, in man, a 'sensation reflex' affecting consciousness and under voluntary control.

There is much evidence to shew that nervous impulses commonly affect consciousness as they pass through the sensory areas of the brain: for example, if part of one of the sensory areas of the cortex is damaged or removed then the corresponding sensations-visual, auditory, olfactory, or the like-can neither be experienced nor imagined*. And there is no evidence that any psycho-physical process takes place as impulses traverse either arcs of the spinal level or the 'higher level' arcs of the cortex which lie outside the sensory areas $\dagger$. In fact, so far as we yet know, all sensation is accompanied by activity in the neurones either of the optic thalami or of one of the sensory areas $\ddagger$ of the cortex.

The further effects of the long loop through the cerebral cortex, and of all the complex connexions which can there be made between various sensori-motor arcs, may be indicated by supposing that the injury to my foot was somewhat sharper than in the case we have just considered. Unless my attention was very much occupied I may have become conscious, not only (as in the case already considered)

* Cf. McDougall: 'So long as any sensory area is intact the corresponding qualities of sensation may be experienced, and even though the sense-organ be destroyed or the sensory nerves severed the sensations may still be experienced in the form of hallucinations; and, on the other hand, if one of the sensory areas is destroyed by disease or a wound, the corresponding sensations are never experienced again though the sense-organ and the sensory nerves remain intact, and their disappearance from the consciousness of the individual is so complete that he is not even aware of the nature of his loss, it is with him as though he had never experienced this kind of sensation.' (Loc. cit. p. 55.) The converse fact that ideas (images) in the mind are accompanied by nervous impulses in the sensory areas of the brain is illustrated by Pawlow's experiments described below (p. 47).

$\dagger$ Higher level arcs may perhaps occur in sensory areas as well as in association areas of the cortex. It will therefore be convenient to describe those cortical arcs the excitement of which affects consciousness as sensory cortical arcs rather than as sensory area arcs. See p. 66 below.

$\ddagger$ See footnote on pp. 31, 32 above. 
of the hurt and of the quick withdrawal of my foot, but also of the fact that I had involuntarily stooped down and clasped the sore toe with both hands, thus easing the pain. In such a case the nervous impulse conveyed by the sensory neurones to the spinal cord is more intense than before. Not only is it able to overcome the resistance of the synapses which lie along the low-resistance path to the muscles that produce the usual reflex already described, but it also breaks down the resistance of synapses by which the afferent path from the sole of the foot (and from the kinaesthetic sense-organs stimulated by the usual reflex) is connected with efferent paths to muscles of the trunk, arms and hands; whereupon, as before, impulses from the kinaesthetic sense-organs in these moving parts not only reinforce the (motor) impulses that originated the movements, but also, on reaching the cortex, make me conscious of these further movements. The synapses of higher resistance to which reference has just been made lie for the most part in the same sensory area of the cortex, but-as in the case of the scratch reflex of the spinal dog-some may lie in the segments of the cord from which issue the motor neurones to the muscles that bend the back and bring up the hands to grasp the injured foot. The conception of series of neurones congenitally linked together by synapses of various degrees of resistance, so as to form 'functional systems of nervous arcs,' is of great importance in our subject.

Once more suppose that, instead of merely causing me a moment's pain and giving rise to a series of involuntary if quite conscious movements, the injury to my foot is sufficiently serious to require treatment. This time I do not let go of my foot, put it to the ground, and resume my walk and conversation almost without interruption. The wound now receives my full attention. I turn the foot over with the help of my hands so as the better to see what harm has been done, I examine it carefully, and perhaps I tie it up so as to keep the wound clean until $I$ reach home. My action is no longer brought about as a mere sensation-reflex, such that the impulses do not traverse any region of the cortex beyond that to which they were brought and from which they returned. On the contrary, the nervous impulses arriving from the sense-organs of the surface and from those of the kinaesthetic sense are this time strong enough to overcome the resistance of the synapses in certain higher level arcs. They therefore spread to other sensory areas of the cortex. Thus they will probably spread to the visual area; and, just as I previously experienced tactile and kinaesthetic sensations when the nervous impulses traversed arcs in the 
corresponding sensory areas, so now, as the impulse begins to traverse a certain system of arcs in the visual area, I experience a 'visual image' of my foot-or, as is commonly said, I see my foot in imagination. This visual image of my foot differs from a visual sensation psychologically in that it appears less vivid, less intense; physiologically the difference probably is that while, in the case of the sensation, the nervous impulse enters the visual area of the cortex by way of an afferent path from the sense-organs of the retina, in the case of the image it is a higher level path which conveys the impulse to the same system of arcs in the visual area*. From the system of arcs the excitement of which gives rise to an image of my foot there are probably many paths in the visual area by which the impulse might proceed, producing as it did so a train of visual imagery. The conditions under which such thinking takes place we are to study shortly. Meanwhile we may take it that, in the case we are now considering, the impulse leaves the visual cortex by a certain low resistance system of arcs: that namely which leads the impulse to cause the focussing of the eyes upon the 'imagined' object-the injured part of the foot. As the sense-organs of the retina are now stimulated by light from the foot, impulses from the retina reach the visual area and the visual image gives place to a visual sensation of the foot and its injury. Now that visual sensations have been added to tactile, the injury may be said to be 'perceived'; for 'perception seems to involve in every case a synthesis of sensations and images of different senses.' $\dagger$ And now at last the process, which up to this point may have remained involuntary, is almost sure to be controlled by my will. The questions how the will's control is exercised and what is the physiological accompaniment of the exercise of the will are to be discussed later. Suffice it here to say that I concentrate my attention upon the wound, probably producing voluntary contraction of the eye muscles so as to improve the focus, and that these contractions stimulate sensory nerve endings from which further impulses pass to the cortex and incrcase the excitement already there. The result is that the impulse can now spread through synapses of still higher resistance. It may, for example, spread to the auditory area and, as it traverses that area, awaken auditory images of lectures on first aid or of advice given to me on some previous occasion. If that advice was to tie up such a wound until it could be properly cleansed, the memory of the advice

* Cf. McDougall, loc. cit. p. 85.

$\dagger$ McDougall, loc. cit. p. 94. It should be added, however, that McDougall's use of the word 'perception' is not universally followed. 
remains in the form of a system of nervous arcs of permanently-at least so long as the memory survives-lowered resistance. Along such a path the impulse passes to the Rolandic area and is there discharged through the pyramidal tract as I begin the train of movements which comprise the tying up of the wound.

All these sensations and images, which we have described in a very much simplified form, are not in the focus of consciousness at the same moment. They follow one another in an orderly sequence. And, as will shortly appear, there is reason to suppose that the nervous impulse meanwhile traverses one after the other, in the sensory areas of the brain, the various systems of arcs the excitements of which correspond respectively to the consecutive states of consciousness.

In the above example of some functions of the nervous system, we have spoken of the different resistances offered by different nervous arcs to the passage of the impulse; and we have assurned that the resistance of an arc-or of a system of arcs-is mainly duc to the synapses which it includes. 'There is,' writes T. Brailsford Robertson*, summarising the conclusions which he draws from some recent experiments, "either in the central nervous system or in the peripheral neuro-muscular system, a resistance analogous to friction, requiring a definite force to overcome it and permit a reaction, just as a heavy body, resting upon a rough inclinerl plane, does not begin to move until the inclination of the plane attains a certain value.'

According to Dr McDougall, 'al though recent rescarches secm to shew that there is protoplasmic continuity in certain cases' of junctions between neighbouring neurones, 'we may confidently believe that in the great majority of synapses there is no simple continuity of substance, but a breach of continuity, or a difference: in the nature of the nervous substance, which prevents the passage of the nervous impulse in the form in which it travels along the nerve-fibres, and renders that passage more difficult. This peculiarity of the synapses may conveniently be expressed by saying that each synapse presents a certain resistance to the passage of the impulse.' $\uparrow$... 'A simple kind of synapse is formed by the division of the end of an axon, or of one of its collaterals, into a number of fine twigs that surround the cell brody of another neurone and terminate in tiny knotss lying close to, or perhaps in contact with, that cell body. Others are formed by the minute terminal branches of an axon or a collateral becoming intertwined with the branches of a dendron of another neurene or, in some

* Folia Neurs-Hislogica, Bd. v1, Nors. 7, 8, Sept.-oct. 1912.

$\dagger$ Loc. cit. p. 28. 
cases, perhaps, with similar fine terminal twigs of the axons or collaterals of another cell. In many cases the terminal twigs of several axons surround one cell body or are intertwined with the dendrons of a single neurone.'* We have thus laid stress upon the structure of synapses because of the very important part which they appear to play in determining the paths to be followed by nervous impulses and thus in governing bodily movements and even psycho-physical processes. For, as we have seen, the passage of a nervous impulse along some particular system of nervous arcs in one of the sensory areasfor example the visual area-of the cortex is a necessary condition of experiencing not only visual sensations but also any kind of visual images.

It is true that most cortical synapses have not been traced by anatomists; and it is true that no direct results of experiment upon the physiology of cortical synapses are as yet available. But 'there is every reason to believe that intrinsically there is no essential difference between physiological dispositions and activities of the lower nervous centres (subcortical ganglia and spinal cord), which condition and determine unconscious behaviour, and those dispositions and activities of the higher centres-the cortex-which condition and determine both conscious and unconscious behaviour.' $\dagger$ Now we know, from experiments upon the synapses of the cord, that 'a feeble stimulus may be just strong enough to excite the sensory neurone, but the excitation may fail to overcome the resistance of the synapse and to spread to the motor neurone and muscle'; and that 'the impulse is delayed at the synapse, a certain time is required for overcoming its resistance, and the delay is briefer...the more intense is the stimulus.' $\ddagger$ It is

* Loc. cit. pp. $27,28$.

$\dagger$ Morton Prince, The Unconscious, p. 230.

\$ McDougall, loc. cit. p. 31, who cites the following further facts which reveal "the resistance presented by the synapse to the passage of the "impulse"'...(4) the application of a continued stimulus, such as a chemical irritant to the sensory neurone results in a rapid succession of impulses in the motor nerve; the energy continuously liberated in the sensory neurone seems to be discharged across the synapse intermittently, just as the electrical energy generated by the friction of an electrical machine is discharged intermittently from pole to pole of the machine causing a rapid series of sparks; (5) the transmission of the impulse in the reverse of the normal or habitual direction, i.e. in the direction from motor to sensory neurone, seems to be very difficult or impossible;... Other characters of the synapses may be inferred from more general considerations; (6) the process of transmission of energy across the synapses is one that readily exhibits fatigue from which recovery is very rapid; the fatigue manifests itself as a temporary increase of resistance;... (7) the synapse is readily affected by changes in the composition of the blood, especially by the presence in it of certain drugs and of waste products of nervous and muscular activity which cause an increase of its resistance; (8) the resistance of the synapse, besides being liable to be increased by fatigue and changes in the composition of the blood, varies from moment to 
clear therefore that the principal cause of the resistance offered by sensori-motor arcs of the spinal level is to be found in the synapse or synapses which such arcs include. Accordingly, it is reasonable to assume* that the resistance offered to the passage of the nervous impulse along cortical systems of arcs is also due to the synapses between consecutive neurones. And we know by introspection that, just as the more we practise the performance of any given action in a certain way the more we tend always to perform it in just that way, so the repeated passage of any given sequence of images through our consciousness makes the sequence tend to recur in the same order. It is therefore reasonable to assume further that the passage of the nervous impulse across any synapse, whether subcortical or cortical, leaves the resistance of that synapse permanently lessened in some degree; and that the more often the impulse crosses the synapse, the lower does the resistance become. This assumption is consistent with all the known facts and is inconsistent with none. Moreover it enables us to resume and predict a large number of facts. It satisfies, therefore, the only conditions required of a 'scientific law' $\dagger$ - which is no more than a hypothesis consistent with experience. We shall therefore make this provisional assumption. It will enable us to 'explain' not only the formation of neural habits but also the association of ideas and even memory itself. For the physiological basis of all these consists in systems of nervous arcs of varying degrees of low resistance.

moment with the state of the neurones between which it forms a junction, being diminished when they are excited and charged with free energy, increasing again when they return to rest; (9) the process of transmission of energy across the synapse leaves its resistance to the passage of the impulse...permanently lowered in some degree.'

* The assumption that the resistance offered to the passage of nervous impulses by nervous arcs is due to the synapses which those arcs contain is not essential to our argument. All that is necessary is to assume that in some way the passage of an impuise along a nervous arc makes it easier for the impulse to traverse the same arc on a subsequent occasion; and we know this assumption to be correct in the case of every arc on which the experiment has been tried. But if, following McDougall, we make the first assumption in the text_-that the resistance is (mainly) due to the synapses-then we must also follow him in making the second-that the resistance of the synapses is reduced by the transmission of impulses. And all the available evidence supports both these assump tions.

$\dagger$ See above, footnote $\ddagger$ on p. 7 . 


\section{CHAPTER 5}

\section{NEUROGRAMS}

\section{$\S$ I. Neurograms or Neural Dispositions.}

WHEN describing reflex movements we said that systems of neurones congenitally linked together by synapses of low resistance would play an important part in our subject*. We may now add that arcs, and systems of arcs, whose low resistance has been brought about by the passage of nervous impulses, will be of hardly less importance in the discussion which follows. The word neurogram has been suggested by Dr Morton Prince $\uparrow$ to describe brain records of life's experience. And we shall find it convenient to use this word, rather than the more usual term 'dispositions,' to denote all low-resistance paths whether among the neurones of the brain or among those of other portions of the nervous system, and to include congenital paths of low resistance as well as those afterwards worn by the impulses which accompany life's experiences $\ddagger$. Our reasons for avoiding, wherever possible, the use of the word 'disposition' in this sense are, firstly, its very different connotation in popular speech, and, secondly, its use in psychological writings to describe what is psychical, as when Dr McDougall speaks of the mind and its dispositions where we should prefer || to speak of the brain and its neurograms.

Examples of neural dispositions, or neurograms, are furnished by the functional systems of nervous arcs one or other of which is excited 9 whenever reflex action takes place. Thus to every simple spinal or sensation reflex there corresponds a neurogram the excitement of which accompanies the reflex act. To every habit also there corresponds a neurogram, or a 'system of neurograms,' as we may more conveniently say if the habit includes in its system a sequence of

* See above, p. 37.

$\dagger$ 'Just as telegram, Marconigram, and phonogram precisely characterize the form in which the physical phenomena which correspond to our (verbally or scripturally expressed) thoughts, are recorded and conserved, so neurogram precisely characterizes my conception of the form in which a system of brain processes corresponding to thoughts and other mental experiences is recorded and conserved.' (Loc. cit. p. I3I.) Neurograms are sometimes called 'engrams.'

$\mp$ Prince uses the word 'neurogram' for acquired neural dispositions only. But we shall find it more convenient to use the same word to describe similar low-resistance systems of arcs, whether congenital or acquired. 'Both instinctive behaviour and acquired behaviour must be conditioned by pathways, and the process in its inner nature must be the same in both.' (Prince, loc. cit. p. 237.)

$\S$ E.g. Psychology, p. 83 .

If I.e. conveys a nervous impulse: see above, p. 27. 
movements which we may want to consider separately. We must here be careful to distinguish the habit itself, a permanent possession of the individual, from the transitory movements to which the habit occasionally gives rise: the neurogram is permanent-or 'canalised'* - and corresponds to the habit, while the passage of impulses through the neurogram corresponds to the habitual movement and takes place only when the movement $\uparrow$ occurs. It should also be clear that the same neurones and systems of neurones may form part of several different neurograms, just as the same movement may occur in the performance of several different reflex or habitual actions: some of the movements of the act (habitual to some people!) of 'dropping a goal' are the same as those of the reflex actions which follow stepping on a hot brick.

The manner in which reflex actions are brought about by congenital neurograms of the spinal and intermediate levels has perhaps been sufficiently illustrated already $\ddagger$. Further examples of habitual movements caused by the excitement of acquired neurograms are afforded by swimming, skating or bicycle riding. When learning to swim or to skate or to ride a bicycle we pay attention to what we are doing. So far as we can, we exercise voluntary control over our movements. But when we are able to swim or skate or ride a bicycle, we perform the requisite movements quite involuntarily and even automatically. The movements proceed in an orderly manner without affecting our consciousness. We may not be able to describe just how we utilise our muscles in order to turn a corner on a bicycle: in fact, we cannot hope to bring back to conscious memory images of movements of which we were unconscious when they took place. Somewhere, however, the record of the movements must be preserved so that they may be reproduced from time to time as occasion arises. And in the conception of a system of neurograms, created as the paths of low resistance were worn (under voluntary control to start with and afterwards by slight involuntary improvements in the movements we first learnt), we have a simple explanation of the facts of 'unconscious memory.'

* See below, p. I 54 .

+ Then only does the impulse traverse the whole neurogram including its motor part; but the sensory part of the neurogram may possibly be excited when the movement does not take place but is only imagined.

$\ddagger$ See above, pp. 34 to 37 .

$\S$ The phrase is used by Dr Morton Prince who adds, 'By repeated experience the neurones become functionally organised in such a way as to acquire and conserve a functional "disposition" to reproduce the movements originally initiated by volition.' (Loc. cit. p. 137.) 
But it is not only of unconscious movements that a record is preserved in the form of neurograms which tend to cause the repetition of the nervous process involved. We have already seen that every state of consciousness is accompanied by the passage of nervous impulses in one or more sensory areas of the brain*. In fact 'it is universally accepted that every mental process is accompanied by a physical process in the brain; that, parallel with every series of thoughts, perceptions, or feelings, there goes a series of physical changes of some kind in the brain neurones. And, conversely, whenever this same series of physical changes occurs the corresponding series of mental processes, that is, of states of consciousness, arises.' $\dagger$ And it is reasonable to suppose that, to different mental processes, there correspond physical processes in different systems of nervous $\operatorname{arcs} \ddagger$. So we may take it that the brain process which corresponds to a particular sensation (or image) and which, we know, involves nervous arcs in certain sensory areas of the brain, consists in the transmission of an impulse by a series of cerebral neurones, which series corresponds to the object of that sensation (or image) and to it alone.

If we describe as the 'depth' or 'intensity' of an acquired neurogram the degree in which the resistance of the nervous arcs composing it has been reduced during the formation of the neurogram in question, we may add that the intensity or depth of the neurogram, the excitement of which corresponds to any particular sensation (or image), depends both upon the number of times that the sensation (or image) has been repeated and upon the intensity of the excitement which accompanied the original sensation (or image) and each repetition of it. We have therefore to recognise that neurograms may possess every gradation of depth or intensity; that some will be so shallow as barely to deserve recognition as neurograms at all; and that the depth of a neurogram-and, still more, of a complex system of neurogranıs-may vary from part to part, as for example when we remember the colour of an object quite easily and clearly but have difficulty in recalling its shape.

Now introspection tells us that, if two sensations or their images are presented to consciousness in quick succession, they are apt to recur in the same succession in future. The first sensation (or image)

* Above, p. 36 . $\dagger$ Morton Prince, loc. cit. p. II 8 .

$\ddagger$ But not necessarily, of course, a wholly different set of neurones: see above, p. 43 .

$\S \mathrm{Cf}$. W. James, who refers to 'the deepest paths' (neurograms). Loc. cit. Vol. II, p. $5^{8} 5$. 
tends to recall the second. As it is usually expressed, they become 'associated' in the mind. If we ask ourselves what this association implies in physiological terms, we notice at once that the necessary and sufficient condition of association must be such that, when the neurogram corresponding to the object of the first sensation (or image) is excited, the impulse shall tend to spread to the second neurogram. And this condition is that the two neurograms should be linked together by a path of low resistance, thus forming a 'system' of two neurograms.

Dr McDougall has considered the question how this linking of the two neurograms is brought about. He points out that the withdrawal of attention from the first sensation (or image) and its transfer to the second must mean that the stream of nervous impulses that was passing through the first neurogram has been diverted to the second; and, after illustrating the manner in which the passage of the impulse through any one system of higher level paths may drain the impulse from every other such system, he gives reasons for supposing that the diversion of the impulse from the first to the second neurogram is effected in this way. The drainage of the impulse from the first neurogram to the second leaves its mark in the shape of a path of permanently lowered resistance connecting the one to the other*.

We must further extend our conception of neurograms. So far we have considered only those neurograms the excitement of which accompanies reflex movements or simple sensations or images. Such neurograms are composed of arcs of the spinal and intermediate levels. We have next to consider neurograms involving arcs of the higher level also.

When we think of a familiar object-any one will do: suppose it is 'Whitehall'-the idea that forms the content of our consciousness is more than a simple sensation or image. Unless we have never seen or heard of Whitehall before, the visual sensation produced by reading the word is followed by the 'meaning' that it has for us. The idea of Whitehall that, at any moment, forms the content of the consciousness of a particular individual includes both a sensation (or image)-in the case before us, the name 'Whitehall'-and the meaning it has for that individual. If he has the mind of a child, the meaning may consist of no more than visual images of the mounted sentries at the Horseguards and auditory images of the roar of the traffic. If he is a historian, the meaning may include images of Charles I and the original White Hall as well as more abstract conceptions of constitu-

* See above, p. 4I, and footnote. 
tional history. If he is a manufacturer of munitions, the master of a workhouse, the: governor of a prison, a director of education, or a company promoter, the meaning of Whitehall will probably centre romnd the: Admiralty, War Office or Ministry of Munitions; the Ministry of Health; the Home Office; the Board of Education; or the Board of Trade as the case: may be. And, if his experience of Government Otfices has been onfortunate, the meaning may include an intense anditory image of the words ' red tape: and, possibly, a regretful vision of the Civil Service in the days of the first Henry, when it consisted of no more than two or three persons who, with a pack horse to carry the ollicial records, accompanied the monarch on his journeys wp and lown the country!

If then the perception produced by reading the word Whitchall maty give: rise: to all the many images which constitute the meaning of the: word, the nenrograms excited by the lirst sensation inust, as we have seen, be linked by paths of low resistance to neurograms to which the impulse spreats as the various associated images arise in consciousnesst. And since the neurograms the excitement of which corresponds to the different visual, auditory or other images must include newones in different sensory areas of the cortex, the conuecting patlis of low resistance must include higher level ares in the association areas of the brain. If now we choose to regard as a single neurogram all that system of ares that may be excited when an idea of Whitchall tills the mind, we must recognise that this neurogram inclucles ares of the higher level. So when I speak of my idea of so-and-so I do not refer merely to some: visual image or 'sign' which may suffice to represent him in most of my thinking about matters with which he is concerned. On the contrary my idea of him includes the imagery by which I represent to myself what. I know of his chanacter as well as my visual images of his appearance, and, perhaps, my anditory images of the somud of his voice. And my neurogram for so-and-so includes not only all the sensory-area arcs that correspoud fo cach of these potential images but also the association-area ares that mite the various sensory-area ares into a single neurogram. livery time I think of so-and-so the impulse traversing this large nemrogram reachess a maximmm in a dilferent part of it: and consefurently the state of my consciousness is correspondingly different,

* Sere aloove, r. 38.

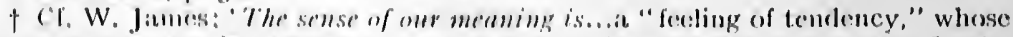

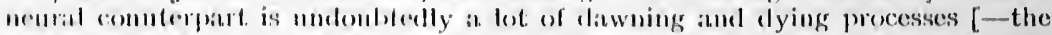
transitery and evanesernt excitement of commected neurograms-] too faint and

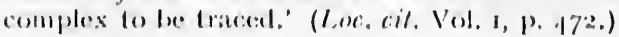


images corresponding to the most exciterl ares of the neurogram being in the focus * of my consciousness and images corresponding to the: less excited parts of the neurogram being more or less on the: fringe. As William James bas it, "no two " ideas" are ever cexactly the same.... A permanently existing" "idea" or "Vorslellun!," which males its appearance before the foollights of cronsciousnes: al periodical interoals, is as mylhological an entily as the Jack of spades.' †...' What is lot twice is the same objecl' + or, as we may now add, the sianc neurograms.

We do woll to rernindl| ourselves again to presserve a clear distinction between the neurogram, a comparatively permanent possession of the nervous systern, and the flecting idea occasioned in the mind by the transitory excitcerrent of the neurogram: a different idea cevery time, as the neurogram is differently excited. 'Nothing but confusion can result from the mixture of "brain-cells" and "irleas". "i An idea. only exists when it is in the mind, and yet a suggestion of permanence is conveyed by the: word 'idea' as used in popular speceh. We can avoid this difficulty by following Dr McDougall and using instearl the phrase 'thought activity.' But, with this warning, we may safely corstinue to employ the: phrase: 'ideaa of an object, as an irclusive term to denote any of the infrnitcly various thesught activitics that result from different distributions of e\%citement in the neareseram of that object-or even in part of the resurogram, provided that the: part is big conough to defire * the; neurogeram (and therefores the: object). A particular joca of a given object thus results from a particular distribution of exciternent in the neasogeam of that objecet.

Having thus extended our conception of neurograms so as to include: these involving, ares of the fighter level, it remains for us tor consider neurograms which also include ares belonging, to the viscoral nervous systern. 'A berutiful illustration' - we guote from Dr Mortern

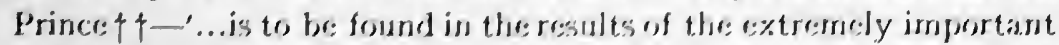
experiments, for psychology as well as physologgy, of Jowlow $4{ }_{4}^{4}$ and

- Sese besisen, $5,6,7$.

\& lose, cit. Vol, 1, j, 23,

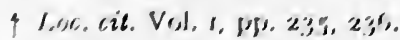

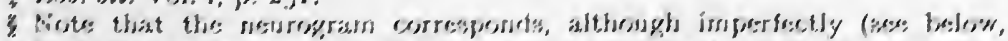

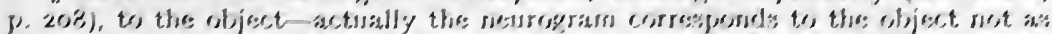

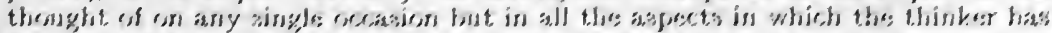

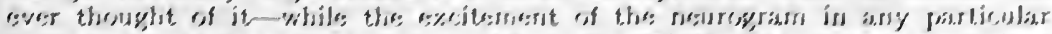

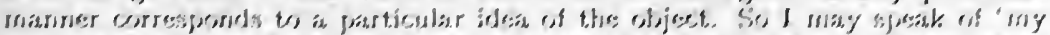

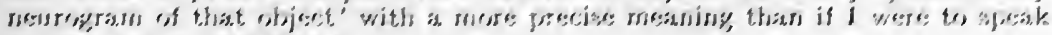
of ' my irleses of that eloject.'

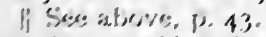

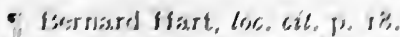

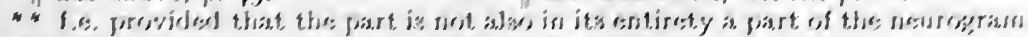
of andether eflijest.

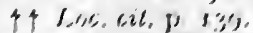

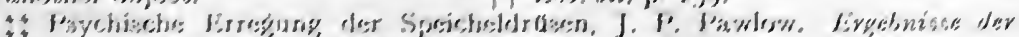

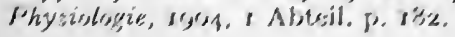


his co-workers in the reflex stimulation of saliva in dogs....It should be explained that it was shewn that the salivary glands are selective in their reaction to stimuli in that they do not respond at all to some (pebbles, snow), but respond to others with a thin watery fluid containing mere traces of mucin or a slimy mucin-holding fluid, according as to whether the stimulating substance is one which the dog rejects, and which therefore must be washed out or diluted (sands, acids, bitter and caustic substances), or is an eatable substance and must as a food bolus be lubricated for the facilitation of its descent. Dryness of the food, too, largely determined the quantity of the saliva.

'Now the experiments of the St Petersburg laboratory brought out another fact which is of particular interest for us and which is thus described by Pawlow. "In the course of our experiments it appeared that all the phenomena of adaptation which we saw in the salivary glands under physiological conditions, such, for instance, as the introduction of the stimulating substances into the buccal cavity, reappeared in exactly the same manner under the influence of psychological conditions-that is to say, when we merely drew the animal's attention to the substances in question. Thus, when we pretended to throw pebbles into the dog's mouth, or to cast in sand, or to pour in something disagreeable, or, finally, when we offered it this or that kind of food, a secretion either immediately appeared or it did not appear, in accordance with the properties of the substance which we had previously seen to regulate the quantity and nature of the juice when physiologically excited to flow. If we pretended to throw in sand a watery saliva escaped from the mucous glands; if food, a slimy saliva. And if the food was dry-for examiple, dry bread-a large quantity of saliva flowed out even when it excited no special interest on the part of the dog. When, on the other hand, a moist food was presented-for example, flesh-much less saliva appeared than in the previous case however eagerly the dog may have desired the food. This latter effect is particularly obvious in the case of the parotid gland".'* *

'It is obvious.' Dr Prince comments, 'that in these experiments, when the experimenter pretended to throw various substances into the dog's mouth, the action was effective in producing the flow of saliva of specific qualities because, through repeated experiences, the pictorial images (or ideas) of the substance had become associated with the specific physiological salivary reaction, and this association had been conserved as a neurogram. Consequently the neurographic residue when stimulated each time by the pretended action of the

* The Work of the Digestive Glands (English translation), p. I52. 
experimenter reproduced reflexly the specific physiological reaction and, so far as the process was one of registration, conservation, and reproduction, it was an act of psycho-physiological memory.

'That this is the correct interpretation of the educational mechanism is made still more evident by other results that were obtained; for it was found that the effective psychical stimulus may be part of wider experiences or a complex of ideas; everything that has been in any way psychologically associated with an object which physiologically excites the saliva reflex may also produce it; the plate which customarily contains the food, the furniture upon which it stands; the person who brings it; even the sound of the voice and the sound of the steps of this person*.

'Indeed, it was found that any sensory stimulus could be educated into one that would induce the flow of saliva, if the stimulus had been previously associated with food which normally excited the flow. "Any ocular stimulus, any desired sound, any odor that might be selected, and the stimulation of any part of the skin, either by mechanical means or by the application of heat or cold, have in our hands never failed to stimulate the salivary glands, although they were all of them at one time supposed to be inefficient for such a purpose. This was accomplished by applying these stimuli simultaneously with the action of the salivary glands, this action having been evolved by the giving of certain kinds of food or by forcing certain substances into the dog's mouth." $\dagger$ It is obvious that reflex excitation thus having been accomplished by the education of the nerve centers to a previously indifferent stimulus the reproduction of the process through this stimulus is, in principle, an act of physiological memory ${ }_{+}^{+}$.

'The experiences of the dogs embraced quite large systems of ideas and sensory stimuli which included the environment of persons and their actions, the furniture, plates, and other objects; and various ocular, auditory, and other sensory stimuli applied arbitrarily to the dogs. All these experiences had been welded into an associative system and conserved as neurograms. Consequently it was only necessary to stimulate again any element in the neurogram to reproduce the whole process, including the specific salivary reaction.' $\S$

* 'Psychische Erregung der Speicheldrüsen,' J. P. Pawlow. Ergebnisse der Physiologie, 1904, I Abteil. p. r82.

$\dagger$ Huxley Lecture, Br. Med. Journ. 6th October, I 906.

$\ddagger$ Dr Prince adds the following footnote: 'Pawlow overlooked in these experiments the possible, if not probable, intermediary of the emotions in producing the effects. The principle, however, would not be affected thereby.'

$\S$ Morton Prince, loc. cit. pp. I39-1 43 .

G. E. 


\section{§ 2. Psychoses and Neuroses.}

These experiments, although undertaken for the purpose of studying the digestive processes only, thus brought to light phenomena which are entirely in accordance with our views of the nature and functions of neurograms. For, since the salivary glands are not subject to voluntary control, the flow of saliva could only have been caused by a nervous impulse reaching the appropriate gland by way of an efferent neurone (of the visceral system). Moreover the action of the gland could not be felt by the dog: it does not affect consciousness. No corresponding mental process can therefore have accompanied the passage of the impulse into the gland. And we cannot conceive that the nervous process began after the mental process had ended, for we know of no cause that could then have started the nervous process. We are therefore compelled to assume-as is, in fact, generally agreed*-that the nervous process had begun before the mental process was over: in short, the mental processes in the case of these experiments, and presumably in every other case also, are accompanied by nervous processes. We have already seen $\dagger$ that certain sensory areas of the cortex are necessary to the experience of certain sensations and images, while certain non-discriminative aspects of sensation are accompanied by reactions of the optic thalami. By assuming that these facts, which have been demonstrated in a limited number of cases only, are universally true, we arrive at the conclusion that the various sensory regions of the brain are the seats of the nervous processes which Pawlow's experiments, among others, compel us to regard as the necessary accompaniment of all mental processes. And, since we know of no nervous process other than the conduction of a nervous impulse along a series of neurones and across the synapses that join them, we cannot but suppose that a nervous impulse traverses the neurones of one or more sensory regions of the brain whenever any mental process occurs. In so doing the impulse must leave its record in the lowered resistance $\ddagger$ of the path it has followed. And this path of lowered resistance is the neurogram-a real physical thing.

In the case of the experiments which shewed, inter alia, that the flow of saliva could be produced by the sound of the steps of the person who habitually brought the food, the nervous impulse, originated by the auditory sense organs and traversing first the neurogram acquired by frequently hearing this person's steps, next traversed the neurogram

* See above, p. 44 and note to p. 31 .

+ See above, p. $4 \mathrm{I}$.

† See above, p. 36. 
(or part of it) corresponding to the person and next that corresponding to the food; for if the whole process took place below the level of the dog's consciousness no other explanation is readily available; and if, on the other hand, after hearing the sound of the steps, the dog had some idea of the person and then some idea of the food, the explanation by neurograms still fits the facts most simply. Indeed, in this second case, the impulse must have passed from one neurogram to the other by way of the low resistance path which we have already seen reason to postulate as the physiological correlative of an association between ideas; or else we must suppose that the passage of the impulse in the first neurogram ceased as the idea of the steps gave way to the idea of the person whose steps they were, and that the impulse in the second and third neurograms was in each case the consequence of psycho-physical interaction. This alternative supposition must be rejected on three separate grounds: (I) it offers no explanation of what happens to the impulse that has been passing in the first neurogram and does not go on to the second, for there is no movement or other evidence of the escape of the impulse by efferent channels; (2) it assumes a clear division between consecutive thought-activities which is entirely inconsistent with William James' notion-now generally accepted - of a continuous stream of thought*; and (3) it involves the unnecessarily clumsy expedient of assuming psycho-physical interaction in cases where a much simpler explanation is available.

Pawlow's experiments therefore afford confirmatory evidence of the existence of neurograms as real physical things; and of real physical links between the neurograms of objects, ideas of which are associated.

\section{§ 3. Instincts and Emotions.}

These experiments also furnish us with excellent examples of neurograms which include visceral, as well as cerebral and spinal, arcs. By far the most important of such neurograms-in many ways the most important of all our neurograms-are those which correspond to our instincts and emotions.

In his work on Social Psychology $\dagger$ Dr McDougall has endeavoured to shew that every emotion is either one of a small group of primary emotions-fear, disgust, wonder, anger, positive and negative selffeeling, tender emotion, and distress $\ddagger$ - or is compounded of some two

* Loc. cit. Vol. I, p. 274.

$\dagger$ Chapter III, pp. 45 et seq.

$\ddagger$ 'Distress' was added later to the original seven, Proc. Avistotelian Society (1915), 'Symposium on Instinct and Emotion,' p. 28. 
or more of them*. Dr Morton Prince $f$ tells us that this view is now generally accepted. Each of the primary emotions is, according to Dr McDougall, intimately linked with one and only one primary instinct in the manner about to be described. For example, the emotion of fear is linked to the instinct of flight + , the emotion of anger to the instinct of pugnacity, and the emotion of wonder to the instinct of curiosity.

Dr McDougall defines a primary instinct as 'an inherited or innate§ psycho-physical disposition [neurogram $\|$ ] which determines its possessor to perceive, and to pay attention to, objects of a certain class, to experience an emotional excitement of a particular quality upon perceiving such an object, and to act in regard to it in a particular manner, or, at least, to experience an impulse to such action.' 9

According to this definition, every instinctive process has the three aspects of all mental process: the cognitive or knowing, the affective or feeling, and the conative or striving to act**. The innate neurogram, which corresponds to an instinct, may also be regarded as consisting of three parts, an afferent, a central, and a motor or efferent part. By suitably subdividing the innate neurogram, we may take the activities of its first or afferent part as corresponding to the cognitive part of the instinctive process; and we might also take the activities of the second (or central) and third (or efferent) parts of the neurogram as corresponding respectively to the affective and conative features of the total instinctive process. This is the description originally given by $\mathrm{Dr}$ McDougall $\uparrow \uparrow$. But, as Dr McDougall has since recognised, 'each

* Dr McDougall has kindly pointed out to me that this statement now requires modification in the light of the new chapter on 'derived emotions' in the I4th edition of his Social Psychology.

+ Loc. cit. p. 446.

$\ddagger \mathrm{Mr}$ A. F. Shand in his Foundations of Character (I9I4) argued that the emotion of fear includes several different 'instincts' in its system. Dr McDougall has met Mr Shand's argument by explaining ('Symposium' quoted in the last note, p. 5 I) that the primary instinct of which the primary emotion of fear is the affective aspect is not simply an instinct of flight but 'a chain instinct of two links': 'it... impels the animal or man to seek cover and there lie hid.'

\& 'The statement that [neurograms] are congenitally organised does not necessarily imply that they are present fully developed at birth, but rather that they have an inherited tendency to develop along certain lines.' (McDougall, Physiological Psychology, p. ro6.)

$\|$ The neurogram is not, of course, psycho-physical; but its excitement may produce psychical processes.

If Social Psychology, p. 29.

** Cf. McDougall, Social Psychology, p. 32; and Psychology, p. 61.

$\dagger^{\dagger}$ 'The innate psycho-physical disposition, which is an instinct, may be regarded as consisting of three corresponding parts, an afferent, a central, and a motor or efferent part, whose activities are the cognitive, the affective, and the conative features respectively of the total instinctive process.' Social Psychology (3rd edition, I9Io), p. 32. 
emotional disposition is so intimately bound up with some conative disposition that we may, for most purposes, regard the innate emotional-conative disposition as a structural and functional unit.'* We shall accordingly describe the central part of an instinct-neurogram as corresponding not only to the emotional part of the instinctive process but also to so much of the conative part as constitutes the tendency to act in such a manner as shall bring about the end towards which the instinct strives. The actual movement made-a much more variable process than the tendency $\dagger$ which leads to it-will then correspond to the excitement of the third (efferent or motor) part of the instinct-neurogram.

Such a neurogram is represented schematically in the following diagram. In this diagram $A$ represents the afferent neurones and the cerebral arcs the excitement of which is affected by the native object of the instinct and corresponds to cognition. The central part $(C)$ of the instinctneurogram is excited by impulses spreading from $A$ and in turn excites, or

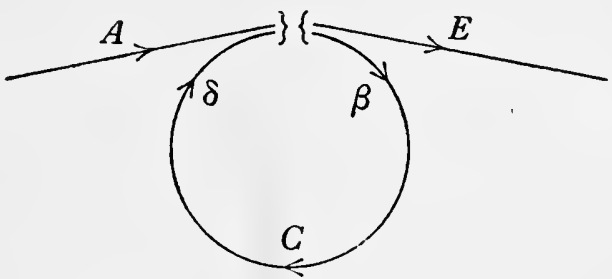

Fig. 2. adds to the excitement of, $E$; it includes $\vdots$ not only arcs in the brain itself, but also arcs which lead from the brain to the viscera and arcs which lead from the viscera back to the thalami, and, finally, arcs which connect the thalami with the cortex of the cerebrum $\S$; p. 3 .

* 'Symposium on Instinct and Emotion,' Proc. Aristotelian Society (I9I5),

$\dagger$ For example, the tendency to escape under the influence of fear may lead either to (multifarious) movements of seeking cover or to the inhibition of all movement when cover has been found. Or the tendency to 'down' the man who angers me, may lead me to injure him with my boot or my tongue, instead of with my uplifted arm.

$\ddagger$ 'The constitution of this part determines in the main the distribution of the nervous impulses, especially of the impulses that descend to modify the working of the visceral organs, the heart, lungs, blood vessels, glands, and so forth, in the manner required for the most effective execution of the instinctive action" (McDougall, Social Psychology, p. 33). In a footnote Dr McDougall adds: 'It is probable that these central affective parts...have their seat in the basal ganglia of the brain.' This probability has been greatly increased, if not transformed into a certainty, by Dr Head's work on the optic thalamus. See above, footnote to p. 31 .

$\S$ In addition to the 'circular nervous process' (cf. McDougall, Physiological Psychology, p. I I6) represented by $C$, there is another circular nervous process (not shewn in the diagram) beginning with $E$ and adding to the central excitement by bringing nervous impulses from the kinaesthetic sense-organs excited by the movements of the muscles stimulated through $E$. 
and we recognise its excitement as an 'affect'* or emotion $\dagger$ and as a conative tendency. Lastly, $E$ represents the efferent or motor arcs along which the excitement is finally discharged to the muscles of the skeletal system, there producing the characteristic instinctive movements $;$. According to our diagram, the excitement of the efferent arcs $(E)$ reaches them in part from the afferent arcs $(A)$ directly, as well as (in much greater quantity) from the central $(C)$ portion of the neurogram. In this respect the scheme represented in the diagram differs from that quoted above from Dr McDougall according to whom 'the excitement of the efferent or motor part reaches it by way of the central part.' $\$$ We have departed from Dr McDougall's description in this particular because introspection tells us that, when three states of consciousness have followed each other in the same order on several occasions, the third state tends to become directly, as well as indirectly, associated with the first. And, as we have seen, this is equivalent to saying that the third neurogram acquires a direct connexion with the first. It follows that, as the $A, C$ and $E$ neurograms are frequently excited together and in immediate succession, the $E$ neurogram must acquire a direct connexion with $A$. Moreover, as William James has pointed out $\|$, it may be that when we meet a bear we begin to run before we are frightened; and most people will agree that, on suddenly becoming conscious of danger-for example, that one is about to step over a precipice-the movement of recoil takes place before the emotion of fear begins to

* In his work on Social Psychology Dr McDougall uses the word 'affective' as the equivalent of 'emotional,' and we shall follow him in this use of the adjective 'affective' and the substantive 'affect.' In the later paper (I9I5) published by the Aristotelian Society (see footnote * on p. 53 above) Dr McDougall proposed to use the word affect to cover 'both the affective aspect and the conative aspect of conscious process.' But when we require an adjective to denote both aspects we shall use the compound form 'affective-conative.' Thus, for example, we shall describe the central part $(C)$ of the neurogram of an instinct as 'affective-conative.'

$\dagger$ According to McDougall our emotions are feelings or 'affects' mainly due to visceral disturbances. He writes for example 'Since the group of visceral adjustments accompanying each kind of instinctive action is peculiar to that kind, and since these adjustments cannot be voluntarily controlled or prevented, whereas the bodily instinctive movements can be, and often are, controlled and modified by the will, we rightly regard the effects of these adjustments as the most reliable symptoms of the emotions.' (Physiological Psychology, pp. Iog, I Io.)

¥ If, ignoring the fact that an instinct, as congenitally organised and before it has been modified by experience, does determine its possessor to act in a particular manner and not merely to experience an impulse to such action, we were to adopt the second alternative at the end of $\mathrm{Dr}$ McDougall's definition of an instinct (quoted above on p. 52), the instinct so defined would be completely represented by the first two parts $(A$ and $C$ ) only of the neurogram we have described.

§ Social Psychology, p. 33.

i) Principles of Psychology, Vol. II, p. 450. 
be felt. We may add that, according to our diagram, the emotion is felt when the final vertical portion of the $C$ neurogram is excited: and that is after the visceral changes, which give rise to the impulses arriving by $\delta$, took place. This agrees with William James' theory of the emotions, according to which the bodily changes take place before the emotion is felt, so that 'we feel sorry because we cry,' instead of crying because we feel sorry as is commonly supposed.

The important function fulfilled by the emotional element in an instinctive process is thus illustrated by $\mathrm{Dr}$ McDougall:

Let us consider the visceral adjustments that result from the excitement of one of the primary instincts, namely the instinct of flight. For effective flight the muscles of the limbs need to receive the greatest possible supply of well-aerated blood; and this can only be secured by an increased force and frequency of the heartbeat, by increased frequency of the respiratory movements of the chest-walls and diaphragm, and by contractions of the small arteries of all the organs that do not immediately aid in flight, driving the blood from all these organs to the muscles of the limbs, especially from the skin and various glands which in their normal state contain large quantities of blood. Hence we find that the excitement of the instinct of flight gives rise to violent action of the heart, to hurried breathing, to pallor and coldness of the skin, to dryness of the mouth and throat, and to those other disturbances of the digestive functions which result from the checking of the blood-supply to the salivary glands and other organs of digestion*.

Dr McDougall goes on to explain that, in addition to assisting in some such manner to further the end-escape, in the case described above-towards which the particular instinct strives, the emotional element in every instinctive process serves the purpose of the instinct by very largely increasing the amount of 'free nervous energy' that tends to escape along the efferent channels and so to produce the characteristic movement. The source of this 'free energy' consists in the impulses reaching the brain by afferent paths from the viscera and from the skeletal muscles. These are the impulses which are felt as the emotion. If the emotional excitement is excessive-if the impulses from the viscera are too intense-they may be able to overcome the resistance of synapses in all parts of the skeletal nervous system and produce general convulsions. But, so long as the excitement is not excessive, 'it renders instinctive action more effective, because it determines the outflow of a greater volume of nervous energy to the muscles. Hence under the strong excitement of an instinct, or, as we more commonly say, under the influence of strong emotion, our muscular system may execute more forcible contractions

* Physiological Psychology, p. I Io. 
than any we can voluntarily produce in the absence of such excitement.'*

Some writers-notably Mr A. F. Shand in The Foundations of Character-have refused to accept Dr McDougall's view that every primary emotion is an essential part of some primary instinct. It is true that the word instinct, as commonly used by biologists, denotes an innate tendency to perceive and pay attention to certain objects and to act in regard to them in a certain way. Thus the behaviour of a newly hatched chick, which pays attention to grains of corn (among all the many objects on the ground before it) and pecks at them, would generally be described as instinctive, although the chick exhibits only a cognitive (perceiving the corn) and a conative (pecking, or trying to peck, at it) process, without giving any evidence of a central emotional process. By accepting Dr McDougall's definition of an instinct we compel ourselves either to describe the chick's behaviour by some other word than instinctive or, as we prefer, to continue to describe innate cognitive-conative neurograms as instinct-neurograms until we know that they do not include a central affective portion which, on being excited, corresponds to an emotion. A justification for leaving the onus of proof to the opponents of the theory is to be found in the fact that, while we cannot know whether emotions do or do not take place in the lower animals or insects, we can, in many instances of instinctive behaviour on the part of the animals most nearly allied to ourselves, 'recognise the symptoms of some particular kind of emotion such as fear, anger, or tender feeling; and the same symptoms always accompany any kind of instinctive behaviour, as when the cat assumes the defensive attitude, the dog resents the intrusion of a strange dog, or the hen tenderly gathers her brood beneath her wings.' $\dagger$

In spite of the limitation which Dr McDougall's theory, expressed in his definition of instinct + , imposes on the use of this word, the theory is coming to be generally adopted because of the simple and consistent account which it gives (or helps to give) of many phenomena both of normal and of abnormal psychology. Thus Professor Stout, in the new edition of his Manual of Psychology, has followed Dr McDougall's lead. And Dr Morton Prince considers that 'this formulation, by McDougall, of emotion as one factor in an instinctive process must be regarded as one of the most important contributions to our knowledge of the mechanism of emotion. It can scarcely be traversed,

* Physiological Psychology, p. III.

$\dagger$ Social Psychology, p. 28 . $\quad$ See above, p. 52 . 
as it is little more than a descriptive statement of observed facts... Its value lies in replacing vagueness with a precise conception of one of the most important of psychological phenomena, and enables us to clearly understand the part played by emotion in mental processes.' *

We have so far spoken of the $A, C$, and $E$ portions of the neurogram represented in our diagram $\dagger$ as not only innate but also innately connected with one another; and in animal life, no doubt, instincts are but little modified by life's experiences. But with men it is different. We have already seen how neurograms that are excited together tend to become permanently connected + , and how neurograms that are linked with the same neurogram also tend to become linked with one another. So both the afferent $(A)$ and the efferent $(E)$ part of the neurogram which represents an instinct may be greatly modified by experience, as may also their connexions with one another and with the central $(C)$ part of the neurogram; but this central part, having its excitement less under voluntary control, changes only slightly if at all $\S$. In other words, we may learn to respond to an object with emotions and conations other than those innately connected with objects of that class: as for instance (to quote an illustration given by Dr McDougall |) when a child, in whom the perception of a dog or cat or other small animal has always excited tender emotion, is once bitten and frightened by a dog and always afterwards has the emotion of fear excited by the very percepts (dogs) which previously excited tender emotion $\uparrow$. And we may learn to give the same emotional and conative responses to new kinds of objects as well as to those whose neurograms are innately connected with the affective-conative ** neurograms in question: for example, a woman in whom tender emotion is congenitally excited by the sight of small children, may learn to respond with the same emotion to small objects of any other kind-lap-dogs, say, or china knick-knacks $\uparrow \dagger$. Finally we may learn to respond to the same cognitive and emotional experiences, if not with a different conative tendency, at least with a movement that has been modified by experience: as when a pugnacious

* Loc. cit. p. 448.

$\dagger$ Above, p. 53.

† See above, p. 54 .

$\S$ Dr McDougall suggests that this persistence of human emotions, and the comparative variability of human instincts, are the reasons why previous writers have not recognised the intimate connexion, which he first pointed out, between instincts and emotions. (Physiological Psychology, pp. 108, 109.)

II 'Symposium on Instinct and Emotion,' Proc. Aristotelian Society, p. 30.

-In this case $A$ (see diagram on p. 53) is given and $C$ is replaced by $C^{\prime}$.

** As explained in footnote * on p. 54 above, this word may be conveniently used to describe the $C$ portion of the neurogram represented in the diagram on p. 53 .

$\dagger \dagger$ In this case $C$ is given and $A$ is replaced by $A^{\prime}$. 
man, who has been insulted and made angry, sends his hand flying to his sword-hilt or to his hip-pocket instead of raising it to strike*.

\section{- § 4. Sentiments.}

When an affective-conative neurogram $(C)$ is not innately connected with a cognitive neurogram $\left(A^{\prime}\right)$ but has become so connected by experience, we do not speak of the whole neurogram $\left(A^{\prime}-C-E\right)$ as the neurogram of an instinct but of a sentiment $\uparrow$. Thus, to take Dr McDougall's illustration just quoted + , the child whose innate tendency was to love dogs but who, as the result of a terrifying experience, has come to fear them is not properly spoken of as possessing an instinctive fear of dogs, but rather as having acquired a sentiment of fear for dogs (or for a particular dog, as the case may be). The essential difference between an instinct and a sentiment, therefore, is that in the former the connexion between the cognitive $(A)$ and the affective-conative $(C)$ neurograms is innate, while in the latter this connexion is acquired $\S$.

But a sentiment also differs from an instinct in that the neurogram of a sentiment may include several different affective-conative neurograms all connected with the same cognitive neurogram. Indeed $\mathrm{Mr} \mathrm{A}$. F. Shand first applied the word 'sentiment' to an organised system of emotional tendencies centred about some object or class of objects $\|$. We must therefore conceive the neurograms of the more complex sentiments as consisting of a number of affectiveconative neurograms connected I with the single cognitive neurogram which corresponds to the object of the sentiment. Such a complex sentiment-neurogram may have no fixed efferent part. The effect of connecting a cognitive neurogram with one or more affective-conative neurograms so as to form a sentiment is that, whenever the cognitive neurogram is excited, the excitement tends to spread to the affectiveconative neurograms; and, if the excitement does so spread, the resulting visceral changes send back to the brain a multitude of impulses which greatly augment the outflow of nervous energy. This outflow may take place almost immediately, giving rise at once to

* In this case $E$ alone is altered. The illustration is given by McDougall, Social Psychology, p. $4 \mathbf{I}$.

$\dagger$ The cognitive neurogram $\left(A^{\prime}\right)$ we may refer to as the cognitive part or element of the sentiment-neurogram: the object to which $A^{\prime}$ corresponds (see footnote $\S$ on p. 47 above) is spoken of as the object of the sentiment.

$\ddagger$ From 'Symposium on Instinct and Emotion,' Proc. Avistotelian Society, p. 30.

$\S$ McDougall, 'Symposium on Instinct and Emotion,' Proc. Aristotelian Society, p. 30. I! Cf. McDougall, Social Psychology, p. I22.

II In Mr Shand's view the connexions are largely, if not wholly, innate. In Dr McDougall's view the connexions are mostly, if not wholly, acquired. 
movements which are all the more powerful because of the large amount of nervous energy available; or the impulses may first pass through further cortical paths, giving rise to a train of thought which is the more intense because of the intensity of the corresponding nervous processes. Moreover, although the direction of this outflow is under the control of the will, it is (as we shall see) in practice largely determined by the conative elements in the sentiment that has been excited.

The power of any idea to direct thought and action thus largely depends on the extent to which the corresponding cognitive neurogram is linked to affective-conative (emotion-instinct*) neurograms. Dr Prince tells us that 'The impulsive force of the emotional dispositions or linked instincts becomes the conative force of the idea, and it is this factor which carries the idea to fruition.' And he adds: 'This is one of the most important principles of functional psychology. Its value can scarcely be exaggerated. Without the impulse of a linked emotion ideas would be lifeless, dead, inert, incapable of determining conduct.' $\dagger$ So Dr McDougall says 'that directly or indirectly the instincts are the prime movers of all human activity' $\ddagger$; while Dr Bernard Hart speaks of 'the great primary instincts which constitute the principal driving forces of the mind.' §

We shall have occasion later to emphasise the pedagogic consequences $\|$ of this 'most important principle.' But we may note here that, just as the cerebral excitement which accompanies and corresponds to an idea in the focus of consciousness is vastly increased when the neurogram of the object of that idea is linked to a primary instinct, so also the cerebral excitement may be increased-although to a much less extent-if the thought-activity in question is giving rise to bodily (skeletal) movements of any kind. For the same diagram (Fig. 2, page 53) will still represent the path of the impulse, $A$ now representing the neurogram whose excitement accompanies the thought-activity, $E$ (and now $\beta$ also) the efferent paths to the muscles, while $\delta$ now represents the path by which the impulses return to the cerebrum from the kinaesthetic sense-organs stimulated by the movement in progress $\uparrow$. The value of the maxim 'learn by doing' lies in

* C.f. Morton Prince, loc. cit. p. 447.

$\dagger$ Loc. cit. pp. $449,450$.

¥ Social Psychology, p. 44.

$\$$ Loc. cit. p. I65. Cf. also Lord Haldane (quoted on page 328 below): 'Without passion nothing great is, or ever has been, accomplished.'

II For example, as Professor Gilbert Murray told the teachers' conference in the University of London on 3rd January, I9I7. 'The teacher who aims at love but not knowledge is a washy educator. But the teacher who aims at knowledge without love is no educator at all.' See also below, p. 345 .

it So $C$ does not now include visceral arcs. 
the fact that the excitement of a cognitive neurogram, which is thus linked from the outset with the neurogram of a circular nervous process whereby impulses from kinaesthetic sense-organs are brought back to reinforce the excitement of cortical neurones, tends to recur more frequently and to be more intense when it does recur*. Just as, in Dr Morton Prince's words, 'the linking of a strong affect tends to safeguard registration and conservation of experience,' so also experiences are more strongly registered and conserved by being linked to voluntary movements.

The formation of a sentiment by linking affective-conative neurograms to a cognitive neurogram not only increases the available energy whenever the latter neurogram is fortuitously excited-whenever the object of the sentiment chances to come to mind-but it also causes the cognitive neurogram to be excited much more frequently, so that the object of the sentiment is often thought of. The explanation will be considered later $\uparrow$. Meanwhile we may note the fact, and observe that it renders still more profound the effect on life and conduct which the primary instincts produce through the sentiments of which they form part.

The formation of a sentiment and its consequences are thus illustrated by Dr McDougall:

I become aware that the man standing beside me has struck me; this cognition evokes in me angry feeling and an irresistible impulse to return the blow...my angry impulse may be checked by one of fear, which prompts me to retreat...as soon as I am out of danger, I may think again of the incident; I live through it again in imagination, as we say. This restores the angry impulse; which, finding no satisfaction, in turn keeps me thinking of my adversary: the insult rankles in my bosom....Quite involuntarily I find myself plotting out schemes of revenge....Up to the time of the incident, I had been, we may suppose, as nearly as possible indifferent to my assailant; that is to say, his presence had evoked in me no well-defined feeling or attitude. But after the painful incident, I cannot think of him without fear, or anger, or both, and without desiring both to avoid him and to get the better of him in some way. Suppose, now, that circumstances repeatedly bring us together, and that his behaviour on such occasions is that of a bully covertly reminding me of the past insult that I dare not avenge. My attitude of blended anger and fear is renewed on each such occasion, and, being thus confirmed and rendered permanent, it becomes a full blown sentiment of hatred [in which the cognitive neurogram corresponds to my assailant and the two affective-conative neurograms, with which the cognitive neurogram is linked, correspond to the instincts of which anger and fear are the 'affects'].... The effect of such linkage is not

* But for the time being-see p. 280 below-the doing finishes the learning.

$\dagger$ See below, pp. 89, $9 \mathrm{I}$. 
only that, whenever the object of the sentiment is forced upon my attention, my thinking of him is coloured or suffused with these emotions, but also that I am rendered peculiarly apt to think of him. If I pass by a crowd of which he is a member, my eye singles him out and watches him furtively; if we both have occasion to attend the same board-meeting, I am acutely aware of him and of all he says and does, though I may avoid glancing at him; if $I$ hear his name mentioned by others in conversation, I am all agog to hear what is said. And this may continue in spite of my best efforts to cast out this demon of hatred and to resume my former attitude of indifference. Again, all my thinking of my adversary is biassed by my attitude; whatever $I$ hear to his discredit I accept and retain, and I attribute his actions to the meanest motives; until, by repetition of this process of selective thinking under the guidance of the specialised conative tendency, I come to think of him as a monster of iniquity*.

The following is another simple example of the effect of a sentiment :

A man walking with a friend in the neighbourhood of a country village, suddenly expressed extreme irritation concerning the church bells, which happened to be pealing at the moment. He maintained that their tone was intrinsically unpleasant, their harmony ugly, and the total effect altogether disagreeable. The friend was astonished, for the bells in question were famous for their singular beauty. He endeavoured, therefore, to elucidate the real cause underlying his companion's attitude. Skilful questioning elicited the further remark that not only were the bells unpleasant but that the clergyman of the church wrote extremely bad poetry. The causal [sentiment] was then apparent, for the man whose ears had been offended by the bells also wrote poetry, and in a recent criticism his work had been compared very unfavourably with that of the clergyman. The [sentiment] thus engendered had expressed itself indirectly by an unjustifiable denunciation of the innocent church bells. The direct expression would, of course, have been abuse of the clergyman himself or of his works $\dagger$.

In the last quotation, Dr Hart wrote 'complex' where we have substituted the word 'sentiment.' But, as Dr McDougall has pointed out $\ddagger$, the morbid complex, which now figures so largely in psychopathology, is essentially of the nature of a sentiment. Indeed, as defined by Dr Hart§, the neurogram of a complex consists of a sentiment-neurogram with a cognitive part to which several other cognitive neurograms have become connected. It follows that every

* Psychology, pp. 105-116.

† Quoted by Dr Hart (loc. cit. pp. 73, 74) from Dr Jung of Zurich. Dr Hart wrote 'complex' and 'rivalry complex' in the two places where we have written '[sentiment].'

$\ddagger$ 'Symposium,' p. 40 .

$\S$ 'A system of connected ideas, with a strong emotional tone, and a tendency to produce actions of a certain definite character... is termed in technical language a "complex,"' loc. cit. p.6r. Neither Dr Hart nor Dr Prince (loc. cit. p. 266) uses the word complex in its morbid sense only. 
sentiment tends quickly to become a complex*, in the extended sense in which Dr Hart and Dr Prince used the word $\dagger$.

This tendency of a sentiment is only one example of the tendency of all neurograms to become linked together as, from time to time, the objects or feelings, to which the neurograms correspond, are thought of or experienced either together or in immediate succession. But the growth of systems of interconnected neurograms is by no means a wholly fortuitous process. Objects that are connected with each other in the 'real' world + -a cause and its effect, for example; or the different properties of the same material; or different objects having the same property or arousing the same emotions-tend to be thought about together, and so to be represented by connected neurograms. The interconnexions of many systems of cognitive neurograms are thus due to the relations which, as we say, actually obtain in the real world. Such 'subject' systems of cognitive neurograms in the brain, corresponding to what is commonly called knowledge in the mind $\S$, will resemble each other in different individuals who have studied the same subject. Other systems, however, are to a great extent peculiar to the individual to whom they belong: for example, a system of neurograms which preserves the record of his personal experiences during a certain period; or a system formed by cognitive neurograms which have, at different times, chanced to become connected with the same affective-conative neurograms the excitement of which occurs whenever he is in a certain 'mood.'

\section{§ 5. Interest-Systems.}

We shall find it convenient to have a name for any system of connected neurograms of which at least oneis a cognitive neurogram $\|$; and we can find no more convenient way of describing such a system

* Whenever any one of the cognitive neurograms is excited the nervous impulse tends to spread-at first through the cognitive neurogram about which the sentiment is centred and afterwards (as we have seen) directly-to the affective-conative neurograms the excitement of which produces both emotional feeling and a tendency to action having a certain definite objective. But every neurogram tends to become linked with others that are often excited about the same time. The cognitive neurogram which is the object of a sentiment is no exception to this rule. Moreover, the sentiment of which it forms part tends to secure its frequent excitement. It follows that every cognitive neurogram about which a sentiment is centred tends to become linked with a rapidly increasing number of other cognitive neurograms. In other words, every sentiment tends to be quickly transformed into a complex.

$\dagger$ But since the usage of the term 'complex' is not yet fixed and since $\mathrm{Dr}$ McDougall, for example, would prefer to keep the word for a pathological fact, we shall as far as possible avoid the term 'complex' in the sequel.

† See Chapter I I below. \& Cf. McDougall, Psychology, p. 83 .

But see footnote $\S$ on p. 163 , below, where this limitation is removed. 
than as an interest-system. This use of the word 'interest'* accords with common speech; for no one ever took an interest in any object about which he had not a goodly number of ideas; nor did any one ever have many ideas about (or associated with any of his thoughts of) an object in which he did not take an interest. A sentiment then is a simple kind of interest, and a 'complex' (in the extended sense in which the word is used by $\operatorname{Dr}$ Hart $\dagger$ ) is another more elaborate kind of interest. A 'hobby' would be represented neurographically by a still more complicated interest-system. We note that an interestsystem of neurograms, as we have defined the term, is a comparatively permanent neural feature whose excitement from time to time occasions mental processes.

In the course of life's experience, then, an individual's neurography -if we may so describe all his neurograms, however distributed and arranged-tends to become organised into interest-systems which increase continually both in extent and in complexity of organisation $\ddagger$. The more perfectly his cognitive neurograms are organised, the more fully do they represent the objects which compose the world, and the relations that obtain between them: the more extensive, in fact, and the more accurate is his knowledge $\S$. But in determining the effect of his interest-systems upon his thought and behaviour-and thus also upon the further growth of his interest-systems-a leading part is played by his affective-conative neurograms and the neurograms of sentiments and complexes in which they become synthesised\|. The harmonious co-ordination of all these interest-systems, and especially

* Cf. Herbart: 'no sooner did the words' of the teacher's anecdote told to mind-wandering schoolboys 'awaken old thoughts, forming strongly-connected series with which the new impression easily combined, than out of new and old together a total interest resulted...' Psychologie als Wissenschaft, § 128. Quoted by William James, loc. cit. Vol. 1, p. 4 1 8.

$\dagger$ See above, pp. 6I, 62 especially footnote $\S$ on p. 6r.

$\ddagger$ We have to regard the brain of the adult as consisting of a great number of such systems and sub-systems of neurones, organised with various degrees of completeness and stability and interconnected with various degrees of intimacy. Some of the perceptual systems [neurograms] are congenitally organised, while others are built up by the course of the individual's experience. The statement that systems are congenitally organised does not necessarily imply that they are present fully developed at birth, but rather that they have an inherited tendency to develop along certain lines. The mental life of most of the animals must be regarded as almost purely perceptual and determined almost completely by congenitally organised perceptual systems. Man, too, has such congenitally organised systems, but he differs from the animals in that his long education greatly modifies his congenital systems and develops many new systems peculiar to each individual.' McDougall, Physiological Psychology, pp. 105, 106.

$\S$ Cf. McDougall, Psychology, p. 83, where however the reference is to the 'mind' and its 'dispositions' instead of the 'brain ' and its 'neurograms.'

\|| Cf. Morton Prince, loc. cit. p. 536. 
of those in which the affective-conative elements are strongest, is a first condition of effective action and consistent behaviour*. It is indeed an essential foundation of character. On the other hand, conflict between different interest-systems or complexes is one of the principal causes of certain kinds of insanity.

We are about to study the spontaneous growth of interest-systems and then their voluntary development in the process of education. Meanwhile we do well to note that the neurograms of interests $\dagger$, complexes and sentiments, permanent as they are when compared with the mental processes that result from their temporary excitement, are not indestructible. They tend, on the contrary, to become gradually disaggregated after they have served their purpose, just as printers' type is distributed when its work is done + .

* See below, Chapter 16.

$\dagger$ The expression 'neurograms of interests' is here used as the equivalent of 'interest-systems.'

‡ Cf. Morton Prince, loc. cit. p. 306. 


\section{CHAPTER 6}

\section{INVOLUNTARY THOUGHT}

\section{$\S \mathrm{r}$. The First Lare: Psycho-neural Parallelism.}

THE neurograms that we have just been studying are the physiological records of states of consciousness and the physiological condition of the repetition of such states. We turn now from psychostatics to psychodynamics, the study of the flow of consciousness. To ascertain the causes determining this flow is, according to Dr Bernard Hart*, the ultimate aim of psychology.

Our problem is to formulate laws according to which our thinking proceeds; without, however, allowing ourselves to forget that a law of nature is no more than a hypothesis consistent with experience.

It is of course obvious that an individual's thought-activities at any moment largely depend upon his environment. Unless he is very busily occupied, a thunderclap close by is sure to make him think of thunder. And, even when sense impressions from outside or from inside his body do not determine his thought-activity, they may still influence it. We shall have more to say later concerning the interruption and modification of trains of thought by incoming sense impressions. First, however, let us simplify our discussion by considering the case of thought-activities that are subject to no outside interference. And let us make it simpler still by speaking, for the present, only of involuntary thinking.

Some of the following statements of these laws of thought have had to be anticipated in order that the preceding account of neurograms and systems of neurograms might not lack some indication of the way in which neurograms are formed and systematised. Thus, for example, we have already $\dagger$ enunciated the law of psycho-neural parallelism + . We now re-state it as follows: A nervous impulse traverses the neurones of one or more sensory regions of the brain whenever any mental process occurs. Employing Huxley's terminology,

* Loc. cit. pp. 6o, $6 \mathrm{r}$.

$\dagger$ On p. 50, above.

$\ddagger$ W. McDougall (Physiological Psychology, p. 167) states the law: 'some neural process invariably accompanies every state of consciousness.' $\mathrm{He}$ warns his readers against confusing the law of psycho-neural parallelism with the hypothesis of psycho-physical parallelism.

G. E. 
we may now express the law more briefly: To every psychosis there corresponds a neurosis*.

This law-that whenever thinking goes on, impulses pass along the brain neurones in such wise that the nature of the thought-activity at any moment is, subject to the fourth law $\uparrow$, determined by the neurones that are then excited and by the degree of their excitement $;$ -is our first law of thought.

The system of brain paths followed by the nervous impulse, and the intensity of the impulse at various parts of the system, thus define the thought-activity at any moment. But we do well to remind ourselves that the system of brain paths excited at the moment in question may include higher level paths the excitement of which does not affect consciousness.

If therefore we describe as 'sensory brain paths' those systems of arcs the excitement of which may affect consciousness, we may say with greater precision, that it is the system of sensory brain paths excited at any moment, and the varying degree of excitement in different parts of this system, that define the thought-activity then in progress. And it is reasonable to assume that the thought-activity which occupies the focus of consciousness, corresponds to the excitement of that part of the system of sensory brain paths that is then most intense§. Moreover, the progressive changes in the distribution of the excitement determine the flow of consciousness.

\section{§2. The Second Law: Diffusion.}

If states of consciousness were discontinuous, it would be difficult to account for the sudden disappearance of nervous impulses from sensory brain paths at the end of one state, or, apart from new sense impressions, for the origination of other impulses when the next state begins. But no such difficulty need trouble us, since introspection tells us that the flow of consciousness is continuous. 'The changes

* Cf. W. James, Principles of Psychology, Vol. I, pp. I 86 and 243. This use of the words psychosis and neurosis is not to be confused with their use in recent medical literature to denote pathological conditions.

$\dagger$ See below, p. 129 .

$\ddagger$ An arc or neurogram that is active, but not excited (see footnote * on p. 29) may affect consciousness if impulses traverse synapses from one constituent neurone to another, although no impulse enter or leave the neurogram or arc as a whole.

$\S$ Cf. W. James, loc. cit. Vol. I, p. 283. If, however, as is not uncommon in pathological cases, there be a second (sub-conscious) neurosis to which no psychosis corresponds, the impulse passing in the second system of nervous arcs, or in some part of it, may be of greater intensity than any in the first system. (See M. Prince, loc. cit. footnote on P. 343.) 
from one moment to another in the quality of the consciousness are never absolutely abrupt.'*

When thought is guided by the will, its continuity is obvious enough. When thinking is involuntary, it is generally easy to trace the connexion between the states of consciousness at two moments separated by a short interval of time. Even when the stream of consciousness-to use William James' phrase-seems to be cut off short by a peal of thunder or other sense impression of sufficient intensity, the transition, rapid though it be, from the interrupted thought-activity to the new thought-activity that arises out of the interruption, is not to thunder pure, but to 'thunder-breaking-uponsilence-and-contrasting-with-it'; for 'into the awareness of the thunder itself the awareness of the previous silence creeps and continues.' $\dagger$

Let us next attempt to make a cross-section of this continuous stream of thought. At any moment there is some thought-activityperhaps an idea of some object-which is receiving most attention, and is said to occupy the focus of consciousness. This thought-activity will ordinarily possess some meaning, more or less; and, as we saw this meaning implies the existence of several associated thoughtactivities of which we may be barely conscious, and which are said to occupy the fringe of consciousness. If, to recall our previous example, an idea of Whitehall is in the focus of consciousness, it is not improbable that some idea of the Civil Service in general, or of the particular Government Office with which the thinker has most to do, will find a place on the fringe, and give to the idea of Whitehall the meaning it then has for him.

But the fringe of consciousness may also contain other thoughtactivities that do not owe their presence to associations with the thought-activity in the focus. It may, for example, include sense impressions: my thoughts must be very interesting if I remain unconscious of a sudden clap of thunder or a twinge of tooth-ache. Indeed, I shall ordinarily be aware of much less violent disturbances or discomforts. Or again, the fringe of consciousness may include elements belonging to some independent and, for the most part, unconscious stream of thought which momentarily obtrudes itself: as when the solution of a problem, pondered but left unsolved overnight, is about to flash into a mind that now seems wholly occupied with other matters.

* W. James, loc. cit. Vol. I, p. 237.

$\dagger$ W. James, loc. cit. Vol. 1, p. 240.

$\ddagger$ Above, pp. 45, 46 .

$\S$ See footnote $\uparrow$ on p. 256 , below. 
So we may describe a typical cross-section of the stream of thought as shewing (a) some thought-activity in the focus of consciousness; (b) other thought-activities, connected with the first, and giving meaning to it, on the fringe; and possibly $(c)$ other independent thought-activities as well.

What now is the neural counterpart of the stream of consciousness, a cross-section of which we have just described? It consists, as we have seen, of a stream of nervous impulses advancing through sensory brain neurones and the synapses that connect them. As the impulses advance and the distribution of excitement among the brain neurones alters-in short, as the neurosis changes-so also does the psychosis change. Our immediate problem is to formulate general laws according to which the neurosis changes, and to describe the psychological consequences of our results.

We may start with a simple neurosis and see how it develops. Imagine a man awakened out of dreamless sleep by knocking on his bedroom door. As he gradually wakes up, he is first conscious of knocking pure and simple. The knocking has no meaning: it is just knocking, that and nothing more. The corresponding neurosis, we may take it, affects no sensory brain paths beyond the auditory centres. As he becomes more and more widely awake, the waking man perceives that the knocking is on the door; that the door is the door of his bedroom; that he is being called; that it is time to get up; that he has this, that, and the other to do to-day. The neurosis extends at the same time, the excitement spreading from the auditory centres to the visual arcs, the excitement of which gives rise, first to an image of the door, and then to images of objects-the bedroom, we saidconnected with the door. And, as the nervous impulses continue to advance along connected sensory arcs in the brain, they give rise to the associated thought-activities: time-to-get-up and day's-workwaiting.

In some such way the simple knocking, the consciousness of which filled the sleeper's mind as he awoke, acquired meaning; and the simple neurosis in the auditory centres extended to a number of connected neurograms-of the door, of the bedroom, and the restthe excitement of which gave rise to this growing meaning*. The development on the fringe of consciousness of thought-activities connected with the thought-activity that for the moment occupies the focus, is similarly accounted for by the extension of the neurosis to which this focal thought-activity corresponds.

* See above, pp. 45,46 . 
This tendency of nervous impulses to spread from the arc in which they are moving to all systems of arcs connected with the first by synapses of sufficiently low resistance is a principle of great importance in our subject. The process is known as diffusion*, or, sometimes, as complication $\uparrow$. Remembering that the synapse between two neurones acts as an insulator, and not merely as a resistance, to feeble impulses, we may state the law of diffusion thus: Excitement in any nervous arc tends to spread to every other arc that is connected with the first through synapses the insulation of which the excitement in question is intense enough to overcome $\ddagger$. We shall refer to this law of diffusion as our second law of thought.

An example of the operation of this law is furnished by the fact that the sufficient excitement of any part of a neurogram-a system of connected low resistance arcs-is ordinarily followed by the excitement of the whole§. Thus, in Pawlow's experiments described above\|, it was only necessary to stimulate again any element in the neurogram to reproduce the whole process, involving the excitement of the whole neurogram which in this case included arcs of the visceral system. The psychological consequence is that the awakening of any imageauditory, visual, olfactory, or whatever it be-of a familiar object tends to arouse all the other images which go to make a percept 9 of the object, an idea of the object as a whole.

But, according to our statement of the law, the extent to which the excitement spreads depends upon the relation which the intensity of the excitement bears to the insulating strength of the synapses; and this varies between wide limits**. If the brain is fatigued, so that the synaptic resistances are high, the diffusion is slight, and the tired thinker finds that his ideas are lacking in wealth of meaning: his

* Cf. McDougall: 'The energy liberated in sensory neurones...diffuses itself.' (Physiological Psychology, p. 38.) Cf. also Bain: 'The organs first and prominently affected, in the diffused wave of nervous influence....' (Emotions and Will, pp. 4, 5, quoted by W. James, loc. cit. Vol. II, p. 373.)

$\dagger$ Cf. McDougall: 'The process of reinstatement of images of other senses by an impression made upon one sense is known to psychologists as complication. and the word may be usefully applied also to the underlying neural process.' (Physiological Psychology, p. Ioo.)

$\ddagger$ Cf. W. James: 'It is hard to doubt the truth of the law of diffusion, even where verification is beyond reach. A process set up anywhere in the centres reverberates everywhere, and in some way or other affects the organism throughout, making its activities either greater or less.' (Loc. cit. Vol. II, p. 38I.)

$\S \mathrm{Cf}$. W. McDougall: 'The perceptual neural system is a complex of sensorimotor arcs of all three levels, and comprises arcs in two or more of the sensory areas of the cortex, and all these arcs are so intimately connected that excitement, initiated in any one of them, tends always to spread throughout the system.' (Physiological Psychology, p. I05.)

T. But see footnote $\uparrow$ on p. 38 above.

II On Pp. 47 to 49.

** See above, p. 4 I. 
vocabulary is restricted, and his imagery poor*. If, on the other hand, the nervous system is in a state of abnormal excitability, as in some cases of strychnine poisoning when the synaptic resistances are abnormally low, 'a stimulus applied to any small group of sensory neurones may initiate an excitement which spreads throughout a very large part of the nervous system and throws almost all the muscles in the body into contraction ' $\uparrow$ : a flash of light may suffice to produce convulsions. Or, if the excitability is normal but the excitement intense, the nervous impulses may be diffused more widely than usual, so that thoughts may become unusually rich in meaning, and capable of expression in uncommon language: under the influence of strong emotion every man may, it is said, become a poet.

Diffusion thus accounts for the presence on the fringe of consciousness, and for the development of, thought-activities that originate from, and give meaning to, the thought-activity in the focus. The presence of other thought-activities of independent origin may be due to the excitement of sensory brain arcs by afferent impulses from the external sense organs or from the viscera.

\section{§3. The Third Law: Inhibition by Drainage.}

Our further account of the neural counterpart of a stream of thought may be simplified by the use of symbols. Let us denote a thought-activity by a letter in Gothic type (e.g. $\mathfrak{A}$ ), and let us use the same letter in ordinary Italic type (e.g. $A$ ) to denote the neurogram the excitement of which corresponds to the thought-activity $(\mathfrak{A})$ in question. It is true that the distribution of excitement in the neurogram, $A$, may be different on different occasions, and that to each different distribution a somewhat different thought-activity $\mathfrak{A}$ will correspond. But, so long as we concern ourselves with comparatively simple neurograms, this source of error need not trouble us; for the simpler the neurogram, the less can the distribution of excitement in it vary. Or, in psychological terms, while I may have many different ideas of my friend so-and-so (corresponding to different distributions of excitement in the complex neurogram of my friend) there is little variation about my ideas of an object like the letter $Z$, of which, as I am neither a lexicographer nor a printer, my neurogram is simple.

* Cf. W. James: "This field of view of consciousness varies very much in extent, depending largely on the degree of mental freshness or fatigue.' (Loc. cit. Vol. I, p. 256.)

$\dagger$ W. McDougall, Physiological Psychology, p. 33. 
Neurograms connected with $A$ we may denote by $A_{1}, A_{2}, \ldots, A_{n}$; neurograms, other than $A$, connected with $A_{n}$ by $A_{n 1}, A_{n 2}, \ldots$; and so on. The thought-activities corresponding to the excitement of these neurograms will then be represented by $\mathfrak{A} ; \mathfrak{A}_{1}, \mathfrak{A}_{2}, \ldots \mathfrak{A}_{n} ; \mathfrak{A}_{11}, \mathfrak{A}_{12}, \ldots$, $\mathfrak{A}_{n 1}, \mathfrak{A}_{n 2}, \ldots$; and so on $*$.

Consider now an involuntary stream of thought: the thinker is making no attempt to direct his thinking. We may make a crosssection of the stream at any point, and follow the stream as it flows on beyond that section. Suppose that the cross-section shews an elementary thought-activity $\mathfrak{A}$ in the focus of consciousness; and, on the fringe, $\mathfrak{A}_{1}, \mathfrak{A}_{2}, \ldots, \mathfrak{A}_{n}$ derived from $\mathfrak{A}$, as well as $\mathfrak{b} \mathbf{j}, \mathfrak{C}, \mathfrak{Z} \mathbb{\mathbb { B }}, \ldots$ of independent origin.

According to our second law, diffusion proceeds whenever the synaptic insulation is weak enough to be overcome by the excitement. Excitement may therefore diffuse from $A_{1}, A_{2}, \ldots, B, C, \ldots$ as well as from $A$. To what extent, if at all, do separate streams of nervous impulses arise from these sources and continue to flow in separate neural channels?

It is clear, in the first place, that if $A$ is connected to no other neurograms $A_{1}, A_{2}, \ldots$ by synaptic insulations sufficiently low for the

* We do well to remind ourselves that our sub-division of the interest-system

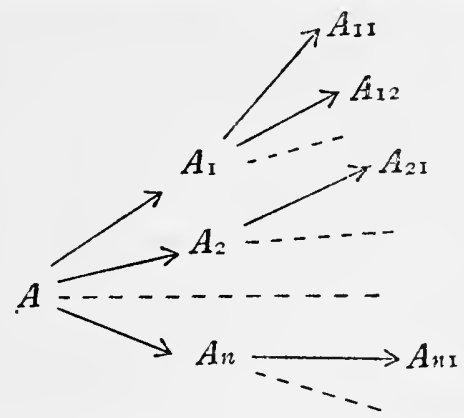

into the elementary neurograms $A, A_{1}, A_{\varepsilon}, \ldots$, is somewhat arbitrary. There may for example be low resistance paths between $A_{1}$ and $A_{2}$, so that $A_{A_{2}}^{A_{1}}$ might be described as a single neurogram, instead of three separate neurograms; but, if so, we are to suppose that the synaptic insulation between $A_{1}$ and $A_{2}$ is higher than any within the elementary neurograms in question. Thus our sub-division of a complex neurogram into elements is not wholly arbitrary, like the mathematician's sub-division of a rigid body into a number of massive particles connected by rigid weightless rods. Corresponding to our elementary neurograms are elementary thought-activities: for example, we conceive a complex thought-activity -an idea of some complex object, suppose-as built up of simpler thought-activities (such as the object's name, its colour, its shape, and so on), and these of simpler thought-activities still, and so on, until we arrive at elementary thought-activities. 
excitement in $A$ to overcome, no separate stream of impulses will start from $A$. What does happen to the excitement of $A$ in such a case will be explained directly*. Meanwhile we may note the same fact in psychological terms: a thought-activity without associations refuses to develop and to originate a stream of thought. For example, the Fuegians, when visited by Darwin in H.M.S. Beagle, betrayed no interest whatever in the brig: they saw it in the offing but did not look at it; it suggested nothing to them; it originated no train of thought. They had never seen its like before, and it could have no associations in their minds. But when they saw the ship's boats, it was quite another story; for the boats suggested their own efforts at canoe building and fascinated them at once $\dagger$.

We next observe that, since the synaptic resistance between $A$ and $A_{r}$ is not likely to be the same as between $A$ and $A_{s}$, diffusion from $A$ does not take place uniformly in all directions. On the contrary, it follows the various and separate paths of least resistance. But if these separate streams of nervous impulses continued indefinitely to go their several ways, they would ultimately issue down the pyramidal tract $\ddagger$, giving rise to disconnected movements having no common end and serving no useful purpose.

It is therefore clear that thought-activities on the fringe of consciousness, whether $\mathfrak{A}_{1}, \mathfrak{A}_{2}, \ldots$ due to the thought-activity in the focus or $\mathfrak{B}, \mathbb{C}, \ldots$ of independent origin, do not give rise to divergent streams of thought that would then issue in disconnected acts. There can however be no doubt that the action which ends a sequence of connected thought-activities is not merely due to the thought-activity that was in the focus of consciousness a moment ago: it is also affected by thought-activities that were on the fringe. For example, I am asked a question, to which, after a little reflexion, I return an answer. My answer is the action that ends a sequence of connected thought-activities, including the logical series on which I have concentrated my attention and which have enabled me to reply to the question. This logical series has occupied the focus of consciousness; but it may not be alone responsible for the answer that $\mathrm{I}$ do, in fact, return. If my interlocutor seems to be heckling

* See below, p. 79. From the 'third law' there stated, it follows that, if $\mathbf{B}$ is the next thought-activity to occupy the focus of consciousness, the excitement of $A$ is drained through $B$.

$\dagger$ See Darwin, A Naturalist's Voyage round the World, pp. 219, 229.

\# That action is the normal ending of every series of connected thoughtactivities or, in neural terms, that all excitement of cerebral neurones tends ultimately to find an outlet down the pyramidal tract, is explained below, in Chapter I 5. 
rather than seeking for information; or if I am busy and dislike being interrupted by his question; or even if I am suffering from some physical ailment causing thought-activities-sensations-which appear on the fringe of consciousness but have no connexion with the thought-activities arising out of the enquiry, then the tone, and perhaps the wording, of my reply may betray an annoyance that is due to thought-activities which never got beyond the fringe of my consciousness while I was thinking out an answer. In other words, if $\mathfrak{A} ; \mathfrak{A}_{1}, \mathfrak{A}_{2}, \ldots ; \mathfrak{b}, \mathfrak{C}, \ldots$ represent the state of consciousness immediately before the cerebral excitement is discharged down the pyramidal tract, this discharge includes the impulses diffused from $A_{1}, A_{2}, \ldots, B, C, \ldots$ as well as that diffused directly* from $A$. The impulses diffusing from all the excited neurograms thus coalesce to produce a single movement or a co-ordinated series of movements.

If the streams of impulses issuing from different excited neurograms thus coalesce at the end of such an involuntary train of thought as we are considering, may they not also converge and coalesce from time to time while the train of thought is in progress? The psychic counterpart of this coalescence of nervous impulses would be the contraction of the field of consciousness from time to time, and the contracted field would consist of little (if any) more than a comparatively intense thought-activity in the focus of consciousness.

Introspection suggests that an alternate contraction and expansion of the field of consciousness does in fact take place. Whoever will recall his experiences during a spell of involuntary thinking, or daydreaming, will probably recognise the phenomenon. At one moment his field of consciousness was gradually expanding, as the thoughtactivity in the focus called up more and more associated thoughtactivities and so increased in meaning. Then one of these derived thought-activities, or some other activity of independent origin, seemed rather suddenly to grow in importance until the focus of consciousness was transferred to it and it filled the whole field, which thus contracted in preparation for the next period of gradual expansion. And it is not generally difficult to see something special about this particular thought-activity that may have caused it thus to dominate the rest. Probably it has some emotional association; or it revived some nonemotional interest (if such there be); or its potency may have been merely due to its having been independently aroused from two or more separate sources.

* I.e. otherwise than through $A_{1}, A_{2}, \ldots, A_{n}$; it may be by a higher level path, the passage of impulses along which does not affect consciousness. 
This pulsation in the stream of consciousness has been described by William James:

As the total neurosis changes, so does the total psychosis change. But as the changes of neurosis are never absolutely discontinuous, so must the successive psychoses shade gradually into each other, although their rate of change may be much faster at one moment than at the next.

This difference in the rate of change lies at the basis of a difference of subjective states.... When the rate is slow we are aware of the object of our thought in a comparatively restful and stable way....As we take, in fact, a general view of the wonderful stream of our consciousness, what strikes us first is this different pace of its parts. Like a bird's life, it seems to be made of an alternation of flights and perchings....The restingplaces are usually occupied by sensorial imaginations of some sort, whose peculiarity is that they can be held before the mind for an indefinite time, and contemplated without changing; the places of flight are filled with thoughts of relations, static or dynamic, that for the most part obtain between the matters contemplated in the periods of comparative rest*.

From the psychological standpoint, therefore, we see reasons for suspecting that the stream of nervous impulses, diffusing from various excited neurograms, are not always divergent but, on the contrary, converge from time to time and even coalesce. Let us examine the neural phenomenon a little more closely. We start, as before, with a field of consciousness represented by $\mathfrak{A}$ in the focus, and $\mathfrak{A}_{1}, \mathfrak{A}_{2}, \ldots$, $\mathfrak{A}_{n}, \mathfrak{b}, \mathfrak{C}, \ldots, \mathfrak{f}$ (say) on the fringe $\dagger$. As excitement continues to diffuse from $A, A_{1}, A_{2}, \ldots, B, C, \ldots$ to $A_{n+1}, A_{n+2}, \ldots, A_{11}, A_{12}, \ldots$, $A_{21}, A_{22}, \ldots, B_{1}, B_{2}, \ldots, C_{1}, C_{2}, \ldots$ the field of consciousness gradually expands, $\mathfrak{A}_{n+1}, \mathfrak{A}_{n+2}, \ldots$ being added to the fringe. But sooner or later $\mathfrak{A}_{r s t \ldots .}$ (or one of the derivatives of $\mathfrak{A}$, or a new thought, say (G), but we will suppose it is $\left.\mathfrak{A}_{r s t . . .}\right)$ begins to increase rapidly in intensity and quickly dominates the whole field. The focus of consciousness is then transferred to $\mathfrak{a}_{r s t . . .}$; and, when this happens, the field changes very rapidly. Meanwhile, by our first law, the excitement $\ddagger$ of $A_{r s t . . .}$ has begun to grow quickly at the expense of the excitement of all the other neurograms that have become concerned, until finally the excitement of the whole system is concentrated in $A_{r s t \ldots .}$. In other words, $A_{r s t \ldots}$ having for some reason or other-

* Loc. cit. Vol. r, p. 243. Cf. also McDougall: 'Mental activity or thinking thus tends to progress in cycles; each cycle begins with knowing, which excites feeling and striving; the striving results in a new knowing, which satisfies the striving; and so the cycle reaches its natural termination in a feeling of satisfaction.' (Psychology, p. 62.)

$\dagger$ See Fig. 5 on p. 80 below.

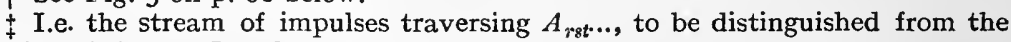
activity of $A_{\text {rst.... }}$ See above, p. 29. 
how we are about to consider*_had its excitement increased beyond that of other excited neurograms, draws to itself the excitement of them all.

Here, then, we have a case in which the intense excitement of one system of higher level nervous arcs causes that system to drain the impulses from all other active arcs of the higher level. Is this what generally occurs? Dr McDougall tells us that it is; and that the same is true of connected arcs belonging to other levels.

William James first suggested that the interruption or ' inhibition' of nervous impulses, traversing one system of arcs, by impulses discharged through another system, was a phenomenon akin to the 'draining or siphoning of certain channels by currents flowing through others.' $\dagger$ But it is to Dr McDougall that we owe the elaboration of the theory of inhibition by drainage. He thus applies it to the case of the reciprocal innervation of antagonistic muscles, e.g. the extensors and flexors of the elbow:

Let us imagine each arc in a simple schematic form as a chain of three neurones afferent $\left(a_{1}\right)$, central $\left(a_{2}\right)$, efferent $\left(a_{3}\right)$, and let us call them $a_{1}, a_{2}$ and $a_{3}$, and $b_{1}, b_{2}$ and $b_{3}$, in the two arcs respectively (Fig. 3). When a

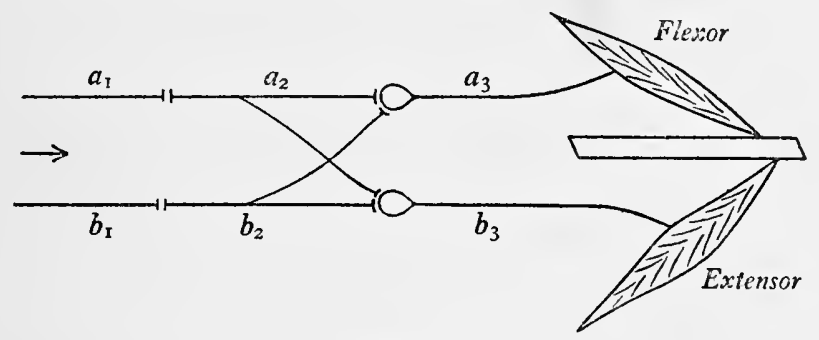

Fig. 3.

strong stimulus is applied to the afferent neurone of arc $a$, it generates neurin rapidly, so that it becomes very rapidly charged, and the resistance of synapse $a_{1}-a_{2}$ is lowered until $a$ series of discharges takes place from $a_{1}$ to $a_{2}$, and again from $a_{2}$ to $a_{3}$. The problem is, then, to imagine such a mode of connection between arc $a$ and arc $b$ as will cause arc $a$ during stimulation to drain off from the afferent and central neurones of $b$ the smaller quantities of neurin generated in them. Several forms of such a connection may be imagined, but I think that probably it takes the form of a collateral fibre coming from neurone $b_{2}$, and taking part with the axone of $a_{2}$ in forming a synapse with $a_{3}$. Whatever the exact constitution of this synapse may be, we may assume that, when its resistance is lowered by the stimulation of $a_{1}$, and consequent charging of $a_{2}$, the collateral of 
$b_{2}$, making connection with $a_{3}$, through this synapse*, becomes the path of least resistance for the escape of neurin from $b_{1}$ and $b_{2}$. These neurones are therefore drained by $a_{3}$, while $b_{3}$ ceases to receive any neurin from $b_{2}$ and the tone of the muscle group supplied by it is abolished. In a similar way, if both $a_{1}$ and $b_{1}$ be stimulated, but one more strongly than the other, the more strongly stimulated arc will drain the afferent and central neurones of the less strongly stimulated arc, because the resistance of synapses of the former will be reduced to a lower level than that of the synapses of the latter $\dagger$.

Professor Sherrington has pointed out that Dr McDougall's scheme fits a number of facts of reciprocal inhibition $\ddagger$, and also accounts 'very lucidly'§ for certain phenomena of alternating reflexes, which are common as spinal reactions. And it accords with the results of

* There is some obscurity here; for, in the first place, the diagram does not indicate clearly that the greater part of the impulses crossing the synapse $a_{1}-a_{2}$ (as well as of the impulses originating in $a_{2}$ in consequence of its stimulation from $a_{1}$ ) tends to proceed by the axone of $a_{2}$ towards $a_{3}$ rather than by the collateral towards $b_{3}$, so that the stimulation of $a_{1}$ will result in the lowering of the resistance of the synapse $a_{2}-a_{3}$ rather than (or more rapidly than) of the synapse $a_{2}-b_{3}$; and, secondly, it is not apparent that the lowering of the resistance of the synapse $a_{2}-a_{3}$ must necessarily involve the lowering of the resistance of the synapse $b_{2}-a_{3}$ between the collateral of $b_{2}$ and $a_{3}$. This obscurity is removed by redrawing the diagram thus:

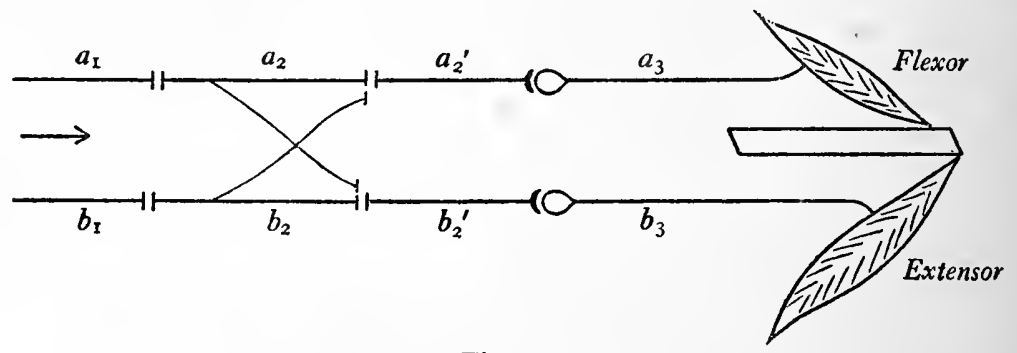

Fig. 4.

The collaterals are here shewn by finer lines to indicate that the greater part of any impulse leaving $a_{2}$ tends to proceed by the axone to $a_{2}^{\prime}$ and so to $a_{3}$ rather than by the collateral to $b_{2}^{\prime}$ and on to $b_{3}$. If now we suppose that the threshold and the resistances of the synapses between $a_{2}{ }^{\prime}-a_{3}$ and $b_{2}^{\prime}-b_{3}$ are greater than between $a_{2}$ or $b_{2}$ and either $a_{2}{ }^{\prime}$ or $b_{2}{ }^{\prime}$, and that the resistances of these last four synapses are approximately equal, the obscurity is removed. For if a stimulus is applied to $a_{1}$, excitement spreads to $a_{2}^{\prime}$ and $b_{2}^{\prime}$ but more to $a_{2}^{\prime}$ than to $b_{2}^{\prime}$ so that $a_{2}^{\prime} a_{3}$ is the more strongly stimulated arc, and its synapse $a_{2}^{\prime}-a_{3}$ is therefore (this is Dr McDougall's hypothesis expressed in our third law) reduced to a lower level than that of the synapse $b_{2}^{\prime}-b_{3}$ of the less strongly stimulated arc $b_{2}^{\prime} b_{3}$. Accordingly any excitement in $b_{1}$ and $b_{2}$ tends to escape by way of $a_{2}^{\prime}$ and $a_{3}$ rather than by way of $b_{2}^{\prime}$ and $b_{3}$.

$\dagger$ Brain, Part cIl (1903), p. r 53. Quoted by Sherrington, loc. cit. pp. 200, 201.

$\ddagger$ Loc. cit. p. $20 \mathrm{r}$.

$\S$ Sherrington, loc. cit. p. 202. Professor Sherrington adds, however, that, in his thinking, certain difficulties still attach to Dr McDougall's view. Loc. cit. p. 203. 
Professor Sherrington's own experiments with strychnine and tetanus toxin*.

But Dr McDougall by no means restricts the application $\dagger$ of his theory to the phenomena of reciprocal inhibition. On the contrary, he has applied it, as we have already indicated, to every case of a thought-activity that comes to occupy the focus of consciousness and, in so doing, drives other thought-activities out of the field of consciousness altogether. Since two unconnected thought-activities cannot both be in the focus of consciousness at the same moment, so that the arrival of the one involves the withdrawal of the other, 'we must suppose,' writes Dr McDougall, 'that systems of arcs of the higher levels are more freely interconnected than those of the spinal level (where, as we have seen, this tendency to reciprocal inhibition obtains only between certain pairs or small groups of systems), so that the free discharge of any one system in the forward direction $\ddagger$ drains any other higher-level system.' $\S$ After describing a hydro-mechanical model to illustrate how the passage of a sufficiently intense impulse through any one system of higher level paths may inhibit by drainage the passage through any other, Dr McDougall proceeds: "There are many good reasons for accepting this view of the inhibitory process. Firstly, it is applicable to the inhibitory processes of the spinal level, and evidence directly supporting it is afforded by the study of such processes in the sensory level. Secondly, if we do not accept it, we have to invoke the aid of "inhibitory centres," and find ourselves in inextricable difficulties....Thirdly, and this is the most important point, the hypothesis of inhibition by drainage offers a satisfactory solution of that crucial problem, the direction of the discharge from the one perceptual system [neurogram] $\alpha$ to any other $\beta$ excited in the succeeding moment. For it is the flow of energy, drained from $\alpha$ by $\beta$, that establishes a path of low resistance between them.' I 'The excitement of one perceptual system thrown into activity inhibits the activity of all others, because it becomes the path of lowest resistance for the discharge of energy from the sensory to the efferent side of the whole cerebral system; and the activity of the dominant system is thus augmented by the energy that it drains to itself from other parts.' 9

* Proc. R. S. LXXVI, pp. I6I and 269 and previous 'Notes' in LII, LIII, LX, LXI, LXIV and LXVI.

$\dagger$ Cf. M. Prince, loc. cit. p. 230, quoted above, p. 40.

I.e. towards the Rolandic cortex. See p. 32 above, and the fifth law on p. 273 below, and the passage quoted from William James on p. 275 below.

$\S$ Physiological Psychology, p. 132.

li Loc. cit. p. 133. Cf. above, p. 45.

II Loc. cit. p. 105. 
We proceed to give some examples of the manner in which the facts of experience are fitted by Dr McDougall's generalisation that, if a nervous arc of the higher level is sufficiently excited, it drains the excitement from all other active arcs. 'A faint tap per se,' writes William James, 'is not an interesting sound; it may well escape being discriminated from the general rumour of the world. But when it is a signal, as that of a lover on the window-pane, it will hardly go unperceived.'* As the longed-for signal and its meaning have been imagined over and over again, the tap neurogram is already excited by anticipation. Accordingly, the tap itself, when it occurs, intensifies the excitement of this neurogram, through which (according to $\mathrm{Dr}$ McDougall's generalisation) the impulses from all the active arcs are then drained: the lover's tap drowns every other thought-activity. 'We easily see now why the lover's tap should be heard-it finds a nerve-centre half ready in advance to explode. We see how we can attend to a companion's voice in the midst of noises which pass unnoticed though objectively much louder than the words we hear. Each word is doubly awakened; once from without by the lips of the talker, but already before that from within by the premonitory processes irradiating from the previous words, and by the dim arousal of all processes that are connected with the "topic" of the talk. The irrelevant noises, on the other hand, are awakened only once.' $\dagger$

Again, if an exciting thought suddenly occurs to one who is engaged in any form of physical activity, his movements become momentarily more vigorous-sometimes with unfortunate results, as when the happy recipient of a bright idea, who is at the moment singing in church, is surprised to find that he has drowned all music but his own $\ddagger$. In such a case the system of arcs, through which impulses are already being discharged towards and down the pyramidal tract, is already excited, and therefore-according to Dr McDougall's theory-the new excitement tends to be drained through that system, the outgoing impulses being correspondingly increased. In this case the path of discharge is already open when the new excitement originates.

* Loc. cit. Vol. I, Pp. 4I7, 4 I8.

$\dagger$ W. James, loc. cit. Vol. I, p. 450.

$\leftarrow$ W. James, Vol. Ir, p. 379. Another example is quoted from M. Féré by W. James: 'The strength of contraction of the subject's hand was measured by a self-registering dynamometer. Ordinarily the maximum strength, under simple experimental conditions, remains the same from day to day. But if simultaneously with the contraction the subject received a sensorial impression, the contraction was sometimes weakened, but more often increased. This reinforcing effect has received the name of dynamogeny. The dynamogenic value of simple musical notes seems to be proportional to their loudness and height.... The dynamogenic value of coloured lights varies with the colour.' 
A similar explanation applies when a sudden stimulus, or some other cause, opens a path of discharge-no matter what-for excitement already existing in the brain: the discharge that would in ordinary circumstances have been due to this cause is augmented by the previously existing excitement that is drained through the same arcs. For example: 'Everyone is familiar with the patellar reflex, or jerk upwards of the foot, which is produced by smartly tapping the tendon below the knee-pan when the leg hangs over the other knee. Drs Weir Mitchell and Lombard have found that when other sensations come in simultaneously with the tap, the jerk is increased. Heat, cold, pricking, itching, or faradic stimulation of the skin, sometimes strong optical impressions, music, all have this dynamogenic effect, which also results whenever voluntary movements are set up in other parts of the body, simultaneously with the tap.'* Or again: 'Everyone knows, that under the influence of powerful emotion, whether of joy, anger, or fear, there is discharged an increase of energy to the muscles, sometimes of an intensity which enables an individual to exert force of which he is ordinarily incapable.' $\dagger$ The path by which impulses are being discharged to the muscles that exert the force, drains the emotional excitement also.

Dr McDougall's theory thus fits and coordinates a number of facts of introspective and experimental psychology, as well as of experimental physiology. We shall shortly see that it resumes and enables us to predict many other facts that we have still to notice. Now, to 'enable us to resume and predict a vast number of facts... is the sole justification which a scientific law is ever required to possess.' + We shall therefore summarise Dr McDougall's theory by enunciating, as our third law of thought, that Any nervous arc of the higher level, if intensely excited relatively to other higher level arcs, tends to drain the impulses from those other arcs; and the same is true of connected arcs belonging to the spinal level. To this third law we may refer as the law of inhibition by drainage, or more shortly as the law of drainage.

The draining of the impulses from all excited neurograms into that ( $X$, say) which-or part of which-is most intensely excited, must, by our first law, result in the corresponding thought-activity $\boldsymbol{x}$ draining all other thought-activities out of the field of consciousness. Our third law thus accounts for the periodic contraction of the field,

* W. James, loc. cit. Vol. II, p. 380 . See also further examples of reinforcement-and especially of 'the overflow of reflex action into channels belonging primarily to other reflex-arcs than that under stimulation'-given by Sherrington, loc. cit. p. 175 .

$\uparrow$ M. Prince, loc. cit. pp. 433, 434.

$\ddagger$ B. Hart, loc. cit. p. $\mathbf{5}$. 
as our second law accounted for its expansion. Using the notation explained above*, we may now represent by means of a diagram (Fig. 5) the alternate gradual expansion and comparatively sudden contraction of the field of consciousness during the progress of an involuntary stream of thought.

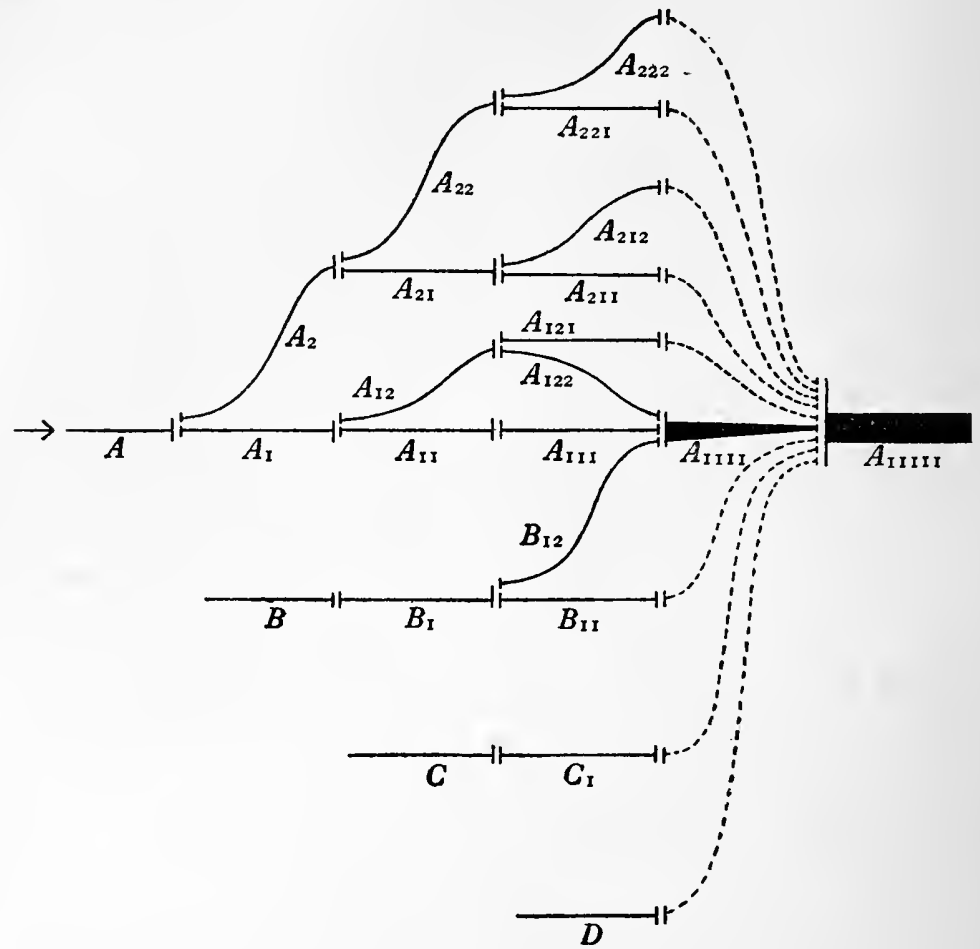

Fig. 5.

The diagram shews, very much over-simplified, the cerebral paths followed by the nervous impulses during a single pulse of the field of consciousness. Each line in the diagram represents a neurogram, or system of connected nervous arcs, such that its internal synaptic resistances $\dagger$ are all less than those of the boundary synapses through which it is connected to other neurograms. As few as possible of these boundary synapses-all of equal resistance, let us suppose, until that resistance is lowered by excitement or increased by fatigue-are

* On pp. 70,74 .

$\dagger$ Or, more accurately, insulating strengths or thresholds: see p. 69 . So long as we remember that a synapse between two neurones acts as an insulator (and not merely as a resistance) to feeble impulses, we may speak of the resistance offered by a synapse instead of speaking of the insulating strength or initial insulation or threshold of that synapse. 
represented in the diagram. Moreover, arcs that do not become active during the pulse under consideration, are not shewn at all.

The pulse begins with the thought-activity $\mathbb{A}$ in the focus of consciousness, $A$ having just drained the impulses from all other active arcs. The excitement next diffuses from $A$ to the connected neurograms $A_{1}, A_{2}, \ldots$ (of which only $A_{1}$ and $A_{2}$ are shewn in the diagram); from them to connected neurograms of the second order, $A_{11}, A_{12}, \ldots, A_{21}, A_{22}, \ldots$; and so on.

Meanwhile new sense impressions, or other thought-activities of independent origin, $\mathbf{t}, \mathbb{C}, \mathbb{1}$..., are arriving. If an incoming sensation $(\mathfrak{B}$, say) were sufficiently intense, the excitement of $B$ would lower its resistance so much that $B$ would drain excitement from all the active arcs, $\mathbf{t}$ thus receiving the whole light of consciousness*. We shall, however, suppose that the stimulation of $B, C, \ldots$ is not strong enough to cause any of those neurograms to become the path of lowest resistance. On the contrary, we shall take it that the excitement of $B, C, \ldots$ is so feeble that, as indicated in the diagram, it diffuses slowly in comparison with that of the neurograms excited through $A$. In other words, the thought-activities $\mathbf{b}$, $\mathbb{0}, \mathbb{Z}$, $\mathbb{1}, \ldots$ develop (acquire meaning) slowly in comparison with $\mathfrak{A}$ and its associations.

The diagram shews $A_{1111}$ as being triply excited $\uparrow$ : by $A_{111}$, by $A_{122}$ and by $B_{12}$ separately. By this means the excitement of the arc

* Cf. W. McDougall: 'It may be said with some plausibility that all of the sense impressions received by our organs solicit our attention. Each one, probably, tends, and tends more strongly the more intense it is, to determine the direction of our mental activity.' (Psychology, p. 57.)

$\dagger$ In our diagram $A_{11111}$ (and even $A_{1111}$ ) is thicker than $A$, suggesting increase of excitement during one pulsation. And this may well occur. When the increase is large, excitement becomes so great that it must quickly find an outletordinarily by way of the pyramidal tract, causing movement. (See below 'fifth law,' p. 273.) But no such increase need take place in the earlier pulsations of a train of involuntary thinking: $A$ may have been as much excited as $A_{11111}$ afterwards becomes. In that case, however, the excitement of. $A$, as it diffused through $A_{1}, A_{2}, A_{11}, \ldots$ must, on the average, have passed through them without increase; and even with loss, if the new excitement ultimately reaching $A_{1111}$ by way of $B, C, \ldots$ be taken into account. It is to be noted that each neurone, and therefore each neurogram, may pass on either more or less nervous excitement than reached it, for each neurone acts-partly at least-as a relay. Chemical changes are set up in it by the incoming impulses, some of the energy of which is thus absorbed; but, on the other hand, the outgoing impulses due to the remain. ing energy that traverses the neurone are augmented by reason of the chemical changes in the neurone itself.

We do well to note, also, that while $A_{1111}, A_{1111}$ drains the 'excitement' (i.e. the nervous impulses-see pp. 28, 29) from the other active neurograms, it does not drain their 'activity.' On the contrary, as will be explained below (p. 85) the activity remains for a while and may give rise to further excitement-impulses traversing the neurograms in question and producing thought-activities-before .finally subsiding.

G. E. 
(or system of arcs) $A_{1111} A_{1111}$ is intensified beyond that of other simultaneously active arcs. According to our third law, it therefore drains* the impulses from them all. But there are many other means by which the intensification of the draining arc might be effected. We proceed to study some that are of special importance.

Among the specially important means of producing relativelyrelative, that is, to the excitement of other active arcs-intense excitement in a particular arc or neurogram, and so causing it to drain the impulses and bring the corresponding thought-activity into the focus of consciousness, we may place first the direct stimulation of sensory end organs. A violent sense impression, $\mathbf{D}$, will always attract attention to itself, away from other objects- $S$, in short, will drain the impulse-unless other thought-activities in progress are of peculiar intensity. That is to say, although the excitement of $S$ may be great, it may not be great relatively to that of other active arcs. My thought, as we have said, must be very engrossing if I fail to notice a thunder-clap nearby. And yet thought-activities may be so intense, so absorbing, as, in William James' words, 'not only to banish ordinary sensations, but even the severest pain.' $\dagger$ He goes on to quote Sir William Hamilton:

Archimedes, it is well known, was so absorbed in geometrical meditation that he was first aware of the storming of Syracuse by his own death-wound, and his exclamation on the entrance of the Roman soldiers was: Noli turbare circulos meos! In like manner Joseph Scaliger, the most learned of men, when a Protestant student in Paris, was so engrossed in the study of Homer that he became aware of the massacre of St Bartholomew, and of his own escape, only on the day subsequent to the catastrophe....And it is reported of Newton that, while engaged in his mathematical researches, he sometimes forgot to dinef.

A second important means of producing relatively intense excitement in a particular neurogram consists in the 'circular nervous process'§ which, as already\| explained, multiplies the excitement whenever the affective-conative portion of an instinct-neurogram is stimulated. Terrify a man, or annoy him sufficiently, or (if he is in

* The dotted paths by means of which the impulses are drained to $A_{1111} A_{1111}$ are for simplicity shewn as being connected with the drained neurograms by means of single synapses only. We are, however, to suppose that impulses may be drained from every part of a neurogram at the same time.

$\dagger$ Loc. cit. Vol. I, p. 4 I9.

‡ Sir W. Hamilton, Metaphysics, Lecture XIV, quoted by W. James (loc. cit.) who adds 'The oft-cited case of soldiers not perceiving that they are wounded is of an analogous sort.'

$\S$ W. McDougall, Physiological Psychology, p. I 6 .

II See above, pp. 54, 55 . 
love) mention the name of his sweetheart; and his emotion-fear, or anger, or tender emotion-and his thought of its object will drive all else from his consciousness. In fact, an instinct-neurogram, whenever and however it is stimulated, tends to drain the impulses and to bring its object into the focus of consciousness.

Thirdly, multiple stimulation of any neurogram produces a higher degree of excitement than would be produced by one of the stimuli acting alone. We have already referred to this means of causing a particular neurogram to drain the impulses from all other active arcs. The arc which drained the impulses in our diagram (p. 8o) owed its relatively intense excitement to this cause. And the profound effect of the lover's tap on the window, as well as other examples quoted above* from William James, further illustrate the influence which the excitation of a neurogram from two or more independent sources may have in bringing the corresponding thought-activity into the focus of consciousness. If, when listening to a lecture, some remark of the lecturer's starts in my mind a train of thought different from that which he is following, I probably pursue my own thoughts until, suddenly, a word or phrase of the speaker's happens "exactly to fit what is passing in my mind at the moment. It is thus, as William James says, doubly awakened; and, as its neurogram drains theimpulse, it fills my field of consciousness, and my attention returns to the lecture.

Professor Sherrington's experiments on spinal dogs $\uparrow$ shew that multiple stimulation may cause an arc of the spinal level to drain excitement from connected arcs. If a sufficient stimulus be applied to the shoulder skin of a spinal dog, there result scratching movements of the hind leg on the same side, but no movements occur if the stimulus has less than a certain minimum intensity. If now an efferent arc, the sufficient excitement of which would result in scratching, be excited by a subminimal $\$$ stimulus, the application of a subminimal stimulus to another efferent arc leading to the same final common (efferent) path, will cause that path to drain excitement from both

* On page 78. The following is another example from the same source: 'In looking for any object in a room, for a book in a library, for example, we detect it the more readily if, in addition to merely knowing its name, etc., we carry in our mind a distinct image of its appearance.' (Vol. I, p. 504.)

$f$ I.e. dogs whose spinal cords have been transected in the region of the neck, so cutting off the brain from direct connexion with the reflex mechanisms of the spinal level. See above, p. 35 .

¥ It would be interesting to know whether the effect of the subminimal stimulus to the first efferent arc could be detected if the stimulation of the other efferent arc were not subminimal. In short, is it possible to detect an increment of the scratching reflex due to a subminimal stimulus? 
excited efferent arcs*. The double stimulation of the final common path results in drainage.

But Professor Sherrington went further, and shewed that the excitement of two neurograms, $A$ and $B$, might combine to stimulate a third connected neurogram, $C$, even though $A$ and $B$ were not stimulated simultaneously, provided that too long an interval did not elapse between the stimulation of $A$ and the subsequent stimulation of $B \dagger$. In other words, $A$ when once excited remains active for a finite period of time. The same fact was clearly proved by experiments on reflex 'after-discharge.' $\ddagger$ When the reflex was evoked by a strong stimulus, the excited arcs would go on discharging the impulses that produced the reflex movement, sometimes for as long as Io or I5 seconds $\S$ after the stimulus had been discontinued. The fact that neurograms of the spinal level remain active for some time after being excited, suggests that the same is true of higher level neurograms also. There is one class of higher level neurograms of which it is obviously true, namely instinct-neurograms. 'In psychological language it is said that the emotion, once excited, tends to maintain itself until exhaustion ensues, or until it has achieved its object.' $\|$ 'Fear, and the bodily symptoms of fear, provoked by a sudden and momentary impression, persist for some little time, though the harmless character of the disturbing incident is at once realised. Hence the sudden anger dies slowly away, though its cause be removed, and is apt to vent itself upon the unoffending. Hence the tears and lamentations may continue to flow though the cause of grief no longer exists. This slow passing away of the emotional state is of course best displayed by children, as by the child who continues to sob in his mother's arms though he has realised that the terrifying face or the growling wild beast was only his elder brother in disguise.' 9 But, while the 'after-discharge' of higher level neurograms is most manifest, and probably of longest duration, in the case of instinctneurograms, there is no reason to doubt its existence in the case of other neurograms. The utterance of the lecturer whose argument I had ceased to follow will still serve to recall my wandering attention if, instead of synchronising with a similar element, $\mathfrak{A}$ say, in my own train of thought, it followed it after not too long an interval: $A$ must meanwhile have continued active. And during the moments that precede falling asleep at night-when, as we say, we have made our

* Sherrington, loc. cit. Fig. 38, p. I19, and p. 123.

\# Loc. cit. Lecture I, pp. 26-35.

$\dagger$ Loc. cit. pp. 123, 124.

II W. McDougall, Physiological Psychology, p. II 5 .

$\S$ Loc. cit. p. 30.

If Loc. cit. p. I 16. 
minds as far as possible blank-it is not only, so far as we can judge, the emotionally exciting events of the day that are revived by the continued activity and renewed excitement of the corresponding neurograms. We may take it, then, that every interest-system which includes affective-conative neurograms*, and probably every other (if there be any other) interest-system also, maintains its activity for some time after being excited. This is a most important principle. For it means that when once any considerable interest-systemespecially if it be rich in emotional elements-has been stimulated, it will tend for some time to reinforce the excitement in any neurograms that are connected with it, and so to make such neurograms drain the impulses. In other words, any considerable interest $\uparrow-$ which is sure to be also, in some measure, an emotional interestcontinues, for some time after it has been awakened, to exercise a selective influence upon thought, tending to bring into the focus of consciousness those thought-activities which have some association with the interest in question + . An excellent example is furnished by Poincaré's§ description how the discoverer's interest in his subject, and especially in the general trend of the argument, exercises a selective influence upon his involuntary thought-activities, tending to bring into the field of consciousness only those that are interesting, that fit in with the general trend of the argument in which he is interested $\|$.

The continued activity of an interest-system once excited has an important bearing on education as well as on mathematical and other

* It is doubtful whether any interest-system exists without affective-conative elements.

+ See the definition of 'interest' on pp. 62,63 .

$\ddagger$ Dr Bernard Hart has already pointed out that an active complex (see above, p. 6r) will thus influence trains of thought. But, as we have seen, the emotional element that is an essential element of a complex is not essential to the exercise of this influence: an active interest-system lacking affective-conative neurograms might still produce the effect. Dr Hart writes: 'Complexes, then, are causes which determine the behaviour of the conscious stream, and the action which they exert upon consciousness may be regarded as the psychological analogue of the conception of "force" in physics. They are not, of course, constantly active, but only become so under certain conditions. These conditions consist in the presence of a "stimulus," occurring whenever one or more of the ideas belonging to a complex is roused to activity, either by some external event, or by processes of association occurring within the mind itself.... So soon as this necessary stimulation has occurred, the complex immediately tends to exert its effect upon consciousness. The effect consists normally in the introduction into consciousness of ideas, emotions, and trains of activity belonging to the complex. Of the ideas, arguments, etc., presented to the individual, those which are in harmony with the complex are reinforced, whereas those not so in harmony tend to be inhibited and to lose their cogency.' (Loc. cit. pp. 62, 63.) 
research. It is essential to good teaching that the teacher awaken his pupils' interest in his subject, so that this interest may cooperate with his exposition in guiding their thoughts, emphasising those that are connected with this interest, and thus harmoniously building up the interest 'so that the mind can, without effort, take in the whole without neglecting the details.'* As Herbart wrote of the teacher's anecdote told to the schoolboys whose minds were wandering: No sooner did the teacher's words awaken the pupils' well-developed interest in the subject of the lesson, an interest containing 'strongly connected series with which the new impression easily combined, than out of new and old together a total interest resulted which drove the vagrant ideas below the threshold of consciousness, and brought ...settled attention into their place.' $\dagger$

A less admirable or useful consequence of the maintenance of activity in an interest-system that has recently been stimulated is the 'bias' it may give to one's thoughts. If we argue with a party politician, his political interest will quickly be excited and will thereafter 'reinforce in his mind those arguments which support the view of his party.' $\ddagger$ And, in a similar way, all my thinking of a man for whom I entertain a sentiment of hatred is biassed by my attitude§.

The foregoing examples must suffice to illustrate this third means - multiple stimulation - by which a neurogram may be made to drain the impulse. Among the most important examples we have given are those in which the neurogram that drained the impulse did so because of its connexion with an active interest-system. We have next to notice that the mere connexion of a neurogram with an inactive interest-system may be sufficient to intensify excitement in that neurogram. This is a fourth important means of intensifying excitement in a neurogram so as to cause the draining of the impulse.

Suppose that a neurogram $A$ is connected, by equal and similar synapses, with two neurograms $A_{1}$ and $A_{2}$; and that these in turn respectively connect, by equal and similar synapses, with two more equal and similar neurograms $A_{11}$ and $A_{21}$. Then, if $A$ be excited, there is no reason why impulses from $A$ should traverse either $A_{1} A_{11}$ or $A_{2} A_{21}$ in preference to the other: if the excitement of $A$ issufficient, $A_{1}$ and $A_{2}$, and afterwards $A_{11}$ and $A_{12}$, will become equally excited.

But now suppose that $A_{1}$ connects not only with $A_{11}$ but also with $A_{12}$. Then, if the initial insulation (and resistance) of the

* Poincaré, loc. cit. p. 59.

$\dagger$ Herbart, Psychologie als Wissenschaft, § 128, quoted by W. James, loc. cit. Vol. I, p. 418 . (See p. 63 above.)

$\ddagger$ Cf. B. Hart, loc. cit. p. 65.

$\S$ See above, pp. 60, 6I. 
synapse $\overline{A_{1} A_{12}}$ be less than that of the synapse $\overline{A_{1} A_{11}}$ or $\overline{A_{2} A_{21}}$, impulses will begin to pass from $A_{1}$ before they pass from $A_{2}$. Suppose now that $A_{1}$ (and likewise $A_{2}$ ) is composed of more elementary neurograms, as shewn in the diagram (Fig. 6), the resistances between neighbouring elementary neurograms-for example, $a_{1}^{\prime}$ and $a^{\prime \prime}{ }_{1}-$ being of course less than those of the synapses joining $A_{1}$ or $A_{2}$ to its neighbours. Then the excitement that escapes from $A_{1}$ must, in part at least, be conveyed through $a^{\prime \prime}{ }_{1}$ to the synapses $\overline{A_{1} A_{11}}$ and $\overline{A_{1} A_{12}}$. Accordingly the transfer of impulses through $a^{\prime \prime}{ }_{1}$, if that elementary neurogram be taken sufficiently small, is greater, in the case supposed, than that through $a^{\prime \prime}{ }_{2}$; and, if this excess is sufficient, $a^{\prime \prime}{ }_{1}$ (or $A_{1}$ ) will, by our third law, drain the impulse from $a^{\prime \prime}{ }_{2}$ (or $A_{2}$ ).

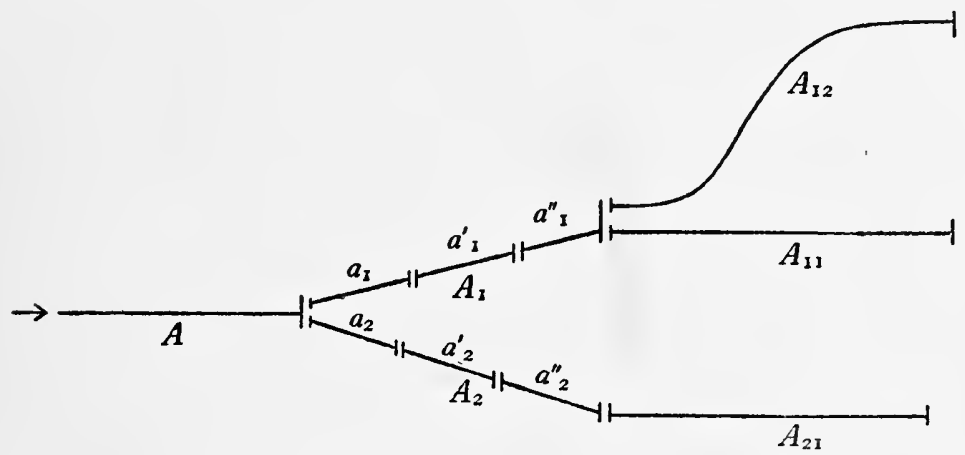

Fig. 6.

If now the initial insulation of the synapse $\overline{A_{1} A_{12}}$ is not less than that of $\overline{A_{1} A_{11}}$ (or of $A_{2}$ ), it is still true that, so soon as the excitement of $A_{1}$ is sufficient to diffuse across the synapse $\overline{A_{1} A_{12}}$ as well as across $\overline{A_{1} A_{11}}$, the excitement of $A_{1}$ will be greater than of $A_{2}$; and $A_{1}$ will therefore (by our third law) tend to drain the impulse, not only from $A$ and $A_{2}$, but from any other independently excited neurogram, $B, C, \ldots$.

In either case $A_{12}$ does not exercise any effect upon the excitement of $A_{1}$ (and thus upon the selection of $A_{1}$ rather than $A_{2}$ as the path to be followed by impulses from $A$ ) until $A_{12}$ has begun to be excited. It might therefore seem that we are again dealing with the influence of an active neurogram on the direction of the stream of impulses; and in a sense this is true. But the case here considered differs from that of multiple stimulation just discussed. For, in that case, the neurogram $(B$, say) whose activity intensified the excitement of the draining neurogram (say $A_{1}$ ) was already active before $A_{1}$ and joined 
with $A$ in stimulating $A_{1}$. Here however the directing neurogram $A_{12}$ derived its activity from $A_{1}$ and was therefore inactive when the excitement began to spread from $A$ into $A_{1}$ and $A_{2}$. This then may be spoken of as the case of an inactive neurogram determining the direction of the flow, causing it to take place from $A$ to $A_{1}$ rather than from $A$ to $A_{2}$.

It follows that, in general, of two neurograms $\left(A_{1}\right.$ and $\left.A_{2}\right)$ connected with an active neurogram $A$, that one $\left(A_{1}\right)$ which is itself connected-through synapses of which the initial insulations and resistances are not too high-to more neurograms, whether active or inactive, than the other $\left(A_{2}\right)$ will, caeteris paribus, attract the impulse from $A$ and drain the system. But observe that, if $A_{1}$ were connected to a third neurogram $A_{13}$ by a synapse of which the initial insulation was considerably higher than that of $\overline{A_{1} A_{12}}$, this third connexion might have little if any influence in directing the flow of impulses and the corresponding stream of thought. First suppose, as before, that the synapse $\overline{A_{1} A_{12}}$ has a lower initial insulation and resistance than the equal synapses, $\overline{A_{1} A_{11}}$ and $\overline{A_{2} A_{21}}$. Then, of these three synapses, the impulse first crosses $A_{1} A_{12}$; and if, as it proceeds along $A_{12}$, it meets no higher insulation than $\overline{A_{1} A_{11}}$, the impulses from $A$ will continue to pass along $A A_{1} A_{12}$. The larger the system of sufficiently low resistance paths into which $A_{12}$ leads, the more likely will this be to occur. Next suppose that the synapses $\overline{A_{1} A_{11}}$ and $\overline{A_{2} A_{21}}$ open first, and that $\overline{A_{1} A_{12}}$ opens afterwards. The amount of impulse crossing $\overline{A_{1} A_{12}}$ will still depend on the resistance of the path $A_{12}$ as well as on that of the synapse $\overline{A_{1} A_{12}}$; and the larger the system of low resistance paths (and the lower their resistance) to which $A_{12}$ leads, the more potent will its influence be in deciding the impulse from $A$ to proceed by way of $A_{1}$ rather than by way of $A_{2}$. If now there were a third neurogram $A_{13}$ into which $A_{1}$ led through a synapse $\overline{A_{1} A_{13}}$ of which the initial insulation were greater than that of $\overline{A_{1} A_{12}}$, we see at once that, provided $A_{12}$ presents a path that remains of sufficiently low resistance until the excitement of $A_{1}$ has begun to wane, the synapse $\overline{A_{1} A_{13}}$ may never open. We conclude that it is the width (or size) and depth (or intensity*) of the interest-system to which $A_{1}$ is connected (through $A_{12}$ ) that influences the attraction of the impulses from $A$ into $A_{1}$ rather than into $A_{2}$. Of course, if $A_{1}$ is directly connected by low resistance synapses to several neurograms, $A_{1}$ forms part of an interest-system and tends all the more to attract the impulse. But the point for us

* See above, p. 44 . 
to note is that the mere fact of $A_{1}$ 's connexion with an interestsystem is sufficient to cause it (rather than $A_{2}$ having no such connexion) to attract the impulse; and the potency of $A_{1}$ in this matter is the greater, the wider and deeper (more intense) the interest-system to $A_{1}$ 's connexion with which this potency is due*.

Generalising this result we see that, of all neurograms $A_{1}, \ldots, A_{n}$ connected with $A$, that one which is itself connected to (or which forms part of) the widest and deepest interest-system, tends, other things being equal, to attract and drain the impulse from $A$. If now we suppose that there are other sources of excitement, $B, C, \ldots$, independent of $A$, we finally arrive at the following corollary to our third law: Of all active neurograms, that one which is connected with (or forms part of) the ridest and deepest interest-system, tends, other things being equal, to drain the impulse from them all. The psychological counterpart of this corollary is: Of all thought-activities on the fringe of consciousness at any moment, that one which is connected with the widest and strongest interest tends, other things being equal, to enter the focus and receive attention; or, more simply and loosely, we tend to think of things that interest us. We observe, in passing, that emotional interests-interests which cannot be excited without arousing emotional feeling-are generally the most intense (deepest) and therefore the most potent in guiding thought. For we have seen that stimulation of affective-conative neurograms generally causes a large increase of the total volume of cerebral excitement $\dagger$ which must result in the deepening of all the connected neurograms to which this intense excitement diffuses. And it follows that the intensity of a system of neurograms is greater, as a rule, the richer that system is in affective-conative elements.

We proceed to illustrate our conclusion that even inactive interestsystems, especially if rich in affective-conative elements, may exercise a directing influence on neural impulses.

William James observed that connexion with an interest-system, even if inactive, tended to make a neurogram drain the impulse. $\mathrm{He}$ points out, for example, that when a sense impression, 'without being either strong or of an instinctively [emotionally] exciting nature, is connected by previous experience and education with things that are

* This result is still true, whether the impulse finally passes by way of $A_{1} A_{12}$ or $A_{1} A_{11}$ : equal external stimuli to $A_{11}$ and $A_{21}$ might necessitate the impulse being ultimately drained by one or other of these arcs, when the influence of $A_{12}$ and its interest-system would secure that the impulse was drained by $A_{1} A_{11}$ rather than by $A_{2} A_{21}$.

$\dagger$ See above, p. 55 . 
so...the result is that it is bruught into the focus of the mind.' * $\mathrm{He}$ cites cases in which a thought-activity ' takes the initiative and draws our attention to itself, not by reason of its own intrinsic interest, but because it is connected with some other interesting thing.' $\dagger$ And, finally, he insists that 'in mature life we never attend to anything without our interest in it being in some degree derived from its connexion with other objects.'...+ 'What-we-attend-to and whatinterests-us are synonymous terms.' $\S$ The facts that attract the attention of physicists and interest them are those...that have an analogy with many other facts and do not appear to us as isolated, but as closely grouped with others.' $\|$

We have already quoted Herbart's description of how the influence of an active interest-system accounts for the maintained attention of disorderly pupils during their teacher's anecdote. At other times, the 'instruction being uninteresting, and discipline relaxed, a buzzing murmur was always to be heard, which invariably stopped for as long a time as an anecdote lasted. How could the boys, since they seemed to hear nothing, notice when the anecdote began?'** We now have the answer. So soon as the neurograms excited (although very slightly, so that the corresponding thought-activities never got beyond the fringe of an inattentive boy's consciousness) by the teacher's utterance began to have connexions with an interest-system, even if inactive, these connexions tended to make the neurograms thus excited drain the impulse from all the other active neurograms. Thus the corresponding thought-activities came into the focus of consciousness. The interest-system was at the same time rendered active and maintained the boys' attention so long as it was, from time to time, re-excited by the anecdote that concerned it.

Or suppose $\uparrow \uparrow$ that my hobby is photography. It is unnecessary for the moment to assume, with Dr Hart, that my photography interest-system is rich in affective-conative elements-in other words, that my interest in photography is coloured by emotion-although this will doubtless be the case. 'It is obvious that the existence of this hobby will continually affect the flow of my consciousness.' Even if I have not been thinking of photography for some time, so that

* Loc. cit. Vol. I, p. 417. He adds: 'The impression draws an interest from them, or perhaps it even fuses into a single complex object with them.'

$\dagger$ Loc. cit. Vol. I, p. 449.

$\S$ Loc. cit. Vol. II, p. 559.

† Loc. cit. Vol. I, p. 434.

If Above, p. 86.

i) Poincaré, loc. cit. p. 27.

** Herbart, Psychologie als Wissenschaft, $\S \mathrm{I} 28$, quoted by W. James, loc. cit. Vol. I, p. 4 I 8 .

†† Cf. B. Hart, loc. cit. p. $6 \mathrm{I}$. 
my photography interest-system is inactive, 'scenes which would otherwise be indifferent to me will frequently arouse interest as possible material for a picture: if I peruse a newspaper an article upon photography will at once arrest my attention.'*

Or suppose, with Dr McDougall $\dagger$, that, being interested in animal behaviour, my glance falls upon a cat seated on the back of a pony. I may not have been thinking of animal psychology for a long time, and my interest-system may be quite inactive, yet, 'out of all the details of the scene presented to my vision, my mind seizes upon these two objects and their relation.' Had the cat 'been seated on the ground at some little distance from the pony, I should have noticed both animals only in the most fleeting fashion, if at all.' It is only because, in their attitude of friendliness, 'they mean $\ddagger$ for me far more than is actually presented to the eye, that the situation appeals to an interest and draws my attention.' $\S$ Or again, to go back to the 'monster of iniquity' described above\|, my sentiments of hatred may be quite inactive, I may not have seen or thought of him for some little time, and yet 'if I pass by a crowd of which he is a member, my eye singles him out'; 'if I overhear his name mentioned by others in conversation, I am all agog to hear what is said.' $q$

We have already** noticed the bearing on the teacher's art of the proposition that connexion with an active interest-system increases the likelihood that any neurogram (directly excited by the teacher) will drain the impulse and so bring the corresponding thought-activity into the focus of consciousness. The more general proposition stated in the corollary to our third law, and illustrated by the foregoing examples, has pedagogic consequences of even greater importance.

That corollary tells us that, in involuntary thinking, with which alone we are as yet concerned, we are more likely to think of objects whose neurograms are connected with interest-systems than of other objects whose neurograms have no such connexions. And we are most likely to think of objects whose neurograms are connected with interest-systems that are most extensive and richest in affective-

* B. Hart, loc. cit. p. 6r. Jung's experiment, cited by Dr Hart (loc. cit. p. 70), further illustrates the influence of inactive interest-systems, for it shews how the connexion of a thought-activity (in this case a word pronounced by the experimenter) with an interest-system may divert the attention of persons experimented on from the business in hand-that of replying with a 'reaction-word '-and so delay the response.

$\dagger$ Psychology, pp. 99, ror.

† I.e. have associations or, in other words, are connected with an interest. See above, p. 45 .

$\S$ Loc. cit. p. ror.

II W. McDougall, Psychology, p. r 6 .

II See pp. 6o, 6r.

** Above, p. 85 . 
conative elements: a wide emotional interest attracts thoughtactivities associated with it into the focus of consciousness.

Now, an 'inactive' interest-system becomes active in the process of causing a connected neurogram to drain the impulse. It follows, as is indeed obvious to introspection, that wide and deep interestsystems are peculiarly liable to be rendered active. So long as the activity of an interest-system continues, other objects whose neurograms are connected with the system are especially likely to receive attention. As their neurograms from time to time drain the impulse, not only do these neurograms become connected with the system (the connecting synapses having their resistances permanently lowered in some degree by the transfer of excitement across them during draining); but the neurograms of other thought-activities, in the field of consciousness at the moment of draining, become connected with the interest-system as all are drained together. Thus an interest-system tends to grow. And the tendency is the greater, the wider and deeper (more intense) the system already is.

The instinct of curiosity, with the emotion of wonder that forms its affective part, influences the growth of interest-systems. It would seem that the appropriate stimulus of this instinctive process is the unlooked-for occurrence together of familiar objects habitually noticed but not previously associated*. So soon then as a neurogram $A$ (especially if it be the netrogram of some simple and familiar object) drains excitement from one or more other neurograms $B, C, \ldots$ (especially if they be neurograms of simple and familiar objects) and so becomes connected with them for the first time, a 'circular nervous process' is set going whereby the excitement of the brain is increased. Thus the connexions between $A$ and $B, C, \ldots$ are deepened (rendered more intense) and the interest-system $A-B-C-\ldots$ becomes more permanent. Moreover, the activity of $A$, as well as of $B, C, \ldots$, is increased; so that not only do $\mathfrak{A}, \mathfrak{b}, \mathfrak{C}, \ldots$ tend to remain in the field of consciousness for some time, but other low resistance paths tend to form between $A$ and the interest-system $B-C-D-\ldots$ as the excitement gradually diffuses throughout that system. This tendency to form other connexions between $A$ and the interest-system with which it has, perhaps fortuitously, become connected is the conative element of the instinctive process.

* Cf. W. McDougall: 'The native excitant of the instinct would seem to be any object similar to, yet perceptibly different from, familiar objects habitually noticed ' (Social Psychology, p. 57); and Poincaré, 'Elegance' (which, as Poincaré explains, causes emotion, doubtless the emotion of wonder) 'may result from the feeling of surprise caused by the unlooked-for occurrence together of objects not habitually associated.' (Loc. cit. p. 3I.) 
For example children who are familiar both with yellowness and with stockings, but in whose minds these two things have not previously been associated, are surprised-wonder-when they see a boy dressed in the uniform of Christ's Hospital. Their curiosity is awakened. Its conative element makes the children want an explanation of the yellow stockings; that is, they want to know the meaning of the yellow stockings; or, in other words, each of them wants to form further neurograms connected both with his yellowness-neurogram and with his stockings-neurogram, and so connecting these two neurograms with each other. If an adult is present, the conative tendency may cause the children to say: 'Why does that boy wear yellow stockings?' But if not, and if the children have not been well brought up, their curiosity may find a less innocent outlet.

Take a more serious illustration. Suppose the anecdote about Newton and the apple to be true. After much reflexion, and ultimately with considerable excitement, Newton has formulated the second law of motion: that force varies as the mass-acceleration it produces. His 'force' interest-system (including an 'acceleration' neurogram) being wide and deep and, we will suppose, active, he goes into the orchard and sees an apple fall with uniform acceleration. 'Acceleration' connects its fall with the 'force' interest. Its fall, rather than any other object in his field of vision, tends therefore* to attract his notice; and the 'fall' neurogram is for the first time connected with the 'force' interest-system. In the unlooked-for occurrence together of the (to Newton) familiar conceptions of 'falling' and 'force,' there is the appropriate stimulus to awaken curiosity. He feels the emotion of wonder $\uparrow$. The conative element in his curiosity makes him seek for an explanation of the connexion between force and falling; he wants the meaning of the connexion; he wants, that is, other associations. And he finds what he wants in the conception of a general law of gravitation. His 'force' interest-system is for ever the wider for this experience. Whenever in future he thinks of falling, he tends to think of force-the force of gravity - and to feel wonder.

And, in general, the cooperation of curiosity-wonder in the development of wide interest-systems must increase their depth (intensity) as well as their width; for, if we have correctly interpreted the part played by this instinct, it must continually introduce affectiveconative elements into every growing interest-system.

* In accordance with the corollary to our third law.

$\uparrow$ Among the visceral arcs included in the affective-conative neurogram are probably arcs the excitement of which causes the erection of the hairs of one's body: the phenomenon known as goose flesh. 
The function of curiosity would thus seem to be to intensify the excitement of a neurogram with which a growing interest-system has just made connexion; and so, if possible, to multiply the connexions between the newly incorporated neurogram and the old interestsystem. Apart from some such effect, the excitement would tend to flow at once away from the newly incorporated neurogram into the centre of the old interest-system: the fall of the apple would have made Newton think again of force and he would quickly have forgotten, if indeed he had ever noticed, that the apple's fall had anything to do with the return of his attention to a favourite topic. For, if we conceive the neurograms of which an interest-system is composed as being on the average more intimately interconnected towards the centre of the system than towards the periphery (and we can hardly conceive them otherwise), the argument on pp. 86 to 88 shews that excitement tends continually to spread in the direction of increasing multiplicity of interconnexions. From any neurogram on the periphery of an interest-system, the excitement tends to flow towards the heart of that system. This conclusion has, as we shall see later*, an important bearing on the problem of training character.

* See below, Chapter r6. 


\section{CHAPTER 7}

\section{WILL AND GENERAL ABILITY}

\section{$\S \mathrm{I}$. Psycho-physical Interaction.}

THE preceding discussion of our third law of thought and its consequences has been concerned with involuntary* thinking. Is this the only kind of thinking? Many psychologists and physiologists believe that if we could completely describe the structure of the nervous system of any man or animal, and had a complete knowledge of the laws of the physical and chemical processes that occur in it, we should be able to account completely for all the conduct of that individual.' $\dagger$ Are they right? Or were we right in adopting $\$$ the 'common-sense' view that human souls exist and influence human behaviour?

The former opinion is held in several forms. Some of its supporters follow Huxley in supposing that our thought-activities-whether perceptions, images, feelings or volitions-as such, have no influence upon our conduct; that they are mere 'epiphenomena,' caused by, but not reacting upon, neural activities in the brain. Others say that the psychoses and the neuroses form two series that run parallel to one another but never meet or interact. And others again hold that the physical and the psychical are two modes in which one series of real events appears to us, and that therefore the two series of appearances run parallel to one another. Hence this view, in its second and third forms, is known as the 'hypothesis of psycho-physical parallelism.' $\S$

The alternative to epiphenomenalism, as well as to psychophysical parallelism, is the hypothesis of psycho-physical interaction. According to this view, soul and body, or psychical and physical processes, act and react upon one another so that psychical processes play a part in determining conduct. And this view is not merely the common-sense view, as we have called it: it is not confined to the man in the street or to old-fashioned philosophers, but is shared by

* See above, p. 65.

$\dagger$ McDougall (Physiological Psychology, p. 7) thus formulates a view which he characterises as questionable and paradoxical. The following paragraph, concerning the hypothesis of psycho-physical interaction and its alternatives, closely follows McDougall's account.

$\ddagger$ See above, p. 9.

$\S$ See footnote $\dagger$ on p. 65 . 
some of those who, in recent years, have done most to advance psychological science.

Thus Dr McDougall* has argued that the hypothesis of interaction is, a priori, more reasonable than that of parallelism or epiphenomenalism. William James also has declared his belief in psycho-physical interaction, but rather for ethical $\dagger$ than for logical or psychological reasons. Dr Bernard Hart and Professor Spearman are on the same side ${ }_{\ddagger}$. Dr Morton Prince agrees that volition can govern conduct $\S$. Mr A. F. Shand must also be reckoned an interactionist, for, although he regards every interest-system as possessing one or more 'wills' of its own $\|$, yet he recognises the difficulty of asserting that there is no will but that which belongs to some emotion or sentiment; and he speaks of choosing between the wills of conflicting sentiments by a will which belongs to neither system $\uparrow$. He adds:

Now supposing that such volitions occur in fact, even if rarely, from what do they proceed?...our personality does not seem to be the sum of the dispositions [systems] of our emotions and sentiments. These are our many selves; but there is also our one self. This enigmatical self which reflects on their systems, estimates them, and, however loath to do it, sometimes chooses between their ends, seems to be the central fact of our personality.

If this be the fact, it is not the kind of fact which we can take into account. The science of character will be the science of our sentiments and emotions [or, more generally, of our interest-systems] - of these many selves, not of this one self.

But it is just this 'central fact of our personality' that we are here concerned with.

It is true that lists of very distinguished names might also be cited in support of parallelism or epiphenomenalism. We are not here

* Physiological Psychology, p. II. The argument concludes: 'We are then logically compelled to believe that neural processes and psychical processes are causally related according to ascertainable laws.'

$\dagger$ Loc. cit. Vol. I, p. 454.

$\ddagger$ 'Many of us,' they write, ' are looking forward to the day when psychological text-books will become less exclusively pre-occupied with sensations and epistemology, but turn rather to appreciating the mind or "soul" as the agent in conduct.' (Bernard Hart and C. Spearman, $B$ J. P. Vol. v (I9I2-I3), p. 77.) They add, in a footnote: 'Significant of the coming change are such definitions of psychology as that of McDougall (Ph. Ps. p. I); also such books as Le Leggi del Lavoyo Mentale by Guido della Valle, or the works of C. Henry (Paris).'

$\S$ Loc. cit. p. 458 .

|| 'Strength or weakness of will, other things being equal, varies with the strength or weakness of the emotion [i.e. in Mr Shand's terminology, a particular kind of interest of which several may be contained in one sentiment] or sentiment to which it belongs; and hence it is that we find the same man strong in some directions and weak in others.' (Loc. cit. p. 65.)

I Loc. cit. p. 66. 
concerned to strike a balance between divergent opinions. We do well, however, to note that neither the interactionists nor those who hold a contrary view are able to give conclusive evidence in support of their opinions. 'The result is two conceptions of possibility'-that interaction does, and that interaction does not, take place- 'face to face, with no facts definitely enough known to stand as arbiter between them.' * Any decision between the opposing theories must therefore be provisional; but some decision, however tentative, is necessary for our purpose, nor can it be further postponed. Following William James, we turn to ethical considerations to incline the balance so as to enable us to decide.

It may be that voluntary and involuntary thinking are essentially the same; that we cannot, if we will, direct or even influence the stream of our thought; that our feeling of effort, when (as it seems to us) we overcome our tendency $\dagger$ to think of something interesting, and, instead, concentrate our attention upon some unpleasant duty, is not more than a passing index of a conflict of interests + . On the other hand, 'the whole feeling of reality, the whole sting and excitement of our voluntary life, depends on our sense that in it things are really being decided from one moment to another, and that it is not the dull rattling off of a chain that was forged innumerable ages ago. This appearance, which makes life and history tingle with such a tragic zest, may not be an illusion.'

If the former theory-that the psychic side of human nature is not free to influence the stream of thought and to direct the accompanying neural activity so as to produce desired movements-be correct, then we need make (or, rather, pretend to make) no further effort, whether to formulate laws of thought or to accomplish any other thing: all will happen as is foreordained. And, if we were to make the mistake of assuming that the psychic side of human natureour souls and their agents, our wills-were free, no harm would be done by our (imaginary) decision to accept the wrong theory; for our mistake (like everything else) would be inevitable. But if the second theory is right, if psycho-physical interaction is a reality and human souls are really free to influence neural activities by the exercise of will and so to modify behaviour, the consequences of assuming and acting upon the opposite theory would be terrible in the extreme. Being born to freedom, we should live as slaves. There is therefore everything to lose, and nothing to gain, by deciding against the

* Cf. W. James, loc. cit. Vol. I, p. 454.

$\ddagger$ Cf. W. James, loc. cit. Vol. I, p. 45 I.

G. $E$. $\dagger$ Corollary to third law: see above, p. 89.

$\S$ W. James, loc.cit. Vol. I, p. 453. 
hypothesis of interaction, unless and until we are forced to do so by the facts. We shall therefore make the assumption that psycho. physical interaction* does take place, that our wills are free to intervene in the routine of involuntary thinking and to influence the direction of the stream of thought; and we shall shortly formulate this assumption in our fourth law of thought.

\section{§ 2. Will-power, the single general factor in human qualities.}

The word 'will' is used in common speech with at least two different meanings. In the first place, we speak of the effort of will by which a man overcomes a temptation or forces up the index of a dynamometer. And, secondly, we speak of the strength of will exhibited by one who, wearing down all opposition, keeps on and on until he has achieved his purpose. But the question how far I am able, by the exercise of my will, to give immediate effect to my present purpose, is quite different from the question whether my motives are so persistent that, without changing my mind, I shall go on making one effort of will after another until I have attained my more remote object. We shall distinguish between the two senses in which the word 'will' is commonly employed by using the word 'Will'-written with a capital ' $W$ ' - only with the former meaning: Will at short range, as exercised in making a sudden voluntary effort. But will in the second sense, will at long range, we shall speak of, not as will, but as purpose. Failure to distinguish Will (will at short range) from purpose (will at long range) is responsible for the statement of Binet and Simon that experimental tests almost completely eliminate individual differences in volition (i.e. purpose) $\uparrow$. But, as we shall see reason to believe, the same mental tests may prove an effective means of measuring differences in Will $\ddagger$.

* The manner of the interaction is discussed below. But it may help the reader if, without prejudice and subject to the more careful statements that follow, we note here that the assumed interaction of soul and body consists in the soul being acted upon by every neural activity that affects consciousness (and possibly by some neural activities that remain unconscious), while the soul acts upon the body and influences conduct only by 'willing,' a process that involves the concentration of excitement in a limited system of neural arcs. It is because, on this view, the soul acts upon the body and produces the results that we observe in conduct only by 'willing,' and not because the Will is the only attribute of the soul, that we shall in the sequel have so much less to say about the soul than about the Will. But see footnote * on p. Ioo, below.

† 'Le Développement de l'Intelligence,' Année Psychologique, I4 année (I900), p. 77 .

$\ddagger$ It is also responsible for Dr Edward Webb using the words 'volition or will " ('Character and Intelligence,' Monograph Supplement to the B.J.P., Cambridge, I9I 5, p. 60) where we should have preferred to substitute the word 'purpose': see below, p. I60. 
We are going to assume that men are free to influence, and even to control, their conduct by the exercise of Will; but we want, if possible, to express this assumption in more precise language. We want, in fact, not merely to say that psychic activity interacts with, and may determine, neural activity, but to arrive at as precise a conception as possible of the manner in which this interaction takes place.

One possible way suggests itself at once. If, by the exercise of Will, it were possible to increase the excitement of a nervous arc of the higher level, then, according to our third law*, that arc would tend to drain the impulses from all other active arcs; and this draining of the impulses would, by our first law $\uparrow$, determine the thought-activity next to enter the focus of consciousness. Now, among those who accept the interaction hypothesis, there is coming to be a general consensus of opinion that this is how Will does act: it reinforces excitement in some particular active neurogram, with the result that impulses traversing other active arcs are drained through that neurogram, and attention is accordingly concentrated on the thoughtactivity to which the neurogram in question corresponds. The following quotations from William James and Dr McDougall illustrate their opinion that the function of Will is to concentrate attention, or (what amounts to the same thing $\ddagger$ ) to reinforce excitement in some particular system of higher level arcs, and so to facilitate the drainage, through that system, of impulses from all other active arcs.

William James describes the essential volitional act either as 'attending' or as 'reinforcing.' He writes:

The faculty of voluntarily bringing back a wandering attention, over and over again, is the very root of judgment, character, and will. No one is compos sui if he have it not. An education which would improve this faculty would be the education par excellence $\$ . . . . A t t e n t i o n$ with effort is all that any case of volition implies. The essential achievement of the witl, in short, when it is most 'voluntary,' is to ATTEND to a difficult object and hold it fast before the mind. The so-doing is the fiat $\| . .$. To sum it all up in a word, the terminus of the psychological process in volition, the point to which the will is divectly applied, is always an idea. There are at all times some ideas from which we shy away like frightened horses the moment we get a glimpse of their forbidding profile upon the threshold of our thought. The only resistance which our will can possibly experience is the resistance which such an idea offers to being attended to at all. To attend to it is the volitional act, and the only inward volitional act which we ever perform $1 . .$. .The soul presents nothing herself;

* See above. p. 79.

$\dagger$ See above, p. 66.

$\ddagger$ In view of our third law of thought. See also footnote $\dagger$ on p. Ioo, below.

\$ Loc. cit. Vol. I, p. 424.

II Loc. cit. Vol. II, p. 567 .

\| Loc. cit. Vol. 11, p. 561. 
creates nothing; is at the mercy of the material forces for all possibilities; but amongst these possibilities she selects; and by reinforcing one and checking others, she figures not as an 'epiphenomenon,' but as something from which the play gets moral support*.

\section{Dr McDougall also speaks of an act of Will both as a 'concentrating of attention' and as a 'reinforcement':}

It would seem, in fact, that the Will concentrates along one system of channels the free nervous energy of all the brain at the moment.... Voluntary attention to any object...is a reinforcement of the idea or percept, in virtue of which it is held more vividly and continuously in the focus of consciousness to the exclusion of other percepts and ideas; and this, too, seems to imply a concentration of nervous energy in the neural systems whose excitement underlies the percept or idea....An effort of Will seems then to be always the voluntary concentration of attention upon some object, the reinforcement of percept or idea, and its essential physiological result seems to be a higher degree of that concentration of the free energy of the brain along one system of paths which, as we have seen, is the physiological condition of all attention $\uparrow \ldots$. The concentration of nervous energy that results from

* Loc. cit. Vol. II, p. 584. We notice the use of the word 'veinforcing.'

Our quotation, here and again on p. I49 of this passage from William James, must not be regarded as indicating that we accept William James' view of the soul as creating nothing and obtaining everything from material sources. We have (see above, p. 95) on the contrary spoken of psychical and physical processes as acting and reacting upon one another. In our view the first cause may as well be psychical as physical. In other words, the soul may have a structure of its own that is no more dependent on the neurography than the neurography is upon the soul. But the action and reaction of soul and neurography upon each other tend to make them correspond each to each, and this correspondence is our justification for saying (on p. 29I below) that a man's character is determined when his neurography and [the strength of] his Will are determinately known.

$T$ See below, page Ir7. Dr McDougall has recently pointed out that an effort of Will may so increase the concentration of excitement in one system of paths as to lower the resistance of-or 'canalise'- that system in a very marked degree, an effect which has so often been overlooked. Thus he writes: '...the laboratory studies of the Würzburg school or type...have made it clear that in a great number of experimental investigations, especially the numerous elaborate experiments on memory and association, a factor of principal importance has commonly been altogether overlooked, namely, the influence of the intention of the subject, of the setting of the will, or as the Germans say, the influence of the Aufgabe (i.e. of the task prescribed and voluntarily accepted by the subject), the determining tendency, the attitude of consciousness, in short of conation. They have made it clear that, in the past, experimenters in this field (accepting more or less explicitly the atomistic psychology and the atomistic physiology of the nervous system) have attributed to links of simple association alone, effects in the way of reproduction which are due to two broadly distinguishable factors, namely, associative links on the one hand and the will of the subject on the other; and it appears clearly enough that in many such cases of co-operation of the two factors, the latter, conation, is of altogether preponderant importance.

'I may illustrate the point very simply from experiments of my own. It is possible to read repeatedly a list of say twelve nonsense syllables in an attitude of indifference, one as nearly as possible passive; and I5O repetitions of such reading may fail to form such associations as will render possible the free reproduction of the series; yet, if the will be bent upon the task of learning the row, it 
volition is unlike the behaviour of all known kinds of physical energy, the universal law of which is diffusion from the place of higher potential to places of lower potential. In volition we seem to concentrate nervous energy from places of low potential into the place of highest potential, and perhaps we shall have to recognise in this concentration of nervous energy a unique effect of psychical activity*.

Dr Morton Prince also speaks of the Will as operating by reinforcing the excitement of some particular neurogram. After pointing out the influence of a sentiment upon a sequence of involuntary thought-activities, Dr Prince adds: '...volition itself can control, reinforce, and determine the particular sentiment and thus govern conduct....' $\dagger$

If the soul has this power-which we agree to call Will-of reinforcing excitement and so concentrating attention $\ddagger$, it should be capable of affecting the stream of consciousness at any moment. Will should therefore be a general factor characteristic $\S$ of the individual and apt to make itself felt in all intellectual processes $\|$. But is there any evidence of the existence of such a general factor?

The existence of many general factors-or rather group factors $\uparrow-$ called faculties, was formerly, and, as it now appears, fallaciously admitted. The fallacy consists in supposing that "whatever is true of any one performance is necessarily also true of all other performances

can be committed to memory by as few as Io or $\mathbf{I} 2$ readings. (American Journal of Insanity, Vol. LXIX (I9I3), p. 869.) See also a paper on 'Some Experiments on Learning and Retention' by May Smith and W. McDougall (B. J. P. Vol. x (March, I920), p. 199).

* W. McDougall, Physiological Psychology, pp. I65, I66, I67.

† Loc. cit. p. 458.

$\ddagger$ Cf. Dr P. B. Ballard: 'Looked at purely from the psychological side, will is hardly distinguishable from attention.' (Handwork as an Educalional Medium, Second Edition (I9I5), p. 64.)

$\S$ Cf. W. James on Will: 'Of course we measure ourselves by many standards. Our strength and our intelligence, our wealth and even our good luck, are things which warm our heart and make us feel ourselves a match for life. But deeper than all such things, and able to suffice unto itself without them, is the sense of the amount of effort [Will] which we can put forth. Those are, after all, but effects, products, and reflections of the outer world within. But the effort seems to belong to an altogether different realm, as if it were the substantive thing which we are, and those were but externals which we carry. If the "searching of our heart and reins" be the purpose of this human drama, then what is sought seems to be what effort we can make. He who can make none is but a shadow; he who can make much is a hero.' (Loc. cit. Vol. II, p. 578.)

!| 'Development of will power in connexion with any activity is accompanied by a development of will power as a whole.' (Scripture, Psych. Rev. Vol. vi, p. 165, quoted by C. Spearman, Am.J.P. Vol. xv, p. 216.)

I 'Group factors' of a series of mental qualities (or other correlated variables) are independent of each other, and each of them is independent of all except the members of a certain group of the mental qualities (or other variables) in question. See Appendix B, $\S \mathrm{I}$, footnote * on p. 476 below. For the definition of a 'single general factor' see below, Appendix B, § 3 , footnote $\S$ on p. 477 . 
usually bearing the same name, such as "apprehension," "discrimination," "judgment," etc.; it was the old theory-so easy to scotch, but hard to kill—of "faculties." This fallacy, though long banished from open daylight, still strangely persists in dark corners; it even secretly governs the thoughts of many who believe themselves to be strongly opposing it....'*

For example, it was once usual to speak of a 'faculty of observation'; and it was implied that an increasing capacity for observing any one class of objects-say, millinery-must be due to the development of this faculty, and that therefore a capacity for observing any other class of objects-buildings, let us suppose-must necessarily be improved at the same time. No evidence has, however, been forthcoming to shew that whoever can bring back from a walk along Bond Street the most accurate description of the hats in the shop-windows will, on the average, also bring back the best account of the architecture of that street. On the contrary, the milliner, just because she gives her attention to the hats, is precluded from observing the buildings; while the architect, whose attention is attracted to the buildings, fails to notice the hats. In short, training persons to observe hats does not result in their observing buildings. Power of observing is not 'transferred' from one class of objects to another. 'To involve transfer,' says Dr Sleight, 'the common elements must be severable from the complex in which they occur.' $\dagger$ There is no general faculty of observation that may be developed by practice on one kind of material and be afterwards employed on quite another. And so with other supposed faculties. 'The balance of expert opinion is now so solidly against the general dogma of formal [faculty] training that as an educational force it must be regarded as moribund.' $\ddagger$

While, however, the old classification of faculties is falling into disrepute, evidence of the existence of one general factor, common to all intellectual processes, is being accumulated.

In an article on 'General Intelligence,' published in the American Journal of Psychology for 1904, Professor Spearman argued that 'all branches of intellectual activity have in common one fundamental function (or group of functions)' $\S$; and even that this fundamental function enters into sensory discrimination no less than into the more complicated intellectual activities of practical life.

* B. Hart and C. Spearman, B. J. P. Vol. v (I9I3), p. 64.

$\dagger$ B. J.P. Vol. IV (I9II-I2), p. 455 .

$\ddagger$ J. Adams, loc. cit. p. 222 (quoted on p. I8 above).

$\S A m . J . P$. Vol. xv (1904), p. 284. 
This conclusion has been challenged*. While, however, an 'absolute identification of General Intelligence and General Sensory Discrimination (if it has ever been suggested by any but its opponents) cannot be maintained,' $\dagger$ the presence of some one quality-general ability, or whatever it be called-as a general factor in all intellectual activity and as the dominant partner in higher intellectual processes is becoming as widely recognised among experimental psychologists as it has always been among those who direct industry, commerce, or other departments of national life $\ddagger$. Indeed, as we are about to seeई, the measure of each of any set of sufficiently dissimilar mental qualities tends to be compounded, according to the vector law $\|$, of a single general factor common to all the qualities and of a specific factor (independent both of the general factor and of the other specific factors) belonging to that one quality alone.

Professor Spearman ף, Dr Bernard Hart**, Mr Cyril Burt $\dagger \dagger$, Dr Webb ${ }_{+\ddagger}^{+}$and others have collected a very great number of facts of which an immediate and complete explanation is furnished by supposing that 'all performances depend in a certain degree upon one and the saine general factor, provisionally termed "General Ability" $§ \S$. No other hypothesis has yet been suggested that so successfully and so simply sums up all the known facts as does the hypothesis of a single

* Notably by Dr William Brown, Mental Measurement, p. 89 .

$\dagger$ C. Burt, B.J.P. Vol. III (1909-10), p. I65. He adds: 'Dr Spearman has written to me: "This conclusion of mine was badly worded. I did not mean (as others have naturally taken it) that general intelligence was based on sensory discrimination; if anything, vice versa. I take both the sensory discrimination and the manifestations leading a teacher to impute general intelligence to be based on some deeper fundamental cause, as sketched in the Zeitschrift für Psychologie, Vol. XLI, p. I Io, para. 5."'

$\ddagger$ For example, Mr W. L. Hichens, Chairman of Messrs Cammell, Laird and Co. Ltd., addressing the Incorporated Association of Head Masters on 9th January, 1917, said that 'the University men he had engaged had all turned out successful up to date. He went to Oxford and had a look round. He did not look out for the fellow who had got a First in Greats or History, but for the fellow who might have got a First-if he had worked,' i.e. for the man with ability. (Quoted from Cassier's Magazine, Vol. 5I, No. 6, I917, p. 87.)

Cf. also Dr William Temple: 'It scarcely matters what subject is taught: the vital matter is that the child should learn "attention" in general.' (The Nature of Personality, p. 28, quoted on p. 137 below.)

$\S$ See below, pp. I Io et seq.

II The law that tells us how far off, and in what direction, lies a not-toodistant ship when we know how far it is north (or south) of us and how far it is east (or west) of us. The law is mathematically expressed in equation (4) in $\S 3$ of Appendix B, below.

TI Loc. cit. and B. J. P. Vol. v (I9I 2-I3), pp. 5I-79.

** B. J. P. Vol. v, pp. 5I-79. †† B. J. P. Voi. IIr (1909-10), pp. 94-I 77 .

$\ddagger \ddagger$ Loc. cit. Dr Webb's work contains a bibliography to which reference may be made for a summary of the literature of this subject.

$\S \S$ Hart and Spearman, B. J. P. Vol. v (I9I2-I3), p. 52. 
general factor: namely, that a single general factor enters into all mental qualities, while group factors, if they are present at all, are, in the case of sufficiently dissimilar qualities, insignificant in comparison with the single general factor.

The methods of mathematical treatment which Galton first proposed to apply to biological investigations have, in the hands of Professor Karl Pearson and his collaborators, established a number of relations between physical characters of human beings. For example, Professor Pearson has shewn that the sons or daughters of a very large number of fathers (or mothers), all of whom exceed the average height of men (or women) by, say, six inches, will on the average exceed the average height by approximately one-half of that amount, namely three inches. The mathematical methods developed by Professor Pearson and those who have assisted him in laying the foundations of the science of biometry, have of late been applied to the investigation of relations between mental characters. Such a relation is measured * by means of the Bravais-Pearson coefficient of correlation, usually denoted by the letter $r$. When two variables increase or decrease in proportion, the correlation between them is said to be complete, and $r=I$. When, however, there is merely a tendency for increase in one variable to be accompanied by increase in the other, then $r$ becomes a fraction, which is smaller in prcportion as the tendency becomes slighter. For example, in the case cited above of the correlation between the height of children and one of their parents, $r=\frac{1}{s}$ nearly. When two variables are quite independent, $r=o$. When ability in one performance goes with inability in another, $r$ becomes negative up to the limit of - I $\dagger$.

Professor Spearman, in his original paper to which reference has already $\ddagger$ been made, shewed that the correlations between the performances of a large number of persons in a set of sufficiently dissimilar mental tests tended, in the various cases which he examined, to be in a certain relation to one another. And he shewed that this relation -which he described as 'forming a hierarchy'-would be accounted for by supposing that the correlations in question were wholly due to

* If $x$ and $y$ are corresponding values of two variables whose Bravais-Pearson coefficient of correlation is $r$, and if $x$ is measured from the mean of the $x$ 's and $y$ from the mean of the $y^{\prime}$ s, then $r$ is defined by the equation $r=\frac{\Sigma x y}{\sqrt{\Sigma\left(x^{2}\right) \cdot \Sigma\left(y^{2}\right)}}$ where, as usual, $\Sigma$ denotes a summation.

$\dagger$ Cf. Hart and Spearman, loc. cit. p. 53. For further particulars of the mathematical theory of correlation, see Introduction to the Theory of Statistics (r9I I), by G. Udny Y'ule; or the chapters on correlation in Professor T. P. Nunn's The Teaching of Algebra and its companion work. A simple geometrical conception of correlation is explained below (p. 484) in Appendix B, § 9. $\ddagger$ See above, p. I03. 
the presence of a single general factor in each of the qualities tested*. But so far the existence of such a single general factor had not been proved.

In I909 Mr Cyril Burt described experiments on two groups of Oxford schoolboys undertaken 'with a view to testing in practice the mathematical methods of Professor Spearman.' $\dagger$ Mr Burt calculated the correlations between the performances of his subjects in twelve different tests, which he classified as sensory, motor, sensorymotor, and association tests, together with one (the twelfth) test of voluntary attention $\ddagger$. Mr Burt's results were consistent with the view that all the correlations were due to the operation of one, and only one, general factor. The following tables, one for each group of boys, shew the observed correlations between the performances of the boys in that group when tested experimentally in each of the twelve different ways. The thirteenth test (Imputed Intelligence) shews the correlations between the boys' performances in the twelve

* See Appendix B, § I.

$\dagger$ Loc. cit. p. 94 .

$\ddagger \mathrm{Mr}$ Burt's tests were divided into five groups as follows:
Lists of Tests
Nature of process tested

I. Sensory Tests:

(I) Discrimination of two points upon the skin

(2) Discrimination of lifted weights

(3) Discrimination of pitch

(4) Comparison of length of Perceptual discrimination lines by eye

II. Motor Tests:

(5) Tapping

(6) Card-dealing

\} Simple reactions

III. Sensori-Motor Tests:

(7) Card-sorting

(8) Alphabet finding

Reactions complicated by discrimination Reactions complicated by recognition

IV. Association Tests:

(9) Immediate retention of

(a) Concrete words

(b) Abstract words

(c) Nonsense syllables

(I0) Mirror Test

(II) Spot pattern Test

V. Test of Voluntary Attention:

(x2) Dotting irregular dots

Immediate memory

Formation of associations during motive activity (progressive process of 'Trial and Error')

Formation of associations during perceptual activity (progressive process of 'Apperception')

Maximal effort of sustained attention 
experimental tests, and the degree of intelligence imputed to them by their Headmaster. The first group, to which Table I relates, consisted of thirty boys in the Oxford Central School; and the second group, the corresponding correlations of whose performances in the several tests are shewn in Table II, consisted of thirteen boys belonging to the Oxford Preparatory School. All the boys of both groups were between the ages of $\mathrm{r} 2$ years 6 months and $\mathrm{r} 3$ years 6 months. The former school was a 'superior Elementary School' and the latter a 'high class Preparatory School.'* Both were exclusively boys' schools. 'In Social Status, the boys of the Elementary School were of the lower middle class, sons of local tradesmen, paying a fee of $9 d$. a week. The boys from the Preparatory School were being prepared for scholarships at one or other of the great Public Schools, and were in nearly every case sons of men of eminence in the intellectual world, that is to say, of Fellows of the Royal Society, University Professors, College Tutors and Bishops.... Within the two schools, however, the social status of the boys was unusually uniform.' $\dagger$

Table I. Hierarchy of Coefficients (Amalgamated Series).

A. Elementary School.

\begin{tabular}{|c|c|c|c|c|c|c|c|c|c|c|c|c|c|}
\hline $\begin{array}{l}\text { Dotting } \\
\text { Apparatus: }\end{array}$ & 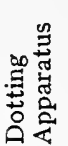 & 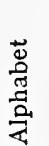 & $\begin{array}{l}\text { 品 } \\
\text { 䓪 }\end{array}$ & 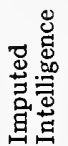 & $\begin{array}{l}\stackrel{\infty}{\Xi} \\
\text { : } \\
\text { ̊ొ }\end{array}$ & 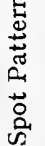 & 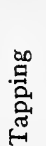 & 岂 & 范 & . & : & 总 & ? \\
\hline Obs. coeff. & - & 77 & 67 & 60 & 69 & 57 & 57 & 50 & 52 & 48 & 38 & 20 & \\
\hline Theor. value $\ddagger$ & - & 80 & 73 & 72 & 72 & 67 & 63 & 49 & 45 & 33 & 28 & 27 & \\
\hline Deviation $\ddagger$ & - & 03 & 06 & I 2 & 03 & Io & 06 & or & o7 & 15 & Io & 07 & \\
\hline P.e. $\S$ of coeff. & - - & 05 & 07 & 08 & 06 & 08 & 08 & 09 & 09 & 09 & I & 12 & \\
\hline \multicolumn{14}{|l|}{ Alphabet: } \\
\hline Obs. coeff. & 77 & - & 74 & $6 I$ & 66 & 59 & 54 & 29 & 52 & I6 & 62 & $3 \mathrm{I}$ & \\
\hline Theor. value & 80 & - & 69 & 69 & 69 & 65 & 60 & 46 & 43 & 32 & 6 & 25 & \\
\hline Deviation & 03 & - & 05 & 08 & 03 & 06 & 06 & I7 & og & I6 & 6 & 06 & \\
\hline P.e. of coeff. & 05 & - & 06 & 08 & 07 & 08 & 09 & II & 09 & 12 & 07 & Io & \\
\hline \multicolumn{14}{|l|}{ Sorting: } \\
\hline Obs. coeff. & 67 & 74 & - & 52 & 72 & 45 & $6 I$ & 34 & 52 & $r_{4}$ & 22 & I9 & \\
\hline Theor. value & 73 & 69 & - & 62 & $6 \mathrm{I}$ & 59 & 54 & 42 & 39 & 28 & 24 & 23 & \\
\hline Deviation & 06 & 05 & - & Io & II & I4 & I3 & 08 & I3 & 14 & 02 & 04 & \\
\hline P.e. of coeff. & 07 & 06 & - & 09 & o6 & Io & 08 & II & 09 & 12 & II & Io & \\
\hline \multicolumn{14}{|l|}{$\begin{array}{l}\text { Imputed } \\
\text { Intelligence: }\end{array}$} \\
\hline Obs. coeff. & 60 & 61 & 52 & - & 44 & 76 & 47 & 67 & 40 & 29 & 13 & 57 & \\
\hline Theor. value & 72 & 69 & 62 & - & 69 & 58 & 53 & $4 I$ & 39 & 28 & 23 & 23 & \\
\hline Deviation & I 2 & 08 & Ic & & I6 & I 8 & 06 & 26 & OI & 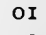 & 0 & 34 & \\
\hline P.e. of coeff. & 08 & 08 & 09 & - & ro & 05 & Io & 07 & Io & 08 & โ2 & 08 & \\
\hline
\end{tabular}

* Loc. cit. p. I02. † Loc. cit. p. Ioo. $\quad$ See below, Appendix B, § 2,

$\S$ I.e. probable error: see below, Appendix B, § I. 
Table I-continued.

Dealing:

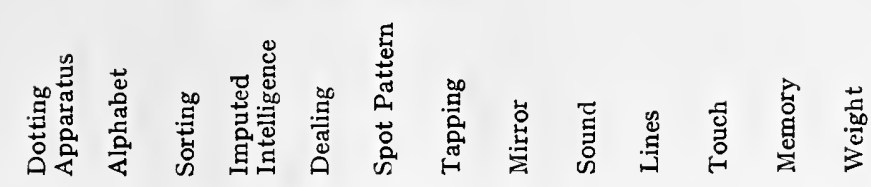

$\begin{array}{llllllllllllll}\text { Obs. coeff. } & 69 & 66 & 72 & 44 & - & 76 & 47 & 67 & 40 & 29 & 13 & 57 & -13\end{array}$

Theor. value $\begin{array}{lllllllllllll}72 & 69 & 6 \mathrm{I} & 60 & - & 58 & 53 & 4 \mathrm{I} & 39 & 28 & 23 & 23 & 04\end{array}$

$\begin{array}{llllllllllllll}\text { Deviation } & 03 & 02 & I I & I 6 & - & 07 & \text { I2 } & \text { or } & 05 & \text { I9 } & 00 & 04 & 03\end{array}$

P.e. of coeff. 06 o7 06 o $09-$ I0 07 II I2 Io II I2 I2

Spot Pattern:

$\begin{array}{lllllllllllllllll}\text { Obs. coeff. } & 57 & 53 & 6 \text { I } & 47 & 65 & - & 4 I & 4 I & 47 & 08 & 26 & -05 & 22\end{array}$

$\begin{array}{llllllllllllllll}\text { Theor. value } & 63 & 60 & 54 & 53 & 53 & - & 4^{8} & 36 & 34 & 25 & 20 & 20 & 04\end{array}$

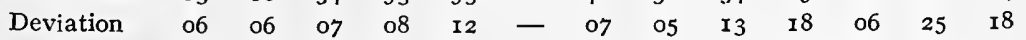

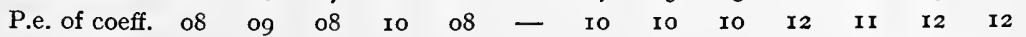

Tapping:

$\begin{array}{lllllllllllllll}\text { Obs. coeff. } & 57 & 53 & 6 \mathrm{r} & 47 & 65 & 4 \mathrm{I} & - & 4 \mathrm{I} & 47 & 08 & 26 & -05 & 22\end{array}$

Theor. value $63 \quad 60 \quad 54 \quad 53 \quad 53 \quad 48 \quad-\begin{array}{lllllllll}36 & 54 & 54 & 25 & 20 & 20 & 04\end{array}$

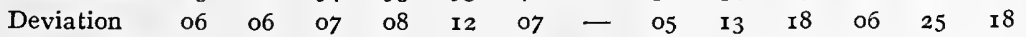

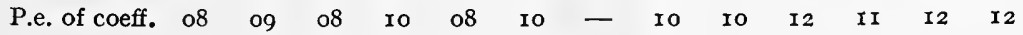

Mirror:

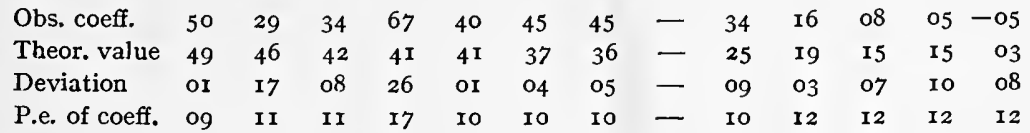

Sound:

$\begin{array}{lllllllllllllll}\text { Obs. coeff. } & 52 & 52 & 52 & 40 & 34 & 47 & 47 & 34 & -0 & -07 & -01 & \text { oI } & -\mathrm{I} 3\end{array}$

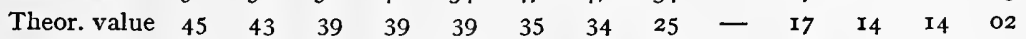

$\begin{array}{llllllllllllll}\text { Deviation } & 07 & 09 & I_{3} & \text { OI } & 05 & \text { I2 } & \text { I3 } & 09 & - & 24 & \text { I5 } & \text { I3 } & \text { I5 }\end{array}$

P.e. of coeff. 09 o9 09 I0 I7 Io $\begin{array}{llllllll}10 & 12 & - & \text { I2 } & \text { I3 } & \text { I2 } & \text { I2 }\end{array}$

Lines:

$\begin{array}{llllllllllllll}\text { Obs. coeff. } & 48 & \text { I } 6 & \text { I } 4 & 29 & 47 & 25 & 08 & \text { I6 } & -07 & - & 26 & 06 & \text { I9 }\end{array}$

$\begin{array}{lllllllllllllllll}\text { Theor. value } & 33 & 32 & 28 & 28 & 28 & 26 & 26 & 25 & 17 & - & \text { 10 } & \text { 10 } & 02\end{array}$

$\begin{array}{llllllllllllll}\text { Deviation } & \text { I5 } & \text { I6 } & \text { I4 } & \text { or } & \text { I9 } & \text { OI } & \text { I7 } & 03 & 24 & - & \text { I6 } & 04 & \text { I7 }\end{array}$

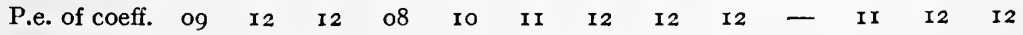

Touch:

$\begin{array}{llllllllllllll}\text { Obs. coeff. } & 38 & 62 & 22 & 13 & 23 & 03 & 26 & 08 & - \text { or } & 26 & - & 16 & 29\end{array}$

$\begin{array}{llllllllllllll}\text { Theor. value } & 28 & 26 & 24 & 23 & 23 & 21 & 20 & 15 & 14 & 10 & - & 08 & \text { or }\end{array}$

$\begin{array}{llllllllllllll}\text { Deviation } & 10 & 36 & 02 & \text { I0 } & 00 & 18 & 06 & 07 & 15 & 16 & - & 08 & 28\end{array}$

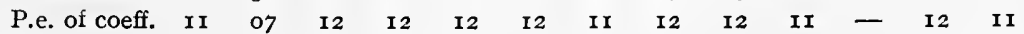

Memory:

$\begin{array}{llllllllllllllll}\text { Obs, coeff. } & 20 & 31 & \text { I9 } & 57 & \text { I9 } & 26 & -05 & 05 & \text { or } & 06 & 16 & - & 05\end{array}$

$\begin{array}{llllllllllllllll}\text { Theor. value } & 27 & 25 & 23 & 23 & 23 & 21 & 20 & \text { I5 } & \text { I2 } & \text { 10 } & \text { I } 8 & - & \text { or }\end{array}$

$\begin{array}{llllllllllllll}\text { Deviation } & 07 & 06 & 04 & 34 & 04 & 05 & 25 & \text { 10 } & 13 & 04 & 08 & - & 04\end{array}$

P.e. of coeff. I2 IO II IO I2 II I2 I2 12 I2 12 - I2

Weight:

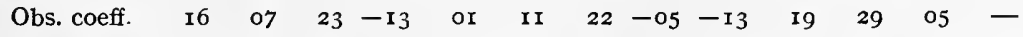

$\begin{array}{llllllllllllll}\text { Theor. value } & 05 & 05 & 04 & 04 & 04 & 04 & 04 & 03 & 03 & 03 & \text { or } & \text { or } & -\end{array}$

$\begin{array}{llllllllllllll}\text { Deviation II } & 02 & \text { I9 } & \text { I7 } & 03 & 07 & \text { I } 8 & 08 & \text { I5 } & \text { I7 } & 28 & 04 & -\end{array}$

P.e. of coeff. I2 12 I2 12 I2 12 II 12 I2 I2 12 II 12 -

Average deviation $=\cdot$ Ioo. $\quad$ Average p.e. $=\cdot \mathbf{I}$ oI. 
Table II. Hievarchy of Coefficients (Amalgamated Series).

B. Preparatory School.

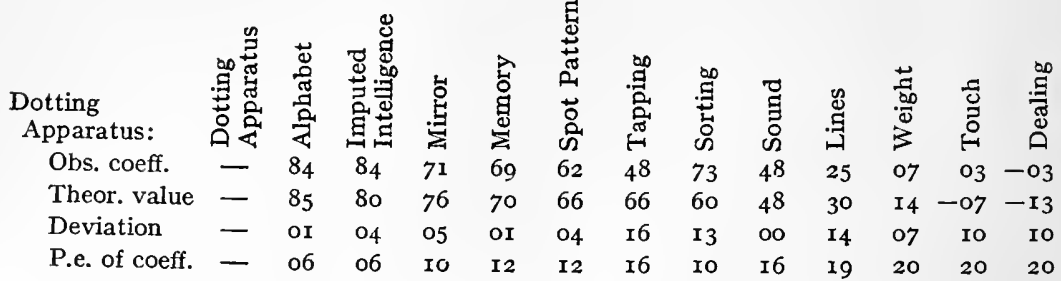

Alphabet:

$\begin{array}{llllllllllllll}\text { Obs. coeff. } & 84 & - & 80 & 48 & 84 & 67 & 57 & 76 & 34 & 22 & -14 & -28 & 45\end{array}$

$\begin{array}{llllllllllllll}\text { Theor. value } & 85 & - & 78 & 74 & 68 & 64 & 64 & 58 & 51 & 37 & 14 & -07 & -12\end{array}$

$\begin{array}{lllllllllllll}\text { Deviation or } & - & 02 & 26 & 16 & 03 & 07 & 18 & 17 & 15 & 28 & 21 & 57\end{array}$

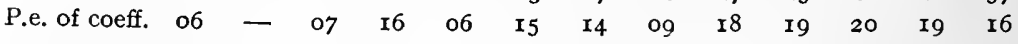

Imputed

Intelligence:

$\begin{array}{lllllllllllllll}\text { Obs. coeff. } & 84 & 80 & - & 54 & 78 & 75 & 43 & 56 & 37 & 17 & -19 & -06 & 29\end{array}$

Theor. value $80 \quad 78-70 \quad 64 \quad 60 \quad 60 \quad 55 \quad 44 \quad 35 \quad 13-06-12$

$\begin{array}{llllllllllllll}\text { Deviation } & 04 & 02 & - & \mathrm{I} 6 & \mathrm{I} 4 & \mathrm{I} 5 & \mathrm{I} 7 & \text { OI } & 07 & \mathrm{I} 8 & 32 & 00 & 4 \mathrm{I}\end{array}$

Mirror:

$\begin{array}{llllllllllllll}\text { P.e. of coeff. } & 06 & 07 & - & \mathrm{I} 4 & 08 & 09 & \mathrm{I} 6 & \mathrm{I} 4 & \mathrm{I} 7 & 20 & \mathrm{I} 9 & 20 & 18\end{array}$

$\begin{array}{llllllllllllll}\text { Obs. coeff. } & 7 \mathrm{I} & 4^{8} & 54 & - & 43 & 3^{8} & 75 & 34 & 57 & 54 & 44 & 3 \mathrm{I} & -44\end{array}$

Theor. value $\begin{array}{lllllllllllll}76 & 74 & 70 & - & 61 & 58 & 57 & 52 & 42 & 34 & \text { I2 } & -06 & - \text { II }\end{array}$

$\begin{array}{llllllllllllll}\text { Deviation } & 66 & 26 & \text { I } 6 & - & \text { I } 8 & 20 & 18 & 18 & 15 & 20 & 32 & 37 & 33\end{array}$

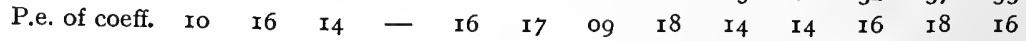

Memory:

$\begin{array}{lllllllllllllll}\text { Obs. coeff. } & 69 & 84 & 78 & 43 & - & 74 & 54 & 64 & \text { I } 7 & 28 & -05 & -35 & 03\end{array}$

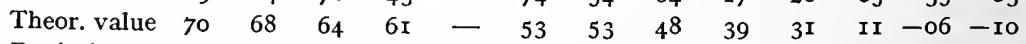

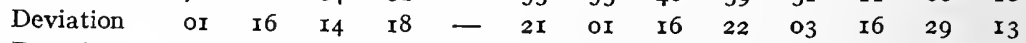

$\begin{array}{llllllllllllll}\text { P.e. of coeff. II } & \text { I } 6 & \text { I } 8 & \text { I6 } & - & 09 & \text { I4 } & \text { II } & 20 & \text { I9 } & 20 & \text { I } 8 & 20\end{array}$

Spot Pattern:

$\begin{array}{lllllllllllllll}\text { Obs. coeff. } & 62 & 67 & 75 & 3^{8} & 74 & - & 38 & 51 & 25 & 34 & 07 & -44 & \text { I9 }\end{array}$

Theor. value $\begin{array}{llllllllllll}66 & 64 & 60 & 38 & 53 & - & 50 & 45 & 36 & 29 & \text { II }-05-10\end{array}$

$\begin{array}{llllllllllllll}\text { Deviation } & 04 & 03 & \text { I5 } & 20 & 2 I & - & \text { I2 } & 06 & \text { II } & 05 & 04 & 39 & 29\end{array}$

$\begin{array}{llllllllllllll}\text { P.e. of coeff. } & \text { I2 } & \text { I5 } & \text { o9 } & \text { I7 } & \text { og } & - & \text { I7 } & \text { I5 } & \text { I9 } & \text { I8 } & 20 & \text { I6 } & \text { I9 }\end{array}$

Tapping:

$\begin{array}{lllllllllllllll}\text { Obs. coeff. } & 48 & 57 & 43 & 75 & 54 & 38 & - & 4^{8} & 28 & 44 & 34 & 07 & -31\end{array}$

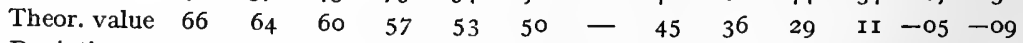

$\begin{array}{llllllllllllll}\text { Deviation } & \mathrm{I} 6 & 07 & \mathrm{I} 7 & \mathrm{I} 8 & \text { oI } & \mathrm{I} 2 & - & 03 & 08 & \mathrm{I} 5 & 23 & \mathrm{I} 2 & 22\end{array}$

$\begin{array}{llllllllllllll}\text { P.e. of coeff. } & \mathrm{I} 6 & \mathrm{I} 4 & \mathrm{I} 6 & 09 & \mathrm{I} 4 & \mathrm{I} 7 & - & \mathrm{I} 6 & \mathrm{I} 9 & \mathrm{I7} & \mathrm{I} 8 & 20 & \mathrm{Ig}\end{array}$

Sorting:

$\begin{array}{llllllllllllll}\text { Obs. coeff. } & 73 & 76 & 56 & 34 & 64 & 51 & 48 & - & 38 & 00 & -22 & -14 & 02\end{array}$

Theor. value $60 \quad \begin{array}{lllllllllll}58 & 55 & 52 & 48 & 45 & 45 & - & 33 & 27 & \text { 10 }-05-09\end{array}$

$\begin{array}{llllllllllllll}\text { Deviation } & \text { I3 } & \text { I } 8 & \text { OI } & 18 & 16 & 06 & 03 & - & 05 & 27 & 32 & 09 & \text { II }\end{array}$

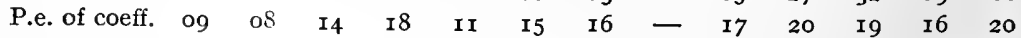

Sound:

$\begin{array}{llllllllllllll}\text { Obs. coeff. } & 48 & 34 & 37 & 57 & \text { I7 } & 25 & 28 & 38 & - & 07 & \text { I4 } & \text { I7 } & - \text { I7 }\end{array}$

$\begin{array}{llllllllllllll}\text { Theor. value } & 48 & 5 \mathrm{I} & 44 & 42 & 39 & 36 & 36 & 33 & -2 \mathrm{r} & 08 & -04 & -07\end{array}$

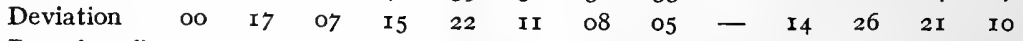

$\begin{array}{lllllllllllll}\text { P.e. of coeff. I6 } & \text { I } 8 & \text { I7 } & \text { I4 } & 20 & \text { I9 } & \text { I9 } & \text { I7 } & -20 & \text { I9 } & 20 & 20\end{array}$ 
Table II-continued.

Lines:

\begin{tabular}{|c|c|c|c|c|c|c|c|c|c|c|c|c|c|}
\hline & 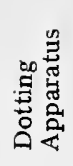 & 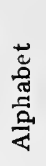 & 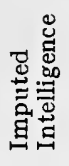 & 岁 & 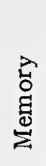 & 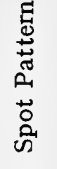 & 苔 & 竞 & $\begin{array}{l}\tilde{D} \\
\text { D̊ } \\
\text { in }\end{array}$ & $\stackrel{g}{\stackrel{g}{a}}$ & $\begin{array}{l}\frac{5}{5} \\
\frac{.00}{00} \\
3\end{array}$ & 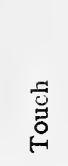 & 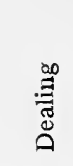 \\
\hline bs. coeff. & 25 & 22 & I7 & 54 & 28 & 34 & 44 & oo & 07 & - & 35 & 19 & - \\
\hline heor. value & 39 & 37 & 3 & 34 & $3 \mathrm{I}$ & 29 & 29 & 27 & $2 I$ & - & 06 & -03 & - \\
\hline eviation & $I_{4}$ & I5 & 18 & 20 & 03 & 05 & I5 & 27 & I 4 & - & 29 & 22 & \\
\hline e. of coeff. & & I9 & & $x_{4}$ & I9 & I 8 & I7 & 20 & 20 & 一 & I8 & I9 & \\
\hline
\end{tabular}

Weight:

\begin{tabular}{|c|c|c|c|c|c|c|c|c|c|c|c|c|c|}
\hline Obs. coeff. & 07 & $-I_{4}$ & -10 & 44 & -05 & 07 & 34 & -22 & 34 & 35 & 一 & $3^{8}$ & -35 \\
\hline Theor. value & $\mathrm{I}_{4}$ & $I_{4}$ & I3 & I 2 & II & II & II & ro & o8 & 06 & - & -or & -02 \\
\hline Deviation & 07 & 28 & 32 & 32 & I 6 & 04 & 23 & 32 & 26 & 29 & - & 39 & \\
\hline P.e. of coeff. & 20 & 20 & I9 & I6 & 20 & 20 & I8 & I9 & 19 & 18 & - & I7 & If \\
\hline
\end{tabular}

Touch:

$\begin{array}{llllllllllllll}\text { Obs. coeff. } & 03 & -28 & -06 & 3 \mathrm{I} & -35 & -44 & 07 & -\mathrm{I} 4 & \text { I7 } & \text { I9 } & 38 & - & -48\end{array}$

Theor. value $-07-07-06-06-06-05-05-05-04-03-01 \quad-\quad$ or

$\begin{array}{llllllllllllll}\text { Deviation } & \text { I0 } & 2 \mathrm{I} & 00 & 37 & 29 & 39 & \text { I2 } & \text { 09 } & 2 \mathrm{I} & 32 & 29 & - & 49\end{array}$

$\begin{array}{llllllllllllll}\text { P.e. of coeff. } & 20 & \text { I9 } & 20 & \text { I8 } & \text { I8 } & \text { I6 } & 20 & \text { I6 } & 20 & \text { I9 } & 17 & - & \text { I5 }\end{array}$

Dealing:

$\begin{array}{lllllllllllllll}\text { Obs. coeff. } & -03 & 45 & 29 & -44 & 03 & \mathrm{I} 9 & -3 \mathrm{I} & 02 & -\mathrm{I} 7 & -\mathrm{I} 3 & -35 & -48 & -\end{array}$

Theor. value $-\mathrm{I} 3-\mathrm{I} 2-\mathrm{I} 2-\mathrm{II}-\mathrm{IO}-\mathrm{IO}-09-09-07-06-02-01 \quad-$

$\begin{array}{llllllllllllll}\text { Deviation IO } & 57 & 4 \mathrm{I} & 33 & 13 & 29 & 22 & \text { II } & \text { I0 } & 07 & 43 & 39 & -\end{array}$

$\begin{array}{llllllllllllll}\text { P.e. of coeff. } & 20 & 16 & 18 & 16 & 20 & 19 & 19 & 20 & 20 & 20 & 18 & 15 & -\end{array}$

Average deviation $=\cdot 165 . \quad$ Average p.e. $=\cdot 162$.

Mr Burt's discussion of the question how far the tabulated correlations satisfy the conditions for a hierarchy* is quoted below in Appendix $\mathrm{B}, \S 2$. His conclusion is that 'in this respect, the results of a wider application of the Test-methods confirm and extend the observations of Professor Spearman, who found in his own experiments "the range of the central function...so universal, and that of the specific functions so vanishingly minute." $\dagger$

Mr Burt's results thus confirm the earlier work of Professor Spearman; for they shew the same tendency of the correlations between dissimilar mental tests to form a hierarchył. Mr Burt's experiments were, therefore $\S$, consistent with Professor Spearman's theory that the correlations between performances in the several tests were mainly, if not entirely, due to the operation of a single general factor. But the existence of the single general factor, while consistent with the facts, and particularly with the new facts obtained by Mr Burt, was still not proved.

* See above, p. I04; and Appendix B, § I.

$\dagger$ Burt, loc. cit. p. 164 .

$\ddagger$ Or, in other words, to satisfy equations (I) in $\S \mathbf{I}$ of Appendix B.

$\S$ See $\S$ I of Appendix B. 
It has, however, since been shewn* that, where the conditions for a hierarchy $\dagger$ are satisfied by the correlations between a set of mental tests, the measure of each of the qualities tested is compounded, according to the vector law $\ddagger$ (which enables a short distance at sea, in whatever direction, to be compounded of so much north, or south, with so much east, or west), of a single general factor $\S$ common to all the qualities and of a specific factor (independent both of the single general factor and of the other specific factors) belonging to that quality alone, no group factors being present.

It follows that, if the correlation between Mr Burt's tests had completely satisfied the conditions for a hierarchy, it would be possible to assert that a single general factor and specific factors, but no group factors\|, entered into each of the correlated qualities tested. Since, however, the conditions for a hierarchy were not completely satisfied by the correlations between Mr Burt's tests, but were only satisfied within the limits of probable error involved, we can assert no more than that a general factor was dominant, and group factors, if present at all, were comparatively insignificant, in each of the tests in question. In other words, Mr Burt's investigation proves the tendency of the measures of dissimilar mental qualities to be functions of a single general factor and specific factors only, without group factors $\uparrow$.

Two years after the publication of Mr Burt's paper, Professor Spearman and Dr Hart proposed ${ }^{* *}$ a new criterion for the existence of a (single) general factor; namely that, in a correlation table such as those $\dagger \dagger$ published by Mr Burt, the correlation between every pair of columns should, with proper allowance for sampling errors + , be $+I$,

* Garnett, Proc. R. S. (A), Vol. 96 (I919), pp. 99-102.

$\dagger$ Equation (I) in $\S$ I of Appendix B, below.

¥ Mathematically expressed in equation (4) of $\S 3$ of Appendix B, below.

$\S$ Defined in $\S 3$ of Appendix B, below. See footnote $\S$ on p. 477 .

II A single general factor and specific factors are defined in the footnote $\S$ on p. 477 ( 33 of Appendix $B$ ); and group factors in footnote * on p. 476 ( $\$$ of Appendix B).

If See Appendix B, $\S 4$ and $\S 5$.

** Spearman and Hart, B.J.P. Vol. v, p. 56.

$\dagger \dagger$ See Tables I and II above, pp. Io6 to Iog.

$\ddagger$ Spearman and Hart's formula for column correlation, corrected for errors of sampling, is

$$
R_{a b}^{\prime}=\frac{S\left(\rho_{x a} \rho_{x b}\right)-(n-\mathrm{I}) \gamma_{a b} \overline{\sigma_{x a} \sigma_{x b}}}{\sqrt{ }\left[S\left(\rho^{2}{ }_{x a}\right)-(n-\mathrm{I}) \overline{\sigma_{x a}^{2}}\right] \sqrt{ }\left[S\left(\rho^{2}{ }_{a b}\right)-(n-\mathrm{I}) \overline{\sigma_{x b}^{2}}\right]}
$$

in which the 's are the correlation coefficients $r$ measured from the mean of the column, and the $\sigma$ 's are the probable errors of the $r$ 's divided by 0.6745 . The bar indicates mean values. The authors decided that, for the purpose of calculating a 'correlation between columns,' only those pairs of columns could be used in which $S\left(\rho^{2}\right)$ is at least twice the correction $(n-1) \sigma^{2}$. 
or, as Dr Webb* pointed out afterwards, - I. Professor Spearman and Dr Hart shewed that the average correlation between columns in such tables should be approximately zero if, as (according to Binet) Thorndike formerly maintained, those are right for whom l'esprit ne serait qu'une collection hétéroclite de facultés qui sont comme juxtaposées mais restent rigoreusement indépendantes $\uparrow$; and that it should possess a low negative value if Thorndike's newer view of 'levels,' or the almost universal belief in 'types, $\ddagger$ is correct. When Dr Hart and Professor Spearinan confronted these three theories by the facts, and applied their formula to the five largest pairs and the five smallest pairs (or as many as were up to the correctional standard§) of columns in fourteen published tables of correlation coefficients dating from various periods up to thirty years back, they found that 'from beginning to end, the correlation between columns is positive and very high; the mean is almost complete $+\mathrm{I}$. This is just the value demanded by the theory of a [single] General Factor.' $\|$ Dr Webb, in his essay on Character and Intelligence, to which reference has just been made*, prepared a further correlation table of five columns (and five rows), the results of five examination tests set to ninety-six training college students. Nine out of the ten pairs of columns were up to the correctional standard of Professor Spearman and Dr Hart, and the application of their formula to those nine pairs yielded $\mathrm{I} \cdot 02 \pm 0.08$ as the average correlation between columns. 'This result,' says Dr Webb, 'is an additional item of evidence in support of the Theory of a General Factor....It takes its place in the huge array of evidence collected by Professor Spearman from experimental tests by many investigatorsthe steadiness of results being such as to rival the niceties which physical measurements reveal. It should be remembered that the raw material for our own (comparatively small) contribution to this total result consisted of test-papers numbering nearly ten thousand.' I

* Loc. cit. above, footnote $\ddagger$, p. 98 .

$\uparrow$ Quoted from Binet by Hart and Spearman, loc. cit. p $5 \mathrm{I}$.

+ When "the very numerous supporters of mental "types"...classify people as "visual," "auditory," "motor," etc., or in any other more ingenious manner, they evidently imply that the different kinds of visual abilities tend to go together (except in so far as their "types" merely refer to habits and preferences, not abilities). It is interesting to notice that even Thorndike appears to be surrendering his former belief in the complete independence of all mental powers, and to be coming round to an opinion of the same class as those just mentioned. In place of "faculties" or "types" he introduces the more cautious term of "levels"; these he declares to be three in number, namely, sensitivity, association, and dissociation; he says that performances correlate highly with those belonging to the same "level," lowly with those belonging to a different one.' (Hart and Spearman, loc. cit. p. 52.)

|| Loc. cit. p. 60.

$\S$ See footnote $\ddagger \ddagger$ on p. IIo.

it Loc. cit. p. 37. 
It is easy to see that, if a number of correlated variables, each of which measures some mental quality*, are dependent upon a single general factor (distributed according to the normal law) and upon specific factors only, and if the correlations between these variables are arranged to form a correlation table, then the correlations between every pair of columns will be $\pm \mathrm{I} \uparrow$. The result obtained by Professor Spearman and Dr Hart, and afterwards confirmed by Dr Webb, is therefore consistent with Professor Spearman's theory of a single general factor as we have formulated it in mathematical terms $\ddagger$.

But the existence of a single general factor as defined above, while it is sufficient to account for the results obtained by Professor Spearman and Dr Hart, and afterwards by Dr Webb, may not be necessary to account for those results. An investigation $\S$ undertaken by the present writer has, however, shewn that if the correlation between every pair of columns in a correlation table, representing the results of a large number of sufficiently dissimilar mental tests, be $\pm \mathbf{I}$, the measures of the correlated qualities can be expressed $\|$ in terms of a single general factor, $g$, and specific factors only. There will be no group factors.

Since, however, the 'correlation between columns' conditionnamely, that the correlation between every pair of columns in the correlation tables should be $\pm \mathrm{I}$-were not completely, but only approximately, satisfied in the mental tests examined by Professor Spearman, Mr Burt and Dr Webb, and in the case of all the mental tests carried out during a period of thirty years preceding the publication of the paper by Professor Spearman and Dr Hart, we can assert no more than that a general factor was dominant and that group factors, if present at all, were comparatively insignificant in each of the tests belonging to sets of tests in which the correlated qualities were sufficiently dissimilar.

Our conclusion may be alternatively expressed in words that differ but little from those $\mathbb{I}$ in which Professor Spearman first described

* And is therefore, we may take it, distributed according to the normal probability law. See Appendix B, § r, below.

$\dagger$ This follows at once from footnote * to p. 476 in $\S \mathrm{I}$ of Appendix B, below.

$\neq$ See $\S 5$ of Appendix B.

$\S$ Garnett, Proc. R. S. (A), Vol. 96 (rgr9), pp. ro2-5. See also $\S 6$ of Appendix B, below.

$\|$ By means of equations (4) in $\S 3$ of Appendix B (p 477).

I The words used by Professor Spearman in summarising the conclusions of his first paper (loc. cit. p. 284) are as follows: 'The above and other analogous observed facts indicate that all branches of intellectual activity have in common one fundamental function (or group of functions) [-our single general factor-], whereas the remaining or specific elements of the activity seem in every case to be wholly different from that in all the others.' 
his theory of the single general factor; for we can now say that all sufficiently dissimilar branches of intellectual activity tend to have in common one fundamental function, the single general factor, whereas the remaining or specific factor of the activity seems in every case to be wholly different from that in all the others and from the general factor.

'So far,' to quote again from Dr Hart and Professor Spearman*, 'all is plain sailing enough. The trouble is not so much in proving the existence of the General Factor as in revealing its precise nature.'

Now we have already $\dagger$ observed that power to reinforce excitement in particular systems of higher level arcs and so to concentrate attention on corresponding thought-activities-a power which William James, Dr McDougall, Dr Morton Prince, Dr Ballard, and others have identified with Will-would constitute such a general factor. Indeed, all the available evidence goes to shew that, if this general factor, Will, exists, it must be the same as the single general factor, which we shall denote by ' $g$,' and its measure by $g$.

Confirmatory evidence that Will is identical with that' general ability whose measure is $g$ is furnished by Mr Burt's investigations. Thus his results proved that the greater the ability (of the boys he tested) to concentrate attention on some new thing, the greater also was the measure of their general factor, ' $g$.' $\ddagger$ This was not a question of the boys' zeal or goodwill§. Their $g$ was great when they possessed great ability to concentrate on something to which they desired to attend. Thus, the 'dotting apparatus' heads the hierarchies in both Tables I and II $\|$ because, in the case of both schools, the boys' performances in the dotting test correlated, on the average, most highly with their performances in the other tests. This " "Dotting Apparatus" is a machine for testing and graphically recording continued maximal voluntary concentration of attention. The method was devised by Dr McDougall; and an improved form of his apparatus has been suggested by Dr Rivers $\$$....In an experiment conducted by

* B. J. P. Vol. v, p. 65.

$\uparrow$ See above, p. ror.

$\ddagger$ Ability to concentrate attention as measured by the dotting test has a high correlation with $g$, according to Table I and Table II above. If suffixes $d, a, s$, indicate the dotting, alphabet and spot pattern tests, the formula (see below, footnote $*$ to p. I I5) $r_{d g}^{2}=\frac{v_{d a} \cdot v_{d s}}{v_{a s}}$ gives $v_{d g}=\cdot 82$ (in Elementary School) and $=\cdot 90$ (in Preparatory School).

$\S$ Burt, loc. cit. p. 166.

i| See above, pp. 106 to 109.

of The earlier form of the same apparatus is described in the $B . J . P$. Vol. II, No. 4 , p. 435. W. McDougall, 'On a New Method for the Study of Concurrent Mental Operations and of Mental Fatigue (Preliminary Communication).' A description of Dr Rivers' improvements will be found in his book on The Influence of Alcohol and Other Drugs on Fatigue, Appendix II, p. I25. (Quoted from Burt, loc. cit. p. 153.)

G. E. 
means of this machine the task of the reagent* is to mark with a pencil or stylographic pen an irregular zigzag row of dots $\dagger$, lithographed in red upon a paper tape, carried past the field of view at an adjustable speed by a small wooden drum rotated by clockwork.... The row of dots upon the paper band (see Fig. 7) is carefully designed so that the succession shall be as irregular as possible, the horizontal distance, however, of each dot from the last (i.e. the interval in the direction of motion) being always $5 \mathrm{~mm}$., the extreme lateral deviation of the dots being $15 \mathrm{~mm}$., and no dot deviating by more than $7 \mathrm{~mm}$. from the line of its predecessor. Sitting at the table, resting his wrist upon

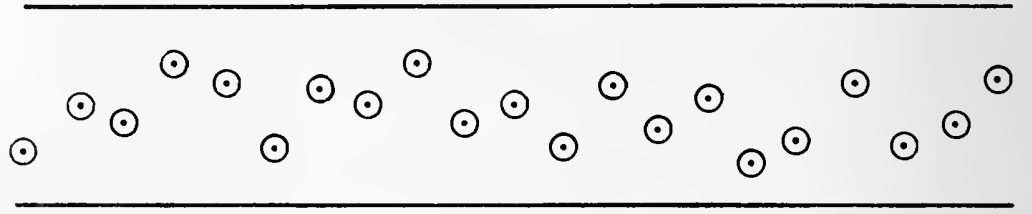

Fig. 7. Portion of Tape to be marked in Dotting Test.

the desk, the reagent watches and marks the dots as they appear through the window, and are carried past towards his left.... Throughout the marking of a series moving at a given rate the subject's task is one of uniform difficulty, and the difficulty of the task depends upon the velocity at which the tape of paper moves. As he has the same fraction of a second for the accomplishment of each hit, he has to make a rhythmical series of strokes with the pencil; but as the position of each dot is unknown till it is seen, each stroke has to be aimed. This requires a sustained effort of attention, the degree of effort depending upon the rate of the rhythm of the strokes, and therefore measured by the rate of movement of the dots upon the paper tape. When marked, the paper furnishes a permanent graphic record of the maintenance of the effort, failure of continuity of attention being indicated by the presence of pencil-marks unaimed, or of red dots unmarked.' 'In the present investigation,' adds Mr Burt, 'the measurement sought was the maximum rate at which each boy could mark the dots correctly.... The speed at which he first made three or more omissions in ten seconds... was accepted as the upper limit of his power of sustained attention.' $\ddagger$

Now if $g$ measures the general factor which, because of the tendency of the correlations to form a hierarchy, tends to enter, according to the vector law in Appendix B, into each of the qualities

* I.e. person undergoing test. $†$ The circles in Fig. 7 . 
tested, then ' $g$,' as Mr Burt says of voluntary attention, 'is the capacity, common to all the functions tested, which enters most into the processes involved.' We have therefore a further reason for identifying $g$ with the measure of power to concentrate attention by an effort of Will.

We have just noticed another reason: the fact that the hierarchies were headed by the dotting test, for the successful performance of which power to concentrate attention was most required, not only in the opinion of the boys tested but also in that of the psychologists interrogated. But, since the dotting test headed the hierarchies, it was, of all the tests, that for the successful performance of which a high degree of ' $g$ ' was most necessary. For its position at the head of the hierarchies implies that, of all the tests, it has the highest correlation with the general factor, ' $g$ '*.

Still further evidence of the identity of ' $g$ ' with power to concentrate attention by an effort of Will was obtained in the course of Mr Burt's experiments. He found, for example, that correlations of performances in motor tests-e.g. card dealing-with imputed intelligence are reduced, abolished, or inverted when motor rapidity is due to frequent practice. On this he comments:

Thus so far as motor rapidity is a function of temporary 'facilitation' of the paths of neural discharge it appears also to be a function of intelligence $\uparrow$, while so far as it is a function of permanent 'canalisation' $\ddagger$ of those paths it [is] but slightly or inversely related to intelligence. Facilitation, however, is a function of operative attention; while canalisation, though due to the operation of attention in the past, corresponds with diminution or absence of attention, as the adaptations of the past become the habits of the future $\S$.

Another indication of the close connexion between ' $g$,' the general factor, and capacity for voluntarily concentrating attention was

* For, denoting the various tests by $a, b, \ldots, s, t, \ldots$ and supposing the whole correlation between performances in any two to be due to a general factor $g$, we have (according to footnote * on p. 476 in $\S$ I of Appendix B, below):

$$
\frac{v_{a s}}{\gamma_{b s}}=\frac{r_{a g} \gamma_{s g}}{\gamma_{b g} \gamma_{s g}}=\frac{\gamma_{a g}}{\gamma_{b g}}=\frac{\gamma_{a t}}{\gamma_{b t}}=\ldots
$$

In Tables I and II these equations are only approximately satisfied by the observed values of the correlation coefficients. But the deviations are in both directions, so that the

$$
\frac{\text { mean value of } v_{a s} \text { for different values of } s}{\text { mean value of } v_{b s} \text { for different values of } s}=\frac{v_{a g}}{v_{b g}} \text { approximately. }
$$

It follows that, if the dotting test be denoted by $d$ and heads the hierarchy because the mean value $r_{d s}$ ( $s$ denoting any other test) is greatest, $v_{d g}$ will also be greatest: dotting will have the highest correlation with $g$.
$\dagger$ Cf. above, p. 99.
$\ddagger$ See above, p. 43 .
§ Loc. cit. p. $\mathbf{1} 36$. 
afforded by Mr Burt's memory test. It will be observed, on reference to Tables $I$ and $\mathrm{II}^{*}$, that the memory test occupies a much higher position in the Preparatory School hierarchy (where it appears among the association tests), than in the Elementary School hierarchy (where it falls in among the sensory tests). It follows that the memory test had a considerably higher correlation with ' $g$ ' in the first case than in the second. Observation of the boys when performing the test $\uparrow$, and an inspection of their papers, suggested the explanation. "The Elementary boys,' instead of reproducing the words in the correct order, 'often wrote down the last two or three as they were ringing in their ears, or reproduced the column in inverted order, and, in endeavouring to complete the number of words, commonly waited for the missing word to "recur spontaneously"; whereas the Preparatory boys seldom wrote the words in reversed or inaccurate sequence, and, on forgetting, muttered the words they had retained in their proper order, in the hope of the chain of associations suggesting the missing link.' + In fact, the Preparatory boys appear to have made, on the whole, greater efforts to concentrate attention on this test than were made by the Elementary group. If ' $g$ ' is to be identified with ability to concentrate attention, the higher correlation of memory with ' $g$ ' at the Preparatory School is at once explained.

After reviewing all his evidence $\S$ on the nature of the general factor, ' $g$,' Mr Burt writes:

We have seen throughout that the greater the change, and the greater the complexity, and the greater the novelty involved in the task performed, the greater also (ceteris paribus) is the Imputed Intelligence [and therefore\|

* See above, pp. ro6 to rog.

$\dagger$ Each boy was shewn (and at the same time read and heard), one at a time, a series of monosyllabic words. He was then asked to write these words down in their original order.

$\ddagger$ Burt, loc. cit. p. 167 . He adds: 'Thus, so far as memory implies mere retentiveness of sensory images, it seems to bear little relation to intelligence; so far as memory implies organisation of new associations, it seems to bear a high relation to intelligence.'

$\S$ This evidence is supplemented below by an investigation in $\S 8$ of Appendix B. And the evidence there obtained from Dr Webb supports Mr Burt's evidence.

II Because, owing to the conditions for a hierarchy being approximately satisfied by Mr Burt's correlations, the measure of imputed intelligence is related to $g$ approximately according to equations (4) in $\S 3$ of Appendix B. Now variation of imputed intelligence with degree of excellence in the several experimental tests cannot be due to variations of the specific factor in imputed intelligence; for that specific factor is altogether independent of the measures of performance in any of the twelve tests in question. This variation of imputed intelligence is therefore due mainly to variations of $g$. (Since these equations are only approximately true in this case, the variation in question is mainly due to variations of $g$, but may also depend, to a comparatively insignificant extent, upon 
also the ' $g$ '] of the performer. To relative novelty all the other attributes are probably secondary. Thus high intelligence [and therefore, as we said, high ' $g$ '] seems to mean high capacity for continually systematising mental behaviour by forming new psycho-physical co-ordinations, older coordinations being retained, so that newer co-ordinations bring with them increased complexity and incessant change. In such progressively integrative actions of the mind the efficient and directive agent is attentive consciousness. And in this sense we may agree that so-called 'Voluntary' Attention is, of all recognised psychological processes, the essential factor' in General Intelligence*.

Mr Burt's conclusion that 'voluntary' attention is the essential factor in 'general intelligence' brings him into line with Binet, who believed the central factor to be 'voluntary' attention, and with Wundt $\uparrow$, who 'would make attention the very essence of intellectual power.' +

Our position is then as follows: we have shewn $\S$ that if human souls exist and are able, by an effort of Will, to influence thought and conduct-a hypothesis which we are about to formulate in our fourth law of thought-the measure of a person's will-power, or in other words the degree in which he is able, by an effort of Will, to influence his conduct (including, for example, his performances in mental tests), must constitute a general factor. We have also found evidence $\|$ of the existence of one and only one general factor, measured by $g$, in large numbers of tests of dissimilar mental qualities. The $\dot{a}$ priori probability that these two general factors-power to concentrate attention by an effort of Will on the one hand, and, on the other, the general factor whose measure is g-are identical, is further strengthened by our investigation in $\S 8$ of Appendix B and has been further increased by Mr Burt's evidence I that so-called 'voluntary attention' is of all recognised psychological processes the essential constituent of the general factor whose measure is $g$. Indeed, all the facts that we have examined are consistent with the view that $g$ measures power to concentrate attention by an effort of Will; or, briefly, that $g$ measures will-power. This theory thus resumes all the facts and is inconsistent with none of them. Pending further evidence we shall therefore assume that $g$ is the measure of Will.

variations of group factors.) We conclude that when Mr Burt, in the above quotation, wrote "the greater also (ceteris paribus) is the Imputed Intelligence of the performer' he might equally truly have written 'the greater also (ceteris paribus) is the ' $g$ ' of the performer.'

* Loc. cit. p. 169.

† Cf. W. Brown, loc. cit. p. 89, and C. Burt, J. E. P. Vol. I (Igri), p. 93.

¥ Spearman, Am. J.P. Vol. xv (I904), p. 208.

$\S$ See above, p. Ior.

|| See above, p. II3.

I See pp. II 3 et seq. 
Dr Hart and Professor Spearman have, however, taken a different view of the nature of ' $g$.' In their view the general factor consists of a common fund of energy; they add that "explanation by "attention" seems inadequate.'* They point out that, as Mr Burt's and other investigations have shewn, the general factor enters most into those performances which require most intellectual effort. Wherever a performance is mechanized by habit, correlation with $g$ tends to disappear. Thus 'of all the features invariably attending the mechanization of any activity, none is more remarkable than the way in which it ceases to interfere with other simultaneous activities.' $\uparrow$ They argue that 'if the non-mechanized activities are thus distinguished by their acute competition with one another, they must be competing for something; if the enhancement of any one such activity can only occur at the expense of all the others, we can scarcely escape the conclusion that all these manifestations of energy derive-to some extent, at leastfrom a general common fund.' $\ddagger$ But if, as Burt, Binet and others have supposed, the general factor measures ability to concentrate attention, or (what amounts to the same thing) to reinforce the excitement in any particular system of higher level arcs, it would still be true that the concentration of activity in one such system could only occur at the expense of all others. For, if we suppose that the Will sufficiently reinforces the excitement in any given neurogram $A$, then, by our third law $\S, A$ tends to drain the excitement from any other active higher level arc; or, to use Professor Spearman's phrase, $A$ drains the whole 'intellective energy' available at the moment. Now, the amount of excitement so drained, the measure of the general common fund of intellective energy, may, so far as we yet kncw, vary in the same individual from one moment to another far more than it varies at the same moment from one individual to another. We cannot, therefore, be sure that the 'fund' is capable of characterising the individual $\|$ as the general factor does. We fall back then on the other view according to which the general factor measures the power to reinforce excitement in the first instance, rather than the very variable amount of energy drained in consequence of this initial reinforcement.

* B.J.P. Vol. v, p. 79.

† Loc. cit. pp. 69, 70.

¥ Loc. cit. p. 70 . See also below, p. 226.

$\S$ See above, p. 79.

II It is possible that a long series of tests applied to an individual at many different times might afford a measure of the average level of his available energy: but no such tests appear to have been applied in the investigations which have furnished the evidence for the General Factor. 
It is, as we have seen*, dissimilar mental qualities that tend to manifest the single general factor, ' $g$,' with specific factors but without group factors. When, however, tests of very similar qualities, such for example as 'ability to translate Latin' and 'knowledge of Latin grammar' are in question, we can no longer be sure that the measure of each quality can be expressed in terms of $g$ and specific factors only $\dagger$. Indeed, the absence of a single general factor within the meaning of our definition $\div-$ or, in other words, the inevitable presence of group factors-when the tests relate to a set of similar qualities, has been repeatedly mentioned throughout the literature of this subject, beginning with Professor Spearman's paper of I904§.

Suppose then that a set of tests is concerned with similar qualities. The measure of each of these qualities, if considered with dissimilar qualities, could be approximately expressed $\|$ in terms of $g$ and a specific factor; and it could be completely expressed in a form $\uparrow$ in which $g$ and a specific factor were dominant but in which group factors were also present to a comparatively insignificant extent. When, therefore, a set of similar tests is in question, it is still convenient to express the measures of the qualities tested in terms of $g$, among other factors. But then these other factors** will include group factors that are no longer insignificant.

Two such group factors have been described: the first by Dr Webb and the second by the present writer. Both will concern us in the sequel. Dr Webb's factor is closely connected with purpose and we shall discuss it in the next chapter. But something may conveniently be said here about the other group factor, Cleverness.

\section{§3. Cleverness, a group factor in intellectual qualities.}

In his paper on 'Character and Intelligence,' to which reference has already $\dagger \dagger$ been made, Dr Webb describes an investigation of fortyeight mental qualities $+\ddagger$. The subjects of his enquiry were ninety-eight men students (average age twenty-one) at a Training College during

* On p. II 3 above. See also Appenđix B, $\$ \S 6$ and 7.

$\dagger$ Cf. p. Ix above. † In $\S 3$ of Appendix B: see footnote $\S$ to p. 477 .

$\S$ Loc. cit. p. 273 . Cf. also Dr Webb, loc. cit. p. 53 (first line).

II By means of equation (4) in $\S 3$ of Appendix B.

T See equation (7) in $\S 7$ of Appendix B.

** Viz. $z_{1}, z_{2}$, etc. in equation (7) of Appendix B. The coefficients ( $n$ 's) of the $z$ 's are now-i.e. in the case of $g$ 's that measure similar qualities-significant.

$\dagger \dagger$ See above, p. 98.

¥ The following paragraphs-as far as $p$. 127 below and including $\S 9$ and $\S$ ro of Appendix B-are taken, with minor changes, from pp. 349-359 of a paper by the present writer entitled 'General Ability, Cleverness and Purpose,' published in the $B . J . P$. for May, 1919 . 
the last six months of their second year of training (January-July, I9I2), and a similar group of ninety-six students during the corresponding period of the following year (January-July, I9I3). Dr Webb also investigated a similar number of mental qualities of four groups of London schoolboys, with whom we shall be only once again concerned*. Of the students' forty-eight mental qualities investigated, forty-three were estimated by pairs of prefects who acted as judges; to each pair a group of twenty (or nineteen) students was assigned $\dagger$. The forty-three mental qualities estimated by the prefects included degree of sense of Humour (No. 8 in Table III on p. I26 below) and two described by Dr Webb as intellectual qualities, namely, Quickness of apprehension (No. 35) and Originality of ideas (No. 38).

Among the five qualities not estimated by the prefects, two were objectively measured, namely Examinational Ability and Professor Spearman's single general factor whose measure is $g+$. The manner in which ' $g$ ' was measured is fully described in Dr Webb's paper. Following Dr Hart and Professor Spearman, Dr Webb described ' $g$ ' as a 'General Factor of Intellective Energy.' But Dr Webb produced no additional evidence for regarding $g$ as a measure of intellective energy rather than as a measure of power of voluntarily concentrating nervous energy; or, what amounts to the same thing, of voluntarily concentrating attention; or, in short, of Will.

The condition that must be satisfied in order that the measures of three or more qualities may be expressed in terms of (the measures of) two independent qualities, just as short distances at sea, whatever their direction, may be expressed $\S$ as so much east or (west) and so much north (or south), has been investigated\|. It has been shewn $\|$ that, among the qualities examined by Dr Webb, the correlations between the three qualities ' $g$,' Humour and Originality satisfy this condition** within the limits of probable error $\uparrow \dagger$, while the correlations

* On pp. I58, 159 below.

$\dagger$ The measurements of the qualities were so chosen as to give the same constant (standard deviation) to the frequency distribution of each. See $\S \mathbf{I}$ of Appendix B.

$\ddagger$ It will be remembered that $g$, like the measures of the other qualities with which we are concerned, is distributed according to the normal law and may have any value from $-\infty$ to $+\infty$, its mean value being zero. We continue (see p. Ir3) to write ' $g$ ' in place of 'General Ability' or 'the quality of which the measure is $g$.' That quality we have identified with Will.

$\S$ By means of the equation $r=x \cos \theta+y \sin \theta$, where $r$ is the distance measured in a direction making an angle $\theta\left(=\tan ^{-1} y / x\right)$ north of east and where $x$ is the distance east and $y$ the distance north, so that $x$ and $y$ are two independent measures. Cf. footnote $\dagger$, p. I 27 below.

I Garnett, loc. cit. (Proc. R. S.), p. Io2.

** Equation (Io) in $\S 9$ of Appendix B.

I! See $\S 9$ of Appendix B.

$\dagger \dagger$ See $\S$ I of Appendix B 
of Quickness with any pair of the other three qualities very nearly do so; so that ' $g$,' as measured experimentally, together with Humour and Originality, as estimated in a very large number of cases by Dr Webb's collaborators, is compounded of two, and only two, independent factors, while Quickness consists almost, if not quite, of some combination of the same independent factors. It further appeared that, if $g$ were taken as one of the two independent factors and measured east, the direction in which the other qualities would be measured would be (in the case of Humour) approximately north by west and (in the case of Originality and Quickness) approximately north-north-east, half east*. We may therefore construct a diagram (Fig. 8) by drawing lines $\mathrm{Og}, \mathrm{Oh}, \mathrm{Oo}$ in one plane, making these angles with each other. And we may add to the diagram another line $O q$, very nearly coincident with $\mathrm{Oo} \uparrow$.

If the training college students who formed the subjects of Dr Webb's investi-

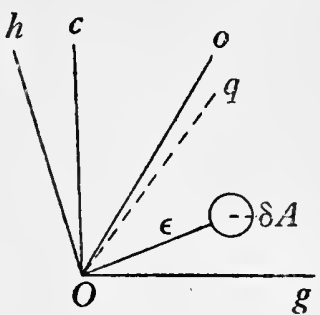

Fig. 8. gation constitute a fair sample of adult Englishmen, the ' $g$,' sense of Humour, Originality, and (probably) Quickness of apprehension of any Englishman can be represented by a single point ( $P$, say) on the plane of the diagram-the 'intellectual plane,' as it has been called; for if $P$ be determined so that the projection of $O P$ on any two of the axes, $O g, O q, O o$ or $O h$ (i.e. the distance from $O$ to $P$ measured in any two of these directions) measures the corresponding two qualities of the subject in question, it follows that the degrees in which the same subject possesses the remaining two qualities will be measured by the projections of $O P$ on the remaining two axes (i.e. by the distance from $O$ to $P$ measured in these other two directions); and the proportion of Englishmen whose intellectual qualities-if for the moment we may confine this term to the four qualities just named-are represented by points lying within any small area $\delta A$ can be calculated§. If $\epsilon$ measures the radius from $O$ to $\delta A$, so that $\epsilon$

* The actual angles are:

$$
\begin{aligned}
& g O h=\cos ^{-1} r_{g h}=\cos ^{-1}(-\cdot 17)=100^{\circ} \\
& h O o=\cos ^{-1} \gamma_{h o}=\cos ^{-1}(\cdot 79)=38^{\circ} \\
& o O g=\cos ^{-1} r_{o g}=\cos ^{-1}(\cdot 47)=62^{\circ}
\end{aligned}
$$

$\dagger$ The correlation coefficient between Quickness and Originality given in $\mathrm{Dr}$ Webb's table is $r_{q o}=\mathrm{I} \cdot 04$.

$\ddagger$ See $\$ 9$ of Appendix B (p. 484 , below).

$\S$ The calculation (Garnett, Proc. R. S., loc. cit. pp. 108, 109) shews that this proportion will be numerically equal to the volume of a cylinder having its axis 
measures the degree in which the subjects represented by points in $\delta A$ are exceptional, the result of the calculation shews* that the number of individuals represented by points in $\delta A$ depends only on $\epsilon$ and is independent of the orientation of the radius joining $O$ to $\delta A$.

With a view to determining the nature of some two independent qualities, or factors, which, combined in different proportions, make up each of the four intellectual qualities, ' $g$,' Humour, Originality and Quickness, we may choose $O g$ as one axis. What, then, is the other axis, represented by a line $O c$ at right angles to $O g$ ? Evidently, since the Humour axis makes with it an angle of only some $10^{\circ}$, it is very nearly identical with the axis of Humour.

Now Dr McDougall has suggested that the process of 'reproduction by similars,' or, as Bain $\uparrow$ called it, 'association by similarity,' is due to 'a partial identity of the complex neural systems involved in the perception of...twoobjects. Each system consists of many sub-systems, and one or more of these sub-systems is common to the two. When the one system is excited, its excitement spreads, not, as is most commonly the case, through some association-path previously established by temporal contiguity, but from the sub-system, which forms also a part of another system, radiates itself through that other system. In the commonplace type of mind this process comparatively rarely occurs. It would seem that in the brains of such persons neural systems tend to become circumscribed and individualised, whereas in a higher type of brain the neural systems are more complexly interwoven, sub-systems becoming freely associated with many principal systems. In a brain so constituted reproduction of similars will frequently occur, causing the dull chain of simple redintegration, the serial reproduction of impressions associated by temporal contiguity, to be broken across. The possessor of a brain so constituted will never be a commonplace person; he may be a crank or an original thinker, or merely a wit.' $\ddagger$

There is no evident reason why such a constitution of brain should have anything to do with capacity voluntarily to concentrate attention, the capacity which Binet and others have identified with the perpendicular to $\delta A$, its base on $\delta A$, and bounded at its other extremity by the surface

$$
z=\frac{\mathrm{I}}{2 \pi \sigma^{2}} e^{-\frac{\mathrm{I}}{2 \sigma^{2}}\left(x^{2}+y^{2}\right)}=\frac{\mathrm{I}}{2 \pi \sigma^{2}} e^{-\frac{\epsilon^{2}}{2 \sigma^{2}}}
$$

where $x$ and $y$ are the measures of the two independent variables of which each of the four qualities is compounded, and where $\epsilon^{2}=x^{2}+y^{2}$.

* Since the equation in the last footnote is independent of the particular axes chosen.

$\dagger$ Cf. W. James, Principles of Psycholggy, Vol. I, p. 578 .

$\ddagger$ Physiological Psychology, p. I39. 
quality whose measure is $g$. Now the quality independent of ' $g$ ' for which we are seeking is very closely connected with Wit or Humour, and also closely connected with Originality*. Let us call it Cleverness. Then Cleverness is defined as a quality which is independent of ' $g$ ' but which, combined with ' $g$ ' in different proportions, wholly constitutes Humour or Originality, and wholly or mainly constitutes Quickness of apprehension. Being closely connected with Humour and with Originality, Cleverness as thus defined is also closely connected with the form of brain constitution described by $\mathrm{Dr} \mathrm{McDougall}$, in the passage we have quoted. Moreover, so far as we can see, this form of brain constitution, like Cleverness as just defined, is independent of ' $g$.' We have therefore grounds for identifying it with cleverness as generally understood.

Let us now consider our diagram (Fig. 8) in the light of this suggestion.

Still having regard only to the five intellectual qualities named in the diagram, we observe that people may be intellectually exceptional in any number of different ways: the radius from $O$ to the small area $\delta A$, while remaining of length $\epsilon$, may make any angle $\theta$ with any one of the fixed axes, say $O g$. If $\epsilon$ remain large and $\theta$ increase, we start with men having great ' $g$ ' -great 'General Ability' $\dagger$; or, as we have said, great Will-power, great power of concentrating attention-but only average Cleverness. Such men will, according to the diagram, possess much more than average Originality and Quickness, but less than the average sense of Humour + . As $\theta$ increases, $\epsilon$ remaining constant, $\delta A$ will come to $O q$ and soon after to $O o$, when $\theta$ is about $60^{\circ} \S$. When, therefore, for a given degree of intellectual exceptionality, Quickness or Originality is greatest, Cleverness is about $\sqrt{3}$ times as great as ' $g$,' while sense of Humour is well above the average. As $\theta$ continues to increase, the rotating radius brings $\delta A$ to $O c$. Then Cleverness is at a maximum ( $\epsilon$ being given); sense of Humour is nearly at a maximum, for the angle $c O h$ is only about $10^{\circ}$; Originality and Quickness are much above the average; but Ability (' $g$ ') is only equal to the average. Finally, when $\delta A$ comes to lie on $O h$ (so that sense of Humour, or Wit, is the most exceptional quality of the

* Its correlation with Humour is, as we see from our diagram (Fig. 8 ), $\cos 10^{\circ}$ or 0.98 and its correlation with Originality is $\cos 28^{\circ}$ or 0.88 .

$\dagger$ See above, pp. 103, II 7 .

$\ddagger$ We here assume the correlation $r_{g h}=-\cdot \mathbf{I} 7$, which is about double the probable error, to be a significant figure; so that the correlation between Ability and Humour is low and negative.

$\S$ For $g O q=\cos ^{-1} \gamma_{g q}=\cos ^{-1} 0 \cdot 53$, and $g O o=\cos ^{-1} \gamma_{g o}=\cos ^{-1} 0 \cdot 47$, according to Dr Webb's table. 
exceptional men represented by points in $\delta A$ ), Cleverness, Originality and Quickness are all much above the average, but Ability is slightly below the average. This does not, of course, mean that very able men (men with very high ' $g$ ') may not have a great sense of Humour; but only that, the greater their Ability (' $g$ '), the greater must be their Cleverness to produce a given degree of sense of Humour.

The distinction between Ability (' $g$ ') and Cleverness has been emphasised by Dr Mercier in an essay on 'Cleverness and Capability.'* He maintains that these two qualities 'are quite different from one another,' and 'may be developed to very different degrees in the same person,' as the point representing that person in our diagram moves from the neighbourhood of $O g$ to the neighbourhood of $O c$. Dr Mercier is further of opinion that 'capability [' $g$ '] ... may be inculcated by a proper training; but no training will make a stupid person clever.' Dr Mercier goes on: 'Capability can be acquired, and it should be one of the main objects of education to see that it is acquired.' The clever man of science 'is fertile in hypotheses.' 'The clever shopman amuses his customers'; he is, in fact, a wit. 'Capable people concentrate their attention on the matter in hand, think it out in all its bearings....' 'Clever [but incapable] people are apt to make mistakes and go wrong because their attention is discursive.' 'From this lack of concentration it results that they do not think matters out.'

William James, after pointing out that there are two stages in reasoning, the first of which-association by similarity-merely operates to call up cognate thoughts (Cleverness), and in the second of which attention is concentrated (' $g$ ') upon the bond of identity between these cognate thoughts, adds: 'So minds of genius may be divided into two main sorts, those who notice the bond and those who merely obey it. The first are the abstract reasoners, properly so called, the men of science, and philosophers-the analysts, in a word; the latter are the poets, the critics-the artists, in a word, the men of intuitions.' $\dagger$ According to this classification, great men of science and philosophers would be represented by points lying far from the origin but near to $O g$, while equally great poets and artists would be represented by points lying equally far from the origin but near to Oc. The importance to the poet of having a large ratio of $c$ to $g$ was emphasised, although in other words, by Schiller in a letter written in 1788 , and quoted by Professor Freud $\ddagger$. Schiller wrote (to a friend who complained of his own lack of creativeness): 'The reason for

* Human Temperament, I91 7, pp. 23-27. † Loc. cit. Vol. II, p. 361.

$\ddagger$ The Interpretation of Dreams, English edition, pp. 85,86 . 
your complaint lies, it seems to me, in the constraint which your intelligence [' $g$ '] imposes upon your imagination.'

We may note in this connexion that the word 'genius' is more commonly used to denote exceptional Cleverness than exceptional ' $g$.' Thus William James writes:

Geniuses are, by common consent, considered to differ from ordinary minds by an unusual development of association by similarity....And as the genius is to the vulgarian, so the vulgar human mind, is to the intelligence of a brute*.

Again, in another place William James says that

Geniuses are commonly believed to excel other men in their power of sustained attention. In most of them, it is to be feared, the so-called 'power' is of the passive sort. Their ideas coruscate, every subject branches infinitely before their fertile minds, and so for hours they may be rapt. But it is their genius making them attentive, not their attention making geniuses of them....It is probable that genius tends actually to prevent a man from acquiring habits of voluntary attention, and that moderate intellectual endowments [moderate $c$ ] are the soil in which we may best expect, here as elsewhere, the virtues of the will [' $g$ ' $\dagger]$, strictly so called, to thrive. But, whether the attention come by grace of genius or by dint of will, the longer one does attend to a topic the more mastery of it one has. And the faculty of voluntarily bringing back a wandering attention, over and over again, is the very root of judgment, character, and will $\ddagger$.

At the same time, it is probable that genius, as the word is commonly used, is more directly measured by $\epsilon=\sqrt{g^{2}+c^{2}}$ than by $g$ (measuring general Ability or capacity to concentrate attention) alone, or even by $c$ (measuring Cleverness, as we have defined it, or tendency to associate by similarity) alone.

If the independence of ' $g$ ' and ' $c$ ' be confirmed by further investigation, and if, of these two, ' $g$ ' alone be educable (although its educability may be innate) $\S$, the distinction between ' $g$ ' and ' $c$ ' should have important consequences for education.

Some further light will be thrown upon the nature of Cleverness, as we have defined it, if we record in conclusion the correlations of its measure, $c$, with the measures of the forty-eight qualities investigated by Dr Webb. Calculated in the manner described in $\S$ ro of Appendix $B$, these correlations are as follows:

* Loc. cit. Vol. II, p. 348 .

$\dagger$ In identifying ' $g$ ' with 'will, strictly so called,' it is necessary, as we have seen, clearly to distinguish the momentary effort of will that we identify with ' $g$ ' from the persistence of purpose that in English is often called by the same name. See above, p. 98.

$\ddagger$ I.oc. cit. Vol. 1, pp. 423-4.

$\S$ See below, $\S 5$ of this chapter. 
Table III.

No. in

Dr Webb's

schedule

I.

Name of Quality

Correlation

with

'Cleverness'

2. Tendency to quick oscillation between cheerfulness and depression (as opposed to permanence of mood)

$-\cdot 05$

$-\cdot 57$

3. Occasional liability to extreme depression $\quad \ldots \quad$...

4. Readiness to become angry $\quad \ldots \quad$.. $\quad \ldots \quad$...

5. Readiness to recover from anger $\quad \ldots \quad \ldots \quad \ldots$

6. Occasional liability to extreme anger $\ldots \quad \ldots \ldots$...

7. Degree of aesthetic feeling (love of the beautiful for its $\begin{array}{llllll}\text { own sake) } \quad \ldots & \ldots & \ldots & \ldots & \ldots & \ldots\end{array}$

8. Degree of sense of humour $\quad \ldots \quad \ldots, \quad \ldots \quad \ldots$

9. Desire to excel at performances (whether at work, play or otherwise) in which the person has his chief interest $\quad \ldots \quad \ldots \quad \ldots \quad \ldots . \quad \ldots \quad \ldots$

Io. Desire to impose his own will on other people (as opposed to tolerance) $\quad \ldots \quad \ldots \quad \ldots \quad \ldots$

I I. Eagerness for admiration $\quad \ldots \quad \ldots \quad \ldots \quad \ldots$

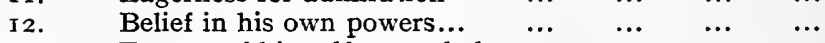

I3. Esteem of himself as a whole $\quad \ldots \quad \ldots \quad \ldots \quad \ldots$

1. Offensive manifestation of this self-esteem (superciliousness) $\quad \ldots \quad \ldots \quad \ldots \quad \ldots \quad \ldots \quad \ldots \quad \ldots$

I5. Fondness for large social gatherings $\ldots \quad \ldots \quad \ldots$

I6. Fondness for small circle of intimate friends ... ...

17. Impulsive kindness (to be distinguished from No. I8)

I8. Tendency to do kindnesses on principle ... ...

19. Degree of corporate spirit (in whatever body interest is taken) $\quad \ldots \quad \ldots . \quad \ldots \quad \ldots \quad \ldots . \quad \ldots$

20. Trustworthiness (keeping his word or engagement, performing his believed duty) $\quad \ldots \quad \ldots \quad \ldots$

21. Conscientiousness (keenness of interest in the goodness and wickedness of actions) ... $\quad \ldots \quad \ldots \quad \ldots$

22. Interest in religious beliefs and ceremonies (regardless of denomination) $\quad \ldots \quad$... $\quad \ldots \quad \ldots \quad \quad \ldots$

$\cdot 15$

$\cdot 33$

$-\cdot 18$

23. Readiness to accept the sentiments of his associates...

24. Desire to be liked by his associates $\ldots \quad \ldots$...

25. Wideness of his influence $\quad \ldots \quad \ldots l \quad \ldots \quad \ldots$

26. Intensity of his influence on his special intimates $\ldots$

Degree of 'tact' in getting on with peopie ... ...

Extent of mental work bestowed upon usual studies

29.

Extent of mental work bestowed upon pleasures (games,

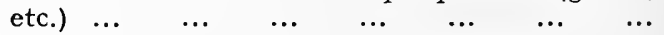

30. Degree of bodily activity during business hours ...

31. Degree of bodily activity in pursuit of pleasures (games, etc.) $\ldots \quad \ldots \quad \ldots \quad \ldots \quad \ldots \quad \ldots \quad \ldots$

32. Degree in which he works with distant objects in view (as opposed to living 'from hand to mouth') ...

Tendency $n o t$ to abandon tasks in the face of obstacles

Tendency not to abandon tasks from mere changeability

$\begin{array}{lllll}\text { Quickness of apprehension } & \ldots & \ldots & \ldots & \ldots\end{array}$

36.

$\begin{array}{lllll}\text { Profoundness of apprehension } & \ldots & \ldots & \ldots & \ldots \\ \text { Soundness of common-sense } & \ldots & \ldots & \ldots & \ldots\end{array}$ $5 \mathbf{I}$

* This figure cannot be calculated from equation (13) in $\S$ Io of Appendix B.

The equivalent formula $v_{c h}=\frac{v_{c h}-v_{g h} \cdot v_{g o}}{\sqrt{\mathrm{I}-\gamma_{g o}^{2}}}$ has therefore been used. 


\section{Table III-continued.}

No. in

Dr Webb's schedule

38.

Originality of ideas Name of Quality

Correlation

with

'Cleverness'

.88

39.

Pure-mindedness (extent to which he shuns telling or hearing stories of immoral meaning) $\quad \ldots \quad \ldots \quad$...

40. Power of getting through mental work vapidly $\ldots$

4r. Physique (estimated by visiting doctor and lecturer in

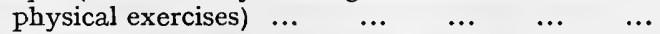

42.

General excellence of character (estimated by lecturers)

43.

Estimate of general excellence of character (supplied by $\begin{array}{llllll}\text { each prefect }) \ldots & \ldots & \ldots & \ldots & \ldots & \ldots\end{array}$

44. $\quad \begin{array}{llllll}\text { Examinational ability } & \ldots & \ldots & \ldots & \ldots & \ldots\end{array}$

45. Athletics (estimated by captains and by a member of
the college staff) $\ldots . . . \quad \ldots$

46. Experimental tests of intelligence, furnishing the correlations of ' $g$ ' with the forty-seven other qualities, as explained on pp. $35-38$ of Dr Webb's

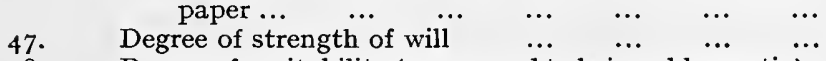

48. Degree of excitability (as opposed to being phlegmatic)

- 0 *

Inspection of these figures indicates that Cleverness may be recognised in practice-as, for example, when interviewing, for an appointment, a candidate to whose general Ability (' $g$ ') testimonials or examination results bear witness - by noting his sense of humour, general tendency to cheerfulness (which is perhaps difficult to judge on the occasion of such an interview!), or quickness of apprehension.

It remains to add that, since $g$ and $c$ both enter $\dagger$ into several intellectual qualities, $g$ cannot be regarded as a measure of general intelligence $\ddagger$. But no harm will be done by speaking of $g$, as we have done, as a measure of General Ability or of Ability, so long as we remember that this is our definition of Ability: the quality whose measure is $g$.

\section{§ 4. The Fourth Law: Free Will.}

We have now to sum up this discussion leading to our fourth law of thought.

* The correlation between the independent variables $c$ and $g$ cannot be calculated from equation (I4). That its value is zero follows from our definition of $c$.

$f$ According to the vector law

$$
q_{i}=g \cos \theta_{i}+c \sin \theta_{i}
$$

where $q_{i}$ is the measure of the intellectual quality in question and $\theta_{i}$ is the angle which its vector would make with $O g$ in Fig. 8.

$\ddagger$ The early papers on the general factor identify it with 'general intelligence' (Spearman, Am.J.P. Vol.xv, p. 20I; Burt, B.J.P. Vol. III, p. 94). But Dr Hart and Professor Spearman have demonstrated that $g$ does not measure 'general intell1gence' as ordinarily understood or imputed by teachers. (See loc.cit.pp.67 and 7r.) 
We began* by considering the fundamental question whether soul and body do, or do not, interact. Recognising that no known facts were conclusive on either side, we determined $\dagger$, on ethical grounds, to assume interaction, an assumption we delayed formulating in the hope that we might be able to say, not only that interaction takes place, but also how it takes place.

The means by which the soul acts (or seems to act) on the body we called 'Will.' Men give this one name to every voluntary effort, whether to worship God or to overpower a foe. But they cannot be sure that two such different efforts of Will have anything in common except the feeling of effort and the name, Will. While awaiting further evidence, we made the simplest assumption possible: namely, that the common-sense of mankind is right in supposing that the process of 'willing' is one and the same in whatever connexion it occurs-or, in other words, that one and the same general factor operates in all voluntary acts-and in marking this supposition by the use of the one word, Will.

We next observed that our third law of thought, derived (as it was) from consideration of involuntary thinking, was ready with the suggestion of a single simple process-reinforcement (or facilitation)by which the soul might exert all its manifold influences upon the body. And we remarked that it was as reinforcement of neural excitement (or, what amounts to the same thing $\dot{+}$, as concentration of attention) that William James, Dr McDougall, and others, writing before the experimental and statistical work of Mr Burt and Dr Webb, described the 'unique'§ means by which the soul affects the body.

Subsequently, as we saw $\|$, the statistical investigations of Professor Spearman and his followers have led to the demonstration $\mathbb{1}$ that a single general factor, ' $g$,' enters into all mental measurements and that, whenever the qualities measured are sufficiently dissimilar, each of these qualities is compounded, according to the vector law, of the general factor ' $g$ ' and of a specific factor that is independent both of ' $g$ ' and of the other specific factors. Each of $n$ sufficiently dissimilar mental tests is thus compounded, according to the vector law, of two out of $n+\mathrm{I}$ independent factors, one of the two being in every case the single general factor ' $g$ ' and the other a specific factor**.

We next $\dagger \dagger$ investigated the nature of this general factor, and we observed that, if we were right in assuming that human Wills exist

* On p. 95. † See above, p. $98 . \quad \ddagger$ See the footnote $\|$ on p. 129 below.

$\S$ See above, p. Ior. II On pp. I03 to I 3 . I Garnett, Proc. R. S. loc. cit.

** I.e. it enters into that test alone. See footnote $\S$ on p. 477 (Appendix B, $\S 3$ ).

†† On pp. Ir 3 et seq. 
and are able to affect thought and conduct by reinforcing neural excitement or by concentrating attention, then the measure, $g$, of the general factor or General Ability also measures the power to concentrate attention by an effort of Will; or, briefly, that $g$ measures Will. Then we saw that the truth of this conclusion was confirmed in several independent ways* by $\mathrm{Mr}$ Burt's Oxford experiments on schoolboys $\uparrow$, and by Dr Webb's research on Training College students. Then we considered some other suggestions concerning the nature of the general factor, and observed, in particular, that 'Cleverness' enters as a group factor into several intellectual qualities so that it would be misleading to describe $g$ as a measure of general intelligence, although it would be convenient to describe as Ability the quality whose measure is $g$. But we found nothing inconsistent with our conclusion $\ddagger$ that $g$ measures power to concentrate attention by an effort of Will.

Our assumption of psycho-physical interaction and of the manner of it, thus fits experience as neatly as could be desired $\S$. These assumptions we now formulate as our fourth law of thought: Will, measured by the general factor $g$, can reinforce the excitement in any excited system of higher level arcs; and so, by our third law, may cause that system to drain the excitement from all other active arcs of the higher level. But it is only if the Will's reinforcement of the excitement in a neurogram is sufficient, that the neurogram in question drains the excitement from other active arcs, so that the corresponding thoughtactivity comes next into the focus of consciousness $\|$.

* The dotting test, the motor tests, and the memory tests.

$\dagger$ See pp. II5, II6.

$\ddagger$ See above, p. II 7 .

$\S$ The present writer is not aware that the alternative hypotheses-whether epiphenomenalism or psycho-physical parallelism in either form-account so simply for these new facts; and in particular for the remarkable fact that each of any number $n$ of sufficiently dissimilar mental qualities appears always to be compounded (according to the vector law) of one of an equal number $n$ of independent (specific) qualities with a single general factor that is independent of each of these $n$ specific qualities and, in fact, to use a geometrical metaphor, belongs to another (or $n+\mathrm{I}$ th) dimension. But he does not therefore claim that the assumption of interaction-namely, that the Will, referred to in the fourth law of thought, is the instrument of soul acting on body-finds its principal support on other than ethical grounds. The fourth law, that the effort of attention that we call an act of Will is accompanied by increased neural excitement in higher level arcs, might indeed be regarded as independent of the interaction hypothesis.

If The fact that power to concentrate attention on any desired thoughtactivity, $\mathbf{a}$, is the same as the power to reinforce excitement in the corresponding neurogram, $A$, follows from our first and third laws of thought. Experimental support for this identification is not lacking. The reagents in Mr Burt's experiments asserted that the dotting test required the greatest effort of attention. It followed that the more they concentrated their attention, the larger was the number of circles which they were able to 'dot' in a given time; or, once more, the greater 
Thus the Will may guide* the stream of thought by concentrating excitement in selected active neurograms. Our fourth law implies that the Will can intensify the stream of thought, as well as guide it. It is true that, when the Will intervenes to inhibit an emotion by concentrating attention on some conflicting thought-activity, this intervention, if successful, may result in a decrease of the total excitement. But for most purposes of voluntary thinking, the Will does intensify the neural processes involved: for example, when Dr McDougall found that the exercise of Will might so increase the intensity of neural impulses that ten or twelve readings of a row of nonsense syllables might effect a greater lowering of resistance-a higher degree of 'canalisation'- than 150 readings 'in an attitude of indifference.' $\dagger$

We are unable to say whence comes the excitement which the Will adds to that of an active neurogram so as to make all the available excitement drain through the neurogram whose activity is thus intensified. It is, however, reasonable to suppose that the guiding excitement is part of the available excitement at the moment, rather than that the Will taps some mysterious outside source for the excitement which it requires for the purpose of guiding thought. It is, in fact, probable that, as Dr McDougall has said, we shall have to recognise in this concentration of nervous energy a unique effect of psychical activity + .

the effort of attention, the shorter the latency-the interval between the stimulus (the appearance of the empty circle on the right of the aperture) and the reaction (the putting of a dot inside the circle). That effort of attention shortens 'reaction time' is, of course, well known: cf. W. James, loc. cit. Vol. I, p. 425, and pp. 427-34.

Now, Professor Sherrington has shewn that, in the case of simple reflexes, short latencies follow strong stimuli. For example: the latency of the scratch reflex in a spinal dog usually lies between $0 \cdot I_{4}$ second for intenser stimulation, and 0.5 second for weaker, but sometimes extends to 2.44 or even to 3.54 seconds. (Loc. cit. p. 2r.) Similarly, the latency in the inhibition of extensor muscle, in the case of the flexion reflex, was as short as .032 second with strong stimuli; but with weak stimuli was occasionally as long as 0.4 second (p. 92).

There is no reason to doubt that the stronger the stimulus voluntarily applied to the boy's motor centres in the dotting test, the shorter would be the latency of the reaction. It appears, then, that a strong feeling of effort of attention accompanies strong reinforcement of neural excitement in the motor centres.

* By our third law and its corollary, neurograms-interest-systems, and especially instinct-neurograms-may also guide thought. So we may now say that thought is guided by neurograms and Will; or, if we prefer, by instincts, interests, and Will.

$\dagger$ McDougall quoted above, p. 10o. Dr McDougall adds this comment: 'Experiments of this class... are bringing home to us the magnitude of the influence of conation (volition) as compared with mere temporal contiguity or succession. Mental process is effective...in proportion as it involves strong conation, strong desire or volition; a fact which implies on the neural side effective concentration of psycho-physical energy in proportion to the strength of the conation.' (American Journal of Insanity, Vol. Lxix, p. 869.)

$\ddagger$ Physiological Psychology, p. 167, quoted above, p. Ior. 
We said that the Will not only concentrates excitement-probably part of the available excitement at the moment-so as to guide thought, but also increases the total quantity of available excitement. The source of the addition to the total available excitement that results from the Will's intervention is less uncertain than that of the excitement which the Will concentrates in a particular neurogram in order to cause drainage through that neurogram; for the intervention of Will-the exercise of voluntary effort-is commonly accompanied by the liberation of neural impulses from some interest-system which the Will renders active. We do well to remind ourselves that, according to our definition*, an 'interest-system' is any system of interconnected neurograms, any system of neurones (not necessarily confined to the brain or even to the spinal cord) which are connected by synapses of low resistance. We may recognise three types of interestsystem, whose excitement may accompany an effort of Will and liberate meanwhile the additional impulses, which are drained through the neurogram selected by the Will for this purpose and the effects of which may then be observed, as in Dr McDougall's experiments on Memory $\dagger$, or as they are marked in every-day life by the increased force of muscular contraction that results from a strong effort of Will.

The first type of interest-system is one of which the excitement results in some characteristic series of bodily movements. The voluntary movements that most commonly accompany the concentration of attention are perhaps those of the muscles of the lips and eyes. Most people who are accustomed to concentrated intellectual effort find that, during every such effort of attention, the eyes, and often the forehead and lips also, are being strained. The position of strain, once voluntarily adopted, maintains itself, and the feeling of strain is accompanied by the inrush of impulses which add to the available neural excitement $\ddagger$. It should be added that the particular muscles affected in the facial expression of attention vary widely with different individuals; but the strain or movement of muscle, that adds to the available excitement during voluntary effort, is by no means necessarily confined to the muscles of the face and head. 'It is a well-known fact that persons striving to keep their attention on a

* See above, P. 62 , including footnote $\|$.

$\dagger$ See above, p. roo.

$\ddagger$ I have often observed that, after going to bed at the end of a hard day's brain work, I have been kept awake by a feeling of continued effort and excitement: but that, no sooner had I realised that the source of the trouble consisted in the continued facial attitude of attention, than the voluntary relaxation of my lip and eye muscles resulted almost immediately in the cessation of the feeling of strain, and so in falling asleep. 
difficult subject will resort to movements of various unmeaning kinds, such as pacing the room, drumming with the fingers, playing with keys or watch-chain, scratching head, pulling moustache, vibrating foot, or what not, according to the individual. There is an anecdote of Sir Walter Scott, when a boy, rising to the head of his class by cutting off from the jacket of the usual head boy a button which the latter was in the habit of twirling in his fingers during the lesson. The button gone, its owner's power of reciting also departed.'* William James regarded these movements as serviceable in fostering thought by draining off superfluous brain currents. Dr Ballard is probably more correct when he writes: 'The real explanation, it seems to me, is that they support trains of thought by providing the necessary degree of present sense stimulation.' $\dagger$ He adds: ' $I$ find that if $I$ wish to concentrate my mind on a difficult problem, I can do so best while rapidly tapping the ground with my right foot.'

Secondly, the interest-system whose activity provides the additional excitement that generally accompanies voluntary effort, may consist principally of arcs of the visceral or 'autonomic' system $\$$. Darwin, for instance, testified to having heard, 'as a proof of the exciting nature of anger, that a man when excessively jaded will sometimes invent imaginary offences and put himself into a passion, unconsciously for the sake of reinvigorating himself; and,' Darwin continues,

* W. James, loc. cit. Vol. I, pp. $457,45^{8}$.

$\dagger$ Loc. cit. pp. 53, 54 .

$\ddagger$ McDougall has suggested that 'the source of the additional motive power, which in the moral effort of volition is thrown upon the side of the weaker, more ideal impulse, is ultimately to be found in that instinct of self-display or self-assertion whose affective aspect is the emotion of positive self-feeling' (Social Psychology, p. 256) and whose neurogram is an important constituent of the neurogram of the self-regarding sentiment (see below, p. 146). Indeed, he defines 'volition as the supporting or re-enforcing of a desire or conation by the co-operation of an impulse excited within the system of the self-regarding sentiment' (Social Psychology, p. 249). He thus makes self-conscious willing continuous with lower forms of conation. McDougall's account of volition is accordingly consistent with our account of (see Chapter 8, below) that kind of will-will at long range-that we decided (on p. 98, above) to call 'purpose.' But our account of will at short range-the 'Will' with which this chapter is concerned-describes every effort of Will as due to psycho-physical interaction, resulting in an increase of excitement in a particular system of nervous arcs. The interest-system whose activity provides the additional excitement may, however, very well be the self-regarding sentiment, as McDougall suggests.

The measure $g$ (see p. II 7 ) of Will would in any case depend upon the interestsystem, whether that of the self-regarding sentiment or any other, from which the additional excitement generally comes. It would also depend upon the relation of that interest-system to the rest of the neurography (see above, p. 63). It would therefore be a function of bodily organisation; but, if we are right in assuming psycho-physical interaction, not necessarily of bodily organisation alone. 
'since hearing this remark, I have occasionally recognized its full truth.'*

The increase of available excitement that results from voluntarily putting oneself into a passion, or from over-exciting oneself by other voluntary means, may be further intensified and prolonged by bodily changes that result from intense excitement. Thus Professor Cannon has shewn that intense excitement stimulates the adrenal glandssmall glands of internal secretion lying anterior to each kidneywhich thereupon increase the percentage of their secretion, 'adrenin,' in the blood. The increased secretion of adrenin produces several bodily changes: 'the cessation of processes in the alimentary canal (thus freeing the energy supply for other parts); the shifting of blood from the abdominal organs, whose activities are deferable, to the organs immediately essential to muscular exertion (the lungs, the heart, the central nervous system); the increased vigor of contraction of the heart; the quick abolition of the effects of muscular fatigue; the mobilizing of energy-giving sugar in the circulation.' Professor Cannon points out that 'every one of these visceral changes is directly serviceable in making the organism more effective in the violent display of energy which fear or rage or pain may involve.' $\dagger$ In short, the presence of adrenin in the blood intensifies and prolongs the bodily changes which gave rise to it. Some of these changes, notably the transfer of blood to the central nervous system, are evidently favourable to an increased output of neural excitement $\ddagger$.

* Quoted from Darwin (The Expression of Emotions in Man and Animals, p. 79) by Professor W. B. Cannon, Bodily Changes in Pain, Hunger, Fear and Rage (I9I5), p. 216.

$\dagger$ Loc. cit. pp. 215, 216.

$\ddagger$ They can be brought about indirectly, it is true, by intense voluntary effort, as was proved by the glycosuria of four out of nine medical students, all normally without sugar in their urine, after a hard examination, while only one of the nine had glycosuria after an easier examination. (Cannon, loc. cit. p. 75.) Professor Cannon adds: 'It would doubtless be incorrect to attempt to account for all the increased strength and tireless endurance, which may be experienced in periods of great excitement, on the basis of abundant supplies provided then for muscular contraction, and a special secretion for avoiding or abolishing the depressive influences of fatigue. Tremors, muscular twitchings, the assumption of characteristic attitudes, all indicate that there is an immensely augmented activity of the nervous system-an activity that discharges powerfully even into parts not directly concerned in struggle, as, for example, into the muscles of voice, causing peculiar cries or warning notes; into the muscles of the ears, drawing them back or causing them to stand erect, and into the small muscles about the lips, tightening them and revealing the teeth. The typical appearances of human beings, as well as lower animals, when in the grip of such deeply agitating emotions as fear and rage, are so well recognized as to constitute a primitive and common means of judging the nature of the experience through which the organism is passing. This "pattern" response of the nervous system to an emotion-provoking object or situation is probably capable of bringing into action a much greater number of neurones in the central nervous system than are likely to be concerned 
In the third place, an interest-system stimulated by the Will and then serving to increase the available excitement, may consist primarily of brain neurones. The activity of such an interest-system need not be accompanied by movement of skeletal muscle or by impulses traversing visceral arcs, unless on account of affectiveconative elements in the interest. Thus, to increase the effect of willing to do some unpleasant duty, one may voluntarily awaken the interests with which the duty in question is in harmony. The awakening of these interests will not only increase the total excitement available, but, by 'multiple stimulation,' in the manner described above*, will involuntarily guide the stream of thought in the direction in which the Will is guiding it voluntarily, namely, towards the performance of the originally distasteful task. The wider and deeper the accessible interest-systems possessed by any individual, the greater the reserves of which his Will can thus make use to reinforce its direct action. The use to which such interest-systems as we are now considering may be put in voluntary thinking is illustrated in scientific research when the researcher desires to associate two hitherto unconnected thought-activities or their corresponding neurograms $\dagger$. For example, Maxwell, having formulated equations for the propagation of electromagnetic waves, and conceiving the possibility of a hitherto unsuspected connexion between electro-magnetic waves and light, would, by voluntarily awakening his interest in optics, add to the excitement available for working out the speed at which, according to his equations, electro-magnetic waves would travel, a speed which turned out to be the speed of light. Or Newton, having conceived the possibility of a hitherto unsuspected connexion between the acceleration of a body falling on to the earth's surface and the central acceleration of the moon in her orbit, may have derived from his interest in astronomy so great an increase of neural excitement that (in fact) he had to get a friend to complete the arithmetic which proved that the same terrestrial gravitation is responsible, both for the weight of bodies on the earth's surface, and for holding the moon in her orbit.

Before leaving this discussion of the increment of neural excitement that commonly accompanies voluntary effort, we do well to

in even a supreme act of volition. The nervous impulses delivered to the muscles, furthermore, operate upon organs well supplied with energy-yielding material and well fortified by rapidly circulating blood and by secreted adrenin, against quick loss of power because of accumulating waste. Under such circumstances of excitement the performance of extraordinary feats of strength or endurance is natural enough.' ( $L O c$ cit. pp. 217, 218.)

* See above, p. 83

+ Cf. below, Chapter I4: 
note one important effect of the increase in question. Suppose that, at any moment, a thought-activity $\mathfrak{A}$ is occupying the focus of consciousness. According to our second law, excitement diffuses from $A$ into as many connected arcs, $A_{1}, A_{2}, \ldots, A_{n}$, as are connected with $A$ through synapses whose insulations the excitement in $A$ is sufficient to overcome; and the greater the excitement in $A$, the greater will be the number, $n$, of associated neurograms that will be rendered active as the excitement drains through $A$. Since, according to our fourth law, the Will can only select (intensify the activity of, and so cause to drain) neurograms that are already active, it follows that increase in the excitement of $A$ increases in general the number of associated neurograms from which the Will can choose one to drain the system. Or, in other words, the greater the neural excitement, the greater the number of thought-activities from among which the Will can choose one and bring it into the focus of consciousness*. The greater, therefore, the neural excitement in a given individual, the greater will be his chance of following a novel train of thought, and so of doing original thinking $t$; or, if he is undergoing examination, the greater will be his chance of bringing to mind some almost forgotten element of his subject; or, if he is making a public speech, the greater will be the choice of language at his command.

\section{§5. Educability of Will.}

We have next to enquire whether the Will-capacity for concentrating and increasing neural excitement-is educable. Mr Burt is inclined to answer this question in the negative. He gives various reasons for believing 'that the superior proficiency at Intelligence tests on the part of boys of superior parentage, was inborn.' $\ddagger$ But the boys 'of superior parentage' were also the boys of a particular school which, if Will is educable, may or may not have been superior to the other in developing ' $g$.' The principal item of evidence cited by $\mathrm{Mr}$ Burt in support of his view consisted in the repetition of his intelligence tests on a number of the Elementary School boys some eighteen months after the series previously described. The average performance of the Elementary School boys at the second test differed,

* See below, pp. I87, I88.

$\dagger$ Webb, in the case of his training college students (loc. cit. Table VI), found that the correlation between measured ' $g$ ' (ability to concentrate attention) and estimated oviginality of ideas was '47. Cf. also W. James' observation that 'If focalisation [concentration] of brain activity be the fundamental fact of reasonable [voluntary] thought, we see why intense interest or concentrated passion makes us think so much more truly and profoundly.' (Vol. II, pp. 366,367 .)

$\ddagger$ Loc. cit.p. 176 . 
in the case of most tests, by less than ten per cent. from the average performance eighteen months previously. One of the two exceptional cases was card dealing; the other shewed a marked improvement, which Mr Burt attributes to the persistence of the practice gained during the earlier experiments. The dotting test shewed a deterioration of three per cent., and this was typical. Mr Burt comments that, though the period between the ages of thirteen and fifteen is for boys one of rapid progress in knowledge, interests, and acquired aptitudes, their capacities-' $g$ '-measured by the tests seemed during the interval to have remained all but stationary. He adds: 'Hence, these capacities appear to constitute a relatively permanent endowment; and consequently it seems legitimate to assume that they depend upon innate differences in the individuals concerned.' *

It is to be remarked, on the other hand, that many of the Elementary School boys subjected to the second test had meanwhile left school and become employed in routine work which, presumably, made no great demand for the exercise of intellectual effort. If practice in the exercise of Will increases the effect of voluntary effort -in other words, if ' $g$ ' can be increased by practice-boys would not be unlikely to suffer, during a period of unintelligent employment, a diminution $\dagger$ of ' $g$ ' at least equal to the increase of their ' $g$ ' in the Elementary School during the first part of the interval between the first and second tests. Had it been possible for Mr Burt to repeat his tests on the Preparatory School boys after an eighteen months' interval spent by them in preparing for public school scholarship and entrance examinations, and affording them, therefore, much practice in the concentration of attention, and had he found that the ' $g$ ' of these boys had not improved appreciably in the interval, the result might well have been conclusive.

Another argument advanced by Mr Burt in support of his view that ' $g$ ' is inherited, rather than developed by education, rests upon the fact that, in most mental tests, the Preparatory boys, whose fathers were 'in nearly every case...fellows of the Royal Society, university professors, college tutors, and bishops,' surpassed the boys of the Elementary School, 'sons of local tradesmen.' But, as we have said, the Preparatory boys on the one hand, and the Elementary

* Loc. cit. p. 176 .

$\dagger$ It is the experience of many large firms of manufacturing engineers in the North of England that such a deterioration does take place. For this reason the firms in question prefer to receive boys into their shops straight from school at fourteen years of age, and to direct their (part-time) education from fourteen to sixteen years, when their 'apprenticeship' begins, rather than to receive them at sixteen after two years' deterioration in uneducational employment. 
boys on the other, not only had different fathers but attended different schools; and it may well be that the Preparatory boys, in writing Latin verses and Greek exercises, and in solving problems in arithmetic, not only in school but in considerable quantity for home-work also, received practice in the voluntary concentration of attention out of all proportion greater than that given by the comparatively playlike exercises of the Elementary School.

A final and more weighty argument in favour of Mr Burt's view, is the low correlation $(0 \cdot 29)$ between the alphabet test of Intelligence and age, in the case of sixty Elementary School boys between ten and sixteen years of age. It would be interesting to see whether the correlations between ' $g$ ' and the length of time during which reagents had been subjected to a strenuous intellectual discipline is equally low.

On the other hand, it is very generally agreed that the Will-or power to concentrate attention*_-is educable, that 'development of will power in connection with any activity is accompanied by a development of will power as a whole.' $\dagger$ For example, Dr 'Temple, discussing the Will and its freedom, writes: 'It scarcely matters what subject is taught: the vital matter is that the child should learn "attertion" in general.' +

Again, Sir Edmund Gosse describes how, as a child, he spent many hours in a stuffy little room making solemn and ridiculous imitations of papers read by his father before the Linnean Society; and he adds:

My labours failed to make me a zoologist, and the multitude of my designs and my descriptions have left me helplessly ignorant of the anatomy of a sea-anemone. Yet I cannot look upon the mental discipline as useless. It taught me to concentrate my attention.....Moreover, it gave me the habit of going on with any piece of work I had in hand, not flagging because the interest or picturesqueness of the theme had declined, but pushing forth towards a definite goal, well foreseen and limited beforehand. For almost any intellectual employment in later life, it seems to me that this discipline was valuable. I am, however, not the less conscious how ludicrous was the mode in which, in my tenth year, I obtained it $\$$.

Once more, C. G. J. Jacobi (I804-5I), the great mathematician, is reported to have said: 'It must not be supposed that it is to a gift of Nature that I owe such mathematical power as I possess. No, it has come by hard work, hard work. Not mere industry, but brain-

* See the fourth law on p. I 29, above.

$\dagger$ Psychological Revıew, Vol. VI, p. 165; quoted by Spearman, Am.J.P. Vol. $x v$, p. 216 .

$\ddagger$ The Nature of Personality, p. 28.

$\S$ Father and Son (I909 edition), p. I76. 
splitting thinking-hard work; hard work that has often endangered my health.'*

The chief Civil Service Commissioner (Sir Stanley Leathes) is of the same opinion. 'What is valuable in historical education,' he writes, 'is not the acquisition of a set of facts...by lectures or systematic instruction, but...the effort to master and understand the books.' $\dagger$

We have also some direct evidence of the educability of Will (measured by ' $g$ ') in the high correlation which, as $\S 8$ of Appendix B shews, Dr Webb's research proved to exist between ' $g$ ' and practice in the concentration of attention. Those students who worked hardest at their studies, 'reckoned in amount of actual energy, not in results of work, or quickness of work,' $\ddagger$ had ceteris paribus the highest ' $g$.'

While then the balance of available evidence seems to indicate that ' $g$ ' is educable, and capable of being increased by practice in concentrating attention by effort of Will, the degree of its educability may well be innate.

\section{§ 6. Will in Everyday Life.}

We have now to examine the part played by voluntary thinking in everyday life. We observe, in the first place, that this part is supremely important: the title rôle in very truth. For it is only by moving the Will to intervene in the stream of consciousness that the soul or ego makes itself felt. Nevertheless the Will is by no means always on the stage.

For example, most of what we do is not done at the direct instance of our Wills. Many of our movements are obviously not 'willed' at all. Of a spinal reflex $\S$ one is not even conscious; and a sensation reflex $\|$ or an instinctive movement may take place for the first time without being preceded by the appearance of an image of it in consciousness $\uparrow$. That is to say, reflexes and instinctive movements may be unforeseen. They may even be unrecognised. For, although the occurrence of any movement that is not a spinal reflex may be felt,

* Professor Sir Richard Gregory, Discovery, p. 5.

$\dagger$ What is Education? p. 82. (Italics mine.)

$\ddagger$ Webb, loc. cit. p. 9r. A further indication that ' $g$ ' is educable is cited below in footnote * on p. I59.

$\$$ See above, p. 35 .

II See above, p. 36 .

- Cf. W. James: 'The other day I was standing at a railroad station with a little child, when an express-train went thundering by. The child, who was near the edge of the platform, started, winked, had his breathing convulsed, turned pale, burst out crying, and ran frantically towards me and hid his face. I have no doubt that this youngster was almost as much astonished by his own behaviour as he was by the train, and more than I was, who stood by. (Loc. cit. Vol. II, p. 487.$)$ 
the feeling need not necessarily occupy the focus of consciousness and so receive attention. In other words, the impulses in the system of brain arcs that corresponds to the feeling of such a movement may not be sufficiently intense to cause that system of arcs to drain the available excitement. This system of arcs becomes a neurogram, however shallow, by reason of all the arcs comprising it having been excited together; and if this neurogram does drain the available excitement, not only is the neurogram of the feeling deepened but it becomes connected with the neurogram of the thought-activity-the stimulus-which led to the movement in question. The next time that the stimulus-neurogram is excited, the excitement tends to spread to the neurogram of the feeling of the movement, which may thus be foreseen and brought under the Will's control. In short, a movement that has once occurred and has attracted sufficient attention becomes, for the future, a movement capable of being controlled by the Will: there is a neurogram corresponding to it, and, whenever this neurogram is excited, the Will can intensify the excitement until the neurogram drains again and the movement is repeated.

Two aspects of this matter require to be noticed. In the first place, our brief account needs qualification unless the movement in question is so simple that a single performance leaves a sufficient record to render repetition possible: the man who has only once had the luck to cut a three on the ice, or to bring off a Telemark swing on skis, cannot yet say that he can perform these feats at will.

Our second remark relates to the repetition of a movement on the sufficient excitement of the neurogram of the kinaesthetic sensation of a previous performance. As we have seen*, the kinaesthetic impulses that reach the Rolandic cortex during the contraction of a muscle appear to return by the pyramidal tract to the motor neurones of that muscle and so to reinforce its contraction. Accordingly, when the kinaesthetic centres (neurones of the Rolandic cortex) are excited afresh, as an image of the feeling of the movement appears in the field of consciousness, the excitement tends to spread in the same direction as before, namely down the pyramidal tract to the motor neurones. Unless therefore the kinaesthetic (Rolandic) portion $\dagger$ of the neurogram of a movement is drained in some other way-for example, in accordance with our third law, as the attention passes involuntarily or voluntarily away from the image of the movement

* See above, pp. 59, 60 .

i A neurogram of a movement may contain arcs in various other regions of the cortex-for example, arcs the excitement of which corresponds to a visual image of the movement. 
to some other thought-activity-its excitement must lead to the movement in question. In other words 'every representation of a [voluntary] movement awakens in some degree* the actual movement which is its object; and awakens it in a maximum degree whenever it is not kept from so doing by an antagonistic representation present simultaneously to the mind.' $\dagger$ Whenever movement follows unhesitatingly and immediately the notion of it in the mind--i.e. when there is no antagonistic thought-activity present-we have what is called 'ideomotor' action $\overbrace{}^{+}$.

Now the voluntary movements which we have just been considering are called 'voluntary,' not because the Will need operate to secure their performance, but because they are subject to the control of the Will. Generally, when they occur it is not because the Will has caused them; and when they are inhibited, their inhibition is not in general due to the Will. On the contrary, these voluntary movements follow, as we have said, when their kinaesthetic images occupy the focus of consciousness unchallenged; and these images generally attain and occupy that position in the course-or, rather, at the end-of a train of involuntary thinking, such as we considered in connexion with our third law

William James has pointed out how apt we are to suppose that the Will intervenes-that sorne fiat or effort of decision is requiredin many voluntary movernents which, in fact, are simple ideo-motor acts. Thus he writes:

We know what it is to get out of bed on a freezing morning in a room without a fire, and how the very vital principle within us protests against the ordeal. Probably most persons have lain on certain mornings for an hour at a time unable to brace themselves to the resolve. We think how late we shall be, how the duties of the day will suffer; we say, 'I must get up, this is ignominious,' etc.; but still the warm couch feels too delicious, the cold outside too cruel, and resolution faints away and postpones itself again and again just as it seemed on the verge of bursting the resistance and passing over into the decisive act. Now how do we ever get up under such circumstances? If I may generalise from my own experience, we more often than not get up without any struggle or decision at all. We suddenly find that we have got up. A fortunate lapse of consciousness occurs; we forget both the warmth and the cold; we fall into some revery connected with the day's life, in the course of which the idea flashes across us 'Hollo!

* Thus the spectator of an exciting high-jump competition accompanies each competitor's jump with an incipient movement of his own legs. Other examples are quoted by W. James (loc. cit. Vol. II, p. 525) from Lotze.

$\dagger$ W. James (lnc. cit. Vol. II, p. 526) who italicises the words quoted.

$\ddagger$ W. James (linc. cit. Vol. iI, p. 522).

$\S$ See above, p. 79. 
I must lie here no longer'-an idea which at that lucky instant awakens no contradictory or paralysing suggestions, and consequently produces immediately its appropriate motor effects. It was our acute consciousness of both the warmth and the cold during the period of struggle, which paralysed our activity then....The moment these inhibitory ideas ceased, the original idea exerted its effects*.

William James concludes that

The immense majority of human decisions are decisions without effort. In comparatively few of them, in most people, does effort accompany the final act. We are, I think, misled into supposing that effort is more frequent than it is, by the fact that during deliberation we so often have a feeling of how great an effort it would take to make a decision now. Later, after the decision has made itself with ease, we recollect this and erroneously suppose the effort also to have been made then $\dagger$.... It may be remarked in passing, that the inhibition of a movement no more involves an express effort or command than its execution does $\ddagger$.

When we come to discuss the fifth law of thought we shall see that action of one kind or another is the normal end of every train of thought. We have already seen that an effort of Will does not ordinarily accompany the final action. Introspection indicates that most of our trains of thought are even more free from efforts of Will than the acts which end them. Or, as we have said, most of our thinking is involuntary, proceeding to its end under the influence of sensations, instincts, and active and inactive systens-especially their affective-conative elements-in the manner described $\S$ in our discussion of the third law.

Among these involuntary thought-activities-whether in the middle of a train of thought or towards the end of it, when the excitement is about to be discharged in producing bodily movement-the soul only intervenes (by means of the Will) in the event of conflict between the involuntary processes: such conflicts often obstruct the fulfilment of the soul's purposes. Or, more briefly, the Will only intervenes to resolve conflicts.

Introspection is our authority for this generalisation. For example, I am never conscious of making an effort of Will except when in the presence of conflicting tendencies of my involuntary thought-activities. Moreover, our generalisation accords with an observation of William James on that part of an involuntary train of thought which is most open to examination: namely, the final act. After multiplying

* W. James, loc. cit. Vol. II, pp. 524, 525.

$\dagger$ Loc. cit. Vol. II, pp. 534, 535.

$\ddagger$ Loc. cit. Vol. II, p. 527 .

$\S$ See above, pp. 81 et seq. 
examples of ideo-motor action, shewing that the final act of a train of thought ordinarily takes place without an effort of Will, he sums up his discussion in words which we have already* quoted and which we may now summarise: The presence in consciousness of an image of a movement brings about the actual movement whenever the image 'is not kept from so doing by an antagonistic representation present simultaneously to the mind.' And he adds: 'the express fiat, or act of mental consent to the movement, comes in when the neutralisation of the antagonistic and inhibitory idea is required.' $\dagger$ In fact, according to William James, the Will only intervenes at the exposed end of a train of thought when there is conflict between the involuntary processes at work.

There is no reason to suppose that the Will intervenes at any other point of an involuntary train of thought, except for the same reason: namely, to resolve a conflict. But for this reason the Will does intervene. Indeed, according to Dr Bernard Hart, the Willthe 'subjective appreciation of the forces at war within us, and deliberate adoption of a consciously selected line of conduct'- 'may be regarded as the rational or ideal solution of a conflict. In fact it may be said to provide the only possible solution in the strict sense of the word.' $\ddagger$ The conflict of which Dr Hart is here writing is due to the existence of an interest-system $\S$-or, at least, a complex\|whose influence upon the direction of the nervous impulses is antagonistic to that of the other interest-systems and neurograms that make up the total neurography of the brain. The manner in which the Will, by intensifying excitement in particular systems of arcs, removes this antagonism, or, as we may say, introduces harmony between such conflicting interest-systems, will be considered very shortly $\uparrow$.

* On p. I40, above.

$\dagger$ Loc. cit. Vol. II, p. 526.

$\ddagger$ Loc. cit. p. 79. Cf. also J. C. Fluegel, B. J. P. Vol. viII (I9I 7), p. $4^{89}$.

\$ Above, p. 63 .

In Chapter 9, below. 


\title{
CHAPTER 8
}

\author{
PURPOSE
}

\section{§ I. Purpose-Neurograms.}

MEANWHILE, something must be said of those neurograms, or systems of neurograms, which correspond to what we have described* as 'the soul's purpose'; for many, if not most, of the conflicts that demand the Will's intervention are due to lack of harmony between these neurograms and other interest-systems.

Mr Lewisham, the youthful schoolmaster in Mr Wells' novel, had pinned on to the wall of his room a list of the South Kensington examinations he was going to pass at various specified dates and of other intended achievements during a period of years. In fact, Mr Lewisham literally 'mapped out his career.' Unfortunately (or, perhaps, fortunately) Love came into his life. The tale describes the conflicts between his carefully and elaborately thought out purposes on one side, and this new interest on the other. Now Mr Lewisham was not exceptional in planning his life ahead, but only in the degree in which he did so. All of us in some degree make or accept plans for the future. But men and women differ greatly in the amount of attention they devote to future events, in the rate at which they discount the future, in the degree in which they work with distant objects in view (as opposed to living 'from hand to mouth'). It is these plans for the future that constitute the soul's purposes.

These purposes may be classified in various ways: according to the manner in which they are formed, according to the matter to which they relate, according to the time when their realisation becomes due, and so on. But all of them have certain features in common. For example, every one of my present purposes, whatever the date for its fulfilment, is represented in my brain by a neurogram $\uparrow$ so

* On p. 141, above. But see footnote * on p. 10o, where we pointed out that the soul may have a structure to which the neurography corresponds only imperfectly, in which case the soul may be said to possess purposes that are not yet represented by neurograms. Since, however, to every psychosis there corresponds a neurosis (according to our first law), every one of my purposes of which I have ever been conscious-or, briefly, every one of my conscious purposes - will be represented in my neurography. And, as stated on p. 144 below, it is with these conscious purposes only that we are concerned in this chapter.

+ See footnote above. 
connected with my neurogram of the signal-whether the arrival of a certain time or the occurrence of a certain event-that, when in due course the latter neurogram is excited, the excitement will tend to spread to the neurogram of the purpose and the purpose will, therefore, tend to be realised. If we speak of all the neurograms connected to my neurogram for future time as forming my 'future' interest-system, we may thus describe this first conmon property of all purposes: Every purpose has a corresponding neurogram belonging to one's 'future' interest-system*.

Then again every purpose must, at some time or other (at least when the purpose-neurogram was formed), have occupied the focus of consciousness $\dagger$. True, the neurography of every young person contains neurograms which may some day be excited through the appropriate signal-neurogram, when (as in the case of the child whom William James saw frightened by a railway train $\ddagger_{\text {) }}$ his unforeseen conduct will cause him surprise. But such conduct, being not only unintentional but unforeseen, cannot be described as belonging to the individual's purposes. The same is true of those actions which may be performed under the influence of hypnotic suggestion without being foreseen by the hypnotised subject. In fact, we restrict our use of the term 'purposes' to conscious purposes: purposes that have at some time or other occupied the focus of consciousness.

We might further restrict 'purposes' in what follows to denote only willed purposes: purposes which the Will originally brought into the focus of consciousness and whose neurograms owe much of their depth to the Will's intensification of their excitement when the purposes in question were first willed and afterwards voluntarily confirmed. Such a further restriction may, at first sight, seem to conform to the use of the word 'purpose' in everyday speech. We have already§ spoken of the common identification of purpose with Will. But, if we reflect upon the origin of the purposes which we hold most dear, we shall find that comparatively few of them are directly due to acts of Will. When the twelve-year-old son of a Cambridge graduate says that he intends in due course to proceed to his father's University, his purpose may be definite enough but is by no means necessarily due to an act of his youthful Will. To follow in his father's steps may well present itself to him as the only right and proper thing to do. He approves the purpose, but does not will it. What

* See below, p. I 46 .

$\dagger$ See footnote * to preceding page.

$\div$ See footnote 9 on p. I38, above.

$\S$ Above, p. 98 . 
this approval involves we shall discuss directly. Meanwhile we observe that the approved purpose to follow his father to Cambridge is no less a conscious purpose, satisfying the conditions we have just laid down, than is a willed purpose to learn to swim next summer or to pass an examination this term.

Purposes, whether willed or merely approved, may be divided into two groups consisting respectively of purposes to do (or at least to think) something and of purposes to abstain from doing (or from thinking) something. The former we may describe as 'positive' purposes, the latter as 'negative.' We shall find it useful to have some notion of the neurographic correlatives* of these two kinds of purposes.

When a hypnotised person is told by the hypnotist (or operator) to do so-and-so at such-and-such a future time and, having recovered from the hypnotic trance, acts at the appointed time in accordance with the operator's suggestion (of which, in his normal conscious state, he has no knowledge), the neurographic mechanism may be very simple. It need consist of no more than the neurogram for the signal and the neurogram for the deed so connected that when, on the occurrence of the signal, the former neurogram is excited, the excitement will spread to the latter, thus bringing the act about. But this system of two neurograms is detached or dissociated from the systems of arcs whose excitement accompanies the main stream of consciousness.

We should expect the neurogram of a conscious purpose to form part of a far more complex system of associated neurograms. For not only must the neurogram of a conscious purpose have drained the impulse from all excited arcs on at least one occasion (when the purpose occupied the focus of consciousness) and so have become connected with all those arcs, but it may also have been often reexcited in whole or in part (as the purpose has from time to time been remembered and reflected upon), and on each of these occasions it will have acquired new connexions. A certain difficulty may trouble us at this point. It may be described and removed by considering a simple illustration. Suppose my purpose is to go for a walk at three o'clock. The corresponding neurogram will have elements corresponding to myself, to a future time (three o'clock), and to a walk. Now why is it that, as I reflect upon this purpose at, say, two o'clock, the excitement spreading to the walk-element of my purpose-neurogram does not result in the ideo-motor act of starting

\footnotetext{
* I.e. 'of the neurograms that correspond to....'
}

G. E. 
for a walk now? The answer is, briefly, because my purpose is not to go for a walk now, but at three o'clock. This answer does not, however, remove the difficulty, which only disappears when we observe that the true purpose is not to-go-for-a-walk but to-waituntil-three-o'clock-and-then-to-go-for-a-walk. The ideo-motor result of giving my undivided attention to this purpose at any time before three is 'to-wait...'; and this is what I do. The apparent difficulty in question then arises from our tendency to economise thinking by substituting the end of a purpose for its entirety: a proceeding which, from our present point of view, is just as foolish as to regard getting up from a meal, to which one has just sat down, as equivalent to eating the meal and then rising from table*. We have, therefore, to recognise that the neurogram of a purpose contains, as an essential part of itself, elements that correspond to the lapse of time (or to the occurrence of a sequence of events) antecedent to the final act, which completes the accomplishment of the purpose. In so far, then, as the lapse of future time is a condition precedent to the fulfilment of all purposes, the neurograms of all purposes have in common elements that correspond to future time $\uparrow$. Thus all purpose-neurograms, since they possess common elements, may be conceived as forming part of a 'future' interest-system. And since some idea of myself enters into all my purposes, the neurogram of my 'self-regarding sentiment' $\ddagger$ also forms part of my future-interest-system. So we recognise, in my purpose-neurogram, elements $\S A, T$, and $S$ (say), corresponding respectively to the proposed action, A (going for a walk, we have supposed), to the future time, $T$, at which the action is to be performed, and to myself, S.

Something must next be said about the neurograms that correspond to negative purposes. What is the neurographic correlative of my purpose to abstain from doing this or to avoid thinking of that? We observe first that the surest way to avoid doing something is to avoid thinking of the deed; for we have seen\| how the thought

* If any reader still feels a difficulty, let him reflect upon the difference between neurograms for 'take the first turning to the right' and for 'take the second turning to the right.'

$\dagger$ We can think of the future, as of the past. To future time, or to past time, there must be systems of arcs that correspond and the excitement of which accompanies thoughts of the future, or of the past. But the arcs, or neurograms (since they have been deepened by experience), that correspond to the past are rich in associations, while those that correspond to the future form an almost isolated system until purposes have been formed.

$\ddagger$ Cf. W. McDougall: 'Symposium on Instinct and Emotion' (Proc. Aristotelian Society), p. 4 o.

$\S$ The following notation is that described on p. 70 above and on p. 190 below.

II Above, pp. I4.0, 142. 
of the deed, if left in undisputed occupation of the focus of consciousness, will quickly be followed by the deed itself, performed as an ideo-motor act. Accordingly, we need not distinguish further between negative purposes whose concern is with action and negative purposes whose concern is with thought. The neurogram that corresponds to my purpose never to think of a certain thing (which we may represent by A) must evidently include the neurogram $A$ as one of its elements. But if my purpose is not-to-think-of-A-at-such-and-such-a-time, then my purpose-neurogram must also include an element corresponding to the time in question. The time element will be related to the remainder of the purpose-neurogram in the manner already discussed. We may, for the moment, leave it out of account, and consider the simpler case of my (negative) purpose never to think of A. For the realisation of this purpose it is necessary that, whenever excitement is about to spread to the neurogram $A$, or at least before the excitement of $A$ has reached a sufficient degree of intensity to make $A$ drain the impulses, and so to make some* thought-activity $\mathfrak{A}$-an idea of A-come into the focus of my consciousness, something should happen in my brain to divert the excitement from $A$.

If now $A$ were intimately linked with some other neurogram $(O$, say) such that its ( $O$ 's) stimulation, even in a slight degree, brought about a quasi-explosive $\uparrow$ discharge of excitement through $O$, so rapid and so violent as to cause $O$ to drain all the active arcs almost instantaneously, the result would be that, whenever an idea of A tended to appear in consciousness, (1) would occur instead. Moreover, on each occasion when $O$ drained the impulses, $O$ would form direct connexions with the other active neurograms besides deepening its connexion with $A$; and, since these other active neurograms would include those through whose connexions with $A$ the excitement had spread to $A$ on that occasion, $O$ would thus gradually become directly connected with all the neurograms $\left(A_{1}, A_{2}, \ldots, A_{n}\right)$ with which $A$ is connected and through which alone $A$ may be excited. The process would continue, $O$ becoming connected to the neurograms $A_{11}, A_{12}, \ldots, A_{21}, A_{22}, \ldots$, connected with $A_{1}, A_{2}, \ldots$. The final result would be that, not only all ideas of $\mathrm{A}$, but all their associated thought-activities would be

* We have pointed out above (p. 47) that, there may be many different ideas (thought-activities) $\mathbf{A}, \mathfrak{A}^{\prime}, \mathfrak{a}^{\prime \prime}, \ldots$ of an object $\mathrm{A}$ corresponding to different distributions of excitement in the neurogram $A$.

+ Cf. the case of the lover's tap on the window quoted above (p. 78 ) from William James, whose explanation how so faint a stimulus to a system of arcs (corresponding to $O$, not to $A$, in the text) may cause that system to drain the impulse, is that 'it finds a nerve-centre half ready in advance to explode.' (Loc. cit. Vol. I, p. 450. Italics mine.) 
excluded from consciousness: the whole interest-system of which $A$ formed part would tend to become 'repressed' or 'censored,' * and $A$ would not become even slightly excited $\dagger$.

Now William James has observed that 'Some persons can voluntarily empty their minds and "think of nothing." With many, as Professor Exner remarks of himself, this is the most efficacious means of falling asleep.' + Let us represent by $O$ the system of arcs whose excitement accompanies the process of 'emptying the mind,' and let us describe the process as the 1 -process. The process, or at least its motor and sensorial elements, differ in different individuals. In some 'This curious state of inhibition can for a few moments be produced at will by fixing the eyes on vacancy.' $\S$ The $\mathbb{P}$-process in another case has been thus described:

When in waking times I thus dismiss a thought I generally make a rapid but suddenly stopped turn of my head from that position which is natural to me in thinking to a rather forced and tense posture and...a number of muscles in the upper part of the body are held rather rigid; and the resulting complex sensory experience is very clearly apprehended. Even upon dismissing insurgent thoughts in order to go to sleep, I notice in myself attention to the sensations arising from respiratory movements and the like $\|$.

In the present writer's own case, the (D-process produces the following sensations: closing the eyelids, turning the eye-balls upwards as if to make doubly sure of not seeing, closing and slightly compressing the lips, sharply inhaling through the nose, and (less certainly) a quick turning of the head to one side 1 .

We have, then, in the 1 -process, whatever its form in anyindividual person, a mechanism sufficient gradually to render him oblivious to an object $\mathrm{A}$ and all its associations in normal consciousness, when once $O$ has been connected, voluntarily or involuntarily, with $A$.

* In a dream, excitement may perhaps spread to $A$ by some other route than those afforded by $A_{1}, \ldots, A_{n}$, the neurograms of the associations of $\mathfrak{A}$ in waking consciousness. Thus $A$ may at least begin to be excited before the (B-process (see below) begins to operate.

$\dagger$ Cf. J. C. Fluegel: 'Repression is apt to extend from the clement of thought which was originally its object to other elements that are associated with it, the associations along which the repression extends being often, moreover, of the superficial type so characteristic of the Unconscious.' (B. J.P. Vol. vili (r9r7), p. 493.)

$\ddagger$ Lec. cit. Vol. I, p. 404 .

$\S$ W. James, loc. cit. p. 404.

II T. Loveday, B. J. P. Vol. viI, No. 2, p. 162 .

If In making voluntary use of this process for the purpose of falling asleep, I am conscious of two stages. In the first I feel the sensations described in the text. In the second stage I relax the effort involved in voluntarily stimulating the (1)-process. 
The neurogram of a negative purpose never to think of A need include no more than $A$ and $O$ connected together; and to $A$ and $O$ must also be connected the neurogram of a future time, $T$, to form the neurogram of a purpose not to think of $A$ at time $T$.

An important question concerning purpose-neurograms is whether or not they tend to be rich in affective-conative elements. Let us alter our illustration of a positive purpose, and suppose that my purpose is to play in an exciting football match at three o'clock, instead of to go for a comparatively dull walk at that hour. Then the element that we have denoted by $A$ in my purpose-neurogram is enriched by affective-conative elements. For that reason both $A$ and the neurogram as a whole are more likely to be excited*, and I an inore likely in consequence to reflect upon and reinforce my purpose. Moreover, when the time comes to change for the game, an idea $\mathfrak{A}$ of it is more likely to remain in the focus of my consciousness and the appropriate movements to follow as an ideo-motor act. For both these reasons, the addition to $A$ of suitable affective-conative elements strengthens my purpose and renders its fulfilment more likely.

On the other hand the reinforcement of $A$ in the negative-purpose neurogram we described, by tending to bring $\mathfrak{A}$ into the focus of consciousness, renders necessary a deeper connexion between $A$ and $O$ to secure the effective exclusion of $\mathfrak{A}$ from consciousness, or in other words, to prevent me from thinking of A. If the inhibition of thought about A is due to an effort of Will-that is, if Will formed the connexion between $A$ and $O$-the effort must be the greater, the richer $A$ is in affective-conative elements.

\section{$\S 2$. Formation of Purpose-Neurograms.}

We have now to consider how purpose-neurograms come to be formed. Already, when defining conscious purposes, we have distinguished $\uparrow$ between ' willed' purposes on the one hand and 'approved' (but not willed) purposes on the other. Let us first consider the formation of willed purposes and their neurograms. We recall William James' dictum, already $\ddagger$ quoted, that 'the soul presents nothing herself; creates nothing; is at the mercy of the material forces for all possibilities§; but amongst these possibilities she selects; and by reinforcing one and checking others, she figures...as something

* See above, pp. 9 I, 92 .

$\dagger$ See above, p. I 44 .

+ Above, p. roo. But see the first footnote to that page.

$\S$ Cf. St Paul: 'How shall they believe [by an act of Will] in him of whom they have not heard? and how shall they hear without a preacher? and how shall they preach unless they be sent?' (Romans x. I4, I5.) 
from which the play gets moral support.' It would follow that a willed purpose depends for its inception upon the simultaneous* excitement, whether from sensory stimuli (derived, for example, from the spoken or written word) or in the course of a train of voluntary or involuntary thought-activities $\dagger$, of the various elements that are to form part of the purpose-neurogram. The Will may then reinforce the excitement in these elements so as to deepen them and connect them together, thus forming the neurogram of a willed purpose $\$$.

Willed purposes, especially if their neurograms are rich in affectiveconative elements, may be of altogether paramount importance. For example, the act of faith by which some people accept religion-the effort of Will by which they decide to act on a hypothesis with a view

* The excitement is not instantaneous but waxes, reaches a maximum, and then wanes. William James has given reasons for supposing that the period'the specious present'-during which a neurogram remains excited is generally about twelve seconds. Our use of the phrase 'simultaneous excitement' therefore means that the periods during which the various elements in question are excited overlap.

$\dagger$ There may also be other ways-hardly to be described in William James' words as 'material forces'-in which the elements of a purpose might be presented to the soul. It is not inconceivable that another Soul-God, if you will-or other souls should communicate directly with our souls. In such a case it is possible to imagine that the other soul which communicates with mine does so either directly, soul to soul, or indirectly, by first creating excitement in my brain. Since, however, my own soul cannot create, but only reinforce, excitement in my brain it is not to be supposed that any other human soul has power to create excitement in my brain; but, if we choose, we may suppose that God has it, and sometimes exercises it. It is, however, simpler (and therefore preferable) to suppose that all purely psychical communications proceed direct from soul to soul. If our first law applies, such a communication reaching my soul must, if and when it affects my consciousness, be accompanied by excitement in some of the neural arcs of my brain. And, if we regard the vision which led to the conversion of St Paul as a psychical communication of the kind now in question, we have in his subsequent blindness some evidence of a neural accompaniment to the psychical process.

$\ddagger$ The connexion is effected by drainage, in the manner already described. We have to suppose that, as the Will intensifies the excitement in the various elements that are to form the purpose-neurogram, the excitement in one or other element becomes sufficient to cause that element to drain the others and so to get connected to them.

$\S$ Negative as well as positive willed purposes may thus be formed by reinforcement. Although in William James' dictum just quoted for the second time, he speaks of the soul reinforcing some possibilities and checking others, our fourth law has only postulated power for the Will to reinforce (but not to $c h e c k$ ). Checking may, in fact, be seen to be a particular case of reinforcing. Thus, when a negative purpose-e.g. ' not to drink alcoholic beverages'-is present to consciousness, there must be an element in consciousness corresponding to 'not' as well as elements corresponding to the beverages, to drinking, and to the thinker himself: otherwise the only purpose that could be formed from these elements would be, ' $I$ will drink alcoholic beverages whenever I get the chance.' We have only to suppose that the 'not'-element in consciousness is accompanied by some excitement of the neurogram we have called $O$. By reinforcing excitement in this neurogram at the same time as in the other elements in question, the Will may form a negativepurpose-neurogram of the kind already described. 
to verifying it - is in many cases the cardinal and culminating fact in their whole lives. It is nevertheless true that, as we have indicated, the great majority of our purpose-neurograms are formed by the flow of neural impulses which, like most neural impulses, are directed by interest-systems, including especially instinct-systems, rather than by Will. In short, most of our purposes are not formed by an effort of Will. They appear to us absolutely appropriate and natural 'and selbstverständlich, an "a priori synthesis" of the most perfect sort,' needing no explanation. 'It takes, in short, what Berkeley calls a mind debauched by learning to carry the process of making the natural seem strange, so far as to ask for the why of any' ordinary human purpose, formed under the influence of instinct or other interest. The common man can only say, 'of course the ordinary little boy purposes to follow his father's trade when he grows up; of course the ordinary little girl purposes to become the mother of a family; of course the youth purposes to win the love of the maiden, that beautiful soul clad in that perfect form, so palpably and flagrantly made from all eternity to be loved!'*

To come within our definition of a purpose $\dagger$, all these 'unwilled' intentions must have at some time occupied the focus of consciousness, receiving the full light of attention. At the moment when any such intention is first recognised, the Will may intervene to repress it if disapproved, and perhaps to strengthen it-to deepen its neurogram-if approved. But, if the Will does not intervene, the mere fact that the purpose has passed the censor without being repressed constitutes it an approved purpose: that is, a purpose conflict with which will cause the Will to intervene $\ddagger$. And this intervention will result from conflict with an approved (or a willed) purpose, whether the antagonistic influence upon the direction of the stream of thought is due to outside events (i.e. external difficulties in the way of the fulfilment of the purpose) or to inside interest-systems.

In this account of the nature and origin of purpose-neurograms, we have distinguished between purposes that originate in an act of Will and those that are approved without effort so soon as they are presented to consciousness. This distinction has facilitated description. In fact, however, the two classes of purposes-those that are willed and those that are merely approved-are by no means inutually exclusive. For, in the first place, purpose-neurograms, the formation

* Most of this wording is taken from William James' account of instinctive (and reflex) acts. (Loc. cit. Vol. II, pp. 386, 387.)

$\dagger$ On p. I 44 above.

$\ddagger$ See above, p. I4I. 
of which was achieved by an effort of Will (reinforcing excitement), include, as a rule, large elements already formed by other means, the effort of Will having achieved no more than the synthesis of preexisting elements into the new purpose-neurograms. We may repeat in this connexion that the willed purpose-neurogram which contains no affective-conative elements will probably be but a poor and transitory affair, representing a feeble and easily forgotten purpose: the ephemeral character of mere good resolutions that are unaccompanied by some instinctive drive-some conative element-is proverbial. On the other hand, whenever a conflict causes the Will to intervene in support of a merely approved purpose, the act of Will (reinforcing excitement) deepens the purpose-neurogram, which thereafter is, partly at least, due to Will. When Professor Ramsay Muir tells us that 'Self-government had throughout the modern age been a matter of habit and practice with the British peoples; now [ $18 \mathrm{I} 5-78$ ] it became a matter of theory and belief,'* he furnishes us with an example of an approved purpose becoming, partly at any rate, willed.

\section{§ 3. Influence of Purposes on the Stream of Thought.}

We have spoken of the future-interest-system which is made up of all purpose-neurograms (except perhaps the neurograms of general negative purposes: ' $I$, will never think of so-and-so') $\dagger$. We have remarked $;$ further that purpose-neurograms for the most part include as part of themselves the 'self-regarding sentiment' neurogram, if we may so describe the neurogram of the self, with all the instinctneurograms to which it has become intimately connected. Moreover, the neurograms of the stronger among willed purposes tend, as we have seen, to include other affective-conative elements in addition to those of the self-regarding sentiment. Finally, purpose-neurograms, the origin of which is due to instinct or to interest rather than to Will, are evidently connected to (and so may be said to include) the affective-conative elements whose excitement first brought them into being. For all these reasons, the future-interest-system is apt to contain a large proportion of affective-conative elements. In other words, our interest in the future, if intense and wide (i.e. if our purposes are strong and far-reaching), will tend to stir our emotions in no small degree.

This future-interest-system shares with our other interest-systems, and with incoming sense-impressions, the business of guiding our

* The Expansion of Europe, p. II5.

$\dagger$ See above, p. 147. $\ddagger$ See above, p. 146. 
thought-activities during involuntary thinking. But the influence of the future-interest-system upon thought, and especially (through thought) upon conduct, is greater than that of most other interestsystems equally deep and wide; and for the following reasons.

In the first place, a future-interest-system influences conduct directly, while the influence of other interest-systems is in general only indirect. For example, suppose that incorporated in my futureinterest is a purpose to be present at Putney on boat race day. During the weeks before the race, my newspapers have directed my attention to the practice and the prospects of the crews; and on each occasion I am reminded of my purpose, on which I see no reason to go back. On each occasion therefore my purpose is deepened, and, as its associations with the various reminders multiply, widened. Finally the day comes. Railway advertisements, newspaper posters, even the horses-decorated with ribbons of one or other of the rival blues-let me know that the time has come for my purpose to be fulfilled. And fulfilled it accordingly is, without effort of Will on my part. Indeed, no small effort of my Will, or some altogether exceptional sense-impression from outside, would then be necessary to prevent my purpose from being fulfilled. On the other hand, an interest in rowing or in our ancient Universities or even in a member of one of the crews, while before the day it might help to form a purpose to see the race, would not, apart from such a purpose and perhaps in face of some contrary purpose for spending the day, take me down the Fulham Road and over Putney Bridge when the day arrived.

In the second place, the future-interest-system exercises a potent influence upon thought-and especially upon conduct-because of its tendency to be associated with the thought-activities that immediately precede, and lead to, actions. We are about to see* that associations of this kind would cause the future-interest-system to influence actions in a high degree. But, first, we must observe that the future-interest-system does tend to become associated with ideas of actions. It does so because action that is neither reflex nor habitual will not follow immediately upon the appearance of an idea of it in consciousness; for in such a case there will be no well-worn channels by which the nervous impulses can escape (down the pyramidal tract, producing movement) from the neurogram whose excitement accompanies the idea in question. In such a case, therefore, the excitement has time to diffuse from this neurogram, thus exciting a number of connected neurograms, before it is

* On the next page. 
finally drained. In other words, associations of the action thought of are awakened before the action takes place (if indeed it ever does take place, for these associations may lead to its being inhibited). Among the neurograms to which the excitement diffuses, are some that correspond to consequences of the action. These consequences are in the future, and their neurograms therefore contain elements that correspond to future time. It follows, from the corollary to our third law, that the future-interest-system-especially if it be wide (covering the whole, or almost the whole, of future time) as well as rich in emotional elements-will tend to attract the impulse and so to influence every action that is not reflex or habitual or otherwise* due to the existence of low-resistance, or 'canalised,' paths (but when such paths exist, the excitement is drained along them towards the pyramidal tract without leaving time for the diffusion process to occur). No other interest-system is in the same position, tending to attract the impulse before any 'uncanalised' action can occur.

Thirdly, on account of its richness in affective-conative elements, the future-interest-system exercises a greater influence upon thought, and so upon conduct, than that of most other interest-systems equally wide and deep. That the presence of affective-conative elements in an interest-system increases the influence of that system upon the flow of excitement through the brain we have already seen $\dagger$. And we have also seen $\ddagger$ that the future-interest-system tends to contain a large proportion of these elements: a fact of which confirmatory evidence is furnished $\S$ by Dr Webb's investigation entitled Character and Intelligence, the most elaborate attempt yet made to assign numerical values to the inter-relations of psychical qualities.

For these reasons, then, a wide and deep future-interest-system is in a peculiarly strong $\|$ position for guiding thought and conduct.

* In particular, actions towards which instincts strive often follow immediately - the excitement pursuing innately canalised paths (i.e. paths whose low resistance is innate)-upon the occurrence of an image of them in consciousness. That instinctive actions are apt to occur without regard for consequences is well recognised.

$\dagger$ Above, pp. 91, 92. $\ddagger$ Above, p. I52. \$ See Appendix B, § I I.

II A further example of the potent influence which purpose-neurograms, and the future-interest-system which they help to form, exercise upon thought and conduct has been noted by Dr McDougall: 'that a conscious conative effort having once been made (an intention or resolution having once been formed, the will having been consciously set towards a given end), the conative process continues or may continue at work sub-consciously for a period of time to which we can set no definite limit. The "Aufgabe," the intention, may continue to play a prominent, a predominant, part, even when it has passed altogether from clear consciousness.' (American Journal of Insanity, Vol. LXIX, p. 870. See also above, pp. I00, Ior.) And Freud and members of his school have demonstrated the immense influence which sub-conscious conations exercise upon our mental life and conduct. 
Where it exists it constitutes, as we shall see*, one of the most important elements in character; and without it strength of character is impossible.

\section{§ 4. Dr Webb's Group Factor in Character Qualities.}

Dr Webb obtained statistical evidence of the high degree in which certain character qualities are influenced by purpose. We have already seen $\dagger$ how his investigation led to the discovery of a new group factor $\ddagger$, Cleverness $(c)$, which along with $g$ enters into several intellectual qualities, some of which are compounded of $g$ and $c$ alone according to the vector law. Dr Webb himself described $\S$ another group factor which, along with $g$, enters into several purpose qualities\|, notably those numbered $34,33, \mathrm{I} 8,20,2 \mathrm{I}, 32$, and 28 in the above Table III $\%$. So far as this group of purpose qualities is alone concerned, neither $g$ nor this new factor is a single general factor within the meaning of our definition**. But any large series of sufficiently dissimilar mental tests may, as we have said, be so expressed that $g$ and specific factors enter into all of them, while this new factor, like our factor Cleverness, enters as a group factor to an extent which is insignificant in all but a small proportion of them.

Dr Webb discusses the nature of his new factor ' $w$,' 'whose generality would appear to extend so widely in character, ' $\dagger \uparrow$ and recognises that it is in some close relation to 'persistence of motives.' He adds that 'this conception may be understood to mean consistency of action resulting from deliberate volition, or will.' ++ But will, used in this sense, is liable to be confused with the word as used to describe an effort of will. It would perhaps be better to describe the new (group) factor as being intimately related to Purpose $\S$.

* See below, Chapter 17 .

$\dagger$ On p. I19 and in $\$ 3$ of Chapter 7 above.

$\ddagger$ In the present section we shall treat a factor (e.g. ' $c$ ') and the measure (c) of that factor as interchangeable terms. Most of the section is taken, with small verbal changes, from a paper by the present writer on 'General Ability, Cleverness and Purpose,' published in the B.J.P. for May, 1919 (pp. 359-362).

$\S$ Loc. cit. pp. 58, 6o. But, as we are about to observe, the factor which Dr Webb described, and measured by $w$, is not the same as the new group factor, $f$, defined overleaf as independent both of $g$ and $c$, that is here in question. We shall however see that $w$ and $f$ have much in common.

\| See below, pp. I $57, \mathrm{I}_{58}$.

If On p. 126. Dr Webb found evidence of the presence of his group factor in qualities numbered 4 , II, and $3 \mathrm{I}$ also. See Garnett, $B$. J. P. loc. cit. pp. 359,360 .

** In $\S 3$ of Appendix B: see footnote $\S$ on p. 477 below.

†† Webb, loc. cit. p. 60 .

$\ddagger \ddagger$ Loc. cit. p. 60.

$\S \S$ It is necessary, as we have pointed out (on p. 98), clearly to distinguish the momentary effort of Will from the persistence of Purpose that in English is so often called by the same name. For example, when in the Lord's Prayer we say 'Thy will be done,' we mean 'Thy purposes be fulfilled.' 
The new group factor, $f^{*}$, as defined in the next paragraph, is independent of $g \dagger$. It is not the same as our new factor Cleverness; for the calculated correlations between the seven qualities in which the new factor has been shewn to enter as a group factor, and Cleverness, are distinctly low, being respectively:

\section{Table IV.}

No. in

Dr Webb's schedule

34 .

33.

I 8 .

20.

2 I.

4.

I I.

\section{Name of Quality}

Tendency not to abandon tasks from mere changeability Tendency not to abandon tasks in face of obstacles ... Kindness, on principle ... Trustworthiness ...

Conscientiousness..........

Readiness to become angry Eagerness for admiration
... $\quad \cdots$

$$
\cdots \quad \cdots
$$$$
\cdots \quad \cdots \quad \cdots \quad \cdots
$$$$
\begin{array}{llll}
\cdots & \cdots & \cdots & \cdots
\end{array}
$$$$
\cdots \quad \cdots \quad \cdots \quad \cdots
$$

Correlation with 'Cleverness' $-\cdot 06$

$-.05$

$\cdot 15$ $\cdot 18$

There is therefore a third factor, $f$, independent both of $g$ and $c$, which enters into the constitution of a number of character qualities ${ }_{+}^{+}$.

We have seen $\S$ how the degree in which a person possesses any quality that depends, according to the vector law, on two independent factors only may be represented by a single point $P$ in a (two-dimensional) diagram such as Fig. 8 . Thus, if the person possesses the two independent factors in degrees $g$ and $c$ respectively, and if the point $P$ is chosen to lie a distance $g$ east of a fixed point $O$ in the diagram and a distance $c$ north of $O$, then the degree, $q$, in which that person possesses any (intellectual) quality that depends on $g$ and $c$ only is the distance of $P$ from $O$ measured in a direction $\|$ which distinguishes that particular quality.

Now, just as in two dimensions-a not-too-large expanse of sea, for example-the position of a ship is known when we know its distance east (or west) and its distance north (or south) of a fixed

* See footnote $\S$ to $p$. $\mathrm{I}_{55}$ above and p. $\mathrm{I}_{5} 8$ below.

+ But, in discussing its nature, Dr Webb was more concerned with the quality which we are going to call Purpose, and of which the axis $O p$ in Fig. 9 below is not at right angles to $O g$, than with the quality, independent of $g$, of which the axis, $O f$ in that figure, is at right angles to $O g$. See also footnote 9 to p. Ior.

$\ddagger$ But the character qualities in question may depend on more than these three independent factors. Suppose we take $g$ and $c$ as the first two independent factors. Then we may express the measure, $q$, of any of these character qualities by means of equation ( $\mathrm{I} 3$ ) in $\S$ ro of Appendix B: namely

$$
q=v_{g q} \cdot g+v_{c q} \cdot c+l_{3} x_{3}+\ldots+l_{N} x_{N}
$$

where $x_{3}, \ldots, x_{N}$ measure the remaining independent factors. We may then define the new factor, $f$ or $x_{3}$, whose existence was proved by Dr Webb, as that which gives the highest average value of $l_{3}$ for all the $q$ 's in question.

$\S$ On p. I21, above. See also $\S 9$ of Appendix B (p. 484 below).

II The direction in question is $\tan ^{-1} v_{c q} / r_{g q}$ north of east, where $\gamma_{c q}$ and $\gamma_{g q}$ are the correlations of the quality in question with ' $c$ ' and ' $g$ ' respectively. 
point, the position of an aeroplane, in three-dimensional space, is known when we know its distances east (or west), north (or south), and above (or, if it happens to be flying in the Jordan valley, below) a fixed point on the sea level. And, just as before, if a person possesses three independent qualities in degrees $g, c$, and $f$ respectively, and if the point $P$ is chosen to lie a distance $g$ east of a fixed point $O$, a distance $c$ north of $O$, and a distance $f$ above $O$, then the degree, $q$, in which that person possesses any quality that depends, according to the vector law*, on $g, c$, and $f$ only, is the distance of $P$ from $O$ measured in a direction $\dagger$ which distinguishes that particular quality.

So we may construct a (threedimensional) diagram, Fig. 9, in which we represent the new group factor $\ddagger$ by an axis $O x_{3}$.or $O f$ at right angles to the plane of our former diagram (Fig. 8) §. Then the axes corresponding to the seven qualities in which the existence of this new factor has been demonstrated, and of some others-notably No. 32 (Degree with which he works with distant objects in view) and No. 28 (Extent of mental work bestowed upon usual studies) into which Dr Webb's new factor also

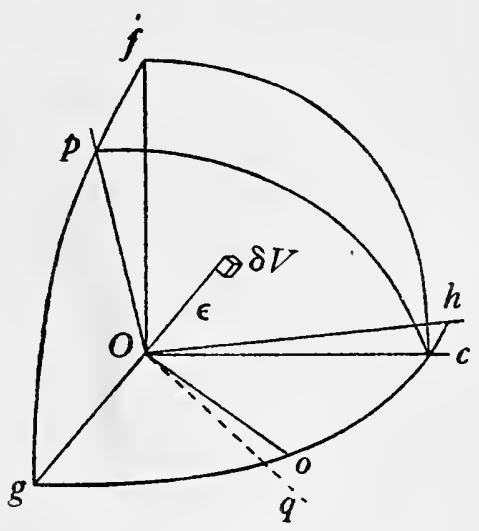

Fig. 9: enters in a high degree $\|-$ will lie in or near the plane $f O g$, since all these qualities have small correlations with Cleverness, the axis $(O c)$ of which is perpendicular to that plane.

Of the seven qualities (Nos. 34, 33, I8, 20, 2I, 4, and II) named in Table IV, the first five have high positive correlations with each other and with the two qualities (numbered 32 and 28 in Table III) to which reference has just been made; and all these seven qualities (Nos. 34, 33, 18, 20, 21, 32, 28) have negative correlations with the last two qualities (Nos. 4 and II) in Table IV $\%$. Let us now for shortness describe the seven qualities (Nos. 34, 33, 18, 20, 21, 32, and 28) having high positive correlations with each other as 'purpose'

* Its expression in this case is $q=r_{q g} \cdot g+v_{q c} \cdot c+r_{q f} \cdot f$ where as before the $r$ 's are the correlations of ' $q$ ' with ' $g$,' ' $c$ ' and ' $f$ ' respectively.

$\dagger$ In the language of solid geometry, this direction is defined by the directioncosines, $\boldsymbol{r}_{q g}, \boldsymbol{v}_{q c}$ and $\boldsymbol{r}_{q f}$.

\pm Defined in footnote $\ddagger$ on $\mathrm{p}$. ${ }_{5} 6$.

if See Webb, loc. cit. p. 59.

$\S$ On p. I 2 I.

I Webb, loc. cit. Table VI. 
qualities, a description suggested by the names of these qualities in Table III above and by the further particulars of the qualities in question given by Dr Webb*. To these seven purpose qualities we may, if we please, add the inverses of qualities No. 4 and No. II $\dagger$, defined as being measured by the measures of No. 4 and No. II respectively with their signs changed. Suppose that $p$ represents the mean position of the points in which the axes of the various purpose qualities meet any sphere described with $O$ as centre. Then, since the average correlation of all these nine qualities with Cleverness is very small (.04), $O p$ lies very near, if not actually in, the plane $f O g$ : and it lies much nearer to $O f$ than to $O g$.

We have next to observe $\ddagger$ that the new group factor $f$ is not identical with the factor for which Dr Webb used the symbol w; for, as we shall see, $w$, although it has much in common with $f$, has still more in common with $p \S$. In the absence of the analysis in Chapter 7 above $\|$, and in Appendix B, Dr Webb saw no use in preserving, in the definition of his new factor, $w$, the qualification that it should be independent of $g$. In fact, the definition which we have just given of $p$ is probably as near as we can get to the definition which Dr Webb would have given to his factor, $w$, had he been concerned with defining it in numerical terms. We shall for the future assume that $p$ thus defined is the measure of Dr Webb's new factor. We substitute $p$ for his symbol $w$ partly because we have defined $p$ while $w$ was not defined, and partly for another reason that will appear later: namely that $p$ measures Purposefulness.

It is interesting to observe that, in the case of the London school boys whose qualities were also investigated by $\operatorname{Dr} W e b b \|$, the correlation of $p$ with $g$ was found to be markedly less than in the case of the men. The number of qualities investigated in the case of the boys was less than in the case of the (men) students. Of the men's qualities shewing marked correlations with Dr Webb's new factor, only six appear in the boys' schedule. The following table gives the (total) correlations of the six qualities with $g$, in the case of the students and of the boys respectively:

* Loc. cit. Appendix II, pp. 84 et seg.

† See Garnett, B. J. P. loc. cit. p. 360.

$\ddagger$ Cf, footnote $\S$ on p. 155 .

$\S$ Cf. footnote $\dagger$ on p. I56.

II See especially $\S 3$ on pp. I 19 et seq.; and Crarnett, Proc. R. S. loc. cit.

If See above, p. 120. 
Number in

Table V.

Correlation

students'

schedule

Quality

with ' $g$ '

Tendency not to abandon tasks from mere changeability $\quad \cdot 45 \quad$. Io

33. Tendency not to abandon tasks in face of obstacles $\ldots . \quad \cdot 25 \quad-45$

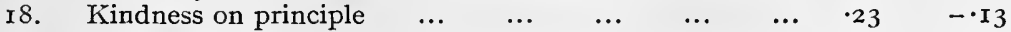

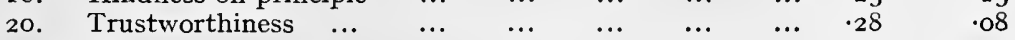

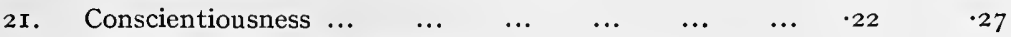

28. Mental work in usual studies $\quad \ldots \quad$.

\begin{tabular}{lllllll} 
Averages & $\ldots$ & $\ldots$ & $\ldots$ & $\ldots$ & $\cdot 38$ & \\
\hline
\end{tabular}

The average correlation of these six qualities with $g$, which is not much more than twice the average probable error in the case of the boys, is thus more than four times the average probable error in the case of the students: a fact which suggests that the correlation between $g$ and $p$ tends to increase as years go on*.

Continuing our investigation of the nature of the quality of which the measure is $p$ and which accounts for the partial correlations $\dagger$ ( $g$ constant) of the purpose qualities named in Table IV and of some others, notably No. 32 and No. 28, we first observe that, in the extent to which a person's future-interest-system-or system of purposeneurograms, where they all form one system-influences thought and conduct, we have a factor which must evidently affect a number of those qualities and which accounts, qualitatively at least, for their mutual correlations in so far as these are not due to their common dependence upon $g \nmid$. A subject whose main interest is in the future, rather than in the present or in the past, will on that account work

* This might indeed have been expected. If $p$ is large in the case of any person, we have (see below, p. r6r) an indication that he possesses a single wide interest of the kind described on p. 244 below and the preceding pages, and therefore (see p. 225) that his $g$ will tend to increase. Moreover, a strong future-interestsystem, the existence of which as we are about to see is indicated by a large $p$ as well as by a large $f$, will be in frequent conflict with the impulses of the moment, and will thus, as we shall also observe in the sequel, have led to the intervention of the Will to resolve the conflict and so to the increase of $g$, if we were right in assuming from the evidence on pp. I37, 138 that $g$ is increased by practice in concentrating attention, or, what amounts to the same thing, by making efforts of will. On the other hand, whoever has strong $g$ will on that account tend to form deep purpose-neurograms (see above, p. I50) and so, other things being equal, to develop a high degree $(p)$ of Purposefulness.

$\dagger$ The correlations of qualities, in so far as those correlations are not due to the correlations of the qualities with another (say, ' $g$ '), are called the partial ( $g$ constant) correlations of the qualities. The partial correlation of $s$ and $t$ with $g$ constant is given by

$$
\gamma_{s t \cdot g}=\frac{\gamma_{s t}-\gamma_{s g} \gamma_{t g}}{\sqrt{\mathrm{I}-\gamma_{s g}^{2}} \sqrt{\mathrm{I}-\gamma_{t g}^{2}}},
$$

where, as usual, $r_{s t}$ denotes the (total) correlation between $s$ and $t$. This is Yule's well-known formula. It follows at once from our equation (8) in $\S 9$ of Appendix B. (See Garnett, Proc. R. S. loc. cit. p. 98.) 
with a distant object in view*; will not abandon tasks that he has once undertaken $\dagger ;$ will act on principle $\ddagger$, his future interest dominating the impulse of the moment; will be consistent (and therefore reliable, trustworthy§ and conscientious\|) owing to the constant influence upon his conduct of the same single future-interest-system; will not readily become angry or otherwise quickly change his mood, because his mental processes are much influenced by a comparatively constant future-interest-system; and will not 'set aside principles for the sake of admiration'** or any other merely present satisfaction.

We have already remarked that $\mathrm{Dr}$ Webb, discussing the nature of his new factor, shewed that it 'is in some close relation to persistence of motives.' That $p$ is a measure of the extent to which a futureinterest-system-or the system of purpose-neurograms, where they all form one system-influences thought and conduct, accords with this conclusion, as we have just seen. But our suggestion that $p$ measures the influence of a single purpose-system upon thought and conduct, accounts for certain other facts that remain unexplained if we regard Dr Webb's factor merely as a general tendency towards 'persistency of motives.' $\dagger \dagger$

Moreover, our suggestion is consistent with Dr Webb's view that the nature of his new 'factor, whose generality would appear to extend so widely in character, is in some close relation to "persistence of motives". + But it carries us further. For, if it describes $p$

* Quality No. 32 in Table III on p. 126 above.

$\dagger$ Qualities Nos. 33 and 34.

$¥$ To act on principle is not, of course, the same thing as to do kindness on principle (quality No. I8). One might conceivably do unkindness on principle. But from the high average partial correlations of 'kindness on principle "we conclude that the principles of most of these training college students in whom 'future objective' (No. 32) and 'religion' (No. 22) were most strongly developed were such as to make them good rather than bad, kind rather than unkind. The same is true of the school boys investigated by Dr Webb, and the same is doubtless true of most inhabitants of Christian countries.

§ Quality No. 20. II Quality No. 2r. If Quality No. 4.

** The words are quoted from a prefect's report on what he understood by 'eagerness for admiration' (Quality No. II). See Webb, loc. cit. p. 86.

$\uparrow \dagger$ See $\$$ I 3 of Appendix B.

¥ We cannot however follow Dr Webb when he adds to the words we have just quoted: "This conception may be understood to mean consistency of action. resulting from deliberate volition, or will.' (Loc. cit. p. 6o.) These words follow immediately upon those quoted in the text. Dr Webb adduces no evidence in support of his statement that the consistency of action (which is marked by trustworthiness, conscientiousness and other qualities having high correlations with these) is due to 'deliberate volition' rather than to one and the same single system of purpose-neurograms in the future-interest-system. The formation of purpose-neurograms and their influence upon the stream of consciousness is (as we saw in $\S 2$ of this chapter) by no means solely due to the operation of deliberate volition, or Will. 
correctly, the presence in a subject's neurography of deep but disconnected purpose-neurograms will not alone suffice to give him a large purposefactor, $p$; but, if all his purpose-neurograms form a single futureinterest-system of the kind that we are about to call endarchical*, dominating his whole neurography in the manner described below $\dagger$, and if these purpose-neurograms are deep, his factor $p$ will then be large. That $p$ measures the influence upon thought and conduct of a future-interest-system, and especially of the purpose-neurograms (which, if very deep, will be its most important constituents), fits all the available evidence. Until more evidence is available we shall therefore assume that this is the nature of $p$ : or, in short, that $p$ measures 'Purposefulness.' So we have, in Dr Webb's investigation, further evidence of the influence of purpose on thought and conduct $\ddagger$.

It follows that $f$, defined as being independent both of $g$ and $c$, measures the influence of purpose in so far as that influence is not due to $g$ or $c$. But it is hardly, if at all, due to $c \S$. So $f$ measures, approximately if not exactly, the influence exercised by purpose upon thought and conduct in so far as that influence is not due to strength of Will $(g)$, but to neurography alone.

If now we repeat for our three-dimensional diagram (Fig. 9) $\|$ the description that we gave for our two-dimensional diagram (Fig. 8) 1 by imagining a small solid element $\delta V$ to move between the three axes $O f, O g$ and $O c$; and if we consider especially positions of $\delta V$ near the plane $C O p$; we shall find that exceptional men, whose mental qualities, in so far as they depend upon the three independent variables measured along $O g, O c$ and $O f$, are represented by the points lying inside $\delta V$, belong to one or other of two well-recognised types**, according as $\delta V$ lies near to $O p$ on the one hand or to $O c$ on the other.

It appears, in fact, that the type of temperament + , which $\mathrm{Dr}$ Webb and others have contrasted with the purposeful temperament, differs from it, not merely in having less than the average degree of Purposefulness, but also in having more than the average degree of Cleverness.

* On p. I63. $\quad \dagger$ In Chapter I2, §4. $\quad \ddagger$ See also Appendix B, § 13.

$\S$ The influence of Cleverness is almost (if not quite) negligible since the correlation-at any rate in the case of Dr Webb's training college students--between $p$ and $c$ is very small, if not zero.

II See above, p. I57.

I See above, p. I2I.

** See Webb, loc. cit. p. 6I ; G. Heymans and F. Wiersma, Beiträge zur speciellen Psychologie, p. 436; G. E. Partridge, An Outline of Individual Study, 1910; and Garnett, B. J. P. loc. cit. pp. 362 to 364 .

†† For example, William James' 'explosive Italian' (loc. cit. Vol. II, p. 538) possessed more than average (i.e. a positive degree of) Cleverness, as well as less than average (i.e. a negative degree of) Purposefulness.

G. E. 


\section{CHAPTER 9}

\section{CONFLICT}

\section{$\S$ I. Opposing Interest-Systems.}

WE have now completed our digression* on the origin, nature and functions of purpose-neurograms and of the future-interest-system in which they tend to become integrated. In the course of this digression we have noted the important influence exerted upon thought and conduct by the future-interest-system, which, as we have said, owes its origin, partly at least, to the operation of Will. We now return to the consideration of the direct action of Will in everyday life.

The normal occasion for the intervention of the Will arises, as we said $\dagger$, when the involuntary guides of the stream of thought are in conflict. The stream of involuntary thought is then obstructed, and the obstruction may be of internal or of external origin. We speak of obstruction originating internally when a conflict occurs between two of the interest-systems that are guiding involuntary thought in accordance with our third law and its corollary: in such a case the rival interest-systems attract the impulse in different directions, and, if the two systems are equally balanced, the intervention of the Will is required to intensify the attraction in one direction or in the other, or possibly in some third direction different from both. And the obstruction originates externally when some sense-impression, arising whether from outside or inside the body, tends to attract the impulse in one direction, while the interest-system that has been guiding thought tends, in an approximately equal degree, to attract it in another.

We may speak of every conflict as a conflict between two opposing interest-systems: it is these interest-systems that attract the impulse in two opposite directions. This attraction of the impulse in two opposite directions is an essential element in every conflict.

In order that two interest-systems may be in conflict, they must be simultaneously excited, and therefore, if for no other reason, they must have some element in common. We can imagine no conflict between two interest-systems which have no common element, no point of contact. Indeed, whoever will reflect upon his recent experiences of conflict will recognise that the interest-systems which

* Chapter 8 , beginning on p. 143 , above.

$\dagger$ Above, p. I4I. 
were opposing one another had something in common. Dr Hart, for example, illustrates a conflict by the case of a lover, the object of whose passion is already the wife of another man. 'The lover's mind will then exhibit two complexes trending in opposite and incompatible directions, on the one hand the desire for the woman, on the other the opposing tendencies constituted by moral education and fear of consequences.' * Here the complexes or interest-systems meet in the lover's neurogram for the woman.

But while two potentially conflicting interest-systems must meet, each must have its own separate organisation; they must have one common element, but not many. Now, we have said $\uparrow$ that interestsystems ordinarily possess such an organisation that the neurograms composing the interest-system are on the average more intimately interconnected towards the centre of the system than towards its periphery; and that therefore $\ddagger$ excitement in a neurogram near the periphery of the system tends to spread in the direction of increasing multiplicity of interconnexions: that is, towards the centre of the system. Since the excitement, wherever it originates in such a system, will tend to flow towards the centre, the neurograms nearest to the centre of the system-neurograms which are on the average the most intimately connected-will also, because they are most frequently excited, tend to be deepest. On both accounts, the influence which any region of the system will exercise upon the flow of excitement will be greater, the nearer that region is to the centre of the system. We shall have much to do with such systems in the sequel, and shall therefore require a name for them. Such a system-and we have just reminded ourselves that every interest-system $\S$ tends to be organised on these lines-we shall describe as an 'endarchy,' because its innermost regions dominate the rest of it: it is ruled from inside. To the consideration of endarchies we shall shortly return. Meanwhile, we have to remark that, if two endarchical interest-systems are in touch at some point on their peripheries, the excitement of the systems of arcs at the point of contact will tend to flow in the opposing directions that lead to the different centres of the two opposing systems. This, then, is the neural condition of a conflict: two distinct interestsystems having a restricted zone of contact and excited from that zone.

* Loc. cit. p. 78 .

$\dagger$ See above, p. 94 .

$\ddagger$ According to the corollary to our third law on p. 89, above.

$\$$ It is no longer necessary to restrict the term 'interest-system' to denote only those groups of interconnected neurograms that include some cognitive elements. See first two lines of $\S 5$ of Chapter 5 on p. 62 , above. 
We may illustrate the conflicting interest-systems by two opposing armies, which resemble the interest-systems in having an endarchical organisation. To the zone of contact between the interest-systems correspond the soldiers in a battle zone of front-line trenches, a comparatively small part of the two hostile forces. Whether these front-line soldiers are fighting, or fraternising (as many did on Christmas day, I9I4), the battalion, brigade, division, corps, army, and general headquarters of the opposing forces are further removed from one another, the higher their places in the rival endarchies. The opposing armies, like the interest-systems, meet at the periphery but have separate foci, separate centres. And it is because the central elements in each of the opposing armies are exercising, upon the peripheral elements in contact, influences which have nothing in common, that the front-line troops on opposing sides are prevented from making friends with each other. Similarly, in the case of the interest-systems, the peripheral elements in contact would quickly become closely interconnected, were it not for the divergent influences exerted upon them by the central elements of the interest-systems of which they respectively form part.

\section{$\S 2$. Resolution of Conficts.}

When two conflicting interest-systems are evenly balanced, the conflict can only be resolved by the intervention of the Will. But when the opposing systems are unequal, the elements of conflict, as we have described them, may be present without occasioning the intervention of the Will. The Will does not ordinarily* intervene unless or until the conflict is incapable of involuntary solution. Not unless the conflicting interest-systems are evenly balanced is the intervention of the Will required to enable the stream of thought to proceed.

When, for example, the expert climber finds it necessary to rely only upon his hand-holds while he swings his body round a buttress of rock that overhangs a precipice, he does not hesitate: he requires no effort of Will. He does not have to weigh his purpose of going on with the climb against the danger of a fall. The question of what may happen if he lets go does not present itself to his consciousness. For the excitement of the corresponding neurogram is so much less than that of the purpose-neurogram of his climb, that the former

* In the extraordinary case, the Will's intervention (if, as we said on p. I4I above, the Will only intervenes to resolve conflicts) takes place because of some conflict other than that between the two interest-systems in question. 
drains involuntarily into the latter (according to our third law), and merely adds to the excitement available for climbing. The novice, on the other hand, who first meets such a place on the mountains, may experience no merely nascent conflict but one that is full-grown. His sense of danger may balance, or even over-balance, his purpose to complete the ascent. It is only by an effort of Will that he increases the excitement of his purpose-neurogram, concentrates upon the climb before him, and so resolves the conflict between the opposing tendencies, to turn back and to go on. When, by an effort of Will, he has decided to go on, the continued activity of his fear-neurogram no longer tends to turn him back, but enables him to grip his handholds all the more tightly*.

Or, to take another illustration, we may compare the Londoner who crosses the Strand oblivious to danger (but in a pleasant state of excitement that helps to make him love London) with his country cousin who cannot face the dangerous traffic without an effort of Will.

Now, the climber who has known the mountains from his youth and has gradually become accustomed to more and more difficult pitches, like the Cockney who has been born and bred in London and has grown up with the increasing danger of street traffic, may never have been called upon to exercise such an effort of Will as is required of one who has to face a great precipice, or a crowded thoroughfare, for the first time. Yet the strong-willed tourist new to the mountains, or the countryman new to the metropolis, may, by an exercise of voluntary decision, beconie as callous to a particular kind of danger as the native has been made by a long series of merely nascent conflicts between purpose, on the one hand, and a sense of that kind of danger, on the other. It follows that a series of efforts of Will, and therefore a single effort of Will, may modify the interconnexion of neurograms precisely as they are modified by the involuntary thought-processes that effect the involuntary resolution of a conflict. For, when the tourist from the plains, after a series of voluntary efforts, has come to behave in the same way as the native mountaineer towards the dangers of the mountains, this result can only have been achieved by the modification of the neurograms of the dweller in the plains into the likeness of those of the native mountaineer. In fact, modification of the interconnexion of neurograms always accompanies the resolution of a conflict, whether the conflict be full-grown and be resolved by an effort of Will, intensifying the excitement in one of the opposing interest-systems so that it

* Cf. above the paragraph beginning 'Secondly...' on pp. 132, I33 above. 
drains the excitement from the other; or whether the conflict be merely nascent, in which case the more powerful interest-system may drain the excitement from the other, without the intervention of the Will. Seeing then that the Will only intervenes to resolve conflicts and that the resolution of a conflict always involves neurographic changes, we conclude that willed concentration of attention always modifies the connexions between neurograms. This conclusion is implied by the words of $\mathrm{Mr}$ Burt, whose experimental tests 'strongly suggest that...one feature or function of attentive consciousness... [is] the power of readjustment to relatively novel situations by organizing new psycho-physical coordinations.' * And, in Dr McDougall's view, the greater the effort of Will, the greater the effect in altering old neural connexions and making new ones $\dagger$.

When two opposing interest-systems are in contact over a restricted zone which at the moment is excited, their conflicting influence upon the stream of thought may be resolved without the intervention of the Will, unless the two systems are very evenly balanced. When the balance is even, the total excitement of the brain appears to increase. This increase of excitement is suggested by introspection. We have already $\ddagger$ seen reason to believe that the intervention of the Will results in an increase of excitement. The increase of excitement here in question may also be partly due to circular nervous processes within the rival interest-systems; for, as we have said, every large interest-system tends to contain affectiveconative elements. Another source of increased excitement may be the activity of the instinct-neurogram which gives rise to the emotion of anger; for, according to Dr McDougall§, the obstruction of any instinctive process (or, what amounts to the same thing, of the discharge of excitement from an excited affective-conative neurogram) is the normal stimulus of the emotion of anger. At all events, if excitement always increases on the occurrence of a conflict, the result may be that the corresponding thought-activity is brought more fully into the focus of consciousness, and that therefore the Will is better able to intervene; for the Will cannot, according to our fourth law, intensify excitement except in arcs that are already active; or, in other words, the Will can only intervene among thought-activities that are already in the field of consciousness. We may add, that an increase of excitement on the occurrence of conflict might suffice to

* Loc. cit. p. I68. See also the passage (loc. cit. p. 169) quoted above, p. I 17.

$\dagger$ See the passage quoted above, in footnote $\uparrow, p$. 130 .

$\ddagger$ See above, p. 130 .

§ Social Psychology, p. 59. 
bring a sequence of unconscious thought-activities into the field of consciousness and so enable the Will to intervene, even in thought which, up to the moment of intervention, had been unconscious.

When, even with the aid of the additional excitement which the anger-process may place at its disposal, the Will is unequal to the task of resolving a conflict between well-balanced interest-systems, there is one other primary emotion-'distress'-that may come in to help. This state of feeling is frequently observed in children. Dr McDougall writes:

Children very early display this reaction, which consists essentially in loud cries, sobbing, and tears....The state of feeling or emotion that accompanies this reaction is, I submit, properly called distress; and the feeling, together with the complex unlearned reaction, common to all members of the species, must be ascribed to an innate affective disposition which, like that of anger, does not seem to be innately connected with any innate cognitive disposition; for, like the disposition of anger, it seems to be excited only secondarily to other impulses. The typical result of the thwarting in the child of any strong impulse is first anger and then, if the impulse thus re-enforced still fails to attain its end, distress. That is to say, we are endowed with this innate tendency to relax our efforts and to cry aloud for help whenever we 'come to the end of our tether,' when we feel that our powers are quite incapable of coping with the situation. The biological value and function of this reaction are obvious; it seems to be primarily a function of infancy and childhood, when the help of older persons is so often required: but it seems to persist in the female sex into adult life; and even strong men, when their utmost efforts prove unavailing, sometimes break down and cry aloud for help on whatever higher powers they may have learnt to conceive, thus showing that in them also this disposition is not wholly transitory.

The two emotions of anger and distress occupy, then, a position unlike any others. They spring from innate affective dispositions and therefore have their specific bodily tendencies and expressions; but they differ from the others in depending for their arousal, not upon any particular objects or sense-impressions, but upon the thwarting of other impulses.

This affect, properly called 'distress' (which I failed to recognise in Social Psychology), must be ranked with the primary emotions*.

The function of distress, then, seems to be to sweep away the anger and the striving-a $\kappa a \theta a \dot{\rho} \sigma \iota \varsigma \hat{\omega} \nu \pi a \theta \eta \mu a \dot{\tau} \omega \nu$-, to absorb the remaining activity of the excited interest-systems, and, even if the help asked for from outside sources does not come in the way the subject expects, to leave him free to try afresh to resolve his conflict, unhampered by the memory of his former failure, or, in other words, by the interestsystems that were rendered active in his previous futile efforts.

* 'Symposium,' loc. cit. p. 28. 
We have now to examine more closely the nature of the alteration of neural connexions which, as we have seen, always accompanies the solution of a conflict, whether voluntary or involuntary. A conflict, we have said, results when excitement traverses a system of arcs that forms the sole connexion between two otherwise separate and distinct interest-systems, to the foci or centres of which the excitement then tends to flow in opposite directions. The resolution of the conflict may be accomplished by disconnecting, from one system or from the other, the excited arcs that are, to start with, on the fringe of both. We shall refer to this process as Disintegration, for it completes the dissociation of one interest-system from the other. Or the conflict may be resolved by introducing connexions between the central neurograms of the opposing systems. Returning to our military illustration, this second method would correspond to a victory that placed the general headquarters of the opposing armies in communication with one another and swallowed up the purposes of one commander-in-chief in those of the other. To this process we shall refer as Integration, for it makes the two interest-systems into one. Or, finally, the conflict may be resolved by a combination of Disintegration with Integration, as when the connected fringes of the two systems are more closely connected to each other and are, at the same time, disconnected from the opposing centres of the rival systems. The corresponding process in our military example would occur if the soldiers in the trenches, and their immediate commanding officers on both sides, were to fraternise, and agree to form an international democratic state from which the influence of discordant autocracies-the higher military and political commanders on both sides-should be removed.

We have, then, a cross-classification of the ways in which a conflict may be resolved. We first saw that conflicts may be resolved either (i) involuntarily, or (ii) voluntarily. And now we have said that conflicts may be resolved by (a) Disintegration (Repression); by (b) Disintegration combined with Integration (Displacement, or, in certain cases, Sublimation); or by (c) Integration (generally involving Conscious Control). Our justification for identifying the processes represented by Freudian terms included in the brackets with the neural mechanisms of Disintegration, Disintegration combined with Integration, and Integration respectively, will appear as we proceed. We may then tabulate the methods of resolving a conflict as follows: 
Table VI.

$a$ (i) Involuntary Disintegration (Repression).

$b$ (i) Involuntary Disintegration combined with Involuntary Integration (Displacement or Sublimation).

$c$ (i) Involuntary Integration. a (ii) Voluntary Disintegration (Repression).

$b$ (ii) Voluntary Disintegration combined with Voluntary Integration (Displacement or Sublimation).

$c$ (ii) Voluntary Integration (generally Conscious Control).

We proceed to illustrate these six processes.

Disintegration (Repression) probably occurs far more often as an involuntary than as a voluntary process. The Freudian literature abounds in examples of Involuntary Repression ( $a$ (i)). We may follow Dr Bernard Hart* in citing a case of Involuntary Disintegration described by Professor Janet under the name of Irène:

Irène had nursed her mother through a prolonged illness culminating in death. The circumstances connected with the death were peculiarly painful, and the event produced a profound shock upon Irène's mind. She had been deeply attached to her mother, and the latter had filled the chief place in all her thoughts, ambitions and activities. Her mother's death, therefore, not only produced a great grief, but it deprived all those ambitions and activities of their main object and end. The ideas connected with the mother's illness and death formed a system or complex intensely painful and repugnant to the personality as a whole. A conflict was thus produced between the complex in question and the personality apart from this complex or interest-system. 'To get rid of this conflict, the mechanism of repression was brought into play. The painful complex was dislocated from the remainder of the mind, and no longer allowed to introduce its constituent ideas and emotions into the field of consciousness.' $\dagger$ Thus an abnormal mental condition developed, characterized by the frequent appearance of symptoms resembling those exhibited by the ordinary sleepwalker. Irène, perhaps engaged at the moment in sewing or in conversation, would suddenly cease her occupation and would commence to live over again the scene of her mother's death, carrying out every detail with all the power of an accomplished actress. While this drama was in progress, she was perfectly unconscious of the actual events happening in her environment, heard nothing that was said to her, and saw nothing but the imaginary scene in which she was living at the moment. This somnambulism would end as suddenly as it had begun, and Irène would return to her former occupation, absolutely unaware of the fact that it had ever been interrupted. After an interval of perhaps several days, a second somnambulism, resembling the first in all respects, would appear in the same abrupt manner. If the patient were interrogated during the apparently normal intervals, it would be found that she had not only entirely forgotten everything which had happened during the somnambulism, but that the whole system of

$$
\text { * Ior. rit. pp. } 28,46,93 .
$$

† Loc. cit. p. 93. 
ideas connected with her mother's death had completely disappeared from her mind. She remembered nothing of the illness or its tragic end; discussed her mother without emotion, and was reproached by her relatives for her callous indifference to the whole subject.

In the case of Irène, the structure of the dissociated system of ideas, whose irruption into the field of consciousness was responsible for the appearance of the somnambulisms, comprised but little beyond the ideas connected with her mother's illness and death. But dissociated interest-systems may attain to any degree of complexity and development*. In cases of so-called double personality, the dissociated system may be comparable in complexity with the remainder of the subject's neurography. In such a case it may be no longer possible to speak of one system as the dissociated system, for the whole neurography is disintegrated into two systems of approximately equal complexity.

An example of Voluntary Repression ( $a$ (ii)) is furnished by the persons who, according to William James, "can voluntarily empty their minds and " think of nothing " $\uparrow$.' When I decide not to entertain certain thoughts, and voluntarily connect them to the (D-process in the manner described above $\ddagger$, I dissociate the neurograms of those thoughts, and, as time goes on, other neurograms forming part of their system, from the remainder of my neurography§. I may thus resist a temptation by connecting the neurogram $P$ of a lower 'propensity,' to use William James' language\|, with the $O$-system. A few such voluntary connexions made between $P$ and $O$, as $P$ is from time to time excited from different sources, and as $\not$ 归 therefore presents itself in various connexions, may effectively dissociate $P$ from my organised interest-systems, and so set me free, at all events during my waking moments, from this particular temptation.

Involuntary Disintegration combined with Integration $(b(\mathrm{i}))-$ Involuntary Displacement-may be illustrated by another case cited by Dr Hart 1 . A patient in whose life a lady of the name of Green played a very prominent part, was asked, 'Do you know a Miss Green?' He replied, 'Green, that's green, that's blue, would you say that water is blue?' Here the neurogram excited by the word 'Green' was involuntarily disconnected from the interest-system corresponding to the lady, and connected instead-or, rather, more deeply connected

* See above, p. I47.

$\dagger$ Loc. cit. Vol. 1, p. 404. $\ddagger$ On pp. I47 to I49.

$\S$ Cf. J. C. Fluegel, B. J. P. Vol. virI, p. 493 (I9I 7 ). We have already (see above, p. I48) quoted Mr Fluegel's view that 'repression is apt to extend from the element of thought which was originally the subject to other elements that are associated with it.'
|| Loc. cit. Vol. II, p. 548.
I Loc. cit. p. I1о. 
-to the colour interest-system, and especially to that part of it corresponding to the colour blue-green. Had the neurogram of green (including both the lady and the colour) been entirely repressed, the patient would not have noticed the word 'Green' in the question. But cases of Involuntary Disintegration combined with Integration are not all pathological. The politician described by Dr Hart* whose political interest-system 'will reinforce in his mind those arguments which support the view of his party, while it will infallibly prevent him from realising the force of the argument propounded by the opposite side,' is a case in point. Dr Hart adds: 'Now, it should be observed that the individual himself is probably quite unaware of this mechanism in his mind.' The political interest-system, on the one hand, exercises a disintegrative action upon the argument that is tending to connect a series of neurograms which it successively excites and to integrate them in a system that would be in conflict with the political interest-system in question; but, on the other hand, it exercises an integrative action by connecting to itself selected elements from the system of neurograms which the argument is successively exciting.

In these examples of Involuntary Displacement $(b$ (i)) - that is, of Involuntary Disintegration combined with Integration-the disintegrative (repressive) part of the process has been far more marked than the integrative part. Indeed, Dr Hart, from whose work they have been quoted, employed them to illustrate Repression alone. But in other cases the integrative action may be far more marked than the disintegrative. Of such a kind are many pathological cases of phobias, obsessions and the like, where the extent of the interestsystem that has gradually been built up around the phobia-neurogram implies far more integrative action than the disintegration involved in dissociating the phobia-neurogram from the system of which it originally formed part. For example: Late one evening a soldier in an Indian frontier station, from which several sentries have mysteriously disappeared at night, hears a slight disturbance outside the compound, and goes out to investigate among the trees near by. He rescues the sentry, who is at grips with two dark figures, when, from a branch overhead, a third black shape falls upon him with outstretched blade. $\mathrm{He}$ is only just in time to avoid the blow. Years afterwards, returning from France with shell-shock, he has no recollection whatever of the Indian incident, but his whole life is coloured and distorted by an unreasoning fear of trees, especially after dark. The fear of trees has 
been disintegrated from its connexions with a certain night in India, but has been integrated with other neurograms to form a far vaster interest-system, so wide as to be excitable through a multitude of everyday happenings.

Such involuntary displacements are usually of slow growth. But they are not by any means always injurious. On the contrary, they may be most beneficial. They are then commonly referred to as 'sublimations' instead of as 'displacements.' A sublimation is, in fact, a displacement in which the prime-moving affective-conative elements are dissociated from an interest-system that is opposed toi.e. potentially in conflict with-the good elements in a future-interestsystem, and then integrated with those good elements. Later on we shall specify more particularly what these good elements are and wherein they differ from elements that are bad in a moral sense. Here it is sufficient to remark that a general consensus of opinion recognises certain purposes as good, and that the elements which we have called good in a future-interest-system are those the excitement of which brings a good purpose before consciousness and tends to its realisation. Mr Fluegel gives as an example of (Involuntary) Sublimation such transformations of neural connexions 'as are effected in a man who, originally endowed with impulses prompting to cruelty and sadism, becomes an efficient surgeon, soldier or butcher.'*...He adds that 'the more advanced forms of Sublimation are in most cases processes of gradual growth through successive steps, each more remote in nature from the original expression of the tendency, and each representing an advance from the moral point of view. Thus we must not grow impatient if in the first stages there is to our eyes still too much resemblance to the original activity, e.g. if...the subsequent surgeon early displayed an undue tendency to pull to pieces flowers, toys or other objects or to behave in an aggressive and cruel manner in his relations with other children. The gulf between the original tendencies and their ultimate expression is obviously far too wide to be bridged by a single step, especially when we are dealing with minds that are still immature. For this reason it would seem very undesirable in educative practice to extend our taboos and prohibitions beyond the sphere in which they are strictly necessary, lest in so doing we interfere with the initial stages of Sublimation. A school or home (or even a country) in which everything is forbidden will probably afford a relatively unfavourable atmosphere for the growth of Sublimation.' $\dagger$

* Loc. cit. p. 485 , where other examples are also given.

$\dagger$ Loc. cit. pp. 486, 487. 
Unless, for example, young men have opportunities of sublimating a tendency to organised fighting into an interest in (playing in) football matches or (rowing in) boat races, their original tendency will probably lead them to a morbid interest in military matters fostered by duelling and ultimately finding its full vent in war.

We have now to illustrate Voluntary Displacement ( $b$ (ii))Voluntary Disintegration combined with Integration. The airman who dodges shells from anti-aircraft guns, may disconnect the problem of anticipating the next burst and keeping out of its way from the fear of the consequences of being hit, and connect it instead with an interest in dodging the opposing back when scoring a try at Rugby football. In short, by an effort of Will he may pretend that dodging 'Archies' is a game. The climber, who succeeds in voluntarily disconnecting most of the effects of fear from his climbing experiences, but retains some of the excitement to add to the energy available for climbing, affords another example of the same kind. But perhaps the best example of Voluntary Displacement is furnished by the process technically called Rationalisation. This process consists essentially in repressing some neural connexion at which conflict is apt to originate between opposing interest-systems, and connecting the systems instead by some roundabout path, the excitement of which will not lead to conflict. For example, to borrow another illustration from Dr Hart, 'The man whose commercial morality differs fundamentally from the code which he practices in his private life, persuades himself that the latter code is not properly applicable to business relations, that to ask a customer three times the value of a certain article is obviously something quite different from thieving, that a man must live, and that the immorality of lying completely disappears when it is necessary for the support of one's wife and family. Whenever, in fact, our actions conflict with our ethical principles we seek for specious reasons which will enable us to regard the actions in question as a peculiar case altogether justified by the circumstances in which they are carried out.... The honest man who swindles the railway company and the government without in any way injuring his sense of personal rectitude, provides an excellent illustration of the process we are now describing.' *

Another illustration is afforded by the puritan councillor whose aesthetic sense was offended by galvanised iron receptacles for sand and gravel placed by the roadside of his city. He would not acknowledge that aesthetic ideas had any part to play in human life. When

* Loc. cit. pp. $85,86$. 
therefore he protested to the city council against the use of the iron receptacles in question, and was challenged for his reason, the only reason he could give was that 'they might afford a murderer an easy means of disposing of the body of his victim.'*

Of a similar kind are all the multitude of rationalisations by which men, ignorant of the true external or internal origin of their opinions, voluntarily inter-connect them so as to form a kind of caricature of a perfectly integrated mind. This caricature differs from the real thing in that the truly reasoned view will fit experience as it grows, while growing experience will continually be in conflict with vain rationalisations such as, according to Mr Trotter, largely constitute the mental furniture of the average man. 'He will have fairly settled views upon the origin and nature of the universe, and upon what he will probably call its meaning; he will have conclusions as to what is to happen to him at death and after, as to what is and what should be the basis of conduct. He will know how the country should be governed, and why it is going to the dogs; why this piece of legislation is good, and that bad. He will have strong views upon military and naval strategy, the principles of taxation, the use of alcohol and vaccination, the treatment of influenza, the prevention of hydrophobia, upon municipal trading, the teaching of Greek, upon what is permissible in art, satisfactory in literature, and hopeful in science.' $\dagger$ In so far as these views are in conflict with each other (internal conflict) or with his experience (external conflict) as it develops, he represses-disintegrates-the disharmony; and it is this admixture of Disintegration with Voluntary Integration which justifies the inclusion of these examples of everyday rationalisation under the head of Voluntary Displacement, or Voluntary Disintegration combined with Integration.

In all these cases of rationalisation, opposing interest-systems are dissociated from one another at the point of conflict, but allowed to come into contact by means of a long-circuit of neural paths, no portion of which is so closely connected to both systems as to give rise to conflict when it is excited; or, in Dr Hart's phrase, the contact between the incompatible systems of ideas is only made 'by means of a bridge of rationalisations which so distorts their mutual significance that conflict is efficiently avoided.' $\ddagger$

We have now to illustrate the process of Integration pure and

* 'The well-known story of the old judge advising the new one never to give reasons for his decisions, "the decisions will probably be right, the reasons will surely be wrong," ' is here in point. Cf. W. James, loc. cit. Vol. II, p. 365 .

$\dagger$ W. Trotter, 'Herd Instinct,' Sociological Review, 1908, quoted by Dr Hart, loc. cit. p. 135.

$\ddagger$ Loc. cit. p. 86 . 
simple. We remark once more that this process is seldom involuntary, and may generally be identified with Conscious Control. We may illustrate the comparatively rare instances of Involuntary Integration ( $c$ (i)) by the case in which a sudden stimulus causes to be performed involuntarily a complex movement which the Will had previously been incapable of carrying out. Suppose, for example, that the novice on skis who has been trying, time after time, but without success, to perform a Christiania swing-a sudden turn by which the ski runner, speeding down a mountain side, may stop himself almost instantaneously - gives it up and goes out for an expedition on the mountains. $\mathrm{He}$ is running swiftly down what he supposes to be a continuous smooth slope, when, suddenly, he is aware of a dangerous crack into which he must fall unless, despite his speed, he can turn to avoid it. Immediately, and without difficulty, he is saved from the danger by a perfect swing*. And the excitement accompanying the involuntary integration that we have described may have been so great that our novice may, for the future, be able to perform the Christiania swing at will. Other similar examples of Involuntary Integration are afforded by one who, having practised the swimming movements on dry land, but never having been able to perform them in the sea for fear of swallowing salt water, is suddenly thrown into deep water to sink or swim; or, again, by the involuntary formation of novel neural connexions in the animal learning a new trick under the influence of fear of pain, in case he does not perform to the satisfaction of his master.

We have said that when a conflict is solved by Integration it is not in general solved by Involuntary but by Voluntary Integration ( $c$ (ii)). And this is the ideal solution of all conflicts $\dagger$. For when a conflict is

* What has doubtless happened is that the sudden fearful vision has excited his fear-system as well as his swing-neurogram; so that, before the turn had well begun and before the semi-circular canals connected with the opposing balanceneurogram had become excited, the inrush of excitement from the fear-system into the swing-neurogram so lowered the resistances of all its synapses that it drained all other excitement arising during the turn. The image of the turn held the focus of consciousness against all comers.

$\dagger$ Cf. Fluegel, loc. cit. p. 489: 'The satisfactory solution of Conflict as revealed by Psycho-analysis would appear to consist in bringing both the opposing tendencies into the focus of consciousness, recognizing them for what they are, both in themselves and in their consequences, without any attempt at covering up or slurring over any of their aspects, either from intellectual laziness or from any painful feeling which they may arouse. The whole experience and the whole of the mental ability of the personality is then available for deciding between the conflicting claims-a state of affairs which is impossible as long as there exists any considerable degree of Repression.'

Cf. also Bernard Hart, loc. cit. p. 79: 'This subjective appreciation of the forces at war within us, and deliberate adoption of a consciously selected line of conduct, may be regarded as the rational or ideal solution of a conflict. In fact it may be said to provide the only possible solution in the strict sense of the word.' 
resolved by so inter-connecting the two opposing interest-systems that the neural conditions of conflict are removed once and for all, the conflict has been for ever ended by a 'fight to a finish.' * No repressed system is preserved to carry on a war after the war. Where, however, Disintegration (Repression) is employed to resolve a confict, the repressed system is preserved, although its dissociation from the remainder of the subject's neurography prevents it from influencing consciousness directly. That such repressed systems are preserved, and that they may influence consciousness indirectly, as in dreams, psycho-analysis affords ample evidence. Moreover, as we saw $\dagger$, such a repressed system tends to grow; and, as it grows, it makes for the disintegration of personality and militates against a consistent and effective life.

Voluntary Integration consists of neurographic changes brought about by the action of Will. We have already $\ddagger$ seen that an effort of Will always produces such changes. According to our fourth law, the only way in which the Will produces this or any other effect is by intensifying the excitement in a system of active arcs so that the remainder of the excitement of the brain shall drain through that system, deepening the path or paths by which the draining takes place.

\section{§ 3. Two Types of Conflict.}

We have now to consider more closely the neurographic changes involved in the solution of conflict by Voluntary Integration. It will be convenient to begin by distinguishing two types of conflict, although we shall see later that the neurographic changes which remove the opposition are essentially the same in both cases. Conflict, as we have defined it, occurs whenever an involuntary stream of thought is blocked; or, what amounts to the same thing, whenever the corresponding flow of excitement through brain arcs is obstructed. The obstruction is always due to competition between two neurograms or systems of neurograms, to which, on account of their competition, we shall refer as the competing or opposing neurograms or systems.

The first of our two types of conflict may be distinguished as unipolar. The conflict is between one neurogram (generally a purposeneurogram) excited by the Will, and some other neurogram or system excited involuntarily through old neural connexions (for example, the neurogram of a habit, instinct, or reflex movement such as may result from a painful stimulus). Conflicts of the unipolar type commonly

* Bernard Hart, loc. cit. p. 79.

$\ddagger$ See above, p. 165 .

$\uparrow$ See above, pp. 147 and 170 . 
arise when some external* obstacle prevents the fulfilment of a purpose.

The second type of conflict is bipolar; it takes place between two interest-systems, originally on equal terms as regards excitement by the Will. Both may be voluntarily excited, as in the case of two purpose-neurograms in conflict; or else neither may be voluntarily excited. Conflicts of internal origin are generally bipolar.

In the first (or unipolar) case, the Will strives to make the excitement go through one neurogram, and one only. In the second (or bipolar) case, the Will strives to make the excitement go through two neurograms by connecting them together. In the first case, the Will may be regarded as a partizan; in the second, as a detached and disinterested judge. These two types of conflict correspond to the two sources of science, the practical source and the theoretical source, described by Professor Whitehead $\uparrow$. 'The practical source is the desire to direct our actions to achieve pre-determined ends. For example, the British nation, fighting for justice, turns to science, which teaches it the importance of compounds of nitrogen. The theoretical source is the desire to understand.' +

Let us now consider in greater detail the solution of unipolar conflicts by Voluntary Integration. We may represent the neural conditions of this type of conflict by Fig. Io on p. I78. In this diagram, $A$ represents the system of arcs maximally excited at any moment, when $\mathfrak{A}$ occupies the focus of consciousness, while $Z$ represents the purpose-neurogram through which the Will strives to make the excitement from $A$ drain. To this end, the Will increases the excitement in $Z$; and, for this to be possible, $Z$ must be active, or, in other words, $\mathbb{Z}$ must be present on the fringe of consciousness. As the excitement in $Z$ rises under the influence of the Will, the excitement from $A$ tends to drain into $Z$ by the shortest available path. Suppose that this path proceeds from the element $a$ of $A$ to the element $z$ of

* See above, p. 162.

$\dagger$ Presidential Address to Section A, British Association, Newcastle, 1916; reprinted in The Organization of Thought, p. Io6.

$\ddagger$ Loc. cit. p. 106. Professor Whitehead adds: "To avoid misconception, I most emphatically state that I do not consider one source as in any sense nobler than the other, or intrinsically more interesting. I cannot see why it is nobler to strive to understand than to busy oneself with the right ordering of one's actions. Both have their bad side; there are evil ends directing actions, and there are ignoble curiosities of the understanding.' In another place (loc. cit. p. I I I), Professor Whitehead holds that 'the first great steps in the organization of thought were due exclusively to the practical source of scientific activity, without any admixture of the theoretical impulse.' We shall have reason to doubt the correctness of this last opinion, for we shall see that theory is as old as the instinct of curiosity, and that therefore it long preceded man. 
$Z$. Since we are considering a case of conflict, we have to suppose that, as the excitement begins to flow from $a$ to $z$, it meets an old path of much lower resistance, leading not to $z$ but to the neurogram, $U$, of some habitual, instinctive or reflex movenient which then takes place and so drains the excitement. The result is to defeat the Will's effort to cause the excitement to drain through $Z$.

Suppose, for example, that I wish to unscrew a metal lid from a glass jar. I am faced by the jar with the lid stuck fast, and this image is in the focus of my consciousness. Its neurogram is represented by $A$ in the diagram. My purpose is to grip the jar tightly with one hand and the lid with the other, and then to twist one hand relatively to the other without my fingers slipping. To this complicated movement corresponds the excitement of $Z$ in the diagram;

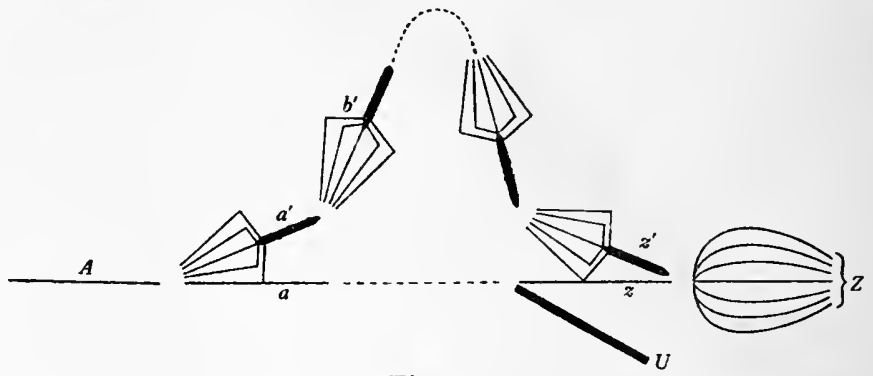

Fig. Io.

and when the movement takes place-when $Z$ drains the excitement - the lid is unscrewed. But if the lid is stuck very fast indeed, a great effort of my Will is required from me in order that I may grip the jar and lid so tightly that, when I twist my hands relatively to one another, my fingers shall not slip. Ultimately, a point is reached when the pressure on my fingers causes me so unpleasant a feeling that my Will is unequal to the task of gripping more tightly. The unpleasant feeling corresponds to the excitement of $U$ in the diagram.

Now, it may happen that the lid is not so tightly stuck but that, with a very great effort, I may be able to make the necessary movement in spite of the unpleasant feeling. In that case I excite $Z$ and the path $A a \ldots z Z$ sufficiently to lower the resistance of the path below that of the path $A a \ldots U$.

Or again $U$ may correspond to some lower propensity of instinct or of habit, while $Z$ corresponds to an ideal impulse. Thus William James writes:

The ideal impulse [when compared with the propensity] appears a still small voice which must be artificially reinforced to prevail. Effort is what 
reinforces it, making things seem as if, while the force of propensity were essentially a fixed quantity, the ideal force might be of various amount. But what determines the amount of the effort when, by its aid, an ideal motive becomes victorious over a great sensual resistance? The very greatness of the resistance itself. If the sensual propensity is small, the effort is small. The latter is made great by the presence of a great antagonist to overcome. And if a brief definition of ideal or moral action were required, none could be given which would better fit the appearances than this: it $i$ action in the line of the greatest resistance. The facts may be most briefly symbolized thus: $U$ standing for the propensity, $Z$ for the ideal impulse, and $W$ for the effort:

$$
\begin{aligned}
& Z \text { per se }<U, \\
& Z+W>U .
\end{aligned}
$$

In other words, if $W$ adds itself to $Z, U$ immediately offers the least resistance, and motion occurs in spite of it. But the $W$ does not seem to form an integral part of the $Z$. It appears adventitious and indeterminate in advance. We can make more or less as we please, and if we make enough, we can convert the greatest mental resistance into the least. Such, at least, is the impression which the facts spontaneously produce upon us*.

In this way, as well as by the method of Repression already described, temptations may be overcome.

But it will often happen that a direct effort of Will is insufficient to cause $Z$ to drain the excitement from $A$. When the Will is incapable of directly exciting $Z$ (and the direct path, $A a \ldots z Z$, from $A$ to $Z$ ) sufficiently to make the excitement drain by that route instead of by the path $a \ldots U$, the excitement may still be drained from $A$ to $Z$ if the Will can make an indirect path, $A a^{\prime} \ldots z^{\prime} Z$, from $A$ to $Z$, avoiding $U$ and its connexions. Reasoning is the process by which the Will $\dagger$ may make a new $\ddagger$ indirect path of this kind. In this process the Will first concentrates excitement in $Z$, although $Z$ fails to drain the excitement. If then $Z$ drain the excitement directly from $A$, the conflict is resolved without reasoning; but, if not, $Z$ remains active and ready to assist in guiding the excitement towards itself. This guiding continues throughout the process; it works by multiple stimulation, in the manner already§ described; and it consists, as we have seen, in the excitement always tending, when other things are equal, to flow through the paths that are most closely connected with the active interest-system, $Z$. diagram.

$\dagger$ We have already (on p. I65) remarked that the operation of the Will always modifies neurographic connexions.

$\ddagger$ W. James (loc. cit. Vol. II, p. 330) makes 'ability to deal with NOVEL data the technical differentia of reasoning' and so marks it off from common associative (involuntary) thinking.

$\S$ See above, p. 83; and below, pp. 257 et seq. 
The voluntary concentration of excitement in $Z$ having failed, we suppose, to cause direct drainage through $a \ldots z$, the Will next concentrates excitement in $A$ : attention is concentrated upon $\mathfrak{A}$. The excitement now spreads to numerous associated neurograms $* a^{\prime}, a^{\prime \prime}, \ldots$, in addition to $a$. Concentration of excitement in $a$ would lead, not to $z$ and so to $Z$, but to $U$ instead. So now excitement is concentrated in one of the other neurograms connected with $A$, say $a^{\prime}$. From $a^{\prime}$ the excitement diffuses to many others $\left(b^{\prime}, \ldots\right)$, some of which are already connected to $a^{\prime}$, and, if the excitement in $a^{\prime}$ is now greater than it has previously been, to some new ones now connected with $a^{\prime}$ for the first time. After diffusion, concentration $\dagger$ : excitement is now concentrated upon some one, say,$+ b^{\prime}$, of the neurograms to which excitement has diffused from $a^{\prime}$. From $b^{\prime}$ the excitement again diffuses to a number of neurograms, in one of which it is concentrated. And so the process proceeds, concentration alternating with diffusion. Finally, if the process is successful, $Z$ is reached through one of its connexions $z^{\prime}$. The excitement then drains from $A$ to $Z$ through the path $A a^{\prime} \ldots z^{\prime} Z$.

The example already $\S$ considered will illustrate the process. As excitement is concentrated in $Z$, my fingers grip and my hands begin to turn in opposite directions. My fingers do not, however, move, and, lest they should slip, my grip increases. As the strain increases, an unpleasant feeling, due to the excitement of $U$, rapidly grows. Moreover, from $U$ the excitement passes on to other arcs the excitement of which leads to the relaxation of the strain $\|$ : the excitement is being drained through $U$ to these arcs instead of through $z$ to $Z$. We are supposing that direct stimulation of my purpose-neurogram $Z$ is insufficient to achieve my purpose. My failure is not due to the tearing of my muscles, which could only result from a state of maniacal excitement incapable of production by voluntary effort. It is due, as we have said, to the excitement draining from $a$ to $U$ instead of from $a$ to $z$.

* Three of these are shewn in the diagram in addition to $a$ and $a^{\prime}$ which are lettered.

$\dagger$ See above, Fig. 5, p. 8 o.

$\ddagger$ In the language we shall use later (e.g. on pp. I87, 190), $b^{\prime}$ corresponds to an essence 'abstracted' from indefinite impressions of the world of experience. Why this particular essence is abstracted will appear when we consider (on pp. 23I et seq.) how new paths made by reasoning must form a certain kind of system (a 'maximal endarchy') corresponding to the systematically arranged, or 'harmonious' world of experience (the 'endarchy of science').

$\S$ See above, p. 178.

II We may imagine neural connexions resembling those shewn in the diagrams (Figs. 3 and 4 ) on pp. 75, 76 above. 
My purpose-neurogram remaining active, I now concentrate my attention on the jar with the stuck lid: excitement is being concentrated in $A$. Among the ideas that enter my field of consciousness are those which correspond to the excitement of the neurograms $a, a^{\prime}, a^{\prime \prime}, \ldots$. Then $a$ represents the idea of 'taking hold and starting to unscrew,' which leads directly to $\mathbb{Z}$, but which leads also to $\mathbf{f}$ (unpleasant feelings and the rest) under circumstances which result in $\mathbf{Z}$, and not $\mathbf{Z}$, coming into the focus of consciousness. We may suppose that $\mathfrak{a}^{\prime}$ represents the thought that the lid is too small for the jar; $\mathfrak{a}^{\prime \prime}$, the thought that the lid is stuck to the jar by some such substance as candle wax; and so on.

Having failed to excite my purpose-neurogram sufficiently to cause the lid to move, and having concentrated my attention on the jar with its stuck lid (A), I realise, not merely that the top is stuck, but that it is stuck at a particular place, or stuck because it is too small $\left(\mathfrak{a}^{\prime}\right)$, or stuck because some adhesive substance joins it to the jar $\left(\mathfrak{a}^{\prime \prime}\right)$. I now concentrate on one of these ideas: for example on $\mathfrak{a}^{\prime}$, that the lid is too small. As the excitement diffuses from the corresponding neurogram $a^{\prime}$ a number of associations of this idea $\left(\mathfrak{a}^{\prime}\right)$ of smallness come before my mind, with vividness depending upon the excitement of the corresponding neurograms, and so in part upon the extent to which these neurograms are stimulated from my purpose-neurogram which continues active all the while. One $\left(\mathfrak{b}^{\prime}\right)$ of these associations of smallness may well be, that the lid has contracted in cooling more than the glass jar: the lid may be too cold, and may expand again on heating. Once more, I concentrate my attention on this idea, and, among the associations to which it gives rise, is the idea that heating may be produced by the friction of my fingers. On this idea I then concentrate, and automatically my fingers rub the lid until it turns freely in my hand. The conflict has been resolved by successive stages of voluntary integration.

We shall shortly* return to this example of the solution of a unipolar conflict by reasoning-a special (and specially important) case, as we have seen, of voluntary integration-in order to illustrate certain characteristics of reasoning in general. Among these characteristics we shall have to consider particularly the alternate concentration and diffusion of neural excitement; the relation of the linked neurograms, successively excited as the chain of reasoning proceeds, to the outside world of things about which one is reasoning; and the influence of the purpose or aim, which overshadows the reasoning

* On p. 186 below. 
process from the start. Meanwhile, however, we have to illustrate the solution of bipolar conflicts, by voluntary integration and especially by reasoning; and then to compare the three* voluntary means of resolving a conflict, by disintegration, by disintegration combined with integration and by integration respectively.

In bipolar conflicts there are two interest-systems attracting the impulse in opposite directions. We proceed to consider how the conflict is to be solved by voluntary integration.

The diagram (Fig. II) represents a case of bipolar conflict; $Z_{1}$ and $Z_{2}$ are the interest-systems in conflict; $A_{1}$ and $A_{2}$ are direct paths leading respectively to $Z_{1}$ and $Z_{2}$. The complete solution of this conflict consists in making $A$ drain into both $Z_{1}$ and $Z_{2}$. But the conflict may be resolved otherwise than by this complete solution. One way of doing so is, as we have already seen, by connecting $\mathbb{Z}_{2}$ to the 1 -process and so repressing it. The conflict is then resolved by Disintegration. Another method, which does not give the complete

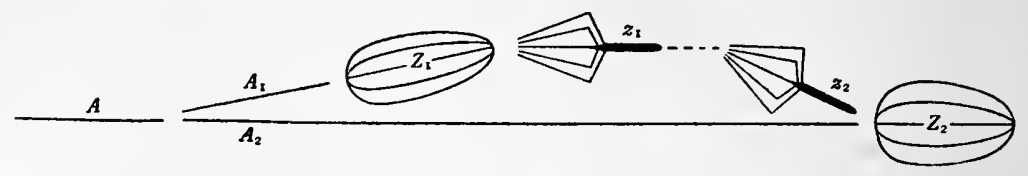

Fig. II.

solution of draining $A$ into both $Z_{1}$ and $Z_{2}$ but which may nevertheless give a solution sufficiently complete for many practical purposes without involving direct Repression, consists in concentrating excitement in one of the opposing systems, say $Z_{1}$, so that the threshold $\overline{A A}_{1}$ is lowered; the excitement will then drain by the path $A A_{1} Z_{1}$ instead of by the path $A A_{2} Z_{2}$. Moreover, the neurography is altered, so that in future $Z_{1}$ will always tend to drain excitement from $A$ without opposition from $Z_{2}$. The conflict has been thus far solved, and this partial solution suffices if all that is required is to choose either $\mathbb{Z}_{1}$ or $\mathbb{Z}_{2}$, no matter which. If, for example, while I am walking in the country without any object but that of enjoying the open air and exercise, I meet a fork in the road, it matters to me not at all which turning I take. My proper course is not to stand hesitating between the two, but-perhaps after tossing a coin-to choose one or the other. My choice consists in concentrating my attention on the thought of the road I am to follow. Conflicts of this type may seem insignificant and unworthy of serious consideration; but, as Mr Fluegel

* See $a$ (ii), $b$ (ii), and $c$ (ii) in Table VI on p. I69. 
has pointed out*, they are distressingly frequent to some minds. But where the conflict is more serious, where $Z_{1}$ and $Z_{2}$ both represent large and deep interest-systems, the device of tossing a coin, followed by the concentration of excitement in $Z_{1}$ (or in $Z_{2}$ ) fails altogether to furnish a satisfactory solution. In such a case we have to rely on reasoning to furnish new paths which shall enable the excitement from $A$ to drain into both $Z_{1}$ and $Z_{2}$.

Suppose, for example, that an old and well-established manufacturing business has of late been going down-hill, and recognises its need of a scientific director or manager, who shall introduce new appliances and processes, and bring back to the firm its old prosperity. Those now in control of the business are anxious to appoint the best possible man and, we may readily suppose, to pay him the least possible salary. Now, the man who will come for a relatively low salary is likely to possess relatively low qualifications. There is, therefore, a conflict in the mind of the present managing director between the desire to appoint a highly qualified man and the desire to pay him a low salary. The neural conditions of the conflict are represented in the diagram (Fig. II, p. I82) where $Z_{1}$ and $Z_{2}$ represent the opposing interest-systems, $Z_{1}$ corresponding to the purpose of getting the best man, and $Z_{2}$ to the purpose of saving the most money. Then the excitement of the direct paths $A_{1}$ (to $Z_{1}$ ) and $A_{2}$ (to $Z_{2}$ ) may correspond respectively to the thoughts $\left(\boldsymbol{A}_{1}\right)$ of paying a high salary to get the best man, and $\left(\boldsymbol{A}_{2}\right)$ of paying a low salary to save the most money. The two opposing purpose-neurograms (or systems of neurograms), $Z_{1}$ and $Z_{2}$, are therefore tending to drain the excitement (from $A$ ) in opposite directions. The complete solution of this conflict is effected by so modifying the neurographic connexions that the excitement from $A$ may drain into both $Z_{1}$ and $Z_{2}$. If, for example, I am able to reason out a connexion $\mathbb{Z}_{1} \boldsymbol{z}_{1} \ldots \boldsymbol{z}_{2} \boldsymbol{Z}_{2}$ between $\mathbb{Z}_{1}$ and $\mathbb{Z}_{2}$, I shall, as we have seen, be forming a neural path $Z_{1} z_{1} \ldots z_{2} Z_{2}$ by which the excitement may drain on from $Z_{1}$ to $Z_{2}$. The completion of this path will at once tend to make the excitement from $A$ proceed to $A_{1}$ and so through $Z_{1}$ to $Z_{2}$, instead of through $A_{2}$ to $Z_{2}$. This follows at once from an argument already $\dagger$ given, when we reflect that the new path $z_{1} \ldots z_{2}$ is of lower resistance than the reverse path $z_{2} \ldots z_{1}+$; and that the existence of conflict entitles us to assume that,

* Loc. cit. p. 495.

$\dagger$ See above, pp. 86 to 88 .

† This is William James' (loc. cil. Vol. II, p. 58I) law of forward conduction. (Cf. W. McDougall, Phy'siological Psycholngy, pp. 136, I37.) The resistance of $z_{2} \ldots z_{1}$ is doubtless lowered to some slight extent by the greater lowering of the resistance of the path $z_{1} \ldots z_{2}$. 
before the opening of the new path $Z_{1} z_{1} \ldots z_{2} Z_{2}$, the rival paths $A A_{1} Z_{1}$ and $A A_{2} Z_{2}$ offered equal resistance to the excitement in $A$. If therefore $Z_{1}$ can be connected to $Z_{2}$ by reasoning, the excitement from $A$ will drain through $A_{1}$ to both $Z_{1}$ and $Z_{2}$, the influences of $Z_{1}$ and $Z_{2}$ on the direction of the excitement having been harmonised by the newly formed neural connexions between them. The conflict will thus have been solved.

In our illustration, where $Z_{1}$ is the neurogram of the purpose to appoint the best man and $Z_{2}$ is the neurogram of the purpose to save as much money as possible, the reasoning process that connects $Z_{1}$ through $z_{1}$ and $z_{2}$ to $Z_{2}$ may take the form of reflecting that a first rate manager may so improve the manufactured article as to increase the demand for it, or its selling price, or both, and thus to increase the firm's receipts by far more than the amount of his salary; so that it will be cheaper $\left(Z_{2}\right)$ in the long run to pay a big salary $\left(A_{1}\right)$ and so to get a good man $\left(Z_{1}\right)$ than to offer a small salary $\left(A_{2}\right)$ and miss both the better man $\left(Z_{1}\right)$ and the big profit that means the greater saving $\left(Z_{2}\right)$.

Let us consider another case of bipolar conflict that will illustrate the differences between the three types of voluntary (willed) solution named in Table VI on p. I69: namely, solution by disintegration (Repression), solution by disintegration combined with integration (Displacement), and solution by integration (Conscious Control or Reasoning). Suppose that, knowing the constitution of the blue cobalt glass that is often used in lamps that mark the entrances to police stations, and knowing also certain optical properties of the constituents in relation to various monochromatic lights, we calculate that the cobalt glass will absorb yellow and green light much more than red light, and red light more than blue or violet light. Our calculation then tells us that white light (containing red, yellow, green, blue, and violet lights in the proportions in which they are contained in sunlight) when looked at through cobalt glass will appear blueviolet, the yellow, green, and red rays having been in large measure absorbed by the glass. Then suppose that, in order to test our calculated colour by experiment, we look at a gas flame through the glass. It appears red, not blue. We have therefore a conflict between the blue of theory and the red of practice. In our diagram (Fig. II on p. I82) $Z_{1}$ may correspond to blue and $Z_{2}$ to red. We want to believe both the reasoned deduction $\left(Z_{-1}\right)$ from well established facts; and we also want to believe the evidence $\left(Z_{\mathbb{F}_{2}}\right)$ of our senses. But the two are in 
opposition, and our desire to believe one conflicts with our desire to believe the other.

We may resolve this bipolar conflict in any one of the three ways of which we have just reminded ourselves. In the first place we may ignore the result of the experiment in which the light appeared red: we try to forget, and in due course succeed in forgetting, that we ever tried the experiment. We then repress $\boldsymbol{Z}_{2}$, disintegrating $Z_{\mathbf{2}}$ from the remainder of the system. Our conflict has then disappeared, for we rest assured that theory is correct in telling us that white light shining through cobalt glass appears blue. (The obvious dishonesty of this solution is probably* a particular case of a general ethical inferiority of this method-repression or disintegration-of resolving conflicts.)

In the second place we may resolve the conflict by disintegration combined with integration: in short, by displacement. I may say to myself-almost in the words of Dr Hart's patient: 'Green, that's green, that's blue...' $\dagger-$ 'Red, that's crimson, that's purplish, 'perhaps quite purple, or say blue.' In this way I voluntarily concentrate upon and extend any 'blue' element there may be in my $Z_{2}$ neurogram, meanwhile ignoring the 'red' elements, until, by a combination of integration with disintegration, I have displaced $Z_{2}$ so as to coincide with $Z_{1}$.

Finally we come to the third-the ideal+-method of solving a conflict: namely by integration. Integration in this case involves linking $Z_{1}$ to $Z_{2}$ by means of a new path; and the process by which the new path is made is, as we said $\S$, that of reasoning. This new path is made by observing that the red colour of cobalt glass seen by gas light is only a particular case of its BLUE-RED-green-yellow colour seen by sunlight; for gas light lacks nearly all the blue of sunlight, and its yellow and its green are largely absorbed by the glass, so that the transmitted portion of gas light must be almost pure red, a deduction which accords exactly with the result of experiment.

* Cf. Fluegel, loc. cit.

$\dagger$ B. Hart, loc. cit. p. xxo, quoted above (p. I 70) to illustrate the solution of a conflict by displacement.

$\ddagger$ See above, p. $\mathbf{I}_{75}$.

$\S$ See above, p. x79. 


\section{CHAPTER 10}

\section{SOME CHARACTERISTICS OF REASONING}

WE have now to examine more closely certain general characteristics of the reasoning process. We might employ any of our examplesthat of unipolar, or either of those of bipolar, conflict-to illustrate these characteristics. We shall, however, find it most convenient to use the first example*-that of a unipolar conflict-because in that case we noted details upon which it was unnecessary to remark again in either of the examples that followed.

We observe, to begin with, that (as we said when first $\uparrow$ using the word) reasoning involves making new association paths. Novelty'ability to deal with NOVEL data'-constituted, in William James' view, 'the technical differentia of reasoning.' $\ddagger$ Moreover, we are about to emphasise the fact that voluntary (willed) concentration of attention is part and parcel of all but the simplest, if not indeed of all, reasoning; and we have already§ quoted the conclusion, strongly suggested to Mr Burt by the results of his Oxford experiments on schoolboys, 'that...one feature or function of attentive consciousness ...[is] the power of readjustment to relatively novel situations by organising new psychophysical co-ordinations.'\| The new association paths made by reasoning are illustrated in our diagrams (Figs. Io and II).

Let us next recognise how important is the concentration of attention (or of neural excitement) during the reasoning process. When, in our example of the stuck metal lid, $\mathfrak{a}^{\prime}$ and $\mathfrak{a}^{\prime \prime}$ both came into my mind together, my reasoning would have ended there had I failed to concentrate on $\mathfrak{a}^{\prime}$ and to leave $\mathfrak{a}^{\prime \prime}$ out of account for the time being. I had, in fact, to concentrate on the idea that the lid was too small, without further considering, for the moment at least, the possibility that the lid might be stuck with candle wax. Thus, in reasoning the neural excitement is more strictly localised than in involuntary (common associative) thinking. 'The first thing,' says William James $\%$,

* See above, pp. 178 and $180, \mathbf{r} 8 \mathrm{r}$.

† See above, p. I79.

$\ddagger$ Loc. cit. Vol. II, p. 330, already quoted above, p. 179 .

$\S$ See above, pp. II6, II 7 .

II Loc. cit. p. I68. (Italics mine.) Cf. also the passage from $\mathrm{Mr}$ Burt quoted above on pp. II6, II 7 .

II Loc. cit. Vol. II, p. 34I. Cf. also p. 330: 'Reasoning may then be very well defined as the substitution of parts and their implications or consequences for wholes.' 
'is to have seen that every possible case of reasoning involves the extraction of a particular partial aspect [say $\mathfrak{a}^{\prime}$ ] of the phenomena thought about $[\mathfrak{A}]$, and that whilst Empirical Thought simply associates phenomena in their entirety, Reasoned Thought couples them by conscious use of this extract*' or abstracted essence. If, for example, in mathematical reasoning we mention 'force,' we mean $\dagger$ (that is, we think only of) force as defined by Newton: that which changes or tends to change a body's state of rest or of uniform motion in a straight line. No idea of force of character, or of a patent food, or of any of the multifarious meanings of the word in common speech, enters our consciousness. The neurogram excited is correspondingly limited. Or again, if, when reasoning about the physical properties of gold, we refer to its 'yellow' colour, no thought of the yellow press enters our minds; and the excited (portion of my) neurogram of 'yellow' is correspondingly limited. (But when we try to reason about 'education' or about 'character' we commonly fail similarly to restrict the excited systems of neurograms; and, in so far as we do so fail, our thinking partakes of the nature of day dreaming-common associa tive thinking-rather than of reasoning.) It is because voluntary concentration of excitement is characteristic of all reasoning, that reasoning is pre-eminently a process of voluntary (willed) thinking.

For another reason also, concentration is of extreme importance in the reasoning process. According to our second law of thought, the greater the concentration of excitement in any one of the linked neurograms-say, in $a^{\prime}$--that make up the new path from $A$ to $Z$ (in Fig. 10, p. 178 ), the greater, in general, will be the number of neural arcs into which the excitement diffuses. The greater therefore will be the number of neurograms $\left(b^{\prime}, b^{\prime \prime}, \ldots\right)$ from among which the Will may choose one $\left(b^{\prime}\right)$ in which to concentrate excitement next. In other

* William James gives another reason why particular partial aspects, or essences, of concrete facts are used in reasoning instead of the complete facts from which they are extracted. Thus he asks ' Why are the couplings, consequences, and implications of extracts more evident and obvious than those of entire phenomena?' And he answers this question by pointing out firstly that 'the extracted characters are more general than the concretes, and the connections they may have are, therefore, more familiar to us, having been more often met in our experience.' He continues 'The other reason why the relations of the extracted characters [thought of as a'] are so evident is that their properties are so few, compared with the properties of the whole [a, for example], from which we derived them. In every concrete total the characters and their consequences are so inexhaustibly numerous that we may lose our way among them before noticing the particular consequence it behooves us to draw. But, if we are lucky enough to single out the proper character, we take in, as it were, by a single glance all its possible consequences.' (Loc. cit. Vol. II, pp. 34I, 342.)

$\dagger$ See above, p. 45. 
words, the greater a man's power of concentration, the greater ceteris paribus will be his choice of new* paths for his reasoned thought and the greater therefore will be his chance of finding a solution for his conflicts, a way out of unprecedented situations $\uparrow$.

Diffusion, as we said, follows concentration. The variety of different ideas that may occur to me during the diffusion stage (from $a^{\prime}$, say) does not depend only upon my Will, my power of concentrating attention on the matter in hand and so of multiplying the excitement as to make it flow into every connected neurogram and into some other neurograms not previously connected with $a^{\prime}$. It depends also upon a general neural feature, which we have defined and called Clevernesst, and which we may here loosely describe as a capacity for forming new associations (due, perhaps, to innately low thresholds between brain neurones). And it depends upon my relevant knowledge: that is, upon the number of my neurograms already connected with $a^{\prime}$. The greater my Will, my Cleverness, and my knowledge of the matter in hand, the greater will be the number of associated thought-activities that may appear on the fringe of my consciousness as I concentrate my attention on $\mathfrak{a}^{\prime}$; or, in other words, the greater will be the number of the neurograms into which excitement diffuses from the neurogram $a^{\prime}$ in which it has been concentrated. My chance of successfully completing the reasoning process by finding a new path $A a^{\prime} b^{\prime} \ldots z^{\prime} Z$ from $A$ to $Z$ therefore depends upon my Will, my Cleverness, and my relevant knowledge.

* On p. r 2 r above we have quoted, from Dr Webb's table of corrected coefficients, $r_{o g}=\cdot 47$ as the coefficient of correlation obtained in his investigation between Originality and ' $g$.' The corresponding coefficient between Originality and Cleverness is given in our Table III, p. 127 above, as $\cdot 88$. We now see why ' $g$ ' should enter so considerably into the constitution of Originality, so that Originality does not depend on Cleverness alone. If, as we have seen reason to believe, ' $g$ ' is educable, Originality can be increased by the development of ' $g$.'

$\dagger$ Cf. above, p. 135 .

$\ddagger$ See above, p. 123. 


\section{CHAPTER 11}

\section{THE ORGANISATION OF THOUGHT}

\section{$\S \mathrm{I}$. The World of Experience.}

Now my knowledge employed in reasoning about the 'real' world, the world of experience, must itself fit experience if the new path, formed by reasoning, is to lead to a solution of the conflict. That is to say-if we follow William James in his lectures on 'Pragmatism,' or Hans Driesch in his 'Gifford lectures,'* and regard that which fits experience as being, so far, true-the steps in our reasoning process must each be true. This characteristic of the reasoning process we have now to examine at some little length.

Let us first illustrate our statement that the steps in successful reasoning about the world of experience must themselves fit experience. If, in our example of the stuck metal lid $\dagger$, the organisation of my neurograms-the neural correlative of my knowledge-had not corresponded with that of the 'real' world, I should have failed to unscrew the lid. Thus, if I had wrongly supposed that the metal of the lid contracted on being heated (whereas it 'really' contracted on being cooled), an idea $\mathfrak{r} \mathfrak{l}$ of the lid being too hot (instead of the idea $\mathfrak{b}^{\prime}$ of its being too cold) would have followed $\mathfrak{a}^{\prime}$ into the focus of my consciousness; and the neurogram $W$ would have diverted the excitement from the true path $A a^{\prime} b^{\prime} c^{\prime} \ldots z^{\prime} Z$ by which $Z$ could be reached. In fact, the failure of my neurograms (where $W$ instead of $b^{\prime}$ was connected with $a^{\prime}$ ) to fit the world of experience (where the object of my thought, $\mathfrak{b}^{\prime}$, was connected with the object of my thought, $\mathfrak{a}^{\prime}$ ) would have prevented me from solving the problem or resolving the conflict. So we repeat that, for successful reasoning about the 'real' world, each link in the chain of neurograms must correspond to a 'real' fact.

There are such facts in the world of our experience. Suppose for example that $E_{p}$ and $E_{q}$ are two successive elements in a chain of neurograms by which, in reasoning about the 'real' world, $A$ has been connected with $Z$. We write $E_{p}$ and $E_{q}$-instead of capital letters (e.g. $P, Q$ ) which we commonly employ to denote neurograms of complete concrete things-in order to remind ourselves of the

* Vol. I, p. 7 . $\dagger$ See above, pp. I78, I80, I8I. 
strict localisation of neural excitement during reasoning: $E_{p}$ and $E_{q}$ are neurograms of 'particular partial aspects' $\left(\mathrm{E}_{p}\right.$ and $\mathrm{E}_{q}$, say) or 'essences'-extracted abstract characters-such as are always used in reasoning*.

It will be convenient to extend the system of symbols already $\dagger$ described by using Roman letters to denote those facts $\ddagger$ or essences to which correspond neurograms denoted by the same letters in Italic type. Thus, if A represents a fact or essence, the thought-activity, or idea of $\mathrm{A}$, which enters my field of consciousness when I think of $A$ is represented by $\mathfrak{A}$, and the neurogram whose excitement, in whole or in part, accompanies my every idea ( $\mathfrak{A})$ of that fact (A) is represented by $A$.

By saying that the link between $E_{p}$ and $E_{q}$ must correspond to a 'real' fact, we mean that, in the world of experience, the essences $\mathrm{E}_{p}$ and $\mathrm{E}_{q}$ 'involve or imply each other. One of them is a sign to us that the other will be found. They hunt in couples, as it were.' $\S$ And the world in which we live does present a number of abstract (general) characters which affect such constant habits of mutual concomitance, or repugnance. In our world 'such a proposition as that $\mathrm{E}_{p}$ is $\mathrm{E}_{q}$, or includes $\mathrm{E}_{q}$, or precedes or accompanies $\mathrm{E}_{q}$, if it prove to be true in one instance, may very likely be true in every other instance which we meet. This is, in fact, a world in which general laws obtain, in which universal propositions are true, and in which reasoning is therefore possible.' $\|$

Now the general characters whose habits of mutual concomitance are best established are those which natural science has defined. And among the expressions of such habits, whether in statements of particular facts (as that ' $\mathrm{E}_{p}$ is $\mathrm{E}_{q}$ ') or of general laws (as that 'what-

* See above, p. 187 .

† On p. 70 above.

$\ddagger$ See below, p. I9I.

$\S$ W. James, loc. cit. Vol. II, p. 337. Cf. also Poincaré (loc. cit. p. I7): 'The most interesting facts are those which can be used several times, those which have a chance of recurring. We have been fortunate enough to be born in a world where there are such facts. Suppose that instead of eighty chemical elements we had eighty millions, and that they were not some common and others rare, but uniformly distributed. Then each time we picked up a new pebble there would be a strong probability that it was composed of some unknown substance. Nothing that we knew of other pebbles would tell us anything about it. Before each new object we should be like a new-born child; like him we could but obey our caprices or our necessities. In such a world there would be no science, perhaps thought and even life would be impossible, since evolution could not have developed the instincts of self-preservation. Providentially it is not so; but this blessing, like all those to which we are accustomed, is not appreciated at its true value. The biologist would be equally embarrassed if there were only individuals and no species, and if heredity did not make children resemble their parents.'

II Cf. W. James, Vol. II, p. 337. 
ever includes $\mathrm{E}_{p}$ includes $\mathrm{E}_{q}{ }^{\prime}$ ), those which best fit experience, and are therefore most true, are those which natural science has taught us. These co-called 'scientific facts' and 'laws of Nature' summarise human experience of our world in carefully defined terms. In short, those statements of fact or law that are most true are also most precisely defined: they are statements of definite facts. They do not deal directly with things as wholes, with concrete things, but with extracted characters, essences, or abstracts. The concrete things of our experience are far too complex, and our ordinary (non-scientific) thoughts of them are far too ill-defined, for us to be able to make true general statements-true 'universal propositions'-about their relations to each other. So we cannot reason about them. Our neurographic imagery will help us here again: for we have already* seen how complex and ill-defined is the neurogram of any familiar concrete thing, and how necessary it is for successful reasoning that we should concentrate excitement in some narrowly defined portion of any such wide system of inter-connected arcs that may be excited during the reasoning process.

We have just been using several different words to describe the world of our experience and its subdivisions: general characters, facts, laws, definite facts, concrete things, essences and abstracts. Some simplification and definition of our terminology is necessary before our enquiry can proceed.

We shall therefore in future use the word thing to denote any portion of the real world, assuming for the purpose of this definition that the world of which we have experience is a reality independent of our sensations or other thought-activities.

We have next to distinguish between a thing in itself-a part (or perhaps the whole) of the real world--and things as we believe them to be. We shall accordingly use the word fact to denote a thing as it is generally assumed and defined to be, after verification that the 'fact' fits all available previous experience of the thing.

The word essence we shall use to denote such a particular partial aspect of a fact as can only be thought of as a whole or not at all: an 'all or none' part of the world of experience $\uparrow$. To an essence there corresponds, in the brain of anyone to whom the essence is known, a neurogram-element, which we define as a system of nervous

* See above, p. 187

$\dagger$ The meaning of 'essence' thus defined will become clearer in the sequel. Meanwhile we may take as an illustration the atom of hydrogen (or of any other element) as first thought of by Dalton and his followers before the disruption and the structure of atoms began to be discussed by modern physicists. 
arcs all of which are excited when the corresponding essence is thought of.

Our reasons for making these definitions and distinctions will appear as our enquiry proceeds. We may however here observe that our object in distinguishing things in the real world from facts in the world of our experience is to avoid assuming the identity of things in themselves with the facts that we know. The identity may exist, but it is unnecessary for our purpose to assume it. According to our definition of a fact, the world of things behaves exactly as if it were identical with the world of facts: the facts, as we said, fit experience and are therefore 'true.' Indeed, everything has happened as if our facts were indistinguishable from the real things they represent. And if some new experience of things does not fit the then known facts, the new experience will alter the old facts*, and the new facts that take their place will again fit all available previous experience.

For the present we shall concern ourselves with facts rather than with the things-in-themselves which these facts represent. And our word 'fact' may denote any portion of the world of experience, however complex or however simple $\dagger$, but always including whatever invariably accompanies it $\ddagger$. So the whole world of experience is a single fact; and so also is every subdivision of it down to the simple fact, which consists of a single essence such as we have just defined, together with whatever other essences invariably accompany it in the world of experience.

Moreover, a fact, as we have defined it, is always definite: it is a strictly limited portion of the world of experience. We shall have more to say on this point later on $\S$. Meanwhile we note that science concerns itself with such definite portions of the world of experience. Facts, as we have defined them, are indeed the material with which science works. If examples are wanted of the manner in which science concerns itself with (definite) facts, and preferably with simple facts or even with essences themselves, rather than with whole concrete

* As, for example, Newton's law of gravitation has been altered by the confirmation of Professor Einstein's prediction concerning the bending of the path of light in passing near the sun.

$t$ It is true that the word 'fact' is often used to denote the relationship between two simpler portions of the real world as when we say 'it is a fact that A is B.' Used in this sense the word fact denotes something in which at least two elements are distinguishable: so that the neurogram in such a fact would be composed of at least two distinguishable elements. But in common parlance the word fact is also used to denote a thing which is not necessarily composite, or in which two or more elements are not necessarily distinguishable. Thus G. K. Chesterton speaks of 'such facts as Death or Daybreak.' (The Nation, February, I918.)

$\ddagger$ See below, p. 213 . $\S$ See below, p. 214 . 
things, they are easily found. The point and line, for example, about which Euclid reasoned are not the point and line as we know them. We have no direct experience of a point without magnitude, or of a line without breadth. But, for the purpose of arriving at true universal propositions through geometrical reasoning, we extract the character of position from the point as we know it, and the character of length from the line as we know it; we define the point or line as being, for all geometrical purposes, equivalent to these simple general characters; and we deal only with the abstracts thus defined. In the same way, the gold (or other substance) whose properties the chemist investigates and about which he reasons, is not the gold which the plain man knows - the measure (as he is too apt to think) both of men's work and of God's gifts-but its extracted character, the element Au, characterised no less by its chemical properties, or by the pink colour of gold vapour or of gold ruby glass or the green translucence of gold leaf, than by the yellow sheen of the minted sovereign.

But the facts of which we have been speaking, although definite and far more simple than the blurred and hazy impressions which are all that men retain of most of their experiences of the real world, may still possess a considerable degree of complexity. The chemist's gold for example, although it is far simpler than that of common speech, possesses multifarious chemical, physical, mechanical and other properties. For reasoning purposes it is necessary, as we said*, to abstract from the impressions we retain of our experience, and to define $f$, not merely a fact, which though definite may be complex, but some essences-particular partial aspects-of the fact $\ddagger$. In this process of abstraction, the impression with which we start is, we suppose, the result of a direct experience of some complex, concrete thing, part and parcel of the real world itself. When we abstract from it some particular partial aspect or essence, we are not of course altering the thing itself, but only our thought about the thing. For example, when we abstract from our impression of the complex concrete thing which we call the 'point of a pin' the essence of its position in space, the pin point still remains an unaltered part of the universe. The process of abstraction may therefore seem to be taking us away from the real world. We may seem to be describing as essences, and therefore as partial aspects of facts, what does not

* On p. 187 above.

$\dagger$ This definition will sometimes involve the invention of a new word which, as Poincaré says (lic. cit. p. 28), will often be sufficient to bring out the newly discovered relation and the word will be creative.

$\ddagger$ Cf. p. 214 below.

G. E. 
belong to the real world as we experience it. But that is not our intention: we only describe as facts or essences what does fit experience. In other words, it is only those abstracted portions of the world of experience which, as we reason about them, yield verifiable conclusions -or, in short, those which fit experience-that we shall speak of as essences or facts.

\section{$\S 2$. The Endarchy of Science.}

The definite facts with which science is concerned are no mere conglomeration of disconnected items, all equally significant or equally insignificant. On the contrary, each has its proper place in an orderly system of relationships. 'Scientists believe,' says Poincaré, 'that there is a hierarchy* of facts.' $\dagger$ Otherwise-that is if all facts and all essences were equally significant, important, or valuable-we should know a continually decreasing proportion of the real world; for, as Poincaré points out, 'However great our activity, facts outstrip us, and we can never overtake them; while the scientist is discovering one fact, millions and millions are produced in every cubic inch of his body.' $\ddagger$ But if, as we said, the facts and essences with which science is concerned are not all equally important, we may select and get to know the more important of them, and so acquire true knowledge of an increasingly valuable proportion of the real world, even though relatively unimportant things are gaining on us all the while.

If now we enquire what exactly we mean by one essence $E_{p}$ being more significant, important, or valuable than another essence $E_{q}$ to any one of us personally, we shall find that the neurogram of the first is deeper than the neurogram of the second: so that, during involuntary thinking, $E_{p}$ is more likely (other things being equal) to be excited than $E_{q}$. But what has made $E_{p}$ deeper than $E_{q}$ ? It may have been that $E_{p}$ has been more frequently excited than $E_{q}$ because, in the world of our personal experience, $\mathrm{E}_{p}$ is of commoner occurrence than $\mathrm{E}_{q}$; or it may have been that $E_{p}$ is more closely connected than $E_{q}$ with affective-conative elements. But, whatever it be, the result is that $\mathbb{E}_{p}$ recurs more frequently than $\mathbb{E}_{q}$. In short, the most important facts for each of us are those of which we think most frequently.

* Or, as we have said (p. 163), 'endarchy.' See below, p. 195.

$\dagger$ Loc. cit. p. 16.

¿ Loc. cit. p. 16. For Poincaré's purpose it was not necessary to distinguish, as we have distinguished, between a fact and the thing it represents. In our terminology it is things that are being produced in such numbers that, however great our activity, they outstrip the facts by which we strive to represent them. 
Now the order of importance of most essences which any science has abstracted, named, and defined, is much the same for every student of that science. The same is true of many facts that comprise large numbers of essences. For example, every student of mechanics would regard Newton's laws* of motion and his laws* of gravitational attraction as being more important than Kepler's laws* of planetary motion; for the former are of far more frequent use-they recur more frequently-than the latter; and indeed the latter may be deduced from the former, although historically Kepler's laws preceded those of Newton. Thus the facts of natural science have much the same order of importance to different persons. We conclude that there is an order of importance belonging to those facts themselves-or at least to the essences $\uparrow$ into which they may be analysed-and independent of the order of their importance to any particular person.

But these essences have to be altered from time to time to fit new experience. Let us however imagine a time when the whole real world is known to science, so that every thing is completely represented by a fact. Let us call the facts of that day complete facts, and essences as they then exist fact-elements. Complete facts and factelements then fit all experience, and not merely experience hitherto available, as is the case with the (incomplete) facts and essences previously defined. The order of importance that we have just seen to belong at any time to the essences then known will alter to fit new experience until, with the arrival of the distant age that we have imagined, it assumes a final form: an order of importance belonging to fact-elements. This ultimate order is implied by Poincaré when he speaks of scientists believing that there is a hierarchy of facts $\$$.

Now we have already§ used Poincaré's word 'hierarchy' in connexion with a series of relations that may be satisfied by the correlations between mental qualities. We shall therefore replace Poincaré's word by our word 'endarchy.' $\|$ That the organisation of thought which Poincaré describes as 'a hierarchy of facts' has the form ascribed to an endarchy by our definition will appear as we proceed. Poincaré's hierarchy of facts we shall therefore describe as 'the endarchy of science,' or sometimes as 'the impersonal endarchy.' We may for the moment define it as consisting of the fact-elements

* All these laws were facts so long as they fitted experience (see p. 192). That they are not, strictly speaking, facts to-day need not impair their usefulness for the illustration in the text.

$\dagger$ See P. 217 below for a definition of the 'value' of an essence.

$\$$ See above, p. 194.

$\S$ On p. I04 above. See also Appendix B, below. \| See above, p. 163. 
of the world of experience arranged in the order of their intrinsic importance. We thus distinguish it from all the various personal endarchies* with which we shall also be concerned. Reserving our definition of these personal endarchies until later, it is sufficient to state here, briefly and vaguely, that they consist of the essences of the world of experience arranged in the order of the importance which they appear to us individually to possess; or, what amounts to the same thing, the order of the depth of the corresponding neurogramelements in our respective nervous systems.

But these definitions of the endarchy of science and of personal endarchies are alike inadequate. The endarchy of science is something more than a mass of disconnected fact-elements graded according to their relative importance. It is rather an organised body of connected facts. It is indeed no less than the world of experience interpreted by science: 'the neat, trim, tidy, exact world which is the goal of scientific thought.' $\dagger$ It is a mode of conceiving our world, a mode which fits experience-enabling future events to be accurately foretold $\ddagger$-and a mode which is therefore true $\S$. But we can no more be sure that the endarchy of science is true absolutely-that the endarchical organisation we are about to describe belongs to absolute things in themselves, as well as to the facts by which science represents them-than we can be sure of absolute truth in any other connexion.

The goal of scientific thought has not yet been reached. The endarchy of science is still very far from being complete\|. But several, more or less separate, partial endarchies-or, as we shall later find reason to call them, 'subject' endarchies-have been developed by particular sciences or branches of science; and these partial endarchies from time to time become linked together, as when Rutherford and Soddy shewed that the chemists' atom had a structure for the physicists to investigate, or when Moseley went

* See below, chapter I2, especially pp. 23 I et seq.

$\dagger$ A. N. Whitehead, The Organisation of Thought (Presidential Address to Section A, British Association, Newcastle, I9I6), p. I Io of reprint.

$\ddagger$ E.g. the endarchy of science is even now sufficiently complete, in one part, to enable astronomical events to be foretold two years ahead in the Nautical Almanac.

$\S$ See above, p. 189 .

II In what follows we shall, however, have occasion to speak of the complete endarchy which is the goal of scientific thought; and, without committing ourselves to the view that the endarchy exists before science has built it up and organised it, we may speak of this development of the endarchy of science as its 'discovery.' Of course, it may exist before and so be truly 'discovered.' Kepler thought so when, after having discovered the laws which bear his name, he prayed: 'We think Thy thoughts after Thee, O God.' But, compare W. James: 'classification and conception are purely teleological weapons of the mind.' (Loc. cit. Vol. II, p. 335.) 
on to prove that the earlier empirical classification of chemical elements depended upon the physical constitutions of their respective atoms. And so we conceive of the complete endarchy of science, the goal of scientific thought, as a single whole built up by uniting the various partial endarchies which particular sciences aim at developing. These particular sciences are not confined to branches of natural science. The discovery of partial endarchies-portions of the complete endarchy-is no less the object of history or of theology than it is of physics, chemistry, or biology.

We have already* remarked that the world of experience does not stand still while we are getting to know it and organising our thought about it. It follows that to-day's world of present and past experience is not the same as the neat, trim, tidy, exact world which will form the complete endarchy of science when the goal of scientific thought has been reached. But the latter includes the former; so that, as we organise our thought about the world of experience as we may now know it, we are approaching the goal of scientific thought and building up the organised system of knowledge which, when complete, will be the endarchy of science.

\section{§ 3. Growth of the Endarchy of Science.}

What we know of the partial endarchies can teach us something of the origin and nature of the complete endarchy of science which is gradually built up into a single organisation as scientific thought advances towards its goal.

The first stage in this advance has already $\dagger$ been indicated. It consists in abstracting, from our blurred and hazy impressions of the world of experience, some clearly defined particular partial aspects: the essences that we have already defined. Each abstracted essence is more general than any one of the impressions from which it is abstracted. For example, when we abstract the' essence 'carnivor' from our impression of a large number of dogs and cats and other flesh-eating animals, the essence carnivor is more general-in that it occurs more frequently in the world of experience-than any one of the dogs, cats, or other animals in question $\ddagger$. The abstraction is effected by thinking of a number of impressions (which may have been obtained by experiment for this very purpose) all of which

* See above, p. I94.

$\dagger$ See above, pp. 187 and 193.

$\ddagger$ Cf. W. James: 'the extracted characters are more general than the concretes.' (Loc. cit. Vol. II, p. 342.) 
contain the same particular partial aspect or essence combined with different (pro hac vice) non essential elements*.

This first stage may be regarded as consisting of analysis pure and simple. But the next stage begins the synthesis of facts by means of the further analysis of (impressions of) experience. In the first stage, a number of separate essences are obtained; and, until this stage has been reached, it is impossible to think of any of these essences except as an indistinguishable part of a much larger total experience. In the second stage, a new (second-stage) essence is abstracted from experience and directly connected - in the intimateand permanent way we are about to describe-with two or more of the essences abstracted in the first stage. Thus the essences abstracted in the second stage of the process connect groups of those abstracted in the first stage.

Let us explain that the connexion between the first and second stage essences is no mere fortuitous association in space or time. The connexion of which we are here speaking is something intimate and permanent. We may make this clearer by describing the second stage in a littlemore detail. Suppose, for example, that, at the very beginnings of language, men's experience had made them familiar with trees of different kinds and with wooden objects of different shapes and uses; and suppose that the first stage of scientific thought about these experiences had been reached, by the separation or extraction of ideas of the several trees and other objects from the total experiences of which a thought of one of the trees or other objects only formed part, and then by further extracting and naming some single essence in the fact of each of these objects as then thought of. And then suppose that the second stage of scientific thought about the experiences in question was reached by the abstraction for the first time of the essence 'wood' from all the pre-existing mass of experience of trees and other wooden objects. The new essence is a particular partial aspect of each of the facts; so it unites all these facts and, at the same time, it connects what were previously only known as separate essences. Or, symbolically, suppose that, in the first stage, a group of independent essences, $\mathrm{E}_{1}, \mathrm{E}_{2}, \ldots, \mathrm{E}_{n}$, has been abstracted and that,

* Cf. W. James' law that 'in order to be segregated [or abstracted] an experience must be repeated with varying concomitants.' (Loc. cit. Vol. II, P. 357.) Cf. also W. James, loc. cit. Vol. I, p. 506.

Or again: 'What does the scientific man do who searches for the reason or law embedded in a phenomenon? He deliberately accumulates all the instances he can find which have any analogy to that phenomenon; and, by simultaneously filling his mind with them all, he frequently succeeds in detaching from the collection the peculiarity which he was unable to formulate in one alone....' (W. James, loc. cit. Vol. II, p. 346.) 
when all the available instances of all these essences are accumulated and surveyed, a new essence, E,-so intimately connected with each of them that, whenever any one of them occurs in the world of experience, it (E) occurs too-is detached from the collection. Then the connexion of the second stage essence, $E$, to each of the first stage essences, $\mathrm{E}_{1}, \mathrm{E}_{2}, \ldots, \mathrm{E}_{n}$, is of the kind we have in mind. In all the available experience, none of the 'derived' essences $E_{1}, E_{2}, \ldots$, or $E_{n}$ occurs without the essence $E$. Whenever any of the essences $E_{1}, E_{2}, \ldots, E_{n}$ appears, it is a sign that the fact, $\mathrm{E}_{1} \mathrm{E}, \mathrm{E}_{2} \mathrm{E}, \ldots$, or $\mathrm{E}_{n} \mathrm{E}$, is present. The second stage essence, $E$, is common to all these facts and so unites* them; it is more general than any of them; it recurs more frequently in the world of experience, and is therefore more important $\dagger$, not only in the personal endarchies of individual men of science, but also in the endarchy of science itself; and, since it is part and only part of each of the facts in question, it is also more simple than they are.

The second stage, therefore, of progress towards the goal of scientific thought consists in abstracting from the complex impressions of experience, and in naming $\ddagger$, new essences which connect together groups of those abstracted in the first stage and which are more important than they are in the (incomplete§) endarchy of science.

* Cf. Poincaré: 'Mathematics is the art of giving the same name to different things' (loc. cit. p. 34 ), and so uniting them.

$\dagger$ Cf. Poincaré: "If a new result is to have any value [or, as we have said in the text, "importance"], it must unite elements long since known, but till then scattered and seemingly foreign to each other, and suddenly introduce order where the appearance of disorder reigned.' (Loc. cit. p. 30.)

If we wish to measure the importance or 'value' of a fact-element in the endarchy of science, we may define it as the sum of the values of the elements which are directly 'derived' (see p. 203) from that fact-element. It is easy to see that this definition accords with our previous statement that the more important (or, as we may now add, the higher the value of) a fact in a personal endarchy, the deeper will be its corresponding neurogram. For the man of science, who has abstracted some essence (or 'reason' or 'law') underlying and uniting a number of previously disconnected phenomena, will in future tend to think of that essence whenever he thinks of any one of those phenomena, and the same will be true of any other scientific thinkers who accept his views. In each of their brains, therefore, the neurogram of the abstracted essence will be deeper than the neurogram of the remainders of the entire phenomena from which the essence has been abstracted. Or, if we imagine a being in whose mind the complete endarchy of science is organised, and who yet possesses a brain on the human model, it would be true of him that the deeper his neurogram of a fact-element the more important (valuable) that fact-element would be. (See below, p. 208.)

$\ddagger$ Cf. Poincaré: "The invention of a new word will often be sufficient to bring out the relation [between facts previously regarded as independent], and the word will be creative. The history of science furnishes us with a host of examples that are familiar to all.' (Loc. cit. p. 28.)

$\S$ We speak here of the incomplete endarchy of science to distinguish it from the complete endarchy which we shall speak of simply as the endarchy of science. The incomplete endarchy of science is at any time an intermediate stage then 
At the end of the second stage, the endarchy that has begun to be formed may be represented by the following diagram (Fig. I2),

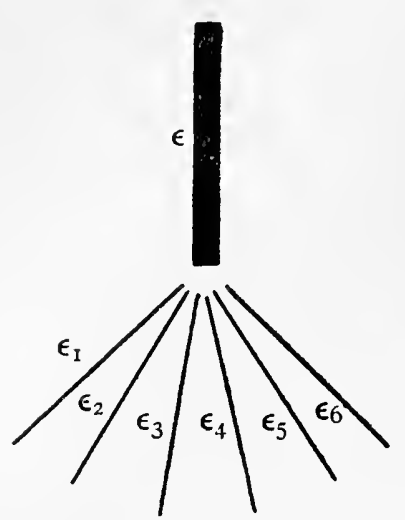

Fig. I2. in which the lower lines represent the essences abstracted in the first stage, the upper line the new essence abstracted in the second stage, while each of the lower lines together with the upper line represent* one of the group of facts which the abstraction of this new essence united.

The third stage in the formation of the endarchy is like the second. It again consists in abstracting an essence which unites some new fact or group of facts to the endarchy that has begun to be formed in the second stage. The essence abstracted in the third stage may be connected-in the sense in which we have just $\uparrow$ been using the wordeither to the essence abstracted in the second stage (represented by the upper line in Fig. I2) or to one of the remaining essences (represented by one of the lower lines in that figure). These two cases are represented in the following diagrams (Figs. I3, I4 and I5 respectively).

The first case may be illustrated by supposing that, in the first stage, the essences canis (represented by the line marked $\epsilon_{11}$ in Fig. I3) and felis $\left(\epsilon_{12}\right)$ have been abstracted from vague general impressions that are denoted in common speech by the words dog and cat respectively, while at the same time the essences equus $\left(\epsilon_{21}\right)$ and bos $\left(\epsilon_{22}\right)$ have been abstracted from impressions of horses and oxen. In the second stage, the common essence carnivor $\left(\epsilon_{1}\right)$, connected to both the essences canis and felis, is further abstracted from impressions of dogs and cats, while the common essence ungulate $\left(\epsilon_{2}\right)$, connected to both equus and bos, is further abstracted from impressions of horses and oxen. In the third stage, the common essence mammal $(\epsilon)$

reached in the development of the complete endarchy of science. The incomplete endarchy is related to the complete endarchy as essences are to fact-elements (see p. I95 above).

* But only so far as this diagram (Fig. I2) goes. Each fact in the group includes not only the essences represented in the diagram by $\epsilon$ and by one of the lower essences, $\epsilon_{1}, \epsilon_{2}, \ldots$, but also every other essence that invariably accompanies these in the world of experience. We shall see later (on pp. 203, 213) that the other essences of the fact that is represented, so far as this diagram (Fig. 12) is concerned, by the two line-elements $\epsilon$ and $\epsilon_{n}$ might be represented by a string of end-on line-elements extending from the top of $\epsilon$ up to and including the central line-elements of the diagram (Fig. I6) on p. 204.

+ From p. 198 on. 
is abstracted again from impressions of dogs, cats, horses and oxen and connects carnivors with ungulates.

As an example of the second case, represented at the close of the third stage in Fig. I4, suppose that, at the close of the second stage when the $\operatorname{dogs}\left(\epsilon \epsilon_{1}\right)$ and cats $\left(\epsilon \epsilon_{2}\right)$ were first united by the abstraction of the essence carnivor $(\epsilon)$ connected both to the essence canis $\left(\epsilon_{1}\right)$ of dog and to the essence felis $\left(\epsilon_{2}\right)$ of cat, only one kind of member of the cat family-the domestic cat, for example-was known; and that afterwards a new kind of cat--the lion, suppose-was discovered. At the end of the second stage the partial endarchy might be repre-

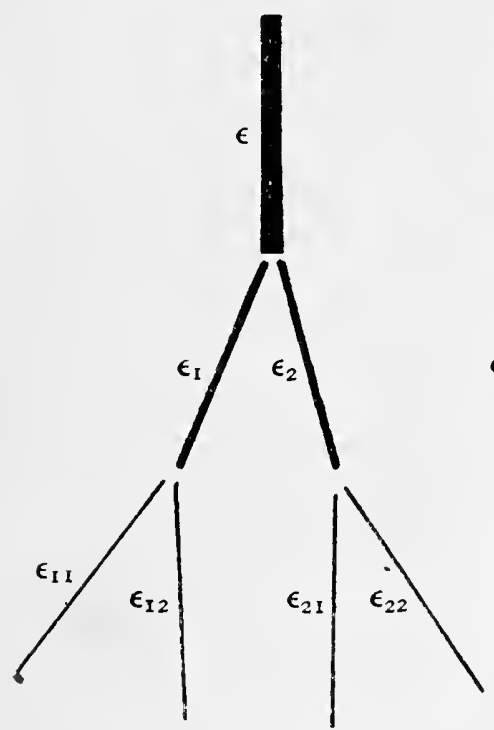

Fig. ${ }_{3}$.

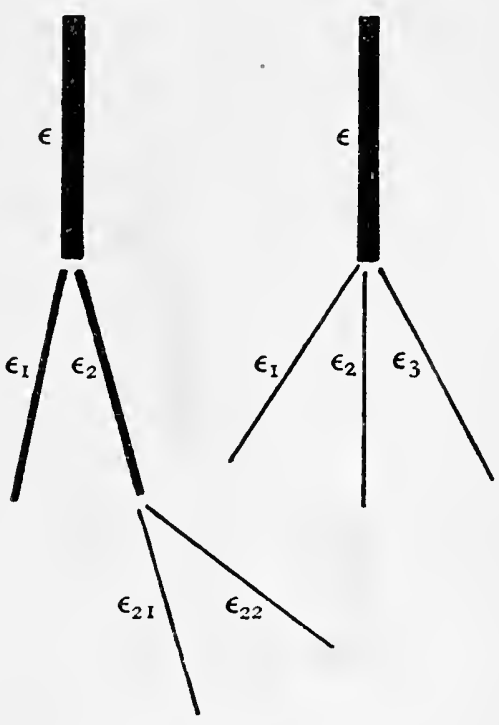

Fig. 15 .

sented by the three line-elements $\epsilon, \epsilon_{1}$ and $\epsilon_{2}$ arranged as in Fig. I4. In the third stage, the lion group is added to the nascent endarchy by abstracting from impressions of cats and lions a new essence, thereafter named felis, and represented in Fig. I4 by the line $\epsilon_{2}$, and connected both to the essence $\left(\epsilon_{21}\right)$ of domestic cat and to the essence $\left(\epsilon_{22}\right)$ of lion. The fitting of the lion group into the endarchy involves a change in nomenclature. The word felis, which we have supposed to have been used before the discovery of lions to represent the (pro hac vice) essence of domestic cats, for the future* represents an essence $\left(\epsilon_{2}\right)$ common to domestic cats and to lions; while the essence

\footnotetext{
* I.e. at the close of the third stage represented in Fig. I4.
} 
of domestic cats is at the same time re-named felis catus and is represented by $\epsilon_{21}$, and the essence of lion, felis leo, is represented by $\epsilon_{22}$.

A third case, represented in Fig. 15, is perhaps better described as a continuation of the second stage than as part of the third stage in the formation of a partial endarchy. For in this third case no new essence is abstracted in order to add the essence of some new impression to the nascent endarchy. For example, the discovery of a new variety (represented in Fig. I5 by $\epsilon_{3}$ ) of an existing species $(\epsilon)$, or the discovery of a new species $\left(\epsilon_{3}\right)$ of an existing genus $(\epsilon)$, requires no new abstraction in order to fit the essence of the new experience into the existing endarchy.

Subsequent stages in the progress towards the goal of scientific thought resemble the second and third stages that we have just described. At every stage new essences are abstracted from (impressions of) experience, and these new essences connect previously existing essences to partial endarchies, or partial endarchies to one another. So the formation of the endarchy of science proceeds until every essence is connected to every other, while every fact is united to every other by the possession of common essences. When at last the goal of scientific thought is reached, the whole knowable universe is thus organised and united.

In Figs. I3 and I4 we have represented two different ways in which the third stage in the formation of the endarchy of science may be accomplished. In Fig. I3 the endarchy is represented as growing upwards or inwards; and in Fig. I4 as growing downwards or outwards. Every subsequent stage may be covered in either' of these two directions. But we have to notice that the former, and not the latter, is the normal development of the endarchy. The reason we shall discuss in more detail later. We may here, however, observe that it is the innermost-or highest-essences in each partial endarchy that are most important and are most likely to receive attention*. For this reason alone they are most likely to become connected through new subsequently abstracted essences that are connected to each of them.

As this normal growth of the endarchy of science takes place, suppose that an essence, $\mathrm{E}_{k_{1} k_{2} \ldots k_{n-1} k_{n}}$, is abstracted in the first stage; that in the second stage a new essence, $E_{k_{1} k_{2} \ldots k_{n-1}}$, is connected to the former in the manner discussed above $\dagger$ so that whenever $\mathrm{E}_{k_{1} k_{2} \ldots k_{n}}$ occurs in the world of experience $\mathrm{E}_{k_{1} k_{2} \ldots k_{n-1}}$ occurs with it; and that,

* See below, $\S 7$ on pp. 216 et seq.

$\dagger$ On pp. 198, 199. 
in the third stage, there is abstracted a new essence, $\mathrm{E}_{k_{1} k_{2} \ldots k_{n-2}}$, connected to $\mathrm{E}_{k_{1} k_{2} \ldots k_{n-1}}$ just as $\mathrm{E}_{k_{1} k_{2} \cdots k_{n-1}}$ is connected to $\mathrm{E}_{k_{1} k_{2} \cdots k_{n}}$; and so on, until at last the central essence, $E$, of the whole endarchy of science is finally abstracted. Then, whenever $E_{k_{1} k_{2} \ldots k_{n}}$ occurs in the world of experience, $\mathrm{E}_{k_{1} k_{2} \ldots k_{n-1}}, \mathrm{E}_{k_{1} k_{2} \cdots k_{n-2}}, \ldots, \mathrm{E}_{k_{1} k_{2}}, \mathrm{E}_{k_{1}}$, and $\mathrm{E}$ occur with it. In other words, wherever the essence $\mathrm{E}_{k_{1} k_{2} \ldots k_{n}}$ appears,

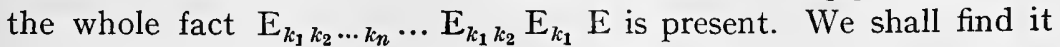
convenient to have a name and a symbol for a fact of this kind. We have already* called it a simple fact; let us now denote it by $\mathrm{T}_{k_{1} k_{2} \ldots k_{n}}$. We observe that whatever includes the simple fact $T_{k_{1} k_{2} \cdots k_{n}}$ also includes each of the simple facts $\mathrm{T}_{k_{1} k_{2} \ldots k_{n-1}}, \ldots, \mathrm{T}_{k_{1} k_{2}}, \mathrm{~T}_{k_{1}}$ and $\mathrm{T}(\equiv \mathrm{E})$. But the converse is not true: whatever includes $T_{k_{1} k_{2} \cdots k_{n-1}}$ does not necessarily include $T_{k_{1} k_{2} \ldots k_{n}}$.

The stages of progress towards the goal of scientific thought are conditioned in two ways by consideration of the use-namely, reasoned thought-to which the endarchy of science is to be put. The first condition is that the essences which, at any stage in the growth of the endarchy, are (directly) connected, as described on p. I98 above, by a single new essence shall be strictly limited in number. And the second condition is that there shall be only one direct path-only one path that does not turn back upon itself and so go over the same ground more than once-from any one essence (or fact-element) in the endarchy of science to any other.

It is not difficult to see why the first of these conditions must obtain. This condition requires that the number of essences which are first connected by the abstraction of a single new essence shall not exceed some maximum number, $m$. Let us speak of the essences $\mathrm{E}_{1}, \mathrm{E}_{2}, \ldots, \mathrm{E}_{m}$ (represented, suppose, by the line-elements $\epsilon_{1}, \epsilon_{2}, \ldots, \epsilon_{m}$ in Fig. I2) which first become grouped together through a new essence $E$ (represented by the line $\epsilon$ ) that is connected to each of them, as essences 'directly connected' to, or 'directly depending on,' or 'derived in the first degree' from, the essence $\mathrm{E}$. Then the first condition requires that the number of essences derived in the first degree from any other essence in the endarchy shall not exceed $m$. The diagram in Fig. 16 on p. 204 represents the central portion of such an endarchy when $m=2 \dagger$ and when every essence has the maximum number $m$ of essences derived from it in the first degree. Any example of scientific classification will illustrate the need for this condition. Thus, when the number of different varieties of any botanical or zoological species becomes excessive, it is usual to group these varieties 
into separate sub-species, so that only a limited number of sub-species may be 'derived' from the single species, while the number of varieties 'derived' from each one sub-species shall also be restricted. The same is true of all scientific organisations: of telephone exchanges, for example, or even of men. As the number of subscribers, whose private

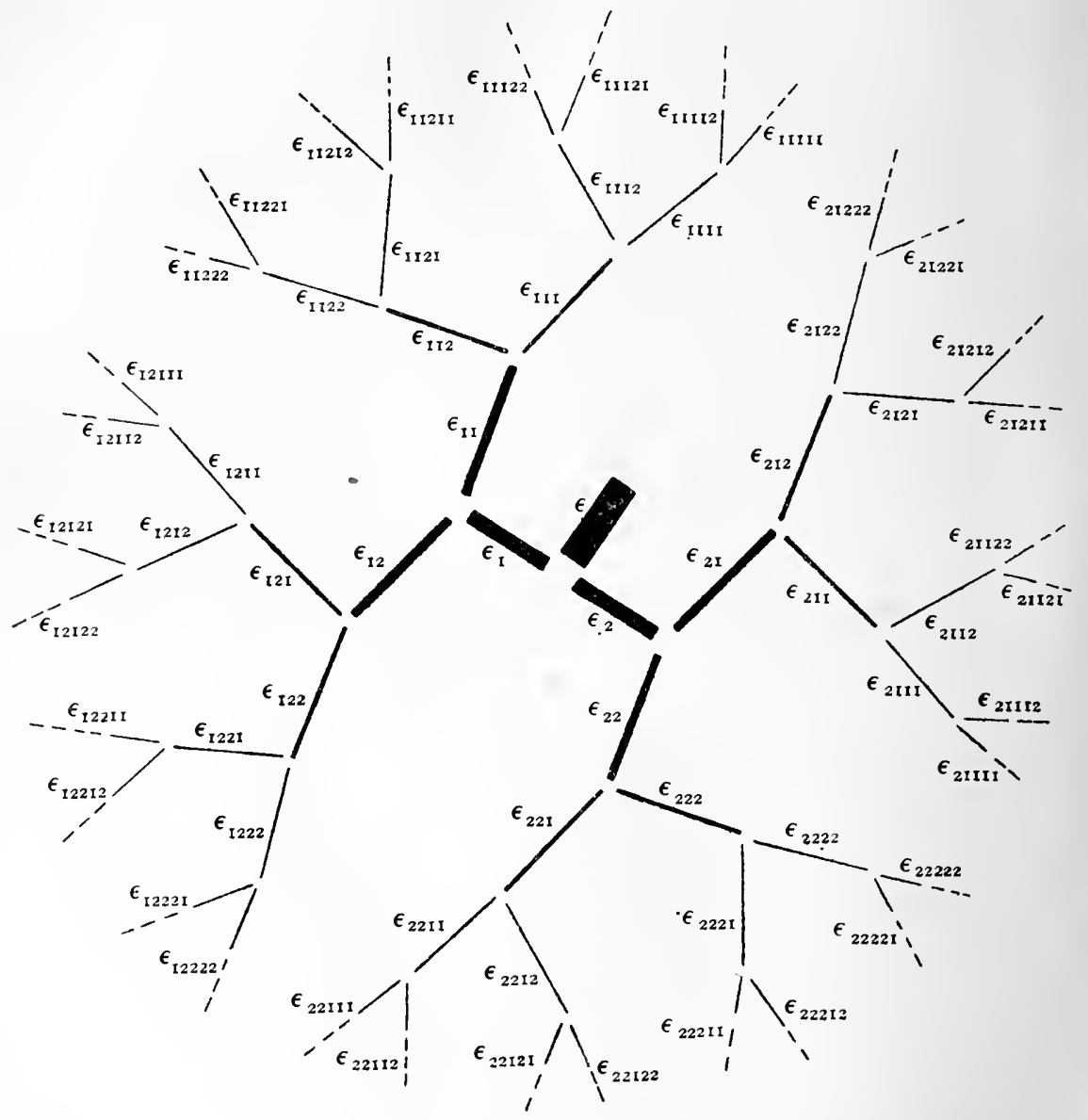

Fig. I6.

lines are brought together in a single exchange, increases, a point is reached when sub-division becomes necessary, and two or more exchanges take the place of one. And, as the number of men in an army increases, there arises a need, not only for more officers, but for more grades of officers, in order that no one officer shall have more than a strictly limited number under his immediate command, 
or, in other words, deriving their authority directly from him. A national army of millions needs more grades of officers than a city police force, and the British Navy than a fleet of merchant liners*. Indeed, a great army that possessed only a commander-in-chief, without subordinate officers or N.C.O.'s, might as well have no officers at all. And an abstracted essence or fact-element, which possessed an almost infinite number of immediate associations or connexions, might as well have none, for any use it or they would be likely to have in rational thinking $\dagger$. We shall return to this point shortly when we come to discuss the use for reasoning purposes of the endarchy of science whose origin and nature we are now considering.

The second condition-that there shall be only one direct path from any one element in the endarchy of science to any other-is illustrated in the diagram (Fig. I6) on p. 204. In this diagram there is only one path, made up of a series of directly connected end-on line-elements, along which it is possible to proceed from any one lineelement to any other without traversing some element or elements more than once. But this merely illustrates what is meant by the second condition as we have stated it. For an example of the operation of the condition, we must look to those portions of the endarchy of science which are most perfectly organised. Thus the science of plane geometry, as formerly studied under the name of Euclid, was highly organised so far as it went, with the result that there was one and only one correct proof of many of the theorems and one and only one correct solution of many of the problems. The same is true in a less degree of other more imperfect branches of mathematics. It is the goal of scientific thought that the same condition should apply to all knowledge $\ddagger$.

* Cf. the second paragraph (on p. 206) of footnote $\ddagger$ below.

$\dagger$ If, for example, in Fig. Io on p. 178 the number of neurograms $\left(b^{\prime}, b^{\prime \prime}, \ldots\right)$ directly connected with $a^{\prime}$ had been very great, the chance of selecting the right one, $b^{\prime}$, for the purpose of reasoning out the new path $A a^{\prime} b^{\prime} \ldots z^{\prime} Z$ would have been so small as to be negligible and the new path would have ceased with $a^{\prime}$. In W. James' words: 'My thinking is first and last and always for the sake of my doing, and I can only do one thing at a time. 'A God, who is supposed to drive the whole universe abreast, may also be supposed, without detriment to his activity, to see all parts of it at once and without emphasis. But were our human attention so to disperse itself we should simply stare vacantly at things at large and forfeit our opportunity of doing any particular act.' (Loc. cit. Vol. II, p. 333.)

$\ddagger$ Another example of the operation of the second condition is furnished by zoological classification. The placental mammal having been separated from other groups (classes and sub-classes) of animals and having been sub-divided into sub-groups (orders), one of which had been named from its carnivorous habits, other flesh-eating animals, which were not placental mammals at all but marsupials, were discovered in Australia. If these flesh-eating marsupials were to be called 


\section{§ 4. Maximal Endarchies.}

Both the first and the second condition that govern the formation of the endarchy of science are fulfilled by the endarchy represented in Fig. I6. But, in the endarchy there represented, every essence (except those in the outermost layer) has also the maximum number $m$ of essences derived from it. We shall want a name for such an endarchy. Let us call it a 'maximal' endarchy. A maximal endarchy may thus be represented, as in Fig. $16 *$, by a single central line-element, $\epsilon$, from which $m$ first ring or zone elements, $\epsilon_{1}, \epsilon_{2}, \ldots, \epsilon_{m}$, radiate; while $m$ second zone elements radiate from each of these first zone elements; and so on, the $p$ th zone containing $m^{p}$ elements of which $m$ are directly derived from each of the $m^{p-1}$ elements in the $(p-1)$ th layer. A $p$ th zone element $\uparrow$ may be denoted by $\epsilon_{k_{1} k_{2} \cdots k_{p-1} k_{p}}$ if directly derived from 'carnivors ' it would be possible to proceed from the element 'placental mammal' in the zoological (partial) endarchy to the element 'marsupial mammal' by two different paths: an upper path through the element 'mammal' from which both placental and marsupial mammal are derived; and a lower path through 'carnivor,' an element that would, under these circumstances, be derived both from placental and from marsupial mammal. Our second condition would then be infringed. In order therefore to satisfy our second condition, the main group of flesh-eating marsupials was described as 'sarcophaga' instead of as 'carnivora.' And, in general, Greek instead of Latin words were used to name sub-groups of marsupials.

Our first condition is further illustrated by the fact that the large main group of flesh-eating placental mammals is said to form an 'order' while the much smaller main group of flesh-eating marsupial mammals is not said to form an order. The smaller partial endarchy of marsupials requires a smaller number of grades than the much larger partial endarchy of placental mammals. (But there is a good deal to be said in favour of using corresponding grades in sub-dividing the marsupial mammals as in the case of the placental mammals, even though they would not be needed for marsupials alone.)

* In this diagram we have taken $m=2$. We have also placed line-elements belonging to the same zone or layer in rings, bounded by two concentric circles having their common centre in the middle of the diagram, so that successive zones of elements are arranged in circles about the centre of the diagram. We might equally well have placed elements belonging to the same zone in a layer bounded by two parallel straight lines, so that successive zones of elements would be placed one below the other. In that case what we shall describe as the most central element would have been described as the highest element; what we shall describe as an inner or outer zone, would have been described as an upper or lower layer; and what we shall describe as the outermost zone would have been described as the lowest layer.

$\dagger$ An element in the pth zone connects any two or more elements in the $(p+I)$ th zone that are derived from it. In order to pass from one of the latter elements to another we therefore reckon it necessary to traverse the $p$ th zone element from which they are both derived. If then we calculate the number of elements traversed in going from every one of the $m^{n}$ elements in the $n$th zone to every other element in that zone, we find that the number is

$$
n m^{2 n}-\frac{1}{2} \frac{m+\mathrm{I}}{m-\mathrm{I}} m^{n}\left(m^{n}-1\right)
$$

for this is the sum of a series of which the $s$ th term is represented by

$$
\frac{2 s-I}{2} m^{n+s-1}(m-I) \text {; }
$$


the $(p-I)$ th zone element $\epsilon_{k_{1} k_{2} \ldots k_{p-1}}$, which is derived through the series of inner zone elements each of which is marked by one less of the suffixes $k_{1}, k_{2}, \ldots$, the second and first zone elements in the series being $\epsilon_{k_{1} k_{2}}$ and $\epsilon_{k_{1}}$ respectively. The essence which is represented by the line-element $\epsilon_{k_{1} k_{2} \ldots k_{n}}$ in the diagram will be denoted by $\mathrm{E}_{k_{1} k_{2} \ldots k_{n}}$ in the endarchy of essences.

We shall have to make further use of the maximal endarchy, the central portion of which is represented in Fig. 16 , for the particular case in which the parameter $m=2$. We shall continue to use Roman capitals to represent facts or essences, and we shall use corresponding Greek letters to denote corresponding line-elements or groups of line-elements in the diagram. The simple fact represented by the group of line-elements $\epsilon_{k_{1} k_{2} \ldots k_{n}}, \epsilon_{k_{1} k_{2} \ldots k_{n-1}}, \ldots, \epsilon_{k_{1} k_{2}}, \epsilon_{k_{1}}, \epsilon$ will, as before*, be denoted by $\mathrm{T}_{k_{1} k_{2} \ldots k_{n}}$; and the group of line-elements by which is the number of elements traversed in going from one $n$th zone element to a second connected with the first by a path that involves ascending through $s$ zones.

If $F_{p}$ represents the frequency with which a line-element of the pth zone is traversed in uniting every element of the $n$th zone to every other it may be shewn that

from which it follows that

$$
F_{p}=m^{2(n-p)}\left(m^{p}-\frac{m+\mathrm{I}}{2 m}\right)
$$

$$
\frac{F_{p-1}}{F_{p}}=m \frac{m^{p}-\frac{m+1}{2}}{m^{p}-\frac{m+1}{2 m}} .
$$

When therefore $p$ is very large, the elements in any zone of the organisation are traversed $m$ times as frequently as those in the zone next below. We have accordingly made the width of the line-elements in cur diagram, which represents the central portion only of an endarchy having a very large number of zones, $m(=2)$ times as great as those of the line-elements of the zone next below.

If $F^{\prime}{ }_{p}$ is the frequency with which a line-element of the pth zone is traversed in connecting any element of any zone further out than the $p$ th to any other element of the same zone

$$
\begin{aligned}
F^{\prime}{ }_{p} & =m^{2 n-p}+m^{2(n-1)-p}+\ldots+m^{2(n+1)-p}-\frac{m+\mathrm{I}}{2 m}\left\{m^{2(n-p)}+n^{2(n-1-p)}+\ldots+m^{2}\right\} \\
& =\frac{m^{2(n-p)}-\mathrm{I}}{m^{2}-\mathrm{I}}\left\{m^{p+2}-\frac{m(m+\mathrm{I})}{2}\right\},
\end{aligned}
$$

which decreases as $p$ increases, so that

$$
\frac{F_{p-1}^{\prime}}{F_{p}^{\prime}}=m,
$$

when $p$ is large. It follows that, when $p$ is large, the relative widths of the lineelements in our diagram represent the relative frequency with which those elements are traversed in proceeding from any element in any lower layer (or outer zone) to any other element in the same lower layer.

If (cf. p. 217 below) the value of an element is proportional to the frequency with which it is traversed, it follows that the value of an element in the $p$ th zone is proportional to $\frac{\mathrm{I}}{m^{p}}$.

* See above, p. 203. 
which it is represented will be denoted by $\tau_{k_{1} k_{2} \ldots k_{n}}$. We shall also find it convenient to denote by $\mathrm{S}_{k_{1} k_{2} \ldots k_{n}}$ the 'branch' endarchy formed by the essence $\mathrm{E}_{k_{1} k_{2} \ldots k_{n}}$ together with all the essences derived from it; and the corresponding endarchy of line-elements will be represented by $\sigma_{k_{1} k_{2} \ldots k_{p}}$. So a 'branch' endarchy is a 'partial' endarchy or a 'subject' endarchy. But a 'subject ' or ' partial' endarchy is not necessarily a branch endarchy, since the latter contains all the essences or fact-elements derived from its highest essence or factelement in the complete endarchy of science. If the complete endarchy of science is a maximal endarchy, so also is every branch endarchy*.

If we imagine an omniscient being to whom the complete endarchy of science is known, but who nevertheless has a brain on the human model $t$, we may speak of neurograms corresponding to the various portions of the endarchy of science; and as before, we shall employ Italic capital letters to represent the neurograms, or systems of neurograms, which correspond to the facts denoted by the same capital letters in Roman type. So $E, T$ and $S$ denote the neurographic correlatives of $\mathrm{E}, \mathrm{T}$ and $\mathrm{S}$. But, when it is necessary to emphasise the imperfect correspondence between $\mathrm{E}, \mathrm{T}$ and $\mathrm{S}$ and the neurograms of an ordinary mortal, these latter may be denoted by $E^{\prime}, T^{\prime}$, and $S^{\prime}$.

Our diagram may therefore be taken to represent an endarchy of neurograms of which the deepest elements-deepest because they are on the average most frequently traversed as excitement passes from any one elementary neurogram represented by a lower, or outer, element in the diagram, to any other-are represented by the widest lines in the diagram $\neq$. It follows from the corollary $\S$ to our third law that, during involuntary thinking, excitement will always tend to flow from any part of such an endarchical system of neurograms towards the centre of the system $\|$.

We propose now to shew that a maximal endarchy results whenever

(a) a multitude of originally disconnected (impressions of) experiences are connected together, first by abstracting from each a single separate essence, and then by successively abstracting new essences directly connected (in the manner we have described I) to each of a number of previously abstracted essences (and so uniting the facts of which they are particular partial aspects); but so that

* See below, p. 2 Iо.

$\ddagger$ See footnote $\dagger$ on p. 206 above.

II Cf. p. 94 above.

$\dagger$ See footnote $\dagger$ on p. 199 above.

$\$$ See above, p. 89.

iी See above, p. 198. 
(b) this number never exceeds a maximum number $m$; and also so that

(c) the average number of essences employed to link any one of the essences to any other is a minimum.

Suppose that there are $m^{n}$ originally disconnected (impressions of) experiences. From each, in the first stage of the process described in paragraph $(a)$, a single separate essence is abstracted. Let us represent these $m^{n}$ separate essences by separate line-elements $\epsilon_{1}, \epsilon_{2}, \ldots$.

In the next stage of the process a new essence is abstracted from every member of a group (not exceeding $m$ in number) of the original impressions; and this new essence forms, according to paragraph $(a)$, a connecting link between the various essences first abstracted from members of the group in question. We may represent this group of essences by the separate lines $\epsilon_{1}, \epsilon_{2}, \ldots, \epsilon_{m}$ (where $m^{\prime}$ does not exceed $m$ ) in Fig. I2 on p. 200. The new essence may be represented in that figure by the line $\epsilon$ connected with each of the lines $\epsilon_{1}, \epsilon_{2}, \ldots, \epsilon_{m}$. We have, at this stage, abstracted from each of the originally disconnected impressions the simple facts partially* represented by two connected line-elements $\epsilon \epsilon_{1}, \epsilon \epsilon_{2}, \ldots, \epsilon \epsilon_{m}$, respectively.

So we may go on representing the abstracted essences by lineelements connected end-on, as in Figs. I3, I4 and I5, so long as we remember that no two of the essences which are 'derived in the first degree' $\uparrow$ from the same essence are connected to each other except through that essence.

Since the various essences with which we are now concerned may thus be represented by line-elements, our proposition will be proved if we can establish the corresponding proposition for line-elements: namely, that a maximal endarchy results whenever

(a) a multitude of disconnected line-elements are connected together by successively positing new line-elements directly connected to each of a number of previously existing line-elements; but so that that

$(\beta)$ this number never exceeds a maximum number $m$; and so

$(\gamma)$ the average number of line-elements employed to link together any two others is a minimum.

This proposition-in terms of line-elements-is easily seen to be true. For the symmetry of a maximal endarchy shews that any two of the elements in the endarchy are, on the average, connected

* See footnote * on p. 200; and also p. 207.

$\dagger$ See above, p. 203.

G. E. 
together by a minimum number of other elements*. And, since the maximal endarchy yields, under the conditions stated in paragraphs $(\alpha)$ and $(\beta)$, the minimum average path from any one element to any other, it appears conversely that, if the average path is to be a minimum, the organisation by which the elements are connected directly or indirectly to one another must be a maximal endarchy.

It follows that the corresponding proposition for essences is also true. (We may however note in passing that, if paragraph (c) had been replaced by the condition that the whole number of abstracted essences was to be a minimum, the resulting organisation would not be a maximal endarchy but an organisation in which the first posited essence connected together $m$ first stage essences, and each subsequent essence connected the last posited essence to $m^{n-1}$ more of the original essences. Such an asymmetrical organisation would not of course satisfy the minimum path condition stated in paragraph (c). Nor would it be a maximal endarchy.)

Now, as we have shewn, the organisation defined by paragraphs $(a),(b)$ and $(c)$ is a maximal endarchy in which, as we have already seen,

(d) the 'number' mentioned in paragraphs $(a)$ and $(b)$ is always the same and equal to $m$; and

(e) one and only one direct path leads from any one essence to any other.

But paragraphs $(a),(b)$ and $(e)$ also define the endarchy of science. It follows that the endarchy of science differs from a maximal endarchy only in so far as paragraph $(c)$ is not necessarily true of the endarchy of science. Since, however, it is evidently in the interest of efficient reasoning that every essence should be connected to every other through a minimum number of intermediate essences we may regard paragraph $(c)$ as being true of the ideal complete endarchy of science which is the goal of scientific thought, although the statement in this paragraph $(c)$ is not necessarily true of any partial endarchy already discovered. Accordingly, we may say that the complete endarchy of science is a maximal endarchy, although the various

* Suppose that there are $m^{n}$ original line-elements. If these be divided into $m^{n-1}$ groups of $m$, and the $m$ members of each group are linked together by the first $m^{n-1}$ new elements posited, and if these $m^{n-1}$ elements first posited are in turn linked together in groups of $m$ by the next $m^{n-2}$ new elements posited, and so on, the resulting organisation is a maximal endarchy. We have said in the text that the symmetry of its arrangement is sufficient evidence that each pair of the original elements in the outermost layer is on the average linked together by a minimum number of the elements subsequently posited. In case this be not evident a demonstration is given in Appendix C, on p. 492 below. 
partial endarchies, which are all that are known of the complete endarchy in its intermediate stages of development, are not themselves necessarily maximal endarchies. These discovered portions of the endarchy of science may not therefore fulfil the conditions stated in paragraphs $(c)$ and $(d)$ although they do satisfy paragraphs $(a)$, $(b)$ and $(e)$.

\section{§ 5. Efficiency and Utility in Thought Organisation.}

It is worth while to observe here that, if we were perfectly free to choose the order in which the essences are abstracted, after the first stage of the process described in paragraph $(a)^{*}$, and so to control the development of the endarchy of science, it would make for the greatest economy of effort spent in thought organisation that there should, at each stage, be one endarchy, rather than several endarchies to be afterwards linked together $\uparrow$. Indeed the efficiency of the effort

* On p. 208 above.

$\dagger$ For suppose that on the average the abstraction of every essence, after those abstracted in the first stage of the process described in paragraph $(a)$, involves an equal expenditure of effort. Then the total effort, $W_{n}$, expended in forming a maximal endarchy having $n$ zones or layers below its central element, will be measured by

$$
W_{n}=m^{n-1}+m^{n-2}+\ldots+\mathrm{I}=\frac{m^{n}-\mathrm{I}}{m-\mathrm{I}} .
$$

If now we write $m^{n}=N$, so that $N$ represents the number of originally separate essences linked up by the endarchy in question, it follows that, when $N$ (or $n$ ) is very large, $W \propto N$.

The number of elements linked together in this endarchy is

$$
m^{n}+m^{n-1}+\ldots+m+\mathrm{I}=\frac{m^{n+1}-\mathrm{r}}{m-\mathrm{I}} .
$$

The utility, $U$, of this endarchy for reasoning purposes may be measured by the number of pairs of elements which it connects together: so that

$$
U=\frac{1}{2}\left(\frac{m^{n+1}-\mathrm{I}}{m-\mathrm{I}}\right)\left(\frac{m^{n+1}-\mathrm{I}}{m-\mathrm{I}}-\mathrm{I}\right)=\frac{m}{2}\left(\frac{m^{n+1}-\mathrm{I}}{m-\mathrm{I}}\right)\left(\frac{m^{n}-\mathrm{I}}{m-\mathrm{I}}\right) .
$$

If therefore $N$ is very large, $U \propto N^{2}$.

The efficiency of the work done in constructing the endarchy is measured by

$$
\frac{U}{W}=\frac{m}{2} \frac{m^{n+1}-\mathrm{I}}{m-\mathrm{I}}:
$$

or, when $N$ is very large, $\frac{U}{W} \propto N$, if $m$ remains the same. The efficiency of the organisation, $\frac{U}{W}$, is then proportional to $N$.

Now suppose that, instead of $N$ originally separate essences being connected in one maximal endarchy, they had been connected in $S$ equally large separate maximal endarchies so as to form $S$ separate branches of science (see below, p. 215) with $N^{\prime}$ of the original $N$ essences in each. We have then, $N=S N^{\prime}$. The utility of each of these separate endarchies is measured by $U^{\prime} \propto N^{\prime 2}$, and the total utility therefore $\propto S N^{\prime 2}$ which $=\frac{N^{2}}{S}$. If $N$ is given, the total utility of the organisation is therefore inversely proportional to the number of separate maximal endarchies in which the original essences are organised.

Meanwhile the work of organising the original essences in $S$ separate maximal 
expended in linking up a large number, $N$, of separate essences in $S$ separate maximal endarchies, or separate branches of science*, is inversely proportional to the number, $S$, of these separate subjects $\dagger$. It follows that the expenditure of effort in organising thought by forming maximal endarchies is more economical if concentrated on the development of a single maximal endarchy than if dissipated in the construction of several maximal endarchies.

The same is true $\ddagger$ of effort expended in organising, not now scientific thought as a whole, but the thought-and therefore the neurography-of some particular person. The efficiency of his reasoning is more increased by a given expenditure of effort upon his education if that effort is directed towards linking up originally separate neurogram-elements into a single maximal endarchy than if it is expended in linking them up into several separate endarchies $\|$. endarchies is the same as that of organising them in a single maximal endarchy; for the work of organising each separate endarchy $\propto N^{\prime}$ and the work of organising the original essences in $S$ separate endarchies $\propto S N^{\prime}=N$ and is therefore the same as that of organising them in a single maximal endarchy. It follows that the efficiency of the work done (or effort spent) in organising a large number of separate essences in equal separate subject endarchies, each of which is a maximal endarchy, is inversely proportional to the number of these subjects.

* See below, p. 215.

$\dagger$ On p. 208 we pointed out that a branch endarchy was a subject endarchy although a subject endarchy was not necessarily a branch endarchy. So a branch of the complete endarchy of science (or, as in the text, a 'branch of science') is a subject, although a subject has not necessarily the form which our definition ascribes to a branch of science. See below, pp. 214, 215.

$\ddagger$ Cf. below, p. 223.

$\S$ See above, p. I91.

II Thus for example, since $U \propto N^{2} \propto W^{2}$, the utility, $U$, of the result produced by a given expenditure of effort, $W$, whether by teacher or by taught or by both, upon a new subject, the study of which begins to form a new separate endarchy in the student's brain, will at first be very small, and will increase rapidly as the study proceeds and the new endarchy grows. If effort is expended uniformly, the total expenditure of effort, $W$, is proportional to $T$, the time given to the new subject. It follows that $U \propto T^{2}$. It is doubtless for this reason that, when Government grants in aid of education are proportional to the number of student-hours (or, for a given number of students, to the number of hours they spend under instruction) - when therefore the amount of the grant is roughly proportional to the effort expended-the Board of Education has to impose the condition that, when the number of hours does not exceed a certain minimum, the grant shall not be in proportion to the number of hours, but shall be nothing at all. This condition is, we suggest, a crude attempt to make the grant approximately proportional, not to the effort expended $(\propto T)$ but to the utility of the result $\left(\propto T^{2}\right)$ or, what amounts to the same thing, to the product of the effort by its efficiency. Such a condition-that no grant will be paid for less than fourteen hours of instruction in a new subject-has figured in the Board of Education's Regulations for Technical Schools for many years past. Of late this regulation has been modified by a reduction of the minimum number of hours from fourteen to ten when the subject studied is not new to the student. This change in the regulations makes them fulfil more nearly the condition in the text, that the utility of the work done depends not only upon the effort expended but upon the size of the endarchy to the further development of which that effort has been directed. 
This conclusion has pedagogic consequences of very great importance*.

\section{§6. Subdivisions of Knowledge.}

Let us now imagine a diagram constructed on the lines of Fig. I6 to represent the complete endarchy of science, each line-element representing an essence or fact-element $\uparrow$; and let us enquire how the facts and essences which science abstracts from complex concrete facts of direct experience are represented in such a diagram.

In the first place, remembering $\ddagger$ that the endarchy of science normally develops from outside (or below) inwards (or upwards), we recognise that the essences, or fact-elements $\S$, in the outermost layer or zone of the completed endarchy, are all that remain to distinguish the particular concrete facts of direct experience after those facts have been united together by successive abstraction of essences. Since every line-element in the diagram corresponds to one and only one fact-element in the endarchy of science, any outermost zone element of the diagram we are imagining will represent an essence, or fact-element, which occurs in only one simple fact $\|$ and so distinguishes a simple fact of direct experience.

It follows at once from the account already given that a simple fact, $\mathrm{T}_{k_{1} k_{2} \ldots k_{p}}$, is represented in the diagram by a series of end-on line-elements, beginning with $\epsilon_{k_{1} k_{2} \ldots k_{p}}$ and ending with the central element $\epsilon$, each element of the series being directly connected $* *$ to its predecessor and to its successor. If $\epsilon_{k_{1} k_{2} \cdots k_{p}}$ belongs to the outermost zone of the diagram, $T_{k_{1} k_{2} \ldots k_{p}}$ will bea 'simple fact of direct experience': the simplest portion of the world of experience that can occur alone. But if $\epsilon_{k_{1} k_{2} \ldots k_{p}}$ belongs to any other zone, $T_{k_{1} k_{2} \cdots k_{p}}$ is a simple abstract fact, which will only occur in the world of experience as part of concrete facts, facts of direct experience $\dagger \dagger$. We note that any simple fact is united to any other by the central essence at least, and in many cases by other essences that are also included in both.

Thirdly, we have used the word fact to denote the whole of any portion of the world of experience; or, as we said before ++ , a fact is any portion of the world of experience but always including whatever invariably accompanies it. Thus, in general, a fact consists of a

* See below, Chapter $2 \mathrm{I}, \S 5$.

+ See above, p. I95.

$\ddagger$ See above, p. 202.

$\S$ Fact-elements are by definition (p. I95) the essences that make up the complete endarchy of science.

I See especially p. 203 above; and cf. p. 207.

$\dagger \dagger$ See footnote $\dagger$ on p. $2 \mathrm{I}_{4}$ below, and footnote $*$ on p. $2 \mathrm{I}_{5}$.

II See above, pp. 192, 203, 207.

$\ddagger$ On p. 192 above. 
combination of 'simple facts.' Since, as we have just seen, all simple facts are united through their central essence or essences, every fact is a continuous portion of the world of experience. But a portion of the world of experience that constitutes a fact, need not be directly experienced, or be capable of being so experienced, by itself alone. In short, it need not be a concrete fact, a fact of direct experience. It may, indeed, be an abstract fact. But it must, we said*, be definite. We may define it by the simple facts which it combines, or even by the outermost (or 'boundary') essences of these simple facts $\uparrow$. Then the fact in question will consist of these boundary essences and of all the more central essences from which they are derived $\ddagger$. For example, the fact of gold with which science is concerned-although it may be an abstract fact, in that it is more simple and more general than any particular concrete lump of gold that might be directly experiencedincludes all the essences that belong to gold in the endarchy of science and that will some day be abstracted from gold, as the chemist now knows it, and will link it to the central essence of the complete endarchy of science. So a fact will be represented in our diagram by a series of line-elements that represent the boundary essences of the fact (and that are not directly connected with each other), together with all those inner line-elements from which the boundary elements are severally derived. Any particular fact, and the particular neurogram that corresponds to it in the omniscient being imagined on p. 208, will be represented by a particular system of boundary line-elements and of inner elements from which the boundary elements are derived. No one of these line-elements can represent the whole fact. In other words, a fact has no single essence $\S$ (unless the fact in question is the central essence of the endarchy of science). We shall return to this point when we transfer our attention from the endarchy of science to personal endarchies $\|$.

A fourth subdivision of thought that will loom large in our enquiry is called a subject $\uparrow$. We shall use the word 'subject' to denote any combination of simple facts of direct experience-or, in short, a

* On pp. I91, I92 above.

$\dagger$ We define $\mathrm{E}_{k_{1} k_{2} \cdots k_{p}}$ as the 'boundary essence' of the simple fact $\mathrm{T}_{k_{1} k_{2} \cdots k_{p}}$. Every simple fact has therefore one, and only one, boundary essence. Only if this boundary essence is in the outermost zone of the endarchy of science, will the simple fact be one of direct experience.

$\ddagger$ See above, pp. 206. 207, where $\mathrm{E}_{k_{1} k_{2} \ldots k_{p-1} k_{p}}$ is not only spoken of as directly derived from $\mathrm{E}_{k_{1} k_{2} \cdots k_{p-1}}$ but also as (indirectly) derived from all the inner essences $\mathrm{E}_{k_{1} k_{2} \ldots p_{-3}}, \ldots, \mathrm{E}_{k_{1} k_{2}}, \mathrm{E}_{k_{1}}, \mathrm{E}$ of the simple fact $\mathrm{T}_{k_{3} k_{2} \ldots k_{p}}$.

§ Cf. W. James: 'There is no property ABSOLUTELY essential to any one thing.' (Loc. cit. Vol. II, p. 333.)

II See below, Chapter 12, 3 .

If See above, pp. 208, 212. 
(complex) fact of direct experience*-as known and studied at any given time before the endarchy of science is complete. It is true that many subjects, as they are known and studied to-day, appear at first sight to consist almost or altogether of abstract facts. While, however, the innermost essences and groups of essences in any subject are, as we have said $\dagger$, most valuable and most frequently thought of, the subject itself has been, as we saw $\ddagger$, built up as a partial endarchy from essences belonging to the outermost zone of the endarchy of science. We shall therefore regard these essences as still belonging to the subject, even when its students are for the most part concerned with its innermost essences. It is important to notice that, while all facts, whether simple or complex, have one or more of the central essences of the endarchy of science in common and are thus connected together, a subject, being a (complex) fact as known when the endarchy of science is incomplete, may be studied as an isolated branch of knowledge. Subjects may therefore be separate from one another, because the central essences of the facts they comprise, and that will ultimately connect them, have not yet been discovered. So subjects, as we have defined them, will be represented in our diagram $\S$ by a partial endarchy, or 'subject endarchy,' $\|$ of line-elements.

The complete discovery of any of the simple facts contained in a subject will connect that subject to the centre of the endarchy of science. It is however conceivable that, at a certain stage of discovery, a subject endarchy might be co-terminus with a branch endarchy $\mathbb{I}_{,} S_{k_{1} k_{2} \ldots k_{n}}$. In that case every essence that belongs to the subject will be related to every other such essence exactly as in the complete endarchy of science. One whose thought is mainly concerned with the subject in question will then be tempted to think that the facts with which he deals are completely discovered. It is therefore important to remember that, however closely any particular partial endarchy may resemble a branch** of the complete endarchy of science, it has still to be connected with other subject endarchies until the goal of scientific thought has been reached. When Laplace, satisfied with the completeness of his account of celestial mechanics, and being asked by Napoleon whether it was true that he had written

* We use this phrase to describe a concrete fact, one or all of whose boundary essences belong to the outermost zone of the endarchy of science.

$\dagger$ See above, pp. 194, 199. See also below, pp. 216, 267.

$\ddagger$ In $\S 3$ of this chapter.

$\S$ Fig. I6 (on p. 204 above) supposed (see p. 2 I $_{3}$ ) extended to represent the complete endarchy of science.

$\|$ See above, pp. 208 and 212.

If See above, p. 208 and footnote $\dagger$ on p. 212.

** I.e. a branch endarchy as defined on p. 208. 
a book about the heavens without once mentioning the name of God, replied 'Sire, je n'avais pas besoin de cette hypothèse-là,' he is supposed to have meant that the facts of the starry heavens had been completely discovered, and did not include any spiritual elements. But his famous epigram referred, not to the real heavens themselves, or even to all available experience of them, but to the facts of mechanical science so far abstracted from this experience. He probably meant no more than that these facts had all been fitted in to such a partial endarchy as we have described.

We may remark in passing that the subdivision of knowledge into separate subjects, and the multiplication of professorial chairs and other teacherships which are concerned with one subject and one subject only, has brought about great progress in the discovery of many of these partial endarchies. But it has tended to obscure the unity of knowledge as a whole. As we multiply specialist thinkers in our universities and elsewhere, we must not forget the need for scientific philosophers who shall aim at discovering inter-relations of the several partial endarchies. There is still room for the Whewells and the Jowetts who take as their portion all 'valuable' knowledge, to whatever branch of science it belongs.

\section{$\S 7$. The Value of an Essence.}

We have already* spoken of essences differing from one another in importance or value according to the frequency with which they occur in the world of experience. It follows that the value of an essence, or fact-element, depends on its position in the endarchy of science. For, in the first place, every experience that includes the essence $\mathrm{E}_{k_{1} k_{2} \cdots k_{p}}$ also includes all the higher essences in the same simple fact $\mathrm{T}_{k_{1} k_{2} \ldots k_{p}}$. Thus it follows from the manner in which the endarchy of science is built up, that the frequency with which any essence occurs in the world of experience is equal to the sum of the frequencies of occurrence of the simple facts of direct experience which that essence first unites, or (what amounts to the same thing) equal to the sum of the frequencies of occurrence of the outermost zone esserices derived from the essence in question; so that, if the value of an essence be measured by the frequency with which it occurs in the world of experience, its value is equal to the sum of the values of the outermost zone essences that are derived from it $\uparrow$. If, as we have supposed, the endarchy of science is a maximal endarchy, and therefore satisfies condition $(d)$ on p. 2 ro, the value of every fact-element is $m$ times as 
great as that of any fact-element directly derived from it. The same would be approximately* true if the value of an essence were measured by the frequency with which it occurred as a link in reasoned thought $\dagger$ between any two other essences in the complete endarchy of science. For the future, therefore, we may measure the value of an essence or fact-element by the frequency with which that essence occurst, whether in the world of experience or in reasoned thought about the world of experience; and the measure is the same as that of the number of outermost zone essences derived from-or simple facts of direct experience united $\S$ by-the essence whose value is to be measured.

This conception of the value of an essence or fact-element in the endarchy of science becomes clearer if we remember that, as the endarchy of science develops from without inwards (or from below upwards), partial endarchies may grow independently and, as we said, be from time to time combined by the discovery of a new essence which unites them. Suppose that, at any stage in the growth of the endarchy of science, a partial endarchy, of which the highest element is represented by $\epsilon_{k_{1} k_{2} \ldots k p}$, has been completely discovered. The partial endarchy in question is then identical with the branch of science

* See footnote $\dagger$ on p. 206.

$\uparrow$ Of the omniscient being already inagined: see footnote $\nmid$ on p. 199 and p. 208 ,

$\ddagger$ Cf. p. 194 above, where we said that the value of an essence to any particular person was greater or less according as the person in question thought of it more or less frequently.

$\S$ We may add here to the passage already quoted from Poincaré in footnote $\dagger$ on p. r99: 'If a new result is to have any value, it must unite elements long since known, but till then scattered and seemingly foreign to each other, and suddenly introduce order where the appearance of disorder reigned. Then it enables us to see at a glance each of these elements in the place it occupies in the whole....Our mind is frail as our senses are; it would lose itself in the complexity of the world if that complexity were not harmonious; like the short-sighted, it would only see the details, and would be obliged to forget each of these details before examining the next, because it would be incapable of taking in the whole. The only facts worthy of our attention are those which introduce order into this complexity and so make it accessible to us.' (Loc. cit. p. 3o.)

Cf. also Dean W. R. Inge on the 'Training of the Reason,' Camtridge Essays on Education, p. $\mathrm{x}_{2}$ : 'The ideal object of education is that we should learn all that it concerns us to know, in order that thereby we may become all that it concerns us to be. In other words, the aim of education is the knowledge not of facts but of values. Values are facts apprehended in their relation to each other, and to ourselves. The wise man is he who knows the relative values of things. In this knowledge, and in the use made of it, is summed up the whole conduct of life. What are the things which are best worth winning for their own sakes, and what price must I pay to win them? And what are the things which, since I cannot have everything, I must be content to let go? How can I best choose among the various subjects of human interest, and the various objects of human endeavour, so that my activities may help and not hinder each other, and that my life may have a unity, or at least a centre round which my subordinate activities may be grouped.' (Italics mine. Cf. the passage quoted from W. James in footnote \|l on p. 238 below.) 
$\mathrm{S}_{k_{1} k_{2} \ldots k_{p}}$ and is represented in the diagram (Fig. I6 on p. 204) by the line-element $\epsilon_{k_{1} k_{2} \ldots k_{p}}$ and all the elements derived from it. Suppose further that other branches of science $\mathrm{S}_{k_{1} k_{2} \ldots k_{p}^{\prime}}, \mathrm{S}_{k_{1} k_{2} \ldots k^{\prime \prime}}, \ldots$, have been completely discovered but are as yet quite separate from $S_{k_{1} k_{2} \ldots k_{p}}$ because the essence $E_{k_{1} k_{2} \ldots k_{p-1}}$ has not yet been abstracted and connected to the $p$ th zone essences, $E_{k_{1} k_{2} \ldots k_{p}}, E_{k_{1} k_{2} \ldots k_{p}^{\prime}}, E_{k_{1} k_{2} \ldots k^{\prime \prime} p}, \ldots *$ * Then suppose that the essence $E_{k_{1} k_{2} \cdots k_{p-1}}$ is abstracted for the first time to connect the $p$ th zone essences of which we have been speaking. The discovery or abstraction of this new $(p-I)$ th layer essences unites all these partial endarchies. It makes a long and valuable step forward towards the goal of scientific thought, the completion of the endarchy of science.

We have followed $\mathrm{W}$. James in avoiding the use of the words conception and concept in this discussion; but we may conclude our description of the endarchy of science by pointing out once more that it is a mode of conceiving the real world. It is not the things themselves that we break up into elements or essences and arrange and connect together in an endarchical organisation. It is rather our thoughts, or concepts, of these things, and the neurograms whose excitement accompanies the thought-activities in question, that are so treated. At the same time we must not imagine that it is only our concepts, or ideas of things, that we are able to alter and arrange. We can and do alter real things themselves. Surely our business here is not merely to know the world but to mould it! Our own neurograms, for example, are part of the world and we change them as we change the organisation of our thought. Or, to take another example, we alter and enrich $\uparrow$ the universal character of colour when, by the discovery of a new dye-stuff or in any other way, we produce a new colour effect. But in the main it is still true that it is not the real world, but our conceptions or thoughts of it and the neurograms whose excitement accompanies those thoughts, that we develop and modify as we gradually construct the endarchy of science for the purpose of economising effort and facilitating reasoned thought.

And this endarchy of science, as we said before, fits the facts of experience and is therefore true. Why the universe, as we know it, fits into this conceptual scheme is another question. Perhaps the

* Of course in practice (and, among other reasons, for that given on p. 194 above) the discovery of the branch of science, $S_{k_{1} k_{2} \ldots k_{p}}$, is not likely to be even approximately complete before the discovery of any of the $(p-1)$ th and inner zone essences, $\mathrm{E}_{k_{1} k_{2} \ldots k_{p-1}}, \mathrm{E}_{k_{1} k_{2} \ldots k_{p-2}}, \ldots, \mathrm{E}_{k_{1} k_{2}}, E_{k_{1}}, \mathrm{E}$ from which it is derived.

$\dagger$ Or, as we lately said, we add to its value when we add to the number of elements derived from it. 
answer is to be found in a progressive differentiation of parts as, according to Kant's nebular hypothesis or Darwin's account of the origin of species, the universe evolves from a common homogeneous origin, and as the parts of it, becoming differentiated, evolve and differentiate further. But probably the answer lies in the constitution of the human brain, which renders thinking most effective when the facts thought of fit in to such an endarchy of science as we have described. Such facts are selected, as Poincaré says, for our attention. Meanwhile our rational thinking is perhaps ignoring other ' unscientific' aspects of the universe.

As we now turn from the consideration of the endarchy of science itself to that of the various personal endarchies, the teleological character of the endarchy of science will become still more apparent. For every fact of the endarchy of science, as it exists at any given time, is or has been part of some thinker's personal endarchy of facts; and of the teleological character of these personal endarchies we shall soon have further evidence*. In W. James' words, 'The conceptual scheme is a sort of sieve in which we try to gather up the world's contents....This whole function of conceiving, of fixing, and holding fast to meanings, has no significance apart from the fact that the conceiver is a creature with partial purposes and private ends.' $\dagger$

* See below, Chapter I2, § I; and p. 234.

$\dagger$ Loc. cit. Vol. I, p. 482. The whole passage is very relevant. 


\section{CHAPTER 12}

\section{NEUROGRAPHIES}

\section{$\S$ I. The Nature of Individual Neurographies.}

WE have roughly described an individual's personal endarchy as the essences of the facts of the world of experience arranged in the order of the importance which they appear to that individual to possess*; or, what amounts to the same thing, in the order of the depth of the corresponding neurograms in his nervous system $\dagger$. But we have not yet seen that everybody's, or indeed anybody's, neurograms are connected together in the order of their depths or according to any other system.

According to Professor Whitehead, the most obvious aspect of the field of actual experience is its disorderly character $\ddagger$. It would follow that the neurographic records of this experience must be equally lacking in organisation until scientific thinking has begun to organise them. Let us then avoid assuming at the outset that people's neurographies are generally organised on the lines of the endarchy of science that we have just been discussing, or indeed in any other manner. For the present, therefore, we shall speak of a person's neurography rather than of his personal endarchy. As we proceed we shall see how far the person's neurography tends, and how far, in the interests both of the individual and of the community in which he lives, his neurography ought to tend, to become organised as a single endarchical system corresponding as closely as possible to the endarchy of science.

First then we have to observe that, for the following three reasons, every individual's ideas of the real world, or rather those of his neurograms whose excitement accompanies these ideas, tend to become organised in a maximal endarchy which corresponds to part of the endarchy of science.

In the first place, the essential parts of the brain processes by which some one pioneer-who first took any particular step towards the goal of scientific thought-discovered some new essence in the

* Above, p. 196. See also below, p. 23I, for our definition of a personal endarchy.

$\dagger$ See above, p. I94. $\quad \ddagger$ Loc. cit. p. I Iо. Cf. footnote $\dagger$ on p. 264 below. 
growing endarchy, must have been afterwards repeated in the brains of those who thought his thoughts after him and accepted his discovery, thereby establishing his new essence as part of the (incomplete*) endarchy of science. The same processes tend to be repeated again and again in the brains of later students. The student whose consciousness is as nearly as possible filled by the essential elements of the thoughts which were present in the mind of the discoverer of a new fact of science at the time of his discovery, may discover the same fact again for himself. This is the heuristic method of education. It leads to the gradual building up in the student's brain of a system of neurograms corresponding to the endarchy of science as it, too, gradually developed. But time will not permit the ordinary student thus to rediscover for himself more than a very small fraction of the partial or subject endarchy which he is studying $\dagger$.

A second reason why every individual's neurography tends to correspond to portions of the complete endarchy of science, is that every boy and girl is likely to be taught, out of school as well as in school, what everyone believes to be true, including whatever is already accepted as part of the endarchy of science. In those branches of the complete endarchy of science whose discovery is most complete and most generally accepted, the correspondence between the complete endarchy of science and the personal neurographies of the students of that science is likely on this account to be most perfect. But, of course, no personal neurography can nowadays correspond even to the incomplete $\ddagger$ endarchy of science as it exists at the present time. Much less possible is it that any individual neurography should correspond to the complete endarchy of science.

In the third place, when we pass from considering the nature of neurographies to discuss how they are formed and organised, we shall find that an important factor in their development is the instinctemotion of curiosity-wonder. And we shall see that this principal prime mover in the multiplication of connexions between the separate neurograms that result from separate and distinct experiences, tends always to link up hitherto separate systems of neurograms by connecting their deepest elements. Thus, as we shall see, there tends to be built up, under the influence of curiosity-wonder, a maximal endarchy of neurogram elements. Suppose, for example, that somebody

* See above, footnote $\S$ on p. r99.

$\dagger$ For this reason alone the heuristic method can nerer wholly replace other methods of education. Another-possibly even more serious--objection to the general application of the heuristic method is noticed below (on p. 224, footnote *).

$\ddagger$ See above, p. 199, footnote $\S$. 
possessed, at a certain stage of the development of his neurography, two quite separate systems of neurograms, each system having the form of a maximal endarchy. Let us, as before, represent these two endarchies by $S_{k_{1} k_{2} \cdots k_{p-1} k_{p}}$ and $S_{k_{1} k_{2} \cdots k_{p-1} k_{p}^{\prime}}$. On the first occasion upon which any element of $S_{k_{1} k_{2} \cdots k_{p}}$ is fortuitously excited at the same time as any element of $S_{k_{1} k_{2} \ldots k^{\prime} p}$, the excitement will tend to move towards the central elements, $E_{k_{1} k_{2} \ldots k_{p}}$ and $E_{k_{1} k_{2} \ldots k^{\prime} p}$, of the respective systems*; and these elements will be more excited than any other elements in the systems. Curiosity-wonder will then, as we saw $\dagger$, tend to connect $E_{k_{1} k_{2} \ldots k_{p}}$ and $E_{k_{1} k_{2} \ldots k_{p}^{\prime}}$ through some new element $E_{k_{1} k_{2} \cdots k_{p-1}}$. Thus, as we shall see in greater detail shortly curiosity-wonder tends to build a maximal endarchy of neurograms.

For these three reasons, then, every individual person's neurography tends, in part at least, to resemble the endarchy of science. We have next to observe that it is in the interests both of the individual concerned and of the community to which he belongs that his personal neurography should so tend.

In the first place, close correspondence between an individual's personal neurography and the endarchy of science will ensure the validity of his reasoning. The endarchy of science fits experience. If therefore the neural path $\S$ followed by the excitement during a train of reasoning corresponds to the endarchy of science, the conclusion reached will accord with the facts of experience. But, if there is no such correspondence between a reasoner's neurography and the endarchy of science, there can be no certainty that his reasoning will be valid $\|$, although it is conceivable that true conclusions may occasionally be reached by such a thinker.

A second reason why an individual's personal neurography should correspond as closely as possible to the endarchy of science, appears when we turn from individual to social psychology and consider the individual as a member of a community. It is only when a particular person's neurography resembles the incomplete - endarchy of science as it then exists-only when it corresponds to the organised body of scientific thought as then generally accepted - that other people will follow his reasoning and accept his conclusions. Thus only will his thought help to build up the endarchy of science; thus only will his

* This follows from the corollary to our third law. See also pp. 94 and 208.

$\dagger$ See above, pp. 92, 93.

¥ See below, pp. 247 et seq.

$\S$ This neural path when completed will consist of a chain of connected elements in the reasoner's neurography.

\|I See above, p. 189 .

I See above, p. I99, footnote . 
experiments be those that are most needed in the then state of scientific enquiry; and thus only will he be sure of avoiding unnecessary repetition of experiments, or of reasoning processes, that have already been adequately performed. (We note in this connexion the difficulties of a new science, when there is as yet no accepted subject endarchy, and when different investigators tend to start from different premises*; so that, instead of gradually building up a single endarchy having its proper place in the complete endarchy of science, they create a number of similar separate subject endarchies, overlapping but not combining. We do well, however, to note also that a personal endarchy which does not, as regards some particular subject matter, correspond with the then accepted endarchy of science, may sometimes be nearer to the goal of scientific thought-may indeed correspond more closely with part of the complete endarchy of science as it will some day be known-than the incomplete science as it appears to its accepted professors.)

Thirdly, an individual's neurography should resemble the endarchy of science as closely as possible in order that his reasoning may be economical or efficient, as well as valid and convincing. Or, at least, economy of effort $\uparrow$ in the organisation of his thought demands that his neurography should form, as far as possible, a maximal endarchy (which, for valid reasoning, must resemble as closely as possible the endarchy of science). For we have already $\ddagger$ seen that, under the conditions stated in paragraphs $(a)$ and $(b) \S$, a maximal endarchy is the organisation which yields the minimum average path-the minimum average number of intermediate elements-between any one element and any other. This therefore is the organisation of neurogram-elements which yields, on the average, the shortest trains of reasoning. We note in passing that, since it is desirable that his reasoning should be as efficient as possible-not only when the organisation of his thought has reached its most complete stage but also at earlier stages of his education, and that probably means throughout the greater part of his life-the effort expended upon his education will, from this point of view, be most effective if it is so ordered that his neurography has the form of a single maximal endarchy of neurograms throughout its

* For example, a reasoned statement about some department of thought which has not yet been scientifically organised, or the scientific organisation of which has not yet been generally accepted, will fail to convince its readers because they have not previously accepted the premises from which the argument starts.

$\dagger$ Cf. Mach's statement quoted by Poincaré (loc. cit. p. 28) that 'the part of science is to effect economy of thought.'

$\ddagger$ See above, pp. 208 to 2 II.

§ On pp. 208 and 209 above. 
development, instead of growing as several separate endarchies* that do not become connected until the process approaches completion.

The fourth reason why the neurography of any person should, as far as possible, be organised in the form of a single maximal endarchy, is that the conditions of conflict cannot occur in a neurography so organised. For, when we were lately $\dagger$ discussing neural conditions of conflict, we observed that two potentially conflicting interest-systems, while they must meet in some common element, must each have its own separate endarchical organisation. This condition of conflict cannot be fulfilled in a single maximal endarchy. In a person whose neurography is organised as a single maximal endarchy, excitement always tends to move in the same direction, namely towards the centre of the endarchy $\ddagger$.

When, later on $\S$, we come to discuss the end of a train of reasoned thought, we shall find that end to consist as a rule in some kind of movement. We shall further observe that a person's conduct will only be consistent and effective if every train of thought has something in common \|. This common property, which will leave its trace on all the individual's behaviour and will thus characterise him, would be furnished by a neurography in the form of a single maximal endarchy, into the centre of which the excitement that accompanies every train of thought would tend to drain $\uparrow$, and the excitement of whose central element or elements would therefore tend to accompany and affect every action. The more nearly a person's neurography is so organised that, whatever elements are first excited, the excitement tends to drain through the same group of central elements, the more likely is his conduct to prove consistent and effective. We have here then a fifth reason why every personal neurography should, as far as possible, resemble a single maximal endarchy.

A sixth reason appears when we consider that a personal neurography in the form of a single maximal endarchy which gives the

* See above, Chapter II, §5. Following Mr Kenneth Richmond (Education for Liberty, p. 202) we may note here that a person's neurography, if it is to be completely inter-connected at every stage of its development, may have to grow differently from the endarchy of science. For the neurographic elements to be connected may be chosen with some freedom: some at least of the elements of a person's neurography are chosen by his educators, and it is conceivable that all might be so chosen. But the endarchy of science starts with given separate experiences that have to be gradually inter-connected. Here is another (see above, p. 22I) reason why the heuristic method of education is not of universal applicability.

$\dagger$ See above, p. $\mathbf{r}_{3}$.

‡ See above, pp. 94, I63, 208.

$\S$ See below, Chapter 15 .

il See pp. 282, 283 below. above.

If In accordance with the corollary (p. 89) to our third law. Cf. pp. 94, 208 
shortest average path from any one neurogram to any other, for that very reason also results in the greatest average freedom of action in any given circumstances. For the only voluntary actions possible at any given moment are those whose images* are then within the field of consciousness; and the organisation of neurograms which results in the shortest average path from any one neurogram to any other is also, other things being equal, the organisation which reduces to a minimum the resistance of the average path from one neurogram to another. In particular, it minimises the resistance of the path from the system of neurograms excited at any moment to the neurogram whose excitement brings an idea of a particular possible course of action into the field of consciousness. Whoever therefore develops a personal neurography in the form of a single maximal endarchy, will, other things being equal, have the greatest possible freedom of action from moment to moment: he will possess the fullest opportunity to choose his own course in life, and to achieve whatever purposes he may have formed. But other things will not be equal in the case of two men who both possess such an endarchy of neurograms, but the endarchy of one of whom includes all, or nearly all, the neurograms likely to be excited by the experiences of his life, while most of the neurograms of the other man's endarchy are remote from his future experiences. Hence the importance in education of securing that the principal interests of the pupil's life, in so far as they can be foreseen, form an integral part of the endarchy which his education should aim at developing.

A seventh reason why every personal neurography should consist of such a single maximal endarchy deserves special notice. Whoever has his thought so organised will tend to have more nervous energy available for all his thought; for, whatever may be the group of his neurograms that is excited during a particular train of thought, the excitement will tend to spread inwards towards the deepest central elements of his neurography $\dagger$. The number of neurogram-elements thus rendered active will, on the average, be far greater than would have been the case had the group of neurograms originally excited been confined to a small separate interest-system. The increase in the average available excitement which therefore results from possession of a single wide interest-system - a single endarchy of neurogramsmust result in an increased power to reinforce excitement, since, as we said above $\ddagger$, the excitement added by the Will so as to guide

* See above, pp. 38,69 .

$\ddagger$ On p. r 30 .

$\dagger$ See above, pp. 94, 163, 208.

G. E. 
thought is probably part of the available excitement at the moment. We notice here a link with Dr Hart's and Professor Spearman's* view, that the general factor, ' $g$, 'consists of a common fund of energy $\dagger$.

We have now considered three reasons why every personal neurography does in fact tend to assume the form of a single maximal endarchy corresponding to the endarchy of science; and seven other reasons why it is in the interests both of the individual and of the community to which he belongs that his neurography should develop along these lines. Reversing our argument, we observe that the endarchy of science itself may perhaps owe its form to the fact that it is gradually built up by the efforts of individual thinkers and observers, each of whom has tended to develop a single maximal endarchy. He has done so in part involuntarily, because of the manner in which his instinct of curiosity operates; and in part voluntarily, not only because he is thus able to organise his thought in a single connected system with a minimum of effort, but also because he thus avoids conflicts, increases the effectiveness of his conduct, secures greater freedom of action, and, by securing a larger average supply of available excitement, increases the effect of every effort of his Will.

\section{§ 2. Individual Neurographies and the Endarchy of Science.}

Returning to the argument interrupted by the last paragraph, we observe that, while every person's neurography tends and should tend to form a single maximal endarchy which corresponds as closely as possible to part of the endarchy of science, no person's neurography can be organised as a whole to form a single maximal endarchy, and no maximal endarchy that forms part of a person's neurography can be so extensive as to correspond, element for element, with the complete endarchy of science $\ddagger$. Even if, at any moment, some individual had organised the whole of his neurography in the form of a maximal endarchy, afferent nervous impulses, stimulated by the évents of his environment, would produce new neurograms and modify old ones faster than he could reorganise his endarchy to include the new or

* See above, p. Ir8.

$\dagger$ Miss M. D. Waller has shewn that, among certain women students, those whose examinational ability is highest, and who, we may therefore be sure, possess the highest ' $g$,' also exhibit the largest relative responses to sudden sensory and emotional stimuli: a result which suggests that they also possess on the average the most available excitement at any given moment. (Lancet, 29th March, 1918.)

$\ddagger$ Cf. above, p. 221. 
altered elements, each in its proper place*. There are indeed whole regions in the endarchy of science, no essences of which are represented in the ordinary man's neurography, regions that correspond to subjects of which he is completely ignorant. And, in regions of the endarchy of science where his neurography does overlap it and correspond to some of its essences, there will be other essences in the complete endarchy that have not been analysed out, so as to have separate elements corresponding to them in his neurography.

So we have to recognise that, however great may be the effort which any individual and his teachers spend upon the organisation of his thought, the volume of his everyday experience will necessarily be so great that it cannot all be organised in a maximal endarchy, or made to correspond, element for element, with the endarchy of science. A selection must therefore be made by every individual and by his teachers. They have to choose experiences to be added voluntarily to those which arrive involuntarily; and their choice may determine not only what lessons he shall learn, but also what physical exercises he shall practise, and, in general, how he shall behave. They have also to decide how the effort available for organising his neurography shall be apportioned among his neurograms, whether formed voluntarily or involuntarily.

We may take it that the person whose neurography we are considering is no Robinson Crusoe living in isolation from his fellows, but one among many other members of a community. He will have his own particular functions to perform in the life of the society to which he belongs. His special activities, social as well as vocational, will bring him specific experiences that are not shared by all his fellowmen. If he is to live his life and to do his work effectively, it is important, alike in the interests of himself and of his neighbours, that he should be able to reason accurately and convincingly, as well as to act effectively, in the matters with which he is every day most concerned. That those of his neurograms which correspond to these matters should form a single maximal endarchy, resembling the endarchy of science as nearly as possible, is therefore more important than that most of the other elements of his neurography should be so organised. These other elements correspond to multifarious and comparatively fortuitous experiences of daily life that have little connexion with the work in which he serves his fellows, and by which he hopes, perhaps, to be remembered. About these comparatively fortuitous experiences he will not need to think often or intensely,

* Cf. above, p. r94. 
and the corresponding neurograms may be comparatively shallow. It is the deeper neurograms that correspond to his principal activities that most need to be scientifically organised in a maximal endarchy, corresponding as closely as possible with the endarchy of science*.

Now it is true that a perfect maximal endarchy is unlikely to be found, even in the most scientifically organised portion of the neurography of the most philosophic of men. But, since we have seen that neurographies tend and should tend to form maximal endarchies, we can simplify our description of individual neurographies by referring them to an imaginary standard-the neurography of some imaginary philosopher--whose deeper neurograms, corresponding to that part of the world of experience with which his thoughts and actions are principally concerned, do actually form a maximal endarchy that corresponds as closely as possible with part of the endarchy of science. Observe, however, that the maximal endarchy, which forms part of the neurography of our imaginary philosopher, will be replaced in some more real and familiar person, whom we may call 'Jones,' by an endarchical organisation, which constitutes the most scientifically organised portion of his neurography and corresponds as closely as possible $\dagger$ with part of the endarchy of science, but which only approximately satisfies the conditions for a maximal endarchy. To this endarchical organisation that may not be, although it tends to be, a maximal endarchy, we shall refer as Jones' scientifically organised endarchy, or, more briefly, his 'scientific endarchy.' But, for the reasons that we have just been studying, the host of other neurograms, records of his experience, that are not organised as part of his scientific endarchy, do, nevertheless, tend, and should tend, to be connected with it so as to form a single system. We have to investigate the nature of the connexion in question.

The systems of nervous arcs that are excited by the fortuitous experiences of every day correspond to essences (or fact-elements $\ddagger$ ) and groups of essences $\S$ distributed over many different branch $\|$ endarchies in the endarchy of science; or, as we said before $\pi$, the

* Cf. above, p. 226.

$\uparrow$ See below, p. 237.

† See above, p. I95. In the next few pages we shall refer to these factelements by their more general name of 'essences' so as to avoid confusion with the (neurogram-) elements that correspond to them in the neurography of the imaginary omniscient being referred trs above on pp. I99 (footnote $\dagger$ ) and 208 .

$\S$ These groups of essences never will be complete facts as we have defined them; for Jones' neurograms that are excited as he experiences a fact, or as he thinks of it, or as an idea of it appears in his field of consciousness, will not include elements that correspond to all the essences belonging to the fact in the complete endarchy of science.

II See above, p. 208.

II On p. I93. See also pp. IgI and 197. 
neurograms of 'concrete' experiences are apt to be complex and illdefined. Among all these essences there may be some that are represented in Jones' scientific endarchy, and this will more likely be the case the more extensive that scientific endarchy is. But the various neural elements whose excitement accompanies a single experienceor a number of simultaneous experiences-are, according to our third law*, empirically connected. Every experience, therefore, that is accompanied by the excitement of elements in Jones' scientific endarchy of neurograms as well as of other elements outside it, will result in the empirical connexion of elements that are organised as part of his scientific endarchy to other elements of his neurography that are not so organised. So we recognise two kinds of connexion between an individual's neurograms. The first corresponds to a rational (or voluntary) connexion of essence to essence, the second to an unreasoning, empirical (or involuntary) connexion of the whole to the whole $\uparrow$. When the connexion is of the first kind, Jones can follow, essence by essence, every link (or step) that connects, one experience $\ddagger$ to the other. But when the connexion is of the second type, he is no longer able to split it into separate essences which might also form part of connexions between other experiences. In short, the several essences that make up the first, or rational, kind of connexion, being usable in many other connexions also (to connect other experiences), are more general than the connecting links of the second kind which connect two particular facts only; and, being essences, they are necessarily also more simple. So we recognise again $\S$ that these last essences, to which Jones' scientific endarchy corresponds, are more simple and more general than those to which correspond the neurograms that form the remainder, or unscientifically organised portion, of his neurography.

We spoke, a moment ago, of a fact of Jones' persunal experience - a fact that had not been analysed, abstracted, and fitted in to his scientific endarchy-as including many essences belonging to widely different branches of the endarchy of science. But different properties

* See above, p. 79.

$\dagger$ Thus William James insists 'that every possible case of reasoning involves the extraction of a particular partial aspect of the phenomena thought about, and that whilst Empirical Thought simply associates phenomena in their entirety, Reasoned Thought couples them by the conscious use of this extract.' (Loc. cit. Vol. II, p. 34r.)

$\ddagger$ The experience in question is, as explained in footnote $\S$ on p. 228 , something less than a complete fact as we have defined it. It is made up of those essences only to which correspond neurogram-elements that are excited when Jones receives the experience in question.

$\S$ Cf. above, p. 199. 
of the same thing, different parts of the same fact-the look of a piece of cloth, for example, and the permanence of its dye*-each have essences which are connected in the complete endarchy of science. Accordingly, any two empirically connected neurograms in Jones' neurography partially correspond to facts, or correspond to parts of facts, which are connected by having some essence in common in the complete endarchy of science. Neurographic correlatives of these connexions through common essences are not, however, to be found complete-but more or less short-circuited-in the unscientific or disorganised portion of the neurography of any person to whom they appear empirical.

These short-circuits, the nature of which we shall shortly examine and illustrate by a diagram $\uparrow$, may connect multitudes of neurograms in the unscientific part of a person's neurography to one or more elements in the scientific part. It is the deepest elements of Jones' scientific endarchy $\ddagger$ which are most often excited, and which, therefore, are most likely to be connected with elements belonging to the unscientific or unorganised part of his neurography. The most central elements of his scientific endarchy will, therefore, tend to have the largest number of connexions with elements outside it. Moreover, since closeness of two fact-elements in the endarchy of science marks a tendency to occur together in the world of experience, groups of essences that are very far removed from each other in the endarchy of science are not likely to be experienced together. Neurograms corresponding to these experiences are therefore unlikely to be empirically connected in Jones' neurography. It follows that elements, and especially the outermost or lowest elements in his scientific endarchy, are not likely to be connected empirically with very distant elements (i.e. elements that correspond to very distant essences in the endarchy of science) in the unscientific part of his neurography. If, however, such distant elements are to be connected to his scientific endarchy, that connexion is most likely to be made empirically through the endarchy's most central elements. Indeed, we shall see later that the central elements of anybody's scientific endarchy are likely

* This illustration is borrowed from W. James: 'Suppose I say, when offered a piece of cloth, "I won't buy that; it looks as if it would fade," meaning merely that something about it suggests the idea of fading to my mind,--my judgment, though possibly correct, is not reasoned, but purely empirical; but, if I can say that into the color there enters a certain dye which I know to be chemically unstable, and that therefore the color will fade, my judgment is reasoned. The notion of the dye which is one of the parts of the cloth, is the connecting link between the latter and the notion of fading.' (Loc. cit. Vol. II, p. 340.)

$\uparrow$ See below, Fig. 17 on p. 235.

$\S$ See below, p. 238 .

$\ddagger$ See above, p. 228. 
to be closely connected with emotion-elements, or even to consist largely of emotion-elements. So there is available a path of connexion between these central elements and the 'unscientific' or unorganised elements of the neurography: it is only necessary to connect these latter elements with certain emotion-elements and they are $i p s o$ facto connected to the central elements of the personal endarchy. Moreover, the more permanent among these outside elements, not being frequently used and deepened by rational thinking, are likely to be already rich in emotion-elements; for otherwise they would rapidly become disintegrated and disappear.

If Fig. I 6 on p. 204 were used to represent Jones' scientific endarchy of neurograms, the neural arcs, connecting an element of this endarchy with the unscientific or unorganised portion of his neurography so as to form a single whole, might be represented by fine lines streaming out like tassels from the various elements of the scientific endarchy. Of these tassels, the largest would be those that came from the most central elements*.

Since, moreover, the whole neurography cannot be organised as a single maximal endarchy $\dagger$, it should, for the last five of the seven reasons given above on pp. 222 to 225 , be the object of every individual's educators, and of himself as chief among them, thus to connect the unscientific part of his neurography as completely as possible with a central scientific endarchy (which, as we said $\ddagger$, corresponds as closely as possible with part of the endarchy of science).

When the whole neurography is thus connected together into a single system, we may speak of it all as forming a personal endarchy $\S$ of neurograms. It is in this sense that the words 'personal endarchy,' whether of neurograms or essences\|, will in future be used. And, since it is through the scientific endarchy that the whole neurography is then connected, we may speak of the scientific endarchy as central, and of the empirically connected portion of the neurography as peripheral.

The personal endarchy, developed by connecting together the whole neurography through a central scientific endarchy, must be distinguished from the scientific endarchy which forms its central portion. While the scientific endarchy corresponds as closely as possible with (part of) the endarchy of science, there are large badly below.

* For the reasons given in the preceding paragraph. See also pp. 242,243 ,

$\dagger$ See above, p. 226.

\pm On p. 228 above.

$\S$ Cf. the definition of an endarchy on p. 163 above.

॥l See above, p. I96. 
organised regions in the personal endarchy where correspondence with the endarchy of science is very slight. Again, while the scientific endarchy approximates to, and in the case of our imaginary philosopher* actually is, a maximal endarchy $\dagger$, we have just remarked that the personal endarchy can never be a maximal endarchy.

\section{§3. Personal Endarchies.}

Let us now suppose that every member of a certain community or Commonwealth has been so educated that his neurography forms a personal endarchy of neurograms. We then observe that the personal endarchies of different individuals will generally differ. For the different offices filled by different members of the same society will demand, as we have seen $\ddagger$, that the scientific endarchy of each should be correspondingly different. The scientific endarchies of the different individuals will thus correspond to different portions of the endarchy of science, according to those individuals' different activities in the life of the community. For example, the endarchies of skilled manual workers will include a large number of elements that correspond to outer zone essences in the endarchy of science, elements whose excitement accompanies immediate practical experience. The personal endarchies of abstract thinkers, on the other hand, will include a much larger proportion of elements that correspond to inner essences in the endarchy of science. The endarchies of experts in particular branches of experimental science will include elements corresponding to all the essences that have so far been discovered in certain branch endarchies§ or parts of branch endarchies. And the personal endarchies of philosophers or theologians will include elements corresponding to as many as possible of the central essences of the endarchy of science, essences that belong to the facts of many different branches of science\|.

* See above, p. 228.

$f$ It is however true that, when any neurogram in the unscientific portion of the personal endarchy is connected to two or more elements of the scientific part, those two or more elements may be empirically connected through the unscientific portion of the neurography, as well as through higher or inner elements of the scientific endarchy. But this will not generally interfere with the use of the scientific part of a personal endarchy for reasoning purposes, seeing that the empirical connexions between elements of the scientific endarchy and elements outside it, being used only on special occasions instead of being simple and general paths of connexion, will not be so deep (or, therefore, so likely to be used in thinking) as the main line connexions in the scientific endarchy.

\$ See above, pp. 227, 228.

$\S$ See above, p. 208.

II Cf. above, p. 2 I6. 
But, however large may be the proportion of 'abstract' elements* in the personal endarchy of any individual, every one of those abstract elements should have one or more outer zone elements in his endarchy derived from it; or, in other words, simple abstract facts should not be studied out of all relation to concrete facts of which they form part. For example, children learning geography ought to have seen at least one river before being taught how rivers are formed or learning lists of rivers in any geographical region $\dagger$. Abstract elements that are not thus supported by elements corresponding to boundary essences $\ddagger$ of facts of direct experience are likely to prove misleading and are certain to lack value§.

Let us now look more closely at the nature of the correspondence between the endarchy of science and the scientific endarchies-the scientific parts of the personal endarchies-of different members of our hypothetical community. While the personal endarchy of any individual will altogether lack elements to correspond to many of the essences in the endarchy of science, and while a single element in a personal endarchy will often correspond to many separate essences in the endarchy of science-essences which the person in question has never distinguished-yet one, and only one, element in his scientific endarchy must correspond to a single essence (or to a single system of connected essences) in the endarchy of science. And if an element, $E^{\prime}$, in the scientific endarchy of any person corresponds to an essence (or to a single system of connected essences), E, in the endarchy of science, then all the elements to which $E^{\prime}$ is connected in that person's scientific endarchy must correspond to essences that are connected with $\mathrm{E}$ in the endarchy of science. In short, it we know that an element, $E^{\prime}$, of a scientific endarchy corresponds to an essence, $\mathrm{E}$, in the endarchy of science, then we know that $E^{\prime}$ does not correspond to any essence in the endarchy of science instead of $\mathrm{E}$, though $E^{\prime}$ may correspond to many essences in addition to $\| \mathrm{E}$.

We have already seen that, when a particular object-and we may now add a particular fact-is thought of on different occasions, the thought-activities, or ideas of it, are different $\uparrow$. Different therefore in some particular must be the system of neurograms excited; or, if exactly the same system is excited on more than one occasion,

* I.e. elements whose excitement accompanies thought of abstract facts (see p. 214 above).

$\dagger$ See also below, p. 343 .

$\ddagger$ See above, p. 214 .

$\S$ See above, footnote $\uparrow$ on p. 206.

II Cf. W. James, loc. cit. Vol. I, p. 46r. We have here written $E^{\prime}$ instead of $E$ so as to mark the possible imperfection of the correspondence between $E^{\prime}$ and E. See above, p. 208.

If See above, pp. 46,47 . 
the relative excitement of different parts of it must differ. It is, however, necessary for rational thinking that different thoughts of the same object should be, to some extent, the same. 'This sense of sameness,' says W. James*, 'is the very keel and backbone of our thinking.' Thus something of the same belongs to every different thought of the same thing or of the same fact. It follows that Jones' every thought of the same fact, $\mathrm{A}$, is accompanied by the excitement of the same neurogram or system of neurograms, although his different thoughts of $\mathrm{A}$ are accompanied by the excitement of different subsidiary (pro hac vice) groups of neurograms giving partially different meanings to the different thoughts. Accordingly, when $\mathrm{A}$ is thought of, other neurograms may be excited in addition to $A^{\prime}$, but not instead of $A^{\prime}$. Whenever $\mathrm{A}$ is thought of, $A^{\prime}$ must be excited, but not necessarily equally in every part.

But $A^{\prime}$ may contain many elements that correspond to, or form part of, neurographic systems that correspond to facts other than $A$. In reasoning about any fact, say $A$, it is necessary, as we have seen $\dagger$, to concentrate attention upon some particular aspect or essence of that fact; and therefore to concentrate excitement in some particular element, or group of elements, that form part of $A^{\prime}$. The element or group of elements, which we select for this purpose, depends upon the purpose for which the reasoning in question is undertaken. Sometimes one element of $A^{\prime}$ and sometimes another will therefore be selected instead of $A^{\prime}$. Or, in other words, A has no single essence, $\mathrm{E}$, and $A^{\prime}$ has no single essential element, $E^{\prime}$. The essence selected for any particular purpose in rational thinking is chosen on purely teleological grounds + .

Now let us consider in more detail how the personal endarchy of some particular person-Jones, say-corresponds to the endarchy of science. Let us begin by considering that portion of Jones' scientific endarchy which corresponds (as closely as possible§) to some particular branch of science, $\mathrm{S}_{k_{1} k_{2} \ldots k_{p}}$. We are thus to consider (the neural correlatives of) Jones' ideas on the subject $S_{k_{1} k_{2} \cdots k_{p}}$. Let us, for simplicity, omit the suffixes $k_{1}, k_{2}, \ldots, k_{p}$, and represent the branch endarchy, S, by line-elements $\epsilon$ (which is equivalent to $\epsilon_{k_{1} k_{2} \ldots k_{p}}$ ); $\epsilon_{1}, \epsilon_{2}, \ldots ; \epsilon_{11}, \epsilon_{12}, \ldots ; \epsilon_{21}, \epsilon_{22}, \ldots ; \ldots ;$ and so on $\|$.

* Loc. cit. Vol. I, p. 459 .

$\dagger$ Above, pp. r86 and 193. We have also seen (on pp. 214, 215 above) how facts and even simple facts overlap, possessing essences in common.

$\ddagger$ Cf. W. James, loc. cit. Vol. II, p. 335, quoted on p. 238 below.

$\S$ See above, p. 228.

II The line-elements in the diagram that correspond to fact-elements in $\mathrm{S}$ or to neurogram-elements in $S$ (or $S^{\prime}$ ), are denoted by Greek letters. 
Now Jones will not know the subject, S, in its entirety. Jones' subject endarchy, $S^{\prime}$, will not therefore contain elements to correspond to all the essences of the complete branch endarchy, S. Let us suppose that it contains only those elements (that correspond to the lineelements) enclosed by the broken line* in Fig. 17.

We note in passing that Brown's ideas on $\mathrm{S}$ will generally differ $\dagger$ from those of Jones. Brown's ideas about $S$ accompany the excitement of portions of Brown's subject endarchy, $S^{\prime \prime}$; and $S^{\prime \prime}$ will commonly differ from $S^{\prime}$ even as both differ from $S$, the system of an

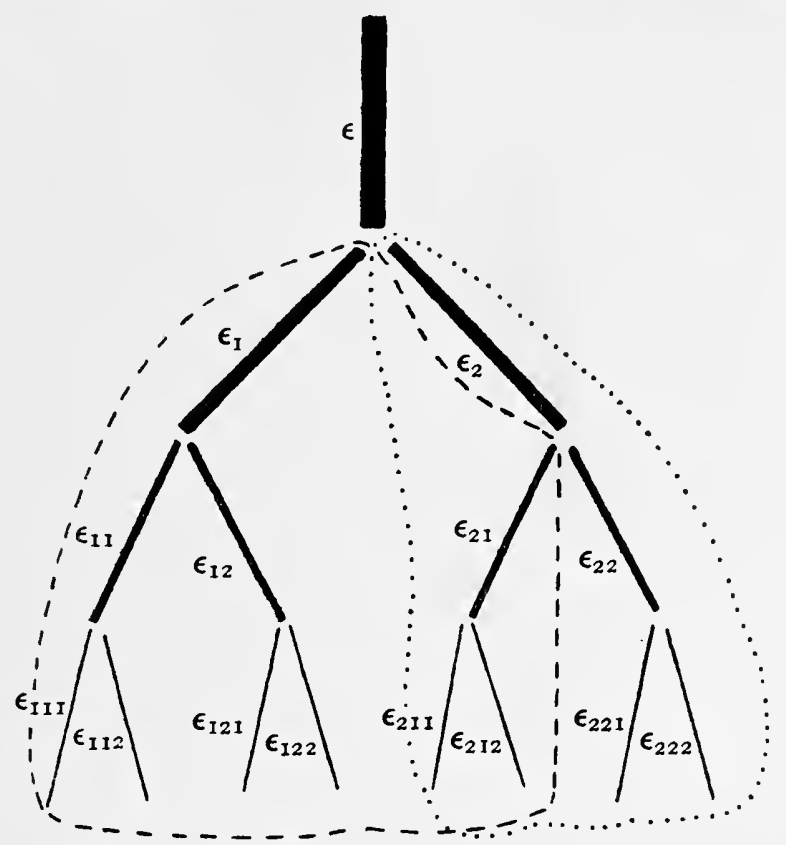

Fig. 17. Diagram representing the branch endarchy S.

* If we are asked why we have chosen to include in the broken line a group of more or less adjacent elements from the middle of the diagram, rather than several separate groups, distributed at random throughout the diagram, we reply that every personal endarchy tends, other things being equal, to include elements corresponding to essences that are near together in the endarchy of science, rather than elements corresponding to those that are far apart in that endarchy. Or more precisely, if a personal endarchy includes an element corresponding to a particular essence of the endarchy of science, the chance that it will include a neurogram-element to correspond to a second specified essence in the endarchy of science is, in general, greater the nearer that second essence is to the first in the endarchy of science. The reason, of course, is that the very habit of mutual concomitance which causes two fact-elements or essences to be near neighbours in the endarchy of science, will also tend to cause the experience of a particular person, Jones, to include both essences if it includes one of them.

$\dagger$ Cf. above, p. 227. 
omniscient being's* neurograms that perfectly correspond to $\mathrm{S}$. $S^{\prime \prime}$ may be represented in Fig. I7 by the elements enclosed by the dotted line.

In Fig. I7, $S_{21}$, the branch endarchy derived from $E_{21}$, is connected with $S_{1}$ by the essences $E$ and $E_{2}$ which are not represented by neurogram-elements in Jones' subject endarchy, $S^{\prime}$. Thus Jones' imperfect subject endarchies, $S^{\prime}{ }_{21}$ and $S_{1}^{\prime}$, may be quite separate as in that part of Fig. I7 which is enclosed by the broken line. But if any element of either $S^{\prime}{ }_{1}$ or $S^{\prime}{ }_{21}$ is excited in Jones' brain at the same time as any element of the other, the excitement will spread towards

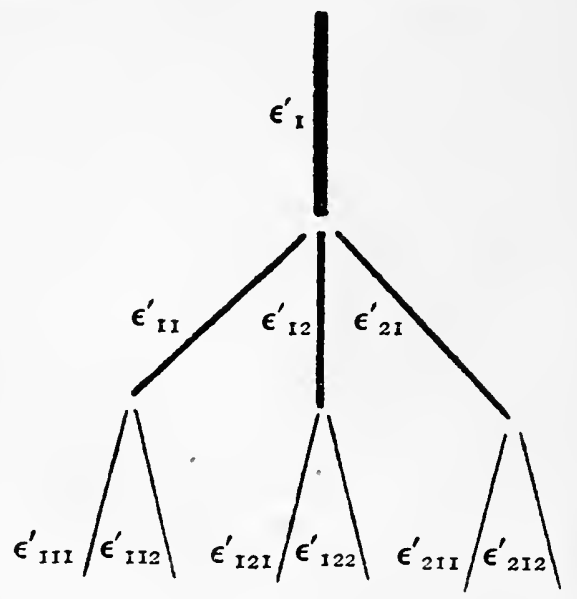

Fig. 18. Diagram representing Jones' endarchy $S^{\prime}$ that imperfectly corresponds to $\mathrm{S}$.

the two central elements, namely $E^{\prime}{ }_{1}$ or $E_{21}^{\prime}$, of the respective endarchies; and, as we have said $\dagger$, the excitement in $S^{\prime}{ }_{1}$ will attain its maximum in the element $E_{1}^{\prime}$, while the excitement in $S_{21}^{\prime}$ will be greatest in the element $E_{21}^{\prime}$. We have then the appropriate stimulus of curiosity-wonder: two elements, $E^{\prime}{ }_{1}$ and $E_{21}^{\prime}$, both corresponding to (particular aspects of) simple and familiar facts, and both excited together for the first time and $E_{21}^{\prime}$. Thereafter $S^{\prime}$ will be the single endarchy (that corresponds to the line-elements) represented $\S$ in Fig. I8.

* See above, p. 208. † See above, p. 222. † See above, pp. 92 and 222.

$\S$ For all that we have said in the text, $E_{21}^{\prime}$ instead of $E_{1}^{\prime}$ might have been placed at the summit of the endarchy in Fig. 18 , in which case $E_{1}^{\prime}, E_{211}^{\prime}$ and $E_{212}^{\prime}$ would have been three elements derived in the first degree from $E_{21}^{\prime}$. But $E_{1}^{\prime}$ having more elements derived from it in Jones' neurography than are derived from $E_{21}^{\prime}$ will be deeper than $E_{21}^{\prime}$; and when the two become connected will therefore, as we shall see directly, be more central than (or above) $E_{21}^{\prime}$ in the combined endarchy. 
We observe that the result of the simultaneous excitement of any two elements in the two separate endarchies, $S_{1}^{\prime}$ and $S_{21}^{\prime}$, is to shortcircuit the true (endarchy of science) path- $\mathrm{E}_{21} \mathrm{E}_{2} \mathrm{EE}_{1}-$ from $\mathrm{E}_{21}$ to $\mathrm{E}_{1}$. What disadvantage does Jones suffer by reason of this shortcircuit in his personal endarchy?

If we regard knowledge as good, useful, or desirable 'for its own sake,' we have our answer at once: Jones suffers because his knowledge is inaccurate or, in other words, because his neurography does not accurately correspond to the endarchy of science. But if we are not content to say that accurate knowledge is to be desired 'for its own sake,' we shall still find that Jones is placed at a disadvantage by reason of the short-circuit between $E^{\prime}{ }_{1}$ and $E_{21}^{\prime}$. In the first place, his arguments will go wrong: he will wrongly suppose, for example, that all that is common to the simple facts*, $T_{11}$ and $T_{12}$, is also common to $T_{21}$. Secondly, if he were suddenly confronted with $E_{2}$, a (to him) new essence abstracted from $T_{21}$, he would have no place for it in his endarchy. Conflict might result in his mind, as in the minds of the chemists of some seventeen years ago to whom physicists presented the new fact of the structure and divisibility of the atom. Moreover, such a short-circuit will tend to make Jones misunderstand Brown when they discuss matters of common interest, matters such as $S_{21}$ $\left(\mathrm{E}_{21}, \mathrm{E}_{211}, \mathrm{E}_{212}\right)$ which form part of the interests of both. When Jones thinks of any fact pertaining to this subject he tends to think at once of $E_{1}$ as its (principal) essence $\uparrow$; but when Brown thinks of the same fact, its (principal) essence is for him $E_{2}$.

\section{§ 4. The Central Elements in Personal Endarchies.}

Finally, what are the central deepest elements in Jones' scientific endarchy, and therefore in his personal endarchy as a whole? When we were lately discussing the endarchy of science, we imagined $\ddagger$ an omniscient being with a human brain, knowing all the facts in the complete endarchy of science. We pointed out that the deepest elements in such a being's neurography would correspond to the central elements in the endarchy of science, for they would be most frequently traversed by excitement as his attention moved from one outer zone fact-element to another. But the neurography of ordinary human beings is by no means wholly the result of the exercise of reasoned thought. We are to discuss in a moment $\S$ how personal

* See above, p. 207.

$\dagger$ I.e. the most central of its essences that is represented in his endarchy.

$\ddagger$ E.g. on p. 208 above. $\$$ See below, Chapters $I_{3}$ and 14 . 
endarchies of neurograms are gradually built up. But we may remind ourselves here that the depths of particular neurograms, say in Jones' neurography, depend, not only on the frequency with which those neurograms have been excited, but also on the intensity of the excitement on each occasion. And we have further seen that neurographic elements which are closely linked to affective-conative elements are on that account likely to be especially deep. We have* gone further, and observed that those elements which belong to the future-interest-system, and to purpose-neurograms in particular, are likely to have most influence of all upon the passage of excitement through the neurography. Since therefore we are supposing $\dagger$ that Jones possesses a neurography of the kind that we have described as a personal endarchy, the central portion in the endarchy must be occupied by purpose-elements $\ddagger$. As William James said, the classification of experience, and, we may add, its record in the form of personal endarchies of neurograms, are 'purely teleological weapons of the mind.' $\$$ Our various personal endarchies characterise us more than they characterise the real world. Different individuals are indeed characterised by very different personal endarchies $\|$.

Now we have considered 9 seven reasons why Jones' neurography should tend to correspond to the endarchy of science. We have seen, too, that the correspondence cannot be complete** and that it should be most marked in his central scientific endarchy where it should be as close as possible $\dagger$. But if Jones is to know as much as possible of

* On p. I53 above; and Appendix B, §§ II and I2. † See above, p. 234.

¥ So, if Kepler was right (see footnote $\|$ on p. 196 above) in saying that he thought God's thoughts after Him, purpose-elements form the central elements of God's universe and therefore of the endarchy of science.

$\S$ W. James, loc. cit. Vol. II, p. 335. He adds: 'The essence of a thing is that one of its properties which is so important for my interests that in comparison with it I may neglect the rest.' Cf. also p. 334: 'Men are so ingrainedly partial that, for common-sense and scholasticism (which is only common-sense grown articulate), the notion that there is no one quality genuinely, absolutely, and exclusively essential to anything is almost unthinkable. "A thing's essence makes it what it is. Without an exclusive essence it would be nothing in particular, would be quite nameless, we could not say it was this rather than that. What you write on, for example, - why talk of its being combustible, rectangular, and the like, when you know that these are mere accidents, and that what it really is, and was made to be, is just paper and nothing else?" The reader is pretty sure to make some such comment as this. But he is himself merely insisting on an aspect of the thing which suits his own petty purpose, that of naming the thing; or else on an aspect which suits the manufacturer's purpose, that of producing an article for which there is a vulgar demand. Meanwhile the reality overflows these purposes at every pore. Our usual purpose with it, our commonest title for it, and the properties which this title suggests, have in reality nothing sacramental. They characterise $u s$ more than they characterise the thing'

\| Cf. above, pp. 227 and 235.

** See above, pp. 221, 226.

If See above, pp. 222 to 225.

t† See above, pp. 228, 231 . 
any part of the endarchy of science, and if $\mathrm{E}_{k_{1} k_{2} \ldots k_{p}}$ is an essence belonging to that part, then-since $\mathrm{E}_{k_{1} \ldots k_{p}}$ never occurs in the world of experience without the inner zone essences $*, \mathrm{E}_{k_{1} k_{2} \ldots k_{p-1}}, \ldots, \mathrm{E}_{k_{1} k_{2}}$, $E_{k_{1}}, E$, from which it is 'derived' $\dagger$-Jones must know as many of these essences as have been discovered; or, in other words, he must have neurogram-elements corresponding to them. Moreover, each of these more central essences that with $\mathrm{E}_{k_{1} \ldots k_{p}}$ make up the simple fact $\ddagger, T_{k_{1} \ldots k_{p}}$, occurs more frequently in the world of experience than any essence derived from it; and it always accompanies any essence derived from it. Each of the more central essences must therefore occur more frequently than their derived essences, including $\mathrm{E}_{k_{1} \ldots k_{p}}$, in Jones' personal experience in so far as it is involuntary. (But we have already§ observed that, for the purposes of reasoning, he may voluntarily restrict the excitement to his neurograms of some particular essences only. The consequences of such restriction upon the organisation of the central elements of his neurography are examined below $\|$.) It follows that the elements of Jones' neurography that correspond to as many of the essences $\mathrm{E}, \mathrm{E}_{k_{1}}, \mathrm{E}_{k_{1} k_{2}}, \ldots, \mathrm{E}_{k_{1} \ldots k_{p-1}}$, $\mathrm{E}_{k_{1} \ldots k_{p}}$ as have been discovered, tend to be in descending order of depth, except in so far as his voluntary thinking modifies this order. Each of these elements tends therefore to be more central in Jones' personal endarchy than its successor in the series. Now we have just

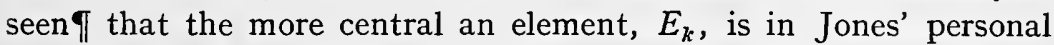
endarchy, the more likely it is to become empirically connected with other elements that correspond to essences which are closely connected with $\mathrm{E}_{k}$ in the endarchy of science. It follows that the central elements of Jones' neurography, and therefore of his scientific endarchy, will tend to include elements that correspond, not only to $\mathrm{E}, \mathrm{E}_{k_{1}}, \mathrm{E}_{k_{k_{1}} k_{2}}, \ldots$, but also to their neighbours in the endarchy of science. Accordingly, given that Jones possesses a scientific endarchy**, it follows that the central elements of his personal endarchy tend to correspond to the most central essences of the endarchy of science.

But if, as we said a moment ago $\dagger \dagger$, the central elements of different personal endarchies correspond to the different purposes of the several persons in question, how can they also correspond to the same central essences in the complete endarchy of science? This difficulty is, from one point of view, unreal, since the central essences of the complete

* See above, p. I99.

\# See above, p. 203.

II See p. 242.

** As we saw on p. 228 above, a scientific endarchy corresponds as closely as possible with part of the endarchy of science. $\dagger$ See above, p. 203.

\$ See above, pp. 186,187 .

I On p. 230 above.

t† On p. 238 above. 
endarchy of science are as yet by no means completely discovered. So far then as reasoned thought is concerned, we need not hurry to find a way out. But, when conduct is in question, an adequate solution of this problem is urgently to be desired.

We have not far to look for a solution. Thus Mr Fluegel, after pointing out that conscious control affords the ideal solution of all conflicts*, argues that the exact nature of the conscious processes whereby particular desires or tendencies are brought into relation with the other dominant tendencies of the personality, 'consists, at any rate to some extent, in the logical process of subsuming the end or goal of each tendency under some higher end or goal.' $\dagger$ In other words, conflicts are best avoided by consciously building up an endarchy of purposes wherein every element (except the central one) is 'subsumed' under some higher or more central element. Mr Fluegel adds:

As a result of this process the mind is able to see clearly the manner in which the particular desires and tendencies in question are related to the other interests of the personality, and these other interests are in turn brought to play upon the particular desires, in a way which is otherwise impossible. It is obvious that in such a case the process cannot logically come to an end at this point and that these higher ends must often be themselves called into question and decisions made between them. This involves the subsumption under still higher ends until we come at last to some conception of the highest good of all, the summum bonum. Theoretically perfect conduct on this principle would thus consist in a completely harmonious series of means to ends, all duly subordinated to the highest end of which the mind in question could conceive. It would in fact be conduct such as would be generally recognised by teleological systems of Ethics as both intellectually and morally the most desirable.

Now we have not hesitated, at an earlier stage $\ddagger$ of our enquiry, to consider what kind of neurography is good for the individual and for the community, as well as what kind of neurography does in fact tend to be formed. If we have regard to similar considerations here, we see at once that it is in the interest of a community that the purposes of its members should be in harmony one with another; or, in other words, that their various ideas of the summum bonum should have much in common. We have now therefore no longer to discover how the central elements in various different personal endarchies may correspond to different purposes, and yet at the same time correspond to the same central essences in the endarchy of science. Our problem is much simplified; for we have only to determine how central

* Cf. p. I 75 above.

$\ddagger$ E.g. on p. 227 above.

$\dagger$ †.J.P, Vol. vili (1917), p. 490. 
elements, belonging to different personal endarchies, but corresponding to the same summum bonum, may also correspond to the same central essences in the endarchy of science. A solution of this remaining problem is readily available in the Christian conception of God, Who is both the highest good*, and in Whom all things consist, even as all the facts in the endarchy of science are ultimately joined together by the single central essence $\dagger$.

We take it then that the central elements of the personal endarchies of all the members of the ideal Commonwealth $\ddagger$ we are considering, will have in common certain purpose-elements that correspond to the central essences of the endarchy of science. This means that any particular member, Jones, of this society will have, among the central elements of his personal endarchy, elements $E^{\prime}$, $E_{1}^{\prime}, E_{2}^{\prime}, \ldots$, which correspond to the central essences $\mathrm{E}, \mathrm{E}_{1}, \mathrm{E}_{2}, \ldots$, in the complete endarchy of science. But in view of his particular activities, social as well as vocational§, in the life of the community, his scientific endarchy should also correspond as closely as possible with some particular part, or 'subject endarchy,' of the endarchy of science\|. Suppose that $\mathrm{E}_{k_{1} k_{2} \ldots k_{p}}$ is the most central element of this subject endarchy. If, for example, Jones is expert in some particular branch of naturalscience, his scientific endarchy will closelycorrespond, not only to the central essences of the complete endarchy of science, but also to some particular branch endarchy, $\mathrm{S}_{k_{1} k_{2} \cdots k_{p}}$, or at least to its most central essence, $\mathrm{E}_{k_{1} k_{2} \ldots k_{p}}$, and its near derivatives $ף$. So, in general, the central essences of Jones' scientific endarchy will include, not only $E^{\prime}, E_{1}^{\prime}, E_{2}^{\prime}$, that correspond to the central essences of the complete endarchy of science, but also the element $E_{k_{1} k_{2} \ldots k_{p}}^{\prime}$ and its

* Because to know Him is, from the Christian point of view, the highest end or goal or purpose of human life: 'This is life eternal' - the attainment of which may well be the most immediately desirable end or goal of every human being'that they might know thee the only true God, and Jesus Christ, whom thou hast sent.' (John xvii. 3.)

$\dagger$ We might borrow a simile from Professor Alexander's recent Gifford lectures, and regard the central essences of the endarchy of science as representing the brain of a being the whole of whose body was represented by the endarchy as a whole, and that is by the whole universe as science hopes some day to conceive it; for the central essences link together and coordinate all the other members of the endarchy. Professor Alexander's simile would make the whole endarchythe whole universe scientifically conceived-denote the God of the Pantheists, while the central essences, in which all the facts of the universe are ultimately joined together, would denote the God of the Theists. (A separate system of essences, apart from the endarchy of science, would be required to represent the God of the Deists.)

$\$$ See above, p. 227.

$\ddagger$ See above, p. 232.

II Cf. below, pp. 266, 267.

If See above, p. 215 , where it is pointed out that specialists in any particular branch of science are apt to be most concerned with the most central or abstract essences that belong to it.

G. E. 
near derivatives which correspond in his brain to $E_{k_{1} k_{2} \cdots k_{p}}$ and its near derivatives in the endarchy of science. In other words, $E_{k_{1} k_{2} \ldots k_{p}}^{\prime}$ will occupy a far more central position in Jones' neurography than that which $\mathrm{E}_{k_{1} k_{2} \ldots k_{p}}$ occupies in the endarchy of science. Or, to put it in another way, while $\mathrm{E}_{k_{1} k_{2} \ldots k_{p}}$ may be far from central in the endarchy of science, the central elements in Jones' neurography will include $E_{k_{1} k_{2} \ldots k_{p}}^{\prime}$ as well as $E^{\prime}$ and its immediate neighbours; but, as we have said*, $E^{\prime}, E_{k_{1}}^{\prime}, E_{k_{1} k_{2}}^{\prime}, \ldots, E_{k_{1} k_{2} \ldots k_{p}}^{\prime}$, tend to be in descending order of depth, so that the deepest of all the elements in Jones' neurography are those which correspond to $\mathrm{E}$ and its neighbouring essences in the simple fact $\uparrow, T_{k_{1} k_{2} \cdots k_{p}}$. We see then how the central elements of different persons' neurographies, while having in common what is most important, may yet differ in matters of secondary but still high significance.

In the endarchy of science, $E_{k_{1} k_{2} \ldots k_{p}}$ is connected with far more essences by paths that traverse essences between $E_{k_{1} k_{2} \ldots k_{p}}$ and the central essence, $E$, than by paths which radiate downward from $E_{k_{1} k_{2} \ldots k_{p}}$ to the fact-elements of the branch endarchy, $\mathrm{S}_{k_{1} k_{2} \ldots k_{p}}$. Suppose now that $F^{\prime}$ corresponds in Jones' neurography, and $\mathrm{F}$ in the endarchy of science, to some essence of a fact that has fortuitously come to Jones' knowledge; and that $F^{\prime}$ is outside his scientific endarchy, while $\mathrm{F}$ is outside the subject endarchy, $\mathrm{S}_{k_{1} k_{2} \ldots k_{p}}$. Then on the average $\mathrm{F}$ is more closely connected to $\mathrm{E}$, and to any one of the essences $\mathrm{E}_{k_{1}}, \mathrm{E}_{k_{1} k_{2}}, \ldots$, between $\mathrm{E}$ and $\mathrm{E}_{k_{1} k_{2} \ldots k_{p}}$, than it is to any essence derived from $E_{k_{1} k_{2} \ldots k_{p}}$. On the average of a number of happenings to Jones, $F^{\prime}$ will therefore be more closely connected to his central elements $+, E^{\prime}, E_{1}^{\prime}, \ldots, E_{{ }_{1} k_{2} \ldots k_{p}}^{\prime}$, than to any other element of his scientific endarchy; and, among the central elements to which $F^{\prime}$ is thus most closely connected, it will in general be connected most closely of all to the most central element, $E^{\prime}$. The large number of elements, in his personal endarchy but outside his scientific endarchy, thus connected to his most central elements $E^{\prime}, E_{1}^{\prime}, \ldots$, will help to keep them deepest; for they will tend to be excited by any chance happening that excites one of the outside elements in question. Multiplication of such connexions, between elements outside his scientific endarchy and his central purpose-elements, will thus tend to prevent any merely rationalई central element such as ${E^{\prime}}_{k_{1} k_{2} \cdots k_{p}}$

* See above, p. 239.

¥ Cf. above, p. 230.

$\dagger$ See above, p. 2 I3.

$\S$ As distinguished from a relatively emotional central element-i.e. an element more closely connected with affective-conative elements-belonging to a purposeneurogram. 
from becoming deeper than the purpose-elements $E^{\prime}, E^{\prime}{ }_{1}, \ldots$ Indeed, were it not for the tendency* of a large part of Jones' experiences to be empirically connected to his scientific endarchy through his central purpose-elements $E^{\prime}, E^{\prime}{ }_{1}, \ldots$, most of his reasoned thinking, while involving the excitement of $E^{\prime}{ }_{k_{1} k_{2} \ldots k_{p}}$ more than of any other element of his scientific endarchy, would not necessarily involve the excitement of $E^{\prime}$ and its neighbours; for these latter elements need not, in the case we have supposed, be traversed in passing from one element of his special subject endarchy $\dagger$ to any other. So the more his thought-activities are concerned with rational or voluntary thinking about his special subject (or branch of science, if his main work is study), the deeper will $E_{k_{1} k_{2} \ldots k_{p}}^{\prime}$ tend to become relative to $E^{\prime}$ and his other purpose-elements. However strong his central purpose-elements may once have been, he may, if much given to reasoned thinking about a special subject, reach the stage when he forgets his higher purposes and echoes Laplace's: 'Je n'ai pas besoin de cette hypothèse-là.' $\ddagger$

Such a tendency is, as we said, obviated if those elements of Jones' neurography (and the more diverse the experiences they record the better) which do not come within the system of his scientific endarchy, are directly connected to $E^{\prime}$ and its neighbours and through them to $E_{k_{1} k_{2} \ldots k_{p}}^{\prime}$. Such connexions will, indeed, tend to form under the influence of involuntary thinking, since every thought-activity tends to become connected to the centre of an interest $\S$. This tendency is greater, the wider and deeper the interest in question: and, in the case we have supposed, the interest-system with $E^{\prime}, \ldots, E_{k_{1} k_{2} \ldots k_{p}}^{\prime}$ at its centre is not only Jones' widest and deepest interest-system, but the only one he has. But his educators, and especially he himself, must not be content to rely upon this tendency of involuntary thinking. They must, on the contrary, make every effort to connect the neurograms corresponding to the whole of his experience (and the wider the better) to his scientific endarchy, for the most part through his central purpose or ideal\|. Thus will the whole flux of his experience be most conveniently arranged for handy reference $\uparrow$. Thus too will

* See above, p. $230 . \quad+S^{\prime}{ }_{k_{1}} k_{2} \cdots k_{p}$ : see above, p. 24 I. $\quad$ \& See above, p. 2 I6. $\S$ See above, p. 9I, where it is shewn that neurograms tend to become connected with interest-systems and therefore with the centres of such systems. And, since interest-systems tend to be endarchical (see above, p. I63), neurograms tend to become most closely connected with the central elements of interestsystems. See also p. 239.

II We thus extend the observation already made (on pp. 86 and 9I) upon the value in education of connecting new neurograms of important facts with deep wide interest-systems. See also p. 223 above.

I Cf. Whitehead, loc. cit. p. III. 
he best secure, so far as the conditions of human life permit, a perfectly integrated mind*.

Wide and diverse experience linked to learning-i.e. scientifically organised knowledge, corresponding as closely as possible to the endarchy of science - that may be narrow, but must form a single interest rather than several disconnected interests, and all subject to a supreme and dominant purpose in harmony with those of one's neighbours $\uparrow$, is therefore to be the aim of education in so far as it is concerned with forming neurograms + : a conclusion that was foreshadowed at the end of Book I§.

Or, as Lord Brougham said a century ago, 'A man should know something of everything and everything of something.' But all his knowledge and all his feelings should be linked together so as to form a single wide interest, whose neurographic correlative-a single wide interest-system-is the kind of personal endarchy that we have described earlier in this chapter. Our conception of a single wide interest will be further considered when we discuss the foundations of character. Meanwhile we shall use the words 'single wide interest' to denote that organisation of thought which not only corresponds to a single personal endarchy of neurograms but is also centred in, and dominated by, a supreme purpose that is in harmony with those of one's neighbours. Such is the single wide interest which education should, as we have just said, aim at forming.

We note, in passing, that the excitement at any part of a single wide interest-system will tend to spread towards the centre of that system \|. This follows from the corollary I to our third law. Accordingly, the possessor of a single wide interest will tend always to be conscious of his supreme and dominant purpose.

* The facts which correspond to a person's unorganised neurograms and which, on account of their disorderly character, he will not be able to explain scientifically, he may describe as 'acts of God,' since their neurograms are directly connected with the central elements in his endarchy. But if, and when, he makes the necessary effort to fit the neurograms of these facts into their proper place in his particular personal endarchy, by reproducing there the connecting elements that exist in the complete endarchy of science, he will have a 'scientific explanation' of the facts in question. The connecting neurograms will still be connected with the central elements, although the connecting path will be much longer since it has ceased to be short-circuited; but, having explained the facts, he may cease to speak of them as 'acts of God.'

$\dagger$ For the sake of the community or Commonwealth as a whole: see above, p. 240.

$\ddagger$ Cf. Morton Prince: 'Every form of education necessarily involves the artificial formation of such complexes....' (Loc. cit. p. 289.)
$\S$ On p. 23 above.
II Cf. pp. 94 and 208.
II On p. 89. 


\section{CHAPTER 13}

\section{CURIOSITY; AND THE INVOLUNTARY GROWTH OF SINGLE WIDE INTERESTS}

WE proceed to consider how far this type of neurography tends to develop involuntarily-for 'The Integrative.Action of the Nervous System'* is not confined to spinal levels-and, in the next chapter, how its development may best be voluntarily secured.

The neural mechanisms of several reflex movements are already developed at birth. These are our first neurograms. For example, a new born baby's iris contracts when a light is held near its open eyes. In lower animals more complex reflexes-sometimes called instincts-also exist from the very beginning. The chicken, that pecks at grains of corn almost immediately after coming out of its egg, has not been taught to do so: the link between its neurogram for the grain of corn, and its other neurogram whose excitement accompanies the series of coordinated movements involved in stooping and pecking, is certainly innate and appears to be fully developed at birth.

Experience, in the form of incoming sense impressions, modifies and adds to our innate neurograms. So great may this modification be amongst human beings that instinctive or other innate processes often cease to be recognisable by the casual observer.

The modification of an innate neurography by subsequent experience is included in the process of education, when that word is used in its widest sense. In the more restricted sense in which the word is commonly used, education includes the modification and development of an innate neurography by the formation of new neurograms corresponding, not to all facts of experience, but only to such as are selected by human educators $\dagger$. But long before the pupil is old enough to take an active and voluntary part in his own education, his neurography-or, if it be preferred, his instincts and interests to which his neurography corresponds-already influences its own further development. It is true that facts which occur together in the world of experience tend, in consequence of our third law + , to be repre-

* Discussed by Professor Sherrington, loc. cit.

$\dagger$ See above, p. 12. 
sented by connected neurograms*. But, according to the corollary $\dagger$ to that law, those experiences which include essences that belong to previously existing interests (including instincts) are selected for attention above all other experiences.

The selective influence of a person's existing neurography, not only upon the direction of his uninterrupted involuntary thinking, but also upon the attention he pays to the various elements of his environment, is increased by the instinct-emotion of curiosity-wonder. The latter effect we have already $\ddagger$ illustrated by the incident of Newton and his apple, when the acceleration of the apple's fall stimulated Newton's curiosity and attracted his attention; and, lest it be complained that this incident was imaginary, we may also remind§ ourselves how, when Darwin sailed to South America on H.M.S. Beagle, he found that the great ship, having nothing in common with the Fuegians' previous experience or interests, aroused no curiosity in their minds, but that the ship's boats and 'simple circumstances-such as the beauty of scarlet cloth or blue beads'being novel examples of a familiar type, caused profound wonder among these savages. So those elements of a given environment that stimulate one man's curiosity will not arouse the curiosity of another, whose interests are different. And this selective influence, which causes one element of the environment to awaken more curiosity than another, is no less marked among the thought-activities that are presented during day-dreaming. Moreover, were it not for the operation of curiosity-wonder, the familiar elements in our thought, as in our environment, would be so vastly the most interesting that we should never notice anything new $\|$. Whenever, therefore, we are engaged in involuntary thinking-whether our thought-activities are stimulated from outside or from within the brain-curiosity-wonder is at work, modifying our thought-activities according to our previously existing interests, making our interest-systems grow in width as well as in depth, and so producing a profound effect upon the development of our neurographies. But what effect? We have now to attempt to answer this question.

Or, rather, since we have already II given our answer-that there tends to be built up, under the influence of curiosity-wonder, a single maximal endarchy of neurograms-we have now to give our reasons for that answer. We shall first carry a little further the deductions we have already** made from our account of the normal stimulus of

* Cf. above, p. 230. $\quad \dagger$ On p. 89 above. $\quad$ † See above, p. $93 . \quad \S$ See above, p. 72.

If Cf. above, p. 94.

** On p. 222 above. 
curiosity, namely, the unlooked-for juxtaposition of simple and familiar facts not previously associated*; and we shall then compare the conclusions so deduced with Poincaré's observations upon the working of the same instinct.

In the first place, then, it follows from the corollary to our third law that curiosity-wonder will tend to connect the central elements of any two maximal endarchies of neurograms not already connected $\dagger$. If a neurography includes a number of maximal endarchies of different sizes, and if for the moment we suppose that the depth of each element is greater or less according as the number of pairs of elements derived from it is greater or less, curiosity-wonder tends most strongly to unite the central elements of the two largest endarchies; for these two elements are the deepest, and so correspond to the most familiar essences not hitherto connected. But the linking up of separate maximal endarchies through their central elements may not lead to the formation of a single maximal endarchy. For if curiosity-wonder did nothing but cause central elements of maximal endarchies, or other deepest elements, to be linked to deepest elements, the resulting organisation would be straggling and asymmetrical, since every new element or group of elements would be added to the central elements, instead of being inserted in the position of the corresponding essence or group of essences in the symmetrical endarchy of science $\ddagger$. But the effect of curiosity-wonder is not confined to making a first connexion between central essences of two hitherto disconnected facts. On the contrary, as we saw instinct strives to concentrate excitement in both groups of neurograms so that further common elements may be abstracted, until at last the two groups become connected according to the connexions of the corresponding (groups of) essences in the endarchy of sciencell.

* See above, p. 92 .

$\dagger$ See above, p. 222.

* Suppose for example that, at a certain stage of its development, a personal neurography contains two separate subject endarchies, $S_{k_{1} k_{2} \ldots k_{p}}^{\prime}$ and $S_{k_{1} k_{2} \ldots k_{p} \ldots k_{t}}^{\prime}$, each of whose elements corresponds to one or more of the essences or fact-elements (denoted by the same letters in Roman type and with the dashes omitted) of the endarchy of science. When, for the first time, any one element of $S_{k_{1} k_{2} \ldots k_{p}}$ is excited simultaneously with any one element of $S_{k_{1}}^{\prime} k_{2} \ldots k_{p} \ldots k_{t}$, curiosity-wonder tends to connect the two subject endarchies through their central elements, $E_{k_{1} k_{2} \ldots k_{p}}^{\prime}$ and $E_{k_{1} k_{2} \ldots k_{p} \ldots k_{t}}^{\prime}$ respectively. The link thus formed short-circuits the corresponding path, $\mathrm{E}_{k_{1} k_{2} \ldots k_{p}} \mathrm{E}_{k_{1} k_{2} \ldots k_{p} k_{p+1} \ldots \mathrm{E}_{k_{1} k_{2} \ldots k_{p} \ldots k_{t-1}}} \mathrm{E}_{k_{1} k_{2} \ldots k_{p} \ldots k_{t-1} k_{t}}$ in the endarchy of science. $\S$ On p. $9^{2}$ above.

$\|$ Thus, in our example, concentration of excitement in $E_{k_{1} k_{2} \ldots k_{p} \ldots k_{t}}^{\prime}$ and its neighbouring elements in the system of neurograms which corresponds to the

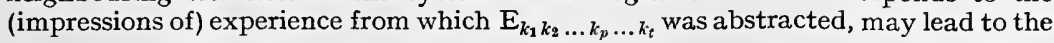

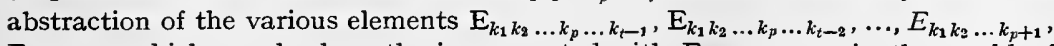
$\mathrm{E}_{k_{1} k_{2} \ldots k_{p}}$ which are, by hypothesis, connected with $\mathrm{E}_{k_{1} k_{2} \ldots k_{p} \ldots k_{t}}$ in the world of experience. 
And, if the involuntary concentration of excitement that is directly due to the operation of the instinct is insufficient to produce this result*, sufficient concentration may result from the intervention of the Will in support of the instinctive process. To this point we shall shortly return. Meanwhile we conclude that the instinct-emotion of curiosity-wonder tends to develop every personal neurography into a single maximal endarchy corresponding to the (complete) endarchy of science; and so leads to the discovery $\dagger$ of the endarchy of science.

This conclusion has now to be compared with Poincaré's observations on his motives for making his scientific discoveries and his feelings when he made them. To this end we first note, as a necessary consequence of our generalisation, that every time the instinctive process achieves its end by further developing a person's neurography into a single maximal endarchy corresponding to the endarchy of science, its achievement will be marked by a feeling of pleasurable satisfaction; for such a feeling follows the successful completion of every instinctive process $\ddagger$ : one's escape from danger by flight, for example, or the response to one's cries for help in distress§. Now

\footnotetext{
* And so to connect $S_{k_{1} k_{2} \ldots k_{p} \ldots k_{t}}^{\prime}$ to $S_{k_{1} k_{2} \ldots k_{p}}^{\prime}$ as $S_{k_{1} k_{2} \ldots k_{p} \ldots k_{t}}$ is connected to $\mathrm{S}_{k_{1} k_{2} \ldots k_{t}}$ in the endarchy of science. $\quad \dagger$ See above, footnote $\|$ on $\mathrm{p}$. I 96.

$\neq$ Cf. W. McDougall: 'We seem justified in believing that the continued obstruction of instinctive striving is always accompanied by painful [or "unpleasant" as we should rather say, since the researches of Head and Holmes on the neurology of pain and " unpleasure" (Brain, Vol. xxxIv, I9I I, pp. IOz et seq.)] feeling, its successful progress towards its end by pleasurable feeling, and the achievement of its end by a pleasurable sense of satisfaction.' (Social Psychology, p. 28 ; italics mine.)

\& I can find no evidence, from introspection or from any other source, that the feelings of pleasurable satisfaction or relief which mark the completion of different instinctive processes are themselves different in any essential respect. But each is accompanied by a vivid memory of the emotion that has just come to an end; and this memory, whose vividness is doubtless due to the continued activity of some of the neurograms whose excitement accompanied the full blast of the emotion, leaves no room for doubt which instinct has been responsible for the pleasurable satisfaction experienced. When that instinct has been curiosity, our satisfaction on its achievement of its end is accompanied by (if it does not include) the feeling that we understand the object of an erstwhile curiosity; that the strangeness has disappeared; that all fits harmoniously together. But if our emotion has not been pure wonder - the affect of curiosity - but wonder compounded with negative self-feeling, a compound which Dr McDougall (Social Psychology, p. I29) identifies with admiration, 'as soon as we feel that we completely understand the object we have admired, and can wholly account for it, our wonder ceases and the emotion evoked by it is no longer admiration' (McDougall, loc. cit. p. I29) but negative self-feeling, to which is added the pleasurable satisfaction due to the successful completion of the curiosity-wonder process. Or, if our emotion of wonder has been compounded with positive, instead of with negative, self-feeling, the feeling of pleasurable satisfaction that comes when we think that complete understanding has been reached will be accompanied by elation or

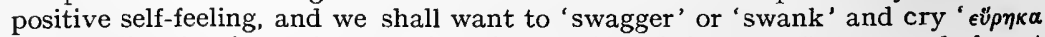
like Archimedes, instead of wanting, like Kepler (cf. footnote \|| on p. I96 above), to reverence the Creator of the object of our emotion.
} 
when we do things under the compelling force of instinct (whether it be to organise-thought-by-developing-a-single-maximal-endarchyof-neurograms, or to run-away-and-hide, or to strike, or to cry-outfor-help), we may say, with Dr McDougall*, that we act so because instinct is driving us; or we may say, as children and other unsophisticated persons commonly do say, that we act so because we feel the corresponding emotion (whether it be wonder $\dagger$, or fear, or anger, or distress); or we may say, with Locke and Hume and the lesser hedonistic philosophers, that we act so for the sake of the pleasurable satisfaction that will follow the results of our action when the instinctive process is complete. If we are neither familiar with modern psychology nor altogether unsophisticated, the third is the way in which we shall probably account for our conduct.

And this, in effect, is just the account that Poincare does give of his motives for organising his thought and making his scientific discoveries $\ddagger$. 'The scientist,' he writes, 'does not study nature because it is useful to do so. He studies it because he takes pleasure in it, and he takes pleasure in it because it is beautiful.' $\S$

In order to make sure that this observation of Poincaré's is the very one which our generalisation || would lead us to expect, and by which it is therefore confirmed, we have to verify (I) that by the 'study of nature' Poincaré means the organisation of thought on the lines of the endarchy of science and so the discovery of that endarchy; and (2) that the pleasure which Poincare observed the scientist to take in the beauty of nature is no other than the pleasurable satisfaction that results when the curiosity-wonder process has achieved its end.

First then we have to shew that, by the study of nature, Poincaré means, not merely to observe natural phenomena more or less at random, but also (and especially) to organise experience of nature; or, in our terminology, to 'discover' the endarchy of science; or, to develop a neurography that shall, so far as possible, correspond to the endarchy of science.

\section{p. 44.)}

* 'Instincts are the prime movers of all human activity.' (Social Psychology,

$\dagger$ A young child would not mention this emotion by name, but would probably describe it by saying that its object 'looked so funny' or (if the child were a little older) 'appeared so strange.'

$\ddagger$ He gives several accounts; or, rather, he describes the same feelings and motives in several different ways. That which follows in the text strikes the keynote of all the rest; and is, moreover, the one which Poincare himself puts first. From some of the others we shall quote in the following pages. The reader who desires still more evidence that Poincaré's observations confirm our generalisation (in italics on p. 248) is referred to Poincaré's book, Science and Method.

$\S$ Loc. cit. p. 22. II In italics on p. 248 above. 
Originally we followed Poincaré in describing our experience of nature as experience of a 'hierarchy of facts.'* For him, as for us, the 'nature' that is studied is a (complete) endarchy of fact-elements which are gradually discovered and organised in a growing (incomplete $\dagger$ ) endarchy as the study proceeds. Indeed, his complete endarchy and ours have much in common; and so have the processes by which, as discovery proceeds, the partial $\ddagger$ endarchies are gradually built up. The resemblance between these endarchies ('nature' in process of discovery), and between the processes (by which nature is discovered or 'studied'), as envisaged by Poincaré on the one hand, and as we have described them on the other, must now be examined more closely.

The hierarchy of which Poincare speaks, like the endarchy of science that we have described, is a mode of conceiving the facts of experience as an 'organised body of connected facts.' § 'Harmonious 'ii is the word Poincare uses to describe the organisation of the endarchy of facts-for the most part still undiscovered-which is the world of experience. It is, therefore, already clear that Poincaré's conception of the world of experience completely accords, so far as it goes, with the endarchy of science that we have described. And it goes further than we have yet said. For the complete endarchy, as it is gradually discovered in the form of an incomplete endarchy, is, according to Poincaré, so designed as to make for 'economy of thought,...economy of effort' $\mid$ in thinking. It must, therefore**, have the form of a maximal endarchy, at each stage of its discovery. Poincaré's complete

* On p. 194 above; and Poincaré, loc. cit. p. I6.

$\dagger$ See above, footnote $\S$ on p. I99.

$\ddagger$ See above, p. I97.

$\S$ Cf. p. I96 above.

II For example; 'Our mind...would lose itself in the complexity of the world if that complexity were not harmonious.' (Loc. cit. p. 30; italics mine.)

Or again; 'What I mean [by the beauty of nature] is that more intimate beauty which comes from the harmonious order of its parts, and which a pure intelligence can grasp.... This special beauty, the sense of the harmony of the world....' (Loc. cit. p. 22 ; italics mine.)

Once more, Poincaré refers to "this feeling, this intuition of mathematical order, which enables us to guess hidden harmonies and relations.' (Loc. cit. p. 50; italics mine.)

The same idea is expressed, although the word harmony (which we have italicised in each of these quotations) is not used, when Poincare speaks of the facts that interest physicists as being 'those that have an analogy with many other facts and do not appear to us as isolated, but as closely grouped with others. ...The isolated fact attracts the attention of all, of the layman as well as the scientist. But what the true scientist alone can see is the link that unites several facts which have a deep but hidden analogy '-i.e. a connexion, as yet undiscovered, in the complete endarchy. (Loc. cit. p. 27.)

Tा Loc. cit. p. 23.

** The proof of our minimum-path proposition (on pp. 208 to 2 Io above) is given in Appendix C, on p. 492 below. 
endarchy-the world of experience, as he conceives it-is therefore a maximal endarchy. The resemblance between nature, as Poincaré conceives it, and our endarchy of science is therefore as complete as could be desired*.

It remains to examine the resemblance between the process by which Poincare observed that nature is studied or discovered, and the process by which we have described the (incomplete) endarchy of science as being gradually built up on the lines of the complete endarchy as that is gradually discovered. For our purpose it will suffice to note that, just as we began with the abstraction of essences $\dagger$, so Poincaré begins with the selection $\ddagger$ of simple§ facts; just as we subsequently abstracted or selected the most general essences, which were the most important or valuable for our purpose the greater the number of facts they united |, so Poincaré observes that the general $\mid$ facts or laws are the most valuable for selection, and that their value depends upon the number of previously known elements which they

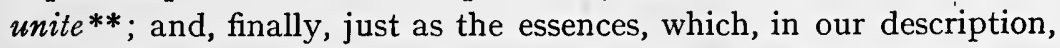
were successively abstracted, were such as to combine with those previously abstracted so as to form a single maximal endarchy, so Poincare observed that the instinct (or, as he puts it, the emotion) which drives men to discover the harmonious endarchy of science

* In case confirmatory evidence be required, Poincaré supplies it by saying that the 'economic'-or, as we should say, 'minimum-path,' and therefore 'maximal'-endarchy that the study of nature tends gradually to build up, produces the same feeling of 'beauty' (p. 23) or 'aesthetic satisfaction' (p. 3I) as is produced by the complete endarchy which is the world of experience; so that this complete endarchy is presumably a maximal endarchy. His words are: 'economy of thought, that economy of effort which...is the constant tendency of science, is a source of beauty as well as a practical advantage. The buildings we admire are those in which the architect has succeeded in proportioning the means to the end, in which the columns seem to carry the burdens imposed on them lightly and without effort, like the graceful caryatids of the Erechtheum.' (Loc. cit. p. 23.) And again: 'This aesthetic satisfaction' - 'the sentiment of mathematical elegance'- 'is consequently connected with the economy of thought. Again the comparison with the Erechtheum occurs to me, but I do not wish to serve it up too often.' (Loc. cit. p. 3I.)

$\dagger$ See above, p. 197.

$\ddagger$ 'We cannot know all the facts....We must make a selection.' (Loc. cit. p. 15.) 'Discovery is discernment, selection.' (Loc. cit. p. 51.)

$\S$ 'Our selection should be made [of] the most interesting facts,...those which have a chance of recurring.... Which, then, are the facts which have a chance of recurring? In the first place, simple facts.' (Loc. cit. pp. I7, I8.)

II See above, p. 199 .

If 'It is necessary, therefore, to think for those who do not like thinking, and as they are many, each one of our thoughts must be useful in as many circumstances as possible. For this reason, the more general a law is, the greater is its value. This shows us how our selection should be made.' (Loc. cit. p. 17.)

** 'If a new result is to have any value, it must unite elements long since known...and suddenly introduce order....' (Loc. cit. p. 30.) 
'makes us select the facts best suited to contribute to this harmony.'*

It appears then that by the 'study of nature,' in the passage which we quoted on p. 249, Poincaré means nothing else than the organisation of thought on the lines of the endarchy of science that we have described; or, what amounts to the same thing, the development in the student's brain of a neurography that corresponds to this endarchy as closely as possible.

We have next to prove that the aesthetic pleasure which Poincare observed the scientist to take in the beauty of nature is no other than the feeling of pleasurable satisfaction that results when the curiositywonder process achieves its end. We first remark that the 'beauty' of nature which gives rise to the feeling observed by Poincare is 'beauty which comes from the harmonious order of its parts.' $\dagger$ The feeling caused by this beauty has therefore the same origin as the 'feeling of elegance' of which Poincaré speaks as being felt by mathematicians who 'attach a great importance to the elegance of their methods and of their results.' $\ddagger$ For that which 'gives us the feeling of elegance in a solution or a demonstration' is again 'the harmony of the different parts, their symmetry, and their happy adjustment; it is, in a word, all that introduces order, all that gives them unity....' $\S$ Moreover, the 'beauty' and the 'elegance' in question are not merely the same in origin; they are, for Poincarés purpose, synonymous. For example, he asks 'what are the mathematical entities to which we attribute this character of beauty and elegance, which are capable of developing in us a kind of aesthetic emotion'? And again, on the same page he writes of 'the feeling of mathematical beauty, of the harmony of numbers and forms and of geometric elegance.' $\|$

So the pleasure which the scientist takes in the beauty of nature, and which, according to Poincaré, causes him to study nature, is the same as the mathematicians' 'feeling of elegance.' But 'elegance,' says Poincaré, 'may result from the feeling of surprise caused by the unlooked-for occurrence together of objects not habitually

* Loc. cit. p. 22. Cf. also, on p. 59, where, after discussing what kind of combination of facts constitutes the kind of discovery to which the same instinct (or, as he says, emotion) drives mathematicians, Poincaré proceeds: 'Now, what are the mathematical entities to which we attribute this character of beauty and elegance, which are capable of developing in us a kind of aesthetic emotion? Those whose elements are harmoniously arranged so that the mind can, without effort, take in the whole without neglecting the details. This harmony is at once a satisfaction to our aesthetic requirements, and an assistance to the mind which it supports and guides.' (Loc. cit. p. 59.)

$\dagger$ Loc. cit. p. 22.

$\S$ Loc. cit. pp. 30, $3 \mathrm{r}$.

$\ddagger$ Loc. cit. p. 30 .

il Loc. cit. p. 59. 
associated '*; in which case the 'feeling of elegance' evidently follows wonder $\uparrow$ (which Poincaré calls 'surprise'). Or elegance may result from 'contrast' $\ddagger$; in which case it again follows the appropriate stimulus to wonder. Poincaré sums up both cases in the words: 'Briefly stated, the sentiment of mathematical elegance is nothing but the satisfaction due to some conformity between the solution we wish to discover and the necessities of our mind.' $§$ These 'necessities' must mean 'instinct': indeed, Poincaré himself uses the word when he speaks of the 'instinctive and unacknowledged preoccupation' $\|$ that drives the scientist to search for truth, and again when he refers to 'our natural instinct for mathematical elegance.' I And there is no instinct that can be meant except curiosity. It follows that the pleasure which, according to Poincaré, the scientist takes in the beauty of nature, and which attracts him to study nature, is a feeling due to the satisfaction of his curiosity.

Poincaré adds that 'this aesthetic satisfaction is consequently connected with the economy of thought.'** We now see how. For the 'aesthetic satisfaction' follows the success of the curiosity-wonder process in organising thought in a maximal endarchy, which we have seen $\dagger \dagger$ to be the most economical organisation possible. It remains to add that we shall in future use the phrase 'aesthetic satisfaction' only to denote the pleasurable feeling that results when the instinct of curiosity achieves its end + .

* Loc. cit. p. 3 I.

$\dagger$ Because it follows the appropriate stimulus to wonder: see above, p. 92.

\$ The contrast between the simplicity of the means and the complexity of the problem presented.' (Loc. cit. p. 31.)

§ Loc. cit. p. 31. (Italics mine.)

II The whole passage is worth quoting, for we shall have to refer to it again. It follows closely on that quoted on p. 249 and it reads: 'If nature were not beautiful it would not be worth knowing, and life would not be worth living. I am not speaking, of course,... of the beauty of qualities and appearances. I am far from despising this, but it has nothing to do with science. What I mean is that more intimate beauty which comes from the harmonious order of its parts, and which a pure intelligence can grasp....It is, then, the search for this special beauty, the sense of the harmony of the world, that makes us select the facts best suited to contribute to this harmony; just as the artist selects those features of his sitter which complete the portrait and give it character and life. And there is no fear that this instinctive and unacknowledged preoccupation will divert the scientist from the search for truth. We may dream of a harmonious world, but how far it will fall short of the real world! The Greeks, the greatest artists that ever were, constructed a heaven for themselves; how poor a thing it is beside the heaven as we know it!' (Loc. crt. pp. 22, 23.)

II Loc. cit. p. 60.

** This is the next sentence to that last quoted from p. 31 .

†† On pp. 208 to 210 and Appendix C, p. 492.

$\ddagger \ddagger$ Aesthetic satisfaction is therefore the feeling that emerges whenever anything that has aroused curiosity, or appeared wonderful, has at last been understood. The understanding for which curiosity strives means the harmonious arrangement 
We have thus verified that Poincaré's observations agree as closely as could be desired with the conclusion* which we deduced from our account $\uparrow$ of the normal stimulus to curiosity-wonder. Our conclusion was that the instinct-emotion of curiosity-wonder urges men to build up their own neurographies in the form of single maximal endarchies corresponding to the endarchy of science, and so to discover that endarchy.

-according to the endarchy of science-of one's ideas about the object of one's curiosity; and the harmony is not only of these ideas with one another (so as to form a scientific endarchy on their own account) but also of the corresponding neurograms with the whole of one's neurography.

* In italics on p. $24^{8}$ above.

$\dagger$ On p. 92 above. 


\section{CHAPTER 14}

\section{REASONING; AND THE VOLUNTARY DEVELOPMENT \\ OF SINGLE WIDE INTERESTS}

\section{$\S \mathrm{r}$. Will and Curiosity.}

WhILE, however, curiosity-wonder makes men want to organise thought in this way, it does not follow that the organisation of thought is a wholly involuntary process. We have, on the contrary, already* pointed out that the intervention of the Will may be necessary to complete the process and to achieve the goal towards which the instinct strives. To this point we now return and ask: How exactly does the Will cooperate with the instinct of curiosity to organise thought in the way we have described?

To answer this question, we take up again the main thread of our argument. Having formulated certain laws that 'govern' involuntary thought, we went on to consider voluntary thinking and so came to the effect of Will in everyday life $\dagger$. Will, we said, intervenes when the involuntary guides of the stream of thought are in conflict $\ddagger$; its intervention makes new paths in the thinker's neurography and, of these new paths, those which yield the ideal solution of a conflict-those which, as Poincare has it, are at once the most

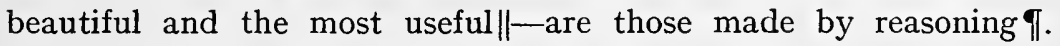
Then we saw that, among other characteristics of reasoning, every step must be true**; and we found this to mean that the new paths made in anybody's neurography as he reasons, must correspond, element for element, with the endarchy of science. Finally, we have just seen that curiosity moves men to strive for this correspondence; but curiosity alone may not achieve it without the Will's aid.

For example, if two elements of anybody's-Jones'-neurography belong to two different maximal endarchies and are both excited at the same time, the excitement will tend to spread to the central elements, $E_{P}$ and $E_{Q}$ say, of each endarchy; and curiosity-wonder will make him want to connect these central elements by a path of

* See above, p. 248.

$\ddagger$ See above, Pp. I4I and 162 .

\| Cf. Poincaré, loc. cit. p. 59.

$\dagger$ See above, Chapter $7, \S 6$.

$\S$ See above, p. 165.

* See above, p. I 80 .

II See above, pp. I75 and $\mathbf{r} 79$. 
linked neurogram-elements which correspond to the chain of successive essences that connect $\mathrm{E}_{P}$ with $\mathrm{E}_{Q}$ in the endarchy of science. Sometimes this connexion may be made unconsciously; or, at all events, without any effort of Will; as, for example, when $E_{P}$ is directly connected to $\mathrm{E}_{Q}$ in the endarchy of science. But, more often, reasoned thought (involving the Will's intervention*) is required to complete the connexion. Indeed, most of us know only too well that curiosity alone, without voluntary effort, will enable us neither to organise our neurographies so as to correspond with discovered portions of the endarchy of science nor to discover new regions of that endarchy. In other words, without an effort of Will we can neither acquire existing knowledge (to any considerable extent) nor discover new knowledge.

We may distinguish two occasions when the Will may support the tendency of curiosity-wonder to connect two (hitherto separate but simultaneously excited) neurograms, $A$ and $Z$, by means of a series of connected neurogram-elements that correspond to essences or factelements in the endarchy of science. The first of these occasions-the first bout of voluntary thinking, the first period of conscious workcommonly precedes the instinctive (curiosity-wonder) process, while the second follows the completion of the process $\dagger$.

\section{§2. The First Period of Conscious Work.}

The first bout of voluntary thinking has the effect of increasing the activity both of $A$ and $Z$, as the thinker, in his efforts to complete the path from $A$ to $Z$, comes back, over and over again, both to $A$ and to $Z$, that he may begin afresh to make a path that shall connect with whatever path he has made from the other end. Now we have

* 'Reasoning is pre-eminently a process of voluntary (willed) thinking': see above, p. I 87 .

$\dagger$ Poincaré quotes examples from his personal experience-and similar examples might be multiplied from the experience of other mathematicians and physicists-shewing how the missing link in a chain of reasoning, which intense and prolonged efforts had failed to complete, would unexpectedly present itself during a holiday excursion or when 'crossing the street,' and would stand the test of subsequent concentrated thought. Such inspirations are, Poincaré supposes (p. 6o), due to the involuntary, or even unconscious, operation of that which we have identified with the curiosity-wonder process. And he adds: 'There is another remark to be made regarding the conditions of this unconscious work, which is, that it is not possible, or in any case not fruitful, unless it is first preceded and then followed by a period of conscious work. These sudden inspirations are never produced (and this is sufficiently proved already by the examples I have quoted) except after some days of voluntary efforts which appeared absolutely fruitless, in which one thought one had accomplished nothing, and seemed to be on a totally wrong track. These efforts, however, were not as barren as one thought; they set the unconscious machine in motion, and without them it would not have worked at all, and would not have produced anything.' (Loc. cit. p. 56.) 
said* that the stimulus to curiosity is greater, the more familiar are the two facts, $A$ and $Z$, between which the instinct strives to make connexions. The repeated excitement of $A$ and $Z$ during the first bout of voluntary thinking (or, as Poincaré calls it, 'conscious work') therefore increases the stimulus to the subsequent instinctive process (or 'unconscious work'). Indeed, Poincaré has' observed that, with this increased stimulus, the instinctive process often plays a most important part in mathematical discovery, when, without it, the 'unconscious machine...would not have produced anything.' $\dagger$ Moreover, we have already pointed out the important involuntary effect exercised upon the reasoning process by the activity of the terminal elements + . These, so long as they remain active, guide the excitement towards themselves or, intermediately, towards neurograms with which they are connected. 'As a rule,' said William James, $Z$ (and similarly, $A$ ) 'overshadows the process from the start.' $\S$ And the active final neurogram, $Z$, overshadowing the reasoning process by which a thinker is to connect an idea of $A$ to an idea of $Z$, determines which particular essence-an essence belonging to which particular subject endarchy-of $A$ he will use in beginning to reason about that fact. For example, according to the purpose of his reasoning about A, his excitement may first be restricted to a physical science group of meanings, in which case the essence $E_{A}$ (of $A$ ) from which he starts will belong to a physical science branch endarchy.

\section{§3. The Interval of Unconscious Work.}

After the first period of conscious or willed work, comes the instinctive process\|. It may be an unconscious process, as in the examples given by Poincaré from his experience of mathematical discovery, examples that might be multiplied indefinitely. And this unconscious process may suddenly burst in upon consciousness, now occupied by quite other matters, with an inspiration-a vivid conviction-that here is the reasoned path between $\mathrm{A}$ and $Z$ that was sought unsuccessfully during the first period of conscious work. How

* See above, p. 92 .

$\dagger$ Loc. cit. p. 56. See footnote $t$ on p. 256.

$\ddagger$ Above, p. 180 . We were there referring only to one terminal element (an element of $Z$ ), but what we said is equally true of the other (an element of $A$ ) also.

$\S$ Loc. cit. Vol. II, p. 338.

|| The following two pages, as far as the end of the section on p. 259, form a digression from our present subject, namely, the function of voluntary thinking in the development of single wide interest-systems. But the matters dealt with in this digression can be most conveniently considered at this point.

II Loc. cit. pp. 52 to 55 .

G. E. 
are we to account for the fact* that the solution-a solution which, as we shall see in a moment, is generally the true solution-of our problem thus finds its way into the focus of consciousness, when all the rest of the process, perhaps including tentative solutions, remained unconscious? The increase of excitement that accompanies this stage of the process at once suggests itself as the answer; and for two reasons. In the first place, the instinct is just achieving the goal of its conation, so that we should expect the accompanying emotional excitement to be greatest. And secondly, the final link in the chain of elements that connects $A$ to $Z$ unites these two active systems, draining excitement from them both $\dagger$. Indeed, this final link commonly corresponds to the highest, or most central + , or most valuable, of all the fact-elements or essences in the endarchy of science, that form the corresponding path of connexion between $\mathrm{A}$ and $Z$ in that endarchy. 'It is certain,' says Poincaré, 'that the combinations which present themselves to the mind in a kind of sudden illumination after a somewhat prolonged period of unconscious work are generally useful and fruitful' $\S$-or, as we have said, valuable.

When one of these sudden illuminations gives us the clue to the solution of our problem, and indeed makes the whole thing that had puzzled us by its complexity seem marvellously clear and simple, we feel inspired, and as if our mental processes were altogether above their normal level. And, since these sudden inspirations often follow an unconscious process that has revealed a connexion (or, as Poincaré says, a 'combination') which the preceding period of conscious work failed to disclose, are we to say that there is an unconscious ego, or a subliminal ego, that is 'superior' to the conscious egoll? Are we to follow Mr Kenneth Richmond and describe the thought processes, which lead to these inspirations, as 'superconscious' '?? We shall not use these words, but we do well to recognise the truth which they are intended to express. It is true that creative thinking, marked by the

* Poincaré records the fact and regards it as 'most mysterious.' (Loc. cit. p. $5^{8 .)}$

$\dagger$ By 'multiple stimulation.' Cf. above, pp. 83 and $\mathbf{3} 34$.

$\ddagger$ Cf. pp. 221 and 247 above. $\$$ Loc. cit. p. $5^{8}$.

il Poincaré, after reviewing the facts which we have summarised in footnote $\dagger$ on p. 256 above, asks approximately the same question; and he adds that he would be loth to accept an affirmative answer. (Loc. cit. pp. 57, 58.) Dr Morton Prince is of the same opinion. (See The Dissociation of a Personality, p. 85.)

If Education and Liberty (1918), p. 221. Again, on p. 237, he writes: 'It is instructive that in the time of the Gothic...buildings were produced which were not only miracles of art but miracles of engineering. It is impossible not to conclude that the extraordinarily complex strains and stresses of Gothic architecture were superconsciously worked out.' 
'discovery' of valuable combinations and so making important progress towards the goal of scientific thought, is often-and perhaps only-accomplished when active consciousness is somehow sidetracked: when, in fact, the Will is not intervening in the train of thought in question, and when there is no interference by extraneous thought-activities, whether due to active interest-systems or to incoming sense-impressions. This removal of normal inhibitions*-if we may so summarise the description we have just given of the conditions that favour creative thinking-occurs when conscious thought is occupied with something quite different from the problem whose solution is being unconsciously discovered. It also occurs when we consciously fix our attention on some object, whether a windmill on the horizon or a pattern on the carpet, so as to leave the involuntary (but now conscious) creative train of thought free from interference. Our ability to secure this freedom depends in part upon the extent to which we have previously succeeded in exciting the activity of. $A$ and $Z$ whose connexion we are seeking to effect; and partly upon the size and activity of extraneous interest-systems. It is doubtless for this latter reason that, as one of the most famous of British physicists has remarked, it is as a young graduate with no responsibilities and no anxieties that one has one's best chance of making discoveries. But let no one suppose that, in attributing our moments of inspiration to the free working of the curiosity-wonder instinct, we are debasing what is noble or confusing the higher with the lower, the 'superconscious' with the subconscious. Because, in various degrees, we share with lower animals some of our emotions, we cannot argue that none of them affects our highest thought-processes. Love $\left(a^{\prime} y a^{\prime} \pi \eta\right)$ itself is an emotion; and there is nothing absurd or inconsistent in the suggestion that divine inspiration may operate by the means that we have described as 'curiosity-wonder.' $\dagger$

\section{$\S 4$. The Second Period of Conscious Work.}

The second occasion $\ddagger$ for the intervention of the Will, in support of the tendency of curiosity-wonder to secure the connexion of $A$ to $Z$, follows, we said, the completion of the instinctive process. It is needed 'to work out the results of the inspiration, to deduce the immediate consequences and put them in order and to set out the

* Cf. K. Richmond, loc. cit. p. 239.

$\dagger$ Cf. K. Richmond: 'If any impatient Christian wants to know why I am leaving divine inspiration out of account, I will explain that I am doing nothing of the kind. I am trying to find out how it works.' (Loc. cit. p. 25I.)

$\ddagger$ See above, p. 256. 
demonstrations; but, above all, it is necessary to verify them.'* For although 'when a sudden illumination invades the mathematician's mind, it most frequently happens that it does not mislead him...it also happens sometimes...that it will not stand the test of verification.' $\dagger$ Hence the need for attempting to verify an inspiration during the second period of conscious thought that should follow it. The inspiration is, for this purpose, voluntarily taken as a hypothesis. If, for example, we have an inspiration that $\mathrm{E}_{M}$ is the fact, or rather the essence, we are seeking to complete the connexion between $\mathrm{A}$ and $Z$, we have to verify, during the second period of conscious thought, every step of the reasoning by which $E_{M}$ is connected to some essence $\mathrm{E}_{A}$ of $\mathrm{A}$ on the one hand and to some essence $\mathrm{E}_{Z}$ of $Z$ on the other. Let us follow the verification of some of these steps $\ddagger$. Suppose that it is Jones who has had the inspiration that $\mathrm{E}_{M}$ is the fact (essence) he is seeking to connect $\mathrm{E}_{A}$ with $\mathrm{E}_{Z}$, and let $E^{\prime}{ }_{M}, E^{\prime}{ }_{A}$ and $E^{\prime}{ }_{Z}$ denote Jones' neurograms for the essences $\mathrm{E}_{M}, \mathrm{E}_{A}$ and $\mathrm{E}_{Z}$ respectively. Then, as we saw§, each of the neurogram-elements $E_{A}^{\prime}, E^{\prime}{ }_{M}$ and $E^{\prime}{ }_{Z}$ may correspond to other essences in the endarchy of science in addition to the one essence, $\mathrm{E}_{A}$ or $\mathrm{E}_{M}$ or $\mathrm{E}_{Z}$, but may not correspond to other essences instead of $\mathrm{E}_{A}$ or $\mathrm{E}_{M}$ or $\mathrm{E}_{Z}$ respectively. Suppose further that $E_{P}^{\prime}, E^{\prime}{ }_{Q}$ and $E_{R}^{\prime}$ denote three neurogramelements which are excited in succession as Jones' thought follows the connexion in his brain between $E_{A}^{\prime}$ and $E^{\prime}{ }_{Z}$. In order to verify the argument by which he connects $\mathrm{E}_{A}$ to $\mathrm{E}_{Z}$, Jones must not only (I) verify every step in the argument but also (2) verify that the several steps-and, in particular, the successive steps-hang together and lead somewhere.

First, to verify that every step in the argument is true by itself, Jones has to make sure that every two successive neurogram-elements, say $E_{P}^{\prime}$ and $E^{\prime}{ }_{Q}$, are connected by no mere fortuitous association, but in the intimate way described on p. I98. In other words, $E_{P}$ and $\mathrm{E}_{Q}$ are essences $\|$ one of which is directly or indirectly derived $\boldsymbol{\|}$ from the other; or, what is, perhaps a simpler way of expressing the same thing, $T_{P}$ and $T_{Q}$ are 'simple facts '** one of which contains or includes

* Poincaré, loc. cit. p. 56.

$\dagger$ Poincaré, loc. cit. p. .6o.

$\$$ Fig. Io on p. 178 illustrates some of the steps by which $A$ is connected with $Z$ and may be referred to in this connexion.

$\S$ On p. 233 above.

$\| \mathrm{E}_{P}$ and $\mathrm{E}_{Q}$ are related to $E^{\prime}{ }_{P}$ and $E^{\prime}{ }_{\ell}$ just as $\mathrm{E}_{A}$ (or $\mathrm{E}_{M}$ or $\mathrm{E}_{z}$ ) is related to $E_{A}^{\prime}$ (or $E_{\mu}^{\prime}$ or $E_{z}^{\prime}$ ).

** The definition of a simple fact is given on p. 203. The notation $\left(\mathrm{T}_{P}\right.$ and $\left.\mathrm{T}_{Q}\right)$ is explained there and on p. 207, and the diagrammatic representation of a 'simple fact' is stated on p. 213 . 
the other. And this relation between portions of the endarchy of science itself is, of course, true.

Secondly, having verified that successive neurogram-elements are thus connected in pairs-for example, $E_{P}^{\prime}$ to $E^{\prime}{ }_{Q}$-according to the endarchy of science, Jones has still to make sure that each step in his argument (corresponding to a single pair, $E_{P}^{\prime}$ and $E_{Q}^{\prime}$ say) is related to the next step (corresponding to the connexion between $E_{Q}^{\prime}$ and $E_{R}^{\prime}$ ), so that these successive steps shall lead somewhere definite. That is to say, Jones has to make sure that some definite relation obtains between every three successive undivided neurograms, as well as between every two. For example, if the relation between $\mathrm{T}_{P}$ and $\mathrm{T}_{Q}$, namely

$$
\mathrm{T}_{P} \text { is (contained by or containing) } \mathrm{T}_{Q}
$$

is related to the relation between $\mathrm{T}_{Q}$ and $\mathrm{T}_{R}$, namely

$$
\mathrm{T}_{Q} \text { is (contained by or containing) } \mathrm{T}_{R}
$$

by the condition that the first, or else the second, alternative shall be taken in both (I) and (2), then the successive steps make a path leading straight downwards or outwards (if the first alternative be selected, or straight upwards or inwards if the second be chosen) in a direct line, such as $\mathrm{E}_{k_{1} k_{2} \cdots k_{p}} \mathrm{E}_{k_{1} k_{2} \cdots k_{p} k_{p+1}} \mathrm{E}_{k_{1} k_{2} \cdots k_{p} k_{p+2}} \ldots$ in the endarchy of science. Moreover, in this case (I) and (2) reduce to one type of deductive syllogism; for, taking the first (or descending) alternative, (I) and (2) may be written

and

$$
\left.\begin{array}{ll}
\mathrm{T}_{P} \text { is (contained by) } & \mathrm{T}_{Q} \\
\mathrm{~T}_{Q} \text { is (contained by) } & \mathrm{T}_{R} \\
\mathrm{~T}_{P} \text { is (contained by) } & \mathrm{T}_{R}
\end{array}\right\}
$$

therefore

and, taking the second (or ascending) alternative, (I) and (2) become

and

$$
\left.\begin{array}{l}
\mathrm{T}_{P} \text { is (containing) } \mathrm{T}_{Q} \\
\mathrm{~T}_{Q} \text { is (containing) } \mathrm{T}_{R} \\
\mathrm{~T}_{P} \text { is (containing) } \mathrm{T}_{R}
\end{array}\right\}
$$

therefore

If then the major and minor premises (i.e. the first two lines) of (3) hold for every three successive simple facts in Jones' reasoning, and so relate every step (connecting two successive simple facts) to its successor, he may be sure that his train of reasoning is leading straight down a direct line in the endarchy of science from one essence $\left(E_{M}\right.$

* The words 'contained by' are placed in brackets so as to conform to the general usage, according to which the elements of a syllogism are connected by the word 'is.' 
perhaps) to another (say $\mathrm{E}_{Z}$ ) that is indirectly derived from it*. Similarly, if the major and minor premises of (4) hold for every three successive simple facts in Jones' reasoning, his train of reasoning is following straight up a direct line of the endarchy of science, perhaps from $\mathrm{E}_{A}$ to $\mathrm{E}_{M}$.

We may remind ourselves here that the essence which comes as an inspiration-the result of the working of the curiosity-wonder process-to complete the connexion between $\mathrm{A}$ and $\mathrm{Z}$, is commonly the highest or most central $\uparrow$ essence $E_{M}$ in the endarchy of science path from $\mathrm{E}_{A}$ to $\mathrm{E}_{Z}$. In that case $\mathrm{E}_{M}$ is connected both to $\mathrm{E}_{A}$ and to $\mathrm{E}_{Z}$ by directly descending paths, so that Jones will have to use only deductive reasoning to connect his inspiration with $\mathrm{E}_{A}$ and with $\mathrm{E}_{Z}$. Thus it is that, in a passage we have already $\ddagger$ quoted, Poincaré speaks of its being necessary to deduce the consequences of the inspiration during the second period of conscious thought.

But it is only when two essences-say $\mathrm{E}_{A}$ and $\mathrm{E}_{Z}$ - of the endarchy of science are such that one of them is directly or indirectly derived from the other, that the path connecting them is wholly a direct ascending or descending line, in which every three successive elements are connected by deductive reasoning. As a rule, the direct path between any two essences- $\mathrm{E}_{A}$ and $\mathrm{E}_{Z}$ - -selected at random from the endarchy of science, consists of two wholly ascending or descending lines united in the most central essence, $E_{M}$, of the whole path. Then $\mathrm{E}_{A}$ may be connected with $\mathrm{E}_{M}$ by deductive reasoning, and so may be $\mathrm{E}_{M}$ with $\mathrm{E}_{Z}$. But the same is not true of the three successive essences with $\mathrm{E}_{M}$ in the middle. Suppose that these are $\mathrm{E}_{L}, \mathrm{E}_{M}$ and $\mathrm{E}_{N}$, where $\mathrm{E}_{L}$ forms part of the descending path from $\mathrm{E}_{M}$ to $\mathrm{E}_{A}$, and $\mathrm{E}_{N}$ forms part of the descending path from $\mathrm{E}_{M}$ to $\mathrm{E}_{Z}$. Then we know that the simple fact $\mathrm{T}_{M}$ forms part of, or is contained by, $\mathrm{T}_{L}$ and all its derivatives including $\mathrm{T}_{A}$, and that $\mathrm{T}_{M}$ also forms part of $\mathrm{T}_{N}$ and all its derivatives including $\mathrm{T}_{z}$. And this is all we know of the connexion between $\mathrm{T}_{L}$ and $\mathrm{T}_{N}, \mathrm{E}_{L}$ and $\mathrm{E}_{N}$, or $E^{\prime}{ }_{L}$ and $E_{N}^{\prime}$.

This series of relation may be put in the form of an inductive syllogism thus

* For, if $\mathrm{T}_{p}$ represents the simple fact $\mathrm{E} \mathrm{E}_{k_{1}} \mathrm{E}_{k_{1} k_{2}} \ldots \mathrm{E}_{k_{1} k_{2} \ldots k_{p}}$, it follows from the first line of (3) that $\mathrm{T}_{Q}$ represents the simple fact $\mathrm{E} \mathbf{E}_{k_{1}} \mathrm{E}_{k_{1} k_{2}} \ldots$ $\mathrm{E}_{k_{1} k_{\mathbf{2}} \ldots k_{p}} \ldots \mathrm{E}_{k_{1} k_{2} \ldots k_{p} \ldots k_{y}}$; and, from the second line of $(3)$, that $\mathrm{T}_{R}$ represents

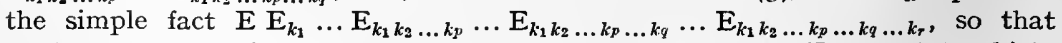
the lowest essences $\mathrm{E}_{p}\left(\equiv \mathrm{E}_{k_{1} k_{2} \ldots k_{p}}\right), \mathrm{E}_{\ell}\left(\equiv \mathrm{E}_{k_{1} k_{2} \ldots k_{\eta}}\right)$ and $\mathrm{E}_{R}\left(\equiv \mathrm{E}_{k_{1} k_{2} \ldots k_{r}}\right)$, to which, inter alia, $E_{P}^{\prime}, E^{\prime}{ }_{\ell}$ and $E_{R}^{\prime}$ correspond, form a descending, but not necessarily consecutive, series of elements in the same direct line, namely $\mathrm{E} \mathrm{E}_{k_{1}} \mathrm{E}_{k_{1} k_{2}} \ldots$ $\mathrm{E}_{k_{1} k_{2} \ldots k_{p}} \ldots \mathrm{E}_{k_{1} k_{2} \ldots k_{q}} \ldots \mathrm{E}_{k_{1} k_{2} \ldots k_{r}} \ldots$.

$\dagger$ See above, p. 258 . See also p. 222 above.

$\ddagger$ See pp. 259, 260 above. 
and every (derivative of)

\section{$T_{M}$ is (contained by) $T_{L}$}

$T_{N}$ is similar to $T_{M}$

therefore every (derivative of) $T_{N}$ is (similar to part of) $T_{L}$

where the words that are not included in the usual statement of the syllogism are enclosed in brackets. If Jones, in attempting to verify the connexion he has made between $\mathrm{E}_{A}$ and $\mathrm{E}_{Z}$, finds that three consecutive neurogram-elements, $E^{\prime}{ }_{L}, E^{\prime}{ }_{M}$ and $E^{\prime}{ }_{N}$, are related to each other by means of the first two lines of (5), he may rest assured that the path in the endarchy of science ascends in a direct line from $\mathrm{E}_{L}$ to $\mathrm{E}_{M}$ and descends in a direct line from $\mathrm{E}_{M}$ to $\mathrm{E}_{N}$; but, as before, $\mathrm{E}_{L}, \mathrm{E}_{M}$ and $\mathrm{E}_{N}$ are not necessarily adjoining essences in the endarchy of science.

If we are asked why we have expressed the relation between three essences $E_{L}, E_{M}$ and $E_{N}$, such that $E_{M}$ is the most central essence in the direct path from $E_{L}$ to $E_{N}$, in the form (5), resembling an inductive syllogism, our answer is threefold. In the first place, this is a convenient form in which to express the relationship. In the second place, every three successive essences $\left(\mathrm{E}_{L}, \mathrm{E}_{M}\right.$ and $\left.\mathrm{E}_{N}\right)$ in the endarchy of science-every three essences, that is, such that the mean $\left(\mathrm{E}_{M}\right)$ is directly connected to each of the extremes $\left(\mathrm{E}_{L}\right.$ and $\left.\mathrm{E}_{N}\right)$ are related either by deductive reasoning, expressed in the first two lines of (3) or of (4), or by the first two lines of (5), which it is therefore natural to identify with induction. And, thirdly, while our usual (conscious) reasoning, as we have illustrated it in Fig. Io on p. I78 and the accompanying text, is wholly deductive in character and follows either an ascending or a descending line in the endarchy of science, the link in a chain of reasoning that is commonly furnished by the involuntary, or even unconscious, working of the curiosity-wonder process-the step, in short, that seems to come as an inspiration-is the transitional one between ascending and descending *. What could therefore be more natural than to oppose the relations in (5) (due to the instinctive process) to those in (3) or (4) (due to ordinary conscious reasoning); and, as the latter represent deduction, to say that the former represents induction $\uparrow$ ?

* See above, p. 258. Cf. also pp. 222, 247.

$\dagger$ Cf. K. Richmond: 'The deductive process is the lower process, because there is nothing creative about it....I am here impelled to rush in where the framers of definitions have feared to tread, and to assert that pure inductive thought is, simply, intuitive thought, or superconscious thought. It is not "the method of reasoning from particulars to generals" as my dictionary puts it; that is the deductive process following, to check or confirm, the first inductive leap.' (Loc. cit. pp. 22 I, 222.) 
If our reasoner, Jones, seeks a connexion between $\mathrm{E}_{A}$ and $\mathrm{E}_{Z}$ in the most general case (where neither $\mathrm{E}_{A}$ nor $\mathrm{E}_{Z}$ is directly or indirectly derived from the other)*, and if the instinctive curiosity-wonder process gives him the highest connecting essence $\mathrm{E}_{M}$ as an inspiration, the rest of the connexion-from $\mathrm{E}_{A}$ to $\mathrm{E}_{M}$ and from $\mathrm{E}_{M}$ to $\mathrm{E}_{Z}$-is made by mere deductive reasoning, as illustrated in Fig. Io on p. I78. This deductive reasoning must, as we said, be performed and that is why the second period of conscious thought is required. 'All that we can hope from these inspirations '- $\mathfrak{F}_{M}$ 's sudden invasion of consciousness-' which are the fruits of unconscious work, is to obtain points of departure for such calculations.' $\dagger$ Moreover, if Jones' inspiration, of which the essence, pro hac vice, is $\mathrm{E}_{M}$, and which he afterwards takes for his hypothesis + , and connects by deductive reasoning with $\mathrm{E}_{\boldsymbol{A}}$ and $E_{Z}$, is outside that portion of the complete endarchy of science which has yet been discovered, while the terminal essences $\mathrm{E}_{A}$ and $\mathrm{E}_{Z}$ are inside it, Jones' connexion of $\mathrm{E}_{A}$ to $\mathrm{E}_{Z}$ through $\mathrm{E}_{M}$ not only extends his own scientific endarchy, but discovers a fresh portion of the endarchy of science.

\section{§5. An Illustration and a Digression.}

This process may be illustrated by a simple example. Professor R. W. Wood of Baltimore noticed the fact, till then unknown to science, that when heat was applied to a pellet of sodium inside a " * The words in brackets are, of course, equivalent to saying that $\mathrm{E}_{A}$ and $\mathrm{E}_{z}$ do not belong to the same simple fact (or are not in the same direct line, $\mathbf{E} \mathbf{E}_{k_{1}}$ $\mathrm{E}_{k_{1} k_{2}} \ldots \mathrm{E}_{k_{1} k_{2} \ldots k p} \mathrm{E}_{k_{1} k_{2} \ldots k_{p} k_{p+1}} \ldots$ ) of the endarchy of science.

$\dagger$ Poincaré, loc. cit. p. 62. The whole paragraph is worth quoting because of its insistence on the disorderly character of purely involuntary thought-a point to which we referred on p. 220 above and to which we shall shortly return-and on the consequent need for (willed) reasoning during the second period of conscious thought that we are now considering. The paragraph reads: 'It never happens that unconscious work' (involuntary thinking) 'supplies ready-made the result of a lengthy calculation in which we have only to apply fixed rules. It might be supposed that the subliminal ego, purely automatic as it is, was peculiarly fitted for this kind of work, which is, in a sense, exclusively mechanical. It would seem that, by thinking overnight of the factors of a multiplication sum, we might hope to find the product ready-made for us on waking; or, again, that an algebraical calculation, for instance, or a verification could be made unconsciously. Observation proves that such is by no means the case. All that we can hope from these inspirations, which are the fruits of unconscious work, is to obtain points of departure for such calculations. As for the calculations themselves, they must be made in the second period of conscious work which follows the inspiration, and in which the results of the inspiration are verified and the consequences deduced. The rules of these calculations are strict and complicated; they demand discipline, attention, will, and consequently consciousness. In the subliminal ego, on the contrary, there reigns what I would call liberty, if one could give this name to the mere absence of discipline and to disorder born of chance. Only this very disorder permits of unexpected couplings.'

$\ddagger$ Cf. p. 260 above. 
glass bulb from which the air had been exhausted, a brilliantly coloured film formed on the inside of the glass; and that the colours changed when the film was heated or cooled. How were these colours to be explained? Or, how was a connexion to be made in the (incomplete) endarchy of science between the (fact of the) cause and the (fact of the) effect? The connexion was made by first abstracting from the whole fact of the sodium pellet, $\mathrm{A}$, whatever its shape or size, the particular essence, $\mathrm{E}_{A}$, of its being a metal known to science; then abstracting from the whole fact of a many coloured film, $Z$, whatever its size or colour, the particular essence, $\mathrm{E}_{Z}$, of the measure of its absorption of the electromagnetic waves that constitute light varying with the length of the waves and so with the colour of the light; then, still in the first period of conscious thought, concentrating attention on $\mathrm{E}_{A}$ and on $\mathrm{E}_{Z}$; then receiving an inspiration (of which the pro hac vice essence was $\mathrm{E}_{M}$ ) that the coloured film was composed of an immense number of minute spheres of sodium that were piled on top of each other as the sodium vapour, produced by heating the pellet, condensed in tiny drops on the cold surface of the bulb, and that such a film would give rise to the colours and changes of colour observed-thus inductively connecting $\mathrm{E}_{A}$ with $\mathrm{E}_{z}$-; and, finally, a second and somewhat prolonged period of conscious thought, in which this inspiration was taken as a hypothesis* and which was then occupied in making, by deductive reasoning, an endarchy of science path, not only between $E_{A}$ and $E_{M}$ (which in this case was very simple), but also between $\mathrm{E}_{M}$ and $\mathrm{E}_{Z}$ (which was not quite so easy) $\dagger$.

It would not be difficult to find many more examples of the way in which the connexion between the terminal essences $\left(\mathrm{E}_{A}\right.$ and $\left.\mathrm{E}_{Z}\right)$, in the most general case of reasoning, is formed by an inspiration - the involuntary invasion of consciousness by an idea, $7 \boldsymbol{F}_{M}$, of the highest essence, $\mathrm{E}_{\boldsymbol{M}}$-followed by deductive reasoning to connect

* Cf. p. 260 above.

$\dagger$ To make the connexion between $\mathrm{E}_{\mathcal{A}}$ and $\mathrm{E}_{\mu}$, it was only necessary to verify that sodium vapour, in a vacuum, would condense in round drops, layer upon layer; but to make the connexion between $\mathrm{E}_{M}$ and $\mathrm{E}_{\mathrm{z}}$, it was necessary to calculate the coefficient of absorption of a medium made up of numbers of small metal spheres and to shew (I) that this coefficient varied with the wave length of the light, (2) that it was not the same as of the solid metal (for otherwise the film would have been throughout of the same colour as a thin film of metallic sodium, which was not the case), and (3) that it varied with the amount of metal per unit volume, thus altering as the film was heated or cooled. These experimental comparisons of the deductions from the hpothesis, $E_{M}$, with the actual films observed, may be described as 'action on the hypothesis $E_{M}$ with a view to its verification.' The calculations, and the whole reasoning, are given in two papers on 'Metallic Films' by the present writer (Garnett, Phil. Trans. Vol. 203 (1904), pp. 385-420 and Vol. 205 (1905), pp. 237-288). 
this highest essence with the terminal essences. Mr Kenneth Richmond finds that children in school can better understand deductive reasoning when it is thus used to follow up and verify an induction, inspiration or guess, than when it is taught as the only process*. Perhaps the reason is that the first period of conscious thought, and the operation of the curiosity-wonder instinct, increase excitement in $E_{A}$ and $E_{Z}$ and their neurographic connexions, and so make them and their connexions into deeper and more active interest-systems than are generally excited by problems in school lessons. But whether this explanation is correct or not, we do well to notice Mr Richmond's observation; for we have seen $\dagger$ reason to assume, pending further evidence on the subject, that anything that makes for the practice of deductive reasoning or other hard thinking increases the thinker's 'general ability,' or ' $g$,' and is therefore of very great importance in education.

\section{§ 6. A Personal Endarchy facilitates Reasoning.}

Returning to the argument interrupted by the preceding section, we remark that a personal endarchy which corresponds, as nearly as the conditions of human life permit, to a perfectly integrated mind $\ddagger$, is not only in large measure the product of reasoning, but facilitates further reasoning. For the more completely Jones' neurograms, $A$ and $Z$, for the facts $A$ and $Z$ of which he seeks to discover or reason out the connexion, are already connected in his neurography by a chain of elements that correspond, element for element, with the chain of fact-elements or essences which connect an essence $E_{A}$ of $A$ to an essence $\mathrm{E}_{Z}$ of $Z$ in the endarchy of science, the less effort will he have to make to complete his connexion between $A$ and $Z$ according to the endarchy of science; and this is true, whether he completes his connexion by reasoning it out for the first time, as illustrated in Fig. Io on p. 178 , or by verifying an apparently spontaneous inspiration.

The facilitation of Jones' further reasoning by that part of his neurography which already corresponds to part of the endarchy of science, is not to be measured simply by the extent of the correspondence, meaning thereby the number of connected essences in the endarchy of science that are represented by correspondingly connected elements in Jones' personal endarchy. It depends on the region of the correspondence as well as upon its extent. That is to say, if the reasoning which Jones has mostly to perform is concerned with the connexion of essences belonging to a particular region of the

* Loc. cit. p. 226.

$\dagger$ See above, p. $\mathbf{I}^{8} 8$.

$\ddagger$ See above, p. 244. 
endarchy of science, it will most help him if his neurography corresponds to that region*, rather than to some other. And, other things being equal, the more central the region to which part of his neurography corresponds, the more this correspondence will assist his further reasoning. We may combine the statements in the last two sentences by saying that the more general any particular group of connected essences in the endarchy of science is going to be in Jones' future experience, the more will his future reasoning be facilitated by correspondence between that group of essences and a group of similarly connected elements in his neurography. We may notice again $\dagger$ that the more abstract-the more central in the endarchy of science--an essence is, the more general it will be in the world of experience; and therefore, other things being equal, in Jones' personal experience. Hence the special importance, in the interest of Jones' future reasoning, of correspondence between his personal neurography and the more abstract portions of the region of the endarchy of science with which his experience will be concerned $\ddagger$. Hence also, in particular, the importance of understanding the principles that underly one's daily practice.

Important as is the extent and region of the endarchy of science for which Jones has corresponding neurograms in his personal endarchy, it would be hard to say whether the intimate connexion of the rest of his neurography to his scientific endarchy was not even more important in rendering his reasoning easier. If, for example, Jones wants a connexion between his neurograms $A$ and $Z$, and if elements $E_{A}$ and $E_{Z}$ of $A$ and $Z$ respectively already form part of his scientific endarchy, he will find the path of connexion that he seeks already completed in his scientific endarchy. And if Jones' scientific endarchy be not wide enough to include both $E_{A}$ and $E_{Z}$ within its borders, yet their inclusion in neighbouring connected portions of his neurography will lessen the amount of new reasoning required to complete their connexion to each other through his scientific endarchy.

* The 'subject' or 'special subject' referred to on pp. 24I, 243.

$\dagger$ See above, pp. 199 and 215.

\$ See above, p. 2 I 5. Cf. also W. James' observation, quoted above (in footnote * on p. I 87), that the extracted essences or other facts are 'more general than the concretes, and the connections they may have are, therefore, more familiar to us, having been more often met in our experience.' (Loc. cit. Vol. II, p. 342.) They are on this account more readily available for building new paths by reasoning. For example, "Think of heat as motion, and whatever is true of motion will be true of heat; but we have had a hundred experiences of motion for every one of heat. Think of the rays passing through this lens as bending towards the perpendicular, and you substitute for the comparatively unfamiliar lens the very familiar notion of a particular change in direction of a line, of which notion every day brings us countless examples.' (W. James, loc. cit. Vol. II, p. 342.) 
The new paths, made by this reasoning, will, moreover, enlarge Jones' scientific endarchy.

Thus it is that the correspondence between a person's neurography and the endarchy of science-a correspondence that is in large measure due to reasoning-facilitates further reasoning. It is indeed a function of reasoned thought, not merely to resolve a unipolar or bipolar conflict for the time being, but to leave a neurographic path of connexion that will prevent the same conflict from occurring again. In short, it is one of the objects of reasoning so to inter-connect neurographic elements that the making of further paths by reasoning shall, as far as possible, become unnecessary.

\section{§ 7. Will, the principal factor in developing Single Wide Interests.}

The reasoning that helps to organise a personal endarchy on the lines of the endarchy of science, and is then helped by the personal endarchy so organised, results from the reasoner's effort to think out the connexion that exists between two facts, $A$ and $Z$, in the world of experience; or, as we have repeatedly put it, to connect his neurograms $A$ and $Z$ according to the connexion between $\mathrm{A}$ and $Z$ in the endarchy of science. But why does he make the original effort? To resolve a conflict is the answer we have already* given to this question; and we have gone a step further back to distinguish these conflicts according as they originate in the curiosity-wonder process or are due to the operation of other instincts or interests-especially, as we may now add, purposes $f$. In particular, a purpose to organise our thought in the manner described in Chapter $12 \ddagger$, may have a profound effect upon the development of our neurographies. But whatever cause, outside or inside the reasoner's brain, may make him want to make the effort required for reasoning in each particular case, without the intervention of his Will there is no effort and consequently no reasoning -not even in the case where an unconsciously performed induction forms part of the reasoning; for even then, as Poincaré says, voluntary efforts are needed to 'set the unconscious machine in motion,' $\S$ and effortful deductive reasoning is subsequently required to verify the results of the inspiration II. Indeed, the scientific organisation of

* See above, pp. I4 1 and $\mathrm{r} 62$.

$\dagger$ See above, pp. I76, I 77. The former cause-curiosity-wonder-is, we said, to be identified with Professor Whitehead's 'theoretical source' of science; while the latter cause-all other instincts and interest (among which purposes are especially important)-may be grouped together and identified with Professor Whitehead's 'practical source' of science.

+ See especially pp. 243,244 .

$\S$ Loc. cit. p. 56. The passage is quoted in full in footnote $\dagger$ on p. 256 above.

If See above, pp. 259, 260. 
thought, by the creation or discovery of the endarchy of science and by the formation of personal endarchies of neurograms which correspond as closely as possible with the endarchy of science, is in the main a voluntary process*.

But the Will that plays so important a part in the organisation of Jones' personal endarchy, so as to make it correspond as far as possible with the endarchy of science, and in other respects conform to our description $\dagger$ of the tidy and (so far as the conditions of human life permit) perfectly integrated mind, may not be Jones' Will alone. For, in its early stages, this organisation is commonly directed by the spoken or written word of a teacher who presents knowledge ready organised on the lines of the (incomplete $\ddagger$ ) endarchy of science. But the best teaching is of little use without a voluntary effort of attention on the part of those who are taught. Dr McDougall's experiment $\S$ of learning nonsense syllables confirms this fact, already so familiar to every educator.

We may here observe that teaching which is to produce a single endarchy of neurograms, corresponding as closely as possible to the endarchy of science, ought not to consist of instruction in several separate subjects by teachers each of whom is working independently

* It may be well to recall in this connexion some points on which we have already touched. First then we have noted (on p. 220) Professor Whitehead's insistence 'on the radically untidy, ill-adjusted character of the fields of actual experience from which science starts.' Its 'most obvious aspect'...' is its disorderly character. It is for each person a continuum, fragmentary, and with elements not clearly differentiated.' (Loc. cit. p. I ro.)

Then we have pointed out, in footnote $\dagger$ on p. 264, how Poincaré insists on the 'disorder' that characterises involuntary thinking, pure and simple. 'Only this very disorder permits of unexpected couplings' that may be most valuable in the organisation of thought if worked out and verified by voluntary thinking.

We have also quoted, on p. I I 7, Mr Burt's conclusion that 'high intelligence" -or, as we said, high ' $g$ '- ' seems to mean high capacity for continually systematising mental behaviour by forming new psycho-physical co-ordinations, older co-ordinations being retained, so that newer co-ordinations bring with them increased complexity and incessant change. In such progressively integrative actions of the mind the efficient and directive agent is attentive consciousness.'

On p. roo we quoted from Dr McDougall a passage which continues as follows: 'Experiments of this class, then, are bringing home to us the magnitude of the influence of conation (volition) as compared with mere temporal contiguity or succession. Mental process is effective in establishing associations (and indeed in all other ways) in proportion as it involves strong conation, strong desire or volition; a fact which implies on the neural side effective concentration of psychophysical energy'-Will, in fact- ' in proportion to the strength of the conation.' (American Journal of Insanity, Vol. LxIX (I9r3), p. 869.)

Finally we saw, on p. 240, how Mr Fluegel also regards the mechanism of the subsumption of lower purposes under higher purposes, and ultimately under the highest purpose of all, as consisting, at any rate to some extent, in a logical, rational, or voluntary process.

$\dagger$ On pp. 243, 244 above and preceding pages.

$\ddagger$ See above, p. I99, footnote $\S$. $\$$ See above, p. 100, footnote $\dagger$. 
of his colleagues*. It is, on the contrary, desirable that the education of any particular pupil should be in the hands of the smallest possible number of teachers who are capable of presenting those portions of the endarchy of science with which the pupil's personal endarchy has next to be made to correspond. A pupil's teachers should, therefore, gradually increase in number as his education advances. And when, from time to time, the number is increased, the work of teaching him should not be equally divided between them. For we have seen $\uparrow$ how much more efficient in organising thought is effort expended in developing a single endarchy, than the same effort expended in producing several separate endarchies. When, therefore, it is no longer possible to find one teacher to direct all a pupil's studies, and a second teacher must perforce be introduced, the latter should be entrusted only with such work as the first is unable to undertake. In like manner, when a third teacher has to be added, he should be given as small a share as possible. And so on. In short, when pupils have advanced beyond the stage when one teacher can be responsible for all their school work, they should still spend as large a proportion of their time as possible with one form-master, whose teaching should be supplemented to the least possible extent by the smallest possible number of specialist teachers. And it should be part of the duty of this form-master (or 'central teacher') to see that the single wide interest-system $\ddagger$, which he is concerned with developing in each of his pupils, contains strong purpose-elements at the centre, and that these purpose-elements are connected, not only with all the school work, but also with the most diverse of the pupils' outside experienceई. But the wise teacher knows well that his best way of developing such an endarchy-or, indeed, of affecting his pupil in any other way-is to enlist the help of the pupil's own Will, as well as of his interests and instincts, among which curiosity is of special importance in building personal endarchies. Thus will the teacher produce most effect while he directs his pupil's education, and thus also-especially if he can get his pupils to share his purposes for their educationwill he best ensure its continuance along the same lines after his responsibility has ceased. In support of such a purpose every pupil's

* In this connexion reference should be made to Integral Education (cf. John Adams, loc. cit. pp. 189-195): see p. 470 in Appendix A, below.

$\dagger$ In Chapter II, $\S 5$, on pp. 2 I I to 213 above.

$¥$ See above, p. 244 .

$\S$ Cf. above, the first paragraph on p. 244 beginning 'Wide and diverse experience....' Cf. also Poincaré: 'Among the combinations we choose, the most fruitful are often those which are formed of elements borrowed from widely separated domains.' (Loc. cit. p. 5I.) 
Will may be expected continually to intervene, analysing his disorderly impressions of experience and integrating the essences so abstracted into simple general facts, and these in turn into laws, so as to build up such a personal endarchy as he has purposed: perhaps such a one as we described in the last two sections of Chapter I2 above*. After all, every pupil, as his education advances, beconies, sooner or later, his own chief educator; and, long before that stage is reached, he begins to have an increasingly important share in his own education.

And so we come back to the supreme part played by one's own Will in organising one's personal endarchy of neurograms. But inheritance and environment (or experience) supply the raw material $\dagger$. Moreover, at any intermediate stage of the organisation, the neurography itself-representing interests (and especially purposes) as well as instincts - cooperates with the Will, and with incoming impressions of the environment, in determining the next stage of its development $\ddagger$ : a development which is of the utmost importance to the individual in question.

* See especially pp. 243, 244.

$\dagger$ See above, p. 245.

$\ddagger$ See above, p. 246 . 


\section{CHAPTER 15}

\section{THE FIFTH LAW}

\section{§ I. The End of a Train of Thought.}

In the preceding pages we have gone on, from the discussion of reflex actions, to consider the flow of conscious thought, first when the Will does not intervene, and afterwards when it does. We have now to ask ourselves how the continuity of the stream of thought is broken; or, in other words, how a train of consecutive thought comes to an end.

Ordinary experience distinguishes three ways in which our thoughtactivities may cease to follow, involuntarily or voluntarily, the associations of some single topic. In the first place, some incoming sense impression may be sufficiently intense to drain-in accordance with our third law-the excitement from those active neurograms which correspond to the topic in question. Secondly, the excitement may simply diffuse, according to our second law, without exciting in the process any system of arcs whose excitement becomes sufficient to attract the impulse from the other active neurograms. And, in the third place, the excitement may be discharged from the brain, in giving rise to bodily movements. Let us briefly consider these three processes in the order in which we have named them.

The interruption of a train of thought by some irrelevant senseimpression is familiar to everybody. A flash of lightning, a cry for help, or a wasp-sting, will generally suffice to distract one's attention from the subject of one's previous thought. But, according to our third law, the greater the excitement that accompanies a train of consecutive thought, the greater must be the intensity of some irrelevant sense-impression in order to interrupt it. Indeed, as we have already observed, one's 'absorption may be so deep as not only to banish ordinary sensations, but even the severest pain.'*

Sometimes a train of thought on some uninteresting topic seems to become less and less vivid, until finally, and for no apparent reason, it ceases altogether. Even when we have several times concentrated our attention by an effort of Will, the resulting increase of excitement in our neurograms may not suffice to prevent the stream of thought

* W. James, loc. cit. Vol. I, p. 4 I9, quoted above on p. 82 . See the examples given by W. James (loc. cit.). 
from simply drying up, as rivulets dry up in sandy channels when the flow of water is not enough. This is an example of the ending of a train of thought by diffusion. When it occurs, our conscious thoughtactivities may cease for a while, until set going again by some new stimulus; or sleep may supervene. The ending of a train of thought by diffusion, implies, as a rule, either that its topic is lacking in interest or is unstimulated by voluntary effort; and, in either case, that the accompanying excitement is slight. For everybody has probably noticed, when trains of thought come to an end in this way, how the whole field of consciousness seems narrowly restricted, its fringe being reduced to very small dimensions, indicating that the number of active neurograms, and consequently the whole excitement of the brain, is small; or else how the subject thought of is connected with no great interests whose systems, rendered active, may pour in new excitement. Tiredness of the thinker, or lack of interest in the object of his thought, combined with insufficient ability to compensate for fatigue and lack of interest by concentrating attention*, are then the chief causes for the ending of a train of thought by diffusion.

When a train of thought is ended by interruption or diffusion, the excitement is drained into new paths, or else is dissipated, but does not leave the brain. The only way in which excitement may escape from the brain is by being discharged down the outgoing nerves and giving rise to some form of bodily activity $\dagger$. And this is the normal ending of every train of thought. It is, indeed, clear enough that trains of thought which end in being interrupted or diffused are inconclusive; and that, if all our thinking ended in one or other of these ways, it would serve no useful purpose, whether biological, sociological or any other. So we repeat that action is the normal end of every train of thought; and this statement we take for our fifth law of thought.

\section{$\S$ 2. The Fifth Law fits Experience.}

It is true that this law does not at first sight appear to fit the case of a man who has been puzzling over some problem for a long

* And so increasing excitement. See below, pp. 28r, 282.

$\dagger$ Cf. W. James: "The reader will not have forgotten, in the jungle of purely inward processes and products through which the last chapters have borne him, that the final result of them all must be some form of bodily activity due to the escape of the central excitement through outgoing nerves. The whole neural organism, it will be remembered, is, physiologically considered, but a machine for converting stimuli into reactions; and the intellectual part of our life is knit up with but the middle or "central" portion of the machine's operations.' (Loc. cit. Vol. II, p. 372.) 
time, when suddenly the desired solution comes into his mind*. $\mathrm{He}$ has thought it out. His feeling of effort, and the unpleasant feeling by which 'instinctive striving is always accompanied,' $\dagger$ give way to a feeling of aesthetic satisfaction so soon as the inspiration has been completely understood $\ddagger$. This change of feeling is, as we saw§, almost certainly due to a visceral change; and the nervous excitement, which was the physiological correlative of the train of thought now ended, has been discharged in producing this visceral change-an internal bodily movement-just as, in the normal case, it is discharged down the pyramidal tract in producing the external bodily movement that the word 'action' in our fifth law commonly denotes. Often both internal and external movements end a train of thought; as when the happy recipient of a bright idea not only experiences a very great change of feeling but also indulges in weird behaviour. When, for example, the idea of specific gravity came to Archimedes in his bath, and solved his problem about the composition of the king's crown, he did not wait to dress, but rushed through the streets of

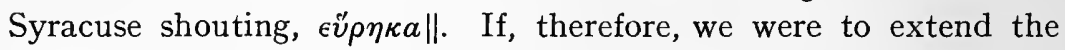
meaning of the word 'action' in our fifth law so as to include all bodily movements, visceral as well as skeletal, the case in which a train of thought ends in a change of feeling 9 would no longer be an exception to our law. We must remember, too, that 'from the physiological point of view a gesture, an expression of the brow, or an expulsion of the breath are movements as much as an act of locomotion

* In the case here supposed there is no interval between 'the first period of conscious thought' and the 'inspiration' (cf. above, pp. 256, 257). Or else it is such a case as Poincaré describes in the words: 'Often when a man is working at a difficult question, he accomplishes nothing the first time he sets to work. Then he takes more or less of a rest, and sits down again at his table. During the first half-hour he still finds nothing, and then all at once the decisive idea presents itself to his mind.'...' It is...probable that the rest was occupied with unconscious work.' (Loc. cit. p. 55.)

$\dagger$ See footnote $t$ on p. 248 above. The instinct here in question is, of course, curiosity.

$\ddagger$ That is to say-if again $\mathrm{E}_{M}$ is presented by the inspiration and the problem was to connect $\mathrm{E}_{A}$ to $\mathrm{E}_{z}$ - -so soon as the connexion between $E_{A}$ and $E_{z}$ through $E_{M}$ has been completed according to the endarchy of science.

$\S$ On p. 53 above.

|| Cf. footnote $\S$ on p. 248 above.

if W. McDougall, in one place, appears to make this the normal case, for he writes: 'Mental activity or thinking thus tends to progress in cycles; each cycle begins with knowing, which excites feeling and striving; the striving results in a new knowing, which satisfies the striving; and so the cycle reaches its natural termination in a feeling of satisfaction.' (Psychology, p. 62.) Elsewhere, however, he supports William James' view that (external) bodily movement-'action' as commonly understood-is the normal ending to every train of thought. For example he writes: 'All mental activity...normally issues in bodily movement.' (Psychology, p. I05.) 
is. A king's breath slays as well as an assassin's blow.'* Indeed, from the standpoint of our fifth law, talking $\dagger$ is so perfect a substitute for other forms of action that it is unfortunately apt to take their place in the circumstances of a fire or of a shipwreck or even of a national crisis when its inferiority to them is altogether obvious.

We have next to see that, with the meaning of 'action' thus extended, our fifth law does fit available experience, and is therefore true.

In the first place, it is consistent with the theory of natural selection. For, in the earlier stages of the struggle for existence, the fittest to survive would be those whose primitive thought promptly led to such action as was best adapted to preserve the individual or the species. In those early times, at all events, thinking was always for the sake of doing; and its value was measurable by its fruits in action. Individuals, or species, whose thinking led to the most effective action for securing prey, for avoiding being preyed upon, and for reproducing their kind, were most likely to survive. This then was the kind of thinking - a kind that promptly issued in appropriate action-that would be developed by natural selection ${ }_{+}^{\dagger}$.

Then again the authorities whose relevant experience is greatestat least among English-speaking people-summarise their experience in conformity with our fifth law. Thus, William James holds that 'Movement is the natural immediate effect of feeling' - that is, of thoughtactivities of whatever kind§-'irrespective of what the quality of the feeling may be. It is so in reflex action, it is so in emotional expression, it is so in the voluntary life.'II In fact, William James' view is that every thought-activity tends to lead to immediate action; and that it is only the gradual making of new paths, of less resistance than the natural or connate paths of motor discharge, that causes our thought-

* W. James, loc. cit. Vol. II, pp. 527, 528. W. McDougall also points out that to speak is to act, so that the expression in words of a conclusion reached by thinking is itself an action that may end the sequence: 'whether or no his words are accompanied by other bodily activity, their utterance is in itself a bodily activity:' (McDougall, Psychology, p. rog.)

$\dagger$ Movements of the tongue are 'skeletal,' not 'visceral,' although the food controller classed tongues with viscera as offal!

$\ddagger$ Cf. W. McDongall: 'Regarded from the biological point of view, the function of all mental process and mental structure is to preserve and promote the life of the race and that of the individual in so far as he subserves the life of the race. The life of the race is preserved and promoted by bodily activities....All mental activity, then, normally issues in bodily movement; since only by promoting and guiding bodily movement can it fulfil its function.' (Psychology, p. 105.)

$\S$ He uses the word 'feeling' as equivalent to our 'thought-activity.' This use he explains in Vol. I, p. I86 (loc. cit.).

\|I Loc. cit. Vol. II, p. 527. The italics are William James'. See also the quotation from William James on p. 286 below. 
activities ever to lead anywhere else*. Such new paths, leading to further thought more readily than to immediate action, were our principal concern when we were lately discussing the organisation of thought by the gradual building up of a personal endarchy of neurograms that should correspond, in large measure, with the endarchy of science.

That Dr McDougall shares William James' view-that 'all mental activity...normally issues in bodily movement' $\dagger$-we have already seen. In another book hewrites: 'Every idea tends to find appropriate expression in movement, the excitement of every neural system tends to issue in motor paths, and whenever ideas are vivid their motor tendencies are clearly manifested.' $\ddagger$

It is not however necessary to take at second hand the experience with which our fifth law has to be compared, if we would be satisfied of its truth. Everybody can supply further experience of his own, as Dr McDougall points out in the passage we have quoted in our last footnote. We can all verify that we tend to make every movement that we think of hard enough§, or imagine vividly enough; that we tend to utter every word that we think of hard enough, or imagine vividly enough-indeed, some people yield to the tendency and 'think aloud'; and, finally, (although this last tendency may become less marked than the others as we become more and more conscious of our inability adequately to fulfil it) we do tend to realise or reproduce every scene or visual object that we think of hard enough, or imagine vividly enough. Thus do all images, whether motor, or auditory, or even visual, tend to lead to movements.

This tendency is so obvious and well known in the case of motor and auditory images that no more need be said about it. But it is necessary to make one or two observations upon the corresponding tendency of visual imagery, since the common experience of adults furnishes comparatively few examples of it. Among such examples we may include those visualisers who never like to think or talk without a pencil and paper to illustrate their ideas\|. Much more

* See, for example, loc. cit. Vol. II, pp. 581, 559.

$\dagger$ McDougall, Psychology, p. 105, quoted in footnote $\ddagger$ on p. 275 above.

$\$$ Physiological Psychology, p. I6r. He adds, by way of illustration: 'if you vividly imagine yourself playing a part in any exciting scene or adventure, a debate, a climb, or a fight, each idea will manifest itself in incipient motions, or at least tensions of muscles.'

§ See below, footnote $\ddagger$ on p. 286.

II Engineers and architects often shew this tendency. Perhaps we may also reckon as examples of the same tendency, the case of the mathematician who, as he calculates and visualises the motion of a spinning top or of an advancing wave, is inclined to illustrate the motion with a gesture. 
obvious, however, are the examples furnished by children. Indeed, it would probably be hard to find a normal child who did not delight in 'realising' the objects of his visual imagery. He may be content to realise or express them in two dimensions, by means of finger and sand-tray, pencil and paper, or paints and brushes; but hewill probably prefer to realise them in three dimensions, by means of wet sand, clay, plasticine, wooden or stone 'bricks,' 'meccano,' a wood or metal workshop, or even a 'science' laboratory. This tendency to construct, in the flat as in the solid, the objects that one sees in imagination, may perhaps be identified with that 'instinct of construction' which, in his book on Social Psychology*, Dr McDougall includes among five instincts whose emotions are less distinctive than those of the seven primary instincts described in that work $\uparrow$.

Whenever the tendency for a thought-activity-say $\mathfrak{A}$-to result in bodily movement is actually fulfilled, the corresponding neurogram, $A$, is deepened by the 'circular nervous process' already $\ddagger$ described. For the excitement then spreads from $A$-or from some kinaesthetic area neurones that are included in, or connected with, $A$-to motor centres, whence it travels down the pyramidal tract and ultimately reaches muscles, whose contraction stimulates afferent neurones, from which the same kinaesthetic area neurones as before are in turn stimulated, thus completing the cycle. The cycle may be repeated any number of times. The kinaesthetic arcs in question are thus deepened. It follows from the corollary to our third law that $A$ is the more likely to attract the impulse on any future occasion of involuntary thinking, and thus to be deepened, not only in its kinaesthetic portion or connexions, but throughout its whole extent. For example, a schoolboy, who is required to prepare a passage in a foreign language so as to be able to translate it in class, will remember the translation better if, when he has prepared it, he reads it over aloud, or, better still, if he writes it out: the reading aloud, or the writing out, deepens his neurograms of his English version in the manner just described. In the same way, children in elementary schools sometimes deepen their neurograms by repeating aloud in chorus statements they are asked to remember.

* Loc. cit. p. 88.

$\dagger$ See above, p. 5I. Dr McDougall tells me (April, 1920) that he did not mean to imply that the five were less important than the seven. W. James (loc. cit. Vol. II, p. 426) also includes 'Constructiveness' in a list of instincts; but he gives no evidence that it satisfies the definition of an instinct, that we have quoted from $\mathrm{Dr}$ McDougall on P. 52 above, by including cognitive and affective as well as conative elements.

$\ddagger$ See above, p. 59 . 
It is, however, possible that, when $\mathbf{A}$ is thought of, the excitement in $A$ may be insufficient to spread through the Rolandic (kinaesthetic) cortex and so to produce movement, even though it suffices to bring $\mathfrak{A}$ to the focus of consciousness. In that case an effort of Will may be necessary to increase the excitement in $A$-or in its kinaesthetic portion or connexions-before the tendency of $\mathfrak{A}$ to be followed by a bodily movement can be fulfilled. In such a case, the fulfilment of the tendency involves the deepening of $A$, not only by the 'circular nervous process' described in the preceding paragraph, but also by the initial effort of Will.

\section{§ 3. Pedagogic Corollaries.}

The deepening of a neurogram that results when the corresponding thought-activity leads to bodily movement has pedagogic consequences of great importance*.

As we have just reminded ourselves, the deepening of a neurogram means that the corresponding fact is better remembered. It is also more likely to become connected with other facts and so to become interesting. That is why, in some schools, practical work may be of so much value in forming a centre of interest to which all the school studies may be connected. Especially in schools where the general ability (' $g$ ') of the children is low, and where they may therefore be unable by an effort of Will to concentrate their attention sufficiently for the learning of their lessons, the creation by practical work of an interest-system from whose activity the neurograms of otherwise uninteresting facts may derive excitement $\ddagger$, is likely to serve a highly useful purpose.

In some cases, the deep interest-system which handwork is capable of creating may be made deeper still by choosing handwork that is closely connected with the pupils' already existing interests (for example, in their present or future occupations), and this is especially true if those other interests are rich in emotional elements. This principle is employed in many Junior Technical Schools $\S$, and in some 'part-time' (day or evening) classes intended for pupils whose ordinary employment occupies the greater part of their time. But, in other cases, this further deepening of the centre of interest created by practical work may not be possible. Even so, however, most young

* Cf. above, pp. 59, 60 .

$\dagger$ It is doubtless for this reason that handwork figures so largely in the curriculum of most modern schools for mentally defective children.

$\ddagger$ See above, p. 85 .

$\S$ See below, Chapter $23, \S$ Io and Chapter $24, \S 5$; especially pp 4I8, 4 I9. 
people between twelve and sixteen years of age will readily find an adequate central interest in handwork*, especially in handwork combined with outdoor activities $\dagger$.

It is not, of course, only because interest in practical work resembles an emotional interest, in tending to deepen the neurograms of facts with which it is connected, that practical work of various kinds plays so large a part in modern education. Among other beneficial effects of practical work in school or college, four may be mentioned here. The first is that some facts can best be learnt, or perhaps can only be learnt, in a laboratory or workshop: the strength and 'feel' of materials, and the structure of plants or animals are examples. The second is that work on the actual things themselves serves as a useful check upon the ideas a student may derive from thinking only of the abstractions or essences with which science mainly deals $\ddagger$. The third is that only by doing things can one learn to do them; and trades may be learnt in some school workshops, even as the technique of a physicist or a chemist may be acquired in some college laboratories. And the fourth is that, if everybody works with his hands at school, manual work will cease-if it has not already ceased-to be regarded as undignified.

Not only, therefore, by deepening neurograms-and so strengthening memory and creating interests-but also in many other ways, may practical studies prove beneficial. But overmuch practical work may also be injurious in education. If, for example, it be true that to increase general ability (' $g$ ') by practice in concentrating attention $\S$ is one of the objects of education, the very same process that strengthens memory and awakens interest in children of low ability, would, if applied to able children, destroy their best opportunities for efforts of Will and so fail to achieve the object in question. In other words, if able children can increase their ability by hard thinking, to make the path of learning too easy, whether by overmuch practical work or in any other way, is to deprive such children of one of the greatest benefits that education can confer. Or again, overmuch practical work in school may be harmful by wasting time and energy. Whoever can understand and remember what he reads without reading aloud, wastes time and energy by pronouncing every word as he reads it. So, too, time and energy that might be devoted to the strenuous

* Cf. Dr G. Kerschensteiner: 'Children do in fact like manual work of the right kind.' (Schools and the Nation, p. I2r.)

$\dagger$ See below, pp. 423,428, where examples are given.

†f. above, pp. $215,233$.

$\S$ See above, pp. I37, 138 and footnote * on p. I59. 
intellectual discipline of mathematics or Latin prose, may easily be wasted in mild laboratory work or other handwork. Or, finally, freedom and encouragement to give rein to one's tendency to follow up every thought-activity by bodily movement, may exercise a more subtly injurious influence upon one's thinking; for to give way to this tendency is to end one's train of thought there and then in action. 


\section{CHAPTER 16}

\section{CONDUCT}

\section{$\S$ I. Conduct as affected by Neurography.}

WE now leave these pedagogic corollaries of our fifth law and proceed briefly to consider what effect upon the ending of a train of thought will be produced by the single wide interest that we have described*. We remind ourselves that every element in the personal endarchy $\dagger$ of neurograms that corresponds to such a single wide interest, is linked to every other through a central scientific endarchy that corresponds as closely as possible with (part of) the endarchy of science; and that the most central, and therefore the deepest, elements in the whole personal endarchy correspond to a supreme and dominant purpose, in harmony with those of one's neighbours. It is true that a perfect single wide interest-coextensive with the whole of the person's experience-may not be realisable in practice. But the more nearly this ideal is approached, the more nearly will the ends of thought be influenced as if it were reached.

In the first place, then, we have already seen that 'when once any considerable interest-system, especially if it be rich in emotional elements, has been stimulated, it will tend for some time to reinforce the excitement in any neurograms that are connected with it.' $\ddagger$ So the possession of a single wide interest makes for the reinforcement of the excitement accompanying every thought-activity-a, for example - connected with it. But it follows from our third law that the greater such intensification of the excitement in the neurogram $A$, the greater also will have to be the excitement of $B$ in order that some irrelevant sense-impression, $\mathbf{B}$, outside the interest in question, may interrupt the train of thought by $B$ draining the excitement from $A$ and its connexions. Consequently a single wide interest tends to prevent trains of thought connected with it from being interrupted by irrelevant sense-impressions, or other thought-activities not so connected. Moreover, the connexion of $A$ with a single wide interestsystem will tend to cause the excitement in $A$ to spread towards the

* See Chapter I2 above, and especially p. 244.

$\dagger$ See above, p. 23 I.

\$ See above, p. 85 . Cf. also pp. 9 r, 92 above. 
centre of that interest-system*. It will thus oppose any tendency for any train of thought to end with $\mathfrak{A}$, through diffusion from $A$. And, in general, the greater the excitement of $A$, the less likely is it that any train of thought will end with $\mathfrak{A}$ by diffusion; for, as the excitement spreads from $A$, the more likely will it be to reach some other neurogram which, on account of its connexion with an affectiveconative neurogram, or because it has been also stimulated from another source, or for some other reason, is capable of draining the impulse to itself and so of starting a new step in the train of thought. Indeed, the process of diffusion is most likely to end a train of thought when the thinker is sleepy or tired; when his field of consciousness is unusually narrowed, no wide or deep interest-system being active; and when, from these and other indications, it seems to him that his excitement is least $\uparrow$. So we conclude that the possession of a single wide interest tends to prevent trains of thought from being ended by interruption or diffusion; and therefore helps to make them end in action.

For another reason also, a single wide interest helps to make its possessor's trains of thought end in action. For, whichever of his neurograms are excited while he thinks, the excitement tends to flow from them towards the centre of his interest-system $\ddagger$, where it excites his purpose-neurograms§. But these purpose-neurograms influence conduct-action-in a specially high degreell. They must therefore be specially closely connected with paths of motor discharge, so that their excitement must tend, not only to influence action, but to initiate it. Again, a single wide interest makes for effectiveness and consistency. For we have already 9 seen that a wide and deep futureinterest-system tends to exert a peculiarly powerful influence upon thought and conduct: an influence that is much stronger than would be exercised by any other interest-system equally wide and deep. But a single wide interest-system, as we have defined it, is no less than the whole neurography organised into a single endarchy that is centred in its possessor's purpose-neurograms. So it is fair to say that a 'single wide interest' is a future interest so wide and deep as to include all other interests within its organisation. And it is evident that a neurography which ensures that the same system of purposeneurograms will influence every action (that is not reflex or habitual**)

* Cf. above, p. 244.

$\ddagger$ See above, pp. 94, 208 and 244 .

II See above, Chapter $8, \S 3$ (pp. 152 et seq.).

II See above, Chapter $8, \S 3$ and especially p. $\mathbf{r}_{54}$.

** Cf. p. 154 above. 
must result in every one of the person's activities being consistent with his supreme purpose, and therefore with each other; and actions that thus cooperate to fulfil a common purpose are necessarily more effective than actions that have nothing in common, and so often undo each other. It follows that the possession of a 'single wide interest' makes for conduct that is consistent and for action that is effective.

We have just spoken of actions or conduct 'being consistent with' a single supreme purpose, as equivalent to their 'cooperating to fulfil' that purpose. And indeed every action taken by anybody-say, Jones -either helps or obstructs the fulfilment of any one of his separate purposes, if he possesses several separate purposes; but, if he has one supreme purpose to which all the rest are subordinate, his every action makes for or against the fulfilment of that purpose. There is no middle course; no neutrality is possible. For the consequences of every action stretch forward into future time, and Jones cannot help thinking of some of these consequences. His corresponding neurograms, say $Q$, either form part of the system, $P$, to which any one of his purpose neurograms belongs; or they do not. When he contemplates another action, the excitement that accompanies his ideas of its consequences in the same future time is attracted both by $Q$ and by $P$. If $Q$ is part of $P$, it helps $P$ to make the consequences of the new action also part of $P$. But if $Q$ has no connexion with $P$, its attraction is in conflict, however feeble, with that of $P$. It follows that an action that is consistent with a given purpose helps, however feebly, to fulfil that purpose; and that an action not consistent with the purpose hinders its fulfilment.

Suppose now that Jones has a single wide interest with a central supreme purpose of which the most central essence is E. Every one of his actions will tend to be consistent with the endarchy of purposes, $P$, of which $E$ is the centre; and, unless his Will prevents it from being so in any particular case, his every action will actually be consistent with $\mathrm{P}$; or, in other words, his neurograms for every action and its consequences will fit harmoniously into his personal endarchy of which $E$ is the centre. But now suppose that his single wide interest is not quite perfect, so that there is some rival purpose, $D$, at work within him. For example, $\mathrm{P}$ may represent the good that Jones would do and $\mathrm{D}$ the evil that sometimes he does; and $P$ may have been built up in the inanner described above on p. 240, so that all his lower purposes-except, for the moment, $\mathrm{D}$-are included in $\mathrm{P}$ and subordinated to $\mathrm{E}$; while $D$ is an inheritance from a long line of animal 
and uncivilised human ancestors. Then there is a continual conflict between $\mathrm{P}$ and $\mathrm{D}$-the conflict so perfectly described by St Paul* and so familiar to everybody-to determine whether each new action shall conform to the one or the other. And Jones will know in each case whether his higher purpose, $\mathrm{P}$, which St Paul $\dagger$ identified with Jones himself $\ddagger$, or his lower tendency, D, has won, by the feeling that he will probably describe as due to an approving or a disapproving conscience.

What is the feeling that conscience approves of an action contemplated or completed? If we have correctly described § the working of the curiosity-wonder process, Jones must experience a feeling of aesthetic satisfaction\|, whenever he first becomes conscious that the consequences of an action fit harmoniously into the system of his central purpose. But whenever the anticipated results of an action are in harmony with the results that it is one's supreme purpose to achieve, one's conscience will approve of that action, provided only that it approves of the supreme purpose in question. Now Jones' conscience will approve of a supreme purpose formed, and related to truth as he conceives it, in the manner described in Chapter I2, § 4: a purpose to achieve 'the highest good of all,' or rather 'the highest end of which the mind in question could conceive.' This highest good, or summum bonum, is the highest good one knows; or, preserving the distinction already** made, it is the 'fact' of the highest good, rather than the highest good 'in itself.' As a rule, the incomplete fact of the highest good-the fact as then known or generally accepted $\uparrow \uparrow$-is that the performance of which conscience will approve as a supreme purpose. And yet, as we have already $\ddagger \ddagger$ remarked in another connexion, the highest good aimed at by some exceptional person-a prophet, if you like, or a conscientious objector to something that most men approve-may be nearer to the 'complete fact' of the highest good than is the incomplete fact that is generally accepted.

If then one's supreme purpose is to realise the highest good of which one can conceive, conscience will approve it, whether it con-

* Epistle to the Romans vii. 15 to 23.

$\dagger$ 'For the good that I would I do not: but the evil which I would not, that

I do. Now if I do that I would not, it is no more I that do it.' (Romans vii. I9, 20.)

$\ddagger$ Cf. our observation on p. $1_{4} 6$ above, that the self-regarding sentiment tends to form part of the future interest and therefore of the interest here called $P$.

$\S$ See Chapter I3 above, and especially p. 248.

II See above, pp. 252, 253.

If 'To fit harmoniously into the system' here means 'to help to build it up into a maximal endarchy': see above, p. 250.

** See above, p. 192 .

$\ddagger \ddagger$ On p. 223 above.

†† See above, p. 222. 
forms to the generally accepted (incomplete) fact of the highest good or no. Suppose that Jones' conscience approves his supreme purpose. It will, as we said, also approve of any action of which the consequences that Jones anticipates are part of, or in harmony with, his supreme purpose. Are we then to say that Jones has two feelings, one of his approving conscience, and the other of his aesthetic satisfaction? There is no evidence from introspection that the two feelings are not the same. We shall therefore provisionally assume them to be identical. In other words, we shall suppose that the feeling that conscience approves of an action is a feeling of aesthetic satisfaction due to the harmony between one's supreme purpose and the anticipated consequences of the action in question. And the feeling that conscience disapproves of an action is doubtless a state of mind caused by conflict between the supreme purpose and the anticipated consequences of the action. That the supreme purpose, where it exists in the form that we have described, should stand in this close relation to conscience will not surprise us if we remember* how the self-regarding sentiment is linked to the purpose in question, and how close the connexion between one's self and one's conscience is generally believed to be.

The effect of a single wide interest, centred (as we saw $\dagger$ ) in a supreme and dominant purpose, in producing consistent and effective conduct, may be increased by the cooperation of the Will. For it follows from our third law that if, when he is about to take action, Jones increases the excitement of his central purpose neurogram, $E$, by an effort of Will + , his chance of acting consistently with his supreme purpose will thereby be increased.

The possession of a single wide interest not only tends to prevent trains of thought from being ended by interruption or diffusion§, and makes for consistent and effective action \|, but also, in the third place, renders possible prolonged continuous thinking. And prolonged trains of reasoned thought often form a necessary preparation for action that is to succeed in attaining one's ends. That the linking up of one's whole neurography, to form a single wide interest-system, must facilitate prolonged thinking, becomes clear when we reflect that, unless low resistance brain paths are available along which

* See above, p. $\mathrm{I}_{4} 6$.

$\dagger$ On p. 244 above.

$\mp$ It is probable that this generally happens, but is by no means necessarily all that happens, when, before taking any important action, the devout man prays to God to serve Whom is his highest purpose. On one occasion, for example, Nehemiah wanted to ask a most important favour from King Artaxerxes. 'Then the king said unto me, For what dost thou make request? So I prayed to the God of heaven. And I said unto the king...' (Nehemiah ii. 4, 5.)
$\S$ Cf. p. 282 above.
II Cf. p. 283 above. 
excitement can continue to flow-and apart from a series of efforts of Will to make such new paths-the excitement will normally be discharged from the brain in giving rise to action. But the linking up of the whole neurography into a single system, provides low resistance paths by which the excitement can pass from any neurogram to any other; and, were it possible for the whole neurography to form a single maximal endarchy, excitement would flow involuntarily from any element-except only the most central element-towards the centre of the endarchy. The possession of a single wide interest thus facilitates prolonged continuous thinking*: indeed, we have already $\dagger$ seen that it facilitates reasoning, which is a particular case of the more general conclusion just reached + .

On the other hand, the man whose neurography is made up of several separate, and therefore small, interest-systems, cannot long continue the same train of thought without much effort of Will. $\mathrm{He}$ therefore tends to allow his thought to pass over quickly into action. $\mathrm{He}$ is the so-called 'practical' man, who, without reasoning about them, simply 'practises the errors of his forefathers.' $\S$ But the truly practical man is he who, while acting automatically in as many as possible of the unimportant affairs of everyday life $\|$, will think out an important course of action to its conclusion before he embarks upon it.

* Cf. W. James: 'sustained attention is the easier, the richer in acquisitions... the mind.' (Loc. cit. Vol. I, p. 423.) † In Chapter I 4, §6, above.

$\ddagger$ That the possessor of a single wide interest will not be under the same obligation as the man with several small separate interests to react promptly, and without much thought, to the stimuli that reach his brain, was noted by William James: 'The reason why that doctrine'- that every idea tends to result in immediate action - ' is not a self evident truth is that we have so many ideas which do not result in action. But it will be seen that in every such case, without exception, that is because other ideas simultaneously present rob them of their impulsive power. But even here, and when a movement is inhibited from completely taking place by contrary ideas, it will incipiently take place. To quote Lotze once more: "The spectator accompanies the throwing of a billiard ball, or the thrust of the swordsman with slight movements of his arm; the untaught narrator tells his story with many gesticulations; the reader while absorbed in the perusal of a battle-scene feels a slight tension run through his muscular system, keeping time as it were with the actions he is reading of. These results become the more marked the more we are absorbed in thinking of the movements which suggest them; they grow fainter exactly in proportion as a complex consciousness, under the dominion of a crowd of other representations, withstands the passing over of mental contemplation into outward action."' (Loc. cit. Vol. II, p. 525. Italics, in last sentence, mine.)

$\S$ Professor Sir Arthur Schuster (Presidential Address to the British Association, Manchester, I915).

II He must not, for example, stop to think what necktie to put on or what fork to use at table, but must do the right thing (in these small matters and others like them) automatically - as a matter of habit-without thinking. Cf. W. James, toc. cit. Vol. II, pp. 370, 37 I. 


\section{§2. Conduct as affected by Will.}

When, however, the conclusion of his train of thought does come, it must be practical. Indeed, not only does every train of thought tend to end in action*-a tendency which is increased when the thinker possesses a single wide interest $\uparrow$-but every train of thought ought to end in action.

So far as the rest of the world is concerned, it is clear enough that the thinker's efforts-whether expended in organising his neurography $\ddagger$ so as to facilitate long continuous thinking, or in making new paths in a badly organised neurography - will be wasted unless some practical result follows from his thinking: especially from his prolonged trains of thought that have cost him most effort. Unless the philosopher communicates, orally or in writing, the conclusions at which he arrives, his philosophy will benefit no one but himself. And the men, or women, who only fight battles, or tend wounded, on the stricken fields of their own imaginations, are equally useless to the world. In short, the interests of human progress--whether towards the goal of scientific thought§ or any other-demand that all human thinking shall have a practical outcome. Otherwise the same ground will have to be traversed over and over again by different thinkers.

It is no less true, if not quite so evident, that the thinker whose thought ends without leading to action, is injuring himself. For, in the first place, by not following up a train of thought with action, he is missing an opportunity of deepening the neurogram $\|$ that corresponds to his conclusion, and so of saving himself the trouble of thinking the same matter out afresh when similar circumstances occur again. And besides, since one must act somehow so long as one continues to live, whoever will not act according to his thinking must

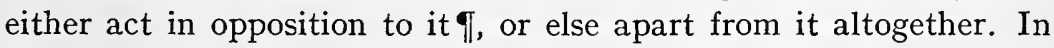
the former case-if he acts contrary to the principles arrived at by his thought-he is sowing the seeds of conflict and of serious interference with his future thinking; while in the latter case-if he tries to keep his actions separate from his thinking-he will not, of course, entirely succeed, since his actions cannot all be performed unconsciously, but he will build up neurograms and systems of neurograms that are altogether separate from the large interest-system by means

* This is our fifth law.

$\uparrow$ Cf. above, p. 282. Will.

$\ddagger$ On P. 269 we saw that this was, in the main, a process requiring effort of

$\S$ See above, p. 196.

\| Cf. p. 277 above. below).

I Like Rousseau, in the following quotation from William James (on p. 288 
of which his principal thinking is accomplished. In either case, therefore, he will be developing a less efficient neurography than if he were to organise his thought as a single wide interest*.

Moreover, every time motor results do not $\uparrow$ follow a train of thought, habit makes it easier for them not to follow when the same thought-activities, or some of them, again occupy consciousness. It follows, as a practical conclusion, that every human being shouldin the interests of economy of thought, his own $\ddagger$ and other people'smake it part of his purpose to end his every train of thought by action§; for then his Will must intervene, whenever necessary to

* As defined on p. 244 and described on the preceding pages.

$\dagger$ See above, pp. 272, 273.

$\ddagger$ So that the maxim even applies to the hermit who chooses, or to Robinson Crusoe who is compelled, to live in isolation from his neighbours (cf. above, p. 227).

$\S \mathrm{Cf}$. W. James: 'Seize the very first possible opportunity to act on every resolution you make, and on every emotional prompting you may experience....' (Loc. cit. Vol. I, p. 124.) He adds, in a well-known passage, 'No matter how full a reservoir of maxims one may possess, and no matter how good one's sentiments may be, if one have not taken advantage of every concrete opportunity to act, one's character may remain entirely unaffected for the better. With mere good intentions, hell is proverbially paved. And this is an obvious consequence of the principles we have laid down. A "character," as J. S. Mill says, "is a completely fashioned will"; and a will [or purpose, as we should say (cf. above, pp. 98, I55)], in the sense in which he means it, is an aggregate of tendencies to act in a firm and prompt and definite way upon all the principal emergencies of life. A tendency to act only becomes effectively ingrained in us in proportion to the uninterrupted frequency with which the actions actually occur, and the brain "grows" to their use. Every time a resolve or a fine glow of feeling evaporates without bearing practical fruit is worse than a chance lost; it works so as positively to hinder future resolutions and emotions from taking the normal path of discharge. There is no more contemptible type of human character than that of the nerveless sentimentalist and dreamer, who spends his life in a weltering sea of sensibility and emotion, but who never does a manly concrete deed. Rousseau, inflaming all the mothers of France, by his eloquence, to follow Nature and nurse their babies themselves, while he sends his own children to the foundling hospital, is the classical example of what I mean. But every one of us in his measure, whenever, after glowing for an abstractly formulated Good, he practically ignores some actual case, among the squalid "other particulars" of which that same Good lurks disguised, treads straight on Rousseau's path. All Goods are disguised by the vulgarity of their concomitants, in this work-a-day world; but woe to him who can only recognise them when he thinks them in their pure and abstract form! The habit of excessive novel reading and theatre-going will produce true monsters in this line. The weeping of a Russian lady over the fictitious personages in the play, while her coachman is freezing to death on his seat outside, is the sort of thing that everywhere happens on a less glaring scale. Even the habit of excessive indulgence in music, for those who are neither performers themselves nor musically gifted enough to take it in a purely intellectual way, has probably a relaxing effect upon the character. One becomes filled with emotions which habitually pass without prompting to any deed, and so the inertly sentimental condition is kept up. The remedy would be, never to suffer one's self to have an emotion at a concert, without expressing it afterward in some active way. Let the expression be the least thing in the world-speaking genially to one's aunt, or giving up one's seat in a horse-car, if nothing more heroic offers-but let it not fail to take place.' '.... if we let our emotions evaporate, they get into a way of evaporating.' (Loc. cit. Vol. I, pp. 125, 126.) 
ensure that all his thoughts shall issue in deeds. This maxim must especially commend itself to any one who recognises his obligation actively to serve his neighbours, or who wants to economise his own thought, or who is not content to spend his life as a mere spectator of a drama in which he plays no part. It will, in particular, be adopted by the Christian who is compelled by the name he bears to aim at being a man of action*. Indeed, just as we saw $\dagger$ that the Christian, even if like other people he does not attain to the ideal $\ddagger$ neurography -a perfect single wide interest-system-must at least fulfil the principal condition of its possession, in that he thinks of the supreme fact, or Truth, in the universe as having the same central essenceGod-as the highest Good; so now we note that he is bound to fulfil this other ideal, of using his Will to make his thinking result in action.

We conclude by repeating $\S$ that action not only tends involuntarily to be the end of every train of thought, but that, in the interests both of the community to which they belong and of themselves, all men should also use their Wills to ensure that every train of thought shall end in action.

\section{§ 3. The Five Laws of Thought.}

All our five laws of thought have now been enunciated. We collect them here for more convenient reference. They are:

I. To every psychosis there corresponds a neurosis (p. 66).

2. Excitement in any nervous arc tends to spread to every other arc that is connected with the first through synapses, the insulation of which the excitement in question is intense enough to overcome (p. 69).

3. Any nervous arc of the higher level, if intensely excited relatively to other higher level arcs, tends to drain the impulses from those other $\operatorname{arcs}$ (p. 79).

4. Will, measured by the general factor, $g$, can reinforce the excitement in any excited system of higher level arcs; and so may cause that system to drain the excitement from all other active arcs of the higher level (p. r29).

5. Action is the normal end of every train of thought (p. 273).

* See below, p. 307.

$\dagger$ On p. $24 \mathrm{I}$ above.

$¥$ See below, first paragraph of p. 3II and p. 303 .

$\S$ Cf. p. 287 above. 


\title{
CHAPTER 17
}

\author{
CHARACTER
}

\section{$\S \mathrm{I}$. Character and Conduct.}

From this account of the five laws of thought and their consequences we proceed to consider the foundations of character. In making the transition we are not, however, going from one water-tight compartment of our subject to another. On the contrary, while investigating the five laws of thought we have been repeatedly compelled to recognise the intimate relation that exists between the trains of fugitive thought that flit through anybody's consciousness from moment to moment, and, on the other hand, the comparatively permanent qualities that characterise the individual in question and so may be said to constitute his character. We have now to enquire what these qualities are.

Chemists distinguish different substances by subjecting them to the same conditions and noticing the differences in their behaviour. So we can hardly find a better means of distinguishing one man from another than is afforded by their different reactions to the same environment, their different behaviour under the same conditions. For, if two men could be found who always did the same thing when placed in the same circumstances, any attempt to distinguish between them would be as unprofitable as (to take Professor Ostwald's example) would have been a discussion, in the days of empirical cookery, whether the dough was raised by an elf or by a brownie: for practical purposes the two men would be identical. In short, if we know how a man will act in any given circumstances, then we know that man; so that a man is defined by his actions and characterised by his conduct*. Or, as the Sermon on the Mount puts it, 'By their fruits ye shall know them.'

Moreover, action of one kind or another is a necessary condition of life. It is true that a man may survive for a while, and even be conscious, after he has lost all power of movement; but such a one can hardly be called alive, except in a very restricted sense. What is

* Cf. Herbart, quoted by Adams: "The tendency...to act in a certain way under a given stimulus...[Herbart] regards... as "the first requisite of character." (Loc. cit. p. 337.) 
it then that defines a man, by determining how he reacts, moment by moment, to his ever changing environment?

Action, according to our fifth law, is the normal end of every train of thought; and, according to our first four laws, a man's thought is determined, apart from incoming sense impressions, by his neurography and his Will. It is true that numerous movements take place without being preceded by conscious thought; but such reflex and instinctive movements are themselves determined, apart from external stimuli, by the arrangement of the nervous system, or, in other words, by the neurography. Indeed, we know no movements that are not wholly determined by the neurography and the Will, along with senseimpressions arriving from outside, or else from inside, the body. It follows that, given a man's environment to which these incoming sense-impressions are due, his reaction will be determined when his neurography and his Will are known determinately. At least, we have no evidence of any other factors, and therefore do well to accept this view as the simplest possible hypothesis that fits all the known facts. If then we were right in saying that a man is characterised by his reactions to every possible given environment, we resume all the available facts by concluding that his character is determined when his neurography and his Will are determinately known*.

Since, however, according to our fourth law, the operation of every man's Will is free and unforeseeable, we cannot be sure that his Will possesses any quality, except only its strength, that characterises him. So we assume, as the simplest hypothesis consistent with all the known facts, that every person is characterised by no more than

* It is argued in the text that, if a person's neurography were completely known-if, for example, the conductivity of every element of his nervous system and of the junction between every pair of adjoining elements were ascertainedand if his Will were also completely known, then the nature of his reaction to his environment (i.e. the kind of movement that would result from given stimulation of his sense-organs, inside as well as outside his body) would also be determined in so far as it depends upon himself; but not necessarily in so far as it may be affected by psychical or other influences which, like his own Will, can affect the conduction of his nervous impulses otherwise than by the stimulation of his senseorgans. (Cf. footnote $\dagger$ on p. I50 above.) What then, it may be asked, is to be said of such factors as Purpose, which we have seen (in Chapter $8, \S 4$ ) to be a group factor 'whose generality would appear to extend so widely in character' (see above, p. 160), or of Cleverness? Our answer is that the influence of Purpose upon conduct is wholly accounted for by the corresponding purpose-neurograms in the neurography; and that Cleverness also affects the growth of the neurography, not only during involuntary thinking, but also, as we saw (on p. I88 above), during reasoned thought; or, if (as we suggested on p. 122 above) Cleverness be a measure of the tendency of nervous excitement to diffuse, and if a positive degree of Cleverness thus marks a general lowness of synaptic resistance, Cleverness, like Purposefulness, is a property of the neurography. The effect of such factors upon character is included in that of Neurography. See also the footnote * on p. roo above. 
(x) his neurography, and (2) the strength of his Will whose measure we identified* with $g$.

Thus, for example, a historian, because he has a different $\uparrow$ neurography from that of a mathematician, has also a different character $\ddagger$. But of course it does not follow that the characters of a particular mathematician and a particular historian may not resemble each other far more closely than those of two specified historians, or of two specified mathematicians.

That a man's character depends in large measure upon his neurography is already widely recognised. Thus 'Herbart maintains that instruction supplies the only force that can modify character.' $\S$ And Dr Morton Prince, after asking 'What makes character?' answers: 'The phenomena of disintegrated personality suggest that our characters are wholly a matter of brain associations and that they may be altered for good or ill by anything that will bring about a rearrangement of these associations.' $\|$ Moreover, 'systems of neurograms...become a part of the personality. Such complexes and systems play an important part by determining mental and bodily behaviour. Amongst other things they tend to determine the points of view, the attitudes of mind, the individual and social conscience, judgment, etc., and, as large systems, may become "sides of one's character".' '

That the strength of one's Will, ' $g$,' or the measure of one's general ability, is also a factor in one's character may not be so readily agreed. But William James implicitly recognised the fact when he wrote: 'The faculty of voluntarily bringing back a wandering attention, over and over again, is the very root of judgment, character, and will....An education which should improve this faculty would be the education par excellence.'** And indeed there can be no doubt that, of two men, one of whom possessed a stronger Will-a greater $g$-than the other, but who were in other respects identical, the former would surpass the latter in the performance of a task to

* See above, p. 129.

$\dagger$ Cf. above, pp. 266, 267.

¥ Cf. Mr Wells' observation, in Joan and Peter, that a man becomes a different man, however slight the difference may be, when for the first time he learns the law of gravitation. $\S$ Adams, The Evolution of Educational Theory, p. 326.

II The Dissociation of a Personality. (I906 edition, p. 299.)

TI The Unconscious, p. 535. Dr Prince adds that 'when such complexes have strong emotional tones they may set up conflicts leading to the inhibition of antagonistic sentiments, and sometimes to the contraction and even disruption of the personality. All these phenomena can be induced by the artificial creation and organisation of complexes and this principle becomes an important one in therapeutics'-and, as we shall see, in education also.

** Loc. cit. Vol. I, P. 424. 
the fulfilment of which both were giving every effort. In other words, their actions would differ because their $g$ 's were different. And, since we have said that both are characterised by their actions, their characters would differ because of the difference in strength of their Wills.

\section{§ 2. Character, Neurography and Will.}

Assuming, then, that character is made up of neurography on the one hand, and of (strength of) Will on the other, we have next to enquire what sort of neurography and how strong a Will should characterise the various individual members of an ideal community*, whether municipal, national or international.

We need not hesitate to answer that the stronger a man's Will is, the better, when once his neurography is all that can be desired for his own sake and for that of the community of which he forms part: it is evidently good for everybody that a man, who wishes to do the right thing in any given circumstances, should be able to make the strongest effort of Will, so as to overcome the intellectual or material obstacles that stand in his way. Moreover, we saw $\dagger$ that the effect of a single wide interest + , in producing consistent and effective conduct, may be increased by the cooperation of Will. We shall presently see that this is the type of neurography that should characterise the various members of an ideal community: a conclusion from which it follows once more that the stronger the Will of the possessor of such a neurography the better.

In order to satisfy ourselves that every citizen should possess the type of neurography which we have described as a single wide interest-system, we have next to remind ourselves that this is the type of neurography that will not only ensure the avoidance of conflict between different members of the cominunity (because they all possess supreme purposes that are in harmony with one another§), but will also come as near as possible to the fulfilment of the seven conditions stated in Chapter I2 above\|. In other words, such a single wide interest will ensure for every man (I) that he and his neighbours shall have harmonious purposes, (2) that his conduct shall be consistent and effective $\uparrow$, (3) that his thought shall be economical**, and (4) that his Will shall tend to be strong $\uparrow \uparrow$. Or, putting the matter

* Cf. p. 232 above.

$\dagger$ On p. 285 above.

¥ See above, p. 244 and preceding pages, especially pp. 24 I to 243.

\$ See p. 244 above.

II See pp. 222 to 225 .

I The fourth, fifth and sixth conditions on pp. 224, 225 above.

** The first, second and third conditions on pp. 222, 223 above.

t† The seventh condition on p. 225 above. 
more shortly, if the conduct of a citizen and his neighbours is to be mutually consistent and effective, and if their Wills are to be strong, their neurographies should be of the kind described in Chapter I2 as single wide interest-systems*. Moreover, as we remarked above $\dagger$ and have just reminded ourselves, every citizen's Will (and the stronger it is the better) should cooperate with his single wide interest in order to increase, as far as possible, the consistency and efficiency of his conduct on the one hand, and, on the other, the strength of his Will.

Now consistent and effective conduct combined with a strong Will marks the strong man, the man with a strong character. The weak man on the other hand is marked by lack of coherence among his interests, and especially among his purposes. He 'has not so much as a purpose, but has only purposes' $\ddagger$, as Carlyle said of the weak government at Versailles in $\mathbf{1 7 8 8}$. Or, as Professor Déjérine and Dr Gauckler say of the neurasthenic, 'He does not lack interest, but rather he is interested in too many things.' $\S$

Every member of the ideal community $\|$ or Commonwealth should therefore possess a strong character, which means a strong Will in combination with a single wide interest $\uparrow$.

* See p. 244 above.

$\dagger$ On p. 285.

$\ddagger$ French Revolution, Ashburton Edition, Vol. I, p. IOI.

$\S$ Psychoneuroses and their Treatment by Psychotherapy, translated by Smith Ely Jelliffe, M.D., Ph.D.

\|I See above, p. 293.

T As defined in Chapter I2: see above, p. 244. Reference to Chapter I2 where the single wide interest was defined will remind (see p. $28 \mathrm{I}$ above) the reader that a perfect single wide interest will hardly be realised in practice, but that, therefore, the more nearly the whole of a person's neurography forms a single endarchical system centred in and dominated by deep and closely interconnected purpose-neurograms, the more nearly will his thought and conduct resemble that which would be due to a single wide interest. It follows (see p. I6I above) that a strong character implies not only a large $g$ (a high degree of Will power) but also a large $p$ (a high degree of Purposefulness), and therefore also a large $f$.

What then, it may be asked, is the relation of Cleverness (measured by $c$ ) to strength of character? (We confine our attempt to answer this question to a footnote because we have seen reason to believe that Cleverness is innate, and in the text we are studying character in its bearing upon the aim of education.)

We have seen (on p. I 88 above) that the possession of a high degree of Cleverness will facilitate the discovery of the endarchy of science, and so render it possible for people to possess more perfect single wide interests which, as we saw, have to correspond, especially as regards their central regions, as closely as possible with the endarchy of science. On the other hand, whoever possesses a high degree of Cleverness, and therefore, if we have rightly interpreted the nature of Cleverness (on p. I22 above), a general tendency to low resistance of the paths of connexion between his neurograms, will on that account require a specially deep central purpose-system if all his thought and conduct is to be influenced by a supreme purpose. (It is perhaps for this reason that persons whose Cleverness is most marked, seem-see p. I6I above-to be lacking in Purposefulness.) A high degree of Cleverness would therefore seem to be, on the whole, inimical to great strength of 


\section{§3. Character in the perfect Commonwealth.}

Let us examine some of the attributes of such a strong character as we have just described-a character that is made up of a neurography in the form defined as a single wide interest-system*, and of a strong Will that cooperates with the single wide interest in guiding thought and conduct. Any one, say Jones, whose character is of this kind, will possess an outlook on life which is no less than a philosophy; for his single wide interest-system is an organisation that includes the whole of his neurography $\dagger$, so that the whole world of his experience is organised as a single whole; and a view of the universe, that shews it organised and shews it whole, is surely a philosophy. But Jones' outlook on life is something more than a philosophy, for it shews everything focussed in a supreme and dominant purpose. This purpose introduces deep emotional elements + into his philosophy and transforms it into a religion: a religion that, so far as we have yet defined it $\S$, may be either good or bad. This result may be expressed by saying that every member of a community, in which all men work together as effectively as possible for a common end, must be religious; and that the central purpose essence of all their religions must be the same. But it does not of course mean that they should all accept in its entirety the same system of theology or ethics.

This conclusion merely echoes the teaching of thinkers and seers in all ages. We cite only a few recent examples. Frederic Harrison thus sums up, in the concluding paragraph of his Autobiographic

character in the same individual. But a high degree of Cleverness, just because it requires a greater effort of Will to direct thought along a particular channel so as, for example, to fulfil a distant purpose, may afford practice in concentrating attention and so make for increase of $g$. Thus it may be true that a clever race of men will, as one generation succeeds another, develop a higher average degree of will-power than might have been the case had it been less clever; and so, as one generation succeeds another, Cleverness, like temptations that are due to some lower propensity of instinct (see above, pp. 283, 284) or of habit (as we said on p. I79 above), may make for increase of strength of character among mankind. It is easy for a dull man to be good.

* On p. 244 above. We remind ourselves again of the harmony that exists between the central supreme purposes of fellow-citizens who possess 'single wide interests.'

$\dagger$ See above, p. 231.

$\ddagger$ Cf. pp. I 52 and I 54 above, and Appendix B, $\S$ II and I2, below.

$\S \mathrm{It}$ is because the philosophy is centred in an emotional purpose that we have called it a religion. All religions-or at least the principal religions of civilised men -fulfil these conditions; and every ethical system that fulfils them might well be called a religion without straining the meaning commonly attached to that word. 
Memoirs, 'all that he has even written or spoken during half a century':

All our mighty achievements are being hampered and often neutralised, all our difficulties are being doubled, and all our moral and social diseases are being aggravated by this supreme and dominant fact-that we have suffered our religion to slide from us and that in effect our age has no abiding faith in any religion at all. The urgent task of our time is to recover a religious faith as a basis of life both personal and social.

Or again, William James opens his volume of lectures on 'Pragmatism' by quoting and approving Mr Chesterton's paradox that the most important thing about a man is his philosophy.* Sir Stanley Leathes expresses the same opinion in another way when he writes, 'Education without religion seems to me impossible.' $\dagger$ And Dr William Temple expressed the same view when he said 'The only religion worth having is one that colours and governs the whole of life and thought....The only religious education which is going to stand the test of an alert criticism conducted by scientifically trained minds is not instruction given in certain isolated periods, but a presentation of the whole universe of being as' subject to a single supreme purpose

So all the members of a community whose conduct is as mutually consistent and effective as possible, and who therefore possess single wide interests centred in mutually harmonious purposes, must, in the first place, be religious. We have, in the second place, to remind ourselves that the development of such a neurography is in the main a voluntary process $§$ : it is largely a matter of Will. In particular, the central elements of Jones' neurography, that correspond to his supreme and dominant purpose, cannot be formed and organised at the centre

* G. K. Chesterton, in the preface to Heretics. The whole passage is worth quoting. It reads: 'There are some people-and I am one of them-who think that the most practical and important thing about a man is still his view of the universe. We think that for a landlady considering a lodger it is important to know his income, but still more important to know his philosophy. We think that for a general about to fight an enemy it is important to know the enemy's numbers, but still more important to know the enemy's philosophy. We think the question is not whether the theory of the cosmos affects matters, but whether in the long run any thing else affects them.'

$\dagger$ What is Education? p. 32.

$\ddagger$ Presidential Address to the Education Section (L) of the British Association (Newcastle, I9I6), p. II. Dr Temple's words that followed immediately upon those quoted, and that formed the concluding words of the passage, were 'filled with the Glory of God.' We have completed the passage with other words because, for the moment, we are not concerned with the question of the kind of religion required by members of an ideal community, but merely with establishing the fact that members of a community whose conduct is to be mutually consistent and effective must be religious.

$\S$ See above, p. 269. 
of his neurography without an effort of his Will. This effort of Will, that forms Jones' central purpose-that makes, or helps to make, his fundamental hypothesis about which he must afterwards reason and on which he must $a c t *$ in order to verify it-is an act of faith. Faith, then, is a matter of Will $\dagger$. Without an effort of Will there can be no faith. Passive acceptance of unverified dogma is not faith at all, but credulity. It is true, of course, as William James has shewn in his book on Varieties of Religious Experience, that the fundamental hypothesis of which we have spoken-the central purpose of a single wide religious interest-may come involuntarily by inspiration $\ddagger$, just as happens with scientific discoveries§. But even so, as we said, an effort of Will is needed \| to act on it, and so to verify it; or, in other words, to bring the inspiration into connexion with the remainder of one's experience, and, indeed, to organise these fundamental purposeneurograms at the centre of a single wide interest-system. And this active faith is always the first step towards religion.

In the third place, the fact that the central essences of Jones' single wide interest constitute a purpose, implies that they are rich in future interest. Moreover, the future in which Jones' single wide interest, or that of any of his neighbours, is centred should be beyond the end of their respective lives. For if not, a time would come in the life of each of them when the future upon which his interest had been focussed would be future no longer. The consequent change in the central elements of his neurography would affect the working of all its parts. Indeed, Jones will only possess a single wide interest so long as it continues to be centred in a purpose $\boldsymbol{\Psi}$, and therefore in the future.

* Experiments, made with a view to verifying that the metal films described on p. 265 did in fact exhibit just those colours and changes of colour which could be deduced from the fundamental hypothesis concerning the structure of the films, would furnish an example of 'action' of this kind. Compare Stanley Hall's observation that 'religion is not theology nor yet ethics, but personal and experimental.' (Adolescence, Vol. II, p. 326.)

$\dagger$ Since ' $g$ ' that measures a man's strength of Will is the measure of his general ability, we are reminded of St Paul's advice to the Christians in Rome, that each of them should be 'measuring himself by the faith which God has allotted to him.' (Romans xii. 3; Twentieth Century New Testament version.) Compare also the statement ascribed to $\mathrm{Dr}^{-}$John R. Mott, the president of the World Christian Student Movement, 'Christianity, gentlemen, is a matter of Will.'

$\ddagger$ See above, Chapter I4, $\$ 3$.

$\S$ Cf. James Clerk Maxwell: "The experimental investigation by which Ampère established the law of the mechanical action between electric currents is one of the most brilliant achievements of science. The whole theory and experiment seems as if it had leaped full grown and full armed from the brain of the "Newton of Electricity.", (Electricity and Magnetism, Vol. II, Chapter III.)

$\|$ During the second bout of voluntary thinking. See pp. 259,260 .

If See above, p. 244 . 
If we suppose, for a moment, that Jones' interest is centred in the immediate future rather than in a future more remote, we observe that his neurography may still satisfy the conditions for consistent and effective action, not only on his own account but in cooperation with his neighbours. His single wide interest may, for example, be centred in a purpose to fulfil a certain ambition. Such a purpose may be intimately linked with the emotion of positive self-feeling. Being central, and being rich in affective-conative elements, it may exert a potent influence upon his thought and conduct, even though he never looks beyond the end of the life which he lives with such zest and vigour. Doubtless he will achieve great things, although his record may be marred by grave faults. But, in his old age, he will not fail to realise that his life has not been a success: whatever else we are intended to do, says Robert Louis Stevenson, we are not intended to succeed. And when the end has nearly come and the emotion that used to spur him on to his greatest achievements is seldom stirred, he will hardly be able to bear the thought of what used to be the centre of all his interest. The stimulus to activity will almost have disappeared, and with activity happiness goes too.... While the absence of interest in anything beyond the present life produces its most marked effect in old age, it is not without result all through life. This was well recognised in Utopia where, in Green's words, 'The disbelievers in a Divine Being or in the immortality of man, who, by a single exception to its perfect religious liberty were excluded from public office, were excluded, not on the ground of their religious belief, but because their opinions were deemed to be degrading to mankind and therefore to incapacitate those who held them from governing in a noble temper.'

It follows that the single wide interest of any member of a community the conduct of all of whose members is as mutually consistent and effective as possible, must be centred in a future beyond the end of his life in the body. And since, as we saw, the central purpose elements of the neurographies of each member must be rich in affective-conative elements, it follows that the typical member, Jones, must have an emotional interest in the future. And these emotional feelings must be feelings of satisfaction rather than the opposite. No one can strive for the fulfilment of a purpose, if its fulfilment will not bring him satisfaction. But anticipated satisfaction is nothing less than hope. Indeed, hope is the imagined, or anticipated, satisfaction that will follow the fulfilment of a purpose. Hope accompanies the thought of every approved purpose into whose 
system affective-conative elements enter: of course I hope to achieve my purposes: if I did not, they would not be my purposes. We conclude that hope-and hope for something that lies beyond his physical death-is one of the qualities that must accompany Jones' type of character: the type of character that belongs to every member of a community the conduct of all of whose members is as mutually consistent and effective as possible. 'At bottom,' says Amiel, 'everything depends on the presence or absence of one single element in the soul-hope. All the activities of man...pre-suppose a hope in him of attaining an end. Once kill this hope, and his movements become senseless, spasmodic and convulsive, like those of someone falling from a height.' * And this hope must, we repeat, be focussed in something beyond the end of human life $\dagger$. 'What makes old age so sad,' says Richter, 'is not that our joys but our hopes cease.' $\ddagger$

So far then our discussion $\S$ of the character of the typical citizen of an ideal community has shewn us that he should possess a single wide interest supported by a strong Will; that he should be religious; and that he should have faith and hope. Such an one possesses what we have called a strong $\|$ character. His thought and conduct will be consistent and effective. Moreover, since the dominant purpose at the centre of his single wide interest is, we said $\%$, in harmony with the purposes of his fellow-citizens, his thought and conduct will tend to be consistent with the thought and conduct of his neighbours. And thus far we have relied upon harmony of central purpose for securing that the conduct of the several members of the community shall be mutually consistent, and therefore effective.

* Amiel, Journal Intime, 5 Juin, I870, quoted by Shand, loc. cit. p. 479.

$\dagger$ The argument in the text requires, in the words we used before (p. 298), that Jones must have a hope and central purposes centred in a future beyond the end of his life in the body. While at first sight it might seem sufficient for these purposes to be centred in other human lives that will continue after Jones' physical death, we have to observe that the central purposes required by the argument in the text, just because they are central in Jones' neurography, must be intimately linked with, or even include, his self-regarding sentiment (see pp. ${ }_{4} 6$ and I $_{52}$ ). Jones must, therefore, think of himself as in some sense surviving until the future with which his central purposes are concerned and upon which his hope is set. We may grant that it is possible to imagine that Jones, while still human, is so selfless as to have little or no self-regarding sentiment: in which case the 'something' (lying beyond his physical death) on which his hope is set need not involve himself. The requirements of the argument in the text might then be satisfied if Jones' self did not in some sense survive, or, in other words, if Jones were in no sense immortal. But, in so far as human evolution does not yet allow us to imagine a Commonwealth composed of such selfless citizens, the conditions, imposed by the argument in the text upon the central elements of Jones' neurography, require for their fulfilment that Jones and his fellow-citizens shall possess personal immortality.

$\ddagger$ Richter, Titan, quoted by Shand, loc. cit. p. 479 .

$\S$ Begun in $\S 2$ of this Chapter. $\quad$ || See p. 294 above. 
Harmony of central purpose would indeed produce this result if the neurography of every member of our community had the form of a single maximal endarchy* corresponding to the endarchy of science and centred in the supreme purpose in question. But such perfectly integrated minds are not yet found in this imperfect stage of human evolution. Jones and his neighbours still inherit selfish animal instincts whose neurograms are hard to organise into a single wide interest-system dominated by a central purpose. So, even when Jones and some of his neighbours set out together to achieve a common purpose-to discover the South Pole, for example; or to map the coastline of the south polar continent-innate selfishness is not wholly swallowed up in unity of purpose (of which, in the case we have imagined, curiosity-wonder supplies the principal affective-conative elements). Members of the expedition do not spend all day and every day in discovery. Cooking and washing-up, as in more humdrum circumstances, occupy a large proportion of their time. And harmony of their main purpose is by no means certain to prevent disputes or conflicts between them in regard to the performance of these little everyday duties. Although each man may have so organised his thought that he involuntarily subordinates selfish motives to the supreme purpose of the little society of explorers, he may not have reached so perfect a stage of thought organisation as involuntarily to think of his companions' minor comforts before, or along with, his own. He may not have learnt to think of his neighbours as himself. He may still have to learn, in St Paul's words, 'not to think of himself more highly than he ought to think, but so to think as to think soberly' $\dagger$; or, as St Peter says, 'with humility.' $\ddagger$ In short, although the common purpose which he shares with his fellow explorers may secure for him considerable freedom $\S$ to do what he likes, because what he likes is what his fellow men also approve, he may not have learnt to treat them in all things as himself-to treat them, that is, with justice, or, as the Samoans say\|, with love.

* See p. 206 above.

$\dagger$ Romans xii. 3 .

$\mp$ I Peter v. 5. Compare the Sermon on the Mount: 'Blessed are the poor in spirit: for theirs is the kingdom of heaven.' (Matthew v. 3.)

$\S$ Cf. p. 225 above.

II Cd. 92 Io a White Paper describing the attitude of the German Colonies as to their future government,' quoted in The Times, 12th December, 19r8. In Samoa, the leading chiefs from every district stated that they were unanimous in wishing that Samoa should remain under British rule, and gave as one of their reasons: "Because the British officials since the occupation have treated them with love (justice), and they now win cases in the Courts, which was unknown formerly.' 
How then are minor conflicts to be avoided, or at least minimised, amongst fellow-citizens, who possess indeed harmonious central purposes dominating single wide interests, but whose single wide interests are not wholly in the form of single maximal endarchieswhose minds, in other words, are only imperfectly integrated? Only by seeing to it that the affective-conative elements that belong to the central purpose of each citizen, and impel him towards its fulfilment, are capable of overcoming his own incompletely subordinated selfish interests when these are opposed to those of his neighbours. The fulfilment of this condition requires that Jones' neurograms for his fellow-citizens shall be as intimately connected as that for his own self-regarding sentiment with his central purpose-system and its affective-conative elements*. But it is clear $\dagger$ that his selfregarding sentiment must be central in his single wide interest. So also therefore must be his sentiments for his neighbours. And therefore his neurogram for humanity + - a common essence abstracted from individual men, women and children-must be central in his single wide interest-system. But, since his thought must be true, his central neurograms must correspond to the central essences of the endarchy of science. It follows, that, if the ideal neurography that ensures justice between fellow human beings is to be attainable without sacrificing truth, humanity must also be central in the endarchy of science. This is a hard saying, but unless it be true-unless human beings really are among the most important things in the universewe cannot see how truth and justice can both prevail among men, even in quite a small society. Somehow, then, the typical character in a maximally effective community requires to have its central purpose intimately linked with men; and not merely with mankind in the abstract, but with one's own well-known neighbours, as well as with all one's other neighbours now living throughout the world and still to be born into it.

* The point is that Jones' brain process is not to be indirect: he is not to say 'If I am unkind or unjust to Robinson, Robinson will be upset and will work less hard for our common purpose; therefore I will treat Robinson with justice and even with kindness.' But Jones' thought is to be direct: 'I will do all I can for Robinson because he is a friend of mine, or (in older phrase) because I love him.' Or, paraphrasing St John's first letter, 'He that loveth not his brother'Robinson-' whom he hath seen, how can he love God whom he hath not seen': how can he work for an abstract purpose of which love is the centre?

$\dagger$ Because the sentiment is closely linked (see p. 146) to the central purpose.

$\ddagger$ This extension of the conception of the typical citizen's neighbours-whom he is to regard with love and treat with justice-from the members of a particular (consistent and effective) community, to every human being, is necessary if that society's thought and conduct is to be uninterrupted by conflicts with other societies, and so to be maximally effective. 
But what is to be said of the affective-conative elements that supply the driving force* of this central purpose, and are strong enough to overcome the typical citizen's selfish motives when these are opposed to his interest in his neighbours' welfare? Dr McDougall, in his Introduction to Social Psychology and in a later essay, has described eight primary emotions $\uparrow$ that form the affective aspects of a like number of primary instinctive processes. The last of these eight emotions, Dr McDougall describes as 'tender emotion,' the affective aspect of the parental instinct. It is fatherly love, brotherly love,

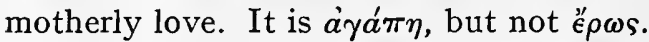

If now we examine this list of primary emotions, it is at once clear that none are so sure to make for human welfare as $\dot{\alpha} \gamma \dot{a} \pi \eta$ on the one hand and curiosity-wonder on the other. Moreover, it is easy to see that curiosity-wonder alone, as the dominant emotional influence upon the central purpose of every human being, would not suffice to secure justice-the equal treatment of all one's neighbours and oneself. Even among our company of explorers, for example, by no means all the activities of every day would be prompted by this instinct-emotion: it would not suffice to ensure harmony of thought and action in minor matters. Indeed, $\dot{a} \gamma \dot{a} \pi \eta$-or rather its linked instinct-alone is capable of defeating self-love and of making one treat one's neighbours as oneself; áyám $\eta$ alone, as the prime mover of the central purpose of every person's character, will ensure justice in human affairs. Thus Professor Zimmern writes:

The inspiration of all sound and enduring political and social construction is what has been called the principle of the Commonwealth....A Commonwealth is a community, designed to meet the common needs of men, founded on the principle of the service of each for all...the perfect Commonwealth, the ideal towards which all political and social endeavour moves forward, is a society of free men and women, each at once ruling and being ruled, each consciously giving his service for the benefit of all.

The principle of the Commonwealth is the application to the field of government and social policy of the law of human brotherhood, of the duty of man to his neighbour, near and far....A Commonwealth is an organisation designed with the ruling motive of love and brotherhood. It seeks to embody, not only in phraseology and constitutional doctrine, but in the actual conduct of public affairs, so far as the frailty and imperfection of man admit, the spirit and ideals of religion. Whosoever will be great among you shall be your minister; and whosoever of you will be the chiefest shall be the servant of all.

The doctrine of the Commonwealth expressed in these words, has been set forth and applied from age after age to the current problems of humanity,

* Cf. p. 59 above.

$\uparrow$ See above, p. 5 r. 
from Plato down to President Wilson. It embodies succinctly and unanswerably, the response of the soul of man to the twin challenge of Prussianism and Revolution*.

\section{$\S 4$. Character and Progress.}

If, therefore, mutual consistency, and therefore efficiency, is to mark the thought and conduct of all the members of our ideal community $\dagger$ or Commonwealth-if, that is to say, they are to advance, or make progress + , in any direction-the character of each of its members should include a strong Will cooperating with a single wide interest, and especially $\S$ with that part of it that is most nearly related to the particular member's service to the community as a whole. This single wide interest, and especially its central essences, should, as we saw in Chapter I2\|, correspond as closely as possible with the endarchy of science, and be dominated by a central purpose in

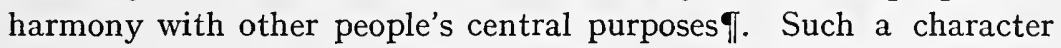
implies religion, faith, hope and, most important of all, love for one's neighbour as the prime mover towards the fulfilment of one's central purpose.

\section{This statement of the character that belongs to every member of}

* Nationality and Government by A. E. Zimmern (1918), p. 355.

$\dagger$ See above, pp. 293, 294.

$\ddagger$ The assumption that to make progress is (at least part of) the aim of this society is consistent with all that we know of living things and groups of living things; for we know none among them that have any other alternative but extinction or continuous change, change that does not repeat itself and is therefore progressive; and we are not here concerned with the education of the members of a society that is content to await extinction. So we assume progressive change, and that as rapid as possible, to be part at least of the aim of the society that is the subject of our enquiry. In so doing we are not restricting the scope of our enquiry to some exceptional or exotic section of mankind. "Advance Australia" is the motto of a continent. And most men in Europe and America would agree that the society to which they belong is also to advance, rather than to stand still and await destruction. It is only concerning the direction of the advance that any considerable doubt exists.

Cf. Mr J. Maynard Keynes' description of Europe before the war: 'Society was working, not for the small pleasures of to-day, but for the security and improvement of the race-in fact for "progress."' (The Economic Consequences of the Peace, 1920, p. 18.)

Cf. also Benjamin Jowett: 'The idea of progress is of modern rather than of ancient date; and, like the idea of a philosophy of history, is not more than a century or two old. It seems to have arisen out of the impression left on the human mind by the growth of the Roman Empire and of the Christian Church, and to be due to the political and social improvements which they introduced into the world; and still more in our own century to the idealism of the first French Revolution and the triumph of American Independence; and in a yet greater degree to the vast material prosperity and growth of population in England and her colonies and in America. It is also to be ascribed to the greater study of the philosophy of history.' (Introduction to his translation of The Republic of Plato, Vol. II, p. I55.)

II See especially pp. 244 and 23 I.

$\S$ See above, pp. 227, 228.

\I See above, p. 244 . 
a maximally efficient and progressive society (whatever be the direction of its progress) is mainly concerned with the central elements of the neurography. And necessarily so, for these are the deepest in a single wide interest-system such as we have defined. But these are also the elements which correspond to the central essences of the endarchy of science, essences that are not yet completely discovered. To discover them and other less important essences, is, we said*, the goal of scientific thought. It is more. To achieve correspondence between the central elements' of one's neurography and the central essences of the endarchy of science is the most important $\uparrow$ step, on the neurographic side, towards the formation of character. From the Christian standpoint, it is, as we shall shortly see $\neq$, no less than the chief end of every human life, determining the direction of the ideal community's progress.

But how is it to be done? In just the same way as the remainder of one's scientific endarchy is developed to correspond with the endarchy of science; and this process we have already§ examined at some length. In brief, if facts are available-that is, if things are generally assumed and stated to be such-and-such, and if these statements fit all available experience of the things in question $\|$-one provisionally accepts, and acts on, these statements of fact with a view to verifying that they fit one's own experience; and, in so doing, one forms one's neurograms of the facts in question, and inter-connects them with the remainder of one's neurography so as to form an organised whole. But, if facts are not available, one seeks to discover them by the process we have called induction: a process in which inspiration is combined with voluntary effort, and which is again completed by action on a hypothesis with a view to its verification 1 .

Accordingly, to achieve correspondence between the central elements of one's neurography and the central essences of the endarchy of science one may begin in either of two ways. The first is by provisionally accepting prevailing opinions, and especially opinions prevailing amongst those people whose views one most respects. The second is to seek an inspiration on one's own account. Some people will prefer to begin, in the first way, with 'authority'; and some will prefer to begin, in the second way, with 'private judgment.' But, in either case, one must proceed to act on one's provisional hypothesiswhether it be the prevailing opinion, or one's own inspiration-with

* On p. 196 above.

¥ On p. 306 below, especially the footnote $\|$.

$\$$ In Chapters $\mathrm{I}_{3}$ and I4. $_{4}$ || Cf. p. I92 above.

I See above, p. 265, footnote $\uparrow$. 
a view to its verification, by seeing whether it fits all one's own experience and so much of the experience of other people as one can disentangle from their interpretation of it.

\section{§ 5. The Christian Hypothesis.}

Now concerning the central essences of the endarchy of science there is one prevailing opinion, accepted by many of the most respected men of our own time and of earlier times, that merits our special attention, because it fits all the conclusions that we have just been drawing concerning the central neurographic elements in the typical character in a maximally efficient, and therefore progressive, society. This account of the central essences of our world, and of their relation to the (not necessarily more familiar) facts of more direct experience, is that given by Christianity; for, after all, as Professor Headlam lately said in an Oxford University Sermon*, there is such a thing as Christianity. And this account, that Christianity gives of the universe, differs only from the hitherto discovered portion of the endarchy of science, in that the Christian account supplies a hypothesis concerning the undiscovered central essences of the endarchy of science and their relation to its discovered portion. In short, the Christian account of the universe-or, as we may term it, the Christian philosophy-completes the discovered part of the endarchy of science with a hypothesis concerning the hitherto undiscovered central essences; and it does so, as we are about to observe, in a manner that enables the corresponding neurography to fulfil the conditions that have to be satisfied by the neurography of the typical citizen of a maximally efficient and progressive community.

Of course it is conceivable that, as scientific thought proceeds towards its goal, it may formulate or discover $\uparrow$ central essences that are at variance with those disclosed by the Christian philosophy as it exists to-day. But we are not, on that account, to wait and see. For unless some men formulate for themselves, or accept from others, and in either case act on, a hypothesis concerning these central essences, they never will be discovered. And, unless every man takes some hypothesis concerning the central essences, and acts upon it with a view to its verification, he will never possess the type of character that belongs to members of a maximally progressive community.

We proceed to notice how the Christian account of the real worldthe Christian philosophy-makes it possible to satisfy the conditions

* Preached on the IIth March, I917.

$\dagger$ Cf. footnote || on p. I96 above.

G. E. 
which our description (in $\S 4$ of Chapter I2, and in $\S 3$ of this chapter) imposes upon the neurography of the typical member of a maximally progressive society. We shall see that, if the Christian hypothesis concerning the central essences of the endarchy of science continues to fit experience, the central elements of a neurography which satisfies the other conditions in question, may also correspond to the central essences of the endarchy of science; so that, in particular, truth as well as justice may prevail in a community all of whose members accept and act upon the Christian hypothesis.

In the first place, then, as we have already* seen, Christianity describes God as having to all else in the universe a relation that closely resembles the relation which the central essences of the endarchy of science bear to all the rest. This suggests a more descriptive name for the 'Christian philosophy.' That more descriptive name was used by its first and greatest teacher, whose teaching was described by his earliest disciples as the 'gospel of the kingdom of God.' 'The kingdom of God' is then the phrase by which we shall in future describe the Christian philosophy: the endarchy of science completed, as regards its undiscovered portion, by the Christian hypotheses concerning its undiscovered central essences.

Secondly, just as we have seen $\dagger$ that our ideal neurography-the neurography of the typical citizen of a maximally progressive Commonwealth-must be centred in a purpose that shall correspond to the central essences of the endarchy of science, so Christianity insists that every man's thought and conduct should be subject to a single supreme purpose that is to be intimately linked with the central essence of the endarchy of science, and therefore, according to the Christian account, with God. 'Seek ye first the kingdom of God' was Christ's exhortation to his disciples to let their lives be governed by a single dominant purpose§. In fact, the advancement of 'the kingdom of God' in the minds of men is the central purpose of every true Christian\|.

Thirdly, Christianity places man in a peculiarly close relationship

* On p. 24 I above.

$\dagger$ On pp. 295 and 244 above.

† Matthew vi. 33. Or again, when some one said to Christ 'Lord, I will follow thee...,' his reply was 'Go thou and preach the kingdom of God.' (Luke ix. 57 and 6o.)

$\S$ Cf. St Paul: 'As many as are led by the Spirit of God '-the central purpose which is God - 'they are the sons of God.' (Romans viii. I4.)

\| This statement is consistent with a suggestion made in footnote * on p. 241 where we said that the central purpose was 'to know God.' For the advancement of the kingdom of God in the minds of men includes its advancement in our own individual minds; and that means organising our neurographies according to the Christian conception of the endarchy of science; and that includes making the 
to God. Jesus taught his disciples to call God their 'father.' Just so we found ${ }^{*}$ that some essence of mankind, and indeed some essences of all individual men, must be near the centre of the endarchy of science, and therefore near the central essence of that endarchy.

Fourthly, we have seen $\dagger$ that active faith is essential to the development of an ideal character; or at least $\ddagger$ of the character that secures consistent and effective conduct among the citizens of an ideal Commonwealth. So, too, Christianity asserts the essential importance of active faith in the Christian character. 'Without faith,' says the writer of the epistle to the Hebrews, 'it is impossible to please God.' $\S$ And the faith that, according to Christianity, is the first step towards knowing God, and to the salvation-eternal lifewhich this knowledge brings with it||, is no passive acceptance of unverified dogma but action on a hypothesis-the hypothesis that Christianity offers concerning the undiscovered part of the endarchy of science-with a view to its verification.

It is true that this faith, on which Christians have always laid so much stress that St Paul even wrote of 'being justified by faith,' is often supposed to be merely passive and not active at all. But this view is surely mistaken. When St Paul told the jailor 'Believe** on the Lord Jesus Christ and thou shalt be saved' he meant 'Go and act upon the fundamental hypothesis of Christianity - that there is a God and that Jesus Christ knew him (or at least knew him far more fully than any man has ever known him before or since) and so taught truth concerning him-and in so doing you will become satisfied that your assumption is correct, and at the same time you will come to love your neighbour and to love God whom to know is life eternal.' But, if St Paul did not continually insist that the faith of which he wrote was essentially active and not passive, Jesus was always quite explicit about it. For Jesus summed up the law and the prophets in the golden rule: 'Whatsoever ye would that men should do to you, do ye even so to them.' He emphasised the same point in the parable of the sheep and the goats, when he made the difference between the saved and the unsaved to consist solely in the

central elements of our neurographies correspond to the central essences of this Christian endarchy, the kingdom of God; and that means knowing God, since God is the centre of the Christian endarchy.

* On p. zor above.

$\ddagger$ See also p. 299.

$\dagger$ On p. 297 above.

$\S$ Hebrews xi. 6.

II 'This is life eternal, that they might know thee the only true God, and Jesus Christ, whom thou hast sent.' (John xvii. 3.)

If Cf. p. 297 above.

** In Greek $\pi i \sigma \tau \epsilon v \sigma o \nu$, the same word as faith ( $\pi i \sigma \tau i s)$. 
fact that the former had actively served* their fellows while the latter had not: no question was raised as to the passive opinions of either. And again, in the Sermon on the Mount, he said 'Not every one that saith unto me, Lord, Lord, shall enter into the kingdom of heaven; but he that doeth....'

Fifthly, just as we have seen $\uparrow$ that hope for something that lies beyond the end of human life is an essential constituent of the character of the typical citizen of our ideal Commonwealth, so Christianity insists on the essential importance of hope, hope of salvation $\ddagger$, hope of eternal life .

Sixthly, we saw $\|$ that brotherly love-árám - -is an essential and central constituent of the typical character among a maximally progressive community. And Christianity makes árám $\eta$ supreme in the kingdom of God; or, in other words, gives àyám $\eta$ the central place in its philosophy. For Christianity teaches that 'God is love.' T Thus Christianity supplies** the dominant emotional element in the Christian character, and it intimately links this emotion with its central purpose that we have described as 'knowing God.' For, if God is love, one cannot know God without loving. Any attempt to form a neurogram of God as the central element of a single wide interest-system and to omit from it this affective-conative element, whose activity accompanies loving thoughts and loving deeds, would ignore the very essence of God. If, therefore, we accept the fundamental teachings of Christianity as our central hypothesis, we must not only seek $\uparrow \dagger$ to know God $\ddagger$, but must also love him and all men, so fulfilling Christ's summary of the commandments: 'Thou shalt

* It is indeed a remarkable circumstance that, insisting as Jesus did on the paramount importance of active service of others-the 'conative element' in Christianity, corresponding to the fundamental 'emotional elenent,' brotherly love-his followers, hardly less than his critics, have commonly regarded his religion as one of suppression rather than of activity and growth. They have laid stress on the need for leaving undone what ought not to be done, instead of on the need for doing what ought to be done. The 'conative element' in Christianity is surely not self-denial but action: the devotion of one's life to the active service of others. Whatever self-denial is involved is secondary, incidental; the things given up are crowded out by action. And activity, especially activity in the service of others, is a chief source of happiness. The mediaeval saints who cut themselves off from the world, underwent great hardship in the quest of their own salvation, and, in some instances, finished by crucifying themselves, were widely mistaken if they thought that they were following in the footsteps of Jesus who went about doing good.

$\uparrow$ See above, p. 299.

$\$$ Titus iii. 7 .

I John iv. 8.

** One cannot reflect upon the character and conduct of Jesus Christ, and especially upon the manner of his death, without loving him.

$\dagger+$ Hebrews xi. 6. $\ddagger \ddagger$ John xvii. 3 . 
love the Lord thy God...' and 'Thou shalt love thy neighbour as thyself.' * Thus Christianity teaches that we are not here merely to know $\dagger$ God, but also to fulfil his purposes: knowing God, loving God and fulfilling his commandments $\ddagger$, all go together.

We thus see how closely the Christian hypothesis concerning the central essences of the real world corresponds to the central neurographic elements in the character of the typical member of a maximally progressive community. Moreover, some such hypothesis must, as we saw $\S$, be acted upon by every one who would possess this type of character. The Christian hypothesis is not necessarily the only possible one from which to start. But if any other that equally well fits the facts we have been considering has ever been formulated, it certainly is not so readily available, or so widely accepted already, as that which Christianity offers. We conclude that every member of a maximally progressive society should begin with the Christian hypothesis. And, since no society can be as progressive as possible if its freedom to fulfil its common purpose is liable to interference from rival societies outside it, a maximally progressive society is one that includes the whole human race. It follows that, if all mankind, or any section of it, is to advance (never mind in what direction) as rapidly as possible, all men should provisionally accept, and, with a view to verifying it, act upon, the Christian hypothesis concerning all that is most important in the universe. We note in passing that, when the Christian hypothesis has been decided upon, the direction of the progress-namely towards the fulfilment of the central Christian purpose $\|$-is also determined.

We must however be careful to realise that the acceptance of the fundamental teachings of Christianity does not mean the acceptance of all the frills that have gradually been added to them, even so as to obscure them altogether. Or, changing the metaphor, we have to be careful to distinguish the foundations of the Christian church from its gargoyles. If, therefore, that is true for any man which fits his personal experience and so much of the experience of others as he can disentangle from their interpretation of it $\Phi$, there is much reason to expect that, whoever will begin to act on the hypothesis that Christianity is true, will find the hypothesis fit his experience, and so will verify it. But his experience, as it develops, will doubtless lead to modifications of any unessential assumptions which he may

* Matthew xxii. 37 and 39.

‡ Cf. I John v. 3 .

II See above, p. 306. $\dagger$ Cf. above, footnote * on p. 24 I, and p. 218.

§ On p. 305 above.

II Cf. p. 305 above. 
include in his first approximation. His Christianity will thus be sure to differ, in some respects, from that of any other Christian. Indeed, it must, if it be a living faith, grow from day to day and from hour to hour.

Moreover, if we can find no better central hypothesis upon which men are to act in the interests of human progress, we can find none better for them to advise their pupils to take as a first approximation, and to act upon with a view to verifying it. But its experimental* character must be made clear from the outset.

* Cf. Dr Stanley Hall: 'Religion is not theology nor yet ethics, but personal and experimental.' (Adolescence, Vol. II, p. 326, quoted above, p. 297.) 


\section{CHAPTER 18}

\section{THE AIM OF EDUCATION}

HERE then at last we have our answer to the question, 'What should be the aim of education?' And our answer is, 'To form Christian characters': characters of the kind described on p. 303, but such that the most central neurographic elements, while fulfilling the conditions there laid down, also correspond to the most important things in the universe as interpreted by Christianity.

But these characters, although they are all to include strong Wills cooperating with single wide interest-systems centred in elements that correspond to the same supreme purpose, are not (we remind* ourselves) to be identical in respect of the remainder of their content. For the many members of the world society are not all to have the same office. Division of labour is as necessary for the effective achievement of the supreme purpose of mankind as for so many of the subsidiary purposes with which every civilised community is familiar. Differentiation of function, partly depending upon innate constitutional differences between individuals, must be prepared for by a world-wide (as by a national or even municipal) system of education.

So the Christian character that education should aim at forming in every human being, should consist of a strong Will cooperating with a single wide interest-system $\dagger$, of which the central elements correspond to the central essences of the Christian hypothesis, while the peripheral elements correspond to the subject's unorganised experience. These peripheral elements are linked to each other and to the central elements by an intermediate system of organised elements - the scientific endarchy - that should correspond as closely as possible with the endarchy of science, and especially with that part of it that is most closely related to the particular group of activities which the individual in question contributes as his share towards the fulfilment of the common purpose of himself and all his neighbours, near and far. And that common supreme purpose of all world-citizensई is-in a phrase to which our enquiry has given precise significancellthe advancement of the kingdom of God in the minds of men.

* Cf. pp. 227, 232.

$\S$ See above, p. 306 . $\dagger$ See above, p. 244 . II See above, p. 309. $\ddagger$ See above, p. 228. 


\section{CHAPTER 19}

\section{THE AIM OF EDUCATION-SUMMARY}

THE results obtained in the foregoing enquiry concerning the aim of education in a World Commonwealth may now be summarised, before we pass on to enquire how this aim can be most effectively and rapidly achieved in the England of to-day.

In Book I we summarised Professor Adams' history of the aim of education in the past. We noticed the absence of any clearly defined and generally recognised aim for education as a whole-a national or world-wide system of education - at the present time. We observed the need for such an aim: an aim provisionally agreed upon and actively pursued, but capable of modification to fit new facts. And, finally, in the light of opinions expressed by great educators before the advent of the experimental science of physiological psychology, we provisionally decided that the aim of education, at least during adolescence and maturity, should be to develop, in every educand, a single wide interest-a unity of knowledge and feeling - that should be the principal factor in determining his thought and action.

In Book II we have sought further light from physiological psychology. We enunciated five 'laws of thought,' of which the first three summed up the leading available facts concerning the physiological processes that accompany thinking, and led to an important corollary concerning the influence of one's neurography-one's system of low resistance paths along which the nervous impulse passes-upon one's thought; namely, that the value of a neurograma system of low resistance paths whose excitement accompanies a thought-activity-measured by the effect it produces upon the stream of thought (and so, as we afterwards saw, upon conduct), depends upon its connexions with other neurograms. This effect, we found, was greater, the wider and deeper the interest-system of which the neurogram in question forms part; and, under certain conditions-if, for example the system is organised according to a particular pattern that we called a 'maximal endarchy'-is also greater the more central the position it occupies in that system. Later we saw that a system of neurograms that corresponds to a purpose tends to have a greater influence on thought and conduct than any other system equally 
wide and deep; and that every such purpose-system tends to be rich in emotional elements.

The fourth law postulated a form of psycho-physical interaction: the freedom of the Will to influence brain processes. We found, in recent experimental and statistical enquiries, evidence of the presence of one and the same general factor in all human qualities. This factor we identified with Will, so that its measure, $g$, measures the extent to which one's Will is able to influence one's thought and conduct. The occasions and manner of that influence were next investigated. We found no evidence that Will intervenes in the stream of thought-activities except to resolve conflicts between rival interest-systems; and we saw that reasoning is the principal means by which Will resolves such conflicts and organises the neurography to prevent their occurrence or recurrence. So we came to examine the reasoning process, especially in its relation to the world of experience with which men's reasoning is chiefly concerned. We were thus led to a definition of the 'neat, trim, tidy, exact world which is the goal of scientific thought,' and we described it as the 'endarchy of science.'

We next asked ourselves how far, having regard to the various activities of different men and women in the life of the community to which they belong, should their several neurographies correspond to this complete endarchy of science. We answered that, for a number of reasons which we enumerated, and, in particular, so as to ensure efficient and consistent thinking that makes for human progress, every citizen ought to develop a tidy and perfectly integrated mind-a single endarchy of neurograms-which should correspond, in so far as the time and effort available for his education and his own 'educability' permit, to the endarchy of science; but which can never correspond to the complete endarchy, nor even to all that part of it which has yet been discovered; and which, therefore, should correspond to part only of the complete endarchy, the part in question depending upon the special activities of the individual citizen in thelife of the community to which he belongs, but should always include central elements corresponding to the central essences of the endarchy of science. Then the personal endarchies of the several citizens would each form a single interest-system consisting, as regards its centre, of elements which correspond to the central essences of the endarchy of science, and, for the rest, made up, partly of a scientific endarchy of neurograms corresponding to part of the endarchy of science, and partly of less well organised neurograms corresponding to the remainder of his experience. Such a neurography we described as a single wide 
interest-system. The central elements in every citizen's neurography, would then, we saw*, correspond to purposes; and, since the central elements of different citizens' neurographies were all to correspond to the same central essences of the endarchy of science, the central dominant purposes of all the citizens would be in harmony with one another. We went on to consider how neurographies of this kind may be developed; and we saw that, while the instinct-emotion of curiosity-wonder has an immensely important part to play, their development is in the main a voluntary process in which the teacher's Will gradually gives place to the pupil's as, in later adolescence, the pupil becomes, and thereafter remains, his own chief educator.

Our fifth law extended from thought to conduct, from intelligence to character, the application of our earlier discussions of the influence of neurography and Will upon the stream of thought. So the five laws $\dagger$ between them summed up, in a manner consistent with available experience, the working of the physical and psychical factorsneurography and Will-that characterise a human being by determining how he reacts to his environment. In particular, the extension effected by our fifth law enabled us to see that the type of neurography that we had described as a single wide interest-system, and that makes for efficient and consistent thinking, will also make for consistent and effective conduct, especially if it be helped by strong Will. And it further appeared that, if the central purpose essences of the single wide interests of a group of people were mutually harmonious (implying some essential purpose common to them all), the conduct of each member of the group-each citizen of the Commonwealthwould not only tend to be consistent with itself, but also with the conduct of the other members. Thus we had defined some of the conditions that should be fulfilled by the character of every member of a community in order to ensure mutually consistent conduct among them all. The type of character that satisfied these conditions we described by the word 'strong.'

Then we attempted a further step, this time into the domain of ethics. Having described a strong character, could we define a higher type that we might reasonably describe as 'good'; or even as 'best,' so that to develop it in every citizen should be the aim of education? For we saw that the aim of education could not be defined until the aim of life had first been determined.

In the absence of any generally accepted view of the aim of human life, we assumed, consistently with a very widespread belief

$$
\text { * See above, p. } 238 \text {. } \quad \text { † See above, p. } 289 \text {. }
$$


among western peoples, that human societies should at least aim at making progress-advancing continuously towards the fulfilment of some far reaching purpose. And we found that, if the Commonwealth, whose citizens' education concerns us, is to make as rapid progress as possible, not only should its typical citizen possess a 'strong' character, but the central purpose-elements of his single wide interestsystem should correspond to a hypothesis concerning the central undiscovered essences of the endarchy of science, and should fulfil four other conditions. These other conditions required that the central purpose-elements in question should be formed by active faith, action on a hypothesis with a view to verifying it; be rich in hope; be stimulated by brotherly love more than by any other primary emotion (since, unless brotherly love is the chief emotional constituent of the common central purpose of all the members of a community, they will hardly avoid conflicts about the minor matters of everyday life, however strongly they strive for the fulfilment of the main purpose that they have in common); and include, among the most central of all, elements corresponding to the essence of humanity. All these conditions are satisfied by the fundamental Christian hypothesis : that God is the centre of the universe, the central fact of the endarchy of science; that knowledge of God begins by faith; that hope of eternal life belongs to people who seek to know God; that brotherly love is of the very essence of God; and that all human beings are in some peculiarly close relation to God.

No other hypothesis that fits the conditions is so readily a vailable, or so widely accepted, or perhaps has ever been formulated; although one could conceivably be imagined. So we concluded that every member of a maximally progressive community-whatever the direction of its progress-will naturally begin with the Christian hypothesis to complete and focus the single wide interest which, in cooperation with a strong Will, will form his character. Such a character we described as a Christian character. And we observed that, the Christian hypothesis having been selected to focus the single wide interest, the direction of the community's progress was determined: namely, towards the fulfilment of the supreme Christian purpose, which we analysed and described as 'the advancement of the kingdom of God in the minds of men.' By the phrase 'the kingdom of God' we denoted the endarchy of science as hitherto discovered, but completed, as regards its central undiscovered portion, by the Christian hypothesis concerning the central essences of the universe and their relations to each other and to all the rest. 
Thus, in a maximally progressive community (and thereforesince no group can be maximally progressive if possible conflict with other groups restricts its freedom to develop-among the whole human race), the common supreme purpose must be this Christian purpose, and the typical character must be the Christian character just defined. The aim of education, the world over, followed at once: 'to form Christian characters,' with all the manifold outer differences that are necessary if their several owners are to cooperate effectively for the fulfilment of their common supreme purpose. 


\section{BOOK III}

A SYSTEM OF EDUCATION 



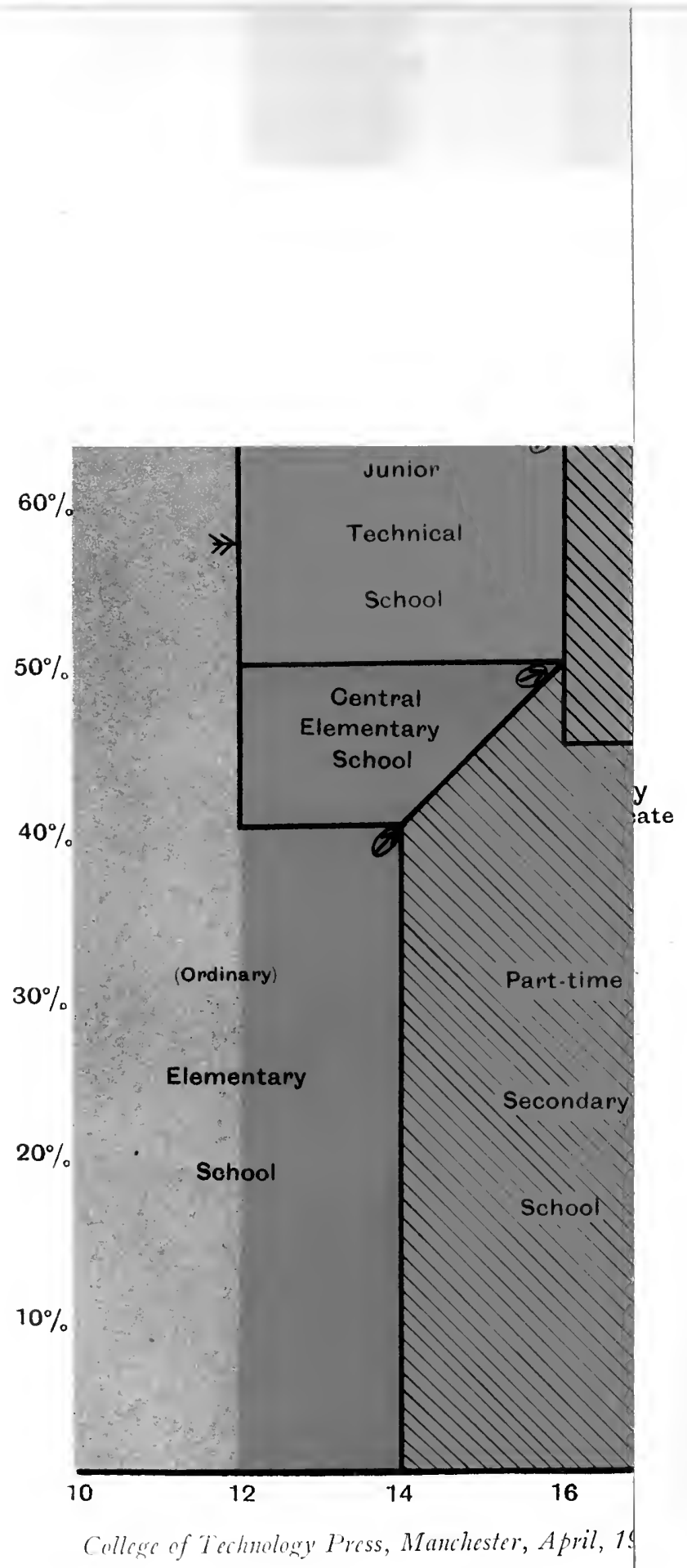




\section{CHAPTER 20}

\section{TYPES OF SOCIAL SERVICE}

\section{$\S$ I. Some Principles of Selection.}

How is this aim of education to be realised? How, in particular, can a world-wide, national, or provincial system of education develop in every citizen this ideal character, with its single wide interest-system that partly depends upon the individual's activities, social as well as vocational? For, until we know the types of activity that a given person is to engage in, the type of character he should ideally possess remains indeterminate*.

The problem how to fashion a perfect system of education-a system that will achieve, in the case of every member of a World Commonwealth, the aim of education that we have defined $\uparrow-$ depends, therefore, on knowing what each person's occupation is going to be. So the far simpler problem with which we shall be principally concerned in Book III--the problem how to adapt and develop (and especially how to begin to adapt and develop) the present provision of education in England, so as ultimately to transform it along the path of least total resistance (the future being discounted at a reasonable rate) into the most perfect system that can at present be conceived for England-depends for its solution upon firstclassifying the occupations of English citizens. Until that has been done, the problem of developing in a sufficient number of future citizens corresponding types of single wide interest, and therefore of character, cannot be solved.

Any attempt to decide beforehand, and without regard to their natural abilities, what positions particular children were to occupy when grown-up, and to educate them accordingly, would probably meet with disaster; but it is by no means certain that this disaster would be greater than that which attends the present practice of educating without regard to future occupation.

It is, however, quite another matter, first to investigate the qualities-especially the kind of single wide interest, and the degree of 'general ability' or ' $g$ ' $\$$-required by those who are to occupy the various positions in industry, commerce, and other essential

* See above, p. 292.

$\dagger$ See above, Chapter 18.

$\ddagger$ See above, Chapter 7 , and especially pp. I I 3 to II 7 . 
A NATIONAL SYSTEM OF EDUCATION

proposed to be brought into operation in England dur
TEN YEARS HENCE

$100 \%$

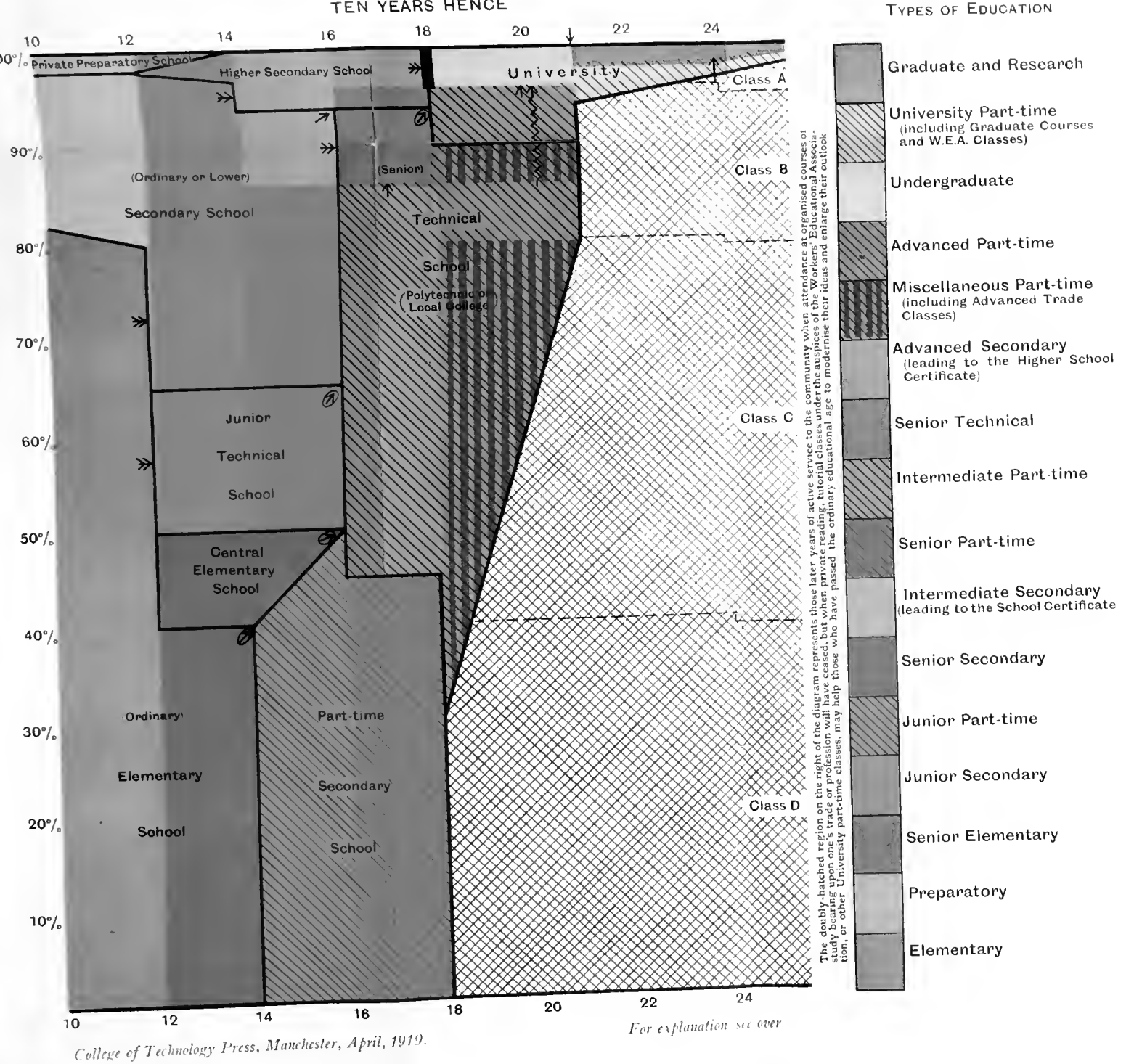

EXPLANATION OF DIAGRAM

The diagram is a 'flow diagram,' the flow being from left to right
Where the national system of education should make different provision for boys and girls respectively, particularly in respect of the types
of education represented on the right-hand side of the diagram, the of education represented the diagram is that proposed for boys and
arrangement shown by the super-normal people; it does not show the special provision require for seriously defective children.

Horizontal Scale: The figures along the top and bottom of the
diagram represent years of age of the ' modal " boy or girl.

Vertical Scale: The figures up the left margin of the diagram
represent percentages of the young people of a given age. represent percentages
The intercept made on any verticial liee, or ordinate, by the enclosed
area which corresponds to a school, or by the coloured area which corresponds to a type of education, is proportional to the number
of pupils of the corresponding age who should be attending the type of
example, the intercept on the 15 -year ordinate shows that, of the chictendary School, 29"., an ordinary or lower Secondary Scho

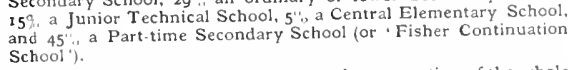
Moreover, the vertical scale indicates the proportion of the whole
number of young people who pass from one type of school to another at any age of transfer. For example, at the age of 12,15 to ordinary or lower Secondary Schools; or, at the age of 18,4 of the young men are shown as passing (possibly, in the case of
engineering students, after a short interval in works, marked by the
thick black line) from Higher Secondary Schools to the University. Colouring represents types of education, unshaded colours represent-
ing whole time education and shaded (singly hatched) colours As a rule, the lighter colours at the top of the diagram represent
more abstract and more chenerent, but less comprehensive, studies gram. For example, junior secondary education is more abstracl and coherent, but less comprehenensive, than senior elementary:
inturmediate secondary than senior secondary; advanced secondary
inum sentior technicalt; and intermediate part-time than senior and the Moreover the more abstract and coherent courses (marked by the
lighter colours) prepare for more concrete and comprebensive studies (marked by darker colours) in the final and most specific
stag of whole-time or part -time education. For example, "inter mediate 'courses, whole-time or part-time. are distinguished from

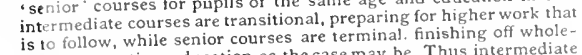

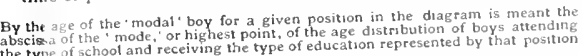

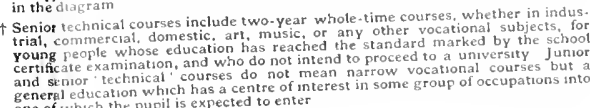

secondary education between 14 and 16 leads on to advance
secondary or senior techrical education between 16 and 8 , an ourses between 18 and 21 ; while senior secondary and seniter

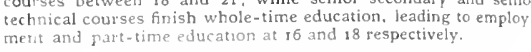

Lettering represents types of school or other educational institution
Indicating types of school by lettering and types of education colouring, the diagram is able to show that one type of school mal
provide more than one type of course of study and also that on type of course of study man be pursued in more than one type
school. Thus, junior secondary yeducation betwern 12 and 14 mat

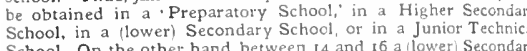

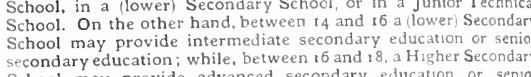

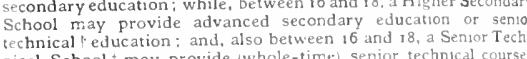

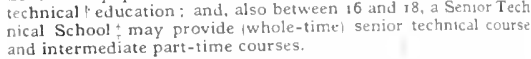

The number of boys or young men who should he attending an

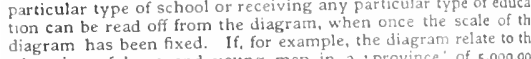

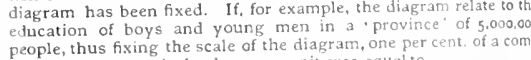
plete two-year vertical column a unit area equal to
-will stand for tr,ooo boys or men attending the eype of school of receiving the type of education represented hy the portion of
diagram in which that unit area is sicuated boys 4.0000 matring ad he the

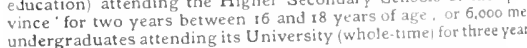
undergra.
from 18.

main-

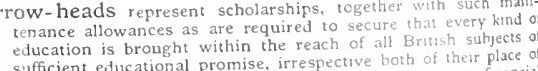

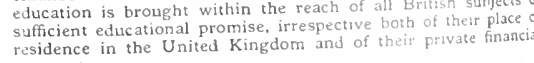

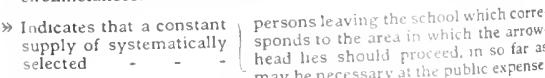

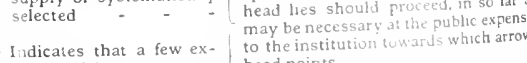

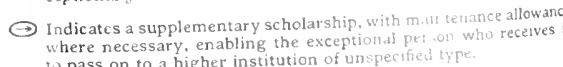

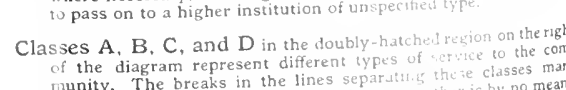

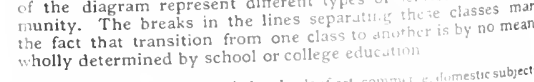

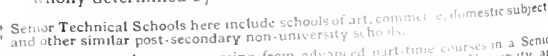

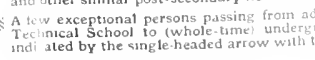


departments of English life; and afterwards to indicate a means of developing the required qualities in a sufficient number of persons selected on account of their innate aptitudes for each different kind of work.

Moreover, selection of this kind makes for the wellbeing of the community and of every individual member of it. Without selection it is impossible to realise the Pauline*-or Platonic $\dagger$-ideal of a commonwealth in which there are many different members together forming one body. Without selection, effective division of labour becomes impossible; and so also does that efficient production which enables a community to exempt from productive labour, and yet to maintain, those discoverers, teachers, seers or prophets on whom it should so largely depend in the weightiest affairs of human life. Or, in the jargon of to-day, square pegs in round holes, with the friction and unrest caused by such misfits, are the inevitable consequences of inadequate selection.

If it be argued, on the other side, that a policy of selection is inconsistent with equality of opportunity for every boy and girl and man and woman, we reply that this would indeed be true if selection were based upon any considerations except those of the personal qualities of the young people selected at several stages of education to receive, if necessary at the public expense, whatever higher type of education is justified by their own educational promise. If, for example, a boy's or girl's selection, for transfer to a school or college giving a higher type of education, depended upon his or her father's ability to pay high fees, or even to do without the money his child might have been earning, a policy of selection would be indefensible. Or, if scholarships and maintenance allowances to enable selected young people to receive higher education varied greatly in different parts of the country, there would still be much to be said against selection. Indeed, the selection of young people for transfer from one type of education to another must be made irrespective both of the young people's place of residence and of their private financial circumstances. A policy of selection will then have nothing to fear from the rival policy of throwing every kind of education open to all boys and girls who, with their parents' consent, prefer the cool and calm of academic groves, however incomprehensible they find the pursuits there, to the feverish strife of the world outside.

For, in the first place, the interests of the individual would obviously suffer were his education to run some uniform course, irrespective of his personal aptitude for the particular studies in

* Romans xii. 4 .

$\dagger$ Republic, Book II. 
question, and quite apart from his liking for the walk in life to which that education had been designed to lead. 'The idea,' wrote Ruskin, "of a general education that is to fit everybody to be Emperor of Russia...is the most entirely and directly diabolical of all the countless stupidities into which the British nation has, of late, been betrayed.'*

In the second place, public interests would certainly suffer if places of higher learning were filled by persons whose intellectual qualities and aims in life did not allow them to profit by the studies there pursued. If, for example, the medical schools of the country ceased to select candidates by a matriculation or entrance examination and instead threw open their doors to all who might like to enter at the public expense, the crowd of incompetents would prevent the better type of student from acquiring the knowledge and experience needed to make him an efficient medical practitioner later on. The medical service of the country would thus be seriously impaired; and other public services would suffer in the same way if the educational avenues by which they are approached were thronged by unqualified and incompetent crowds. Even if these avenues were so widened as to make ample room for everybody to pass from the elementary school to the secondary school and so to the university, the abstract studies of the higher educational institutions would not only fail to train, but would actually impair, the less able intellects. Indeed, the children for whose education so much has lately been done in schools for mentally defective children, would lose these benefits, and get nothing instead, were they expected to follow the same educational paths as had been prepared for other people; and it is to be remembered that the low grade intelligences here in question do not form a well defined class by themselves, but are merely the extremes in an approximately normal distribution of ability $\dagger$. Whoever recognises that so-called mentally defective children should be selected from the rest for special treatment, ought therefore also to recognise that separate treatment is equally desirable for every grade of subnormality or supernormality: and this means selection. So we shall expect that in the words of the prophecy of Mr Wells' modern Job, "The qualities that serve the great ends of the race will be cherished and increased; the sorts of men and women that have these qualities least will be made to understand the necessary restraints of their limitations.' +

* Fors Clavigera, p. 254.

$\dagger$ Cf. C. Burt, The Distribution and Relations of Educational Abilities. See also p. 475 below.

$\ddagger$ The Undying Fire, p. $2 \mathrm{Ir}$. 
It is, indeed, obvious that educational, as well as economic, considerations forbid the provision of a uniform system of education that would require, encourage, or even enable all young people, no matter how inferior their ability, to receive a type of education designed to fit the ablest of them to render to the community the best service of which they were capable. We should neither ignore the value of education altogether*, nor attempt to provide all men and women, whatever their ability and inclination, with identical education, but rather bring every kind of education within the reach of all citizens whose educational promise is sufficient to justify their selection to receive, if necessary at the public expense, that type of education which, having regard to their ability and inclination, will best fit them to serve their fellows and to find their own happiness in that service $\dagger$.

Recognising then that children and young people who are to receive a higher type of education, if necessary at the public expense, must be selected from among their fellows, we have to answer the question how this selection can best be effected. The nature of the selective test, with the part to be played in the selection by teachers, parents, pupils, external examiners and education authorities, is to be discussed in Chapter 25 below. At this point we need only consider some underlying principles.

We take first the quantitative aspect of the matter, and ask how many children and young people are to be selected, at each stage of education, for transfer to higher studies? The answer to this question depends upon the number of people required to render to the community the various types of service of which it stands in need, including those services of discovery, authorship, preaching and teaching that minister to the spiritual and intellectual needs of mankind but do not contribute directly to the production of material wealth.

Having decided approximately how many men and women are

* Cf. Winston Churchill, A Far Country, p. 455. 'It is difficult to answer a man who denies the cardinal principle of American democracy-that a good mayor or a governor may be made out of a dog-catcher. He called this the Cincinnatus theory, that any American, because he was an American, was fit for any job in the gift of state or city or government from sheriff to Ambassador to Great Britain. Krebs substituted for this fallacy what may be called the doctrine of potentiality. If we inaugurated and developed a system of democratic education, based on scientific principles, and caught the dog-catcher young enough, he might become a statesman or thinker or scientist, and make his contribution to the welfare and progress of the nation: again, he might not; but he would have his chance, he would not be in a position to complain. Here was a doctrine, I immediately perceived, which it would be suicidal to attempt to refute....'

$\dagger$ Cf. Ruskin: "The education that makes men happiest in themselves is that which also makes them most serviceable to others.' 
required for each different type of service, the community must next take into account the numbers that are already being privately educated for it. Of course the private provision of education, including those great private institutions that in England we know as 'public schools,' will especially cater for persons who hope to undertake the most influential, interesting and remunerative forms of service. But the public provision of education is not, on this account, to leave those occupations as the preserve of families distinguished by wealth or social position. On the contrary, it is of great importance to the community that the national system of education should supply ambassadors, diplomats, bishops, financiers, captains of industry and other leaders in church and state, better qualified for their responsible positions than were many or most of those who occupied these positions in the past. This statement by no means implies that the private provision of education in England will cease to supply any, or even many, leaders of the nation's thought and action; but it does imply that privately educated men will in future secure only those positions for which they are better qualified than the best men that the State has been able to select and to educate for the same kind of work. In other words, the number of people who should be selected, reselected, and selected again to receive, so far as is necessary at the public expense, the kind of education that will best prepare them for a particular kind of service, is the number which, when added to the number of those whom private education will have prepared as well or better for the same kind of service, will suffice to supply so much of the demand as is also a need for service of the type in question. Healthy competition between a national system of education on the one hand, and efficient private schools and colleges on the other, will facilitate educational experiments, make for progress and a high standard of work in the public as well as in the private educational institutions, and so benefit the nation; provided always that the private schools and colleges are subject to inspection, and are only permitted to survive so long as they do not waste the human or material resources with which they are entrusted*. On this condition, it should be open to anyone to be trained, according to his own choice, either in State-aided institutions, partly at the State's and partly at his own expense, or else privately, at no cost to public funds.

* The improvement of public secondary education has compelled many private schools of an old-fashioned type to close their doors, and will probably result in the closing of a good many more when they have been publicly inspected in accordance with Mr Fisher's Education Act of r9r8. 
Candidates who obtain the highest places in the examination for the higher division of the Civil Service do not all give the same order of preference to the various government offices which they might enter. They differ, for example, on the question whether the Indian or the Home Civil Service is to be preferred. So it sometimes happens that a candidate, who is placed second or third or even lower on the list, may secure the unique billet which he would have sought had he obtained the highest place. In the same way, it will not infrequently happen that a boy or girl who, having reached a stage of education when two or more alternative courses are open, and having been selected to follow the highest course that leads to more responsible work, but work involving greater intellectual effort, than that approached through the lower alternative course, will elect to enter the latter rather than the former. Thus, if a selective test is applied to boys in public elementary schools just before they reach the age of twelve, and if those who acquit themselves most creditably are free to go on to ordinary secondary schools, while those who do next best are entitled to promotion to junior technical schools, experience shews that several of the ablest boys (who might, if they chose, enter ordinary secondary schools) will prefer to enter junior technical schools instead, with the result that an equal number of less able boys will become entitled to promotion to ordinary secondary schools.

No method of selection will be able to compel selected boys and girls to proceed, against their will, to the higher type of education which they have been selected to receive, if necessary at the public expense; and this is especially true of selection applied to young persons who have passed the age of compulsory education. It is, however, to be hoped that the provision of adequate maintenance allowances will remove the economic causes of parental opposition to the advancement of able children. It is even more important that the parents and their children should realise that it is a public duty to cooperate whole-heartedly in any national system for the selection of the ablest young people to receive the highest education. Indeed, the future of the country, and, in particular, of the national industries, depends in no small degree upon the willingness of the ablest young Englishmen to subject themselves to the strenuous intellectual discipline demanded by a university course of study. When once this fact is generally realised, there will be no reason to fear that the kind of young men who responded so magnificently to their country's call to fight for her will refuse to work for her.

When we come to classify the types of service which England 
(for example) needs, we shall find that a higher degree of 'general ability' or ' $g$ ' is required for some occupations than for others. Selection for promotion to a higher type of education will therefore depend, inter alia, upon the degree of 'general ability' possessed by the candidates at the stage at which that selection takes place. This does not of course imply that, when the very first selection takes place, say just before the age of twelve, it will be necessary for an able boy to decide whether he aims at becoming a judge or a professor or a general (although of late it has been necessary for him to make up his mind, even at this early age, if he wishes to become an admiral); for the educational avenues to all these careers, and to many humbler ones, do not begin to fork until a much later age. Indeed, as we shall later observe*, boys who are to enter different occupations at, say, twenty-two years of age may well have more in common, as regards the type of education best suited to their needs at the age of seventeen, than have two seventeen-year-old boys of whom one is to enter mechanical engineering at eighteen and the other at twenty-two; for these last two are really aiming at different occupations, although in the same department of industry. That is why a junior technical school, intended for boys who are to become apprenticed to engineering trades at sixteen, is less suitable than an ordinary secondary school for boys who will go on to a senior technical school at sixteen with a view to entering engineering works two years later.

\section{$\S 2$. Classification of Services.}

For the same reason, as we now enter upon the classification of the various kinds of public service, with a view to discussing later on $\dagger$ how best to educate a sufficient number of persons selected on account of their innate aptitudes for each different kind of work, we reject the classification that will probably first suggest itself: namely, to classify work of public utility either as industrial or commercial, and then to sub-divide industry into its various branches, such as

* See Chapter 23, below.

$\dagger$ See below, Chapters 23 and 24. The Council for Organising the British Engineering Industry, established in Manchester in I9I5 and subsequently amalgamated with the British Engineers' Association, approved a report on Engineering Education and Research which was prepared by Mr A. P. M. Fleming of the British Westinghouse Electrical and Manufacturing Company, Dr Miles Walker, Professor of Electrical Engineering in the University of Manchester, and the present writer, and which followed the method in the text of first classifying occupations and the qualities required in each, and then describing a system of academic and practical training designed to develop the required qualities in a sufficient number of persons selected on account of their innate aptitudes for each different kind of work. 
engineering, building, chemical manufacture, the textile trades and the like. Indeed, the qualities required in the manager of an engineering works have more in common with those needed by the manager of a chemical works or of a cotton mill, than have the qualities sought for in the lowest grades of labour employed in any of these industries. In the same way, the designer of electrical machinery will generally have less in common with the engineering tradesman* who makes what he designs, than with the professional physicist, who would never be classed as an engineer.

We shall find it convenient to adopt the following classification:

CLASS A. Leaders in thought and action, including statesmen, captains of industry, discoverers and other persons distinguished in science or arts or letters: in fact, the modern counterparts of the oldtime prophets, priests and kings. (In organised industry, 'industrial statesmen, ' chief designers, research engineers or chemists, consulting engineers and the like, should as a rule belong to this class.)

Class B. Managers, officials, and others of intermediate rank, who occupy positions of responsibility, but not of the first importance to the community or to mankind. (Among industrial workers, many works managers and heads of departments, together with junior technically trained members of the designing, testing, and managerial staffs, belong to this class.)

Class C. Craftsmen, skilled tradesmen and leading hands, including the non-commissioned officers of industry, commerce, government, or other branches of national or international service. (In organised industry, foremen as well as leading hands and skilled tradesmen, will for the most part belong to this class.)

CLASS D. Labourers, repetition workers and other unskilled persons without specific training for any particular walk in life.

No essential discontinuities are to be imagined between these classes; nor are the occupations named to be regarded as forming a complete list of the classes of work they are intended to indicate.

We have already $\dagger$ remarked that the purposes of the various members of every community, and indeed of all communities, should be in harmony one with another, and that to this end the central elements of the neurography of every citizen should correspond to the same central essences of the universe. Moreover, since every occupation includes that of citizen, it is clear that, however the single

* I.e. the skilled craftsman.

$\dagger$ See above, pp. 240, 316. 
wide interests of different citizens will have to differ according to their different occupations*, the single wide interests will also have to overlap, so that different individuals may share as far as possible each others interests and have at least their interest in the State in common. For the present, however, we are not so much concerned with whatever is common to our four classes as with their special or distinguishing features.

In order better to focus our discussion on the distinguishing features of each of our four types of occupation, it will be convenient to pay special attention to the qualities required in industrial occupations. But it should be noted at the outset that the characteristic qualities, which we shall describe as belonging to those members of each class who are engaged in industry, will also belong to those members of the same respective classes who are engaged in other forms of public service $\dagger$.

It is clear that each class is concerned, in the course of daily work, with a greater variety of ideas than the class next below it. Accordingly, trains of thought of members of class A must, on the average, be fresher, and therefore less governed by habit, than those of members of classes B, C or D. 'The controllers of the Great Industry,' writes Mr Graham Wallas, 'are always on the look out for that type of man whom Americans call "a live wire." For such a man secretaries and typists and foremen carry on all that punctual performance of habitual acts which took up so much of the time and labour of a merchant or manufacturer even fifty years ago. He is set to form a habit of non-habituation.' $\ddagger$ Such a man requires more emotional drive than one who is engaged in mere routine work. And, since his ideas cover so wide a range, they are not so naturally associated together as those which their daily work brings to members of classes $\mathrm{B}$ or $\mathrm{C}$ or $\mathrm{D}$. He therefore needs to weld his various neurograms into a single wide interest-system by making voluntary connexions between them; and, in order to make such associations, especially between dissimilar ideas, he needs 'skill in thinking,' power to concentrate, Will, ' $g$ ' or general ability. So class A requires a wider interest and more ability, or skill in thinking-but not necessarily more paythan class $B$, class $B$ than class $C$, and class $C$ than class $D$.

* See above, p. 232 and pp. 3II, 313 .

+ The remaining paragraphs of this Chapter are taken, with but slight modification, from a paper on Education and Industry read by the present writer to the Education Section (L) of the British Association on IIth September, 1915, in Manchester.

$\ddagger$ The Great Society, p. 87 . 
Let us now look more closely at each of these classes. The first named on our list is that of the industrial statesman. We know him already as the captain of industry. But he may shortly change his name, for the title of captain does not indicate with sufficient clearness the fact that the head of a great industrial firm must needs concern hirnself with much that is happening outside the establishments which he controls. Not only must he be familiar with the state of the markets from which he draws his supplies and in which he disposes of his products, but, by grasping the significance of economic, social and political changes all over the world, he must be able to foresee opportunities for developing his business according to a far-reaching policy, and to indicate the lines of technical research which are most likely to lead to such developments. Work of this kind involves the widest sort of knowledge. But beware of the professional administrator who is prepared to administer anything at a moment's notice! The statesman-whether industrial or not-must possess, in addition to a wide range of knowledge and much ability, a very special interest in the particular concern he is directing, whether that concern is his own small business or an Empire whose destinies are under his control. He must see that concern as a whole, and must love it. 'Without passion,' said Lord Haldane to the students of Edinburgh University, 'nothing great is or ever has been accomplished.' *

Lord Haldane went on to compare the statesman with the expert, greatly to the latter's disadvantage $\uparrow$. We shall, however, place the expert in the same class as the industrial statesman, because the former requires an equally high degree of ability or Will, and at least as complex (if not so widely varied or so emotional) an interest as the latter. The industrial statesman may perhaps be compared to the astronomer who uses his telescope to increase his grasp of the universe as a whole, while the expert rather resembles the naturalist whose microscope enables him to see the parts in great detail. It is evident that the successful development of industry demands not only the expert in special branches of science or technology (even in so narrow a field as that of the methyl-blue chemist whom Lord Moulton recently described $\ddagger$ ), but also the industrial statesman who coordinates the work of experts in different fields, being himself enough of a specialist fully to understand his experts, to command

* The Conduct of Life (1914), p. 25.

† With Lord Haldane's Edinburgh address should be contrasted his speech (reported in The Times of r 3 th July, 1916) in the House of Lords on this country's lack of experts.

Journal of the Society of Dyers and Colourists, Vol. XxxI, p. Io. 
their confidence, and, when necessary, to decide between them. Whoever has authority must also have knowledge.

The members of class B have less need voluntarily to connect by reasoning the neurograms which constitute their 'scientific endarchies*.' Their skill in mathematics, for example, need only extend to what is commonly used, or is likely to bo used, in the routine of their daily duties: they must be able to follow a paper on some subject connected with their work, but need not have had enough practice in the use of mathematics to employ it freely upon investigations originated by themselves. They need less ability or 'skill in thinking' than members of class A. They require, on the other hand, a very wide descriptive knowledge of material things; and as much of this knowledge as possible they should have acquired at first hand from direct sense impressions. Last, but by no means least, the works manager and his immediate assistants need to interest themselves in the social and economic welfare-including the further education, recreation, and housing - of all their employees; and this interest will help to form the nuclei of the single wide interests which are to include all the activities of members of class $B$.

Foremen and leading hands have hitherto been generally recruited from among skilled tradesmen $\dagger$. They are therefore presumed to be qualified themselves to perform every task they have to supervise, and even to perform it better than the men who are actually doing the work. Upon this presumption is based the claim that the shop foreman must be paid a higher wage than any workman under him. This view, accepted as it generally is by employers and employed alike, is responsible for no small restriction of output. But it is based on a misconception, since the foreman is paid for supervising men, and the workman for manipulating material--two quite incommensurate processes. There are, however, signs of change. Technically trained foremen, whose wages may (to start with) be less than those of the men they have to look after, are already being employed, especially in shops where much repetition work is done. Yet it remains true that the qualities now most sought for in foremen and leading hands are those of the craftsman whose interest is centred in his manual work.

The operative skilled tradesman, whom, for this reason, we have placed in the same class as his foreman, is distinguished from the machine man in class $D$, in that the craftsman in class $C$ has a variety of skilled work to do, while the members of class $\mathrm{D}$, who may do

* See above, pp. 228, 231. † See the footnote * on p. 326 above. 
skilled work, repeat the same process over and over again until its performance is governed by habit, when it almost ceases to receive attention. On the one hand, ideas connected with doing, like ideas associated with a strong instinct, are peculiarly liable to receive attention*, so that the work of the skilled tradesman in class $C$ is well able to form a strong centre for his single wide interest. On the other hand, ideas connected with the repetition work of class D tend to become circumscribed and cut off from other interests. There is, however, reason to believe that repetition work is not altogether uninteresting to a certain type of mind. It is indeed actually preferred by some people, including many women; so that such work may form a substantial, if not a dominant, part of an interest that is not rich in exciting ideas.

The remaining part of the single wide interest is of special importance in the case of class D. Now that an eight-hours day is becoming universal $\dagger$, it may be that the artisan or labourer, who leaves work with much of his day still before him and feeling pleasantly exercised rather than unduly tired by his somewhat monotonous but by no means exacting labour, will devote himself increasingly to the affairs of the community: helping, for example, to solve from inside such urgent social problems as that of housing. With that end in view, we must see to it that the average member of class $D$ receives -not only in maturity through the Workers' Educational Association, but also in youth through vocational part-time classes-the kind of training which shall best develop a single interest, wide enough to include an interest in human brotherhood the world over. So may we ensure that the activities which he contributes towards the fulfilment of the common purpose of himself and his neighbours, are directed, nat merely to the prosperity of a particular industrial class, but to the well-being of mankind through the advancement of the kingdom of God in the minds of all men $\ddagger$.

* This follows from the Corollary to our Third Law (p. 89 above), in view of pp. 59,60 .

$\dagger$ The International Labour Organisation of the League of Nations met for the first International Labour Conference at Washington in November, rgrg. The organisation consists, according to Chapter xirr of the Peace Treaty, of two government representatives, one employers' representative, and one workers' representative from each State Member of the League. The Conference adopted six draft conventions and nine recommendations. One of the conventions, probably the most important, dealt with the question of the eight hours day and forty-eight hours week in industry. This convention was carried in the full conference by a majority of eighty-two votes against two. (These facts are quoted from a lecture on the International Regulation of Conditions of Labour delivered by Sir Malcolm Delevingne at the College of Technology, Manchester, on roth February, 1920.)

$\ddagger$ See above, pp. 306, 3 II. 


\title{
CHAPTER 21
}

\section{APPLICATIONS OF PRINCIPLES}

\author{
FORMULATED IN BOOK II
}

\section{$\S \mathrm{I}$. The Power of Education.}

THE principal conclusions of our Second Book have now to be applied to the problem of developing, in a sufficient number of suitably selected persons, the qualities which we have seen to be specially needed in each class of public service, so that the system of education with which we are concerned in this our Third Book shall produce men and women who will be able and anxious to serve each other and the World Commonwealth*, and who will realise their best selves in that service. So we shall now review some of the principles enunciated in Book II and cite further evidence that they fit experience, and especially the experience of teachers, and of those men of affairs whose work has brought them into close touch with human nature.

First then we would insist once more upon the power of education; and, in particular, that education really is able to achieve its aimthe aim which we have described as that of forming Christian characters.

We have already $\dagger$ quoted Dr Morton Prince's summary of his work on The Unconscious, where he says that systems of neurograms become a part of the personality, play an important part in determining behaviour, and, amongst other things, tend to determine one's point of view or attitude of mind, and, as large systems, may become sides of one's character. At the same time we quoted from another work of Dr Prince the suggestion 'that our characters are wholly i matter of brain associations and that they may be altered for good or ill by anything that will bring about a rearrangement of these associations,' and in particular by education. Education is concerned with bringing about a rearrangement of associations. Indeed, the process by which dissociations due to shell-shock $\ddagger$ have in so many cases been successfully treated in recent years, is often described as that of re-education.

* Cf. pp. 319, 363. In this Chapter we are not, as in the remainder of Book III, focussing our attention on England. † See above, p. 292.

$\ddagger$ Cf. Shell Shock, by Professors G. Elliot Smith and T. H. Pear. 
But it is not in medical literature alone that we have to look for evidence of the power of education to make or modify character. Lord Sydenham has said* that those who remembered the simple, kindly people of the southern German states forty or fifty years ago must have been astonished at the radical changes the War had revealed.

The utilization of a large system of education for political purposes has (he argued) enabled the ruling classes of Germany to Prussianise the whole country within two generations....The lesson we derive from this sad experience is that the power wielded by education is enormous for evil, but it must be equally powerful for good, properly handled.

Dr Lyttelton, when headmaster of Eton, urged that this power of education should be more widely recognised in England.

One of the most staggering facts about the Germans (he said), is that they give proofs of the power of a lie if it is taught with unanimity and consistency and for several years on end. Bad education, in short, if it is thorough, is a mighty power for playing havoc with human life. But we English, for the last three hundred years, have not really believed that education is a power at all....If Louvain, Reims, \&c., are the outcome of falsehoods thoroughly taught, what might be the power of truth if taught with equal thoroughness? $\dagger$

Or again, in the words of one of the greatest of English educators:

The war has proved the enormous power of education over the minds and souls of men. Applied with persistence and pedantic pertinacity, it is the most formidable engine in the modern world for controlling conduct and swaying purpose. England has shrunk from using this power in a masterful way.... Our reasons for not putting the power of education to its full and most effective use have been partly sound and partly stupid; stupid, in so far as we have failed to realize how powerful a dynamo education may become; sound, in so far as, for fear of its being misapplied by the State, we have deliberately foregone the advantages of using an instrument which can be set to cut deep into moral freedom and into private judgment on fundamental questions of right and wrong. $\ddagger$

\section{§ 2. Training the Will.}

If education is to exercise its great power so as to secure the greatest good for this nation, or for the whole world, it should aim, we said§, at cultivating strong Wills and at cooperating with

* In January, I9I 7 .

† Times Educational Supplement, 1st August, I916.

† Sir Michael Sadler, K.C.S.I., C.B., Vice-Chancellor of Leeds University, Times Educational Supplement, I 4 th January, 1916.

$\S$ See above, pp. 31r, 270. 
them to develop neurographies in the form of single wide interestsystems. Of these two consistent aims-that can best be realised by being pursued together*--we first remind ourselves of the cultivation of strong Wills by practice in hard thinking. We illustrated $\dagger$, by quotations from Jacobi, Sir Edmund Gosse, Dr Temple and Sir Stanley Leathes, how widely accepted is the view that Will, or power to concentrate attention, can be increased by practice; or, in other words + , that ' $g$ ' is educable; and we noted $\S$ Dr Webb's direct evidence that $g$ can be increased by practice in concentrating attention. Further evidence might be multiplied that the increase of $g$ by practice in concentrating attention fits the experience of practical teachers. For example, 'Care must be taken so that at every stage the children have work to do, something to bite at that is difficult but just not too difficult for them...' $\|$ Or again, the dominance of classics in English 'public schools' is justified by a public schoolmaster on the ground that 'it is easier beyond all question to make a form of thirty boys think hard and continuously over a Latin Prose than over any other subject.' 'f Mr Robinson speaks for many public schoolmasters besides himself when he adds that up to the ages of sixteen or seventeen, the time will be spent most profitably in training a boy to think.'

But one needs to concentrate upon a subject of study, not only in order to increase $g$, but also in order to deepen one's neurograms of that subject. Thus Mr Robinson says, in the article from which we have just quoted, 'the quickest way to instruct a boy in the careful and considered use of words and sentences is translation from another language,' because 'English books...will demand no true steady mental effort...offering...merely a soft attractive diet with nothing in particular to masticate or digest. No effort of attention or concentration is called out by their simple logic.' $\dagger \dagger$ Or again, Sir Stanley Leathes, tells us that what he found valuable in historical education 'came...not by lectures or systematic instruction, but in the effort to master and understand the books we were set to read.' $+\ddagger$

* See above, p. 225, where it is shewn that, other things being equal, Will or ' $g$ ' is best developed alongside of a single wide interest.

$\dagger$ See above, pp. 137, 138 .

$\ddagger$ The identification of the quality whose measure is $g$ with Will, or power to concentrate attention, is discussed in Chapter 7 above.

$\S$ See above, p. $13^{8}$; and below, Appendix B, $\S 8$.

II The New Teaching, p. 262. (Article by James Fairgrieve, M.A., F.R.G.S., on Geography.)

If Mr C. E. Robinson of Winchester in The Nineteenth Century and After, June, I9I7, p. I324.

t† Loc. cit. pp. 1320, 1321.

** Loc. cit. p. 1326 .

$\ddagger \ddagger$ Loc. cit. p. 82, italics mine.

G. E. 


\section{§3. The Central Purpose.}

The object of education is, we said, to form a single wide interest centred in a Christian purpose; and we pointed out that the single wide interest that education has to develop is not to be shapeless, but to have the very definite form which we described* as endarchical. This single wide interest is in fact to be a shapely well-planned structure, conforming to a well-thought-out design and, especially, it is to be focussed in a central purpose. It is to have, not several foci, but one focus $\uparrow$. It is to consist, not of several subjects, but of a single comprehensive unity.

Here too we are in agreement with the experience of leading educators. Thus Professor T. P. Nunn observes:

The architectural consistency and completeness of a curriculum is a matter of high importance; its development from the lowest form of a school to the highest should be like a piece of continuous thinking, forming a logical, well-rounded-off whole. One of the worst results of organising the teaching in terms of subjects is, indeed, that it makes architectural consistency so difficult; that it produces isolated groups of apartments but no unitary buildings $\ddagger$.

We have seen $\S$ further that, in this shapely structure, this single wide interest-system, the central elements are the most important, for they correspond to the supreme and dominant purpose, to which the whole single wide interest should, as we said, be subject. The special importance that we have attached to the central Christian purpose $\|$ is recognised, not only by accepted religious leaders, but also, and very widely, by public men who lead in other branches of the nation's life and thought. When, for example, the present writer was recently discussing the question of engineering training with a group of a dozen or more Manchester engineers, and had in passing expressed the opinion that the function of the secondary school, through which most of the future leaders of engineering industry should pass on their way to college, was to foster the growth of true religion, one after another of the members of the group took up this question and asserted that, in his opinion, religion was intimately connected with all that was best in his own business. One of the engineers present went so far as to say that he never made any important advance in his designs or methods of manufacture without feeling its close

* See above, Chapter 18; and p. 244.

$\uparrow$ The bearing of this observation upon hobbies and holidays is discussed in Appendix D.

$\ddagger$ The New Teaching, pp. 173, 174. (Article on Science.)

§ On pp. 244 and 3 I I above.

II See above, pp. 306 to 309 . 
relation to his religious life; and it appeared that most, if not all, of the other members of the group shared the same experience.

And the Christian purpose must be central in the single wide interest; it must be related to all the rest of that interest, instead of forming a separate interest as is too often the case in practice. Accordingly, the managing director of Messrs Cammell Laird and Company has thus criticised modern education:

No one, I think, can fail to notice the tendency to keep moral education apart from the rest of the curriculum. The former is confined almost entirely to the Sunday sermon in chapel, with possibly an occasional shy reference to the subject during Scripture lessons. The consequence is that a boy's religious life and his ordinary every-day life are in two separate water-tight compartments, and this fatal doubleness of life-this duplicity - pursues him to the end of his days, producing that shapelessness of life which Plato regarded with so much horror. The code of honour that regulates his every-day life is derived, not from religious teaching, but from the cricket field or the football ground*.

\section{§ 4. The Scientific Endarchy and Organised Thought.}

We turn next to the intermediate zone $\dagger$ of the single wide interestsystem. This zone corresponds to one's organised thought. Connecting the peripheral to the central elements of one's neurography, it forms the framework, and, with the central elements, determines the form, of the whole structure. After the central elements themselves, it is these of the intermediate zone that are the most important; or, what amounts almost to the same thing, scientific arrangement of knowledge is more important than knowledge of any particular concrete facts $\ddagger$. Education is more concerned with values-the more central among endarchically ordered essences-than with facts as such .

The special importance which we were led to attach to this intermediate zone-the scientific endarchy, as we previously\| called it-in the singlewide interest-system, accords with the opinions of experienced educators.

Thus Cardinal Newman, writing in 1852 , insisted that this framework, this philosophy which links all one's knowledge and experience together and (especially) to one's central purpose, far surpasses in importance any particular section of one's knowledge or experience regarded by itself alone. So he writes, with reference to the knowledge

* Mr W. L. Hichens, in an address on 'Education and Business' delivered to the Incorporated Association of Headmasters, on 9th January, I9I 7.

+ See above, p. 3 II. † See above, Chapters II and I 2.

$\S$ See above, p. $2 \mathrm{I} 7$, especially footnote $\S$. II On p. 228 above. 
which is to be pursued in education, that 'philosophy must be its form, or, in other words, that its matter must not be admitted into a mind as so much acquirement, but must be mastered and appropriated as a system consisting of parts related one to another in the unity of the whole.'*

Another publicist, whose writings are probably exercising a greater influence on English thought about education than those of any other modern author, expresses a similar view when he says:

What should constitute the education of the public man? This is something above and outside his attainments, his accomplishments, his business equipment....These are secondary things.... What is the backbone stuff? The answer to-day is surely not essentially different from the answer a Greek would have given in the time of Plato. He would have said nothing of the importance of compulsory Egyptian or Sansk1it, and equally nothing of a knowledge of simples or metal working. But he would have said that the backbone stuff must be a clear and critical knowledge of oneself in relation to God and to the universe. That, we submit, under modern conditions means for an Englishman a thorough study of philosophy and of history $\dagger$.

It is indeed from such studies, and especially from the study of the history of his own particular kind of service to the community, that the public man links his scientific endarchy, his expert knowledge, to his central Christian purpose, humanity and God.

\section{§ 5. A Single Wide Interest: not Separate Subjects.}

Now we have seen that education has not only to aim at producing a single wide interest $\ddagger$, as its ultimate goal on the neurographic side, but has also to give the same form to the growing neurography at each stage of its development§. In particular, knowledge is not to be imparted to the pupil as several separate subjects to be ultimately welded together so as to form a single wide interest; rather is the unity of knowledge, as far as possible, to be insisted upon throughout the educative process. Thus we pointed out that the value of any given expenditure of effort, whether by teacher or taught or by both, decreased rapidly with the number of separate subjects

* Idea of a University, Discourse 6. Cf. the quotation from G. K. Chesterton on p. 296 above.

$\dagger \mathrm{Mr}$ H. G. Wells' Articles on 'The Elements of Reconstruction,' The Times, August, I916.

$\ddagger$ See above, pp. 3II, 244

$\S$ See above, pp. 223, 224; especially footnote * on p. 224. 
upon which that effort was expended*. We even proposed a formula for ideally simple cases, and said that, in such cases, the educational value of the study of a new department of knowledge was proportional to the square of the time during which the study was continued, and inversely proportional to the number of separate subjects into which that branch of knowledge was sub-divided $\dagger$. This formula apparently fits Sir Stanley Leathes' experience, for he writes: 'If there is historical material on the one hand, and literary material on the other, the range, interest, and variety of the problems that can be set without leaving well-trodden ground may be four times as great as when literature is separated from history.' $\ddagger$

In fact, the single wide interest is not a structure which, like some motor cars and other standardised products of manufacture, may have its several parts formed separately and assembled only in the last stage of the process. Its development is rather to resemble that of an organism, the structure remaining single throughout, but becoming increasingly integrated, as well as increasingly complex, as its development proceeds. Here too we are in accordance with educational experience. We have already§ seen that many educators, from Milton and Goethe downwards, have pointed out that the greatest mistake in education consists in teaching subjects in water-tight compartments. So soon, in fact, as the period of childhood is ended, the school curriculum should combine with experience out of school to form a comprehensive unity. To 'assimilate knowledge as part of the personality,' $\|$ to form a single wide interest, is better than to amass knowledge of manifold separate subjects. It is the former, not the latter, that makes for an ideal character.

It is more than half a century since Newman expressed the same idea in the words:

I will tell you what has been the practical error of the last twenty yearsnot to load the memory of the student with a mass of undigested knowledge, but to force upon him so much that he has rejected all. It has been the error of distracting and enfeebling the mind by an unmeaning profusion of subjects, of implying that a smattering in a dozen branches of study is not shallowness, which it really is, but enlargement, which it is not .

* See above, Chapter II, §5, especially p. 212.

$\dagger$ Our formula (see pp. 2 II and 2 I 2 above) was $U \propto \frac{T^{2}}{S}$, where $U$ is the educational value of an expenditure of effort continued uniformly during a time $T$, and equally distributed between a number, $S$, of separate subjects.

$\ddagger$ What is Education?, p. 137.

$\S$ In Appendix A (see especially p. 470 below) referred to above on p. 21.

II Times Educational Supplement, I4th January, I9I6.

II Idea of a University, Discourse 6. 
So again the headmaster of Rugby (Dr David) told the Consultative Committee of the Board of Education in IgII that a boy who had passed the School Certificate examination*, as we now know it, 'could then be put through a real educational course, without reference to examination needs for the rest of his school life' $\dagger$; or, in other words, that when boys had passed the proposed fifth form examination, it would no longer be necessary for them to study a number of separate subjects for examination purposes, so that a higher ideal-'a real educational course'--would become possible.

Dr David is by no means alone among the headmasters of public schools in holding this opinion. For example, the present headmaster of Eton, when headmaster of Shrewsbury, wrote: 'It is obvious that the ordinary public school curriculum is grossly overcrowded.' $\ddagger$ And yet the number of separate subjects to which approximately equal weight is attached, is by no means so great in the public schools, where form-masters still flourish, as in those secondary schools of recent foundation where, for the most part, specialist teachers take the place of form-masters or form-mistresses.

We have already seen that Sir Stanley Leathes, who after teaching history in Cambridge became chiefly responsible for. the Civil Service examinations, is impressed by the waste of educational effort that results from separating subjects that ought to be taught as one, instead of as many. Thus he points out that 'A man may take many "subjects," but every subject will suffer if they are not made to interpret and strengthen each other.' § And again he writes:

Why do we value the best Classical education? Not because Latin and Greek are superior to all other languages.....Nor because of the excellent drill provided by Greek and Latin grammar and composition...but...the humanists of the Renaissance were enthusiasts at once for language, literature, and history.... That tradition survives in the best Classical School of to-day ll.

Sir Stanley Leathes' experience accords with the findings of Sir Joseph Thomson's Committee on 'The Position of Natural Science in the Educational System of Great Britain.' I Their report to the Prime Minister states that 'the classical sides have all the advantages of a more effective working of the form system than is easily attainable

* See below, footnote * on p. 387 .

$\dagger$ Report of the Consultative Committee on Examinations in Secondary Schools.

$\ddagger$ A Schoolmaster's A pology, by the Rev. C. A. Alington, p. 35 .

$\S$ Loc. cit. p. IIo.

\| Loc. cit. pp. II6, II7.

Report of the Committee appointed by the Prime Minister to enquire into the position of Natural Science in the Educational System of Great Britain, I918. [Cd. $901 \mathrm{I}$. 
on the modern sides, where the work is so much distributed among a larger number of masters that no one master sees any group of boys for a large part of the school week. The modern sides suffer, in fact, from diversity of effort and indefiniteness of aim, and these conditions do not make for strenuous work.'*

Once more, Professor R. L. Archer has recently pointed out that courses of study, leading to pass degrees in British universities, commonly differ from honours courses, in that the former are equally concerned with several separate subjects, while the latter are more coherent, having a single centre of interest, however wide may be the range of subordinate, illustrative and connective matter that is included in the course. Professor Archer concludes that the education provided by pass courses is chiefly inferior to that of honours courses, in that 'existing pass courses disperse interest and so destroy it.' $\dagger$

Our quotations indicate that the importance we have been led to attach to the scientific arrangement of knowledge, so as to form a shapely $\ddagger$ single wide interest, fits the experience of educators far and wide. In short, mere volume of knowledge is comparatively valueless unless it is scientifically arranged for handy reference§. Comprehensiveness and coherence of interest are both desirable, and both should be pursued together. But, if for a time the two cannot be reconciled, coherence must never be sacrificed to comprehensiveness: coherence at seventeen is a sure way to comprehensiveness at twenty-seven.

But while the organisation of knowledge, by building up a shapely single wide interest, is the main concern of education on the neurographic side, education is also concerned with providing new knowledge, supplying new material, and so widening, as well as arranging, the pupil's interest. In other words, we are not to forget that, even after the elementary or preparatory education of childhood is at an end, education has some concern with the unorganised fringe, as well as with the intermediate zone (or scientific endarchy), of the growing single wide interest. We repeat $\|$, however, that whole-time education in school or college during adolescence and maturity, should be more concerned with the organisation of thought than with the widening of experience.

In other words, the concern of education is not with knowledge for its own sake. Indeed, to say that anything-education or whatever it may be-is desirable 'for its own sake,' is no more than a lazy

* Loc. cit. p. I2.

$\dagger$ The Passman, p. 55.

$\ddagger$ I.e. 'endarchical': see Chapter 12 above, especially pp. 23I, 244.

$\S$ Cf. Chapter II, § 7, and p. 335 above.

\|l See above, $\S 4$ of this Chapter. 
device for avoiding deep thinking. So Dr Alington says that he 'can see no reason for wanting to teach either [grammar], or composition in foreign languages, to a scientific boy for its own sake.* Or, as Sir Stanley Leathes has it, 'book-learning is only a means to an end.' $\dagger$ 'True Education,' said Emerson, 'is what remains behind, when all that was learnt at school has been forgotten.' $\ddagger$

\section{$\S 6$. The Growth of a Single Wide Interest.}

We have already repeatedly observed $\S$ that the organisation of a single wide interest or 'personal endarchy' is, in the main, a voluntary process, in which the teacher's Will gradually gives place to the pupil's as the latter's education proceeds. We here remind ourselves of some aspects of the growth of a single wide interest according to the principles arrived at in Book II, and these we then proceed to compare with further records of experience.

The beginnings 'of' a single wide interest are made, as we said\|, during infancy and childhood. To this raw material, out of which the single wide interest is gradually formed, the manifold experiences of adolescence and maturity go on adding. But childhood is mainly concerned with the collection of the raw material, 'the recording of disconnected facts.' II It 'has few organised interests.'** The organisation of a single wide interest makes, in fact, very little progress until adolescence has begun. But even during childhood, when education is more concerned with the acquisition of knowledge than with its arrangement, it is also concerned with the formation of the central Christian purpose, and with linking that purpose, by means of emotional elements, to the unorganised fringe that makes up the remainder of the childish neurography: there is little or no intermediate zone, corresponding to organised thought, at this stage.

The age at which the miscellaneous interests of childhood begin to become organised varies, like other human characters, from one individual to another. There is, however, a general consensus of opinion that the change begins with most people somewhere between the ages of twelve and fourteen. When, early in the teens, purpose begins to come into its own, formal education in school or college has to take account of it. It is at this stage that the main concern of education on the neurographic side begins to be with the central

* Loc. cit. pp. $39,40$.

$\dagger$ Loc. cit. p. $4^{8}$.

‡ Quoted in The Ninteenth Century and After, for June, I9I7, p. I3I5. See above, e.g. pp. 269 and 271.

II See above, p. 245.

If $\mathrm{Cf}$. Robert Louis Stevenson in The Wrong Box.

** W. James, loc. cit. Vol. I, p. $4 \mathrm{I} 7$. 
purpose and the intermediate zone of organised thought that is centred in it. Thus the medical officer* of the Manchester Grammar School told the Consultative Committee of the Board of Education that 'The age of fourteen was a very important epoch in a boy's life.... He now began really to use his previous acquirements for his own conscious ends.' $\dagger$ Dr Rivers, giving evidence to the same Committee said that, at about the age of fourteen, a child would be likely to consider how far a particular school subject-manual training was the subject in question-'stood in any relation to his future career, and if he recognised no such relation, the work would probably fail to arouse his interest.' $\ddagger$ Again, Sir Daniel Hall told the Headmasters' Association that, beyond the age of fifteen, 'it was necessary to rely on the interest that depended on the subject studied having some bearing on the boy's future life.'§ Once more, one of His Majesty's chief inspectors of schools\| has said that 'A child's interest up to twelve is in amassing information; afterwards he may take an interest in scientific method.' Finally, Mr James Strachan, discussing the teaching of mathematics, says that 'In the highest classes of our schools and universities... as in the ordinary school classes, the process of attaching a purpose to the work is adding interest and sanity to the whole study.' I

The development of a single wide interest, begun during childhood, should make increasingly rapid progress as adolescence proceeds. This development should, as we said ${ }^{* *}$, be accompanied by an increase of $g$ that measures power to concentrate attention. And, as we also pointed out, the increase of $g$ and the formation of a single wide interest are mutually consistent processes that should accompany and assist each other. For the concentration of attention upon the subject of any thought-activity not only increases $\uparrow \dagger g$ by practice in concentrating attention-the only way in which, so far as we could see, $g$ can be increased-but also deepens the neurogram of that subject and so renders more permanent that part of the growing single wide interest-system $+\ddagger$.

* A. Mumford, M.D., M.R.C.S.

$\dagger$ Report on Practical Work in Secondary Schools, p. 358 [Cd. 6849] (evidence given on zoth December, 19II).

$\$$ Ibid. P. 366 (evidence given on 5 th June, 1912). W. H. R. Rivers, M.D., F.R.S., Fellow of St John's College, Cambridge.

\& A lecture before the Headmasters' Association Sir Daniel Hall, F.R.S., reported in The Christian Science Monitor, 21st March, Igr7.

I) Mr H. Ward.

T The New Teaching, p. 229.

** See above, pp. 225 and 333 .

$\dagger+$ The justifications for this assumption are discussed on pp. 137, 138, 333 above.

$\div$ Cf. Dr McDougall, quoted above, p. roo, footnote $\dagger$. 
The corollary to our third law of thought told us that, of all active neurograms, that one which is connected with the widest and deepest system tends, other things being equal, to drain the impulse from them all*. It follows that connexion with a deep and wide interest-system increases the likelihood of a particular neurogram becoming excited and so deepened. A pedagogic maxim follows at once: namely, that a teacher who wants his pupil to remember any fact should see to it that the corresponding neurogram is connected to a deep and wide interest-system. Or, since, as we have reminded ourselves, no fact is wanted ' for its own sake,' but rather to strengthen Will and to help in forming an appropriate wide interest, we should rather say that the teacher who is helping his pupil to develop a single wide interest, will best ensure this result if he remembers that the value $\dagger$ of each new element added depends upon the number of previously existing elements which it connects together for the first time.

It follows that the process of forming, deepening, and connecting together those elements which are to form the most central region of the single wide interest-system, and which, on this account, will be both deepest and most closely inter-connected, should be begun as early as possible. By beginning early in life with a central purpose (corresponding to the central elements just discussed), the pupil has a reason for all his subsequent studies; and not merely a reason for studying, but, as we have just said, an aid to remembering. When once this central portion of the growing single wide interest-system has begun to be formed, the process of connecting up the whole neurography to form a single wide interest-system will better proceed by linking (the central and deepest elements of) subsidiary interestsystems to the elements of this central region, than by linking the subsidiary systems directly to each other.

We have next to observe that, since the collection of the raw material of a single wide interest-system proceeds at first far more rapidly than the growth of the organised region of the system, the unorganised fringe of every person's neurography is relatively larger in early youth, while the organised central region and intermediate zone become relatively larger in maturity. The scientific endarchy, that forms the inner zones of the complete single wide interest-system, will be formed by growth that takes place both inwards and outwards. The former is, as we saw $\ddagger$, the normal method of growth of the

* See above, p. 89 .

$\dagger$ See above, Chapter II, $\S 7$ See also $\S 8$ of this Chapter, especially pp. 35 I to 353 below.

$\ddagger$ See above, p. 202. 
endarchy of science, the organised system of human thought to portions of which the scientific endarchies of particular individuals more or less correspond. This is also the principal direction in which every personal endarchy should grow. In other words, the pupil, especially in early youth-before he has developed any considerable interest in abstract principles which lie nearer the centre of the endarchy of science than does the raw material of experience that accumulates during childhood-should, in general, approach abstract principles through the practical applications of those principles. In short, his interest in the abstract should, as a rule, grow out of his interest in the concrete: he should generally proceed from concrete to abstract. Thus Sir Joseph Thomson's Committee consider that:

In framing a course in Science for boys up to the age of sixteen, it should be recognised...that the course should...give special attention to those natural phenomena which are matters of every-day experience, in fine, that the Science taught in it should be kept as closely connected with human interests as possible. The keen interest which many boys feel at this age in the applications of science, such as aeroplanes, steam engines, wireless telegraphy, motor cars and the like should be utilized to the fullest extent. There are great advantages in introducing the study of the principles of Science by starting from a machine or a striking physical phenomenon and working backwards to the principle, rather than by starting from the abstract and proceeding to the concrete; such a method is in no way inconsistent with a logical and continuous development of the subject. The advantage of the method is that the impetus due to a boy's interest in the subject helps to carry him through the lessons, while in the ordinary method interest may be aroused [-i.e. connexion may be made between the subject studied and the boy's pre-existing interests-] only after the lessons are nearly completed*.

But the proverbial pendulum must not be allowed to swing too far away from the older method of studying the exact sciences; namely, that of proceeding from abstract to concrete; or, in other words, of first laying down the abstract principles of the subject studied, and afterwards deducing the practical applications of these principles. Indeed, when once a sufficiently wide and deep interestsystem, corresponding to the principles of a subject, has been developed in a pupil-probably by proceeding inwards from a limited number of practical applications of those principles-his knowledge of the subject can be more rapidly and effectively extended by proceeding downwards and outwards $\uparrow$ from these central principles to their further applications, than by first studying these further applications and from them proceeding, over and over again, inwards to the same
* Loc. cit. [Cd. gor 1], p. 23.
$\uparrow$ See above, p. 202. 
central principles. Thus, in the earlier stages of the development of an interest in a new subject, and therefore in general during childhood and early adolescence, the interest should grow from outside inwards, or from concrete to abstract; while, as adolescence proceeds, and the better organised inner regions of the growing single wide interest become relatively wider and deeper, the growth of the interest-system should, to an increasing extent, take place from inside outwards, or from abstract to concrete. In other words, the normal method of developing a single wide interest (or that part of it which corresponds to any particular subject of study) will consist in beginning with a few examples, applications, or concrete experiences*; proceeding from them, by abstraction, to generalisations, laws, principles, and on to the highest principles in the single wide interest; and finally deducing further applications of these principles and comparing them with observations or concrete experiences. We thus summarise, in more general terms, the account we have already given of the voluntary development of personal endarchies $\dagger$ by the creation of reasoned paths of connexion between apparently disconnected facts.

We note, in passing, that the differences that exist between the mental qualities, and especially between the g's, of different persons, cause the relative stress that ought to be laid upon the inwards and outwards growth of the personal endarchy to vary widely between different individual pupils. There are, for example, pupils nearly all of whose knowledge of mathematics or mechanics should be acquired by the inwards growth of their interests: that is, by proceeding from the concrete to the abstract. And there are others-generally persons with higher $g$ 's, greater powers of concentrating attention-whose time would be largely wasted were they compelled to trouble themselves with the practical details of manifold applications before proceeding to abstract the principle or law that all these applications are intended to illustrate, and are indeed required to illustrate before less able boys and girls can fully grasp the meaning of the laws or principles in question. When we come to discuss the types of education that are best adapted to pupils of different grades of ability, we shall illustrate this difference in the method by which the same subject, and the same part of it, should be studied in different educational courses $\$$.

* See above, p. 233, where we noted that all abstracts oughit to connect to some concretes. For example, as we said, children should have seen at least one river before learning about rivers in general. † See above, Chapter $x_{4}$.

$\ddagger$ See below, $\S 8$ of this Chapter. For example, the Pythagorean theorem may be differently approached, on the one hand by thirteen-year-old boys receiving senior elementary education, and, on the other, by boys of the same age receiving junior secondary education (see Chapter 23 ). The former will be likely 
While, as we have more than once repeated*, the development of a single wide interest-system is in the main a matter of Will-at first the teacher's and later the pupil's-we must not forget the exceedingly important part which predominantly emotional interests play in involuntary thinking. The voluntary development of a single wide interest-system must therefore take account of these emotional interests, and see to it that they are made to form part-and a very important part-of the single interest which education is concerned with developing; for any portion of the growing neurography-and, in particular, the organised scientific endarchy, with the formation of which secondary and university education is so largely concernedmay be deepened by being connected with emotional interests $\dagger$. Thus the student of the mathematical theory of probability who is concerned with problems about drawing marked balls from a bag, will think more effectively about his problem if the balls he imagines are attractively coloured; or a student of hydrodynamics may make more progress with his study if he works near a window overlooking a seascape that fills him with emotion. Or, to take a less trivial example, it is well, other things being equal, to study a subject in the closest possible relation to one's main purpose in life; for this, as we saw, should be the widest and deepest emotional interest of all. Thus the student whose ordinary employment occupies the greater part of his time-or, as we shall call him for brevity, the part-time student-will generally be well advised to study mathematics, or any other subject, in close connexion with the applications of that subject to the employment in which he is contributing to the progress of the Commonwealth. Of the value of this maxim there can be no doubt in the mind of anyone who has personal experience of the extraordinary efficiency that so often marks parttime education as hitherto provided in England. For part-time education that is intimately related to concurrent part-time employment ministers to the same growing interest: the interest awakened in school is kept awake during the remainder of the week by the experiences of the workshop or other field of service, and so goes on developing without interruption. Moreover, sucli part-time education explains much that would otherwise be meaningless in the student's

to treat it as the $47^{\text {th }}$ proposition of the first book of Euclid; but the latter may be willing to accept as an empirical fact that the square on the longest side of a right-angled triangle is as big as the sum of the two squares on the shorter sides, at least in all the cases they have weighed and measured, and therefore presumably (and they may be content to leave it at that) in every other case as well. $\quad$ * From p. 269 above.

$\dagger$ See above, p. 89. 
employment; he is therefore anxious to obtain all that he can from his part-time teacher, and so gives him his concentrated attention.

But, if the fullest advantage is to be taken of the close connexion that may obtain between part-time studies and the student's interest in his daily work, it is necessary that the part-time teacher should share that interest, and if possible have had personal experience of the work to which his students are giving the greater part of their time. This principle is of more importance* in its application to junior part-time students who expect to be taught by their teachers, than in its application to advanced part-time students who do not so much expect passively to receive instruction as actively to seek it from a master of the subject.

It is for a similar reason that, as we have said $\dagger$, the younger pupils in a secondary school should spend a large proportion (which should, in the lowest forms, exceed one-half) of their school time with the same form-master or central teacher, between whom and his pupils close personal relations will thus be likely to develop, so that the knowledge he imparts will automatically and immediately be connected to wide and deep interests in his pupils' minds. Moreover, if this form-master or central teacher himself possesses a single wide interest, so that he sees life steadily and sees it whole, the connexions that exist between his thought-activities are likely to produce corresponding connexions in his pupils' neurographies, and so to assist in developing in each of them an interest that will be single as well as wide. It is, of course, true that this important means of securing unity or coherence of interest, instead of the multiplicity of separate subjects that too often takes its place in modern secondary schools, may involve some sacrifice in the proficiency of the teacher in some of the subjects which he has to teach. But, important as is the closest possible correspondence between the endarchy of science and the pupil's scientific endarchy, it is by no means necessary to ensure this correspondence by requiring that the beginnings of any subject-peripheral essences of a particular subject endarchy, or sector, of the endarchy of science $\$$-should be presented by a teacher who has specialised upon the inner essences of the same sector, the most abstract principles of the subject taught.

The future historian, for example, will not necessarily best secure

* Compare Section $\mathrm{V}$ of the Board of Education memorandum on the staffing of continuation schools, where emphasis is laid upon the importance of the personal relation between teacher and taught, especially in the earlier years ( 14 to 16 ) of continuation school work. [Circ. IrO2, dated 4th April, I9r9.]

$\uparrow$ See above, p. 270.

$\ddagger$ See above, Chapter II, $\$ 6$. 
a knowledge of mathematics, that will take its properly subordinate place in his single wide interest-system and have its right relation to the central purpose of his life, by studying under the most eminent mathematician available; for the latter may well have almost lost whatever interest he ever possessed in any mathematical knowledge that the historian is ever likely to have time to acquire. This is no reason why the mature historian should not endeavour to understand, for example, the problem of relativity that may be exercising the minds of the most eminent among mathematicians. But it is a reason why a schoolboy whose main interest is in history, should learn mathematics from a teacher who is qualified rather by his sympathy with the boy's interests than by his distinction as a mathematician. Unfortunately, however, many modern secondary schools altogether ignore this principle, actually refusing to allow boys of thirteen to be taught history and English by the same master, even when the teacher of history-or the teacher of English-happens to be called their form-master (merely because they keep their books in his classroom, although they do not spend more time with him than with any one of five or six other specialists)!

The most familiar example of the principle here in question is furnished by so-called religious instruction. Very few teachers will nowadays fail to recognise that the development of the most central elements of a pupil's single wide interest-system, the elements that correspond to the central spiritual essences of the universe, can better be presented by a parent or house-master or form-master who shares the boy's interests, than by an expert in theology, or a religious enthusiast, who gives religious instruction and nothing else, and who does not know the boy. Indeed, religious teaching-teaching with the aim of forming the central Christian purpose--must belong to parents and to central teachers; it must permeate the whole teaching* until the pupil reaches an age at which he, rather than his teacher, directs his education; and it must include the practice of the dictates of brotherly love, not only in loyalty to schoolfellows, but, as time goes on, in service to a wider society outside school or college.

Another example of the same principle is furnished by the teaching of English, a subject which should always be taught to younger boys and girls by their form-master or form-mistress rather than by a specialist who teaches English and nothing else. As Sir Stanley Leathes says, 'Every don should constitute himself a professor of

* Cf. Chapter 17 above, especially p. 296. Cf. also Dr Temple's Presidential Address to Section L of the British Association (Newcastle, I9I6), p. II. 
English.'* Or, as Sir Joseph Thomson's Committee have it, 'The science master cannot be allowed to repudiate responsibility for the English in which the work of his class is written.' $\dagger$

So, too, when the teaching of natural science has become more general, so that classical form-masters are less ignorant of it (and more appreciative of the intense human interest that belongs to it), the physical and biological sciences taught at school to those boys in whose interest-systems the elements that correspond to natural science will never be central, should be taught by the form-master, even a classical form-master $\ddagger$. And, as Sir Joseph Thomson's Committee point out, it is to be hoped that in future every boy will study science at least until he reaches the age of sixteen.

Without insisting, as we have insisted, that the greater part of the curriculum in the earlier teens should be entrusted to a single teacher§, Professor Nunn applies the same principle in a less exacting way by urging close cooperation between persons who teach different subjects to the same boys. Professor Nunn goes on to say that

The curriculum in science, geography and mathematics should be thought out as a whole. There is only one way of bringing about effective co-ordination of this kind; the teachers of these subjects must meet from time to time in conclave to adjust their frontiers and regulate their politics. It should be regarded as unthinkable that any one of them should go his own way without constantly taking account of the needs and proceedings of his colleagues $\|$.

* Loc. cit. p. I22.

$\dagger$ Loc. cit. p. 26.

\$ That classical form-masters can, even to-day, successfully undertake the teaching of 'science for all' has been proved by Mr Eggar, of Eton, who, at a recent meeting of the Association of Public School Science Masters described how a block of 200 boys had been taught astronomy, light and sound by classical masters. Two hours a week had been given to these studies. One of these hours had been occupied by the classical form-master. During the remaining hour the whole block, including the classical form-masters, came together to the big lecture room for joint instruction. The classical masters had, according to $\mathrm{Mr}$ Eggar, taken very kindly to the experiment, and the boys and masters had alike taken a great interest in it. Mr C. L. Bryant, of Harrow, added that he had examined these 200 Eton boys, and that, for boys at the age of fourteen and a half, their examination papers were extraordinarily well done. 'So far as the experiment went the result had been a success.' (See The Times Educational Supplement, 2nd January, r9r9.)

$\S$ Sir Joseph Thomson's Committee 'agree...with the view expressed in one of the answers to a questionnaire which we sent to the Mathematical Association that the best method of securing coordination of the work in mathematics and science is to assign the teaching of mathematics and physics argely to the same teachers.' (Loc. cit. p. 33.)

|| Loc. cit. p. I79. Such meetings as Professor Nunn suggests, least frequently occur, but are by no means least required, in the case of part-time education, where it often happens that the teachers, as well as the students, are engaged during the greater part of the week in some other employment, and where the several teachers of different subjects to the same group of part time students pursuing the same course of study, may not even know each other by sight! 


\section{§ 7. Continuity in Education.}

A system of education that extends from the nursery to the university, and includes within its scope the practical training that is given by works or business houses or public offices to their younger employees, will not be likely to develop a single wide interest, unless the education it provides is continuous. Each type of education should prepare for that which the pupil will next receive. The curriculum in every school or college should shade into that which follows in the next educational institution, while that of the last whole-time school or college should prepare specifically for the occupation that comes after, continuity between whole-time school or college and whole-time employment being preserved by means of a transitional period of part-time education.

The principle of continuity in education follows from the principle of the single wide interest, which in turn follows, as we said, from the corollary to our third law of thought. And this principle of continuity is widely recognised in the abstract. Thus, for example, the Consultative Committee of the Board of Education, after stating that manual work in modern infants' schools is suddenly discontinued, remark that 'this abrupt transition cannot be sound.' *

Continuity in education has a two-fold aspect: looking forward and looking backward. The educator who knows what kind of experiences his pupils are likely to receive when soon they leave his care, can prepare each of them to absorb these new experiences into his single wide interest, so as to widen and deepen that interest, instead of producing the beginnings of new and separate interests. The university lecturer to engineering students, who keeps himself in close touch with works practice, can prepare his undergraduate students to make the most of the practical works training that is to follow their graduation. The same principle is as applicable to the education of boys or girls who are about to be transferred from school to college, or from one school or course to another, as to that of young people whose whole-time education is about to cease. Thus the sixthform master of a higher secondary school who preserves close touch with university work, and is qualified at any time to accept a university lectureship $\dagger$, can make the advanced secondary education with which

* Report on Practical Work in Secondary Schools, r9r3 [Cd. 6849], p. 6.

$\dagger$ More than twenty years ago such transfers already occurred. The present writer remembers the two masters who taught the highest classical forms at his school, leaving in quick succession, one to become an Oxford college tutor, and the other to become a fellow and lecturer of a Cambridge college.

G. $\mathbf{E}$. 
he is concerned lead continuously on to the undergraduate honours courses which his boys are about to enter.

But the preservation of continuity demands that educators shall take account of the work their pupils have lately been doing, as well as of that which they are about to do. So the sixth-form master of the higher secondary school must take account of the fifth-form work that has preceded, as well as of the undergraduate studies that are to follow, his teaching. In the same way, the works training that follows an undergraduate course in engineering is most effective when, as in some modern engineering works, it is directed by an Apprentice Master* who is himself in close touch with the universities from which his 'college apprentices' have come. And, in general, the more a teacher knows of the interests with which his pupils come to him, and the more use he makes of that knowledge in connecting and widening those interests, the better progress will he make towards the goal of developing a single wide interest in each of his pupils.

The principle of continuity, especially in its forward aspect, was recognised by the Consultative Committee of the Board of Education who wrote: 'The nearer a pupil is to his entrance into life, the more steadily must the actual practical needs of his occupation be kept in view, and the more decided therefore must be the bent of his education to that end.' $\dagger$ Or, in the words of a report $\ddagger$ adopted by the Council for Organising British Engineering Industry in I9I6, 'The last two or three years of every person's whole-time education should have the specific aim of preparing him for his particular walk in life so far as that can be foreseen. We are not asking for purely vocational training, and we do not suggest that during these last two or three years education should be unduly specialised. Our desire is rather to see a coherent curriculum in which every subject is linked to every other because they are all taught in close relation to the student's future activities, both vocational and social'§ (including not only the work by which he earns his living, but all the work that is his life-the whole of what Kim would call his 'Great Game'); and, as we may add, to the central purpose of his life that should direct all these activities.

* 'It must be remembered however that the great defect of the pupil system in engineering works is that as a rule no one is responsible for the necessary theoretical training; it is often left to chance.' Sir Joseph Thomson's Committee's Report, p. 48. (See also below, p. 408.)

$\dagger$ Report on Higher Elementary Schools (1906), p. II.

$\ddagger$ See above, footnote $t$ on p. 325 .

$\S$ Report on Engincering Education and Research, p. I4. 


\section{§ 8. Contrasted Types of Study.}

The principle of continuity in education thus demands a difference between the type of study pursued by those, on the one hand, who intend to make its pursuit their principal occupation for many years to come, and by those, on the other hand, who are about to discontinue the study in question, or at least to devote to it only a comparatively small proportion of their time.

It is important to discover wherein this difference of type consists. To this end we first remind* ourselves that the personal endarchies of different individuals are often very different. So different indeed may these personal endarchies be that an element, which is near $\dagger$ the centre of one man's single wide interest-system, may correspond to an essence of which the neurographic correlative is peripheral in a second man's neurography, while it may be altogether absent from that of a third. Thus, to the same essences of the endarchy of science + there may correspond elements having very different positions in different personal endarchies, and therefore different values $\S$ to the several persons in question. Clearly then the value of an essence to a particular person depends, not only upon the (impersonal) value of the same essence in the endarchy of science, but also upon the particular regions of the endarchy of science that are selected $\|$ and more or less short-circuited If for neurographic reproduction in the particular personal endarchy in question.

So we distinguish types of study, not only according to the (impersonal) values of the essences studied, but also according to the (personal) values which those essences are going to have to the students. In other words, a type of study is marked, not only by the values of

* See above, Chapter I2, §§ 3, 4, especially pp. $23^{2}$ and 235.

$\dagger$ See above, p. $24^{2}$.

$\ddagger$ See above, p. I95.

$\S$ See above, p. I94. It will be remembered that the value of an essence to any one of us personally was there stated to be greater or less according as our neurogram for it was deeper or shallower; and therefore, in the case of persons whose neurographies possess the endarchical organisation of single wide interestsystems, according as the corresponding neurogram is more or less central; and, in any case, according as the essence in question is more or less frequently thought of by the particular person concerned. Similarly on a later page (2I 7 ) we concluded that the (impersonal) value of an essence in the endarchy of science was measured by the frequency with which it occurred in the world of experience or in reasoned thought about it. Since the relative frequency with which a particular essence occurs in the world of experience, or in all men's reasoned thought about it, is by no means equal to the relative frequency with which that essence is thought of by any particular person, it is clear that, as stated in the text, the value of an essence to any particular person is by no means necessarily proportional to the (impersonal) value of the essence in the endarchy of science.

II See above, pp. I94 and 267.

If See above, pp. 230 and 237. 
the essences studied, but also by the values that the corresponding elements are going to have in the students' single wide interestsystems. For example, we recognise a difference in type between the study of abstract principles and the study of concrete facts of direct experience, the essences studied in the first case being more central in the endarchy of science, and therefore more valuable, than the essences studied in the second; and we also recognise a difference in type between the study of one and the same group of essences by one student, in whose personal endarchy the corresponding elements are going to be more or less central, and by another student, in whose personal endarchy the corresponding elements will remain peripheral*. In either case, the difference in type depends only upon differences of value, and not upon other differences between the facts studied $\dagger$. In short, as we said before in another connexion, the kind of difference in type of study with which we are here concerned is a question not of facts but of valuesł.

Since, however, the intermediate zone that corresponds to the organised thought of one who is expert in some particular branch of knowledge, will, as we said $\S$, correspond as closely as possible to a branch of the endarchy of science, those elements which are more central in our expert's scientific endarchy will, in so far as they correspond to essences of his own special subject, be more or less central according as the corresponding essences are more or less central in the endarchy of science. And, in general, elements of high value in that region of the endarchy of science which is most nearly reproduced neurographically in anybody's single wide interestsystem, will there be represented by elements of high value; so that, whoever studies the more central abstract essences of a subject in which he is to become expert, will be studying essences that are not only of high (impersonal) value, but will also be of high value to him personally. So the type of study that is specially concerned with the more central essences of a subject in which the student is to become expert, is a type that is marked by the essences' high values,

* E.g. the principles of biology are more central in the single wide interest of a naturalist than in that of a theologian, and the principles of economics ought perhaps to be more central in the single wide interest of a Chancellor of the Exchequer-or, rather, of his permanent advisers in the Treasury-than in that of the Prime Minister for the time being.

$\dagger$ Or, using our diagram (Fig. I6) on p. 204 to represent the endarchy of science, and employing polar coordinates with the centre of the diagram as origin, we may express this result by saying that a difference in type of study depends upon a difference of zone rather than a difference of sector-a difference in the radial, rather than in the angular, coordinate.

$\ddagger$ See above, footnote $\S$ on p. 217 and p. 335 .

$\S$ See above, p. $23^{2}$. 
both impersonal and personal. Being near the centre of the student's single wide interest-system, the corresponding elements will be deep, closely inter-connected, coherent; and, being comparatively central in the endarchy of science, the same essences will be abstract and will belong to a narrow region of that endarchy. On the other hand, the essences that form the unorganised periphery of a single wide interest will, as we saw, belong in large measure to concrete facts of direct experience, facts that may have little coherence but that will cover a very wide region of the endarchy of science.

Here we pause for a moment to illustrate the use of contrasted types of study in the system of education with which we are concerned. We have said* that, as education builds up a single wide interest in the pupil, he should first pay attention to facts of experience. From these he should be led, as soon as possible, to abstract laws and principles, the more central essences in the endarchy of science. From these again he may be led to abstract still more central essences: laws, principles, and generalisations. All the time he is to be connecting these abstractions with his central purpose, on the one hand, and with facts of experience, on the other. As the end of whole-time education approaches, the abstractions to which he has been giving so much attention (and so has had the best opportunity of increasing $\dagger$ ' $g$,' his power of concentrating attention) have to be closely related to his particular purpose in life, and to those principles that underlie the practice of the occupation he is about to enter.

Thus, for example, the undergraduate course in engineering that is being followed by one who is about to enter the works in which, as a college apprentice + , he is to continue his engineering training, should be concerned with the relation of engineering to human society (in overcoming material obstacles to human progress), and so to God. It must also include a study of the principles of mechanics, physics and other pure sciences that have been applied to engineering. That is to say, it should connect to one another, and to the central purpose of the student's life-his purpose to serve God and man as an engineer -a number of essences that belong to different branches of the endarchy of science, and whose connexions in that endarchy may not have been completely discovered, or, if they have, may have to be short-circuited $\S$ in the engineer's scientific endarchy\|. Such short-

\footnotetext{
* See above, pp. 233 and 344 .

$\dagger$ See footnote $\dagger \dagger$ on p. $34 \mathrm{I}$ above.

† See below, pp. 408, 409, 431 .

$\S$ See above, pp. 230 and 237 .

II See above, p. 228 .
} 
circuiting is justified at the conclusion of whole-time education, and at whatever age whole-time education ends, by the fact that time does not permit the future engineer (or indeed the future citizen of whatever occupation) to master in its entirety all that has been discovered concerning every branch of knowledge with which his future occupation will have some connexion*. Moreover, this process of short-circuiting that the student of engineering must apply to the principles, laws, and generalisations of those branches of natural science into which he will have to go relatively deeply, should also be applied to his studies in other fields of knowledge with which his acquaintance has to be wide rather than deep. Such time as he can devote to the literature of ancient Greece and Rome may exercise a greater influence upon his character and conduct if that literature is welded into his single wide interest by being studied in good English translations than if it is confined to a separate interest, of which the greater part is made up of Greek and Latin grammar and rules of syntax that form essential parts of the school studies of the classical boy. And 'could not the classical or literary student learn more of the scope and utility of Mathematics if the subject were presented to him with fewer artificial divisions, and if he were not required to work through several hundred examples in Algebra before he is permitted to know the meaning of Trigonometry, or the application of the simplest branches of Mathematics to the everyday problems of mechanics or physics? By the student who aims at classical scholarship or at high attainments in Mathematics, years may profitably be devoted to the grammar of the subject; the application and the reward will follow later.'

The summary of Sir Joseph Thomson's Committee's Report, from which this quotation is made, continues:

Precisely the same argument may be applied to the teaching of Natural Science as it has been practised during the last few years in most of the schools. Teachers have had in view exclusively the preliminary training of scientific men, and not the desirability of inspiring students of Classics, Mathematics, English or other subjects-students who will form the majority of future statesmen, politicians, public administrators and men of business-with an appreciation of science for the sake of its human interest and its vast services to societv. The teacher of Science has followed too closely the example of the classical master. The foundations of the subject are laid with equal care, whether any super-structure is to be erected upon them or not; and it has not been fully considered that a cottage completed and ready for occupation may be of more value to the 
community than the foundations of a palace which have never been carried above the damp-proof course*.

So much then by way of illustrating $\dagger$ our principle that, if education is to develop a single wide interest, there must be two distinguishable types of education, and two distinguishable ways + in which one and the same subject should be studied by those, on the one hand, whose whole-time education, or whose study of that particular subject, is to continue for many years to come, and by those, on the other, who are about to discontinue whole-time education, or their study of the particular subject as the case may be. While, as we have just

* Ministry of Reconstruction Pamphlet, entitled Reconstruciion Problems 26: Natural Science in British Education, p. 2.

$\dagger$ See above, p. 353. We here resume the discussion there interrupted for purposes of illustration.

$\ddagger$ This distinction is emphasised in the following reply (dated 1917 ) by the present writer to a question circulated by Sir Joseph Thomson's Committee before issuing their Report.

Question. To what extent is it desirable that a somewhat prolonged elementary training in Science, with laboratory work, should have been an obligatory part of the Secondary School Education of all students at some time previous to their entering the University, and does this apply to the needs of all Faculties?

ANSWER. It is desirable that all freshmen, entering a university through the normal avenue of the secondary school, should have received some training in Science, including laboratory work.

But the science training given at school to those who are to study Science or Applied Science (including Medicine) in the university should differ in kind, as well as extent, from that of the students entering other Faculties. The school Science of the former should, after an introductory course of the kind described below, include a large proportion of laboratory work, and a more exact study in a limited range under teachers who have specialised in that limited range of study and are qualified to teach it in a university. The school training of the latter-the non-science boys or girls-should be extensive rather than intensive, inspirational rather than informational. It should be concerned with a few wide generalisations, and the particular phenomena discussed should be such as best illustrate these general ' laws,' and, as far as possible, such as also make a strong emotional appeal. Thus, the school study of Science by boys (or girls) on the classical or other nonscience 'sides' should cover a wide range of Sciences-Astronomy, Geology, Physiography, and Biological Sciences, and even Experimental Psychology, as well as the Physics and Chemistry intensively studied on the Science side-and should emphasise the bearing of natural knowledge on all branches of human activity. A similar course adapted to the interests of the younger students would well serve as an introduction to the study of Science by boys (or girls) on the science side.

The Joint Matriculation Board of the Northern Universities has recently decided to provide a paper in General Physical Science alternative to papers in Mechanics, Physics and Chemistry. For some years past the Board has provided a paper in General Biological Science alternative to papers in Botany and Zoology. Thus provision is made for testing in two different ways the knowledge of Science which, on the one hand, literary candidates should possess as part of their general education, and, on the other, that introductory systematic knowledge which Science candidates ought to possess of their own special subjects.

It has in the past been too commonly supposed that Science for literary boys should consist of the science boys' course, so truncated as to deprive it of almost all interest. 
seen, the concluding stage of whole-time education should be concerned with the relation and application of earlier abstract studies to the central purpose and the practical work of the student's future walk in life, the middle period of whole-time education-especially in the case of people whose whole-time education is to be so prolonged that this middle period occurs in later adolescence-should be more concerned with the intensive study of a limited region of the endarchy of science, and with the mastery of the laws, principles, and generalisations that belong to a particular branch of that endarchy, than with the short-circuiting of endarchy-of-science paths (so as to enable the varied experiences of the prospective walk in life to be readily welded into the single wide interest that has begun to be formed by the preceding study of principles and their relation to the most central essences of all). In other words, the special concern of the middle period of whole-time education, at least if it occurs in later adolescence, is to secure coherence in the educand's* growing interest by making it correspond as closely as possible with one of the must central and most completely organised regions of the discovered portion of the endarchy of science: we are reminded $\dagger$ of the special importance of correspondence between the educand's neurography and the more abstract portion of the region of the endarchy of science with which his experience will be concerned. In developing a single wide interest, this 'transitional' type of education is to emphasise the singleness rather than the width; it is to aim at coherence $\ddagger$ rather than at comprehensiveness; its concern is with the central (rather than with the peripheral) portions of the single wide interest that it is beginning to develop; with the effective handling of large general ideas; with giving the same name to different objects, Poincaré's definition of the function of mathematics§; with abstract conceptions; and with the roots of things. But they must be the roots of the right things. Nothing is more deadening than the acquisition of a mass of abstract ideas to which subsequent experience never connects a large body of illustrative concrete facts that give life and meaning to the abstractions $\|$. Nothing is more deadening than the abstract study of several separate subjects having no relation to the centre of one's interest.

* We use the word 'educand' here and on the following pages rather than the more familiar word 'student' because the latter word suggests a person who has passed the age of secondary education, whereas Professor Adams' word 'educand' (see above, p. I2) is equally applicable to the person being educated whatever his or her age may be, and whatever the stage of education he or she may have reached.

$\dagger$ See above, p. 267; also p. 215.

§ Poincaré, loc. cit. p. 34. Cf. p. 199 above.

$\ddagger$ See above, p. 353 .

|| Cf. p. 233 above. 
And, as we said, the more learnedly such subjects are taught by expert but independent teachers, the more deadening their influence is likely to be. Or, as Professor Whitehead observed in a remarkable presidential address to the Mathematical Association:

Education with inert ideas is not only useless: it is, above all things, harmful-Corruptio optimi, pessima. Except at rare intervals of intellectual ferment, education in the past has been radically infected with inert ideas. That is the reason why uneducated clever women, who have seen much of the world, are in middle life so much the most cultured part of the community. They have been saved from this horrible burden of inert ideas.

To attempt, in (whole-time) school or college, to grow the abstract roots of several disconnected subjects that will not go on growing when once that school or college has been left behind, is to multiply the inert ideas that prevent the realisation of one's best self and that hinder effective service to the community.

On the other hand, abstract conceptions, acquired at school or college by young people whose walk in life brings them experiences to which those abstractions are naturally related, will form the very roots and branches of the single wide interest that education should aim at developing*. In fact, the right kind of abstract study during whole-time education unifies the miscellaneous experience of later life and makes it usable. It coordinates masses of practical details by exhibiting them as particular cases of a few underlying principlest.

So there is one way of studying matters from which the single wide interest is to go on growing outwards for a long time to come, until the matters in question lie near the intense centre of that interest; and there is quite another way of studying whatever is to form the interest's fainter fringe. The latter type of study, though it may be nearer its beginning than the former, is certainly much nearer its end. Or, taking the materio-centric instead of the paedocentric point of view, one and the same subject should be studiegd in two quite different ways, by those, on the one hand, who are to become expert in it and make it their own, and by those, on the other, who are content to remain amateurs in regard to it.

The difference between the learned way in which one should know a subject in which one has to be expert, and the amateurish, cultured way in which one has to know other subjects, is illustrated by Sir Stanley Leathes' observation that 'Professors are men of learning, but they should not aspire to create men in their own image -to usurp the Divine prerogative.' $\ddagger$ To put the matter in another

* Cf. above, p. $3 \mathbf{I r}$.

$\dagger$ Cf. above, p. 267 .

$\ddagger$ Loc. cit. p. $15 \mathbf{1}$. 
way, it is the business of an expert in a subject to have it near the centre of his interest and to know it; while an amateur in regard to it need not know the subject, but should know about it, being content to give it a less central place in his single wide interest. One important neurographic difference between knowing a thing and knowing about a thing is that the expert's neurograms correspond to many, if not all, of the discovered essences of that thing, while the amateur's neurograms correspond only to comparatively few of these essences.

This difference may be further illustrated* from the personal experience of the present writer. When he was transferred from the classical to the mathematical side of his school, he did not begin to study algebra at the stage he had reached in the mathematical forms of the classical side, where the study of mathematics was nearing its end. Instead, he went back to the beginning of algebra and set about mastering abstract conceptions, which would have formed mere inert ideas in the mind of a classical boy, but which the future student of mathematics would be frequently using for many years to come. The way in which mathematics should be studied on the classical side, is an example of the 'terminal' way in which it should be studied where its study is approaching completion. The very different way in which the same subject should be studied on the mathematical side, illustrates the different method of studying central subjects in the coherent curriculum that ought to characterise the middle, or 'transitional,' period of whole-time education, a coherence to which subsequent terminal courses and part-time classes will add comprehensiveness.

Still further to illustrate the difference between the finishing whole-time courses that we have described as terminal, and the transitional courses intended for boys or girls of the same age and previous education who are likely to continue their whole-time education beyond the age at which the terminal courses end, we first note that, while terminal courses are more practical, concrete, wide and comprehensive, transitional courses are more abstract, deep and coherent. That education suffers when the difference between terminal and transitional courses is overlooked, is shewn by the findings of Sir Joseph Thomson's Committee, who report that the modern sides of higher secondary schools $\uparrow$ commonly suffer in comparison with the classical sides, because the latter provide definitely transitional courses, while the former provide courses that aim at being terminal

* Other illustrations have been given above on pp. 353, 354 .

$\dagger$ See below, Chapter $24, \S 4$, especially p. 443. 
as well as transitional. The teaching of classics, say the Committee, looks forward to Oxford 'Greats' or to the Cambridge Classical Tripos, while 'the modern side teachers, on the other hand, have to deal not only with the future candidate for a university honours degree... but with a large number of boys who will pass directly into the ranks of commerce or industry.' The result is that, while the classical sides have the coherence that belongs to transitional courses, 'the modern sides suffer...from diversity of effort and indefiniteness of aim.'* In another place the same Committee remark that the (advanced secondary $\dagger$ ) course which they sketch for boys who are about to enter university courses in engineering, may want some modification $\ddagger$ to transform it into the (senior technical§) course that will best suit boys who are leaving school at about eighteen to go into works without intending to proceed later on to a university.

The comparative width, or comprehensiveness, of terminal courses, as compared with transitional, is illustrated by the subjects studied in modern secondary schools that expect to lose most of their pupils at sixteen years of age. Thus the principal assistant secretary of the Secondary Branch of the Board of Education said, in evidence to the Board's Consultative Committee, that

The curriculum of the [secondary] schools was [in I9II] much fuller than it had been. In many schools more subjects had been added, and in many, even where certain subjects were not new, they had assumed a much more important position in the curriculum....In 1902 it was chiefly in connexion with the instruction in natural science that attention was being drawn to the necessity of making education much more practical. But now [in I9II] the same principles were being devised in regard to other subjects, for example practical mathematics, the teaching of geography and the introduction of domestic subjects $\|$.

So the tendency of these modern secondary schools is to substitute for courses in which the study of a group of subjects is abstract in character, other courses in which there is less of abstract study, and the aim of which is to produce width rather than singleness of interest: the newer courses are rather multi-focal, while the older courses tend to be unifocal. Or, in the language we have just been using, these secondary schools appear to be tending to replace transitional by terminal courses. In a later chapter we shall conmend the substitution of terminal for transitional courses in ordinary

* Loc. cit. p. I2.

$\$$ Loc. cit. p. 48 .

$\dagger$ See below, Chapter 23, $\S 3$.

II Report on the Consultative Committee on Examinations in Secondary Schools, I9I I, pp. 429, 430. (Evidence of the Hon. W. N. Bruce.) 
secondary schools* for boys and girls who intend to leave school on reaching the age of sixteen. But there is a danger that the changes described in Mr Bruce's evidence, while they tend in this direction, will not result in satisfactory terminal courses, unless indeed regard be had to the need for connecting every subject studied with the pupil's interest in some group of occupations, one or other of which he expects soon to enter $\dagger$.

To the preceding discussion of the features that distinguish terminal from transitional courses we have now to add that part-time education should, in general, partake rather of the nature of terminal than of transitional whole-time courses; and whole-time education, as compared with part-time, should be more transitional than terminal. In other words, part-time education should be chiefly concerned with the widening of an existing interest: it should make for comprehensiveness. Largely by means of descriptive lectures, it should add to the more concentrated and more abstract study of whole-time school or college, and to the experiences of daily work, a multitude of new facts that give meaning to the abstract study as well as to the routine practice. It should make for continuity, both in series with preceding whole-time education, and in parallel with concurrent practical training in works or offices. It should add a fringe of culture to the single wide interest whose develupment, begun at school, is being continued at work. So part-time classes, in which whole-time education is continued and made to overlap and fit on to the practical training that every employer should give to his younger employees, is an essential part of a perfect system of education. Its most important function is, we repeat, to preserve continuity in the development of the educand's single wide interest, as he passes from whole-time school or college to whole-time employment in the service of the community. The need for such a system of part-time education will not have disappeared, even when the last two or three years of everybody's whole-time education has the specific aim of preparing him for the occupation that comes next. Moreover, part-time education is necessary at whatever age the transition from whole-time education to employment takes place. There is need for the post-graduate evening classes, in which the young university-trained engineer-now engaged in manufacturing, say, Diesel engines or rotary converters-extends and brings up to date his knowledge of these special departments of mechanical or

* See below, Chapter 24, § 4 .

$\dagger$ Cf. p. 353 above. 
electrical engineering, as well as for the part-time secondary education of boys and girls who enter employment direct from elementary schools at the age of fourteen.

We have observed* parenthetically that $g$ is in general more likely to be increased by the abstract studies that characterise transitional courses than by the more concrete, comprehensive, and descriptive matter that characterises terminal courses. We repeat this observation here because of its great pedagogic importance. We have seen $\dagger$ how large is the part played by the Will in the voluntary development of personal neurographies, so as to make them correspond as closely as possible with the endarchy of science. If, therefore, we have been right in saying that a man's Will, or power to concentrate attention, or $g$, is increased by practice + , it follows that the effort to make one's neurography correspond with the endarchy of science, and especially with the more central essences in that region of the endarchy of science with which the individual's occupation is likely to make him most concerned $\S$, will make for the increase of his $g$. And this effort is typical of the abstract studies with which transitional courses are principally concerned. This conclusion may also be reached by observing that concentration of attention can best be practised upon a number of closely associated thought-activities-a coherent interest-because, unless the thought-activity before consciousness at any moment calls up many others from which the Will can select that which is next to receive attention, this faculty cannot be practised; and, without practice, power to concentrate attention cannot be developed.

\section{§ 9. Handwork.}

The last application of the principles discussed in Book II that we have here to note is concerned with the effect of connecting bodily movements with thought-activities. We have already, in Book II, considered the widening and deepening of interests that handwork may produce $\|$. We have noted also that handwork may be employed for the creation of an interest-system, to which neurograms corresponding to other school activities may be attached, and be thereby deepened and increased in value $\%$. We have here only to emphasise a remark already ${ }^{* *}$ made, that children whose $g$ is small can be stimu-

* See above, pp. 34I (especially the footnote $\dagger \dagger$ ) and 353 .

+ See Chapter I4, §7.

$\S$ See above, pp. $266,267$.

If See above, p. 278.

\footnotetext{
¥ See above, pp. 137,138 .

II See above, pp. 59, 60, 277 .

** See above, p. 278.
} 
lated to make an effort of Will in manual training classes, when they will make no such effort to master more abstract studies. In fact, handwork of the right kind increases $g$ because, in many cases, it cannot be done at all without some concentration of attention*; so that subnormal children may secure through handwork an increase of $g$ which could not be effected by more abstract studies $\dagger$.

The Report of the Consultative Committee of the Board of Education on Practical Work in Secondary Schools, which supports the preceding $\ddagger$ conclusions concerning the importance of handwork in the education of subnormal children, produces no evidence that handwork-except perhaps such practical work as is included in the general description, 'laboratory work'-should form part of the regular curriculum provided in secondary schools§ for pupils who are over the age of fourteen. We have indeed remarked already\| upon the danger of injury to able children through overmuch compulsory practical work in school. We conclude that while woodwork, and in favourable cases metalwork also, should form part of the optional studies, handwork of this kind should not be included in the compulsory curriculum of secondary schools attended by pupils over the age of fourteen.

* Cf. above, p. 278. This statement is confirmed by the Consultative Committee of the Board of Education in their Report on Practical Work in Secondary Schools: 'Handwork...gives the opportunity for each individual pupil to deal with and conquer an outside force or forces over which he can only gain the mastery by the deliberate effort to gain mastery over himself. The definitely active attitude which he must take up if he is to do the work at all has been found in practice to react favourably on all sides of his work [by increasing his $g$ ]. Work of this kind has to be done by the pupil himself; it forces him to observe and to think...' (Loc. cit. p. 7.)

$\dagger$ Cf. the Report of the Consultative Committee of the Board of Education quoted in the previous footnote: 'The experience of our witnesses...shows that Handwork develops a side of him [the backward child] that would not be developed by other methods.' (Loc. cit. p. 9.)

$\ddagger$ See the last two footnotes.

$\S$ And therefore for children who are not subnormal.

II On pp. 279, 280 above. 


\section{CHAPTER 22}

\section{A FLOW-DIAGRAM OF EDUCATION}

\section{§. Introduction.}

In the preceding chapter we have considered the applications of some of the principles enunciated in Book II. In the present and succeeding chapters our endeavour will be to take these applications of general principles and to apply them to the problem of education in England*: namely, the problem of adapting the present English provision of education so that it shall realise the aim of education indicated by our enquiry in Book II. It will be remembered that this ain will only be realised when every member of the community develops that particular kind of single wide interest, and that degree of general ability, 'skill in thinking,' or ' $g$,' which his particular service to the community most needs for its efficiency $\dagger$, and which will enable him to realise his best self in that service. While the central purposes of all English citizens are, as we said, to have the same most central essences, and so to be in harmony with one another (and, as far as possible in harmony with the central purposes of men and women throughout the world), the intermediate zone of each individual's neurography-his scientific endarchy that corresponds to his organised thought - is to vary according to the particular activities by which he serves his fellows. As the labour of ministering to the spiritual and material needs of a community is divided amongst its several members, different types of service being assigned to each, different types of single wide interest must be developed, the differences being especially marked in those intermediate zones of which we have just spoken. While, therefore, the education of every citizen is alike concerned with giving him a central purpose in life, kindling in him what Mr Wells calls 'the undying fire,' inspiring him with the spirit of the Lord + , and while this supreme function of everybody's education can only be fulfilled if the teachers share that central purpose $\S$ and are inspired by that same spirit, the business of forming aright the various types of single wide interest, and of developing the various degrees of

* See above, p. 319 .

$\dagger$ See above, pp. 3 I I and 316 .

¥ For, as St Paul said, 'Where the Spirit of the Lord is, there is liberty' ( 2 Corinthians iii. I 7): cf. above, p. 300. $\quad$ See above, pp. $270,346$. 
general ability required by different citizens according to their different types of service, cannot be accomplished without organisation of different types of education in the various schools and colleges of this (or any other) country. If, therefore, we are concerned in this chapter and the next with the differences between the various types of education required by members of the different classes described in Chapter 20*, and with the differences between the several types of education required by the same individual at different stages, it must not be supposed that we are for a moment forgetting that all education has alike to aim at the creation of harmonious central purposes in all men, and at the relation of all the thought and feeling of every individual person so as to form in him a single wide interest dominated by his central purpose.

For the present then, we are concerned with different types of education. Sixteen different types are represented in the diagram $\dagger$ facing p. 3I9. This number might be increased indefinitely as the organisation of a national or world-wide system of education becomes more and more perfect; but this number at least should be recognised in England within the next decade.

The differences that here concern us between various types of education are differences of the kind that distinguish transitional from terminal courses $\ddagger$. They are differences between alternative modes of presenting the same subject, rather than between different subjects similarly presented. From our present standpoint, a descriptive account in English of a Greek play belongs to much the same type of education as a descriptive account of an industrial process; an evening spent in solving, or trying to solve, mathematical problems belongs to much the same type of education as an evening spent in composing Latin prose; or, again, discussions between graduate students engaged on similar researches belong to the same type of education, whether those similar researches are in natural science or history or philology. We have already§ referred to the different types of education to which the study of the Pythagorean theorem may belong, according as it is approached deductively, as would normally be the case in a junior secondary $\|$ course, or inductively, as might well happen in a senior elementary $\|$ course; and, just as the

* See above, p. 326 .

$\$$ See above, Chapter $21, \S 8$.

$\dagger$ See footnote $\ddagger$ on p. 457 below.

$\S$ See footnote $\ddagger$ on p. 344 above.

I| A further account of these types of junior secondary and senior elementary courses follows below in Chapter $23, \S \S 3$ and 12 respectively. Junior secondary education is represented by the upper half of the second (reading from the left) two-year column of the diagram facing p. 319; and senior elementary education is represented in the bottom half of the same column. 
same subject may be presented in different ways to different students of the same age, so also it ought, in general, to be differently presented to the same students at different ages*.

The diagram to which reference has just been made and which we shall employ in the next chapter to illustrate the inter-relationships of the several types of education that will concern us, will later $\uparrow$ be used to shew the inter-relations of different types of schools and colleges ;; and, still later , the same diagram will be employed to illustrate a system of scholarships, together with maintenance allowances, that would bring every kind of education within the reach of all British subjects of sufficient educational promise wherever they may happen to live and however little they, or their parents, may be able to pay. It will, however, be convenient to explain the diagram once for all, rather than piecemeal as we have to put it to its several uses. In the course of our description of the diagram, we shall have to refer to the several types of education described in Chapter 23 and to the several types of school or college described in Chapter 24 .

\section{§2. The Province as a Unit.}

The diagram is described as representing a 'national' system of education designed with a view to English needs and possibilities. Apart from these special needs and possibilities, there is nothing about the diagram that renders it more applicable to a national, than to a world-wide, or merely a provincial, system of education; and, indeed, within England itself the needs and possibilities vary so greatly between, say, Lancashire on the one hand, and Wessex or East Anglia on the other, that somewhat different diagrams, corresponding to somewhat different educational systems, might well be drawn for different English provinces. On this understanding, we shall use the diagram before us to represent, either a system of education for all England, or, alternatively, a system for one of the ten or twelve

* Cf. Mr James Strachan: 'Just as there were certain assumptions it was natural for Euclid to make, and these are more numerous and less explicit than those made by the modern philosophic mathematicians, so there are assumptions which it is natural for the school boy or girl to accept, and these are still more numerous and still less explicit than those made by Euclid. They form the natural basis of geometry for the young.' (Article on Mathematics in The New Teaching, p. 206.)

$\dagger$ See below, Chapter 24.

$\ddagger$ For the future we shall print the several types of school and college-but not 'school,' or 'college,' or 'university' alone-with capital initial letters, in order to distinguish them from the several types of education with which we also have to do.

$\S$ See below, Chapter 25.

G. E. 
educational provinces into which England may one day* be divided. But the diagram will not serve to represent a system of education that can be maintained or administered by an authority concerned with less than one of the provinces of which we have just spoken; for the diagram cannot be used to represent less than one complete educational unit, although it may be used to represent any number of such units. In the latter case, the units are not to be supposed to be entirely self-contained. For example, the pupils from the Higher Secondary Schools $\dagger$ of one province should be free to enter the university or universities of another province. The position we are here concerned with establishing is that an authority, if responsible for Elementary Schools alone, may fail to secure continuity, or adequate means for transferring pupils, from elementary to secondary education; or again, an authority that is concerned with secondary education but within whose area no university is situated, may altogether neglect to provide advanced secondary education that prepares for undergraduate courses; or, if it does provide advanced secondary education of a sort, it may ignore the need for making the advanced secondary courses of study continuous with the undergraduate courses to which theyshould lead; and, in any case, it may fail, as some local authorities to-day do fail $\ddagger$, either to contribute anything whatever to the funds of the local university, or to make any scholarship or maintenance grant to a qualified boy or girl (from its own district) who, without such aid, is prevented from entering the university through lack of means. So, then, the diagram cannot be employed to represent a system of education in any area less than that of a province which includes one or more universities as part of its educational system§.

The diagram is a 'flow-diagram,' the flow being from left to right as age increases. The flow upwards and downwards is allowed for, since any region of the diagram that has the same colour and is enclosed by the same black lines, is designed to represent a pool, in which all the streams, coming in along the left-hand boundaries, are intimately mixed before the outflow takes place uniformly over the right-hand boundaries. Thus, for example, a pupil, entering a junior

* And the sooner the better for the reasons given here and on pp. 450, 45I, and in Chapter 25, $\$ 2$ below. There are several other purposes of local government, notably housing, for which sub-division of the country into provinces is hardly less urgent than it is for education. Cf. an unsigned article on 'The Machinery of Government' in Nature for 3rd April, I9I9.

$\dagger$ See below, Chapter $24, \S 4$ and footnote $\ddagger$ on p. 365 .

\$ See below, Appendix H, pp. 502 et seq.

$\S$ This suggests that the English educational provinces might be constituted on the lines roughly indicated in Appendix E, on p. 497 below. 
secondary course in an ordinary Secondary School at about the age of twelve, is shewn in the diagram as having the same chance of being transferred, two years later, to an intermediate secondary course in a Higher Secondary School, whether, before the age of twelve, he attended a Public Elementary School on the one hand or a Secondary School on the other.

Where the proposed system of education, whether of the whole nation or of the inhabitants of a particular province, should make different provision for boys and girls respectively, the diagram represents the system proposed for boys and young men rather than that proposed for girls and young women. Note will be taken as we proceed of some of these differences*. Here it is sufficient to observe that the differences will, in general, be more marked in the more advanced work represented on the right-hand side of the diagram.

The diagram relates only to the education of normal and supernormal pecple: it does not shew the special provision that is required for seriously defective children $\dagger$.

\section{§3. The Horizontal Scale.}

The figures along the top and bottom of the diagram represent years of age. But-although there are hard and fast vertical lines $\ddagger$ in the diagram, as for example that which separates the ordinary Elementary School from the Part-time Secondary School at the age of fourteen-it is not to be supposed that each child, who is attending an ordinary Elementary School on his fourteenth birthday, will sever his connexion with the school precisely fourteen years from the moment of his birth. According to $\S 9$ (r) of the Education Act of r9r8, obligation to attend whole-time day school no longer ceases at a stated age, but at some convenient season, such as the next end of term following upon the fourteenth birthday. Thus the exact age of

* For an example, see below, p. 386 footnote $\dagger$.

$\dagger$ Mr Cyril Burt, reporting as psychologist to the London County Council, states that 'A child who is retarded by more than three-tenths of his age is regarded as qualified for a special school.' Thus, for example, children who, at the age of ten, are doing the work that most children would be doing at the age of seven or earlier, and again children who, at the age of fifteen, are doing the work that the most normal children would be doing at the age of ten and a half or earlier, should not be admitted to that part of the system of education which is represented in the diagram, but should have provision made for them in special schools and classes for mentally defective children.

The proportion of the population thus excluded from the diagram on account of mental deficiency is probably rather less than $\mathrm{I} \cdot 5$ per cent. Mr Burt estimates that, according to the scale just quoted, 6,504 out of 454,297 London children between the ages of eight and thirteen are deficient. (See Fig. 7, between pp. 42, 43 of the Report on The Distribution and Relations of Educational Abilities quoted above.)

$\ddagger$ Or, more precisely, 'ordinates.' 
transfer of different children from one kind of school to another will be different. But the statistician may represent these different ages by a curve of frequency distribution*, the highest point of which corresponds to the age at which most children experience the transfer in question. Professor Pearson calls that point the mode of the frequency distribution. It is therefore the age of the 'modal' boy that is represented by the figures along the top and bottom of the diagram $\uparrow$. It is, however, to be noted that the beginning of the diagram at the age of ten does not indicate any transition from one type of education to another, or from one type of school to another, at that age: it is only because we shall be most concerned with education that follows the age of ten that we have not extended the diagram to the left to represent the education which the modal child should receive before that age.

\section{§ 4. The Vertical Scale.}

The vertical scale of the diagram is marked along its left-hand margin, where the figures represent percentages of normal and supernormal boys and young men $\ddagger$. The intercept made on the vertical

* If, for example, $y d x$ measures the number of boys who are transferred from Elementary Schools between the ages of $x$ and $x+d x$, and if $x$ and $y$ be measured in directions that are at right angles to one another, then $y d x$ is the area of a column bounded by the $x$ axis at the bottom, by the frequency distribution curve at the top, and by the ordinates $x$ and $x+d x$ respectively on the two sides: so that, when $d x$ is very small, the tops of these columns for different values of $x$ or the locus of the column tops-is the curve of frequency distribution. Then the highest point of this curve, where $y$ is at its maximal value, is called the mode of the frequency distribution. The value of $x$ that corresponds to this value of $y$, marks the age at which most children experience the transfer in question.

$\dagger$ Thus, for example, according to the diagram, the mode of the frequency distribution for boys transferred from Elementary to Secondary Schools occurs at the age of twelve. We may call this the modal age of transfer between these two types of schools. In the same way the other sharp vertical lines in the diagram correspond to the ages at which ' modal ' children and young people would, according to the system of education which the diagram represents, pass from one type of education to another. In particular, the advanced part-time courses provided by Senior Technical Schools or Polytechnics do not cease at the age of twenty-one for everybody; some students may not, indeed, complete these courses until they are more than sixty years of age. What the diagram represents is that more young men complete advanced part-time courses at the age of twenty-one than at any other age. In fact, according to the system of education represented in the diagram, it is not only university part-time classes, to which the diagram sets no upper limit of age, but also all the miscellaneous part-time classes, provided by Senior Technical Schools or Polytechnics, that will continue to be attended by a diminishing proportion of the population up to, and beyond, middle life.

$\ddagger$ As we said above, the same figures will very nearly represent the corresponding proportions for girls as for boys, so far as the left-hand side of the diagram is concerned; but, to the right of the diagram, the figures for young men become increasingly different from the figures for young women. (Cf. footnote $\dagger$ on p. 386 below.) 
line, or ordinate, by the coloured area which corresponds to a type of education, or by the black-bounded area which corresponds to a type of school, is proportional to the number of (male) pupils of the corresponding age who should be receiving the type of education, or attending the type of school, in question. For example, the intercepts made by the coloured areas upon the ordinate that corresponds to the age of fifteen years, divide that ordinate from the top to the bottom of the diagram in the following proportions: $\mathrm{I} 4,36,5$ and 45 per cent. These figures shew that, under the system of education represented in the diagram, of normal and supernormal boys completing their fifteenth year, $\mathrm{I} 4$ per cent. would be receiving intermediate secondary, 36 per cent. senior secondary, 5 per cent. senior elementary, and 45 per cent. junior part-time education. The intercepts made upon the same ordinate by the black-bounded areas, that correspond to types of schools, divide that ordinate in the following proportions: $6,29,15,5$ and 45 per cent. As before, these figures shew that under the scheme of education represented in the diagram, of normal and supernormal boys of fifteen years of age, 6 per cent. would be attending a Higher Secondary School, 29 per cent. an ordinary or Lower Secondary School, I5 per cent. a Junior Technical School, 5 per cent. a Central Elementary School, and 45 per cent. a Part-time Secondary School (or Part-time Continuation School, as some prefer to call it).

Moreover, the vertical scale indicates the proportion of the whole number of boys who, on attaining a given 'age of transfer,' should pass from one type of education, or from one type of school, to another. For example, at the age of twelve, I5 per cent. of the children are shewn as passing from Elenientary Schools to ordinary or Lower Secondary Schools, I5 per cent.* to Junior Technical Schools, and Io per cent. $\dagger$ to Central Elementary Schools. Again, 3 per cent. of

* These figures accord with those recommended to the Manchester Local Education Authority in a recent (December, I9I8) report on 'Manchester's Education Problem' by Mr Spurley Hey, the Director of Education. It is to be hoped (see below, p. 4I5) that within ten years Manchester will have given effect to this proposal, so that, as represented in the diagram, ten years hence, 50 per cent. of the population will be receiving whole-time secondary education up to or beyond the age of sixteen. It is true that Mr Hey recommends that the transfer of the I 5 per cent. of children from Elementary to Junior Technical Schools should take place at a later age than that of the 15 per cent. from Elementary to ordinary Secondary Schools, while our diagram represents the transfer from Elementary to Junior Technical Schools as taking place at the same age as the principal transfer from Elementary to Secondary Schools. We are here in accordance with a resolution adopted by the Association of Technical Institutions at its annual meeting in London early in the year 1918.

$\dagger$ This figure might be considerably increased up to an upper limit of 50 per cent. But see below, p. 423 and the footnote on p. 452 . 
the boys attaining the age of fourteen are shewn as transferring at that age from ordinary or Lower Secondary Schools to Higher Secondary Schools, there to receive intermediate secondary education; at the same age 8 per cent. enter intermediate secondary courses in the ordinary or Lower Secondary Schools; 2 I per cent. enter senior secondary courses in the same schools; and all the 15 per cent. of the boys who are in Junior Technical Schools pass, at the same age, from junior secondary on to senior secondary courses. These figures will probably suffice to shew how the vertical scale in the diagram is to be interpreted.

It is to be noted that the units in terms of which the vertical scale of the diagram is measured are percentages, and not absolute numbers of boys whose age is given. It was therefore unnecessary to make the top and the bottom lines of the diagram converge to allow for the fact that, owing to the normal operation of the death-rate, there would be fewer boys of seventeen than of fifteen in the area-whether a province or the whole country-whose system of education is represented by the diagram.

We have already* pointed out that the diagram is concerned with university education as well as with other types, so that it cannot represent the education of a district or province that is inhabited by much less than two million people; for the provision of separate university institutions for smaller groups of population would be prohibitively expensive. Let us then suppose, for the moment, that the diagram relates to the education of boys and young men in a province inhabited by five million people. We thus fix the scale of the diagram, and so become able to read off the number of boys or young men who should be attending any particular type of school or college, or receiving any particular type of education. The number of boys or young men who are in any one year of school or college age is about I per cent. of the population; so that 100,000 boys or young men would be represented by any two-year column in the diagram; and I per cent. of a complete two-year column, a unit area equal to $\square$, will stand for 1,000 boys or young men attending the type of school or receiving the type of education represented by the portion of the diagram in which that unit area is situated. Thus, in a population of five million people, there should, according to the diagram, be 6,000 boys (4,00o receiving advanced secondary and 2,000 senior technical education) attending the Higher Secondary Schools of the province in the two years between sixteen and eighteen

* On p. 366 above. 
years of age; or 6,000 men undergraduates attending its university (whole-time) for three years from eighteen.

It will be asked why the diagram has been drawn to shew these particular numbers, instead of numbers that are either more or less than these. On the right-hand side of the diagram, the scale is mainly determined by the need and demand for university education. According to the figures published by the British Science Guild, the number of whole-time students in English universities was, just before the war, 5 per I0,000 of the population. The corresponding figure for Scotland was I7 per I0,000; and, for America, Io per I0,000, or, including all four-year colleges, 20 per I0,000. The corresponding figure for Germany was I4 $_{4}$ per ro,ooo. Since positions of authority and responsibility tend more and more to be occupied by university graduates, it is of special importance to a nation that its university education should be adequate in quantity as well as in quality. Now that the supply of university education in England is far less than the demand-a demand not merely from young men whose university education has been deferred by the war but, if we may judge from the experience of a single University College*, from boys approaching the university directly from Secondary Schools-it is reasonable to propose that the supply of university education in this country ten years hence should be at least equal (quantitatively, in proportion to the population) to that which, twenty years earlier, was already available in the greater part of the English-speaking world. The accompanying diagram therefore represents university undergraduates as constituting 18 per Io, ooo of the population of England ten years hence, assuming that half as many women as men will then be entering English universities $\dagger$.

The scale on the right-hand side of the diagram having thus been determined, the scale in the middle and left of the diagram was fixed

* Applications from school boys and on behalf of school boys for admission to the College of Technology, Manchester, at the beginning of the following academic year (October, I9I9) were two and a half times as numerous during one month (May-June) of the preceding spring as they had been in the corresponding period of the year before the war.

$\dagger$ According to the diagram, 4 per cent. of the male population enters the university, and spends there an average of three years; so that, as 50,000 men (or I per cent. of the population of $5,000,000$ ) attain university age in any one year, there will every year be 2,000 freshmen undergraduates entering the university of a province of this size. If, as in the diagram, the undergraduate courses extend over three years, there will therefore be 6,000 men undergraduates. And, if about half as many women as men should receive a university education, and if the average university course lasts for three years, 9,000 students, or I 8 per I0,000 of the population will be attending a university at any one time. Then 3 per cent. of the whole population will at some time or other enter a university. 
from the fact that at least one local education authority * is considering practical proposals for retaining half the population $\dagger$ in whole-time attendance at school until the age of sixteen. This is the proportion shewn in the diagram, which also shews the existing proportion of the population in attendarice at Public Elementary Schools below the age of ten.

\section{§5. Types of Education.}

Colouring is used in the diagram to represent types of education, unshaded colours representing whole-time education, and shaded (singly hatched) colours representing part-time education that is received, either in the day or in the evening, by people whose ordinary employment occupies the greater part of their time.

The doubly hatched region on the right of the diagram represents those later years of active service to the community, when attendance at organised courses of study bearing directly upon one's trade or profession may have ceased, but when private reading, and tutorial classes $\ddagger$ under the auspices of the Workers' Educational Association, or other university part-time classes§, may help those who have passed the ordinary educational age to modernise their ideas and to enlarge their outlook. Classes A, B, C and D in this doubly hatched region represent the different types of service described in Chapter $20 \|$. The breaks in the lines separating these classes mark the fact that transition from one class to another is by no means wholly determined by school or college education!

It will be observed that the central singly shaded area, that represents part-time education in the diagram, forms a kind of bridge between whole-time education to the left, and whole-time employment to the right. We have already pointed out that part-time education is no less necessary at the top than at the bottom of such a system of education as the diagram is designed to represent; but,

* See above, footnote * on p. 369 .

$\dagger$ I.e. half the whole number of boys and girls who live to be sixteen years of age.

$\ddagger$ If the diagram extended further to the right, patches of the light blue singly hatched colour, which represents university part-time studies, would appear in each portion of the doubly hatched region. But the modal age at which the attendance of men, whose formal education has been discontinued at an early age, enter classes conducted under the joint auspices of universities and the Workers' Educational Association is very little less than thirty, and therefore beyond the age range of the diagram.

$\S$ E.g. such as are provided by the People's High Schools in Denmark: see a paper by J. S. Thornton that forms Chapter xvir of Sir M. E. Sadler's Continuation Schools in England and Elsewhere.

I| See above, p. 326 . Concerning the relative sizes of these classes, see below, p. 4I 5 , especially footnote $\|$.

II See above, p. 360 . 
of course, part-time education will begin and end at different ages for different types of students.

As a rule the lighter colours at the top of the diagram represent transitional types of education, while the darker tints below them represent terminal types. We have already* noted some of the leading characteristics that should distinguish transitional from terminal types of education. In particular we have observed that transitional should, in general, be more abstract and more coherent than terminal types of education. For example, in the diagram junior secondary education is more abstract and coherent than senior elementary; intermediate secondary than senior secondary; advanced secondary than senior technical; and intermediate part-time than senior part-time education. Moreover, as their names imply, the more abstract and coherent transitional courses (marked by the lighter colours) prepare for more concrete and comprehensive terminal studies (marked by darker colours) that are to follow in the final $\uparrow$ and more specific stage of whole-time or part-time education.

\section{§ 6. Types of School.}

Lettering represents type of school, college, or other educational institution. Indicating types of school by lettering and types of education by colouring, the diagram is able to shew that one type of school may provide courses of study of more than one type; and also that one type of course of study may be pursued in more than one type of school. Thus, junior secondary education between twelve and fourteen may be obtained in a Preparatory School, in a Higher Secondary School, in a Lower Secondary School, or in a Junior Technical School. On the other hand, between fourteen and sixteen, a Lower Secondary School may provide intermediate secondary education and senior secondary education; while, between sixteen and eighteen, a Higher Secondary School may provide advanced secondary education and senior technical $\ddagger$ education; and, also between sixteen and eighteen, a Senior Technical School§ may

* See above, pp. $35^{8}$ et seq.

$\dagger$ But see footnote $\ddagger$ on p. 372 .

‡ Senior technical courses include two-year whole-time courses in industrial, commercial, domestic, or any other vocational subjects (including art and music), for young people whose education has reached the standard of the school certificate examination, and who do not intend to proceed to a university. Senior secondary and senior technical courses do not mean narrow vocational courses, but a general education which has a centre of interest in some group of occupations into one of which the pupil is expected to enter. (See below Chapter 23, especially $\S 8$ and $\S$ Io.)

$\S$ Senior Technical Schools here include schools of art, commerce, or domestic subjects, and other similar post-secondary non-university schools. 
provide (whole-time) senior technical courses and intermediate parttime courses.

We may anticipate a later chapter* by noting here-what is too often overlooked-that a school or college may, and ought to, exercise upon its pupils or students an educational influence that is no less strong and no less characteristic than that of any particular curriculum. The school or college influence of which we are here speaking is one of the kind which Oxford and Cambridge associate with residence and tutorial superintendence: it belongs, not so much to the lecture room or laboratory, as to the students' societies, the playing fields, the summer camp, and the services in chapel or hall.

\section{§7. Scholarships and Maintenance Allowances.}

Arrow-heads represent scholarships, together with such maintenance allowances as are required to secure that every kind of education is brought within the reach of all inhabitants of the area-whether province or country-whose educational system is represented in the diagram. The question of scholarships will be further considered below in Chapter 25. It is sufficient to observe now that these scholarships, with whatever maintenance allowances are necessary in each case, are to be equally available for all persons wherever they may happen to live in the area to which the diagram relates-say, England-and however little they may be able to contribute towards the cost of their own education $\uparrow$.

The main flow of selected scholars is marked by double-headed arrows. The single-headed arrows indicate the flow that results from a supplementary selection of a few exceptional persons who, for one reason or another, have missed being chosen when the main selection took place. Thus the double-headed arrows indicate a constant supply of systematically selected persons-and the single-headed arrows a few exceptional persons-leaving the school which corresponds to the

* Chapter 24 below.

† It will be noted in Chapter 25 that the cost to this country of a national system of scholarships and maintenance allowances based upon this principle, and sufficient to enable the numbers of boys and young men over fourteen years of age shewn in the diagram, however impecunious their families, to attend the various types of school and college represented in the diagram, would be well within the bounds of financial possibility. Even if maintenance allowances for boys who need them had to be I5s. per head per week between fourteen and sixteen, 3os. per head per week between sixteen and eighteen, and $£ 4$ per head per week between eighteen and twenty-one, the whole cost of scholarships and maintenance allowances would probably be less than the expenditure out of public funds on elementary education before the war; and less than one-sixteenth of the nation's annual drink bill. See Appendix G below, especially p. 501 . 
area in which the arrow-head lies, and proceeding, in so far as may be necessary at the public expense, to the institution towards which the arrow-head points. A single arrow-head enclosed within a ring indicates a supplementary scholarship, with maintenance allowance where necessary, enabling the exceptional person who receives it to pass on to a higher institution of unspecified type*.

* The single arrow-heads enclosed in rings are employed in cases where it was not possible, except by giving long shafts to the arrows-as, for example, where a few exceptional persons, passing from advanced part-time courses in a Senior Technical School to whole-time undergraduate courses in a University, are indicated by the single-headed arrow with the wavy shaft at the twenty years ordinate-and so impairing the legibility of the diagram, to shew whither a supplementary scholarship would enable a pupil to transfer. Thus, the ringed arrow-head on the fourteen years ordinate represents supplementary scholarships that enable a few exceptional Elementary School children of the age of fourteen to transfer to ordinary Secondary Schools or to Junior Technical Schools. 


\section{CHAPTER 23}

\section{TYPES OF EDUCATION}

WE have now to employ our diagram* to illustrate the various types of education required by members of the different classes described in Chapter $20 \dagger$. It will be convenient to consider first the several types of education which successively make up the normal education of members of Class $\mathrm{A}$, and then to describe the types of education that normally prepare for Class B, Class C, and Class D, in that order. We shall thus describe some characteristic features of each of the sixteen types of education represented in the diagram. Finally, we shall indicate how some of these types of education, and especially those provided by part-time courses, may be employed in an order different from the normal so as to afford alternative educational paths, differing from the normal avenues of approach, to the several classes of occupation.

\section{$\S$ I. Nursery Education.}

The beginnings of formal education-that part of the whole educational process in which, as we said $\ddagger$, human educators intervene -take place in the nursery or, at least, at home. Education in the nursery, the nursery school, and the infant school, need make but little progress with the building up of the single wide interest, or with the increasing of ' $g$ ' by practice in concentrating attention: aims with which the types of education that belong to later adolescence are so much concerned. Rather is it the function of the earliest education to increase the unorganised fringe that is relatively so large in the neurography of infancy and early childhood§; to form the central nucleus of the future single wide interest by familiarising the child with the idea of God as the source of all good gifts, and the beneficent central power of the universe; and to connect this central nucleus directly to as many as possible of the elements in the unorganised fringe of the childish neurography, and especially to those concerned with right conduct.

* Facing p. 319 above.

¥ See above, Book I, Chapter 2, p. I2.

$\dagger$ See above, p. 326.

\$ Cf. William James' observation, already quoted, that 'Childhood has few organised interests.' (Loc. cit. Vol. I, p. 4I7.) 
The growth of the unorganised fringe, that corresponds to manifold and disconnected experiences, is to be fostered and guided in two (by no means mutually exclusive) ways. The first may be called aural or visual instruction, and consists of making the child acquainted with some new thing: a new colour, for example, or a new story, or a new country. The second may be called practical instruction, and consists in giving the child the use of some material object (such as a boat, or a pony, or a dog, or, in general, a toy) with which he may become familiar by handling it and by other bodily movements. Such practical instruction enables the child to learn for himself to behave intelligently, and ultimately automatically, in the presence of objects with which accident or aural or visual instruction has first made him acquainted.

But it is not long before abstraction and analysis* have to introduce the beginnings of thought-organisation into the education of even very young children; for this process is involved in naming particular objects, selected parts of the whole field of consciousness. In fact, learning to talk and to count is the beginning of organising thought and of forming that intermediate zone-the scientific endarchy surrounding the central purpose-system - with which subsequent types of education, and especially the transitional types that belong to later adolescence, have so much to do.

\section{$\S 2$. Elementary and Preparatory Education.}

We pass from the education of very little children to the types of education-preparatory and elementary-which, with the 'modal' $\dagger$ child, should end at about the age of twelve. The last two years of education of these two types are represented in the first two-year column of our chart.

In this stage of education, that finishes at the age of twelve the beginnings of thought-organisation, or abstraction in the direction of forming a single wide interest, should receive more attention than in the earlier stage, but not nearly so much as in the following stages. For elementary and preparatory education should still be largely concerned with increasing the unorganised fringe of the neurography, and with connecting the elements of the fringe directly to the central nucleus of the future single wide interest-system; so that, in our view,

* See above, pp. I98, I99.

$\dagger$ See above, p. 368.

$\ddagger$ Throughout the present chapter we shall, in the interests of brevity, use this or an equivalent phrase to express the fact that the mode of the frequency distribution of ages, at which the type of education in question should be completed, occurs at the age (of twelve, or at whatever age may be) mentioned in the text. In other words, when we say that a type of education is to begin or end at $n$ years of age, we mean that the modal boy or young man is to begin or end the type of education in question on attaining $n$ years of age. (See above, p. 368.) 
religion should not merely enter preparatory and elementary education as one of many equal subjects, but it should enter into every lesson on the time-table: it is not merely religious instruction but religious education-education inspired by the Spirit of the Lord, education centred in spiritual things-that is wanted*. For the rest, instruction, pure and simple-aural, visual and manual $\uparrow$ instructioncontinues to play an important part at the stage to which elementary and preparatory education belong. The information acquired at this stage through ears and eyes, as well as the results of the practical instruction, are, in the main, 'knife and fork studies $\ddagger$ '.

It may be convenient briefly to indicate how the headings under which we considered the concern of nursery education with widening the unorganised fringe of neurography, and with introducing the first beginnings of organisation, apply to some of the subjects commonly studied in the course of elementary and preparatory education. These groups, which are by no means mutually exclusive, were:

(a) instruction, aural or visual, concerned with forming the first elements of a child's neurograms for new facts-or, in short, acquainting him with new facts-that would not ordinarily come within his infantile experience so as to attract his attention, but which he will nevertheless need to know before he is much older;

(b) manual or other practical instruction, concerned with widening and extending neurograms so as to include elements (probably belonging to the Rolandic cortex) whose connexion with one's neurogram of an object enables one to act intelligently in the presence of that object; and

(c) comparatively abstract studies, among the first of which are learning to talk and learning to count, concerned with the beginnings of abstraction and thought-organisation.

In the elementary or preparatory stage of education, history, geography, and nature study will, in the main, belong to group (a). For history, as studied by children, consists of stories that acquaint the children with the basic facts of sociology. It is studied because

* See above, pp. 296 and 347.

$\dagger$ We use this word here instead of the word 'practical' because the practical instruction defined above, although it is concerned with all voluntary movements and could therefore best be described by the impossible word 'skeletal,' has after all most to do with the hands.

$\ddagger$ Cf. Professor Adams' Evolution of Educational Theory, p. I6. These 'knife and fork studies'-the phrase is Lord Avebury's - are a necessary preliminary to the later stages of education. For example, unless a child can read simple words in his own language, his further education is hampered, even if reading could be a wholly unnecessary accomplishment in his particular walk in life. 
of the high place which the central essences of sociology occupy in the endarchy of science as completed by the Christian hypothesis. But the information conveyed by the stories, which are all that a child learns of history, does not form part of his single wide interest until, later on, the central essences of sociology are abstracted. To become acquainted with the stories and never, not even at political meetings in later life, to study the principles, will not contribute to a single wide interest. In fact, history at this stage is not concerned with thought-organisation, but with instruction about apparently disconnected facts. Geography, too, at this stage is only concerned with basic facts, whether of sociology or of natural science. Later on, one's interest in one's British citizenship suffers unless it includes some knowledge of the geography of the British commonwealth, and one's interest in one's world citizenship suffers if one is ignorant of world geography; and, in either case, the motive to achieve a common purpose suffers too. But the connexions which weld together the miscellaneous facts that form one's childish knowledge of geography, so as to include it in its proper place in one's single wide interest, hardly begin to be established until childhood is at an end. Nature study, again, is, at this stage, concerned with basic facts. As with history or geography, its study does not contribute to the single wide interest unless and until it is continued and integrated by the abstraction of central essences.

Reading, writing, the study of a foreign language, and handicraft, will, at this stage of education, mainly belong to group $(b)$, although, as we said, overlapping between different groups is bound to occur. Reading, pure and simple-reading about familiar subjects with the aim, not of acquiring new information, but of becoming familiar with the written symbols for facts with which the reader is already acquainted-we have to include in group $(b)$ because reading of this kind aims at widening the reader's neurograms for facts already known to him, by extending those neurograms to include elements that correspond to the visible written symbols for the factsin question, as well as elements whose activity accompanies the oral expression of those symbols. In the same way, writing, pure and simple-writing words which one has already learnt to read, and with some meaning of which one is already acquainted-belongs to group $(b)$; for such writing involves manual and other bodily movements, and thus adds to existing neurograms the elements whose activity accompanies the movements in question. At the elementary and preparatory stage of education, the study of various foreign languages also belongs to 
group $(b)$, for it consists mainly in learning more names for familiar facts. Moreover, a foreign language studied at any later stage of education, even in a university by students whose only object is to understand the expression in the foreign language of thoughts which they would understand if expressed in their own language, or else to express in the foreign language thoughts which they are already able to express in their own, would also belong to group $(b)^{*}$. It should be added that this kind of study of a foreign language ought to form part of the education of childhood, rather than of adolescence or maturity. In the words of Sir Stanley Leathes: 'If a parent wants his boy to learn French and German, he had better see that he learns them before he is ten; if possible, before he is eight.' $\dagger$ Handicraft, again, evidently belongs chiefly to group $(b)$. For many pupils, however, and especially for those who become craftsmen, handicraft will later on form a central interest, closely connected with their supreme purpose, and forming the link between that purpose and most of their other school studies. Moreover, as Rousseau pointed out $\ddagger$, school handicraft may extend the interests of pupils who will not become craftsmen, so as to make those interests overlap the interests which their fellow citizens take in their own craftsmanship.

Of the studies that commonly form part of elementary education, mathematics alone-arithmetic, some geometry, and perhaps the elements of algebra-belongs mainly to group $(c)$. The same is probably true of preparatory education also, although it has been the usual practice of Preparatory and other Secondary Schools to lay more stress than is cornmonly laid in Elementary Schools on the aspect of education that we have connected with group $(c)$. But the difference between preparatory and elementary education, as hitherto provided in this country, is more a matter of practice in the power of concentrating attention, and so of increasing $g$, than of neurography or subject matter. It is in private study, and especially in homework exercises upon such subjects as Latin and Euclid, that the Preparatory

* As in the case of every other subject mentioned in this and the preceding paragraph, it is not the subject, but the subject as studied during elementary or preparatory education, that belongs, in the main, to the particular group to which it has been assigned in the text. For example, at a more advanced stage a foreign language may be studied in connexion with sociology, attention being given to the Sittlichkeit of the foreign people; or from the point of view of philology. It may, however, be remarked that, where the study of a foreign language is not to be carried to an advanced stage, the sociological (Sittlichkeit) interest of the study can generally be better served by the study of the history and institutions of the foreign people, and of translations of their literature, than by studying only the elements of their language: cf. p. 354 above.

$\dagger$ Loc. cit. p. 95.

$\ddagger$ See above, p. 279. 
School boy has better opportunities than the boy from the Elementary School* to practise concentrating attention and so to develop $\dagger$ ' $g$.' And indeed subjects of this kind have probably been selected for the curricula of Preparatory Schools, or at least for the curricula of the Higher Secondary Schools to which Preparatory Schools are intended to lead, for the sake of the high degree of concentration of attention required for studying them as they usually are studied on the classical sides of English 'public schools,' rather than for the sake of making the pupil acquainted with their particular subject matter. At all events, the thirteen Oxford Preparatory School boys, whom Mr Burt examined, possessed on the average a considerably higher degree of ' $g$ ' than was possessed by the thirty Elementary School boys whom he examined at the same time. As has already $\ddagger$ been said, Mr Burt attributed the superior general ability of the Preparatory School boys to the fact that they 'were in nearly every case sons of men of eminence in the intellectual world, that is to say of Fellows of the Royal Society, university professors, college tutors, and bishops,' while 'the boys of the elementary school were of the lower middle class, sons of local tradesmen.' But, for the reasons already stated, we incline to attribute the superior general ability of the Preparatory School boys, partly at least, to the fact that their education was far more likely to increase ' $g$,' than were the more comprehensive, but less concentrated, studies of the Elementary School.

The differences we have observed to exist between elementary and preparatory education, as hitherto provided in England, may, to some extent, be justified by the fact that, hitherto, children who have attended private Preparatory Schools or Secondary Schools before the age of twelve have generally had a much longer school life still before them than children who have attended Public Elementary Schools up to at least the age of twelve. For the fact that an Elementary School child in his twelfth year was, as a rule, approaching the end of his whole-time education, was a sufficient reason for his education being more terminal in character than that of the Secondary School boy of the same age. But when the duration of a child's whole-time education depends only, or chiefly, upon his own personal qualitiesand especially upon his general ability and ambition or aim-the justification for this particular difference between elementary and

* In many if not most parts of England-in striking contrast with Scottish practice-Elementary School children are not even allowed to take books home, on the ground that the school-books belong to the local education authority and not to the children!

$\dagger$ See above, pp. $137,138$.

$\ddagger$ See above, p. 136 .

G. $E$. 
preparatory education will have disappeared. Indeed, had our diagram been designed to represent education in England fifty instead of ten years hence, the first two-year column of the diagram might well have been printed in the same colour from top to bottom. But it is too much to hope that, within the next ten years, such differences as remain between elementary and preparatory education will wholly depend upon the qualities of the children receiving education of these respective types. In particular, it is too much to hope that, within ten years, the language spoken by children attending Elementary Schools on the one hand, and Preparatory Schools or ordinary Secondary Schools on the other, will have become indistinguishable; and, until this language difficulty has been overcome, it will be very hard to remove those other differences between preparatory and elementary education that do not depend upon the intellectual qualities of the children. This and other differences that we have still to note as existing between elementary and preparatory education, resemble the differences that distinguish terminal from transitional courses at a later stage.

Thus elementary education has been more concerned than preparatory education with widening the child's interest by acquainting him with new facts: in short, by the studies that mainly belong to our group $(a)$. The reason is doubtless to be found in the different nurture that has hitherto marked children following preparatory and elementary courses respectively; for the children in Preparatory and other Secondary Schools have, for the must part, developed wider interests than are possessed by the average Elementary School child of the same age, because of their less restricted home surroundings, and especially because of their better educated parents. Then again, Elementary Schools have, as a rule, given more attention than Preparatory Schools to handicraft and other practical instruction. It has been assumed that children attending Preparatory Schools and other Secondary Schools would be able to obtain at home, and especially in the holidays, that practical experience of constructive work in wood, metal, or other material*, which the child receiving elementary education could only receive at school. On the other hand, preparatory courses have tended to include a larger proportion of abstract study, work that is not closely connected with the practical experiences of home or school life. Latin and Greek, for example, are

* For example, concrete. The pleasure which some children derive from making, or helping to make, concrete structures is described by M. E. Francis in The Things of a Child; and by Professor E. B. Poulton, F.R.S., in his life of his son Ronald Poulton. 
often taught to children under twelve in Preparatory Schools because these are instrumental subjects-'knife and fork studies'- that prepare for junior secondary education of a particular kind that is offered by Higher Secondary Schools. But, as we said, the chief difference between preparatory and elementary education in England to-day consists in the greater importance attached by the former to increasing general ability, or ' $g$,' by hard study of whatever subject.

\section{§3. Secondary Education: Junior, Intermediate, and Advanced.}

According to the plan laid down at the beginning of this chapter*, we have next to consider the further education of the future member of Class A who is following the normal avenue of approach to his profession. From the age of twelve to the age of eighteen he will receive secondary education, of which we may recognise three successive types, junior, intermediate, and advanced, each occupying two years. All of these belong to the transitional category described in an earlier chapter $\dagger$, for the aim of each of them is to prepare for higher whole-time education that is to follow. To the terminal type, on the other hand, belong senior secondary education which, as we shall see $\ddagger$, will form the greater part of the provision of the ordinary or Lower Secondary School, as well as of the Junior Technical School, for pupils between fourteen and sixteen years of age; and the senior technical courses which Higher Secondary Schools may provide for the last two years of the school life of those young people who are not going on to the universities, are also of the terminal type.

If the future member of Class A, with whose secondary education we are now concerned, has attended an Elementary School nearly up to the age of twelve, he should, for reasons which we shall further consider in the next two chapters, receive no more than his junior secondary education in an ordinary or Lower Secondary School. That is to say, he should enter his Higher Secondary School by the age of fourteen and there receive the intermediate and advanced secondary education that will occupy the remaining years of his school life until, at or soon after the age of eighteen, he enters a university to read for an honours degree.

It is during the period of junior secondary education that, as we have seen $\S$, a central purpose in life may begin to become dominant in the growing wide interest. From that time onwards, the chief concern of transitional secondary education-including junior, inter-

* See above, p. 376 .

¥ See below, Chapter $23, \S$ ro.

$\dagger$ See above, Chapter $21, \S 8$.

$\S$ On pp. 340, 34I (Chapter 2I, §6). 
mediate, and advanced secondary courses-is with the structure* of a pupil's single wide interest as a whole. Of transitional secondary education it is far more true than of elementary or preparatory courses which precede it, or of terminal courses which follow it, that the curriculum is an organic whole, rather than an assemblage of standardised parts. It resembles a living organism or a work of art, rather than a Ford motor car or a standard ship $\dagger$. It does not consist of the independent study of many branches of knowledge; and its effectiveness is not to be tested merely by an external examination of proficiency in separate subjects $\ddagger$ regarded as isolated units. Rather, as we said $\S$, is its first concern to form and, by means of strong emotional associations, to deepen the pupil's central purpose. The chief function of secondary education, and especially of transitional secondary education, is, in fact, to foster the growth of true religion-' not theology nor yet ethics, but personal and experimental.' $\|$

Around this centre it has also to build up, out of the miscellaneous information obtained in childhood and its own coherent curriculum, some of the most valuable and important parts, particularly in the intermediate zone**, of that single wide interest which should continue to grow throughout maturity. This intermediate zone has to correspond as closely as possible with some region of the endarchy of science that shall extend to the limits of the discovered portion of the endarchy. Thus will the intermediate zone of the pupil's growing single wide interest include elements that correspond to some of the most valuable of the discovered essences of the endarchy of science. We are thus applying our general principle $\dagger \dagger$ by starting from practical, concrete experiences, and from them building up a single wide interest by abstraction so as to reach as near the centre of the discovered portion of the endarchy of science as the available time and the ability of the student permit; or, in other words, so as to reach the highest principles, laws, and generalisations which subsequent terminal (whole-time and part-time) courses will afterwards apply to the field of experience where the student's walk in life will, for the most part, probably lie.

Another reason why transitional secondary education, especially in later adolescence, should include and, subject always to the most central purpose, be centred in the highest, and, as they often are, the

* See above, pp. 334 to 336 .

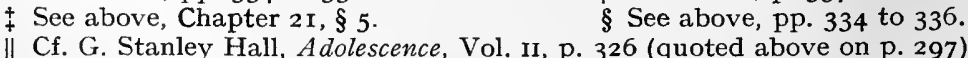

$\dagger$ See above, p. 337 .

If See above, p. 340 .

$\dagger$ See above, Chapter 2 I, $\S 6$, p. 342 .

** See above, Chapter $2 \mathrm{I}, \S 4$. 
most recent, generalisations of organised thought*, is that these generalisations appeal to the emotions $\dagger$ of later adolescence and awaken the enthusiasm of young people who are on the threshold of manhood or womanhood. The result is that the student's neurograms for such generalisations acquire the depth and permanence that fit them for the central position they should occupy in the growing single wide interest-system.

Still another reason why the secondary education of future members of Class A, who, as we saw $\ddagger$, require a specially high degree of ' $g$ ' or 'skill in thinking,' should thus concern itself with abstractions, is that progress in such abstract studies demands concentrated attention and so, as we have already pointed out§, is peculiarly well fitted for increasing ' $g$ ' $\|$.

We have seen 9 that the greater the general ability, or ' $g$,' possessed by a pupil, the longer in general should his whole-time education extend. The longer also will it be before the education, which he receives in common with other boys of like ability who will ultimately engage in different forms of Class A service to the community, should cease, not merely to be of the same type as that of the other boys, but actually to include the same subjects studied in the same way. But when the age for discontinuing whole-time education is imminent, terminal courses will provide different education for boys of like ability who are to find employment in different groups of occupations. While, at their commencement, terminal courses must all be continuous with the transitional courses that precede them, they will, towards their close, involve the study of different subjects, or at least a different study of the same subjects. So there will have to be many varieties of terminal courses. Moreover, the transitional courses that prepare for, and are continuous with, terminal courses that are different, not merely at the end but also at the outset, must themselves differ (not in type but) in subject matter**. And, in general, the normal $\uparrow \dagger$ educational paths that lead to different fields of service should fork with increasing frequency as the end of whole-time education approaches $\$+$. It follows that the different types of education which a world-wide,

* I.e. the incomplete endarchy of science: see above, footnote $\S$ on p. I99.

$\dagger$ Cf. p. 345 above.

$\ddagger$ On p. 327 above.

$\S$ On p. $36 \mathbf{1}$ above.

II See above, p. 333 and footnote $\dagger \dagger$ on p. $34 \mathrm{I}$.

I See above, p. 325.

** See above, p. 356 , where emphasis is laid on the right hind of abstract study.

t† See above, p. 376 .

$\ddagger$ Cf. p. 325 above. 
national or provincial system provides for future members of Class $\mathrm{A}$, should offer a progressively increasing variety of subject matter*.

Remembering what has already been said of the supreme importance of making the central spiritual purpose central in secondary education, and distinguishing the different varieties of junior, intermediate, and advanced secondary education, not by any difference in emphasis on the central purpose, but by the region of organised thought which they respectively make next most central, we may therefore suggest that a complete system of education should offertogether with ample opportunities for physical exercise, handicraft, drawing, and (for girls) domestic subjects - the following varieties of junior, intermediate, and advanced secondary courses to every boy or girl $\dagger$ who is selected to receive education of those types $\ddagger$ :

JUnior Secondary Courses, that form a single wide interest having:

I. Classics nearest the central purpose, but also including English, mathematics, natural science, history, geography, and such other languages as will be studied at least up to the standard marked by the School Certificate examination $\S$.

* Thus university graduates, who are being trained in methods of research during.the two or three years that follow their first graduation, may each be following a different course of study adapted to his individual needs. Of undergraduate honours courses preparing for graduate study and research the variety, while still large, will be less than that of graduate studies. The advanced secondary courses that prepare for undergraduate studies must again be of several different kinds, but the variety need not be so great as of university 'honours schools' or 'triposes.' The variety of intermediate secondary courses may be smaller still, while of junior secondary courses one or two kinds will probably suffice.

$\dagger$ Since girls in their early twenties are far more mature than boys of the same age, girls may be said to have more growing-up to do in their teens. Their school routine should therefore allow them more leisure for their own thoughts, especially between the ages of fourteen and sixteen. It is accordingly suggested that girls should take three years over the intermediate secondary studies that boys will usually complete in two years; and that therefore the usual age at which girls enter universities should be at least one year in advance of the age at which boys come up to universities direct from school.

$\ddagger$ Later in the day on which the outlines of these ten courses were dictated, there came into the writer's hands a note by the headmaster of Sherborne School entitled Subjects of Study in a Public School. In this pamphlet Dr Nowell Smith provides one (junior secondary) course in the Lower School; three (intermediate secondary) courses in the Upper School; and four (advanced secondary) courses in the Sixth Form. He thus makes the number of sides of the school (or, as we say, varieties of courses) increase as the work becomes more advanced.

$\S$ The examination for the School Certificate-the School Certificate examination, as it is called in the text and in the regulations of the Joint Matriculation Board for the Universities of Manchester, Liverpool, Leeds, Sheffield, and Birmingham; or the First School Examination as it is described in official literature-is an examination conducted by a university examining body for pupils who are completing their intermediate secondary education. 
II. Natural science nearest the central purpose, but also including English, mathematics, history, geography, and such other languages as will be studied at least up to the standard marked by the School Certificate examination.

InTERMEDIATE SeCONDARY Courses, that form a single wide interest having:

I. Classics and ancient history nearest the central purpose, but also including mathematics, natural science, and such other foreign languages as will be studied at least up to the standard marked by the School Certificate examination.

II. Modern studies (English and foreign languages, literature, and history) nearest the central purpose, but also including Latin, natural science, and mathematics; and possibly -if it can be studied up to the School Certificate standard-Greek, among subsidiary subjects.

III. Natural science and mathematics nearest the central purpose, but also including a modern foreign language, and-if they can be studied up to the School Certificate standard-Latin and Greek as subsidiary subjects.

Advanced Secondary Courses*, that form a single wide interest having:

I. Classics and ancient history nearest the central purpose, but also including English and other subsidiary subjects.

II. Modern humanities, principally languages, nearest the central purpose, but including modern history, and also including English and other subsidiary subjects.

III. Modern humanities, principally modern history, nearest the central purpose, but also including languages and economics, as well as English and other subsidiary subjects.

IV. Natural science nearest the central purpose, but also including mathematics and English and other subsidiary subjects.

* The following five varieties of advanced secondary courses correspond approximately with the three divisions into which the Higher School Certificate examination-or Second School Examination-is generally divided, our second and third varieties being generally included in the second group of Higher School Certificate subjects, and our fourth and fifth being included in the third group. But the Joint Matriculation Board of the Northern Universities divide their second group approximately as we have divided it. See Appendix $F$ for particulars of this examination as conducted by the Joint Matriculation Board in 1919 . 
V. Mathematics nearest the central purpose, but also including natural science (astronomy, mechanics, physics, and chemistry), and English and other subsidiary subjects.

In accordance with what has already* been said about the importance of giving form-masters a dominant place in secondary education, especially in its earlier years, it is desirable that at least half the teaching week should be given by every boy, at any rate during the junior and intermediate courses, to work under one formmaster. It is the form-master whose principal concern it will be to relate the pupil's growing single wide interest to a central Christian purpose $\uparrow$. Throughout junior and intermediate secondary education, it is also the form-master who should teach English $\ddagger$. During junior secondary education, the form-master should teach history and geography, and, on the classical side, undertake part, at least, of the teaching of natural science. During intermediate secondary education, the classical or modern side form-master should still take part in the teaching of natural science to the boys in his form. At the same stage, the form-master on the science side should teach his boys, not only all their English as we have already said, but, if possible, all their mathematics§ as well.

During advanced secondary education, it is still desirable that the pupils should give more than half the week to those subjects on which their coherent curriculum is focussed, but it is no longer as important as in the earlier\| stages of secondary education, that these focal subjects should be taught by one form-master. Indeed, as we said $\Pi$, an increasing degree of interchangeability between university lectureships and sixth-form masterships in Higher Secondary Schools is much to be desired. To this end, sixth-form masters might well be enccuraged to engage in researches under the general direction of a neighbouring university. In so far as geographical considerations permit, these investigations should involve work in the university laboratories, library, or elsewhere within the precincts of the university; but, even when the distance between the School and the university is too great for such an arrangement to be practicable, the possibility of a schoolmaster engaging in research, and of his research keeping him in touch with university life and thought, is by no means wholly precluded.

In case it be again objected that, when form-masters teach subjects other than those in which they have specialised, their

* See above, pp. 270,346 et seq.

$\ddagger$ See above, pp. 347,348 .

II See above, p. 346 .

$\dagger$ See above, p. 347 .

\$ See above, p. 348 .

if See above, p. 349. 
teaching will necessarily suffer in comparison with that of experts, we remind ourselves once more* that the same subject should be studied in different ways by boys on different sides of their school; or, in other words, by boys who are following different varieties of the junior, intermediate, and advanced secondary courses outlined above: in short, by boys who are looking forward to walks in life that are very different although they may all belong to Class $\mathrm{A}$.

We have retained classics as the focal subject of one of the alternative secondary courses at each of our three stages, junior, intermediate, and advanced; and for two principal reasons. The first is that the university study of classics will always be necessary for the purpose of keeping the thought of our time in touch with the best of the thought of ancient Greece and Rome; while the study of classics at this stage will be sure to suffer if it has not been approached by strenuous classical study in Secondary Schools. For some time to come, however, the proportion of boys whose interest will be centred in classics throughout their secondary education will probably exceed the proportion of adults whom the community will need as classical scholars. This leads us to our second reason for demanding the retention of classical sides in Higher Secondary Schools: namely, that the teaching of classics for many generations has resulted in a comparative perfection of method that enables form-masters to secure far more concentrated attention from their classical forms than the various specialist masters, who are responsible for modern studies, can generally obtain from their pupils $\dagger$. Moreover, the classical studies are by tradition more closely welded together, and are thus more effective in developing a single wide interest, than is the case with the work done on the modern and science sides of most Secondary Schools $\$$. There does not, however, appear to be any inherent reason why modern humanities or natural science or mathematics should not be so taught, by suitably trained form-masters, as focal subjects, and so related to a central purpose and to subsidiary subjects of a coherent

* See above, Chapter $21, \S 8$, especially pp. 355 and 357 .

$\dagger$ Cf. Mr Robinson's justification of classical studies, quoted above on p. 333 . See also the quotation, on the same page, from Mr Fairgrieve.

$\ddagger$ See above, pp. 338 and 359. To the passages quoted on these pages from Sir Joseph Thomson's Committee's Report, we may here add the following sentences: 'It cannot however be said that up to the present there has been either in the schools or in the universities any clear conception of modern studies which might give these sides a meaning and an aim. The teaching of Classics has behind it a longer tradition and is on the whole better understood, with the result that teachers are on surer ground. It looks forward to an end which is clearly marked by the Oxford School of Literae Humaniores or the Cambridge Classical Tripos.' (Report, loc. cit. p. I2.) 
curriculum, as to make them no less effective than the traditional discipline of classical sides in practising concentration of attention and in developing single wide interests. This reform of junior and intermediate secondary education on modern and science sides-in our plan the mathematical side does not begin before the sixth form, or, as we have called it, the period of advanced secondary educationis indeed urgently needed. Until it has been effected, many able boys, whose future walk in life could better be approached by transitional secondary courses focussed in modern humanities or natural science, will rightly continue to spend much of their school time on classics, with the result that, for the sake of the advantages in developing ' $g$ ' and in forming a single wide interest that are not obtainable in the lower forms of modern and science sides, many of the ablest boys will still be compelled to cumber their minds with what Professor Whitehead calls a 'horrible burden of inert ideas.' *

It remains to add that the subsidiary subjects, which should be included in the coherent curriculum of any of the ten $\dagger$ kinds of secondary education that we have outlined, need by no means be confined to those that we have named. In particular, organised games and social activities of various kinds, to some of which we shall have occasion to refer in the next chapter, have their places in all secondary education.

\section{§ 4. University Entrance.}

The future member of Class A should, as a rule, remain at his Higher Secondary School until he has spent two years (receiving advanced secondary education) in the sixth form, and has reached the standard of the HigherSchool Certificate in the branch of knowledge which he wishes further to pursue as an undergraduate in a university 'honours school.' He should, as indicated in our diagram, enter the university at or soon after the age of eighteen. It is true that the future engineer often spends some time in an engineering works between school and college, but there is a growing consensus of opinion that this period should not be too long $\ddagger$; perhaps from Christmas until

* See above, p. 357.

$\dagger$ Five advanced, three intermediate, and two junior.

\$ 'Something may be said for the view that the time spent in works between school and college is of great use in bringing the student into contact with things about which he will study at college, in broadening his outlook upon the world, and in subjecting him to works discipline. As, however, he has not yet learnt [from advanced secondary studies] the principles involved in the machinery and processes displayed in the factory, he cannot hope to acquire knowledge at as great a rate as during the two years' apprenticeship following his college course. 
the following October* would be ideal if both school-leaving and university-entrance scholarships could be awarded, as those of some Oxford and Cambridge colleges already are, just before Christmas $\uparrow$. In fact, as Sir Joseph Thomson's Committee point out, 'It is desirable on educational and other grounds that boys who intend to pass on to a university should as a rule remain at the secondary school up to the age of eighteen, provided that the school is so organised as to furnish satisfactory [advanced secondary $]_{\ddagger}^{+}$courses in the various groups of subjects appropriate for them.' § The Committee accordingly recommend that 'Eighteen should be the normal age of entry from secondary schools to the universities.'\| It is, however, still the practice of most English universities to make sixteen the minimum age for entry. So long as this minimum is only intended to mark the

For this reason, the time spent in works between school and college should not extend over too great a period, and this period should not reduce the essential training in works after the college course has been completed. The abler the student, the less necessary in general is the short works course between school and college. Perhaps an ideal arrangement would be for a boy to win his university scholarship in December, leave school at Christmas, spend nine months in the works, and enter upon his college course in the following October.' (Report on Engineering Education and Reseavch approved by the Council for Organising British Engineering Industry, since combined with the British Engineers' Association, in 1916 ; p. ro.)

* The interval that, in some cases, may be spent in works between school and college is marked by the thick black line at the top of the eighteen-years ordinate in the diagram. The width of the black line is less than proportional to the Christmas to October period suggested in the text, for it is only a comparativcly small number of boys who should interrupt their academic training by spending nine months or a year in works, or in otherwise obtaining practical experience, between school and college.

$\dagger$ This suggestion is at variance with a finding of Sir Joseph Thomson's Committee, namely that: 'The interval between the date of the [university entrance] scholarship examination and the time of coming up into residence is too long, and we recommend that the examinations should be held not earlier than Ist March.' (Report, loc. cit. p. 6r.) If this recommendation of Sir Joseph Thomson's Committee were adopted, the difficulty alluded to in the text might still be metbut not, it is submitted, so satisfactorily-by adopting a suggestion made by the Consultative Committee of the Board of Education in their 'Interim Report on Scholarships for Higher Education' [Cd. 829I]: namely, by allowing a scholar to postpone taking up his scholarship. They write: 'Our conclusion is that if a Scholar desired to defer his entry to the University for the purpose of acquiring experience in works, permission should not be refused on proof that some suitable establishment was willing to take him and give him an adequate chance of acquiring the appropriate experience.' (Report, loc. cit. p. 40.)

$\ddagger$ See above, pp. $3^{83}$ and 387 .

§ Loc. cit. p. 27.

II Loc. cit. p. 27. The Report adds: 'In making this recommendation we are supported by all the witnesses who have given evidence to us on the subject.'

Compare the findings of the Consultative Committee of the Board of Education in their 'Interim Report on Scholarships for Higher Education': 'For those who are to go to the Universities the normal age for completion of Secondary Education should be not less than seventeen; eighteen or even nineteen is better.' [Cd. 829I, p. I5.] 
practical limit of an age-of-entry frequency distribution whose mode* is at eighteen, this lower limit need not be altered; but that eighteen, rather than seventeen or less, should be the modal age of entry to a university from the normal avenue of approach to it, needs to be more generally recognised. The fact that, of the very small number of men who still enter universities under seventeen years of age, an unduly large proportion fails to make good, is not sufficiently widely appreciated.

For reasons that we are about to consider $\dagger$, the courses of study followed by most undergraduates in English universities should resemble the work of 'honours schools' rather than that now required for pass degrees in these universities. In general, therefore, undergraduate courses should not be expected to introduce the student for the first time to work below the standard marked by the Higher School Certificate + , although they may well include the revision of such work under the direction of university teachers who are able to place familiar fundamental matter in a new perspective; and indeed this business of placing familiar matter in a new perspective should be undertaken at the earliest possible moment after the undergraduate has entered his university. In his first year, and even in his first term, he should become acquainted with university teaching at its best§. But, since some years must elapse before the present provision of advanced secondary education in England is increased sufficiently to enable every boy, who is approaching the university by the normal path, to have spent two years in the sixth form of a Higher Secondary School before beginning his undergraduate studies, it will, for the present, be necessary for universities to provide preliminary one-year courses preparatory to the three years undergraduate course in an honours school\|. To these preliminary courses will be admitted boys who have completed their intermediate secondary education in a Lower Secondary School (together with a few exceptional boys from other institutions whose work does not reach the Higher School

* See above, p. 368 .

$\dagger$ See below, $\S 5$ of this chapter on pp. 397 et seq.

$\ddagger$ Only so can the universities 'be freed from most of the elementary teaching that has up to now tended to distract their attention from their proper province.' (Sir Joseph Thomson's Committee's Report, p. 58.)

$\S$ 'The student, on coming to the University, should come under the influence of the great teachers of the subject (instead of being placed, as is sometimes the case, in the hand of junior lecturers or demonstrators), and should be inspired with the views and the spirit of those teachers.' (Education, Secondary and University, A Report on Conferences between the Council for Humanistic Studies and the Conjoint Board of Scientific Societies, p. 33, by Sir Frederic G. Kenyon, President of the British Academy, 1919.) See also below, p. 400.

\| This principle has been accepted by Manchester University for the Faculty of Technology. 
Certificate standard) and who have satisfied such other conditions* as may be imposed by the university concerned. It will then be the object of each preliminary course to enable these boys, after one year's work, to enter undergraduate (honours) courses as well prepared as boys who have satisfactorily completed advanced secondary courses up to the standard marked by the Higher School Certificate examination.

At the present time it is usual for English universities to require candidates for admission to degree courses to have reached a certain standard of proficiency in five or six separate subjects. Very recently this uniform requirement has been modified in two ways. First, less specialised knowledge of some subjects has been allowed to be compensated for by more expert knowledge of other subjects $\dagger$, a change that renders it possible for secondary education to ain at developing a single wide interest in the inanner that we have described, without imperilling the pupil's chance of passing a university entrance test that requires him to have specialised in six separate subjects. No narrowing of secondary education is likely to result from this change; for, as we have seen, the more a student, young or old, cares for and believes in his own focal subject, the more he will desire to make the whole world tributary to it. Or, as Professor Campagnac has put it, 'The more ardent his specialism, the more catholic will be his tastes.' The second alternative was first devised by London University for the purpose of testing candidates who had not passed through English secondary schools. In a modified form this second alternative has recently been adopted by the Northern Universities $\ddagger$ for the purpose

* It is now usual for English universities, other than Oxford and Cambridge, to require candidates for admission to degree courses either to have passed a special 'matriculation examination,' or to have passed the School Certificate examination 'with credit' in certain separate subjects; or, in other words, to have reached a higher standard than is necessary for passing the School Certificate examination in those subjects. For example, the Universities of Manchester, Liverpool, Leeds, Sheffield, and Birmingham admit to their degree courses candidates who have passed the School Certificate examination with credit in English, Mathematics, History, and three other approved subjects, of which one must be a language. See also the next footnote.

$\dagger$ Thus the Joint Matriculation Board of the Universities of Manchester, Liverpool, Leeds, Sheffield, and Birmingham will accept, as equivalent to a pass in the six-subject Matriculation examination, a pass in the School Certificate examination (requiring a less expert knowledge of five subjects) together with a pass in the Higher School Certificate examination. Moreover, a sufficiently satisfactory performance in the Higher School Certificate examination, although it may not secure the certificate, will, if supplemented by a "pass 'with credit' in four subjects of the School Certificate examination, be accepted under certain conditions as equivalent to a pass with credit in the six subjects of the Matriculation examination.

$\ddagger$ Manchester, Liverpool, Leeds, Sheffield, and Birningham. 
of testing the qualifications of ex-service men who wish to enter upon degree courses.

These changes are, as we said, beginning to reduce the demand for the six-fold specialisation that, until recently, was a necessary condition of admission to degree courses in the newer universities of this country. But the entrance conditions of English universities need to be further modified in the same direction. Indeed, entrance tests for undergraduate courses need to be distinguished from tests of the efficiency of Secondary School teaching. The object of the former is to prevent the damage to the university and to the individual student that would result if he were admitted to an undergraduate course without either a sufficiently wide knowledge to enable him to take his part in the educative influence which undergraduates should exercise upon one another, or a sufficient special knowledge of the subject, or group of subjects, the further study of which he wishes to pursue in the university. A university entrance test should therefore consist of two principal parts, namely a test of general education* and a test of special knowledge.

While it must remain open to every university, and even to every Faculty or honours school, to decide what degree of special knowledge is required before students can be regarded as qualified to profit by the undergraduate courses they wish to pursue, there is much to be said in favour of all British universities, including those of the overseas Dominions as well as those of the United Kingdom, agreeing that whatever evidence of general education is satisfactory to one British university (whether in the United Kingdom or in the Dominions) shall be accepted by every other British university. The Canadian or Australian who, after qualifying to enter one of the universities of his Dominion, discovers that the particular studies he wishes to follow could better be pursued in some university of the Mother country, will then be qualified to enter upon his studies in the United Kingdoin so soon as he has brought his special knowledge up to the required standard. And he will not, as at present, too often happens, have to return to general school studies in order to pass a new test of general knowledge.

* Having regard to the distinction we have drawn between transitional and terminal types of study, and to our observations on p. 347 above, we suggest that the examiners concerned with the general part of a university entrance test had better not be persons who are learned (see above, p. 357) in the subjects in which they are examining. Professors of the natural sciences might well examine in English those matriculation candidates who are not intending to make a special study of English in the university, while professors of history or literature should be able to examine the non-scientific candidates in science. 
So far as students attending Secondary Schools in England are concerned, the minimum standard of general education required might well be marked by the School Certificate*. Or, alternatively, and with a view to avoiding such evils as are inherent in any system of external examinations, the university or universities of any province might well accept, as evidence of general education, a school leaving certificate awarded by the authorities of certain Secondary Schools recognised by the university or universities for this purpose. Such certificates might be awarded on the pupil's school record combined with his performance in an internal examination in which his schoolmasters would be assisted by an external assessor appointed by the university of the province. Any tendency of particular schools to abuse this privilege could be counteracted by striking those schools off the recognised list.

Special arrangements are required for testing the general knowledge of candidates, especially those of mature age, who are approaching the university otherwise than through the normal avenue of the Secondary School $\uparrow$. For any school-boy examination $\ddagger$ - the School Certificate examination or any other-is, by its very nature, unsuited to older men who, since leaving school, have spent several years in obtaining a wide experience of life. The alternative examination here in question should have much in common with the London University examination referred to above, or the Joint Matriculation Board's alternative examination for ex-service men. In other words, it would

* Compare Sir Joseph Thomson's Committee's Report: 'It is not to be expected that the universities will be content with a mere pass in the First School Examination, but we think that any university could obtain sufficient evidence for general education by demanding either a pass with credit in a certain number of subjects or the passing of the First School Examination together with some measure of success in the Second Examination.' (Loc. cit. p. 59.)

+ Among such candidates will be those who, as we shall see later, should be given facilities for entering undergraduate courses many years after leaving school, because, during those years, they have proved themselves to possess very exceptional qualities. Some of the most successful students of applied science and technology are already to be found among men who were enabled by scholarships (represented in our diagram by arrows on the twenty-years ordinate) to enter the university direct from industry. Increased facilities of this kind are needed.

‡ Compare Sir Joseph Thomson's Report, p. 6o: 'It happens not infrequently that in consequence of some change of plans candidates wish to enter a university long after they have left school, perhaps after a period of occupation in some kind of technical work. It seems unreasonable to expect such candidates to shew the same knowledge of the subjects of a secondary school course as may fairly be required of school boys. We therefore recommend that the universities should not require them to pass the ordinary matriculation examination, but should make special arrangements to test their fitness to enter on university work. We have ascertained that most of the universities would favour such a change. The number is not likely to be so large that a special test of each individual candidate would present serious difficulties.' 
be largely viva voce in character, and aim specifically at testing the width of the candidate's knowledge and his power of expressing it in English, rather than his familiarity with any particular branch of school study, whether it be history, mathematics, geography, or anything else. Care must be taken to ensure that the alternative examination shall be no easy option that will encourage boys, who have some hope of ultimately entering a university, to leave school before they have passed the School Certificate examination*. When such an alternative test of the general education of men of riper years has become operative, existing matriculation examinations, in so far as they are intended to test general knowledge, might well come to an end, as Sir Joseph Thomson's Committee have suggested $\dagger$; for the (external) School Certificate or the (internal) leaving certificate will suffice as evidence of the general education of boys who are entering the university direct, or almost direct, from Secondary Schools.

But, as we have said $\ddagger$, before entering upon an undergraduate (honours) course, a candidate, by whatever path he is approaching his university, should be required to have reached the standard of the Higher School Certificate examination in the subject, or group of subjects, which he is going to make his special study in the university. We are here in agreement with the Royal Commission on University Education in London, who go so far as to say that 'The university should draft its regulations for admission with a view to making the Higher School Certificate...the normal qualification for registration.' § Our contention that the test for special knowledge should depend upon the particular Faculty or honours school which the candidate desires to enter, is supported by another finding of the same Commission: it suggests that students who stay at school long enough before going on to the university might 'make some definite preparation for the Faculty they intend to enter.'

* We suggest, however, that the scope of the School Certificate examination should be extended so as to form a final test for senior, as well as for intermediate, secondary education; so that, by supplementing these extended School Certificates by passing at the Higher School Certificate level in the subjects which they wish to study in a university, men who are approaching the university by way of senior secondary education and part-time classes instead of by the normal avenue of the Higher Secondary School, would be able to qualify for admission to honours courses without first having to pass a matriculation examination in general subjects some years after they have left school.

† See Report, p. 59.

$\ddagger$ See above, p. 392 .

$\S$ Final Report, p. 40. This has now (1920) been done in the Faculty of Technology of Manchester University.

II Final Report, p. 40. A similar opinion was expressed in evidence given to the Board of Education's Consultative Committee and recorded in that Committee's Report on 'Examinations in Secondary Schools' by Sir William M'Cormick who 


\section{§ 5. Undergraduate Studies.}

These observations upon the conditions of admission to undergraduate courses were introduced by our remark* that the cuurses of study followed by most undergraduates in English universities should resemble the work now required for honours, rather than for pass, degrees. To this point we now return in order to justify our claim that the former rather than the latter should be the staple provision made by universities for their undergraduate students.

Honours courses in English universities to-day differ from pass courses in two principal respects. In the first place honours courses are, as a rule, of higher standard than pass courses. It is true that many undergraduates find it harder to obtain a pass degree at Cambridge or Oxford than to obtain a third class in some of their triposes or honours schools. But, taking English universities as a whole, it may be said that in order to obtain a degree in honours it is necessary to study a single subject, or group of subjects, for three years from the standard marked by the Higher School Certificate examination, while the pass degree can be secured after three years university work by a student who has matriculated, but has not reached the Higher School Certificate standard in any of his subjects before entering the university. The first difference, then, between honours and pass degrees is the far higher standard of knowledge of some subject, or group of subjects, required for the former than for the latter.

This difference in standard is, as a rule, much greater than the difference between the standard of knowledge required of the honours man and that required of the passman at the outset of their university courses; for, while the former concentrates his attention upon his special subject or group of subjects, the latter concerns himself equally with several separate branches of knowledge, and so fails to reach high attainment in any of them. Indeed, this lack of coherence of pass courses in English universities separates them from honours courses by a far wider gulf than the difference in standard to which we first drew attention. Professor Archer $†$ has recently emphasised

said that he did not want to 'impose the same examination on each profession and each Faculty.... While specialising to some extent the student should certainly continue to receive [in a Higher Secondary School] an all-round education.... Probably, however, it would not be necessary to demand the same high level of attainment in the general subjects as in the special ones.'

* See above, p. 392 .

$\uparrow$ See The Passman by R. L. Archer, Professor of Education in the University College, Bangor.

G. E. 
this latter distinction. After describing how, during the seventeenth and eighteenth centuries, the universities, not only of England but also of France and Germany, were out of touch with the most progressive thought of the nation and with its general aspirations, Mr Archer continues:

The decay affected the Universities of France and Germany as well as those of England; but in both those countries an earlier effort was made to counteract it. [In England] the Nineteenth Century Revival represents in many respects a return to the older traditions. It was largely effected by the institution of honours courses, which rejected the principle of balance and reverted to the principle of unity.... We have only to think what the influence of the Universities would be if it depended solely on their pass courses, and we shall see that these courses have been a comparative failure. It is the success of the honours courses which has led us to regard this failure with indifference. It has concealed their failure from the general public, which even now hardly realises the enormous differences covered by the letters M.A. But are we justified in attributing their failure to the principle of balance [between several separate subjects]? A crucial instance exists which appears to supply an answer; the pass B.Sc. courses of our modern Universities, where this principle has been abandoned, have succeeded*.

Mr Archer, after speaking of the success of certain foreign university courses in stimulating their students, proceeds:

From what does this stimulus arise? As in all the other cases where Universities have succeeded, it springs from inspiring unity of purpose... but beyond furnishing another illustration of this fundamental principle, the system does not help us... [In fact] little help will be obtained by looking abroad for models, since the distinction between honours and pass courses is peculiar to our islands. Nor will it be of great assistance to collate the various courses which exist at the individual Universities of the United Kingdom. Fundamentally they are all based on the same principle. London University set the pattern. Its aim was to widen the scope of studies, and thus it brought into even greater prominence than before the idea that a pass course should be composed of a variety of separate subjects, to each of which the student should be expected to devote an equal amount of attention. The newer Universities in the main followed the London lines, and the older Universities have almost unconsciously followed in the same direction. The differences between one pass course and another are not differences of principle. Those of the older Universities may be easier; the three final subjects may in one case be taken successively and in another concurrently; in one University each subject may be passed separately, whereas in another a pass is awarded on the joint results in all subjects; one University may examine at the end of each of the three years, another only at the end of the first and third. These are matters of detail; on more fundamental matters all are agreed. All assume (I) that a pass course for

* Loc. cit. pp. 9, 1о, II. 
the B.A. degree should include not less than three subjects; (2) that, while some subject or subjects may be compulsory, others may be chosen by the student from a given number of alternatives; (3) that the different subjects are intended to train the student in different ways, not to combine to produce a single effect; (4) that some at least of the subjects should therefore be quite unlike the rest in general character*.

Thus the honours courses of English universities differ from pass courses not only in being of higher standard but also in being far more coherent, and therefore better adapted to increasing the general ability $\dagger$ of the most able $\ddagger$ young men whom the country has been able to select to receive the highest type of education. For both these reasons, honours courses make better use of the resources of English universities§; but it is doubtless true that some of them would do well to aim at forming a wider single interest without sacrificing the essential singleness of aim.

It is therefore to honours courses, rather than to pass courses, that we shall principally look in the future for an essential part of the training of members of Class $\mathrm{A}$, for the men with creative minds, inventors of new appliances and processes, men who shall not merely be able to follow existing practice but also to cope with new problems and even to lead in new lines of advance. And our undergraduate courses, if they succeed in producing men of this type, will do so, not because of the knowledge they impart, wide though it be, but because of the stress they lay on the acquisition of skill in thinking $\|-$ a high degree of ' $g$ '-along with knowledge. It is general ability, skill in thinking, skill in applying old knowledge to new situations, rather than knowledge itself without such skill, that now as always marks the really practical man. If, in fact, his undergraduate course can, in Huxley's phrase, 'give him real, precise, thorough and practical knowledge of fundamentals,' the candidate for membership of Class A may well wait for the comparatively terminal courses provided by subsequent practical experience (whether in works, law chambers, hospitals or elsewhere), post-graduate evening classes, and private reading to develop further his technical and professional information to a marketable standard. Whatever letters he may be able to write after his name, his undergraduate course should have aimed at making him a Bachelor of Arts, skilled in the art of learning, and only

* Loc. cit. pp. I3, I4 (italics mine).

$\dagger$ See above, pp. 353, 36r.

$\ddagger$ We have seen on p. 327 how specially important is the development of ' $g$ ' in the case of future members of Class A.

$\S$ The deficiency of these resources, not in quality but in quantity, was referred to on p. $37 \mathrm{I}$ when we were discussing the vertical scale of our diagram.

|| See above, p. 327. 
incidentally have given him a body of scientific knowledge capable of immediate application. Indeed, English universities would do well to consider whether, as with medicine so with other branches of applied science*, they should not postpone giving technical degrees until undergraduate courses have been supplemented, and their scope widened, by subsequent professional experience combined with university part-time classes. Technical degrees, like degrees in medicine, might then be recognised as qualifications to practise a profession $\dagger$.

This view just expressed of the chief aim of an undergraduate course insists that there shall not be overmuch lecturing. Every student should keep his chief energies for his private study, since it is his own rather than his teacher's activity that will develop his skill in thinking. At this stage of his education he is, as we have said, his own chief educator $\ddagger$. He must look upon his university teacher as someone to be 'learnt from,' rather than as someone to be 'taught by.' And yet every undergraduate should attend lectures by the most distinguished professors who should take part in the teaching of undergraduates, not because of the knowledge they are peculiarly able to impart, but because, as the Royal Commission on University Education in London has remarked, 'it is the personal influence of the man doing original work in his subject which inspires belief in it, awakens enthusiasm, [and] gains disciples.' $§$

All undergraduates should also be afforded opportunities for studying subjects outside their own specialisms. In particular, students of technology and applied science should be encouraged to hear lectures on economics, applied physiology, and other aspects of the nascent science of industrial administration. Lectures by experts from industrial concerns and by business men should be provided in their interests. Visits to works, mills, and factories ought also to be arranged. Moreover, no student should be given his degree unless he can read his own special subject in at least one foreign language. But, despite all these facilities for, and encouragements to, studying subsidiary subjects so as to widen the students' single interests, it remains true that the chief aim of an undergraduate course should be to cultivate skill in thinking by concentrated study of deep lying principles in some particular branch of knowledge.

In urging that the chief aim of an undergraduate course must be to cultivate skill in thinking, or, as we formerly said, to strengthen

* Including not only physics, chemistry, metallurgy and other physical sciences but also economics, law, history. (See above, p. 197.)

$\dagger$ See below, p. 409.

$\S$ Loc. cit. p. 29. Cf. also above, p. 392.

$\ddagger$ Cf. p. 27 I above. 
Will or increase* ' $g$,' rather than to impart information, we do not mean that such a course must be at all detached from practical things $\dagger$. It is no more possible to develop skill in thinking without knowledge than to acquire skill in the use of tools without material to work upon. Technical knowledge is, in fact, a most excellent foundation and medium for cultivating skill in thinking $\ddagger$.

To the technical knowledge which the future member of Class A has acquired as an undergraduate, he should go on adding after taking his degree; and this is true, not only of the future engineer or research chemist, but also of the future theologian, or barrister, and indeed of the future members of all other learned professionsई.

While however the need of the country-a need that is no longer far in excess of the demand $\|$-for men who have received the kind of training provided by the honours schools of English universities, requires that honours, rather than pass, courses should be the staple which the universities of this country offer to their undergraduates, passmen should not be excluded from these universities. While therefore the majority of undergraduate courses should, as we have said, lead to honours degrees, and, according to our diagram, be open only to a small $\uparrow$ percentage of the ablest young people, some provision must be made for less able men and women whose future walk in life makes it nevertheless desirable that they should spend at least three years under the influence of university students, and especially of their fellow undergraduates. In particular, such provision is needed

* See above, pp. 137, 138 .

† It is more than half a century since J. S. Mill told St Andrews that: 'A university is not a place of professional education '; and, whatever may have been the case in 1867 , it is certain that professional education is one of the functions of a university to-day. Thus the Royal Commission on University Education in London write in their final report: 'Both the history of educational organisation and a right view of the methods of university work appear to us to justify the inclusion of professional and technological studies within the University.' (Loc. cit. p. 33.) But the popularity of Mill's view is shewn by the following newspaper comment upon Sir Alfred Ewing's appointment as Principal of Edinburgh University: 'Without any suggestions to the detriment of Sir James A. Ewing, I think his appointment as Principal of Edinburgh University most undesirable. Academic circles generally will be of the same opinion,... he is an excellent man at his job. But his job happens to be engineering, explosives, and all that sort of thing, and such knowledge had better be kept, particularly in war time, in the departments where it can be of practical use. In any university it is out of place.' (Daily Sketch, 24th May, r916.)

$\ddagger$ It will be observed how far we are from sharing the view quoted from a popular journal in the preceding footnote.

$\S$ Cf. p. 197 and footnote * on p. 400 above.

II I.e. the need is increasingly realised by large employers who are thus increasing the demand. Cf. Sir Joseph Thomson's Committee's Report, pp. 72, 73.

If But much larger than the percentage of young people who at present enter universities in England. See p. $37 \mathbf{I}$ above. 
for those who are to teach boys and girls between twelve and fourteen years of age, whether in the lower forms of Secondary Schools or in the highest standards of Elementary Schools, and for those who will take the first two years (fourteen to sixteen) of the work in the Part-time Secondary Schools that are now being established in accordance with the Education Act of r9r8. A large majority, if not all, of these teachers should have received a university training and have obtained a university degree. But they will not ordinarily be able to continue their university studies beyond the three years of an undergraduate course; nor will it be possible for most of them to postpone to a graduate part-time course the acquisition of practical professional experience, such as the university-trained engineer obtains in a works during his college apprenticeship, or such as the university-trained physician or surgeon obtains during the last two or three of the five or six years' course that begins when he enters* his university. For these men and women, therefore, there is needed a special type of undergraduate course, more terminal and less transitional in character than those of the honours schools. These non-honours courses should be the pass courses of the future, but they must differ from the pass courses of to-day by becoming more coherent in character. Indeed, as Professor Archer has urged $\dagger$, it is necessary to provide pass courses that shall form organic wholes, in place of the assemblages of heterogeneous studies that make up the pass courses of to-day. The standard of special knowledge required for admission to these pass courses may well be far below the Higher School Certificate standard which, we said $\ddagger$, should generally mark the beginning of an undergraduate course.

But, while coherent pass courses, such as we have indicated and such as are more fully described in Professor Archer's book, must be provided, honours rather than pass courses should, as we said§, be regarded as the staple teaching which English universities offer to their undergraduates.

\section{§6. Graduate Study and Research.}

After taking their degrees in honours, the abler men will do well to devote two years, either immediately following their graduation

* This entry should, in future, normally take place at the age of eighteen and at the Higher School Certificate standard in some branch (preferably biological) of natural science. Cf. Sir Joseph Thomson's Committee's Report, p. 45.

$\dagger$ Loc. cit. Chapter IV, especially p. 54 -

$\$$ See above, p. 390 .

$\S$ On pp. 392, 4 OI above. 
or after a period of practical professional experience*, to further whole-time study combined with training in methods of research. In their provision for organised graduate study and training in the methods of research, English universities have, for the most part, lagged behind those of America and central Europe. But they are now alive to the deficiency and, with the aid of increased government grants, are taking steps to make it good. The establishment by most English universities during the last two years of a new research degree, to mark the satisfactory completion of at least two years of graduate study and research, is evidence that the universities now fully realise the need for courses of this kind. It is to be hoped that these courses will receive graduates from other universities at home, as well as from British and foreign universities overseas. Interchange of students between different universities, with all its attendant advantages for the students and for the universities concerned, can best take place at the stage marked by graduation $\uparrow$. Moreover, while every university in England may well attempt to provide the various undergraduate courses required by the province which it principally serves, no university can attempt to provide every necessary course of graduate study and research $\ddagger$. Transfer from one university to another on graduation should therefore be a feature of a national or international system of education. For example, Cambridge or Oxford graduates in engineering will often do well to spend two years of graduate study and research in a university that is geographically nearer to some centre of the engineering trade than are the older universities, and under the direction of professors and lecturers who are in intimate and continuous touch with neighbouring engineering works. Or again, any able young graduate will do well to devote two years to graduate study and research in Sheffield University before entering whole-time employment in a Sheffield steel works. Once more, the British undergraduate who develops exceptional capacity for mathematics will do well to proceed from his own university to

* The diagram shews a single-headed arrow on the twenty-four years ordinate, to indicate that a few exceptional persons should be offered scholarships, with whatever maintenance allowance is necessary, in order to enable them to return from whole-time employment to graduate study and research in a university.

$\dagger$ We notice in this connexion the single-headed arrow on the twenty-one years ordinate at the upper edge of the diagram. If we use the diagram to represent a national system of education, this arrow-head represents an influx of students from overseas; or, if we use the arrow-head to represent the system of education belonging to a particular English province, the arrow represents a transfer of graduate students from other universities, whether at home or abroad, to the university (or universities) of the particular province in question.

$\ddagger$ Cf. footnote $*$ on p. $3^{86}$ above. 
Cambridge, there-perhaps after taking the second part of the mathematical tripos-to devote some time to research work in the principal British centre of mathematical learning.

Courses of graduate study and training in methods of research are represented in our diagram as part of a system of education. But it is not on this account to be supposed that the training of students in methods of research is the only object of research work in universities. For, in the first place, universities have for many centuries been the principal homes of research, both in this country and throughout Western Europe. As Sir Joseph Thomson's Committee report 'Great discoveries...have for the most part been made in the laboratories of the universities and, we think, will continue to be so.'* It is true that research upon certain industrial problems cannot be effectively completed outside the mills, factories, or workshops in which the problems have arisen; unless indeed research laboratories be provided in which industrial processes can be carried out, either on the scale of twelve inches to the foot, or with a sufficiently close approximation to actual works conditions. Many private industrial concerns are therefore establishing their own research departments. Meanwhile, the government Department of Scientific and Industrial Research is encouraging firms engaged in certain industries to form industrial research associations for each of the industries in question. Several of these new research associations contemplate the establishment of research institutes, in which industrial research on a works scale will be possible. It is, however, important that these new research institutes should, as far as possible, have their roots firmly planted in universities which have hitherto carried out researches of the kind in which the new associations are interested. Moreover, the industrial research institutes of the future must look to the universities, not only for the training of their researchers, but also for the investigation of many borderland problems that lie on the boundaries between different sciences, and that cannot be dealt with effectively in the research institute, either because it has not been equipped for the purpose, or, much more frequently, because its staff is qualified to deal with a narrower field of knowledge than that which universities make their own. Experience, both in this country and, especially, in America $\dagger$, has shewn how productive of valuable results may be the

* Loc. cit. p. 66. See also the quotation from p. 29 of the Final Report of the Royal Commission on University Education in London on p. 400 above.

$\dagger$ See for example Mr T. L. Humberstone's paper on the Mellon Institute in the University of Pittsburg, published by the Board of Education's Department of Special Enquiries and Reports. 
cooperation between universities and manufacturing firms in industrial research. In the laboratories of English universities, many investigators are already engaged upon industrial research, more or less according to the scheme associated with the name of the Mellon Institute*, Pittsburg. And sometimes investigations are carried on partly in university laboratories and partly in neighbouring works: an arrangement which perhaps affords the best means of training an investigator for industrial research, if only the professor responsible for the investigation, has free entry to the works, and a position there of sufficient authority to enable him to direct that part which is carried out in the works, just as he directs the remaining part in his own university laboratories $\uparrow$. And sometimes, too, a course of this kind, although it has been primarily designed as a training in methods of research, leads to valuable results that are capable of immediate industrial application.

In fact, universities should assist these new research associations by training researchers; by forming the soil on which a nascent institute may begin to grow (its first researches being carried out, either wholly in the university laboratories, or partly there and partly in neighbouring works, mills, or factories); and, when the growing research institute has become big enough to extend into separate buildings of its own, by continuing to cooperate with the institute's researchers, especially where they are concerned with the borderland of the field with which the research institute is principally concerned.

So the first reason why the training of students in methods of research is not the only object of research work in universities, is because one of the chief functions of a university will always be to extend and organise knowledge, or, in other words, to undertake research. And the second reason, to which we have already $\ddagger$ alluded, is that the university professor or lecturer who is doing original work in his subject is best able to inspire his students diligently to study it and to make the necessary effort for its mastery. A third reason appears when we reflect that, if an attempt were made to train the

* 'Over there the manufacturer who requires research into some industrial problem pays the main cost of the investigation, either at the special laboratory belonging to the university or sometimes, on a large scale, at his own works. If a valuable discovery is made a Board of Arbitration determines the fee or donation payable to the researcher. This question of reward, it must be remembered, constitutes one of the difficult problems of research here as elsewhere.' The Marquis of Crewe in Ways and Means, Vol. I, p 44I, 2 Ist June, I9I9.

$\dagger$ Arrangements of this kind have been made between the College of Technology and certain engineering works in Manchester.

$\mp$ See above, p. 400 . 
future leaders of industry, as well as of other departments of public service, in an institution where research was neglected, and to train research workers in separate institutions elsewhere, a valuable opportunity would be missed of making our future leaders in politics and government, as well as in industry and commerce, realise the importance of encouraging research, and of appreciating and applying its results*.

\section{§ 7. University Part-time Studies.}

We have said $\uparrow$ that the future member of Class A should go on adding to his technical knowledge after he has taken his degree. We

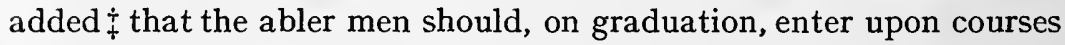
of advanced study and training in methods of research. The less able graduates should, however, pass directly into employment; but their continued education should not on this account be neglected. Already we have observed that part-time studies should, at every stage of education, preserve continuity $\S$ between whole-time education on the one hand, and whole-time employment on the other. Accordingly, the future member of Class $A$, who has entered employment on graduation, should continue to add to his knowledge and to widen his interest, not only in the practice of his daily employment, but also by attending post-graduate evening classes $\|$ from time to time. So will he add what we have called 'terminal' studies to the comparatively ' transitional' type of education which his honours undergraduate course has provided. So, too, will he keep up to date his knowledge of new advances outside his own particular line. And, if he is able to attend such classes taken by his former professor, his attendance will benefit his university as well as himself. At all events, the professors and lecturers who take university part-time classes in technological subjects, find that evening students who are engaged all day in industrial practice are hardly less effective than their own consulting

* Compare the following quotation from Sir Joseph Thomson's Committee's Report: 'But it is useless to speak of particular reforms unless the need for reform is recognised. That scientific research and the scientific study and direction of industrial processes are necessary for the development of our industries and even for their maintenance in the face of foreign competition, is a proposition which in educated circles will not in these days be denied. But as one of our correspondents writes: "Scientific research on industrial problems is of no use whatever to an uneducated trade. Such a trade can neither state its needs with definiteness or accuracy, nor can it interpret into practice and utilise the results of research. Indeed, it does not feel the need for research and cannot therefore make a demand for it....In some trades it will be necessary to wait for the full development of research schemes until we have a generation of leaders qualified to demand and make use of industrial research".' (Loc. cit. p. 75.)

$\dagger$ See above, p. 399.

$\S$ See above, pp. 360 and 372.

$\ddagger$ On p. 402 above.

If See above, pp. 399, 400 . 
work* in keeping them in touch with recent industrial developments. Indeed, a university situated in a great centre of population-and especially a university whose whole-time courses, graduate or undergraduate, in applied science and technology are designed to prepare future members of Class $A$ for positions of responsibility in the industries of its neighbourhood-cannot hope to retain the sympathy and good will of the community to which it must look for material as well as moral support, if it neglects to provide terminal part-time courses for graduates and other men and women whose days of regular study are past, but who wish to keep abreast of the demands made upon them by their professions. The provision of part-time courses is, in fact, an essential function of a university in a great centre of population $\dagger$.

* The following extract from a reply by the present writer to a question (No. I5 $(b)$ in the questionnaire) circulated by Sir Joseph Thomson's Committee in 1917 , is relevant in this connexion:

'It will be well known to members of the Committee that the practice of most Universities in Great Britain and abroad is to allow professors and heads of departments-and sometimes their lecturers also-complete freedom to undertake private consulting work. In my view, this practice is preferable to any other, provided that every professor and lecturer furnishes the Vice-Chancellor, Principal, or other official representative of the Governing Body, with full particulars of all the private work that he undertakes and of the fees which he receives in respect of it. Obligation to furnish such reports will tend to check any tendency to repetition of routine work, which supplements academic incomes but adds little to the experience of the investigator or the reputation of the University. The Vice-Chancellor or Principal will recognise the confidential conditions under which private consulting work is undertaken, and the submission of his colleagues' reports to him, instead of to a committee of the Governing Body, will prevent industrial concerns who wish to obtain the confidential opinions of university professors from fearing that their private business may come to the knowledge of competitors who might possibly serve on such a committee. On the other hand, the Vice-Chancellor or Principal should be free to consult the Governing Body whenever, in his opinion, it is necessary to do so. With such safeguards against abuse, the value to a University of its professors obtaining a varied consulting practice is, in my opinion, so great as to render it undesirable that the University should ask professors to pay over [to it] their consulting fees in whole or in part, even in cases where their work involves the University in some slight cost for materials and apparatus.

- It has been argued, on the contrary, that professors or lecturers should transfer to the Governing Body the whole of the proceeds of their private practice. In my view, such a policy must tend to discourage professors of technological subjects from developing a private practice, without which all their university work will suffer.'

+ Compare the Final Report of the Royal Commission on University Education in London: "Although it may be true that the first and most urgent call upon the University is that made by its regular students, it could not hope to retain the sympathy and support of the community to which it must look for material as well as moral assistance, if it refused help and guidance to men and women who, though the days of regular study were passed, wished to keep abreast with the demands made upon them by their professions. A university in a great centre of population must be prepared to provide advanced instruction of a specialised kind for all classes of the community who are willing to receive it. A great deal of this work must be done in the evening.' (Loc. cit. p. 33.) 
Moreover, these university part-time classes should include within their scope, not only advanced and specialist studies bearing upon the professional work of graduates and other persons of similar education, but also extension lectures and tutorial classes-the latter perhaps under the auspices of the Workers' Educational Association-designed for members of Class B or C or D who, although they have passed the ordinary educational age, desire to modernise their ideas or enlarge their outlook.

But we must not suppose that the continued education of university graduates who are just entering employment should be confined to university part-time classes. On the contrary, to assist with the further education of young graduates, who have just entered their employ, should be recognised as part of the duty which every business concern or government office owes to the community; just as it has long been recognised to be one of the most important duties of a physician on the staff of a great Metropolitan hospital to assist in the professional training of students who, in many cases after completing a three-year undergraduate course in one of the older universities, are attached to the hospital medical school. The young barrister, also commonly a graduate, receives a similar professional training from the older member of the profession for whom he 'devils'; and the young curate from the vicar or rector under whom he works. Similarly, it is becoming more frequent for great engineering manufacturing concerns to include among their staff an 'Apprentice Master,' part of whose business it is to supervise and direct the practical training of men who, after graduating in engineering, have entered the works for a two or three years' college apprenticeship*, which, combined with university part-time classes, will form the terminal courses of their professional training. We have already $\dagger$ indicated

* Not long ago it was usual for manufacturing engineering concerns to demand a premium from the college apprentices as payment for the practical training here in question, and in certain parts of England engineering firms still ask for these premiums. In other districts, however, the practice of requiring premiums has been discontinued for some years, and of late, following the American practice, leading engineering firms have been willing to pay to college apprentices wages sufficient to maintain them during their college apprenticeship. In this way the firms in question have secured the pick of college graduates, and have thus been placed in a favourable position for the selection of permanent members of their designing, managerial, and commercial staffs. It is to be hoped that this practice will extend in England, and in this expectation we have excluded from our scholarship proposals in Chapter 25 those industrial bursaries by means of which the Commissioners for the $185 \mathrm{I}$ Exhibition have enabled college graduates with no private financial resources to enter upon college apprenticeships in engineering, or other industries, in works where no wages were paid to college apprentices.

$\dagger$ See above, p. 350. 
that, in the interests of continuity of education, it is hardly less important that the Apprentice Master and his assistants should be in close touch with the universities from which the college apprentices come, than that the professors of these universities should be in intimate contact with the works into which their students go. It remains to add that the systematic training of young graduates, entering employment for the first time, should be entrusted to responsible officers like these Apprentice Masters, not only in engineering works, but, as we have said, in every department of public and private business. When arrangenients of this kind for the practical professional training of young graduates are in more general operation, it will be easier than it is now to give effect to the suggestion, made above*, that technical degrees should be instituted or reserved for men who, after satisfactorily completing an undergraduate honours course, have also satisfactorily completed an approved course of practical training, combined with part-time university study.

\section{$\S$ 8. Senior Technical Education.}

The education of future members of Class A has been considered at some length because of the special importance of the service which members of this class may render to the community. Paradoxical though it may appear at first sight, there is yet good reason to believe that every individual member of Class $\mathrm{B}$, or Class $\mathrm{C}$, or Class $\mathrm{D}$, will be more effectively helped towards the fulfilment of the main purpose of his life, by the perfection or the improvement of the education of the leaders of the community to which he belongs, than by the improvement or perfection of the education which he himself receives. If, for example, national leaders plunge the world into war, the lifelong purposes of everybody else are likely to be frustrated. Or, to take a less glaring instance, if the leaders of an industrial concern are incompetent, the work, however perfect, of the draughtsmen, undermanagers, craftsmen, or repetition workers-in fact, of all that firm's workers, whether in Class B, C, or D-may be rendered futile. Good craftsmanship is wasted on an article of bad design, while good craftsmanship, cheapness, and every other attribute that belongs to effectively organised production, is wasted if the products meet no human need.

But, when once future members of Class A are being effectively selected $\dagger$ and educated for their responsible work, the effective selection

* On p. 4 oo.

$\dagger$ So (it will be remembered) that equal opportunity is afforded to all, quite irrespective of their parents' economic or social class. 
and education of the future members of our other classes become of very great importance. Relatively to that of members of Class $A$, the education of persons who are to occupy less responsible positions (and especially, as we shall see, the education of the future members of Class $C$ and Class $D$ ) has received far too little attention in the past. Indeed, many authorities seem to have neglected it altogether; or, rather, to have been content with a system of education described by the metaphor of a ladder, or of a single broad highway, along which all men would proceed together, but for very different distances, so that only the few would reach the goal, while the vast majority-instead of following to its end an educational path specially designed to lead to their own field of activities: social, be it remembered, as well as vocational-would fall by the wayside (or, in the case of the ladder metaphor, fall off with more or less of a crash), leaving incomplete* a course of study planned for the others who had gone further.

If, then, in the following paragraphs we discuss the education of the future members of Classes B, C and D in less detail than we have employed for describing the education of future members of Class A, it is not because of any underestimate of the immense importance of rightly educating the great majority of citizens, but because the results so to be obtained may be destroyed by defective education of the leaders of the people.

It is the general experience of university teachers, and especially of those who are concerned with the more abstract studies undertaken for honours degrees, that only a small proportion of men are fitted by nature and nurture (including their previous education) for studies of this kind. Principally for this reason, but also because the community could not afford to provide a university training for all its members, the majority of the future members of Class $B$ will enter employment before they are twenty years old, and without first having received a university training. We have, in our diagram, taken the age of eighteen as the modal age at which members of this class will discontinue whole-time education, and pass into employment combined with part-time study. This has long been the age at which most of the best educated boys and girls who are not going on to the university first enter employment, whether in private businesses or public departments. And, in recent years, a number of industrial concerns, who lay themselves out to continue the education of young

* We are reminded of the following words quoted above $($ pp. 354,355$)$ from the Ministry of Reconstruction's Pamphlet: 'A cottage completed and ready for occupation may be of more value to the community than the foundations of a palace which have never been carried above the damp-proof course.' 
people as, at different ages and with different qualifications, they enter the firms' employment, recognise eighteen as the normal or modal age at which apprenticeship begins for the class of apprentices or learners coming next below those who have completed university or college courses.

According to our diagram, boys and girls who are to become the members of Class B should devote the last two years of whole-time education to a course of study that is more terminal in character than the transitional (advanced secondary) course followed by young people of the same age who are preparing to enter university honours schools. Senior technical education is the name by which we have described courses of the terminal type here in question. We have already* observed that the future member of Class A should be offered an increasing variety of courses of study as his education proceeds from junior secondary, through intermediate secondary and advanced secondary, to undergraduate studies. So, too, the variety of senior technical courses must greatly exceed that of intermediate secondary courses. It must also exceed that of advanced secondary courses. Indeed, different varieties of senior technical courses must prepare for different industries, for several branches of commerce, for domestic occupations, for fine art, for music, as well as for other kinds of occupation. That does not mean that the courses in question must be narrowly vocational in character. Indeed, as we saw $\dagger$, terminal courses should include a wider range of subjects than are included in transitional courses for pupils of the same age and previous education. But the English studies, and the other subsidiary (or, as Professor Campagnac calls them, 'tributary') subjects that form part of these senior technical courses, must centre around, and be tributary to, the central interest which the student will have in the occupation that he is about to enter $\ddagger$.

It is to be carefully noted that the proximate aim of senior technical education is not to prepare for further whole-time study, but for employment combined with part-time study. The importance of not losing sight of this difference in aim is well brought out in a report by Dr C. A. Prosser on the Boston Mechanic Arts High School§.

* See above, p. 386 , footnote *.

$\dagger$ See above, p. 359 .

$\ddagger$ Cf. above, pp. 353,360 .

$\S$ 'A Study of the Boston Mechanic Arts High School, being a Report to the Boston School Committee by Dr C. A. Prosser' published by Teachers' College, Columbia University, New York City, 1915. Dr Prosser finds that the Boston Mechanic Arts High School, that was intended to provide what we have called senior technical education for boys between the ages of sixteen and eighteen, was instead endeavouring to prepare boys to enter the Massachusetts' Institute of 
At the same time, as indicated by the ringed single arrow-head on the eighteen-years ordinate of our diagram, a very few of the ablest young men completing their senior technical education should be enabled, by scholarships and maintenance allowances, to proceed to undergraduate studies in universities*.

When, in the next chapter, we discuss the functions of schools, colleges, and other educational institutions, as distinguished from the aims of the various types of education which each institution may provide, we shall observe that the greater part of the provision of senior technical education should be made by Polytechnics, local colleges or, as they are most frequently called, 'Senior Technical Schools,' rather than by the Higher Secondary Schools that provide advanced secondary education for pupils of the same age. One reason is that, if all the (one in ten) boys between the ages of sixteen and eighteen who, according to our diagram, should be receiving senior technical education were to receive it in the same schools that provide advanced secondary education for only 4 per cent. of the boys of the same age, the outlook of the Higher Secondary Schools towards the universities would be spoiled, and the aims and ideals of advanced secondary education would be in danger of being obscured by the equally worthy, but quite different, proximate aim of senior technical education.

Moreover, senior technical education commonly requires more specialised, and often more costly, equipment, for which the funds of Higher Secondary Schools are altogether inadequate; whereas, if the equipment in question is provided in Senior Technical Schools, it can

Technology or the engineering departments of other institutions of university rank. Dr Prosser writes: 'The School fails to meet the needs of the 85 per cent. of its pupils who do not go to the engineering college, because it serves primarily the interests of the 15 per cent. of its pupils who do.' Or, again: 'The Committee has said it is not the function of the school to prepare for the engineering college.... [But] instead of aiming to prepare boys for advantageous entrance into industry on completing the high school course, there is every evidence that the controlling aim of the school is...to fit them to enter the engineering college....The course of study is not the right kind to give the training desired by the School Committee. It is too abstract and too far removed from the practical experiences the pupil will meet when he goes into industry. The academic part of the course is essentially the same as that of any general high school.' (Loc. cit. pp. 8, 9.) In fact, the course fails as a senior technical course because it possesses too much of the character of an advanced secondary course.

* Thus the Consultative Committee of the Board of Education in their 'Interim Report on Scholarships for Higher Education' [Cd. 8291] write:

'Though it is improbable that in the immediate future there will be a larger supply of well-qualified candidates from Higher Secondary Schools, useful recruits for the Universities may be found among the day-students of Senior Technical Schools, and among those who have obtained instruction by evening study and part-time study in works.' (Loc. cit. p. 64.) 
also be used for the education of part-time students, whose admission to Secondary School buildings is undesirable for reasons that will be considered in the next chapter. Accordingly, our diagram shews that four-fifths of the provision of senior technical education is made by Senior Technical Schools while only one-fifth of it is made by Higher Secondary Schools. It follows that the intermediate secondary education which should prepare for senior technical courses should, for the most part, be provided by ordinary or Lower Secondary Schools, rather than by Higher Secondary Schools which would otherwise be attended by the majority of their fourteen-to-sixteen years old pupils for two years only, to the very great disadvantage of the Higher Secondary Schools and of all their pupils. But those boys or girls who receive their senior technical education in Higher Secondary Schools-as for example in the secretarial or domestic science form of a girls' high school*, or in the engineering form of a 'public school' $†$ should, as indicated in our diagram, have received their intermediate secondary education in the same Higher Secondary School.

The very important place which senior technical education has to fill in a national or provincial system of education is as yet hardly realised. The ro per cent. (of sixteen-to-eighteen years old boys), which our diagram represents as receiving education of this type, will number no less than 10,000 in a province inhabited by $5,000,000$ people. Of this ro,ooo, our diagram shews that there should be 8,000 attending whole-time two-year courses in the Senior Technical Schools of the province. And yet, even in the Manchester province, the present number is barely 5 per cent. of that which our diagram proposes for ten years hence!

\section{§ 9. Advanced and Miscellaneous Part-time Studies.}

Just as the future member of Class A, who enters employment after completing his undergraduate course at the modal age of twenty-one (or, if he proceeds to graduate study and research, at, say, twenty-three or later), should combine university part-time studies with the further education which his employment should give him, rather by design than at haphazard, so the boy who enters employment on completing

* According to our diagram, the completion of intermediate secondary education, marked by the School Certificate, should be a condition of entry to senior technical as well as to advanced secondary education. But the region to the right of our diagram, as has been explained, is concerned with the education of boys and young men rather than of girls and young women. The question whether girls attending Higher Secondary Schools should be required to obtain a School Certificate before being admitted to secretarial, domestic, or other senior technical forms we leave open; but we suggest an affirmative answer.

$\dagger$ E.g. at Marlborough; or the engineering forms of Oundle.

G. E. 
his senior technical education at the age of eighteen, should combine attendance at part-time classes with directed* experience in the practice of his occupation. For the majority, these part-time studies should take the form of organised advanced part-time courses extending over some three years, and, while retaining the terminal and descriptive character that belongs to part-time courses in general, carrying a student's knowledge of his specialism up to the standard nowadays marked by an ordinary degree. The provision of part-time classes of this kind, meeting either in the evening or (preferably) on two half-days a week, is in our view part of the function of every university that is situated in a great centre of population $\dagger$. Our diagram therefore shews the university as providing those advanced part-time courses which are specially designed to meet the needs of persons who have received whole-time education up to the age of eighteen. Other advanced part-time courses, for future members of Class B who are approaching their field of service otherwise than by the normal path of senior technical education, will need to be provided by Senior Technical Schools. Moreover, there will be a certain proportion of future members of Class $B$ for whose part-time education, on first entering employment, some alternative to advanced part-time courses will need to be provided. Such provision is described as miscellaneous part-time classes in our diagram.

In conformity with a principle already $\ddagger$ laid down, the variety of senior technical courses required by future members of Class B will be exceeded by the variety of part-time courses upon which most of these young people will next enter.

Our diagram shews twenty-one as the modal age at which organised courses of part-time study are discontinued by future members of Class B. It is not on this account to be supposed that the education of the average member, or even of any member, of this class finally ceases at this early age. Not only will he remain subject to the educational influences of the practice of his employment, but by private reading, attendance at occasional university lectures $\S$ or

* See above, p. 408, where the importance of directing the practical experience of young graduates, and of employing an Apprentice Master or other official mainly or partly for this purpose, is urged. Here we emphasise the importance of similarly directing the practical experience which the future member of Class B, or indeed of any other class, obtains on first entering employment.

$\dagger$ Cf. the passage quoted on p. 407 (footnote $\dagger$ ) above from the Final Report of the Royal Commission on University Education in London (p. 33).

$\$$ See above; pp. $3^{86}$ and $4 \mathrm{II}$.

$\S$ For example, a number of works managers in the Manchester district have, for the last two academic years (I9I8-20), been attending late afternoon lectures delivered in the College of Technology on various aspects of industrial administration. 
courses of lectures, as well as in other ways, he will have opportunities of modernising his ideas and enlarging his outlook long after he has passed the ordinary educational age*.

\section{§ ro. Senior Secondary Education.}

According to the system of education represented in our diagram, one-half $\dagger$ of the young people of this country are, within ten years, to receive whole-time secondary education until the age of sixteen. If this proportion is considered in connexion with such statistical enquiries as that which $\mathrm{Mr}$ Rowntree carried out at the beginning of the century in York + , and from which it appears that the proportion of men, who are engaged in the kind of occupations that in our classification $\S$ belong to Class C, is approximately\| that shewn on the right of our diagram, and, if we assume that the ratio, which the number of repetition workers in Class $D$ will, ten years hence, bear to the number of craftsmen and others of Class C, will not greatly differ from the ratio that obtained in York at the time of Mr Rowntree's enquiry, it will follow that the majority of future members of Class $\mathrm{C}$

* Cf. p. 372, especially footnote + , above.

$\dagger$ See above, pp. 37 I, 372 and footnote $*$ on p. 369 . Our diagram represents 72 per cent. of the young people who continue in whole-time attendance at school up to the age of sixteen (or $3^{6}$ per cent. of the whole population of the same age) passing into employment at that age, while only 28 per cent. (or I 4 per cent. of the whole population of the age in question) continue to receive whole-time education for two more years at least. At present the majority of pupils in Secondary and Junior Technical Schools discontinue their attendance before attaining the age of sixteen. But the Education Act of r9r 8 encourages continued attendance up to the age of at least sixteen so as to obtain exemption from obligation to attend part-time day classes during the following two years. On this account, and because of the general awakening throughout the country to the advantages of remaining at school at least until sixteen years of age, illustrated by the fact that the Manchester Local Education Authority is treating the provision of whole-time education for 50 per cent. of the population up to the age of sixteen as a practical proposition, it is not unreasonable to suggest, as in our diagram, that sixteen rather than an earlier age will, ten years hence, mark the earliest period at which secondary education is discontinued by large numbers of pupils. But for reasons, including economic reasons similar to those which we have already given for not proposing that everyone should receive a university education, we are not proposing that within the next ten years more than I 4 per cent. of the young people between sixteen and eighteen years of age should be receiving whole-time education during that period. That is why our diagram shews 36 per cent. of the population, or 72 per cent. of the young people who receive whole-time secondary education up to the age of sixteen, as passing into employment at that age.

‡ See Poverty by B. Seebohm Rowntree.

$\S$ See above, p. 326 .

II We are, for the purpose of the approximation that we require here, identifying with the members of Class $C$ the heads of those families in York whose incomes exceeded thirty shillings a week in the year I900, but who did not keep domestic servants. This was Mr Rowntree's Class D who formed 32.4 per cent. of the whole population. At the time of his enquiry the unskilled labourer's wage in York varied between eighteen and twenty-one shillings a week. 
will not enter employment before the modal age of sixteen. In other words, the normal educational path that leads to Class $C$ will begin with elementary education up to the age of eleven plus (or, as our diagram shews, twelve), will then follow a transitional course of junior secondary education up to fourteen, and then a terminal course of senior secondary education up to the age of sixteen. Whole-time education, ending at sixteen, will in turn be followed by part-time education, supplementing practical training in the actual practice of a trade or other employment.

Of elementary, and junior secondary, education we have already spoken. It is at the conclusion of junior secondary courses that the normal educational paths followed by future members of Classes A and $\mathrm{B}$ diverge from that followed by future members of Class $\mathrm{C}$. While the former proceed to intermediate secondary education between the ages of fourteen and sixteen, the latter enter senior secondary courses covering the same age range. The difference between senior and intermediate secondary education is that between terminal and transitional courses. It has already* been considered at some length. Suffice it here to repeat that, while the transitional course is concerned with making the intermediate zone of the pupil's growing single wide interest-system correspond as closely as possible with a number of the most central, most valuable, and most closely inter-connected essences of the endarchy of science, the terminal course makes the innermost group of elements in the pupil's intermediate zone correspond, not so much to a group of innermost essences of the endarchy of science, as to a group of essences that will form a natural centre for the interest which the student will increasingly take in the trade processes or craft which will form his principal service to his fellow-citizens. In other words, the terminal course short-circuits $\dagger$ connexions in the endarchy of science in order that, subject always to his supreme central purpose, the pupil's interest may be centred around his particular service to the community, rather than around some region of the front line of the (incomplete) endarchy of science as it gradually extends inwards towards the attainment of the goal of scientific thought.

We have already insisted upon the practical importance of distinguishing between the aims of transitional and terminal courses for persons of the same age and previous education. This distinction is likely to meet with more opposition, when applied to differentiate

* See above, Chapter $2 \mathrm{I}, \S 8$.

$\dagger$ See above, pp. 230, 237 and 35I. 
between intermediate and senior secondary courses, than when used to mark the distinction between advanced secondary and senior technical education at a later stage. And yet it is hardly less necessary, in thought and practice, to separa te intermediate from senior secondary education in the last two years (fourteen to sixteen) of an ordinary (or Lower) Secondary School, than to separate advanced secondary from senior technical courses in the last two years of a Higher Secondary School. In particular, Dr Prosser's arguments* for distinguishing between advanced secondary education and senior technical education in Boston are equally applicable to the case of intermediate and senior secondary education in this country. Sir Joseph Thomson's Committee were clearly of that opinion. Speaking of the work of Junior Technical Schools which, in their view, should be regarded (as in our diagram) as belonging to secondary education $\dagger$, the Committee quote, apparently with approval, the view of the Board of Education, that 'pupils should not be diverted to Junior Technical Schools [or, as we should rather say, not receive senior secondary education] if they are intended ultimately to pass on to more advanced full-time technical courses' because 'it is not possible to provide a curriculum that is suitable both for the boy who is passing directly to industry [at sixteen] and for the boy who is to continue his education' $\ddagger$ beyond that age. Moreover, as we have already $\S$ seen from evidence given by $\mathrm{Mr}$ Bruce to another committee, the curricula of ordinary (or Lower) Secondary Schools are, in fact, being altered so as to approximate more closely to the terminal type, rather than to the transitional type to which belong the older classical sides of Higher Secondary Schools.

In conformity with our principle that the more nearly the end of whole-time or part-time education approaches, the greater should be the variety of available alternative courses - a principle which we applied when we said that a much larger number of senior technical

* See above, p. 4 II , especially footnote $\S$.

$\dagger$ Thus the Committee write: 'We think, as we have already said, that there is room in our scheme of education for those between the age of twelve and sixteen for schools where no foreign language should be compulsory and where a definite bias should be given towards practical education in connection with Science and Mathematics, but we see no reason why these schools should not be regarded as part of our secondary system even when their practical work is organised with a view to the predominant industry of a neighbourhood.

'We recommend that Junior Technical Schools should be strengthened and developed into such a form of secondary school, retaining some of their distinctive features, e.g. that a reasonable proportion of the staff must have had practical trade experience of occupations for which the school prepared.' (Report, loc cit. p. 43.)

$$
\text { † Report, p. } 43 \text {. }
$$$$
\text { § Quoted above on p. } 359 .
$$ 
courses were needed than the five varieties of advanced secondary course which we have previously outlined *-there is need for a much larger variety of senior, than of intermediate, secondary courses. Indeed, in so far as geographical considerations permit, every province should provide senior secondary courses preparing for each of a large number of different groups of trades or crafts practised in the neighbourhood. But, we repeat, the differences between the several varieties of the same type of education-even where these differences are most marked (as in the case of terminal courses that belong to senior secondary education)-do not imply that the whole subject matter of the courses should be different. Many so-called general subjects will form part of all or of many of the different courses, although these subjects may be differently taught in each. The writing of English $\uparrow$, for example, is to receive attention throughout every course represented in our diagram, from the Elementary School child's essay to the graduate's thesis for a research degree. In the same way, physical exercises $\ddagger$, of one kind or another, ought to form part of the education provided by every school and college and might well be compulsory for those who do not voluntarily, and to a sufficient extent, take part in organised games.

It is unnecessary, for the purpose of the present enquiry, to attempt a description of all the many varieties of senior secondary courses that need to be provided by the Secondary and Junior Technical Schools of every English province. Suffice it here to say that, while senior secondary courses should, subject always to the supreme central purpose, have as their central subject the craft or trade or other employment which the pupil shortly hopes to enter§, it is no part of their business to attempt to teach a trade. Their object is rather to prepare a boy for apprenticeship (in its widest sense, and by no means limited to industrial crafts) by teaching every subject in close relation to the interest which the boy feels in his future calling; just as at Osborne and Dartmouth the boys' interest in the Navy is used to awaken and keep alive their interest in every lesson, whatever its subject may nominally be. Experience has shewn\| that boys will thus make more progress in these general or 'tributary' subjects than if they were studying them in a school which had no specific aim.

We may conclude our account of senior secondary education by

* See above, pp. 386, 387, 388, 4II and $4 \mathrm{r} 4$.

+ Cf. pp. 347,348 above.

$\ddagger$ See above, p. $3^{86}$.

$\S$ Cf. p. 353 above.

\| Cf. the reference to the Bootle Junior Technical School on the following page. 
the following extract from a paper* on 'Junior Technical Schools' prepared by the present writer in July, I907:

A brief review of the common characteristics of these schools may help to elucidate the cardinal elements which should be reproduced in each new Junior Technical School.

In the first place, the schools are not intended to teach a trade....It is commonly accepted that the right place to learn a trade is in the workshop, but that the workshop training can be both facilitated and increased by the right kind of previous education. Thus, taking Professor Hopkinson's illustration, students may properly make experiments on the flow of water in pipes, and so get a knowledge of the fundamental physical laws governing such flow; but a school is not the place in which to teach students how much fall experience has shewn to be desirable in a drain or sewer. In the Junior Technical Schools, therefore, attention is given rather to Mathematical Drawing and Practical Plane and Solid Geometry than to the memorising of conventional representations used by an engineer's draughtsman. Similarly in a commercial school, in lieu of teaching any particular method of book-keeping, Arithmetic is taught with a special reference to the keeping of accounts.

The various subjects of the curriculum are tanght in relation to the occupations for which the school is preparing, and thus in relation to one another. For example, in the Shoreditch School, preparing boys for woodworking trades, Geography is taught by telling the boys where the various woods on which they have been working come from and the way in which they are brought to this country; and History is taught, I understand, by going backwards from the present time and tracing the Industrial History of England and of the other countries in which the Geography has aroused an interest. Again, in the Bootle School, the subjects dealt with in the lessons on Manufacturing Processes and Workshop Practice have so improved the English composition that...' some of the essays were on a totally different plane from those usually met with at this age.' Further, the practical work is not primarily intended to develop skill in trade processes, but is rather used to illustrate the theoretical teaching and to awaken the interest of adolescent boys; and, once awakened, the interest can be extended to other subjects. In this way the [pupils] are given a practical turn of mind, by being enabled to perceive a connection between theory and practice, so that book-knowledge becomes to them a useful tool rather than something to grow rusty on a shelf and only to be taken down on those rare occasions when they realise its need. At the same time a wide interest is developed, due to the association in the scholar's mind of a large number of ideas of different kindst.

* The paper was printed for private circulation. At the time only thirteen Junior Technical Schools had been established in England, most of them during the preceding two years.

$\dagger$ Further evidence of the efficiency of senior secondary education as hitherto provided in Junior Technical Schools is furnished by the suggestion of the committee appointed by the Council of the North-east Coast Institution of Engineers and Shipbuilders that 'Much greater use should be made of Junior Technical 
We have already* seen how important it is that a teacher who is seeking to develop a single wide interest in each of his pupils should himself possess an interest that is both single and wide. We went further and pointed out $\nmid$ that the teacher of young part-time students engaged in the same or similar occupations should share his students' deep interest in their daily work, and, in fact, possess a single wide interest resembling as closely as possible the particular kind of single wide interest that he and his colleagues, who are teaching the same group of students, are endeavouring to form in each of them. In other words, the teacher of young part-time students should have a single wide interest-system, of which the intermediate zone, immediately connected with the most central purpose-elements, corresponds to the particular form of service which his students are rendering to their fellow men. This principle need not, however, be restricted in its application to the case of part-time students. Thus we have already urged that, in the interests of continuity in education, the sixth-form master + , concerned with advanced secondary education in a Higher Secondary School, should be in close touch with the university work for which he is preparing his pupils; while the university lecturer in engineering or medicine or any other branch of applied science should, in turn, be in close touch with the works or hospital or other establishment in which the young graduate will continue his professional training. So also is it desirable that the form-masters $\S$-and not merely such specialist teachers of technical or commercial subjects as may then still be required-who take part in senior secondary education, should have had practical experience of, and still take a lively interest in, the group of occupations into one or other of which their pupils are about to pass.

\section{$\S$ Ix. Intermediate Part-time Studies.}

As in the case of future members of Class A and of Class B, preservation of continuity in the normal education of future members of Class $C$ requires, not only that those who taught them during their

Schools in pre-apprenticeship education' (Sir Joseph Thomson's Committee's Report, p. 43); and by the prominence which the Council for Organising British Engineering Industry in I9I6 gave to a similar recommendation (loc. cit. p. 2).

* See above, p. 346 . † See above, p. 346. † See above, p. 349.

$\S$ Thus Dr Prosser in the Report from which we have already quoted (on p. 4II) urges that, in the case of senior technical education leading to industrial occupations in Massachusetts, 'all instructors be required to have some industrial experience as a qualification for service and those who do not have such contact or cannot acquire it be gradually transferred to other High Schools [or as we should say Higher Secondary Schools] and replaced by those who do possess such qualifications.' (Loc. cit. p. Io.) 
last whole-time terminal course should be in touch with the business concerns or public departments which are going to employ them from the age of sixteen, but also that these firms or departments should provide 'Apprentice Masters' or Welfare Workers thereafter to direct the young people's daily work. By contact with the Secondary Schools or Junior Technical Schools from which the young people come, as well as with the part-time teachers whose classes they attend from the time of entering employment, these Apprentice Masters will help to preserve continuity in their education as gradually they pass from whole-time study to whole-time employment.

The part-time classes to which we have just referred play an essential part in the education of future members of Class C. Soalthough it is true that the Education Act of $19 \mathrm{r} 8$, even when towards the end of the next ten years its provisions become fully operative, will not compel young people, who have received satisfactory (wholetime) secondary education until the age of sixteen, to continue in attendance at part-time day classes until the age of eighteen-we have, in our diagram, represented part-time education as continuing at least until the age of eighteen for everybody who is not receiving whole-time education. While, for some time to come, many of the part-time classes at which attendance is not compulsory may have to be conducted after working hours, evidence is accumulating to shew that the more progressive industrial and commercial concerns will allow all their employees, who are under eighteen, to attend day, instead of evening, classes; so that it may soon be usual for all young persons under eighteen employed in the chief industries-at least of the Manchester district-to attend part-time classes during working hours, whether or not they are compelled to do so. And it is most unlikely that any considerable number of young people engaged in these industries will abstain from attending part-time courses while they are under eighteen years of age.

Again, the future member of Class $C$ will not ordinarily discontinue attendance at part-time classes even on reaching the age of eighteen. $\mathrm{He}$ will, instead, continue for several years to attend classes of a more or less advanced character that bear upon the practice of his trade or other employment. Accordingly, it will be the function of the part-time studies which he pursues during the first two years (sixteen to eighteen) of his employment, to prepare him for the more advanced studies that come later. The first part-time course which he will follow should therefore differ from that which he would follow were he expecting to discontinue part-time studies on reaching the age of 
eighteen, and the difference will be of the kind that distinguishes transitional from terminal whole-time education. To the more transitional of these two types we have given the name of intermediate part-time education, and to the more terminal type that of senior part-time. While therefore, as we said, part-time education as a whole is more terminal in character than whole-time, intermediate part-time differ* from senior part-time courses much as, in the case of whole-time studies, intermediate secondary differs from senior secondary education.

Our principle $\nmid$ requires that the necessary varieties of intermediate part-time courses will exceed in number the varieties of senior secondary courses, and will in turn be exceeded in number by the varieties of advanced trade courses and other miscellaneous part-time classes, which are represented in our diagram as providing the final part-time education of future members of Class C. Our use of the word 'final' must not, however, lead us to forget that the young man or woman who discontinues attendance at organised courses of parttime study, at or soon after the age of eighteen, should have an opportunity of returning later $\rho$ n to part-time studies that may have no direct bearing upon the particular kind of service that he or she is rendering to the community $\ddagger$.

\section{$\S$ 12. Senior Elementary Education.}

When future members of Classes $\mathrm{A}, \mathrm{B}$, and $\mathrm{C}$ have been transferred from the Public Elementary Schools at about the age of twelve to follow the normal§ educational paths that lead to their respective fields of service, the work of the future members of Class $\mathbf{D}$ who are left behind should differ in type from the elementary education received by all children below that age. It might well be centred in, but not of course confined to, handwork\| during the remaining two years of compulsory school attendance, and so have much in common with the training of boy scouts or girl guides.

* For a further account of this difference see $\$$ I 3 of this Chapter on pp. 424 et seq. below, especially p. 428 .

$\ddagger$ Compare footnotes $\ddagger$ and $\S$ on p. 372 above.

$\uparrow$ See above, p. 386 .

$\S$ See above, p. 376 .

I| Compare the reference to practical instruction in The Education Act, I9I8:

'Clause 2. (I) It shall be the duty of a local education authority so to exercise their powers under Part III of the Education Act, I902, as

(a) to make, or otherwise to secure, adequate and suitable provision by means of central schools, central or special classes, or otherwise;

(i) for including in the curriculum of public elementary schools, at appropriate stages, practical instruction suitable to the ages, abilities, and requirements of the children.' (Italics mine.) 
Education of this type is described in our diagram* as senior elementary, in order to distinguish it from elementary education that we have described as appropriate for children under twelve. Mr Fisher's Education Act makes it the duty of a local education authority to provide this senior elementary education either in central schools or in central or special classes or otherwise. In large centres of population, there is indeed much to be said for not extending the work of the ordinary Elementary Schools beyond the modal age of twelve, when the elementary education of children should come to an end: the less able children who are not transferred to junior secondary education elsewhere would then proceed to Central Elementary Schuols in which they would receive the whole of their senior elementary education. But a large proportion of the child population of this country is to be found in rural or semi-rural districts where separate Central Elementary Schools for children over twelve cannot conveniently be provided. Our diagram therefore shews that the greater part of the provision of senior elementary education is made by ordinary Elementary Schools.

Senior elementary education is, as we have said, terminal in type. We have already $\dagger$ illustrated its difference from the transitional junior secondary education for children of the same age. Being terminal, senior elementary education should be provided in more varieties $\ddagger$ than are required in the case of junior secondary courses.

Wherever the conditions of a district render it probable that a considerable proportion of the children receiving senior elementary education in the same school will enter employment of a particular kind, the handwork that forms the central interest of the senior elementary curriculum may well be related to that type of employment. It must, however, be clearly understood that the object of this connexion between school handwork and subsequent employment is not the production of efficient wage slaves, but, as in the case of senior secondary education to which we have already $\S$ alluded, to increase the children's interest in all their school work. For example, in a Lancashire town where it is certain that most of the children will enter some branch of the cotton industry, the school handwork, in which the senior elementary education of the district is centred, might well involve handling and observing the cotton plant and samples of yarn and fabric in various stages of production. No attempt need be made to develop manipulative skill, for example, in

* Facing p. 3 I 9 above.

\$ See p. $3^{86}$ above. $\uparrow$ See above, p. 344 and pp. $35^{8}$ et seq.

$\S$ See above, pp. 418, 419. 
piecing ends. But the children might receive object lessons in the course of which they would measure various geometrical, botanical, mechanical, physical, chemical, and other properties of cotton. In so far, then, as their other studies-natural science, mathematics, and even geography and history-were made tributary to this central interest, so far would their interest in their whole curriculum be deepened and become more likely to stimulate them to efforts at concentrating attention.

\section{§ 13. Junior and Senior Part-time (Secondary) Education.}

One of the most serious, if not the most serious of all, discontinuities in the education of English boys and girls is that which results when pupils pass from the Elementary Schools at about the age of fourteen-and hitherto in many parts of England before that age-into whole-time employment unaccompanied either by attendance at part-time classes or by educational supervision in their daily occupations. Such supervision the trade apprentices in bygone days used to obtain by close association with a master-craftsman, and it is now provided by the Apprentice Master* and his assistants for boys employed in some of the most progressive manufacturing concerns of this country.

Evidence has multiplied to prove that this discontinuity has had serious consequences for the education of a majority of English men and women. The increased interest in education, that began in 1914-15, encouraged several groups of educators and other people to publish programmes of educational reconstruction. Indeed, the present writer, in the course of an address delivered in February, r9I7, was able to say that several of these recent programmes

agree in proposing that nobody's whole-time education should cease before the first end of term after the attainment of the age of fourteen; and that every employed person should be required by law to attend parttime classes during working hours for not less than one whole day or two half days per week throughout the following three or four years. The Education Reform Council, the Workers' Educational Associationt, the

* See above, pp. $350,408,409$.

$\dagger$ The paper from which this quotation is made continues: 'The programme of the Workers' Educational Association asks for compulsory attendance at parttime day classes for not less than twenty hours per week between the ages of fourteen and eighteen. The chairman of the Education Reform Council (Dr W. Garnett) suggested, in a recent speech, what seems to me an admirable way of giving effect to the wishes of the Workers' Educational Association without necessarily going beyond what the other bodies, including the British Engineers' Association, would be prepared to support. His proposal is that all employed persons between fourteen and eighteen years of age should be required to attend 
Association of Technical Institutions, and the Council for Organising British Engineering Industry* have all issued reports which include these recommendations....

Mr Fisher was quick to seize the opportunity afforded by this remarkable consensus of opinion. The Bill which he proposed to Parliament in the autumn of I9I7, and the revised Bill which ultimately reached the Statute Book in the following year, made parttime education - to be provided during working hours on the equivalent of one day per week-compulsory up to the age of eighteen for those who had not received satisfactory whole-time education at least until the age of sixteen. In view, however, of the difficulties inherent in making so great a change $\dagger$, the Act provides that part-time education between the ages of sixteen and eighteen shall not become compulsory until seven years after all young people up to the age of sixteen have been compelled to receive either whole-time or part-time education.

The continuation schools-or Part-time Secondary Schools as we shall prefer to call them--that will be established in accordance with this Act will make the principal provision for the education of the future members of Class D between the ages of fourteen and eighteen. When, as indicated in our diagram, most future members of Class C receive whole-time secondary education until the age of sixteen, the Part-time Secondary Schools will be mainly, although not exclusively, attended by future members of Class D. Even then, however, it will be necessary for Part-time Secondary Schools to meet the needs of two different groups of students. To the first group will belong those

part-time classes in working hours for not less than sixteen or twenty hours a week; but that Local Education Authorities should be authorised to reduce this number of hours to, say, eight in cases where they are satisfied that the young people in question are receiving suitable training during the actual practice of their employment. Such an arrangement would do much to discourage unnecessary entry into blind-alley occupations.'

* See above, footnote $\dagger$ on p. 325.

$\dagger$ According to our diagram a province of 5,000,000 people, in which 50 per cent. of young persons remain in whole-time attendance at Secondary or Junior Technical Schools until the age of sixteen, will require accommodation in its day continuation schools, or Part-time Secondary Schools as they are called on the diagram, for 90,000 boys, and for a similar number of girls; or a total of r 80,000 boys and girls between fourteen and eighteen years of age. Assuming that onefifth of this number attends the part-time secondary schools at any one timefor attendance is only required on the equivalent of one whole day per weekone-fifth of 180,000 , or, say, 36,000 places, will need to be provided in the Parttime Secondary Schools of the province. In Birmingham, or in Manchester and Salford, where the population slightly exceeds $1,000,000$, the number of Part-time Secondary School places needed within ten years will therefore be at least 7,200; and, if by that time the proportion of the population receiving whole-time secondary education up to the age of sixteen is less than the 50 per cent. in our diagram, the necessary number of Part-time Secondary School places will be correspondingly increased beyond 7,200. 
who, like many of the young persons engaged by manufacturing engineering concerns in repetition work, or like many of the boys and girls in the spinning rooms or weaving sheds of cotton mills, are employed in connexion with a great industry with which they may remain connected during the greater part of their lives and which in any case is capable of forming the centre of an interest which it is the function of the part-time school to widen, and, widening it, to make not only better workmen, but also better citizens. To the same group will also belong those future members of Class $\mathrm{C}$ for whose whole-time education until the age of sixteen provision has not yet been made. The second group will contain boys and girls in 'blindalley' occupations, or, in other words, whose employment is of a temporary character or has little bearing upon the work which they afterwards intend to do. The same school will often provide for both classes of students: it is, as we shall see more clearly in the next chapter, the school rather than the curriculum that is the unit of corporate life.

It will be convenient to describe as 'vocational' the courses provided for the first of our two groups. But, as will shortly appear, our use of this word does not imply that the young people are being educated for anything less than their whole lives. The school is to fit them for all their activities, social as well as vocational. Such a 'vocational' course should be conducted under the supervision of an advisory committee, containing representatives of employers and work-people. The same committee might supervise several similar courses in the same school or in several schools. On the other hand, one and the same school might have several advisory committees according to the different groups of trades or employments in which its students are engaged.

A 'vocational' course provided in a Part-time (day) Secondary School for persons belonging to our first group, whether their employment be industrial, cornmercial, domestic, or any other, should include:

(I) some practical work connected with their employment;

(2) a study of the principles underlying the practice of that employment;

- (3) a study of the bearing of the employment in question upon the well-being of the whole community, together with other so-called 'citizenship' subjects, including especially English.

The intention of the practical work is not primarily to teach an employment or to develop manipulative skill. It is rather to give the more varied practical experience that the students want but cannot 
obtain in the ordinary practice of their vocation; and so to connect the students' employment with all their continuation school work. The stronger this connexion is, the greater the interest that the students will find in their vocation, in their school, and in their whole lives; and the more likely will their education be to make them happy and effective servants of each other and the community.

The second element in the curriculum is the study of principles underlying this practice. Of the usefulness of such a study we have already* spoken. We may add here that 'an interesting example has been furnished by Judd $\uparrow$, who found that children could be taught to hit a mark lying in water just as readily by rule of thumb as by a clear method based on the knowledge of refraction of light. When, however, he changed the depth of the mark below the surface of the water, the rule-of-thumb scholars had to do all their learning over again, whereas those who had learnt [the principle involved]...were soon as successful under the new conditions as under the old ones.'

But perhaps the most important section of the 'vocational' curriculum is that which bears directly upon citizenship. This third section should, first of all, relate the whole curriculum to the central purpose of the students' lives, and should therefore include some reference to the ethics and ideals of their employment, which, to focus our ideas, we may suppose to be industry or commerce. It should also include an elementary study of the economic conditions which govern wages and hours of labour; economic geography, beginning with an account of the origin of the materials which the students handle or with which they are otherwise concerned in the practice of their craft; economic history, consisting mainly in biographical studies of the great men and women whose lives have been of special importance in the development of the employment group in question; and, possibly, other subjects studied from a similar point of view. Such studies will render the pupils less liable to be misled in later life by unsound economics or sociology; and may also enable them to take an active and intelligent part in solving social problems from inside.

For persons belonging to our second group, employed in occupations which cannot form the centre of their interest,Part-timeSecondary Schools must provide the centre of the interest as well as its periphery. Most young people between fourteen and sixteen years of age will, as

* E.g. on pp. 353, 357 above.

$\dagger$ Educ. Rev. June, I908, pp. 36, 37.

¥ Quoted by Professor Spearman, Journal of Exp. Ped. Vol. II, I9I3-I4, p. 252. 
we have said*, find such a centre of interest in the handwork which secures for boy scouts and girl guides the various stars and badges that mark their proficiency in cookery, splicing, astronomy, carpentry, or what not. Such handwork, especially if combined with outdoor activities, is well adapted to form the centre of interest of nonvocational courses, both because boys and girls like it $\dagger$, and also because interest in doing things resembles an emotional interest in that both are apt to intensify and render permanent intellectual ideas with which they are connected $\ddagger$.

Since many of the employments practised by young people belonging to our first group are not entered until the age of sixteen, the junior part-time courses that form the first two years work of the Part-time Secondary Schools will more often be non-vocational in character than the senior part-time courses that form the concluding two years work of these schools§. Accordingly, and in conformity with a principle of which we have already made several applications $\|$, there needs to be a greater variety of senior than of junior part-time courses.

It remains to remark upon the difference between senior part-time courses-and especially perhaps the vocational courses where the distinction is most likely to be overlooked-that are terminal, and the intermediate part-time courses that must be more transitional in character because they prepare for more advanced part-time work that is to follow. In the senior part-time course, what we have called citizenship studies are, as we have just seen, of paramount importance. But the intermediate part-time courses of ten years hence are, according to our diagram, to be attended by young people most of whom will not only have previously received four years of wholetime secondary education, but will further widen their interests by subsequent terminal courses of study. In common with other transitional courses of study, intermediate part-time education will therefore tend to go more deeply into a small group of comparatively abstract studies, so that the students may become familiar with principles, laws, and generalisations, many of the applications of which to the students' principal interests will be deferred until after the period of intermediate part-time education.

It is much to be regretted that most of the provision of part-time education for young people between sixteen and eighteen years of

* On p. 279 above.

$\ddagger$ See above, pp. 59, 60, 278 .

II See above, p. 386 , footnote
† See above, p. 279, footnote *.

$\S$ See the diagram, facing p. 319 above. 
age has, hitherto, been intermediate rather than senior-transitional rather than terminal-in type*. The consequent omission of citizenship subjects from senior part-time courses has resulted in the neglect of a great opportunity of educating the majority of part-time students during the critical years of adolescence.

\section{$\S$ I4. Byways of Education.}

In the preceding sections of this chapter we have briefly described the normal $\uparrow$ paths by which future members of Class A, Class B, Class $\mathrm{C}$ and Class D should, ten years hence, reach their respective fields of active service to their fellows and the community. In the course of this description we have referred to each of the sixteen types of education represented in our diagram. We have now to observe that the system of education, represented in our diagram, includes alternative or abnormal educational ways in which future members of Classes A, B and C may be educated. Indeed-as we shall again point out when, in the next chapter but one, we discuss a national scholarship system-account must be taken of the fact that some young people will, on account of illness, late development, or other exceptional circumstances, miss being selected at the normal time for transfer to a higher type of education, although their subsequent achievements, either during their whole-time education or when later on they are combining employment with attendance at part-time classes, may fully justify the transfer of these few exceptional people to higher education that will prepare them for a higher class of service later on.

Thus, if we may take engineering training as typical, our diagram shews that, of the boys who pass, at the age of fourteen or at least before the age of sixteen, from senior elementary education into the ranks of 'boy labour' in manufacturing works, Io per cent. will, by excellence in their employment combined with good work in their junior part-time studies, secure promotion at the age of sixteen to intermediate part-time studies, designed to prepare for Class $C$, while the remaining 90 per cent., most of whom will ultimately belong to

* The central and local education authorities were, until recently, in the habit of denoting successive years of part-time education by successive numbers from one to seven inclusive. The text, however, is concerned with emphasising the distinction between intermediate courses (say, $\mathrm{T}_{3}$ and $\mathrm{T}_{4}$ ) that aim at preparing for advanced courses (say, T 5, T 6 and $T$ 7), and senior courses. The latter should not be described as $\mathrm{T}_{3}$ and $\mathrm{T}_{4}$, if by these symbols are understood courses that prepare for $\mathrm{T}_{5}$; for senior courses, unlike intermediate courses, terminate (but see pp. 372, 422 above) organised education for the time being.

$\dagger$ See above, p. 376 .

$\ddagger$ See above, pp. 374, 375 .

G. E. 
Class D, will transfer at the same age to senior, instead of to intermediate, part-time studies. This promotion is to be accompanied by promotion from the ranks of 'boy labour' to the higher class of 'trade apprentices,' most of whom follow the normal educational path to Class $\mathrm{C}$ and so enter employment from Junior Technical Schools at the age of sixteen. Again, the best boys of this (trade) apprentice class, including a sprinkling of the boys who have been promoted into it from the ranks of boy labour, will obtain promotion into the still higher class of 'special apprentices,' most of whom, on their way to Class B, have entered employment at the age of eighteen after completing (whole-time) senior technical courses of study. Moreover, a few exceptionally able trade apprentices may be enabled, by means of scholarships combined with maintenance allowances, to enter upon a whole-time senior technical course at an age* which will exceed by a year or so that at which senior technical education is begun by those who approach it by the normal way of intermediate secondary education. But those future members of Class B who will not have the benefit of whole-time senior technical education between the ages of sixteen and eighteen, will pass directly from intermediate part-time to advanced part-time courses of study, which will, as a rule, be provided in Senior Technical Schools on two half days $\dagger$, or on three evenings, a week. (An alternative to these advanced part-time courses is open to engineering students in certain districts. The alternative, on the North-east coast + , consists of 'sandwich' courses of study provided by Senior Technical Schools, and occupying three consecutive winter sessions of five months each, following upon two years of works training combined with intermediate part-time studies. And, just as students who follow advanced part-time courses on one day a week or in the evening are generally able to do so without suffering any reduction in wages, so the engineers and shipbuilders on the North-east coast, whose apprentices receive their training on this 'sandwich system'§, pay their apprentices wages during the five

* These scholarships are represented in our diagram by the single-headed arrow on the seventeen-years ordinate.

$\dagger$ See above, p. 4 I 4 .

F For example, at Sunderland.

$\S$ This 'sandwich system,' which is alternative to advanced part-time courses in Senior Technical Schools, should be carefully distinguished from the better known 'sandwich system' in vogue in Scottish universities where, in view of the fact that the academic year in Scotland used to extend over little more than half the calendar year, engineering students were able to intersperse their undergraduate courses with long summers spent in obtaining practical experience in works. It has been suggested that the training of engineering students in English universities should follow this Scottish practice. Such a change would, however, result in engineering students keeping different terms from those kept by all the 
months that they are in the Senior Technical School, as well as during the seven months that they spend in the works or in the shipyards.) Once more, a small proportion of the ablest of these 'special apprentices' will, by excellence in their employment as well as in the advanced part-time courses* which most of them should be attending on one day a week or in the evening, secure scholarships $\dagger$ transferring them at about twenty years of age to (whole-time) undergraduate courses in a university or college of university rank. Having finished their undergraduate courses and taken their degrees, they will return, as 'college apprentices,' to practical work at their profession for the completion of their technical training for membership of Class A.

other members of their university; with the result that engineering students would tend to become a class by themselves and so be debarred from obtaining their full share of those most important university influences that are discussed in our next chapter.

* Organised advanced part-time courses will not be available for every possible variety of Class B occupation for which these 'special apprentices' are preparing. Accordingly, the diagram represents 25 per cent. of them as attending miscellaneous rather than organised advanced part-time classes.

$\dagger$ Represented in our diagram by the two arrows, one with a wavy shaft, on the twenty-years ordinate leading from advanced part-time to whole-time undergraduate courses. 


\section{CHAPTER 24}

\section{TYPES OF SCHOOL AND COLLEGE}

\section{§. Public Spirit.}

IN the preceding chapter we have been concerned with types of curriculum; with organised courses of study; with the educational influences of the schoolroom or lectureroom, laboratory or workshop; and, most of all, with the strenuous intellectual discipline involved in private study of the right kind. We have now to take note of those other educational influences that ought to be no less strong, and no less characteristic, than that of any particular curriculum. The influences with which we are concerned in this chapter are of the kind that the older universities associate with residence and tutorial superintendence. They belong especially to the playing fields and the river; the school or college societies; the school or college chapel; the boarding houses, hostels, or rooms in college or out; the byways and purlieus, buildings, grounds, or neighbourhood of a school or college or university. In other words, we have now to consider what is sometimes called 'atmosphere'; the thing that peculiarly belongs to the school or college as a whole and not to any one side or section of it; the thing that results in a characteristic point of view, and sometimes in distinctive mannerisms or even a distinctive manner; the thing that may even remain the same or nearly the same from one generation to another, while the curriculum may have been altered almost out of recognition (so that some, at least, of the pupils or students are pursuing studies which, in the days of their fathers or grandfathers, were altogether outside the scope of the school or college in question). Let us call this thing the 'spirit of the school' or college or university.

The emotional appeal of some of these influences first demands our attention, for we have seen* how specially important in the neurography are those elements which correspond to strong emotional interests. As we have also seen $\dagger$, great use may be made of these emotional elements in building up a single wide interest-system. And so strong is the emotional interest which many famous schools and colleges are capable of arousing in their members, that it may be made to play a very important part in the educational process. Indeed, 
just as we said* that the teacher of part-time classes should share his pupils' principal interests, and just as we went on to say, almost paradoxically, that the school boy whose main interest is in history should learn mathematics from a teacher who is qualified rather by his sympathy with the boy's interest in history than by his distinction as a mathematician $\dagger$, so now we observe that the master who is likely to be best able to form single wide interests in the younger pupils of a Higher Secondary School, requires, as his chief (but not of course his only) qualification, a full share of the spirit of the school, a deep understanding of its aims, a profound sympathy with its ideals, and that measure of athletic prowess which young school boys are apt to regard as an indispensable means of increasing the honour and reputation of their school.

There is, however, some danger lest the feeling which a good school should arouse in all its pupils may be used, not merely as a means of deepening and helping to form their single wide interests, but actually as the centre to which all else is made subsidiary. But every good thing may be abused; and the danger to which we have just alluded does not excuse any neglect to increase the emotional appeal which every school or college ought to make.

This appeal is due, in part, to associations with the past. To belong, for example, to a school which was founded by John Colet, the daily prayers of which were written for it by Erasmus, which produced Milton and Marlborough and other famous Englishmen, must make a deep impression upon every Pauline; while membership of a college that produced Francis Bacon, Isaac Newton, Clerk Maxwell and J. J. Thomson, may stimulate a Trinity man, and especially perhaps a Trinity physicist, to an extent that it would be hard to over-estimate. Venerable buildings, too-chapels, halls, great courts, and dreaming spires $\$$-deepen the emotional appeal made by the memories of great and famous men. It is true that a historic past and ancient buildings are beyond the reach of schools and colleges of recent foundation. But they too can strive to produce great men, and have in many cases already done so; they too, as they extend their buildings, can build beautifully, and that means, in the first place, simply and serviceably .

* See above, p. 346 .

+ See above, pp. 346,347 .

$¥$ Mr Compton Mackenzie's title for the third, or Oxford, section of his novel Sinister Street.

$\S$ Compare the remark, quoted at a recent conference of English universities: 'I know of no reason why a Gothic building should not be well adapted to serve the purposes of a university, but I never saw one that was.' 
We have been speaking of the emotional elements which membership of a great school or college renders available for inclusion in the single wide interest-system there being built up. We have now to speak of the direct educational influence that may be exercised by school or college life. We shall do so under three heads: mass influence, team work, and personal relationship.

Under the name 'mass influence' we allude to the effect of living for a period of years in the closest touch with an organised society whose ideals and outlook may remain the same or nearly the same for generations at a time. Sometimes there results an antiquated standpoint. Sometimes, however, a small school or college society succeeds in preserving ideals of heroic conduct, high thinking, patriotism, and search for knowledge, when, all around, moral and intellectual standards are temporarily depressed. 'Continuity, tradition, and the trusteesense which you find in an Oxford college or an Inn of Court-the sense that you hold for those who come after you what was held for you by those who went before,'* these it is, and the ideals of the public service connected with them, that tend to become impressed upon every member of a school or college in which they flourish. In short, hardly any man can spend long years in the company of people who share a common purpose without himself coming to share it too.

By 'team work' we describe another direct influence of school and college life: namely, training in self-government; social service, both to the members of the school community and, through school or college missions, to the outside world as well; loyalty to a small society, that best prepares for loyalty to a larger national or international group later on; and unselfish cooperation as an insignificant member of a cooperative body $\dagger$.

Lastly, under the heading of 'personal relationship,' we have referred to those educational influences which a school or college exercises by bringing together a number of boys or young men from different home surroundings and, in the case of many Higher Secondary Schools and universities, from different parts of the country and even from overseas. It is to her 'public schools,' whose pupils come from all parts of Great Britain and Ireland, that the United Kingdom is largely indebted for its unity. Of late years the ancient universities

* Midas and Son, by Stephen McKenna, 1919, pp. 220, 221.

$\dagger$ This aspect of school and college life has been well put by a former college tutor who has also seen service as a headmaster: the Rev. Canon W. Temple, in a presidential address to the Education Section (L) of the British Association at Newcastle-upon-Tyne in 1916. 
have also succeeded in bringing together young men who come, not merely from different counties, but from schools and homes of the most varied types. And at a recent* conference, called by the Imperial Education Comnittee of the War Office, it was stated that Oxford had done much towards unifying the English-speaking world by bringing together, under the terms of the Rhodes Trust, a number of scholars from all parts of the British Empire and the American Commonwealth. It is to be hoped that every British and American university at home, and overseas, will have a share in this work of harmonising the purposes and outlook of English-speaking people, so that they may work together for the good of the whole world. This aspect of university work was well put by Cardinal Newman who, writing in $185^{2}$, went so far as to say:

I protest to you, Gentlemen, that if I had to choose between a so-called University, which dispensed with residence and tutorial superintendence, and gave its degrees to any person who passed an examination in a wide range of subjects, and a University which had no professors or examinations at all, but merely brought a number of young men together for three or four years, and then sent them away as the University of Oxford is said to have done some sixty years since, if I were asked which of these two methods was the better discipline of intellect,... which of the two courses was the more successful in training, moulding, enlarging the mind, which sent out men the more fitted for their secular duties, which produced better public men, men of the world, men whose names would descend to posterity, I have no hesitation in giving the preference to that University which did nothing, over that which exacted of its members an acquaintance with every science under the sun $\uparrow$.

\section{§ 2. Universities.}

In applying these considerations to the system of public education that we hope to see brought into operation in England during the next decade, we shall not follow the order of the flow in our diagram $\ddagger-$ from elementary and preparatory education at its beginning to graduate study and research at its end-but the reverse.

Beginning then with the university, we first remark how fortunate it is for England that, with only one important exception, her most advanced study and research in applied science and technology form part of the work of her universities $\S$. Anything which tends to

* June, Igrg.

$\dagger$ Quoted in the Final Report of the Royal Commission on University Education in London, pp. 26, 27.

$\ddagger$ Facing p. 319 above

$\S$ Compare the following extracts from a memorandum by the British Science Guild dated April, 1919: "Technical Education is intimately connected with the teaching and development of pure science. Higher Technical Education in 
segregate particular classes of students in separate institutions according to their different special studies, impairs the influence which a university is, as we have just seen, capable of exercising, and which it ought to exercise, in harmonising the ideals and purposes of the leaders of the people.

The extent to which a university can exercise upon its students the kind of influences that specially concern us in this chapter depends, as Newman* saw, on the prevalence of residence and tutorial superintendence in that university. The majority of the students entering English universities at the present time have been pupils of day schools where these influences are generally less than in the great boarding schools. And the homes in which these students have lived during their school life, and where they go on living as undergraduates, are too frequently lacking in intellectual interest: their home environment has not familiarised them with big ideas, broad generalisations, or organised thought about life as a whole.

While a university cannot immediately and directly alter the home conditions of its undergraduates, it can still do much to increase those of its educational influences which do not mainly belong to courses of study that lead to degrees or diplomas. Tutorial superintendence, for example, can be exercised in a non-residential university; and the effect of long talks with one's tutor, either alone or in company with a small group of other undergraduates seated round the tutor's fire, may be very great indeed. A fortnightly essay $\dagger$ may afford the occasion for gatherings of this kind; but, in some of the most profitable of these tutorial hours, the written essays may hardly be mentioned, almost the whole time being occupied with a discussion that widens the student's interest and helps hin to see the relation of his principal studies to modern life as a whole. Such informal talks afford senior members of a university an opportunity of sharing with their juniors the business of 'training, moulding, enlarging the mind' which, in

particular, therefore, must be in intimate contact with the Universities and vice versa. The question of the further provision of Higher Technical Education cannot be considered apart from the question of the development of existing Universities and the formation of new Universities.' Cf. also the quotation from Mr Michael Longridge's presidential address to the Institution of Mechanical Engineers on p. 44 I below.

Already before the War the Verein Deutscher Ingenieure was pointing out the injury done to Germany by the separation of her Technische Hochschulen from her universities. In most German states the Technische Hochschulen (or technical universities) are not merely independent of the universities proper, but are actually located in different towns; and the Handels Hochschule at Cologne illustrates a tendency to separate the highest commercial, as well as the highest technical, education from the work of German universities.

* See p. 435 above. $\dagger$ Cf. p. 400 above. 
Newman's view, was so important a part of the duty that a university owes to its undergraduates.

But there is no sufficient reason why universities that are at present mainly non-residential should not take steps to increase their influence by causing most of their undergraduates to reside, at any rate for a time, in colleges or hostels. Under a residential system, as we have already indicated, students have far better opportunities of educating each other than is otherwise possible: they may, in particular, develop qualities of leadership in connexion with debating, literary, scientific, technical and athletic societies, which never flourish so well under a non-residential system.

The end we have in view does not necessarily require that every undergraduate should reside in a college or hostel during the whole of his undergraduate life. A long step, even if only a first step, will have been taken when at least one year's residence is required from every undergraduate. The great increase of hostel accommodation which this long first step would render necessary in the case of most modern English universities, outside London, could probably best be provided at some distance from the centre of the large town where the university's buildings are, for the most part*, situated. A group of residences placed within easy distance of the university town might provide the nucleus of a real university colony. The rapid improvement of electric transport that is to be expected within the next few years, will bring suitable country sites within less than half an hour's run from the middle of the city where the university buildings stand. Those modern universities which are situated in the largest centres of population would probably be well advised to consider the purchase (before improved means of transport increase the price) of land in the neighbouring country, for the purpose of providing residential accommodation.

It will, of course, be objected that the increased cost of university education involved in our proposal would be prohibitive. But, even if the whole of this increase of cost had to be met from public funds, the additional burden would not be excessive. Thus, it is safe to estimate that an undergraduate member of a modern university can reside in a university's hostel, instead of living at home, at an increased cost of less than $£ 50$ a year. If then every undergraduate member of, say, the University of Manchester were compelled to reside in a hostel

* It may well be that the increased hostel accommodation required can best be provided in the immediate neighbourhood of the university buildings where these have already been removed from a central site, as has happened in Birmingham. 
throughout his undergraduate life, and if all the increased cost were borne by public funds, the charge upon them would be far less than the present expenditure of the University, including both Owens College and the College of Technology. And if the number of undergraduates were increased to $6,000^{*}$ men and 3,000 women, making a total of $9,000 \dagger$ undergraduates, and if every undergraduate were required to reside in a hostel for one year, the cost $\ddagger$ of this requirement, reckoned as before at $£ 5^{\circ}$ per student per annum, would still be less than the present expenditure of Owens College and the College of Technology combined. When we reflect that the total annual income of all the universities and university colleges of the British Isles was, in I9I4, about $£ 2,000,000$, and that the Universities of Oxford and Cambridge account for about one-half of this amount (so that the total annual income of the comparatively non-residential universities and university colleges is about $£ \mathrm{r}, 000,000)$ while that of the universities and colleges of the United States is $£ 20,000,000 \S$, we realise that it is by no means out of the question to propose to spend an additional $£ \mathrm{I}, 000,000$ per annum in order to ensure that every English undergraduate shall spend at least one year in residence at a university or college.

We may here note that, while private benefactions to universities and colleges in the United States amount to more than $£ 5,000$, 000 annually, in the United Kingdom they do not average one-twentieth that sum ||. In fact the wealthy people of England have not, during the last century, endowed education as the richer citizens of the United States are now doing, or as was done by the rich ecclesiastics and nobles of the Middle Ages. When sums of money are given, they are frequently ear-marked for particular buildings and equipment; but, necessary as these undoubtedly are, they are not needed so much as the provision and proper payment of a personnel adequate for research, for teaching, and for the leading of public opinion. While it is probable that larger gifts will be forthcoming in the near future, it is doubtful whether the increase that may be expected will be

* This is the number of undergraduates to be expected under the system represented in our diagram from the population of the Manchester province, estimated at 5,000,00o persons. (Cf. pp. 370, $37 \mathrm{I}$ above.)

$\dagger$ That is 18 per Io, ooo of the province referred to in the preceding footnote, a figure approximately equal to the percentage in Scotland immediately before the War: see above, p. 37 I.

$\ddagger$ ir5o,000 a year.

$\S$ These figures are taken from p. Io of the Report of the Education Committee of the British Science Guild upon the Position of Universities and Technical Institutions.

II See p. 2 of the British Science Guild Report. 
equal to the greatly increased needs of the universities of this country. When the provision of university education in England has become equal to that represented in our diagram, it is probable that most English universities will have to depend for the principal part of their revenue on public funds that must be made available on an altogether new and greatly enlarged scale.

This increase of national and local grants to universities will doubtless be accompanied by some increase in the part played by the nation and the locality in university government. We would suggest that this change can best be brought about by associating the provincial universities of England more closely with the districts which they specially serve. The establishment of provincial education authorities* (preferably as federations of existing local education authorities for higher education, but including representatives of the provincial university or universities and of some other autonomous educational institutions) would not only be the best means of ensuring adequate local support for the universities $\dagger$, but would also, as we have said $\ddagger$, secure (and for the first time) an adequate provision throughout the length and breadth of England of all forms of higher education $\S$, and especially of the higher secondary education that

* See above, p. 366, and Appendix E.

$\dagger$ Compare the following extract from a memorandum by the British Science Guild dated April, 1919: 'In dealing with all education of university grade or of the type provided by higher technical institutions, it is necessary to establish local authorities for much larger areas than the majority of counties and of county boroughs. It is true that in some cases a county borough (i.e. Manchester) has provided and largely maintained a technical institute of first rank, but the institute is always attended by pupils from the districts of other authorities, who contribute either nothing or an inadequate share towards the cost of maintenance, and the financial injustice involved threatens the security of these institutions, while in the matter of the award of scholarships by the existing local education authorities we are very far from securing equal opportunity for residents in different areas.'

$\ddagger$ See above, p. 366 .

$\S$ Compare the following extract from a paper published by $\mathrm{Mr}$ Cloudesley Brereton entitled 'A Bird's-Eye View of Educational Reform ': 'One does not believe however that the present-day University or the present-day institution of higher learning will ever be able to realise its true local function, leaving out those of national or imperial importance, until we have boldly adopted larger educational areas, thus bringing together town and country in a new and fruitful union; while one is equally confident that unless these areas are also adopted for other political purposes, such as the solution of proper means of water supply, of communication, and other problems of local administration that at present cry for solution [cf. footnote ${ }^{*}$ on p. 366 above], the requisite genius loci, or spirit of regionalism, will not be developed and we shall be still far from the ideal of the local University being not only the spiritual head of the hierarchy of the schools, elementary, secondary, technical, agricultural and the like, but also the main organ for reintegrating communal feeling and humanising commercial life and giving the proper inspiration to every art and craft in the locality. Rightly understood the local University should be a focus of local patriotism and local enlightenment.' (The Nineteenth Century and After, June, 1917, p. I311.) 
best prepares for university studies*. It is true that a good deal has already been accomplished by voluntary cooperation between existing local education authorities and universities. But, with the increase in the volume and scope of the work of modern English universities, voluntary cooperation between small authorities can no longer be relied upon for the local support of one of these universities, whether by supplying its students with the necessary preliminary education, or by supplying its coffers with the necessary funds. Indeed, a system which can be upset by the refusal of a single authority to play its part, will not suffice to bring university education within the reach of every young person of sufficient educational promise. To this question we shall return in the next chapter.

\section{§3. Senior Technical Schools, or Polytechnics.}

Senior technical education, on the need for which we have already $\dagger$ insisted, must, as we also observed $\ddagger$, be clearly distinguished from the work for an honours degree that ought to form the staple studies of most undergraduates in every university. To the reasons, economic as well as educational, then given for this observation, we have now to add that the influences which a university ought to exercise upon its undergraduates along the lines indicated in this chapter, would be seriously impaired were the university to include, in addition to the number of undergraduates indicated by our diagram, the larger number of boys between sixteen and eighteen years of age represented as following senior technical courses of study; for the number of undergraduates between the modal ages of eighteen and twenty-one is, according to our diagram, only three-quarters of the number of sixteen to eighteen-year old boys from the same province who should be receiving senior technical education in the Technical Schools of the province, and only three-fifths of the whole number of boys under the modal age of eighteen who should be receiving senior technical education. Thus, for example, our diagram shews that in a province of $5,000,000$ people there would be 6,000 men under-

* The change here anticipated would involve some change in the present system of government of most English provincial universities. It may be suggested that the Courts of these universities should, in future, represent:

(a) the teaching body;

(b) the graduates;

(c) the provincial education authority;

(d) persons co-opted on account of their special interest in, and knowledge of, university problems.

Such a change in the constitution of a University Court might in some cases involve little if any alteration in the personnel of the executive or, as it is often called, the University Council.

$\dagger$ See above, p. 4I3.

$\ddagger$ See above, p. 4 Io. 
graduates over the modal age of eighteen, and Io,ooo boys between sixteen and eighteen receiving senior technical education, 8,000 of them in the Senior Technical Schools or Polytechnics. It is clear that the undergraduate life of the university of such a province would be swamped by the inclusion of these large numbers of younger students receiving education of a different type. We hold, therefore, that, as indicated by our diagram, senior technical education, except in so far as it is provided for a comparatively small minority in Higher Secondary Schools, should be recognised as belonging, not to universities and colleges of university rank, but to institutions of a different kind* that we describe as Senior Technical Schools $\dagger$. While one, or at most two, universities may well suffice for each of the ten or twelve English provinces + , there is no reason why the number of Senior Technical Schools should not be very much greater. One, at least, should be available in every county borough or other large town that owes its size to its own industry or commerce§.

When the position that senior technical courses of study ought to occupy in a national system of education has been generally recognised, and when such courses are provided on the scale indicated by our diagram, it may be possible to restrict the use of Senior Technical School buildings to those whole-time students who are receiving this type of education, and so to foster those educational influences that we are specially considering in this chapter. But in the meanwhile-and for many years to come-the present practice of using for the benefit of part-time students the special qualifications of the members of the teaching body of a Senior Technical School, and the costly equipment of the workshops and laboratories that are needed

* We are here in agreement with Mr Michael Longridge who, in the course of his presidential address to the Institution of Mechanical Engineers, said:

'I think, also, that a clear distinction should be made between technical colleges of university rank on the one hand and senior technical schools, whose entrance standard is lower than university matriculation, on the other. The interests of engineers require two classes of institutions giving technical instruction, each with its definite purpose:

(I) Technical "colleges";

(2) Technical "schools" (senior and junior).

"The technical "colleges" should be of university rank, and should be departments or faculties of universities, like the [College] of Technology in Manchester, not independent of them, as in Germany.' (Engineering, p. 412, 27th April, 1917.)

$\dagger$ In London these institutions are generally known as Polytechnics, while the regulations of the Board of Education describe them as Local Colleges.

$\ddagger$ See above, pp. 366,367 , and Appendix E.

$\S$ We are not saying even that every county borough ought to have its own Senior Technical School providing organised two-year courses of study for boys between sixteen and eighteen years of age. Canterbury, for example, is a county borough because of its cathedral, rather than because of its industrial or commercial importance. 
for many of its whole-time courses, will be continued; so that, as our diagram shews, Senior Technical Schools will provide, not only wholetime two-year courses for young men between sixteen and eighteen years of age*, but also intermediate and advanced part-time courses, together with miscellaneous part-time classes, including advanced trade classes. Most of the part-time classes intended for persons over the age of eighteen will meet in the evening; but, even in the evening, the day students' common-room should be reserved for their sole use, so that these day students may feel that their Senior Technical School is, after all, their own institution, rather than a heterogeneous aggregate in which their share is quite small.

It remains to add that, according to the figures given in our diagram, and supposing that all the intermediate part-time classes meet in the day-time $\uparrow$ (on not more than two half-days a week), the students present on any one day will be equally divided between whole-time and part-time students; while the whole number present on any one day will be approximately equal to the whole number present on any one evening if we further suppose that all the students over eighteen are evening students, and that, on the average, each of them attends the Senior Technical School on two evenings in each week during the winter session + . The approximation to equality will be still closer if we suppose that, of the students over eighteen, those following advanced part-time courses attend on the equivalent of one day a week instead of in the evening.

* Compare the findings of the Consultative Committee of the Board of Education recorded in their 'Interim Report on Scholarships for Higher Education': 'We conceive that the chief function of Senior Technical Schools-besides providing evening instruction-should be to provide continuous technical and scientific instruction from about the age of sixteen to that of eighteen or nineteen. They would thus link up naturally-where they exist and they are not so numerous as they should be-with those pupils of Secondary Schools who conclude their Secondary studies at about the age of sixteen.' (Report [Cd. 829I], p. 20.)

$\dagger$ Cf. p. 421 above.

$\ddagger$ Our diagram shews that, so far as boys and men are concerned, the Senior Technical Schools of a province of $5,000,000$ people will be attended on any one day by 8,000 whole-time students receiving senior technical education, and by 8,200 (being one-fifth of the $4 \mathrm{I}, 000$ ) part-time students following intermediate part-time courses; and on any one evening, if we suppose that every part-time student over eighteen attends on the average on two evenings a week, by 21,000 (being two-fifths of the whole number of 52,500 represented in the diagram). Thus the number present in the evening will not be very different from the number present in the day-time.

If the advanced part-time classes in the Senior Technical Schools meet in the day-time on the equivalent of one day per week and the other hypotheses in the text remain unaltered, the number of students present in the Senior Technical School on any one day will be 18,000 while the number present on any one evening will be $\mathrm{I} 7,5 \mathrm{Oo}$. This would be a most economical arrangement so far as buildings and equipment are concerned. 


\section{§4. Secondary Schools.}

Having thus distinguished between universities on the one hand, and Senior Technical Schools on the other, we have next to observe the differences between Higher Secondary Schools and those other Secondary Schools that are described in the diagram as ordinary or lower. The distinction we draw between these two types of Secondary Schools is clearly shewn in the diagram: it is that, while Higher Secondary Schools retain almost all their pupils until the age of eighteen (or, if all the pupils of Higher Secondary Schools were able enough and well enough educated to pass the First School Examination at the normal age of sixteen*, for two years work above the standard marked by the School Certificate $\dagger$ ), ordinary or Lower Secondary Schools part company with their pupils at the modal age of sixteen. In other words, it is the Higher Secondary Schools alone that undertake sixth-form work; or, in the terminology of our diagram, it is only the Higher Secondary Schools that provide advanced secondary (together with a comparatively small amount of senior technical) education.

Many educators, it is true, believe that it is a mistake to collect all the advanced secondary education of a province into a comparatively small number of Higher Secondary Schools. They would prefer that every Secondary School should have its own sixth-form, however small; a little leaven, they say, of pupils who intend to proceed to a university, after reaching scholarship standard or the standard of the Higher School Certificate $\leftarrow$, will leaven the whole lump. But, when we look at the figures presented by our diagram, we cannot share this view, except in so far as it applies to a rural district in which Higher Secondary Schools cannot be made available. A certain proportion of the qualified boys and girls will always prefer to attend Higher Secondary Schools, where very few pupils leave before the age of eighteen. If, then, we were to assume that half $\S$ the number of

* This is very far from being the case in many of the 'public schools' to-day. But many of the boys admitted to these schools would not be there if a competitive test were applied to all English children for the purpose of selecting the number shewn in our diagram to be educated in Higher Secondary Schools, and if all the less able boys and girls were excluded from these schools.

$\dagger$ See footnote $\S$ on p. 386 above. $\ddagger$ See above, p. 387 , and Appendix F.

$\S$ A large proportion of the boys who intend to proceed to a university will in any case desire to receive their advanced secondary education in Higher Secondary Schools where it has long been the practice for most boys to remain at least until the age of eighteen. Thus the Consultative Committee of the Board of Education in their 'Interim Report on Scholarships for Higher Education' [Cd. 829I] write: 'The Public Schools have a great tradition; a tradition of character, a tradition of manners, a tradition of physical excellence, a tradition of selfgovernment. They do in fact supply the boys of the country with more than half the Higher Secondary Education that they receive' (loc. cit. pp. 18, 19). 
young people, shewn in our diagram as attending Higher Secondary Schools between the ages of sixteen and eighteen, were transferred instead to Lower Secondary Schools and evenly distributed between them, so that every Secondary School should have its own sixth-form, however small, the result would be that every Secondary School that contained 300 pupils between the ages of ten and sixteen would have a sixth-torm of only twelve pupils who had passed the School Certificate standard, and were either preparing to enter the various honours schools of a university, or were receiving senior technical education. Of these twelve, no more than two or three would ordinarily be following the same variety of advanced secondary, or senior technical, course. The cust of making appropriate provision for each of these sixth-form boys and girls would be altogether prohibitive. The formal education of these boys and girls-the ablest, be it remembered, in the whole school - would therefore be sacrificed, in order that the school as a whole might be able to look to a sixth-form for its monitors or prefects, and perhaps for the leaders of its athletics.

The injury that would thus be done to nearly half the ablest boys and girls-not merely the ablest in the school, but in the whole land-during the two most valuable years of their school life, would not, however, be limited to defective education in the classroom or the study. Those other educational influences that we discussed in the first section of this chapter, and that should be so powerful at the top of a Higher Secondary School (where, during the last years of their school life, able boys and girls are surrounded by schoolfellows of the same age and similar attainments), would be replaced, in the ordinary or Lower Secondary School (where the sixth-form would be less than 4 per cent. of the whole school), by temptations to behave like demi-gods or eastern potentates lording it over a mass of inferior beings.

In the interest, then, of the ablest of the children-and also, be it added, of the training in self-government which boys and girls who are about to leave an ordinary Secondary School at the age of sixteen will have more opportunity for practising if they are not governed from above by a very small sixth-form aristocracy -it is desirable that as far as possible Higher Secondary Schools should be distinguished from other institutions that provide the greater part of secondary education*.

* Professor John Adams writes: 'My experience backs up your argument. In the Scottish schools a good deal of harm was done in the post parish-school period by teachers trying to carry on a "top" of a few boys of ability." 
Moreover, we should deprecate any arrangement for transferring more than a very small number of pupils from Lower to Higher Secondary Schools at or about the age of sixteen. If the Higher Secondary School is to exercise its fullest influence upon its pupils, it ought to have them for at least four years. Accordingly, the transfer of the ablest children from Lower Secondary Schools, into which some children pass below the age of twelve and into which a large additional number is transferred from Elementary Schools at that age, should take place, as indicated in our diagram, not later than the age of fourteen. Those pupils of the Lower Secondary Schools who, on account of late development or other exceptional circumstances, fail to secure transfer at this age, but are afterwards qualified to receive university education (if necessary at the State's expense), should, as we said, for the most part be admitted to universities for a preliminary year's work* in preparation for an honours school, after matriculating direct from their Lower Secondary Schools, and.without proceeding to a Higher Secondary School for two years of advanced secondary education. Only a very few exceptional persons should be transferred from Lower to Higher Secondary Schools on the completion of their intermediate secondary education.

\section{§5. Junior Technical Schools.}

In our diagram we have represented Junior Technical Schools as providing the same type of education-namely, junior secondaryfor all their pupils between twelve and fourteen years of age as is provided in all Secondary Schools for pupils of the same age. And we have represented Junior Technical Schools as providing, for all their pupils between fourteen and sixteen years of age, education of the same type - namely, senior secondary - as should be provided by Lower Secondary Schools for the majority of their pupils of the same age. Our discussion $\dagger$ of the nature of senior secondary education shewed no essential difference in type of the senior secondary education that should occupy the last two years of the whole-time education of boys who are passing directly into employment at the age of sixteen, whether that education is provided in a Junior Technical School on the one hand, or in an ordinary Secondary School on the other. Moreover, we have pointed out that, in the view of Sir Joseph Thomson's Committee, as in that presented by our diagram,

* See above, p. $39^{2}$.

$\uparrow$ See above, Chapter $23, \S$ Io.

G. E. 
the work of Junior Technical Schools ought to be regarded as belonging to secondary education*.

Is there, then, any adequate reason for perpetuating the present distinction between Junior Technical Schools and ordinary or Lower Secondary Schools? Or is it not preferable that the function now fulfilled by Junior Technical Schools should, in future, belong to ordinary Secondary Schools, to which should be added new 'sides' concerned with those varieties of senior secondary education that have hitherto been provided in the Junior Technical Schools? Our answer is that in the future-and perhaps, although our diagram does not indicate it, within the decade with which it is especially concernedthe work that is now done in separate Junior Technical Schools should be undertaken by new 'sides' of Lower Secondary Schools. For, just as we have seen that the influences discussed at the beginning of this chapter will be more effective at the university stage when the highest teaching and research in applied science and technology form part of the activities of the universities than when they are confined, as in Germany $\dagger$, to separate institutions, so will the same influences be better fostered, at any early stage of education, by bringing together in the same schools the work that has hitherto been undertaken in separate buildings by Junior Technical Schools on the one hand, and by ordinary Secondary Schools on the other. It will, however, be observed, from the description we have already $\ddagger$ given of the character of senior secondary education, that, if regard be had rather to the type of education provided than to the particular subjects taught, the change we are here suggesting consists, not so much of turning Junior Technical Schools into modern Secondary Schools of the usual type, as of making ordinary Secondary Schools adopt, for the majority of their pupils, the ideals and practice of the best Junior Technical Schools.

Although we are thus recommending the ultimate fusion of Junior Technical and ordinary Secondary Schools, we must point out that the beginnings and early development of the former have taken place outside organised secondary education, and also outside the Elementary School system. In fact, the growth of Junior Technical Schools towards an established position in English education has not only been primarily due to a definite purpose, clearly realised and enthusiastically pursued by their teachers, their headmasters, and their inspectors§,

* See above, p. 4I 7. † See above, p. 435, footnote $\S$. ‡ In Chapter $23, \S$ ro. $\S$ Of the technical branch of the Board of Education. Compare Sir Joseph Thomson's Committee's Report, p. 43. 
but has been fostered and facilitated by the extra measure of freedom to expand and to experiment that was allowed to them by the single paragraph* of departmental regulations under which they worked: a freedom that would have been denied to them under the more specific and complicated regulations for Public Elementary Schools $\nmid$ or stateaided Secondary Schools $\ddagger$.

Moreover, it was not until the theory of the terminal, as distinguished from the transitional, course had first been thought out in its application to this stage of education, that it began to find acceptance among those Secondary School masters and those officers of local and central education authorities who still believed that all secondary education ought to be transitional in character and suitable for children who intended, not only to complete secondary courses, but to pass on from them to university work. Accordingly, the new terminal type of education, especially designed to meet the needs of boys who were about to enter industrial occupations, was not provided by Secondary Schools but by new Junior Technical Schools for which accommodation was generally found in the buildings of Senior Technical Schools. These buildings were, for the most part, nearly empty in the day-time because the need for senior technical education had not been realised, and because the practice of providing intermediate part-time courses in the day-time, instead of in the evening, was still in its infancy.

But the original separation of Junior Technical Schools from Secondary Schools was due to inability, as well as to unwillingness, on the part of the pioneers of the Junior Technical School movement to make their schools comply with the regulations for Secondary Schools as they then existed. For, in the absence of the encouragement, afforded by the Education Act of 1918, to boys and girls to continue to receive whole-time secondary education until the age of sixteen, the promoters of Junior Technical Schools, in rgo6-7 when the number of these schools first began to expand rapidly, did not think

* Section 42 of the Board of Education's Regulations for Technical Schools.

† For example the Public Elementary School Code laid down definite regulations concerning the qualifications of teachers, while the early promoters of Junior Technical Schools attached more importance to the kind of qualifications described on p. 420 above than to the qualifications required by the Code.

$\ddagger$ For example, the promoters of Junior Technical Schools, when the number of these schools first began to expand rapidly about the year 1906, were unwilling to insist that every Junior Technical School boy should study a foreign language, however little chance there might be of his acquiring a useful knowledge of it; for, in those days, the boys generally passed from Junior Technical Schools into industry at fifteen years of age, and seldom spent more than two or three years altogether in Junior Technical Schools. 
it practicable to retain pupils in a Junior Technical School much beyond the age of fifteen, or to admit them to these schools before the age of thirteen or, at the earliest, twelve. In these circumstances Junior Technical Schools could not supply the four-year courses required of every Secondary School in receipt of government grant.

\section{§6. Part-time Secondary Schools.}

Of the high importance of part-time secondary education, both junior and senior, we have already* spoken. We return to the question in this chapter in order to point out how necessary it is that, in the development of part-time secondary education, the influences with which this chapter is especially concerned shall not be neglected. For the age range of part-time secondary education-from fourteen to eighteen-is the same as that of the so-called 'public schools': the age range of higher secondary education. These part-time day schools must be the 'public schools' of the great majority of our fellowcountrymen. They must form the young people's characters, as well as develop their intellects. No part of English education has, on the whole, been more successful than our 'public school' system. Whatever good we may get that is new, we must not lose the good that is old. We must therefore endeavour to introduce into the part-time secondary education of the many what have hitherto been the best features of the whole-time secondary education of the very few. In fact, the Part-time Secondary School of the near future must be possessed of school societies and be governed, as far as possible, by the boys and girls themselves. Its buildings should be open for recreative purposes at times when classes are not being held. It should have its own teams for football, cricket, and other sports, and it should have its Sunday service, and perhaps its summer camp.

The fostering of public spirit in a part-time school attended by different pupils on different days is a difficult, but not an insoluble, problem. The difficulty will not be so much felt in those new Parttime Secondary Schools that are being provided by large industrial or commercial concerns for their own employees $\dagger$. It is not indeed

* See above, Chapter 23, § 13 .

$\dagger$ Not all of the new continuation schools that will be needed to comply with section Io of the Education Act of 19I8-a section that requires that every young person (between fourteen and sixteen years of age at first, and, seven years later, up to eighteen years) shall attend school for at least 320 (or, if the local education authorities so resolve, for 280 ) hours every year-will be provided by local education authorities. Section Io (3) (ii) of the Act provides that a young person who is under obligation to receive part-time education may receive it outside the local authority's continuation schools; and section ro (8) requires that young 
to be understood that more than a small proportion of the provision of part-time secondary education required by the Education Act of I9I8 will be made in such 'works schools.' It is, however, contended that the best works schools may be the best of all the new day continuation schools, because of the very special degree in which they are able to exercise the kind of influence here in question. Thus, one who had been a teacher in a local education authority's continuation school and who had recently removed to take charge of a works school in the neighbourhood of Manchester, informed the present writer how sure he felt that his opportunities of really getting hold of his boys and influencing them aright were far greater in the works school, where he saw his boys every day, than in the local education authority's school, where he only saw them on two half-days a week. Another works school in the same neighbourhood has already some 400 members. It is self-governed. It has its own magazine. It is divided into houses that correspond to different groups of trades. The house matches awaken the greatest enthusiasm. The captain of the school, himself a senior trade apprentice-for the school is concerned with trade apprentices only-fills his high office as effectively as any chairman of a board of directors. One of the firm's managers keeps in close touch with the senior boys who wield authority in the school. He may help them with advice, but he does not interfere. His function is that of a wise headmaster. Thus an intimate contact between employer and workman is established at the very outset of their mutual connexion, and should be greatly to the advantage of both in all their subsequent relations with each other.

It is true that, in two obvious respects, a works school must be at a disadvantage compared with schools provided for all comers by a local education authority. In the first place, there is the danger that compulsory inspection by the central or local education authority may not suffice to prevent ill-disposed industrial concerns from misusing works schools on their premises, so as to increase their employees' productive efficiency at the expense of their education as citizens. In the second place, while the local authority's schools ought, as far as possible, to provide different teaching for boys or girls engaged in different groups of educative crafts, they ought also to afford opportunities of bringing together, for purposes of recreation and social

people, while they cannot be compelled by the local authority to attend a continuation school 'at or in connection with the place of their employment,' may attend such a 'works school' instead of one of the local authority's continuation schools, provided that the works school is open to inspection either by the local education authority or by the Board of Education. 
life, young people who may not only (as in the case of the works school) be engaged in different crafts, but also be employed by different firms and in different branches of industry. But the works school will, at its best, more than make up for these disadvantages by the unique facilities it affords for corporate life, social organisation, and self-government among young people who have far more than their part-time school in common.

The continuous presence in the works of a number of cultivated teachers cannot fail to exercise a beneficial effect upon the social life of the whole works, and not only of the pupils of the works school. For the latter, the benefit will be especially marked. The educative value of the four and a half days a week that they spend in the practice of their employment will be increased. In some cases, the craft teachers of the workshops will also be teachers in the works school; and, in every case where the works school is properly organised by an 'Apprentice Master,'* who is intimately connected with the management of the works, the contact between those who teach apprentices in the school and those who instruct them in the workshops will be very close. The harmony of purpose between the school and the works that can thus be secured will go far to obviate the danger of conflict between the lessons the young person learns in the works and those that are taught him by his master in the school. In particular, the teachers in the works school are more likely than some of their fellows in the schools outside to realise how great a public service is performed by productive industry.

Then again, although it is no more the main business of the works school than of any other Part-time Secondary School to select the abler apprentices for special promotion and higher training, there can be no doubt but that the management of the works, being in closer touch with the apprentices' school work if it is done in a works school than if it were done in a local authority's school, will be kept better informed of the school progress of the young people.

It has been argued that the education provided in a works school, not being under the direction of a local authority, may lack coordination with the system of education in force in the locality. This argument will have greater weight when effective steps have been taken to coordinate the work of the various schools conducted by the several separate local authorities in the same educational district. When once the system of schools and colleges throughout an educational area that is large enough to form a natural unit, with its centre in the

\footnotetext{
* See above, pp. 350, 420 and $42 \mathrm{I}$.
} 
local university (or-as in the case of Lancashire and Cheshireuniversities), has been coordinated by a single provincial authority, it will be time to complain if any works schools then remain outside the provincial authority's jurisdiction. At the present time, it is often easier for a central college to secure coordination with a works school than with the schools conducted by adjoining, and sometimes rival, local education authorities.

In these circumstances, we can hardly insist on works schools being placed under the (sometimes quite small) local authorities of to-day until these in turn have been combined into (or placed under the direction of) authorities for a complete educational area, of which, as we said*, there should be about a dozen in England. Meanwhile, however, the closer the cooperation $\dagger$ between the works school and the existing local authorities-and there may be many of themfrom whose areas it draws its pupils, and with the authority in whose area it is situated, the better for all concerned.

\section{§ 7. Elementary Schools.}

The practicability of separating ordinary Elementary Schools, for children under the age of twelve, from Central Elementary Schools, which provide senior elementary education for those children who have been left behind when their abler schoolfellows have been transferred to junior secondary education elsewhere, has already $\ddagger$ been discussed. Wherever this separation is practicable, it might well be made in the interests of the influences described at the opening of this chapter. Indeed, as the proportion of abler children who receive secondary education after the age of twelve increases up to or beyond the 50 per cent. shewn in our diagram, it will become less and less desirable that the ordinary Elementary School, attended alike by children of every grade of ability, should be dominated, not by the ablest, cleverest, or most virtuous children, and not even by the children who will later on be recognised as the most athletic, but by those who, although possessed of less than average ability, are biggest, and strongest, and most mature, simply because they are oldest. And yet the change we are here suggesting, desirable as it is and still more desirable as it will become when a great increase takes

* See above, p. 366, and Appendix E.

$\dagger$ Examples of such cooperation are furnished by cases in which the works school is conducted in a Technical School building used by the authority only in the evening and rented from them in the day-time by the factory concerned. In another case, the local authority provides the teachers and organises the school, while the firm concerned finds the building and all the pupils.

$\ddagger$ See above, p. 423. 
place in the number of children transferred from elementary to secondary education at the age of twelve, is by no means so urgently needed as some of the other changes proposed in earlier sections of this chapter.

\section{§ 8. Summary.}

Particularly pressing is the need for more residence and tutorial superintendence in modern universities; for a clear distinction in thought and practice between Higher Secondary Schools on the one hand, and Lower Secondary Schools* on the other; for replacing separate Junior Technical Schools by new 'sides' of ordinary Secondary Schools (and by seeing to it that the education provided by these schools for those of their pupils who are between fourteen and sixteen years of age, and who intend to enter employment on reaching sixteen, shall be of the senior secondary type which has in the past been provided by most Junior Technical Schools but by comparatively few Secondary Schools); and last, but by no means least, for introducing into the Part-time Secondary Schools of the future the atmosphere of public spirit, loyalty, and self-government that has hitherto belonged almost exclusively to whole-time Secondary Schools of ancient foundation.

* It is held in some quarters that Higher Secondary Schools alone should be recognised as Secondary Schools, while Central Elementary Schools should suffice to do for boys and girls above the age of twelve all that we have described as done by Lower Secondary Schools. In view of the different traditions of elementary and secondary education in this country, such a proposal would almost inevitably lead to a lowering of the standard of the work of the whole-time education of the large proportion of boys and girls who, before long, will stay at school until they reach the age of sixteen. In these circumstances, we cannot commend the proposal to replace Lower Secondary Schools by Central Elementary Schools, although at first sight it appears economical because the Board of Education are satisfied although Central Elementary Schools possess smaller playgrounds and inferior laboratories than are required of grant-aided Secondary Schools. 


\section{CHAPTER 25}

\section{A NATIONAL SCHOLARSHIP SYSTEM}

\section{$\S$ I. Maintenance Allowances.}

THE bringing into operation in England of such a system of education as is represented in our diagram* requires, not only the provision of the various types of education and the several types of school or college described in the two preceding chapters, but also the selection of a sufficient number of able young people, qualified by natural gifts and previous education to make the fullest use of this provision, and enabled to do so by scholarships and maintenance allowances. Indeed, the systematic selection of children and young people on account of their personal qualities alone, and irrespective of their place of residence or their financial resources $t$, to receive, if necessary at the public expense, whatever type of education will best fit them to serve their fellows and the community as a whole, is of the very essence of the system of education designed in our Book III $\ddagger$ for the purpose of realising the aim of education that we set forth at the end of Book II.

It is true that the Education Act of I9I8 obliges local education authorities 'to secure that children and young persons $\S$ shall not be debarred from receiving the benefits of any form of education by which they are capable of profiting through inability to pay fees.' $\|$ But this obligation refers only to young people under the age of eighteen; and, what is more, it refers to fees alone. Inability to pay fees is not the principal obstacle in the way of able young people of slender means who desire, and are otherwise qualified, to receive higher education. Until adequate maintenance allowances are added to the remission of fees, the father's income will be a more potent factor than his child's qualities in determining the education the child is to receive. It is therefore, as we said, an essential condition of bringing into operation in England the system of education represented in our diagram that every scholarship awarded shall be supplemented,

* Facing p. 319 above.

$\dagger$ See above, pp. 320 and 374 .

‡ The first chapter of this book (Book III, Chapter 20) makes this clear. See especially p. 322 above.

$\S$ The expression 'children and young persons' covers all young people under the age of eighteen.

II Section 4 (4) of the Education Act, I9r8. 
wherever necessary, by a maintenance allowance proportioned to the scholar's needs.

The Consultative Committee of the Board of Education in their Interim Report on Scholarships for Higher Education put the matter thus:

Roughly speaking, no Local Education Authority should give any aid to any candidate to go to the University unless it were prepared if necessary to pay the whole of his expenses; and the like is true of the Government in the case of Scholarships granted by Government. The main question to be decided by the awarding body with regard to each candidate is: Is this candidate fit to be assisted by public funds to receive University instruction? If he is not so fit, there is no need to give him any assistance at all*.

The principle thus applied to university scholarships is equally applicable in every other department of a national scholarship system. If a scholar is fit to be assisted by public funds to receive education of whatever type, and in whatever type of educational institution, the ainount of the assistance given him should be sufficient to enable him to derive the fullest advantage, not only from those direct educational influences discussed in Chapter 23, but also from those other influences considered in Chapter 24.

It may however be objected that, if maintenance allowances were added to the remission of fees, the cost of such a scholarship system as is represented by the arrows $\dagger$ in our diagram would be prohibitive. The cost would indeed be very great, but not, it is submitted, beyond the resources of this country. Even the most extravagant estimate + , based upon the high prices ruling in July, IgIg, shews that the whole cost of the scholarships and maintenance allowances would probably be less than the public expenditure on elementary education in England and Wales before the War, and less than one-sixteenth of the country's annual expenditure on alcoholic drink! $\S$ Moreover, there is no investment of public funds that produces so splendid a return in the material prosperity, and in the spiritual well-being, of the people, as results from expenditure on the right kind of education.

\section{§ 2. Provincial Authorities.}

The administration of national scholarships, together with such maintenance allowances as are required to secure that every kind of education is brought within the reach of all children and young people

* Loc. cit. pp. 58,59 .

† See above, Chapter 22, $\S 7$ (pp. 374, 375).

$\ddagger$ Such an estimate is given in Appendix G.

$\S$ See above, p. 374 . 
of sufficient educational promise, irrespective both of their place of residence in England or Wales and of their private financial circumstances, cannot be directly undertaken by the Board of Education; nor can such a system be effectively administered by existing local education authorities*. It is true that the Education Act of I9O2 entrusted the duty of supplying or aiding the supply of higher education to larger local education authorities than some of those $\dagger$ which it established for elementary education. But these higher education authorities have not proved large enough to envisage their responsibility for the highest education of all. The result is that, although responsibility for the highest education rests equally upon all the higher education authorities, a boy's chance of winning a university scholarship depends upon the particular town or county in which he happens to live $\ddagger$. Thus, for example, when in 19r3-I4 the city of Manchester was giving university scholarships costing .23 of a penny rate (and this in addition to other expenditure on university education amounting to a rate of $I \cdot 38$ pence), at least one county borough in Lancashire offered no university scholarships, and contributed nothing from its rates for university education either in Manchester or in Liverpool. And, indeed, a local education authority within whose borders there is no provision of some particular type of education is apt to neglect its responsibility for education of that type: it is likely to refuse to contribute to the cost of this type of education, whether provided by some other local authority or by an independent governing body; it may also refrain from offering scholarships that will enable young people resident in its area to obtain education of the type in question. We have seen§, too, that it would be out of the question for every existing higher education authority in England and Wales to attempt to secure the provision in its own area of every type of education represented in our diagram. Even the great county areas like that of Lancashire are, from our present point of view, as inchoate as a railway system all of whose junctions are under the control, if not of an enemy, at least of rival companies. Except only London, there is no administrative county in England that has a university or university college within its

* See above, Chapter 22, §2.

$\dagger$ Namely, non-county boroughs with a population over I0,000 and urban districts with a population over 20,000 .

$\ddagger$ See Appendix $\mathbf{H}$ for a statement (published in 1916 by the Council for Organising British Engineering Industry) of the scholarships tenable in universities and institutions of university rank offered by the higher education authorities (namely the county councils and county borough councils) in Lancashire and Cheshire.

$\S$ See above, p. 365 . 
area*. Moreover, non-residential Higher Secondary Schools, as well as Senior Technical Schools, are, for the most part, located in large towns, although a very considerable proportion of their pupils or students reside in the surrounding county areas.

If then we are right in supposing that equal educa tional opportunity will only be afforded to children of equal merit throughout the length and breadth of England when the administration of scholarships and maintenance allowances is entrusted to authorities large enough to envisage a complete system of education from the Elementary or Preparatory School to the University, it follows that new provincial education authorities $\dagger$ are needed, each concerned with one of the ten or twelve provinces into which we have already $\ddagger$ suggested that England and Wales should be subdivided for educational purposes.

While the first business of these provincial education authorities should be gradually to bring into existence a national scholarship system that will fulfil the conditions we have laid down§, they should, to an increasing extent, exercise a coordinating influence upon the activities of the various local education authorities and independent educational institutions in their respective provinces. For, in order to secure a smooth and continuous flow from the beginning to the end of our flow diagram, the amount of the flow at any point, and the educational channel that is to receive it, must be adapted to each other's dimensions. In other words, the provision of the various types of education in the various types of school or college represented in our diagram, and the number of young people selected and enabled by scholarships and maintenance allowances to follow each course of study in each educational institution, require mutual adjustment. Accordingly, the authority that administers the scholarships and maintenance allowances, and so aims at supplying the demand of the community-not only for the several classes (A, B, C, and D) of service represented in our diagram, but also for the many varieties of each class of service-by creating a sufficient flow of pupils into each

\footnotetext{
* If Cambridge and Durham are exceptions, they are exceptions of the kind that prove the rule.

$\dagger$ We have not followed the Education Reform Council (see Education Reform, being the report of the Education Reform Council published for the Teachers' Guild by P. S. King and Son, 19I7) in describing these authorities as 'Provincial Councils.' For reasons explained in an article that appeared in Nature on 3 rd April, 1919 (see above, footnote *, p. 366), we believe that Provincial Councils are needed for many purposes of local government in addition to education, and that, when such Councils come into being, each provincial education authority may be a department of one of them.

$\$$ See p. 366 above, and Appendix E on p. 497.

$\S$ See above, p. 374 .
} 
educational channel, ought also to be in some measure responsible for the channels into which they direct the flow*.

We would therefore gradually extend the functions of provincial education authorities, until each provincial authority becomes responsible, through the Board of Education, to Parliament for ensuring the provision, in its own province, of a system of education that will fulfil the conditions described in our preceding chapters, and that will make the scholarships and maintenance allowances (being considered in the present chapter) equally available for all young people of sufficient educational promise. This proposed extension of the powers and duties of provincial education authorities, established in the first instance for the administration of a national $\dagger$ scholarship system, does not mean the abolition of existing local education authorities; and still less does it imply the abolition of university autonomy. But it does mean that, in each province, there shall be one authority representative of the universities and of the existing local education authorities + , coordinating their work, and

* Compare, for example, the Education Reform Council's discussion of the training of teachers (loc. cit. pp. 21, 22). Compare also the following extract from a memorandum by the British Science Guild dated April, I919: 'Proper provision for the training of teachers also involves a task too great for the majority of the present authorities. These difficulties would be surmounted if for the purpose of Higher Education the country were divided into about ten Provinces roughly corresponding to the areas of the Universities, and Provincial Authorities were created out of the existing Local Authorities with the addition of University and Technical members.' And it is not only the training of teachers, but the training of all future members of Classes A and B that is beyond the power of the small higher education authorities of the present day. For, as we have seen, universities and Senior Technical Schools have an essential function to perform in the training of future members of these respective classes, and most higher education authorities are unrepresented on the governing bodies of universities, while many such authorities are even without a Senior Technical School.

$\dagger$ In order that the scholarship system administered by ten or twelve provincial education authorities may be a national system, each provincial authority should, whenever necessary, allow its scholars to hold their scholarships in schools or colleges located in a different province. Moreover, the scholarships awarded by a provincial education authority should not cease to be tenable with the removal of the scholar or his parents to another province.

$\ddagger$ And possibly also of the teaching profession in the province; and possibly of organised industry. Thus the recently established Federal Council of Lancashire and Cheshire Teachers' Associations, representative of the teaching profession in the two counties, might be given the duty of nominating the representative or representatives of the teaching profession on the provincial education authority for the North-western Province. Similarly Whitley, district councils for leading industries, or rather federations of all such councils within the province, might nominate representatives to serve on the provincial education authority.

The Federal Council of Lancashire and Cheshire Teachers' Associations, after a series of committee meetings held during the spring and summer of 1919 , under the present writer's chairmanship, passed a number of Recommendations concerning 'A National System of Education' having much in common with the proposals 
ensuring the adequacy of scholarships and maintenance allowances (as well as of the provision of each kind of educational institution and of each type and variety of education) for every inhabitant of the province, wherever he happens to live and however poor his family may be, provided only that his own educational promise is sufficient.

The executive power of these provincial authorities should only grow gradually. But from the first they should advise the existing education authorities, including the governing body of the local university. It should also be their duty to advise the Board of Education upon the education schemes submitted by the several authorities in accordance with Section 4 of the Education Act of I9r8; and in doing so they should have special regard to the inter-relations of these several partial schemes that together constitute the plan for the province as a whole. The executive power of the provincial authorities should however be increased, as their experience fits them for it, by the transfer of power to the provincial authority, both from the Board of Education and from the local education authorities. If experience justifies, that development might proceed until the existing education authorities, whether for elementary or higher education; become, in effect, local committees of the provincial authority, and until the Board of Education, while continuing to exercise some measure of control (by means of the government grants which it will then disburse through the provincial authorities), becomes chiefly concerned with the coordination of the work of the several provincial authorities, with the private provision of education in England, and with national systems of education abroad. To this end, the Board's department of Special Inquiries and Reports will need to be very greatly expanded and strengthened, until its work is recognised as second in importance to none of the other functions of the Board.

We have said* that the growth of the demand-determined, as it must largely be, by the provision of scholarships and maintenance allowances-for various types of education, and the increase of the supply of education to meet this demand, should be adjusted to one another. But the need for this adjustment is not to be made an excuse for unduly postponing the creation of a national scholarship system

contained in the present book. These Recommendations, with a Prefatory Note and an Introduction by the present writer summarising Chapters 22 to 25 of this book, and with a copy of his diagram (facing p. 319 above), have now (March, 1920) been published by the Manchester University Press.

* See above, pp. 456,457 . 
on the one hand, or, on the other, for delaying the necessary increase of the public provision of all kinds of education. On the contrary, each reform needs to proceed apace, and so to stimulate the other to keep up with it. And, if for a time one is to get ahead of the other, the lead should be taken by the national scholarship system. For nothing will so vividly bring home to local education authorities, and to governing bodies, the need for increased teaching strength, or for new buildings and equipment, as their inability to cope with the demand of qualified pupils for admission to their respective schools and colleges.

\section{§3. Multiplicity of Scholarships.}

Turning now to some other aspects of the national scholarship system, represented by the arrows in our diagram, and briefly described above, we first remind ourselves that these arrows mark selection for transfer to a higher type of educational institution, together with such money payments, or such increased money payments, as are necessary to enable the selected candidates to enter the higher school or college*. The ages at which the various selective tests should be applied and the scholarships awarded have also been considered in an earlier chapter $\uparrow$. It remains for attention to be here directed to the multiplicity of single- and double-headed arrows, corresponding to the many educational paths by which it should be

* Where selection takes place without differentiation in money payments between the selected candidates on the one hand, and those not selected on the other, there are no arrows. For example, where some pupils of a Lower Secondary School are selected at the age of fourteen to receive intermediate secondary education, while those not so selected enter upon senior secondary studies, there are no arrows. Again, where an increase of money payment takes place without renewed selection there are no arrows. For example, we have suggested in Appendix $G$ that at the age of fourteen the money value of maintenance allowances paid to necessitous scholars in Lower Secondary Schools be increased from a maximum of five shillings a week to a maximum of fifteen shillings a week, but no arrows mark this change.

† See above, Chapter 23. The scholarship selections represented in our diagram as taking place at or after the age of sixteen correspond closely with those recommended by the Consultative Committee of the Board of Education in their 'Interim Report on Scholarships for Higher Education' [Cd. 8291]. The Committee recommend:

'(I) That, in framing schemes for Scholarships, the following ends be kept in view: the training of men and women according to their capacity that they may serve the needs of the nation in the manner for which they are best fitted; the reward of merit and the encouragement of learning; and the provision of equal educational opportunity: the furtherance of industry, agriculture, and commerce, being regarded as a principal need of the nation, and Higher Education being regarded as a means to this end among others.

(2) That, for the furtherance of Higher Scientific and Technological Education, Scholarships from Secondary Schools to Universities and the Highest Scientific and Technical Colleges be still accepted as the principal means.' (Loc. cit. p. 7o.) 
possible, in any democratic system of education, for the various fields of useful service to be reached. In this connexion we emphasise again* the importance of such scholarships as are represented by the two vertical arrows on the twenty-years ordinate, by means of which an able boy, who may have passed directly from an Elementary School into employment at the age of fourteen, may later obtain admission to undergraduate studies in a university. Such a transition would have been impossible in Imperial Germany where the road to the university was rigidly defined. But English education must remain democratic. The loss to our national life that would result from refusing to admit to full university privileges students who approach the universities otherwise than through the normal avenue of the Higher Secondary School would indeed be deplorable.

\section{§4. Methods of Selection.}

The provincial education authority, charged with the business of awarding scholarships and maintenance allowances, will have carefully to consider the best means of making the necessary selection at the various points marked by arrows in our diagram. Many ways of making each selection will have to be tried before the best is discovered. The following suggestions are merely intended as a first approximation to the methods that may ultimately be employed.

Pending the results of further investigations into the nature and uses of such mental tests as $\mathrm{Mr}$ Burt $\dagger$ and other experimental psychologists have employed for the purpose of determining general ability, an essay and an arithmetic paper should remain the principal means of selecting boys and girls of about the age of twelve for transfer (with such scholarships or maintenance allowances as are required) from Elementary to Secondary or Junior Technical Schools; for $\mathrm{Mr}$ Burt, Dr Webb, and others have shewn how high is the correlation between general ability and success in examinations. Wherever the very large numbers $\ddagger$ involved in this scholarship test do not render oral examination impracticable, it would be desirable to combine an interview, such as forms part of the entrance test to

* See above, p. 395, especially footnote $\ddagger$.

$\dagger$ See above, pp. I05 et seq.

$\$$ The numbers submitted for the final test might be reduced by adopting a suggestion made by the Incorporated Association of Headmasters that a preliminary selection should first be made in the Elementary Schools. The Association recommend that 'Every elementary school should be required to furnish a list of boys of not more than eleven years of age who are of more than average ability and fitted to pursue a secondary education; and all of these boys should be submitted for examination.' 
Osborne, with the written examination at this stage*. And, when such an oral examination cannot be held, the examiners should be required to take into account a written report from each candidate's headmaster $\dagger$. In view of the high importance of 'purpose' among human qualities; the selectors of candidates for transfer might also do well to have before them short formal statements, from each candidate and from his parents, concerning theirwishes for his ultimate employment; but formal statements of this kind would be much more valuable in the case of selections of young people at and after the age of fourteen, since purpose does not begin to play its leading part in character until adolescence has set in $\S$. The duty of recommending to the provincial education authority, for selection, the names of candidates approaching the age of twelve might be left, in view of the large numbers involved, to a body of external examiners. The same examiners might also recommend the award of supplementary scholarships (represented by the ringed single arrow on the fourteenyears ordinate) to children approaching the age of fourteen who missed being selected at the age of twelve. But, in the case of all the other selections represented in our diagram, the teaching body of the institution to which each scholarship is intended to lead should play the chief part in recommending candidates for that scholarship; and they should always have interviewed the candidates they recommend. The selectors should, moreover, have before them reports from each candidate's headmaster, as well as formal statements of 'purpose'of the kind just alluded to-from the candidates and their parents, unless such statements prove in practice to be unreliable. And it should always be open to a Higher Secondary School, a Senior Technical School, or a university or college of university rank, to choose between selected scholars when the number presenting themselves for admission exceeds the available accommodation.

If, as has just been suggested, the test for the selection of scholars for transfer from Lower to Higher Secondary Schools at the age of

* It was suggested on p. 127 above that the principal object of such an interview is to determine the cleverness, $c$, of the candidate whose general ability, $g$, is approximately determined by his written examination papers. When $c$ as well as $g$ is determined in a scholarship test, any attempt to place candidates in order of merit should be based upon the sum of the squares of the measures of their cleverness and ability, rather than upon the simple sum of the marks obtained in the tests of these two independent qualities. In other words, the final mark that should determine a candidate's position in the order of merit should be proportional to $(\epsilon=) \sqrt{g^{2}+c^{2}}$ and not to $g+c$. Cf. p. I25 above.

$\dagger$ The usefulness of the headmasters' reports would be increased by their being drawn up in some standard form such as experience may shew to be the most suitable.

$\ddagger$ See above, p. 244 .

$\S$ See above, pp. 340, 34I.

G. E. 
fourteen, should rest in the first instance with the masters of the Higher Secondary Schools concerned, care must be taken to prevent the scholarship test having too close a relation to particular studies pursued in the Higher Secondary School. Even as the arithmetic and essay papers set to children approaching the age of twelve do not demand special knowledge that any of the candidates are likely to lack, so the selective test applied to candidates who are just completing their junior secondary education should not unduly favour those candidates who have made a special study of such subjects as Greek and Latin, that may be carried further by intermediate and advanced secondary studies on the classical sides of Higher Secondary Schools, but that form no necessary part of the future studies of those others who will proceed from junior to senior secondary education.

On the other hand, when boys or girls who are completing their intermediate secondary education are tested with a view to the transfer of selected candidates to Senior Technical Schools (by means of the scholarships represented in the diagram by the double-headed arrow on the sixteen-year ordinate), the test should not be wholly or mainly concerned with such general knowledge as is required for the School Certificate examination, although to have obtained this Certificate may well be a condition precedent to the award of a scholarship. Special excellence in the group of subjects which the candidates wish to pursue further during their subsequent whole-time educationand which should therefore have been studied in the more abstract ('transitional') way that is most likely to develope general ability or ' $g$ '*_-should more than compensate for mediocrity in other (comparatively 'terminal') subjects of the School Certificate examination. And this special excellence and ability had better be tested by means of special papers than by using papers set for the purpose of a pass examination $\dagger$. In particular, candidates who excel in an English essay or in a mathematical problem paper-excellence which requires ability, and cannot be attained by knowledge alone-should be preferred to the possessors of masses of miscellaneous information. The same is true of the means employed for selecting the few exceptional boys and girls who are to be transferred (by means of the scholarships represented by the single-headed arrow on the sixteen-years ordinate of our diagram) from Lower Secondary Schools

* See above, Chapter $2 \mathrm{I}, \S 8$.

† Compare the finding of Sir Joseph Thomson's Committee that: 'It is not desirable to use large pass examinations like the Local Examinations or Matriculation Examinations for the award of scholarships.' (Loc. cit. p. 6r.) 
to Higher Secondary Schools, or to the preliminary one-year university courses whose functions we described above*.

This principle is still more important in the case of selection for university entrance scholarships, whether the selection be made from boys or girls who are completing advanced courses in Higher Secondary Schools $\dagger$, or whether it be made from among older students attending part-timeclasses $\ddagger$. In either of these cases, the test of general knowledge, which, as we have said§, forms a necessary part of the test of fitness to enter a university, should be regarded as altogether subsidiary, if indeed it is taken into account at all, in deciding the order of merit of competitors for university entrance scholarships.

Research studentships, whether for students who have just graduated at another university (represented by the vertical arrow on the twenty-one-years ordinate) or for men and women who have already spent some years in whole-time employment (represented by the vertical arrow on the twenty-four-years ordinate), should not be awarded by examination at all, but after careful consideration of all the circumstances of each particular case, and, in the case of young graduates, having special regard to the opinions of their abilities formed by the professors under whom they have just been working II.

* See above, p. 392. The question whether the few exceptional young people who hold the scholarships here in question should hold them in the sixth-form of a Higher Secondary School or in a preliminary one-year university course must depend in part upon local circumstances and in part upon the age of the individual scholars at the time of their selection. Experience shews (see above, p. 392) that very few students who enter a university before the age of seventeen are sufficiently mature to profit by the comparative freedom of study that marks university courses, or by those other university influences that were discussed in Chapter $24, \S 2$. It follows that, other things being equal, a scholar selected appreciably before the age of seventeen for one of these supplementary (i.e. marked by a single-headed arrow) scholarships, had better hold his scholarship for two years in a Higher Secondary School, while successful candidates of seventeen years of age or upwards had better proceed direct to preliminary studies in a university. It should be added that the maximum annual value of the scholarship and maintenance allowance will, as pointed out in Appendix G, have to be greater if it is held at a university than if it is held at a Higher Secondary School.

$\dagger$ This selection is represented by the double-headed arrow at the top of the eighteen-years ordinate in our diagram (facing p. 3 rg).

$\ddagger$ This selection is represented by the two single-headed arrows on the twentyyears ordinate in the diagram.

$\S$ See above, pp. 394 and 395 .

II Thus the Consultative Committee of the Board of Education recommend in their 'Interim Report on Scholarships for Higher Education' that every holder of an undergraduate scholarship who satisfactorily completes his undergraduate studies should be allowed, on the recommendation of a professor, to remain up at his university for one or two years of advanced study and training in methods of research (loc. cit. p. 72). 


\section{CHAPTER 26}

\section{TEACHERS}

A PUPIL as he advances in years should, as we have observed*, gradually become his own chief educator. Accordingly, the university teacher need not be so much one who can teach, as one who knows and discovers, and who inspires with a love of knowing and discovering $\dagger$. Advanced study, and experience in research, are therefore the special qualifications of a university teacher. And, as we saw $\ddagger$, these should be combined, in the case of the professor or lecturer in a department of applied science or technology, with continued close personal experience of industrial practice: experience of a kind that can only be secured by a consulting practice $\S$ that brings the professor or lecturer into practical touch with works, mills, or factories, and places him in a position to command the fullest information concerning the processes and methods of the concerns that he advises.

The junior members of the teaching body of a university should possess qualifications similar to those expected of their seniors, although they may not have so long or so distinguished a record $\|$ as original investigators. It is true that in the past many of the lecturers and assistant lecturers in modern universities have not recognised that the advancement of knowledge is one of their functions. They have been content to prepare their students for university examinations, and in this work some of them have proved to be exceedingly effective teachers; but the number of lectures which they have been expected to deliver, added in some cases to an excessive amount of evening teaching, has prevented them from undertaking original research, even if experience of, and capacity for, research had been among their qualifications when first they joined the university teaching bodymanyyears ago. It would be impossible now, for the first time, to require original

* See p. 27 I above.

$\dagger$ Cf. p. 400 above.

$\ddagger$ See above, p. 349 .

$\S$ See above, pp. 406, 407 .

II Degrees and diplomas at best afford imperfect evidence of the qualifications of a university teacher or of any other member of the teaching profession. But, if a university professor may ordinarily be expected to be qualified for the Doctor's degree of an English university, according to the conditions under which such degrees have hitherto been conferred, the junior member of the teaching body of a university should be expected to possess the qualifications that are to be demanded from candidates for the Ph.D. degree as newly established in England (see above, p. 403). 
work from men who have grown old in the profession of university teaching. But the mistake must not be repeated. Care must in future be taken that even the most junior member of the teaching body of every university recognises that research and teaching are equally his duty. To this end, his teaching duties must allow him ample leisure for research.

Sixth-form masters, concerned with advanced secondary education in Higher Secondary Schools, should possess the same qualifications as junior university teachers. We have already* suggested that the sixth-form master should preserve close touch with university work, and that he should be engaged in original work on his own account, perhaps for this purpose spending part of his time in the precincts of a neighbouring university. But the schoolmaster, even more than the university teacher, needs to share his pupils' interests $\dagger$, and, by commanding their respect and even their admiration, to add an emotional interest to the studies with which he is concerned. For this reason, athletic prowess + , to which few English boys are indifferent, is a more important attribute of the schoolmaster than of the university teacher.

Members of the teaching body of a Senior Technical School require much the same qualifications as sixth-form masters in Higher Secondary Schools. But teachers concerned with senior technical education, whether in a Senior Technical School or in a Higher Secondary School, resemble university teachers of applied science and technology in requiring an intimate personal acquaintance with the group of employments into which their pupils are about to enter.

The same is true of teachers responsible for advanced part-time classes, whether provided in a university or in a Senior Technical School. For teachers of trade classes, on the other hand, practical experience of the trade, combined with knowledge of the principles underlying the trade practice, is the most important special qualification.

Schoolmasters who take the middle forms of a Higher Secondary School, or who are elsewhere responsible for intermediate secondary education, should generally possess an honours degree as evidence of a focussed single wide interest. They need not however undertake, or have undertaken, original investigations on their own account. On the other hand, the athletic and other qualities that will enable them to appeal to their pupils' interests and awaken their enthusiasm, are

\footnotetext{
* See above, p. 349 . $\quad \dagger$ See above, p. 346.

$\ddagger$ Cf. above, p. 433
} 
more important in their case than in the case of sixth-form masters. Moreover, some training as a teacher is a desirable, if not a necessary, qualification; since fourteen year old boys, who are just beginning their intermediate secondary education, are often very far from anxious to obtain the best education that their schools are able to give. They need to be taught.... And intermediate part-time classes should be taught by teachers possessing intellectual qualifications similar to those required for intermediate secondary education.

Teachers who are concerned with senior secondary or senior parttime education, require much the same qualifications as the formmasters responsible for intermediate secondary education, although an honours degree is less necessary for them. But, as in the case of members of the teaching body of a Senior Technical School, these teachers ought also to have had direct personal experience of the employment into which their pupils are about to enter, or (in the case of senior part-time classes) have already entered. It may be suggested that teachers of senior part-time classes attended by boys engaged in some particular industry, or group of industries*, would do well themselves to have entered employment in such an industry at an early age-say, after completing senior secondary education at the age of sixteen-and, four or five years later, to have been transferred by means of scholarships to an appropriate honours course in a university. The most suitable course will only be discovered by experiment and experience, but it is not improbable that the group of subjects that are coming to be known as the science of industrial administration might well form the staple of the honours course to be pursued by future teachers of industrial students in senior part-time courses. The training in teaching, which, as we have indicated, is desirable in the case of these teachers, ought immediately to follow their graduation.

In the case of teachers concerned with junior secondary and junior part-time education, an ordinary, instead of an honours, degree should suffice; but the course for the ordinary degree should be planned on the lines indicated above $\dagger$, so as to form an interest that is single and wide. For the rest, the qualifications of these teachers are much the same as for those concerned with intermediate secondary education, except that professional training is more necessary.

Senior elementary education requires much the same teaching qualifications as junior secondary, intended for boys and girls of the same age. But teachers engaged in senior elementary education ought, 
as far as possible, to have had some experience of the employment into which most of their children are likely to enter. Moreover, training as a teacher should be regarded as indispensable.

For the success of elementary and preparatory education, training as a teacher is of the first importance. A university degree is desirable, but by no means essential, for teachers of children under twelve years of age. Among the teachers of the younger children in Elementary Schools, there are, and always should be, many kindly women whose successful teaching is indisputable, but who would never pass the final examination for a university degree.

These brief notes on the qualifications of teachers engaged in providing the various types of education represented in our diagram, are little more than a summary of observations that have been made from time to time in the course of our enquiry. One further observation must be repeated and emphasised in conclusion. It is that the form-master's neurography tends, as we said*, to be reproduced in his pupils. Hence the supreme importance of training, appointing, and retaining, teachers of the right kind: teachers whose own education has brought them into close and equal fellowship with many sorts of men, has taught them to subordinate their personal interests to those of the society to which they belong, and has in fact been rich in the influences discussed in Chapter 24, as well as adequate from the standpoint of examination tests. In short, a perfect system of education requires, above all, perfect teachers; and perfect men and women, whatever their walk in life-be it teaching or any other-require a perfect education, an education that achieves its aim and so forms Christian characters $\uparrow$. 



\section{APPENDIX A*}

\section{The 'Broad Foundation' Metaphor; and some consequences}

OF ITS PREVALENCE AT THE PRESENT TIME.

So general is the use of the broad foundation metaphor in one form or another that, more than any other simple formula, it represents, not indeed the aim of English education as a whole (for as a whole, English education is, according to Chapter 3 above, characterised by aimlessness), but the partial aim of a large section of stateaided education. Its influence upon secondary education has been especially marked. It has, moreover, a semi-official character; for, although no unequivocal statement of even the proximate or provisional aim of our national system of education is contained in any official publication, the Consultative Committee of the Board of Education make free use of the metaphor of the broad foundation of liberal education.

Thus, in their 'Report on Examinations in Secondary Schools.' the Committee record their opinion that

Every Secondary School should provide, for pupils up to an average age of sixteen, a sound basis of liberal education which, though not necessarily of the same type in all schools, would serve as a foundation upon which varieties of further education could be based $\dagger$.

In their next report, the Committee repeat

It must... be held to be one of the functions of the Secondary School... to provide those of its pupils whose future callings may involve manual work...or the utilisation and control of such work with a foundation on which technical instruction may subsequently be built $\ddagger$.

If this building metaphor be considered merely as a metaphor, it is inferior to the plant metaphor of Pestalozzi and Fröbel. Indeed 'in the case of the Plant Metaphor which has had such a wide influence in modifying views of education...it may almost be said that we are not here dealing with an analogy at all, but with a type. The plant does not merely stand for an organism. It is an organism. If it be asked why, in that case, it is necessary in order to illustrate the development of the child, the answer is that the child as possessing

* See above, p. 5.

$\dagger$ Report (I91 I), p. IO4.

$\ddagger$ Report on Practical Work in Secondary Schools (19r3), p. 5. 
self-consciousness is an organism of a higher grade than the plant, is in fact a hyper-organism.... Organism is a better metaphor by far than machine; but it is a metaphor, and an inadequate one in every way.'* And the building metaphor is even worse than machine. It involves the heresy of the passivity of the educand, 'one of the most pestilent heresies in education.' $\dagger$

The building metaphor of the 'broad foundation,' on which any sort of superstructure may afterwards be built, further disregards the need for continuity in the development of an organism like the human brain or of a hyper-organism like the human soul. It presumes that, up till, say, the age of sixteen, a number of educands, destined for all kinds of different walks in life upon which they may have entered a year later, can well be studying exactly the same subjects in exactly the same waył.

Perhaps, however, the metaphor is not to be taken literally. How then does it work out in practice? The Board of Education requires that certain 'subjects' shall be taught in all Secondary Schools in receipt of government grants.

The emphasis upon subjects, and especially it would seem upon separate subjects, that appears to be the inevitable result in practice of aiming in theory at a broad foundation (on which, it is understood, any kind of superstructure may afterwards be built), has not lacked condemnation by leading educators.

'Milton' said Professor Perry from the presidential chair of the Education Section of the British Association, 'Milton first taught me the true notion of education, that the greatest mistake is in teaching subjects in water-tight compartments.' § 'Thorough knowledge of one subject, and practice in it,' said Goethe, 'produces higher culture than incomplete knowledge of a hundred subjects.' $\|$

In fact, since the theory of a general (as opposed to a specific) education, was first formulated, there have never been wanting warnings 'that we ought to abstain from dividing and parcelling out (morceler) what nature has made one and indivisible.' 9

Why then has the application to Secondary School curricula of the metaphor of a broad foundation produced instead a number of loose stones?

* Adams, loc. cit. pp. $285,286$.

$\dagger$ Adams, loc. cit. p. I 8.

$\ddagger$ This presumption is examined and condemned in Chapter $21, \S 8$. See, especially, p. 355 above.

$\S$ Nature, Ist October, I9I4, p. I3I.

II Quoted by Dr Georg Kerschensteiner, The Schools and the Nation, p. 256.

If A. Bertrand, L'Enseignement Intégral, p. 53. Quoted by Adams, loc. cit. p. I90. 
It may be that, like Latin in mediaeval schools, or like reading, writing, arithmetic and other subjects taught tochildren in Elementary Schools, the various subjects taught (under the influence of the broad foundation metaphor) to adolescent boys and girls are regarded as 'instrumental' subjects: subjects without which further education in the university, in the works, or in business would be impossible. But this explanation is not satisfactory. For Latin, a common enough subject in the Secondary Schools, is no longer an essential instrument for everybody. And, in view of the different methods by which Latin and modern languages are respectively taught in most English schools, if Latin be replaced by a modern language, the substitution will result in less demand for concentrated attention on the part of the educand and so, as we have seen, will interfere with the training of his character. The modern language may, however, be taught so as to be of use as an instrument in life and in further learning; but, if it is so taught, it will not be so tested. Many a man who can read and master foreign works upon a subject in which he is interested, and who can understand, and be understood, when he travels in the foreign country, would yet fail hopelessly to pass a School Certificate or Matriculation examination in that subject, an examination which might nevertheless be passed by one who could not use the language either to read or to converse with profit on any serious subject. It is equally certain that the study of a special period of history for some school examination is not an economical way of providing oneself with an 'instrument.'

If the 'instrument' explanation fails, can it be that certain kinds of knowledge-some of the loose stones of 'liberal education' which together go by the name of a broad foundation-are thought to be of value for their own sakes? Matthew Arnold defined culture as 'the acquainting ourselves with the best that has been known and said in the world.' * But unless, in Herbert Spencer's phrase, we 'turn fact into faculty,' unless our knowledge makes us act differently, it is of no value to us. There is no use in knowing all good if we are not educated to seek the highest when we see it.

The underlying ideal of the humanists (writes Professor Adams) is a liberal education, the education suited for a free man. When we go into the matter we find that what is understood by a free man is one who is not compelled to acquire any special skill for purposes of utility, but can give his time to subjects that are regarded as of special culture value. He can take up his time for example with literature, music, the fine arts. But in the last resort he is not regarded as a man who is cultured because

* Literature and Dogma, XIII (1876). 
he has studied those subjects, but because he is familiar with them. It is not that they have left a residuum of training and that he is therefore a better man than those who have not had the opportunity of being trained in the same way. He is esteemed because he knows the particular thing he has studied. If, as Ruskin assures us, a man damns himself for ever as a man of culture when he speaks of Iphigenía as Iphigénia, it is not because his ear has not been trained in general, but because he has not learned how to accent that particular word and others like it; in short, because he does not know things that people of a particular set do know. To be able to distinguish a Botticelli from a Fra Lippo Lippi marks a man as a person of culture....It is true that many men pass for cultured who do not know the subjects they are supposed to know, but still they are supposed to know them. The very fact that they must pretend to know certain things is a confession that a knowledge of some specific facts is regarded as in itself essential to culture. In plain English, the man of culture is taught certain things that are regarded as necessary to be known in the particular circle in which he moves. The training of a man of culture is as technical as the training of a civil engineer*.

Confusion between the acquisition of knowledge, necessarily the means of education, with the true end of education is by no means confined to the humanists. Professor Perry, in his address to the British Association in Australia, complained bitterly that there is not more natural science taught in our Secondary Schools and in our universities:

It is exasperating that all the most important, the most brilliant, the most expensively educated people in England, our poets and our novelists, our legislators and lawyers, our soldiers and sailors, our great manufacturers and merchants, our clergymen and schoolmasters, are quite ignorant of natural science.... The university man, ignorant of science, becomes a ruler of our great nation....and without turning a hair he fraudulently accepts this important duty for which he is utterly unfit....But I get too angry when I think of what our universities might do in the great world of natural science and of the futility of almost all their studies.... The general higher education of the community is being altogether neglected $\uparrow$.

Professor Perry's view appears to be shared by a number of influential men of science who have joined him in signing a recent memorandum on the 'Neglect of Science.' They urge the reform of the competitive examination for the higher appointments in the Home and Indian Civil Services, by giving 'a preponderating-or, at least, an equal-share of marks...to natural science subjects, with safeguards so as to make them tests of genuine scientific education.' $\dagger$

* Adams, loc. cit. p. 240.

$\ddagger$ Times, 2nd February, I9r6.

$\dagger$ Nature, ist October, I914, pp. I26, I27. 
A grateful nation should welcome any reform which would secure that all candidates for Civil Service appointments possessed a sufficient (instrumental) knowledge of natural science to enable them to discharge their duties effectively, and any reform which would secure that certain posts in the Civil Service were filled by men who had achieved distinction in the appropriate branch of natural science should be no less welcome. But the present method of selecting and educating civil servants has produced such satisfactory results from certain points of view that the reforms which are undoubtedly necessary ought not to be made without considerable circumspection. In securing the new that is good we are, in this connexion, warned by Lord Cromer* to be careful not to sacrifice whatever of the old is even better. The importance of Lord Cromer's warning is the more apparent when the memorandum we have quoted thus describes the consequences of the particular reforms proposed by the signatories:

...the object we have in view would be obtained. Science would rise in our schools to a proper position...our officials of all kinds, no less than members of Parliament, would come to be as much ashamed of ignorance of the commonplaces of science as they would now be if found guilty of bad spelling and arithmetic $\dagger$.

Such language gives rise to a fear lest knowledge about natural science be supposed to be of value 'for its own sake.' One needs to be ashamed of ignorance only in so far as it connotes incapacity to act as one should. The acquisition of knowledge, whether of natural science, or of 'the best that has been known or said in the world' is not the object of education. Mr Gradgrind was wrong in saying that 'what we want is facts,' and meaning that he preferred the knowledge about horses possessed by one child who could not behave intelligently $\ddagger$ in the presence of horses but could quote a long definition beginning 'Quadruped graminivorous,' to that of another who, although unable to define a horse, had spent her whole life with a travelling circus. And we shall be equally wrong if we suppose that mere knowledge of isolated facts that can be reproduced in a competitive examination, is comparable in importance with character, one of the most important constituents of which is, as we have seen, the kind of knowledge that is related to daily experience and modifies conduct in all its vocational and social aspects.

* In an address delivered to the Royal Society of Arts, following a paper by Mr P. J. Hartog entitled: 'Examinations in their bearing on national efficiency' (Journal of the Royal Society of Arts, 3rd and Ioth February, I9II).

$\dagger$ Loc. cit. italics mine.

$\ddagger$ Cf. Adams, loc. cit. p. 198. 
If now the various separate subjects, which, in practice, the 'broad foundation' metaphor gets taught in secondary schools, are not taught because they are going to be directly useful as instruments of further education; and if they are not taught because they are of value for their own sakes, whether as humanities or as sciences; are they perhaps taught in the hope that each subject will develop some faculty which, if not so cultivated, would be for ever stunted and thus leave the whole personality irreparably mutilated? If so, 'The breakdown of the doctrine of formal training'* dooms this hope to disappointment.

We have thus been unable to discover any intelligible educational aim which can justify the prevalent use of this metaphor or its consequences in the schools. Aimlessness alone can account for either the one or the other. This, or any other, product of aimlessness in education is not a matter of indifference. What is not good in education is very definitely bad, if for no other reason than that the time available for the education of even the most promising educand is so short. Of the particular product of aimlessness which we have considered as typical-the broad foundation metaphor-one of the greatest of living educators $\dagger$ writes: 'The most fatal demand which the higher schools have ever had to face is the demand for general allround education.' $\ddagger$

* Adams, loc. cit. p. 215. See also p. I7 above.

$\dagger$ This judgment is based on Dr Kerschensteiner's work before I9I4.

$\ddagger$ Kerschensteiner, loc. cit. p. 255. 


\section{APPENDIX B}

\section{Mathematical Appendix to Chapters 7 and 8.}

\section{$\S$ I. Frequency Distributions and Hierarchies*.}

Suppose that, in a series of mental tests, the performances of one of the persons, or subjects, tested are measured by $q_{1}, q_{2}, \ldots, q_{n}$ respectively in the several tests, $n$ in number. Then all available evidence goes to shew that, if the number of individuals tested is very large, the number, $p$, of subjects, the measures of whose performances in a given test lie between $q$ and $q+\delta q$, will be given by

$$
p=\frac{\mathrm{I}}{\sigma \sqrt{2 \pi}} \cdot e^{\frac{-q^{2}}{2 \sigma^{2}}} \cdot \delta q .
$$

That is to say, each of the $n q$ 's will be distributed according to the normal probability law. In this equation $\sigma$ measures the standard deviation of $q$ : the probable error divided by $\cdot 6745$. We shall suppose that the various performances are measured in such units that the $\sigma$, or standard deviation, is the same for all the $q$ 's in question.

If now we denote the correlation between any two $q$ 's, say $q_{s}$ and $q_{t}$, by $r_{s t}$, the relation which Professor Spearman found to obtain between the correlations in sufficiently dissimilar mental tests may, as Mr Cyril Burt $\uparrow$ has pointed out, be conveniently expressed by the equation

$$
\frac{r_{a s}}{r_{a t}}=\frac{r_{b s}}{r_{b t}} \quad(a, b, s, t=\mathrm{I}, 2, \ldots, n) \ldots(\mathrm{I}) .
$$

When the relations $\ddagger$ expressed by this equation obtain between the several correlations $(r$ 's), these correlations are said, in Professor Spearman's phrase $\S$, to form a 'hierarchy.' In actual practice, Professor Spearman found that the conditions for a hierarchy, expressed by equations (I), were approximately satisfied by the correlations between the performances recorded in his first paper. Professor

* See above, p. ro5.

$\dagger$ $\dagger$. J.P. Vol. III, p. I59, where Mr Burt says that equation (I) is immediately deducible from an equation $(f)$ previously given by Spearman and Krüger, Zeit. f. Psych. Vol. 44, p. 85. In a subsequent paper (B. J. P. Vol. v, I9I2-13, p. 65) Professor Spearman and Dr Hart refer to the equation as 'Burt's equation.' For convenience of reference it has also been described by $\mathrm{Mr}$ Burt's name in papers by the present writer, Proc. R. S. (A), Vol. 96 (I9I9), pp. 9r et seq., and B. J. P. Vol. IX (1919), pp. 345 et seq., although, as Mr Burt acknowledged, it follows directly from Professor Spearman's earlier work.

$\ddagger$ The number of independent relations, expressed by equation (I), between the $\frac{1}{2} n(n-\mathrm{I}) r$ 's is $\frac{1}{2} n(n-3)$. See Garnett, B.J.P. Vol. X (I920), pp. $245,257$.

$\S A m . J . P$. Vol. I5 (1904), p. 274. 
Spearman pointed out that a perfect hierarchy would result-or, in other words, that the conditions for a hierarchy would be completely satisfied-if the correlations in question were wholly due to the presence of a single general factor*. Accordingly, the tendency of the correlations, in the cases described by Professor Spearman in his first (I904) paper, to satisfy equations (I) and so to form a hierarchy, was consistent with Professor Spearman's theory that these correlations are principally due to the operation of a single general factor.

\section{§2. Mr Burt's Correlation Tables $\uparrow$.}

The following is Mr Burt's discussion of the question how far the correlations recorded in his tables satisfy the conditions for a hierarchy set forth in equation (I) above:

How far [he writes] ...do these observed correlations form an ideal hierarchy? They clearly do not fit into the proposed scheme with perfect precision. Nor indeed can we expect them to do. Like all empirical observations they are subject to error. What we have to demand is the following.

Firstly, the deviations from the ideal hierarchy should, on the whole, be neither more nor less than the probable erroneousness of the observations. The theoretical values for the ideal hierarchy... are given in the tables, together with the deviations of the observed coefficients from them, and the probable errors for the coefficients are given in two decimal places only. For the Elementary School group, the average deviation works out at $\cdot$ Ioo, while the average probable error comes to -IOI. For the Preparatory School group the average deviation works out at $\cdot{ }^{1} 6_{5}$, while the average probable error comes to $\cdot{ }^{\prime} 62$. So far, then, a neater agreement between observation and theory could scarcely be desired.

* The proof is as follows: It has been shewn (Garnett, Proc. R. S. (A), Vol. 96 (I9I9), equations (7) and (8), p. 94; see also Garnett, $B . J . P$. Vol. x (I920)) that each of the $n q$ 's may be expressed as a linear function of a number $N$, where $N \Varangle n$, of independent variables $x_{1}, x_{2}, \ldots, x_{N}$, each of which is distributed according to the normal law with the same standard deviation as before, by means of equations of the form

where

$$
\begin{gathered}
q_{s}={ }_{s} l_{1} \cdot x_{1}+{ }_{d} l_{2} \cdot x_{2}+\ldots+{ }_{s} l_{N} \cdot x_{N} \\
{ }_{s} l_{1}^{2}+{ }_{s} l_{2}^{2}+\ldots+{ }_{s} l_{v^{2}}=\mathrm{I}
\end{gathered} \quad(s=\mathrm{I}, 2, \ldots, n) \ldots(2),
$$

It has also been shewn (Garnett, Proc. R. S. Vol. 96, equation (I6), p. 96) that

$$
r_{s t}={ }_{s} l_{1} \cdot l_{1}+{ }_{s} l_{2} \cdot l_{2}+\ldots+{ }_{s} l_{N} \cdot l_{N} \text {. }
$$

If all the $q$ 's have only one common factor, say $x_{1}$, and no group factors-i.e. $x$ 's having finite coefficients in more than one of the equations (2)-we therefore have

so that

$$
\begin{array}{cc}
r_{s t}=l_{1} \cdot l_{1} l_{1} & (s, t=\mathrm{I}, 2, \ldots, n) \\
\frac{r_{a s}}{r_{a t}}=\frac{r_{b s}}{r_{b t}} & (a, b, s, t=\mathrm{I}, 2, \ldots, n) \ldots(\mathrm{I}) .
\end{array}
$$

$\dagger$ See Tables I and II on pp. Io6 to Iog. 
Hardly less reassuring is the accordance disclosed on turning from the average deviation to the extreme deviations. In a 'normal'* chance distribution, we should expect a deviation three times greater than the probable error to occur about once in 24 times. Here we have 78 coefficients for each group. Here, then, we should expect such a deviation to occur about three times in each. Actually, it occurs four times in the Elementary School, and twice in the other. Again, a deviation four times as great as the probable error may be expected to occur by mere chance about once in I 24 times. Here it occurs twice in the Elementary School, and not at all in the other. Some of these deviations are themselves suggestive. At the Elementary School, three of the four large deviations occur with Imputed Intelligence...; such irregularities are here quite natural, since the method of estimating intelligence was not homologous with the methods of estimating the other capacitiest.

\section{§3. Hierarchical Correlations}

On p. IIo above, reference is made to a paper by the present writer in which he investigated the consequences that follow whenever the correlations between the measures of mental tests-or between any other variables that are distributed according to the normal law* with the same standard deviation-satisfy the conditions for a hierarchy given in equations ( $\mathrm{I}$ ) on p. 475. Mathematically expressed, the result is that, if the correlations between $n$ variables, $q_{1}, q_{2}, \ldots, q_{n}$, each of which is distributed according to the normal law with the sane standard deviation, satisfy the conditions for a hierarchy expressed in equations ( $\mathrm{I}$ ), the $q$ 's may be expressed, by means of 'vector' equations of the form

$$
q_{s}=r_{s g} \cdot g+\left(\mathrm{I}-r^{2}{ }_{s g}\right)^{\frac{1}{2}} \cdot x_{s}=g \cdot \cos \theta_{s}+x_{s} \cdot \sin \theta_{s}, \quad(s=\mathrm{I}, 2, \ldots, n) \ldots(4)
$$

as linear functions of $n+I$ independent variables, each of which is distributed according to the normal law with the same standard deviation as that of the $q$ 's, and among which one $(g)$ is a single general factor $\S$ while each of the $n$ others $\left(x_{1}, x_{2}, \ldots, x_{n}\right)$ is a specific factor, no group factors being present. In the above equations (4), $r_{s g}$

* For the definition of the 'normal' probability law or normal chance distribution, see above, $\S$ I of this Appendix.

$\dagger$ Loc. cit. pp. 163, 164.

$\ddagger$ See above, p. Iro.

$\S$ In what follows we shall use the term 'single general factor' to denote only that one of $n+I$ independent factors (each distributed according to the normal law with the same standard deviation) which-when $n$ correlated $q$ 's (each again distributed according to the normal law with still the same standard deviation) are expressed in terms of them alone, and therefore (see footnote * on p. 476 and equations (4) above) as linear functions of them-enters into each of the $q$ 's, while the remaining $n$ independent factors are specific, each entering into a different $q$. This definition and reasons for the need of some such definition of a 'single general factor' are given in a recent paper on 'General Ability, Cleverness and Purpose' (Garnett, B. J. P. Vol. Ix (1919), p. 347).

G. $\mathbf{E}$. 
is the coefficient of correlation between $q_{s}$ and the general factor, $g$; and $\theta_{s}=\cos ^{-1} r_{s q}$. There are no group factors.

\section{$\S 4$. Dr Thomson's Dice.}

It is true that Dr Thomson (B. J. P. Vol. virl (Sept. I9I6), pp. 27 I et seq.) has constructed correlation tables shewing the correlations between dice-throwing tests among which at first sight there appears to be no general factor but only group factors, and that Dr Thomson's correlation tables correspond as closely with perfect hierarchies as do the correlation tables (Tables I and II) obtained by Mr Burt. It has, however, been shewn (Garnett, B.J.P. Vol. x (March, I920), pp. 242 et seq.), firstly, that the chance of obtaining, by means of group factors and without a general factor, so close an approximation as that obtained by Dr Thomson to the satisfaction of the conditions for a hierarchy in a set of experimental tests such as those of Mr Burt is less than one in one hundred thousand million; and, secondly, that the correlated variables in such dice-throwing tests as those of Dr Thomson, although they appear at first sight to possess no general factor but only group factors, do, in fact, become expressible in terms of a single general factor with specific factors only, when the number of independent variables, and therefore the number of dice, become infinite; and it is only when the number of independent variables, and therefore the number of dice, is infinite that perfect hierarchies can be obtained in dice-throwing tests of this kind.

\section{$\S 5$. Imperfect Hierarchies*.}

The tendency of the measures of dissimilar mental qualities to be linear functions of a single general factor and specific factors only, without group factors, may be expressed by saying that, if $q_{1}, q_{2}, \ldots, q_{n}$ are the measures of the several qualities tested, then

$$
q_{s}=l_{s} \cdot g+m_{s} \cdot x_{s}+{ }_{s} n_{1} \cdot z_{1}+{ }_{s} n_{2} \cdot z_{2}+\ldots,
$$

where $q_{s}$ is the measure of the sth quality tested; $g$ is the general factor (which would be a single general factor, as defined above $\dagger$, were the coefficients of the group factors all zero, instead of merely being on the average very small); $x_{s}$ is a specific factor; $z_{1}, z_{2}, \ldots$ are group factors whose coefficients ( $n$ 's) tend severally and in the aggregate to be very small compared with the coefficients ( $l$ 's) of $g$ or ( $m$ 's) of the specific factors; and, as before, each of the $q$ 's and other variables is distributed according to the normal law with the same standard deviation. 


\section{§6. Professor Spearman's 'Correlation between Columns' Conditions*.}

On p. II 2 above, reference $\dagger$ is made to the present writer's investigation of the consequences of the fulfilment of Professor Spearman's 'correlation between columns' conditions. The result of that investigation is to shew that, if the correlations between columns conditionsnamely, that the correlation between every pair of columns in a correlation table is $\pm \mathrm{I}$-are satisfied by the correlations between $n$ variables, $q_{1}, q_{2}, \ldots, q_{n}$, each of which is distributed according to the normal law with the same standard deviation, and each of which, we may suppose, measures some mental quality tested, then the difference between each of the correlated $q$ 's and a constant multiple of another variable $y$ (that is independent of the $q$ 's but distributed according to the normal law with the same standard deviation) may be expressed in terms of a single general factor and specific factors only. Put mathematically, the consequence of the satisfaction of the correlation between columns conditions is that $\ddagger$ $q_{s}^{\prime} \equiv\left(q_{s}-y . k\right)\left(\mathrm{I}+k^{2}\right)^{-\frac{1}{2}}=g \cdot \cos \theta_{s}+x_{s} \cdot \sin \theta_{s} \quad(s=\mathrm{I}, 2, \ldots, n) \ldots(6)$,

where $k$ is a constant, and where $x_{1}, x_{2}, \ldots, x_{n}, g$ are $n+\mathrm{I}$ independent variables, each of which is distributed according to the normal law with the same standard deviation as that of the $q$ 's (or of the $q^{\prime \prime}$ s defined by this equation), and among which $g$ is a single general factor while all the $x$ 's are specific factors, no group factors being present. When $k$ becomes zero (as happens whenever the conditions for a hierarchy in equations ( $I$ ) are satisfied), equations (6) become the same as equations (4); in which case the $q$ 's will be expressible, according to the vector law of equations (4), in terms of $n+\mathrm{I}$ independent variables, each of which is distributed according to the normal law, and one of which is a single general factor while the others are specific factors. And this happens when the $q$ 's measure sufficiently dissimilar mental qualities; for $k$ then becomes zero and the conditions for a hierarchy are satisfied by the correlations of the $q$ 's.

\section{\$7. Imperfect Fulfilment of the Conditions §.}

The consequences of the imperfect fulfilment of the 'correlation between columns' conditions are stated above on p. II2. They may be otherwise expressed as follows: The fact that the correlations

* See above, p. II 2 .

† Garnett, Proc. R. S. (A), Vol. xcvi, pp. I02-5. $\cos \dot{\theta}_{2}$.

$\ddagger$ Garnett, loc. cit. equation 53, p. 105, $l_{s}$ in that equation being replaced by § See above, p. II2. 
between every set of mental tests approximately satisfy the "correlation between columns' conditions or the conditions for a hierarchy, is evidence that the measures of the qualities tested (or, if the correlated qualities are not sufficiently dissimilar so that the conditions for a hierarchy are not satisfied, the difference between these variables and a real multiple of an $\overline{n+\text { Ith }}$ variable independent of them all) are made up, firstly and principally, of a single general factor and specific factors, and secondly and to a comparatively insignificant extent, of group factors. Or, put mathematically, our result is that*

$$
q_{s}^{\prime} \equiv\left(q_{s}-k y\right)\left(\mathrm{I}+k^{2}\right)^{-\frac{1}{2}}=l_{s} \cdot g+m_{s} \cdot x_{s}+{ }_{s} n_{1} \cdot z_{1}+{ }_{s} n_{2} \cdot z_{2}+\ldots
$$

where $q_{s}$ is the measure of the sth quality tested in such units that all the $q$ 's have the same standard deviation; $k$ is a real quantity which vanishes when the conditions for a hierarchy are satisfied; $g$ is the general factor (which would be a single general factor as defined above $\dagger$ were the coefficients of the group factors all zero instead of only being on the average very small); $x_{s}$ is a specific factor; and $z_{1}, z_{2}, \ldots$ are group factors whose coefficients ( $n$ 's) tend severally and in the aggregate to be very small compared with the coefficients $(l$ 's) of $g$, or ( $m$ 's) of the specific factors. In this equation, $g$, the $x$ 's, and the $z$ 's are independent variables, each of which is distributed according to the normal law with the same standard deviation as that of the $q$ 's.

The same result may be expressed by saying that the measure, $q_{s}$, of the sth of $n$ sufficiently dissimilar mental tests tends to be cornpounded, according to the vector law of equation (4), namely

$$
q_{s}=g \cdot \cos \theta_{s}+x_{s} \cdot \sin \theta_{s}, \quad(s=\mathrm{I}, 2, \ldots, n) \quad \ldots(4),
$$

of a single general factor $(g)$ common to all the tests, and of a specific factor $\left(x_{s}\right)$ independent of the general factor and peculiar to the sth test, the specific factors ( $x$ 's) entering into the several tests being also independent of each other; but that, in the case of a set of insufficiently dissimilar mental tests, $q_{s}$ in the above equation must be replaced by $q_{s}^{\prime}$ or $\left(q_{s}-k y\right)\left(\mathrm{I}+k^{2}\right)^{-\frac{1}{2}}$, where $y$ and $k$ have the same meanings as before + .

* See Garnett, B. J. P. Vol. Ix, pp. $365,366$.

$\dagger$ In $\S 3$ of this Appendix: see footnote $\S$ to p. 477.

$\neq$ In equations (4), as in equations (7), the $q$ 's, the $x$ 's and $g$ (and $y$ when present) are each distributed according to the normal law, and measured in such units that their standard deviations are the same. 


\section{§ 8. Will and General Ability*.}

Mr Burt's tests furnish the following further item of evidence that, if Will exists as a general factor $\uparrow$, it must be the same quality as that which is measured by $g$, the single general factor, and which, following Professor Spearman and Dr Hart + , we have called 'General Ability.'

If the Will exists and ever enters into anything, then the Wills of the subjects of Mr Burt's tests $\S$ certainly entered into many, if not into all, of these tests.

Of the two groups [of reagents, says Mr Burt], the Preparatory School boys were perhaps slightly superior in conscientious steadiness and care; while the Elementary School boys (who were markedly pleased at the interruptions of their regular routine, and were further fortified in their specially prolonged examination by the promise of a prize for the best) were perhaps slightly superior in spontaneous interest and attention.

It is clear then that the boys of both groups did try. Whoever will repeat, say, the alphabet test against the clock will be convinced that they must have tried, for he will be conscious of his own effort of Will. Moreover, we have already cited the evidence of DrMcDougall, to whose suggestion Mr Burt's research owed its origin\|, concerning the profound effect of Will in mental tests, and especially in memory tests $\uparrow$.

If then, we suppose that the measure, $W$, of a subject's Will is, like the measures of other mental qualities, distributed among different subjects according to the normal law, and that the scale on which $W$ is measured is such as to give to $W$ the same standard deviation as that of the measures of ' $g$ ' and of the mental qualities tested, it follows, as we have seen $* *$, that $W$ may be expressed as a linear function of a number of independent variables among which we may include $g$ and the specific factors of Mr Burt's tests. And, since $W$ enters most obviously into those tests that have the highest correlations with each other, and therefore $\dagger \dagger$ with $g$, it is reasonable to assume that $W$ has a finite correlation with $g$. We may then, if we please, express $g$ as a linear function of $W$, and of any number of independent variables $x_{1}, x_{2}, \ldots, x_{n}$, each of which is independent of $W$ and distributed according to the normal law with the same standard deviation as that of $W$ and of $g$. So we may write

$$
g=L W+l_{1} x_{1}+l_{2} x_{2}+\ldots+l_{n} x_{n},
$$

* See above, p. I16, especially footnote §.

$\ddagger$ See above, p. I03.

II C. Burt, loc. cit. p. 95 .

** On p. 476 , footnote *, equation (2). $\dagger$ See above, p. Ior.

$\S$ See above, pp. I05 et seq.

Tा See above, p. roo, footnote $\uparrow$. †† See above, p. II3. 
where $L, l_{1}, \ldots, l_{n}$ are constants, independent of the particular subject. (person) tested and connected by the relation

$$
L^{2}+l_{1}^{2}+l_{2}^{2}+\ldots+l_{n}^{2}=\mathrm{I},
$$

and where $x_{1}, x_{2}, \ldots, x_{n}$ are the measures of general qualities which belong to the same person but are independent of $W$ and of each other. Moreover, if the same person's performance in any one of a set of sufficiently dissimilar mental tests-for example, in the alphabet test-is measured by $q_{s}$, then approximately*

$$
q_{s}=g \cos \theta_{s}+\xi_{s} \sin \theta_{s}
$$

where $\theta_{s}$ is a constant for that test, being as before independent of the particular person tested, but where $\xi_{s}$ measures a specific quality of the person which enters into that test but into no other.

We have to shew that $l_{1}=l_{2}=\ldots=l_{n}=0$. If possible, suppose that $l_{1}$ is not zero. Then $x_{1}$ measures some general quality of the person tested which is independent of his Will, but which enters into each of the tests along with his Will, and in a degree which bears a constant ratio $\left(l_{1}: L\right)$ to the degree in which his Will enters into the same test. Some group factors, independent of Will, have been discussed above; but none of them satisfies these conditions; and we have no knowledge of any other general factor that might do so. If, for example, we return to Mr Burt's 'so-called "voluntary" attention' and observe that it may include 'spontaneous interest' as well as 'willed' attention, we cannot identify 'spontaneous interest' with our hypothetical factor whose measure is $x_{1}$; for the spontaneous interest of any individual tested, must vary from one test to another according to that particular individual's interest in the subject matter of each test, and this variation is quite independent of the variation (measured by the variation of $\theta_{s}$ in equation (4) from one test to another, and therefore quite independent of the particular person tested) of the degree in which his Will can influence the result of the test. In short, we know of no general quality $x_{1}$ that can satisfy the prescribed conditions. It is therefore in accordance with all the facts known to us to assume that $l_{1}=0$; and similarly that $l_{2}=l_{3}=\ldots=l_{n}=0$. It follows that $L=\mathrm{I}$ and therefore from equation (4) that $g=W$.

So our two assumptions, one on this and one on the preceding page, lead to a further indication that Will is identical with the general factor whose measure is $g$.

The view that $g$ measures power to concentrate attention by an effort of Will, or, in neural terms, power to reinforce excitement in * Equation (4) in $\S 7$ of this Appendix (on p. 480 above). 
any active system of higher level arcs, finds further confirmation in Dr Webb's elaborate investigation. Of the forty-three correlations of ' $g$ ' with the forty-three qualities estimated by the prefects*, the greatest is 0.60 which measures the correlation between ' $g$ ' and 'extent of mental work bestowed on usual studies (No. 28 †).' The correlation of ' $g$ ' with the objectively measured Examinational Ability is 0.67 . The only other correlations of ' $g$ ' that exceed 0.50 are those with three of the intellectual qualities, namely, Quickness of Apprehension, Profoundness of Apprehension, and Power of getting through mental work rapidly.

Dr Webb has supplied further particulars of the estimated quality - 'extent of mental work bestowed on usual studies'-which the general factor correlates most highly. In an appendix he has collected selections 'from the reports of the observers as to what they understood by the terms used in the schedules, and what guided them in marking their subjects.' $\ddagger$ The following are some of the entries under the head 'extent of work bestowed on usual studies':

The thoroughness with which he attended to his work.

The + men possessed power of concentration-difficulties did not throw them off a task.

The + men worked hard and conscientiously; their work was well prepared and evidenced much thought and reading.

The + men, even when they failed, seemed to have been thoughtful.

Comparison of these entries with those under the other heads makes it clear that this quality,- 'extent of mental work bestowed on usual studies' - which, of all the estimated qualities, correlates most highly with ' $g$ ' ' is also the quality which most involves Will, or power to concentrate attention: an observation that is in accordance with the conclusion-namely that $g$ measures Will-to which we have already been led §.

\section{§ 9. The Co-planar Condition $\|$.}

The condition that the measures of three or more correlated qualities should depend on two, and two only, independent variables may be obtained as follows.

We have already $\uparrow$ seen that the measurements $\left(q_{1}, q_{2}, \ldots, q_{n}\right)$ of the qualities tested in Dr Webb's investigation may be expressed as linear functions of a number $N$ (where $N \nleftarrow n$ ) of independent variables,

\footnotetext{
* See above, p. I20, and footnote $\ddagger \ddagger$ to p. II 9 .

$\dagger$ In Table III, on p. I26 above. $\quad \ddagger$ Loc. cit. (supra, p. 98) p. 84

$\S$ See above, p. II 7 .

I See footnote * to p. 476 in $\S$ I of Appendix B.

II See above, p. I20.
} 
$x_{1}, x_{2}, \ldots, x_{N}$, each of which is distributed according to the normal law with the same standard deviation as that of the $q$ 's. For example,

where

$$
\begin{aligned}
q_{s}= & { }_{s} l_{1} \cdot x_{1}+{ }_{s} l_{2} \cdot x_{2}+\ldots+{ }_{s} l_{N} \cdot x_{N} \\
& { }_{s} l_{1}^{2}+{ }_{s} l_{2}^{2}+\ldots+{ }_{s} l_{N}^{2}=\mathrm{I}
\end{aligned}
$$

It may also be shewn that the correlation, $r_{s t}$, between any two of the $q$ 's, $q_{s}$ and $q_{t}$, is given according to the cosine law* expressed by

$$
r_{s t}={ }_{s} l_{1} \cdot{ }_{t} l_{1}+{ }_{s} l_{2} \cdot{ }_{t} l_{2}+\ldots+{ }_{s} l_{N} \cdot{ }_{t} l_{N}
$$

However great may be the number, $N$, of independent variables we can, by a linear transformation, choose new independent variables $y_{1}, y_{2}, y_{3}, \ldots$ such that two variables, $q_{s}$ and $q_{t}$, depend on two and two only among them, say $y_{1}$ and $y_{2}$. Equations (2) may then be replaced by

so that

$$
\left.\begin{array}{l}
q_{s}=y_{1} \cos \theta_{s}+y_{2} \sin \theta_{s} \\
q_{t}=y_{1} \cos \theta_{t}+y_{2} \sin \theta_{t}
\end{array}\right\}
$$

This equation may be interpreted by saying that, if we measure the $y_{1}$ and $y_{2}$ of any person along two axes $O y_{1}$ and $O y_{2}$ at right angles to one another, and plot the point, $P\left(y_{1}, y_{2}\right)$, corresponding to that person, so that $y_{1}$ and $y_{2}$ are the projections of $O P$ on $O y_{1}$ and on $O y_{2}$ respectively, and if we then draw a line $O q_{s}$, which we may call the axis of $q_{s}$, making with $O y_{1}$ an angle $\theta_{s}$ (measured in the direction from $O y_{1}$ to $O y_{2}$ ), the degree in which the person possesses the quality measured by $q_{s}$ will be equal to the projection of $O P$ on $O q_{s}$. Moreover, the correlation between $q_{s}$ and $q_{t}$ is equal to the cosine of the angle $q_{s} O q_{t}$; so that this correlation represents the average deviation in $q_{s}$ (or $q_{t}$ ) corresponding to unit deviation in $q_{t}$ (or $q_{s}$ ). We are thus afforded a very simple geometrical conception of the measure of correlation.

The condition that three qualities $q_{1}, q_{2}$ and $q_{3}$ should be expressible in terms of two, and only two, independent factors follows at once. It is the condition that $O q_{1}, O q_{2}$ and $O q_{3}$ should lie in a plane; or that the sum of the angles $q_{2} \hat{O} q_{3}, q_{3} \hat{O} q_{1}$, and $q_{1} \hat{O} q_{2}$ should be zero; or that

or that

$$
\cos ^{-1} r_{23}+\cos ^{-1} r_{31}+\cos ^{-1} r_{12}=0
$$

$$
r_{23}^{2}+r_{31}^{2}+r_{12}^{2}-2 r_{23} r_{31} r_{12}=\mathrm{I}
$$

* Garnett, Proc. R. S. equation (I6), p. 96 


\section{$\S$ 10. The Correlations of Cleverness*.}

The correlation of $c$ (the measure of Cleverness, as we have defined it $\dagger$ ) with the forty-eight qualities investigated by Dr Webb may be calculated as follows. Let us take the general factor measured by $g$, and Cleverness measured by $c$, as two of the independent variables in terms of which any of Dr Webb's qualities may be expressed. Then the measure of any of his qualities is given by equation (2) $\ddagger$, which now becomes

$$
q=r_{g q} \cdot g+r_{c q} \cdot c+l_{3} x_{3}+l_{4} x_{4}+\ldots+l_{N} x_{N}
$$

where $x_{3}, x_{4}, \ldots, x_{N}$ are the remaining independent variables. Any of the qualities represented in our diagram, say Humour, is measured by

$$
h=r_{g h} \cdot g+r_{c h} \cdot c=r_{g h} \cdot g+\sqrt{I-r_{g h}} \cdot c,
$$

so that, according to the cosine law in equation $(8) \S$,

$$
r_{q h}=r_{g q} \cdot r_{g h}+r_{c q} \cdot \sqrt{\mathrm{I}-r_{g h}} \text {, }
$$

from which we obtain

$$
r_{c q}=\frac{r_{u h}-r_{g q} \cdot r_{g h}}{\sqrt{\mathrm{I}-r_{g h}^{2}}}
$$

All the $r$ 's on the right-hand side of this equation are given in Dr Webb's table\| of corrected coefficients for the students. If we substitute Dr Webb's values and calculate $r_{c q}$ for all values of $q$, except $q=g$ and $q=h$, we obtain the series of coefficients of correlation printed in Table III on pp. I 26 and I 27 above.

\section{§ II. Evidence that a future-interest-system tends to be rich in affective-conative elements $\$$.}

On p. 152 it was argued that the future-interest-system is apt to contain a large proportion of affective-conative elements. Dr Webb's figures, so far as they go, support this conclusion. For if we examine Dr Webb's schedule** of forty-eight qualities to discover which single one of them is most akin to our future-interest-system, we shall, at first sight, select quality number 32-'Degree in which he works with distant objects in view (as opposed to living from hand to mouth).' And if we then examine the appendix to Dr Webb's paper, giving 'a selection from the reports of the observers as to what they understood by the terms used in the schedules, and what guided them in

* See above, pp. I25 to I27 and footnote $+\ddagger$ on p. II9. † On p. I23, above.

$\ddagger$ See above, pp. 476 and 484 .

if Loc. cit., Table VI.

$\S$ In $\S 9$ of this Appendix (p. 484).

** Quoted above in Table III on pp. 126, 127, 
marking their subjects,'* our selection of this thirty-second qualitywhich we may for shortness describe as 'future objective'-will be justified. The measure of 'future objective' was not, of course, a direct measure of the extent to which a subject's thought and conduct were guided by his future-interest-system; such a measure the observers did not attempt to make. But the observers' reports $\dagger$ make it abundantly clear that, in measuring 'future objective,' they came near to measuring the influence on thought and conduct exercised by the future-interest-system.

Let us next enquire which of Dr Webb's forty-eight qualities most nearly measures wealth of affective-conative neurograms; or, in other words, which corresponds most closely to emotional interest. Once more there can be little doubt about our answer, whether we make it after merely inspecting the schedule or after carefully examining the observers' reports on the meanings they attached to the scheduled names of qualities. The quality the measure of which comes nearest to measuring richness in affective-conative neurograms is the seventh in the schedule-'Degree of aesthetic feeling (love of the beautiful for its own sake).' $\ddagger$

* Loc. cit. Appendix II, pp. 84 to 96.

t The following is Dr Webb's 'selection from the reports of the observers as to what they understood by' this quality (Number 32) 'and what guided them in marking their subjects':

'(a) "Did he always live for the present, or did the future ever trouble him, with regard to (i) his pleasures, (ii) his studies or duties?" (b) "The + men showed forethought, the - men were happy-go-lucky." (c) "One who lives and works now in order to enjoy results in the future." (d) "The capacity for acting in the present strongly guided by what may happen in the future, however distant." (e) "The + men worked hard to educate themselves or to fit themselves for their career, as opposed to working to get top in an examination." ( $f$ ) "The + men work looking towards the future; in fact T-'s religious convictions cause him to work wholly and entirely to fit himself for the life after this." $(g)$ "The extent to which he works as a neans to an end." $(h)$ "The extent to which he looks forward to and prepares for the future with a view to improving his lot":" (Loc. cit. p. 93.)

¥ The following is Dr Webb's 'selection from the reports of the observers as to what they understood by' this quality (Number 7) 'and what guided them in marking their subjects':-

'(a) "If he loves Art and all that is clean and pure for its own sake, then he has the true aesthetic feeling." (b) "Admiration for a poem, picture, book, etc., for its inherent beauty." (c) "Appreciative enjoyment of the beautiful as shewn in poetry, art, etc." (d) "Desire to perceive beauty in all its forms because of the pleasure brought by the perception." (e) "Love of the beautiful because it appealed to their finer nature and to their finer emotions." $(f)$ "Way in which subject would involuntarily attend to or ignore things which are calculated to appeal to the aesthetic feelings":' (Loc. cit. pp. 85, 86.)

If we remember that ' what we attend to' is ' what interests us' we see how clearly the last two quotations-and especially the last $(f)$-from the observers' reports shew that they were measuring some kind of emotional interest; in other words, wealth of some kind of affective-conative neurograms. 
What then is the correlation between these two qualities-No. 32 and No. 7 ? The corrected coefficient obtained from Dr Webb's table* is 79: the highest but two (and within .02 of the highest of all) of the forty-seven correlations of No. 7 and the highest but three of the forty-seven correlations of No. 32. Here then we have direct numerical evidence of the tendency of a strongly developed future-interestsystem to be accompanied by a wealth of affective-conative neurograms.

Now it is difficult, if not impossible, to see any psychological reason why a particular kind of interest-system should generally be accompanied by neurograms of a particular kind, unless indeed the interest-system is, at least in part, composed of the neurograms in question. This conclusion is supported by statistical considerations; for it has been shewn $\dagger$ that, if $q_{32}$ be the measure of the first quality (No. 32) and $q_{7}$ of the second quality (No. 7) and $r_{(32)(7)}(=\cdot 79)$ the coefficient of correlation between then, we may write

$$
q_{32}=r_{(32)(7)} q_{7}+l_{2} x_{2}+l_{3} x_{3}+\ldots+l_{n} x_{n}
$$

where $x_{2}, x_{3}, \ldots, x_{n}$ measure qualities that are independent of $q_{7}$ and of each other, and are distributed according to the normal probability law with the same standard deviation $\ddagger$ as $q_{32}$ or $q_{7}$; and where

$$
r^{2}{ }_{(32)(7)}+l^{2}{ }_{2}+l^{2}{ }_{3}+\ldots+l^{2}{ }_{n}=\mathrm{I} \text {, }
$$

so that

$$
l^{2}{ }_{2}+l^{2}{ }_{3}+\ldots+l^{2}{ }_{n}=\mathrm{I}-(\cdot 79)^{2}=\cdot 38 \text {, }
$$

only. The form of the first of these equations shews that four-fifths $\left(r_{(32)(7)}=\cdot 79\right)$ of $q_{7}$, enters into the constitution of $q_{32}$; according with the view that the affective-conative neurograms which tend to accompany 'future objective' largely enter into its constitution.

\section{§ I2. Evidence that purpose-neurograms tend to be rich in affective-} conative elements.

Our argument $\S$, that a future-interest-system influences conduct in a specially high degree because a future-interest-system is rich in affective-conative elements, depends, as we said\|, upon our previous conclusion 9 that the presence of affective-conative elements in an interest-system increases the influence of that system upon the flow of excitement through the brain. Dr Webb's figures are in agreement with this earlier conclusion; for they shew that certain purpose

* Loc. cit. Table VI.

$\uparrow$ See above, equation (2) on p. 476 .

$\ddagger$ Dr Webb's qualities were all distributed according to the normal probability law and with the same standard deviation.

\| See above, p. I54.

$\S$ See above, p. 154 .

ๆ See above, pp. 91, 92. 
qualities* which stand in a specially close relation to character (and therefore, as we have seen $\dagger$, to conduct) have also specially high correlations with the quality (No. 7 in Table III) that most nearly measures wealth of affective-conative neurograms.

Thus Dr Webb found that the group factor that we described as purposefulness + was contained in a high degree by the following qualities $\S$, whose correlations with 'profoundness of apprehension' (No. 36 in the schedule I) and with 'common sense' (No. 37) most exceed their correlations with 'quickness of apprehension' (No. 35) and with 'originality of ideas' (No. 38):-

On the positive side (having positive correlations with No. 36 and No. 37):

34. 'Tendency not to abandon tasks from mere changeability.'

32. 'Degree in which he works with distant objects in view.'

33. 'Tendency not to abandon tasks in the face of obstacles.'

I8. 'Tendency to do kindnesses on principle.'

20. 'Trustworthiness (performing his believed duty).'

2I. 'Conscientiousness (keenness of interest in the goodness and wickedness of actions).'

22. 'Interest in religious beliefs and ceremonies.'

39. 'Pure-mindedness.'

28. 'Extent of mental work bestowed upon usual studies.'

On the negative side (having negative correlations with No. 36 and No. 37):

2. 'Tendency to quick oscillation between cheerfulness and depression (as opposed to permanence of mood).'

4. 'Readiness to become angry.'

I I. 'Eagerness for admiration.'

I3. 'Esteem of himself as a whole.'

I4. 'Offensive manifestation of this self-esteem (superciliousness).'

I5. 'Fondness for large social gatherings.'

3I. 'Degree of bodily activity in pursuit of pleasure (games, etc.).' T

The nature of the group factor 'purpose' has been discussed above**. Suffice it here to repeat, in Dr Webb's words, that the nature of this factor, "whose generality would appear to extend so widely in character, is in some close relation to "persistence of motives". ' $\dagger$

* See above, pp. $155,{ }_{5} 57$ and ${ }_{15} 8$

\$ See above, pp. 158 and 16r.

\| Quoted in Table III on pp. 126, 127 above.

I It should be added that the negative correlations of these 'negative' qualities with No. $3^{6}$ and No. 37 are all less-and generally much less-in absolute magnitude than $-\cdot 53$, while all but one of the positive correlations exceed $\cdot 50$ (Webb, p. 54).

** In Chapter $8, \S 4$ on pp. I 55 et seq.

†† Loc. cit. p. 60. 
In fact, these purpose qualities* (into which the group factor, $f$, defined above $\dagger$, enters in the highest-positive or negative-degree), are those which represent the observed effects of interest-systems that most influence thought, and therefore conduct. And it is these very qualities which, along with No. 36 and No. 37 that led to their selection, form, with the quality (No. 7) that most nearly measures wealth of affective-conative neurograms, eight $\ddagger$ out of its nine highest positive correlations and its three $\S$ highest negative correlations. Here then is statistical evidence that, at any rate in the case of the estimated qualities of some 200 training college students, the purpose interest-systems-those that most influence conduct-are those which are richest in affective-conative elements.

\section{§ r3. Purposefulness \|.}

We have here to notice certain facts which are accounted for if Dr Webb's new factor $w$-or rather $p$, that we have defined and identified with $w$-measures the "influence of a central purposesystem of neurograms on thought and conduct,' but which remain unexplained if $p$ measures a general tendency towards 'persistency of motives.'

In the first place, Dr Webb points out that the high partial ( $g$ constant) correlations of such moral qualities as 'trustworthiness' or 'kindness on principle' with his new factor, are consistent with the view that this new factor is in close relation to 'persistence of motives ' I: for, he observes, 'the persistence of a motive in consciousness, and its power to appear in consciousness at any time, even when

* See above, pp. 155 $_{5}, 1_{57}, \mathrm{I}_{5} 8$.

$\dagger$ On p. 156. Cf. also, footnote $\S$ to p. I55.

$\ddagger$ The correlations between quality No. 7 and the eight qualities are:-

$\begin{array}{lll}\text { No. } 20(\cdot 8 \mathrm{I}), & \text { No. } 32(\cdot 79), & \text { No. } 18(\cdot 76), \\ \text { No. } 21(\cdot 76), & \text { No. } 22(\cdot 76), & \text { No. } 37(\cdot 76), \\ \text { No. } 28(\cdot 7 \mathrm{I}), & \text { No. } 36(\cdot 7 \mathrm{I}), & \end{array}$

the correlations being given in brackets. The other high correlation of No. 7 is that between it and 'general excellence of character,' No. $43(\cdot 8 \mathrm{I})$.

$\S$ The correlations between quality No. 7 and the three qualities are:-No. 4 $(-.54)$; No. $2(-\cdot 49)$; No. $5(-\cdot 38)$, the correlations being given in brackets.

II See pp. 160, 161 above.

If The partial ( $g$ constant) correlations of the qualities, 'trustworthiness' (No. 20), 'conscientiousness' (No. 21) or 'kindness on principle' (No. I8) with each other and with 'tendency not to abandon tasks' (Qualities Nos. 33 and 34) are all high. So are their partial ( $g$ constant) correlations with 'future objective' (quality No. 32, 'degree in which he works with distant objects in view as opposed to living from hand to mouth'). These partial correlations are:

$\begin{array}{llllllll}\text { Quality No. } \ldots & \ldots & \ldots & 20 & 2 \text { I } & \text { I } 8 & 33 & 34 \\ \begin{aligned} \text { Partial Correlation }(g & \text { constant) }\end{aligned} & & & & & \\ \quad \text { with No. } 32 \ldots & \ldots & \ldots & .62 & .77 & .4 \text { I } & .74 & .9 \text { I }\end{array}$ Suppose these partial correlations are wholly due to $p$. Then, if $x, y$, measure any 
the field of ideas occupying consciousness at the moment is little, if at all, related to it, seems quite reasonably to be at the base of moral qualities. Trustworthiness, conscientiousness, kindness on principle, fair-play, reliability in friendship, etc. are lessons derived from social education. These lessons will be learnt more effectively in proportion as they persist long and recur readily.* On this we have to remark that the persistence of a motive (or as we should rather say, the persistence of the neurogram of the motive, for that alone persists) is quite distinct from its power to recur readily whenever action is about to take place and whatever may be the thoughtactivities then in consciousness. Both these distinct attributes belong as we have seen, toa strong future-interest-system or system of purposeneurograms at the centre of a single wide interest-system $\uparrow$. But it is by no means clear that any motive which leaves a strong and relatively permanent impression among the neurograms of the brain will on that account alone recur as readily as if its neurogram were less deep, but closely connected with neurograms of frequently recurring experiences.

Again, it is not easy to see why an effectively learnt lesson concerning trustworthiness, conscientiousness or other moral quality should make one work with a distant object in view, and so account for the high (partial) correlations between 'the degree in which a subject works with distant objects in view' (No. 32) and the moral qualities, trustworthiness, and the rest. But these high correlations are at once explained if the second factor, measured by $p$, be what we have suggested.

Once more, 'interest in religion' is one of the qualities into the constitution of which Dr Webb's factor entersł. This means that, if the other factors that help to make up this quality be kept constant, Dr Webb's factor, or $p$, and 'interest in religion' should increase or decrease together. Now it is not easy to see why a higher degree of interest in religion should increase 'persistence of motives' in general: the persistence of some motives it should increase, but surely not the persistence of all. On the other hand, a high degree of interest in two of these five qualities having high partial correlations $\left(v_{x y . g}\right)$ with each other, Yule's equation gives $r_{x y, g}=r_{x p}, v_{y p} \gamma_{y p}$; so that, if $r_{x y \cdot g}$ is nearly unity, both $r_{x p . g}$ and $r_{y p . g}$ must also be nearly unity. In other words, the qualities $x$ and $y$ must have high correlations with the new factor, $p$.

* Webb, loc. cit. p. 60.

$\dagger$ See above, Chapter I2, especially pp. 240 to 244.

\$ See Webb, loc. cit. p. 59, where he states that interest in religion (Quality No. 22 in Table III on pp. 126, r27) and Puremindedness (Quality No. 39) give quite similar results to the other seven positive purpose qualities (Nos. $34,33,18$, $20,2 \mathrm{r}, 32$ and 28 ) named on $\mathrm{p}$. 157, above. 
religion is bound to result in a high degree of interest in the futureespecially the remote future-and therefore to increase the extent to which future interest influences behaviour. Here then we have another reason for supposing $\mathrm{Dr}$ Webb's factor, or $p$, to be what we have suggested, rather than 'persistence of motives,' pure and simple. 


\section{APPENDIX C*}

\section{A Note on Maximal Endarchies.}

Suppose that the originally disconnected elements have at last been linked together, directly or indirectly, by positing new elements according to paragraphs $(\alpha),(\beta)$ and $(\gamma)$ on p. 209 above; and let us examine the resulting crganisation. For this purpose we may reverse in imagination the order in which the organisation developed, and begin with the last element added to complete the linking up of the original elements.

From this element, $\epsilon_{1}$, not more than $m$ elements may (paragraph $\beta)$ be derived. Consider then how the next $m$ elements, $\epsilon_{2}, \epsilon_{3}, \ldots, \epsilon_{m+1}$, will be placed in order that paragraph $(\gamma)$ may be satisfied when $m+\mathrm{I}$ elements have been posited in the reversed process that we are considering.

Evidently $\epsilon_{i 2}, \epsilon_{3}, \ldots, \epsilon_{m+1}$ are all derived directly from $\epsilon_{1}$. For if not, suppose that any one of them, say $\epsilon_{m+1}$, is derived from $\epsilon_{2}$ instead of from $\epsilon_{1}$. Then the number of intermediate elements linking $\epsilon_{1}, \epsilon_{2}, \ldots, \epsilon_{m}$ together in pairs remains the same as if $\epsilon_{m+1}$ had been derived directly from $\epsilon_{1}$; the number of intermediate elements between $\epsilon_{m+1}$ and $\epsilon_{2}$ is reduced by one; and the number of intermediate elements between $\epsilon_{m+1}$ and each of the remaining $m-\mathbf{I}$ elements is increased by one. Thus the whole number of intermediate elements employed in connecting the $m+$ I elements to one another in pairs is increased by $m-2$. If therefore $m>2$, paragraph $(\gamma)$ would be infringed by the removal of $\epsilon_{m+1}$ from a position in which it was derived directly from $\epsilon_{1}$ to a position in which it was derived directly from $\epsilon_{2}$ (or $\epsilon_{3}$ or $\epsilon_{4} \ldots$ or $\epsilon_{m}$ ).

Consider now the position of the next $m^{2}$ elements, $\epsilon_{m+2}, \epsilon_{m+3}, \ldots$, $\epsilon_{m^{2}+m+1}$ to be posited in our reversed process. The first of them, $\epsilon_{m+2}$, cannot be derived from $\epsilon_{1}$ and must therefore be derived from one of the other elements. Suppose that $\epsilon_{m+2}$ is derived directly from $\epsilon_{2}$. Then $\epsilon_{m+3}$ is also derived from $\epsilon_{2}$. For if not it must either be derived from one of the other first zone elements, say $\epsilon_{3}$, or else from $\epsilon_{m+2}$. But if $\epsilon_{m+3}$ were derived from $\epsilon_{3}$ instead of from $\epsilon_{2}$, the number of intermediate elements between $\epsilon_{m+2}$ and $\epsilon_{m+3}$ would be increased from one to three, while the average number of intermediate elements between the remaining pairs would be unaltered, the whole number of intermediate elements employed in connecting $m+3$ elements

\footnotetext{
* Appendix $\mathrm{C}$ is a note upon the proposition set forth on pp. 208 to 2 ro above.
} 
together in pairs being thus increased by two. And if $\epsilon_{m+3}$ were derived from $\epsilon_{m+2}$ instead of from $\epsilon_{2}$, the number of intermediate elements between $\epsilon_{m+3}$ and $\epsilon_{m+2}$ would be reduced by one; the number of intermediate elements between $\epsilon_{m+3}$ and all the other $m+\mathrm{I}$ elements would be increased by one; and the number of intermediate elements between the remaining pairs would be unaltered. Thus the whole number of intermediate elements employed in connecting the $m+3$ elements would be increased by $m$. In either case, then: the average

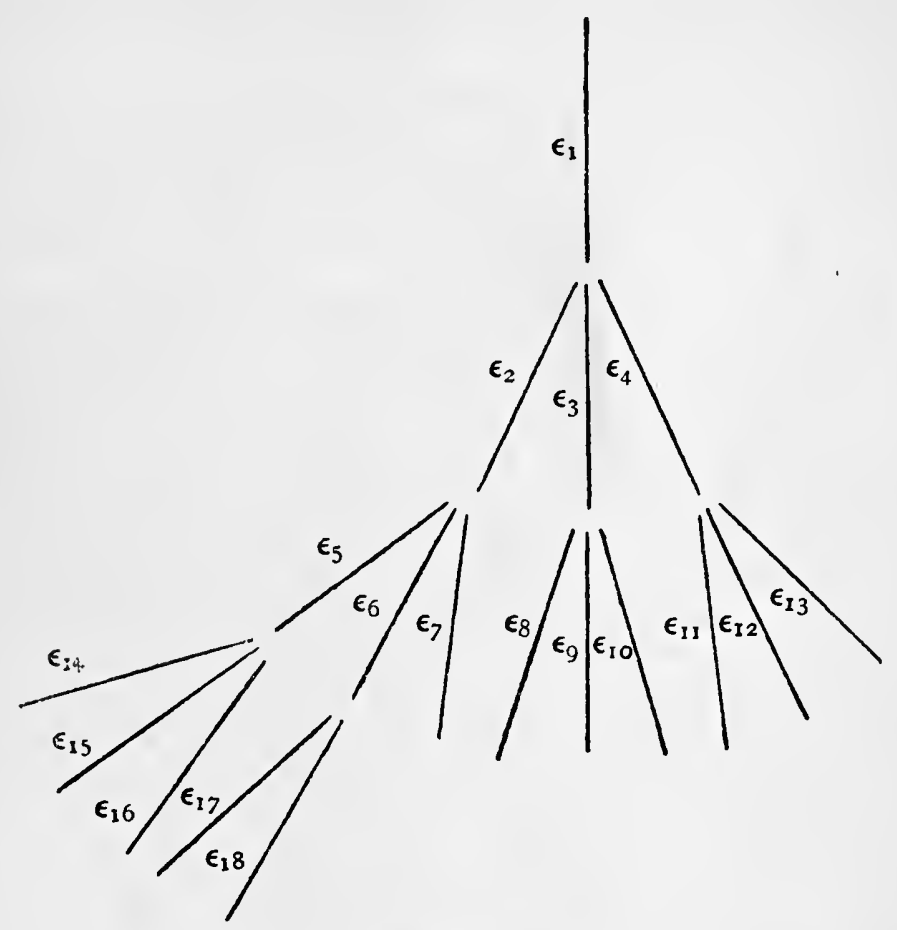

Fig. 19.

number of intermediate elements would be increased by the change. So that, to satisfy paragraph $(\gamma), \epsilon_{m+3}$ must be directly derived from $\epsilon_{2}$.

Similarly it may be shewn that every element from $\epsilon_{m+2}$ to $\epsilon_{2 m+1}$ is derived from $\epsilon_{2}$, every element from $\epsilon_{2 m+2}$ to $\epsilon_{3 m+1}$ from $\epsilon_{3}$, and so on.

The $m^{3}$ element in the third zone must similarly, if paragraph $(\gamma)$ is to be satisfied, be derived in groups of $m$ from the $m^{2}$ elements in the second zone; and so on.

G. E. 
Our proposition is therefore true when the whole number of elements is $\mathrm{I}+m+m^{2}+\ldots+m^{n}=\frac{m^{n+1}-\mathrm{I}}{m-\mathrm{I}} \equiv \Sigma_{n}$, say. Repeating the argument by which we shewed that the $(m+3)$ rd element to be posited in the reversed process must be derived from the same first zone element as the $(m+2)$ nd element posited, we may shew that, if the whole number $N$ of elements is intermediate between $\Sigma_{n}$ and $\Sigma_{n+1}$, the endarchy is maximal down to the $n$th zone and the remaining $N-\Sigma_{n}$ elements are derived in groups of $m$ from $n$th zone elements, except that from one $n$th zone element is derived the number equal to the excess of $N-\Sigma_{n}$ over the positive integral multiple of $m$ that is next less than $N-\Sigma_{n}$.

Thus, if $m=3$ and $n=2$, so that $\Sigma_{n}=\mathrm{I} 3$ and $\Sigma_{n+1}=40$, and the whole number of elements is $N=\mathrm{I} 8$, the arrangement determined by paragraphs $(\alpha),(\beta)$ and $(\gamma)$ is as shewn in Fig. I9 above, being a 'maximal endarchy' down to the $n$th (second) layer which includes $\epsilon_{5}, \epsilon_{6}, \ldots, \epsilon_{13}$. 


\section{APPENDIX D*}

\section{A Note on Hobbies and Holidays.}

Among the pedagogic applications of the principle of the single wide interest arrived at in Book II, consideration should be given to hobbies and holidays. A hobby is an interest more or less disconnected from the main interest of one's life. To it, therefore, there corresponds an interest-system which, if not altogether separate from one's principal interest-system, has at least a separate centre or focus. The synapses between the principal system and the focal elements of a subsidiary interest-system that corresponds to a hobby, will be of higher resistance than those that connect any neighbouring elements of the principal system, and in this respect they will differ from synapses near to the centre of the single wide interest-system that we were discussing in Chapter I2 above; for these latter, as we have seen, always form part of paths that go on deepening as the centre of the single wide interest-system is approached. In general then, the possession of a hobby implies the existence of a subsidiary centre of interest in its possessor's neurography; so that the whole neurography cannot have the form of such a single wide interest-system as we have taken to be the goal of education on its neurographic side. It would therefore seem at first sight that logical consistency would require educators to discourage hobbies. But further reflexion reminds us that no one, even in the most critical stages of his education, can devote the whole of every day to the voluntary development of his single wide interest-system; and that, therefore, it is generally preferable that part of the time necessarily available for involuntary thought-activities should be devoted to a hobby, that may later be absorbed into the growing single wide interest-system, rather than that it should leave no neurographic record of which use is ever likely to be made. Only, no hobby interest should become so wide or so deep as actively to interfere with the growth of the single side interest-system.

Another reason for encouraging hobbies, within the limits set by the preceding sentence, is that the possession of strong interests, subsidiary to and centred in one's central religious purpose, but not otherwise closely connected with one's everyday activities, may add

* Appendix D is a note for which room could not be found on P. 334 above. 
to one's happiness and increase the usefulness of one's public service by making holidays possible. Unless there exists some such subsidiary interest, preferably associated with physical activity, it will not be so easy to withdraw attention from the habitual subjects of one's thought, and thus to allow the activity of the corresponding well-worn nerve tracts to subside when these neurograms need rest.

How far hobbies should be reserved for holidays depends upon the number of these holidays. Where they are few and far between, hobbies cannot be confined to them. But it is probably preferable that people who have original and constructive work to do-members of Class A described in Chapter 20*-should have long enough holidays to permit of hobby interests remaining inactive between holidays, except indeed such hobbies as demand and will receive but little time and no effort. Our reason for this view is that, if only a few hours, perhaps eight in number, of every day are given to the main work of one's life and to interests connected with it, that work will lose much of the interest and meaning that might attach to it. It will be in danger of beconing too narrow in scope, and of being less well done than would otherwise be the case; and, at the same time, the activities of leisure hours will lose much of their efficiency and interest by not being associated with the activities that are called work.

* See above, p. 326. 


\section{APPENDIX E*}

\section{Provinces of England and Wales}

\section{Suggested sub-division of England and Wales into tenor twelve educational provinces that might reith great advantage become administrative units for several other purposes of local government.}

Province and University Centre(s)

I. Northern

2. Yorkshire

Leeds-Sheffield

3. North-Western

4. Wales

5. North-EastMidland

6. Eastern

7. West-Midland

8. Western

9. South-Midland

ro London

II. Southern\|

I2. South-Western I
Newcastle-Durham

\section{Geographical Counties}

Northumberland, Durham, Cumberland (and perhaps the Cleveland District)

Yorkshire (except perhaps Cleveland)

Lancashire, Cheshire, West. morland

Wales and Monmouth

Lincoln, Nottingham, Derby, Leicester, Rutland, Northampton (except Soke of Peterborough)

Norfolk, Cambridge, Huntingdon, Suffolk, Northampton (Solee of Peterborough only)

Stafford, Salop, Warwick, Worcester, Hereford

Gloucester, Wiltshire, Somerset

Oxford and Berkshire

Essex, Hertford, Bedford, Buckingham, Middlesex, Kent, Surrey, Sussex§

Hampshire, Dorset

Devon, Cornwall

* See above, p. 366 ; and Chapter 25, § 2.

$\dagger$ Norwich is only now contemplating a university college that may become the provincial university of East Anglia. Cambridge is not reckoned as the university centre on account of its national and non-local quality.

$\ddagger$ Oxford is not reckoned as the university centre on account of its national and non-local quality.

$\S$ Sussex might later become a thirteenth province, with Brighton as its university centre.

II As Reading forms the natural university centre for the northern parts of Hampshire, and as Bristol serves parts of Dorset better than does Southampton, it might be well to omit this sub-division for the present; to include Dorset with the Western province centred in Bristol; and to include Hampshire with the SouthMidland province, making Southampton a second university centre to Reading.

If Pending the further development of university education in Exeter, and the establishment of the proposed British-American University in Plymouth, the South-Western province should be merged in the Western province, having its university centre in Bristol, with Exeter as a subsidiary centre. 


\section{APPENDIX F*}

\section{Particulars of the Higher School Certificate Examination as held by the Joint Matriculation Board in I9I9.}

Group I. Greek, Latin, Greek and Roman History.

Group II (A). English Literature, French, German, Italian, Russian, Spanish, History, Latin, Pure Mathematics.

Group II (B). Economics, Geography, History, French, German, Italian, Russian, Spanish.

Group III. Pure Mathematics or Higher Pure Mathematics, Applied Mathematics, Physics, Chemistry, Botany, Zoology, Geography.

Candidates must pass in one of the four Groups (I, II (A), II (B), III), and

in one subject (Subsidiary) which is not included in that particular Group, except that

if Group II (A) is presented, either English Literature or Latin or Pure Mathematics may be taken as the Subsidiary subject when not taken as a Full subject of the Group;

if Group II (B) is presented, either Economics or Geography may be taken as the Subsidiary subject when not taken as a Full subject of the Group.

To pass in Group I, candidates must satisfy the Examiners in the three subjects of the Group taken as a whole.

To pass in Group II (A), candidates must satisfy the Examiners in three subjects of the Group taken as a whole, in which a language other than English must be included, but not more than two such languages may be taken.

To pass in Group II (B), candidates must satisfy the Examiners in three subjects of the Group taken as a whole, in which a language and either Economics or Geography must be included.

To pass in Group III, candidates must satisfy the Examiners in three subjects of the Group taken as a whole.

The Oral Examinations in French, German, Italian, Russian, and Spanish, and the Practical Examinations in Science subjects, will be held at the University Centres, and may be held elsewhere at the discretion of the Board. The Board will require the Laboratory notebooks of any of the candidates who present themselves for examination in a Science subject, and the Examiners may take account of them.

* Appendix $\mathrm{F}$ is a note for which room could not be found on $\mathrm{p} .387$ above. 


\section{APPENDIX G*}

\section{An Over-Estimate (made in July, I9I9) of the Annual Cost of a National Scholarship System.}

In order to estimate the maximum sum required to provide scholarships and maintenance allowances that will bring every kind of education-when already provided according to our diagram (facing p. 3I9 above)-within the reach of all children and young people of sufficient educational promise, irrespective both of their place of residence in England and Wales and of their private financial circumstances, we consider first the case of boys and young men belonging to a province inhabited by $5,000,000$ people.

Our diagram shews 50,000 boys receiving secondary education between the ages of twelve and fourteen. Of this number, 30,000 have been transferred by means of scholarships and maintenance allowances from Elementary Schools which they attended up to the age of twelve. If we suppose that every one of these 30,000 boys will require a maintenance allowance of five shillings a week in order to enable his parents to meet the extra cost-including travelling expenses, but not fees (since, as we observed in $\S$ I of Chapter 25 above, the Education Act of 1918 prevents young people from being debarred by inability to pay fees from receiving a type of education by which they are capable of profiting) - of sending him to a Secondary or Junior Technical School instead of to an Elementary School, we shall have over-estimated rather than under-estimated the cost of the scholarship system, so far as junior secondary education is concerned. For we shall assume that there is no question of providing maintenance allowances to boys and girls who remain at ordinary Elementary Schools or transfer to Central Elementary Schools there to remain at least until the age of fourteen, when the Education Act of I9I8 ceases to oblige them to remain whole-time at school; and we may assume that those boys who were receiving preparatory education before the age of twelve will not need scholarships or maintenance allowances in order to enable them to receive junior secondary education between the ages of twelve and fourteen. Scholarships and maintenance allowances sufficient to enable half the male population of the province to receive secondary education between the ages of twelve and fourteen will therefore cost, at most, $£ 390,000$ a year.

The scholarships and maintenance allowances necessary to keep

* Appendix G is a note on $\S 1$ of Chapter 25; see especially p. 454. 
the 50,000 boys at school from fourteen to sixteen will, in the case of the poorest boys, have to be considerably increased in value beyond the five shillings a week that should suffice between the ages of twelve and fourteen. For the alternative to continued secondary education between fourteen and sixteen is not, as in the case of secondary education between twelve and fourteen, whole-time education in an Elementary School, but lucrative employment. The poorest parents, if they are to be able to give up their son's weekly earnings in order that he may continue to receive secondary education, must therefore receive a maintenance allowance comparable with, but certainly not greater than, the money he might be earning. If we take fifteen shillings a week as the upper limit of the scholarship and maintenance allowances at this stage, and if we assume that, of the 50,000 boys in question, 44,000 will need scholarships and maintenance allowances of this amount, we shall again have over-estimated the total sum of money needed for scholarships and maintenance allowances between the ages of fourteen and sixteen. The upper limit of the annual sum at which we thus arrive is $£ I, 7 \mathrm{I} 6,000$.

The number of boys or young men selected from a province of $5,000,000$ people to receive whole-time education between the ages of sixteen and eighteen is, according to our diagram, 14,000. The earning power between these ages is again greater than the earning power of boys between fourteen and sixteen years of age. If we assume that thirty shillings a week will suffice to enable a boy between sixteen and eighteen, however poor his parents, to continue to receive the advanced secondary or senior technical education for which he has been selected, we shall not be under-estimating the sum required. If then we further assume that 10,000 of the 14,000 boys in question will require maintenance allowances of this amount we shall again be over-estimating. The maximum sum at which we thus arrive is $£ 780$, 000 a year for the province in question.

The earning power at undergraduate age is greater still. Moreover, there is no obligation upon local authorities to pay the fees of necessitous qualified students beyond the age of eighteen. The amount of an undergraduate scholarship must, therefore, be sufficient to meet the cost of fees as well as of other expenses of life in a university. It is, however, safe to assume that $£ 4^{*}$ a week, or $£ 208$ a year, is sufficient to enable any young man, however poor his parents, to enter fully into the undergraduate life of any university except Oxford or

* Note added in January, 1921.-Principally because of the general rise in university fees since July, IgI $9, £ 4$ here replaces the original estimate of $£ 3$. The subsequent figures have been amended accordingly. 
Cambridge. If we assume that all except 1,000 of the 6,000 men undergraduates selected according to our diagram from a province of $5,000,000$ people will require the full amount of this maintenance allowance, we shall again be over-estimating rather than underestimating. The annual cost for undergraduate scholarships in the province would, on this assumption, be $£ I, 040,000$ a year.

Finally let us suppose that, of the 6,000 undergraduates, 2,000 remain up for, on the average, a further two years of advanced study and research, and that all these men require scholarships and maintenance allowances at the rate of $£ 4$ a week in order to enable them to postpone entering remunerative employment until the age of twentythree or thereabouts. The annual cost for research scholarships will then be $£ 4 \mathrm{I} 6$, ooo, of which a considerable proportion may be defrayed by industrial research associations, as well as by individual industrial and commercial concerns in whose interests it is necessary that the present supply of university trained researchers be increased.

The maximum total annual cost of scholarships and maintenance allowances for the boys and young men of a province of 5,000,000 people is thus $£ 4,342$,ooo. The maximum total annual cost for the whole of England and Wales may be reckoned at seven times this figure, or $£ 30,394,000$. Be it remembered, however, that this is a maximum, and that it may very greatly exceed the sum required. Indeed it is not improbable that half this sum would suffice. In that case the whole cost of the scholarships and maintenance allowances for England and Wales would only be a little more than $£$ I5,000,000 for boys and young men, and say $£$ I0,000,000 for girls and young women: or a total of about $£ 25$,000,000, being less than the annual public expenditure* on elementary education in England and Wales in the year I9I3-I4. So we may take it that the whole cost of the scholarships and maintenance allowances (but not of the teachers, the sites and buildings, or the equipment) that would be necessary to bring into operation the system of education represented in our diagram for boys and young men, and a similar system for girls and young women, would probably be less than the sum annually spent on public elementary education in England and Wales before the War. We need not therefore regard it as prohibitive. Indeed, as Mr Fisher has lately (January, I92I) observed, a Nation that can afford to spend $f_{4} 400,000,000$ a year on drink, and $f 100,000,000$ a year on tobacco, is not quite at the end of its resources.

* $€_{25}, 856,000$, according to the Seventh Report from the Select Committee on National Expenditure (2rst December, 19ro). 
APPENDICES

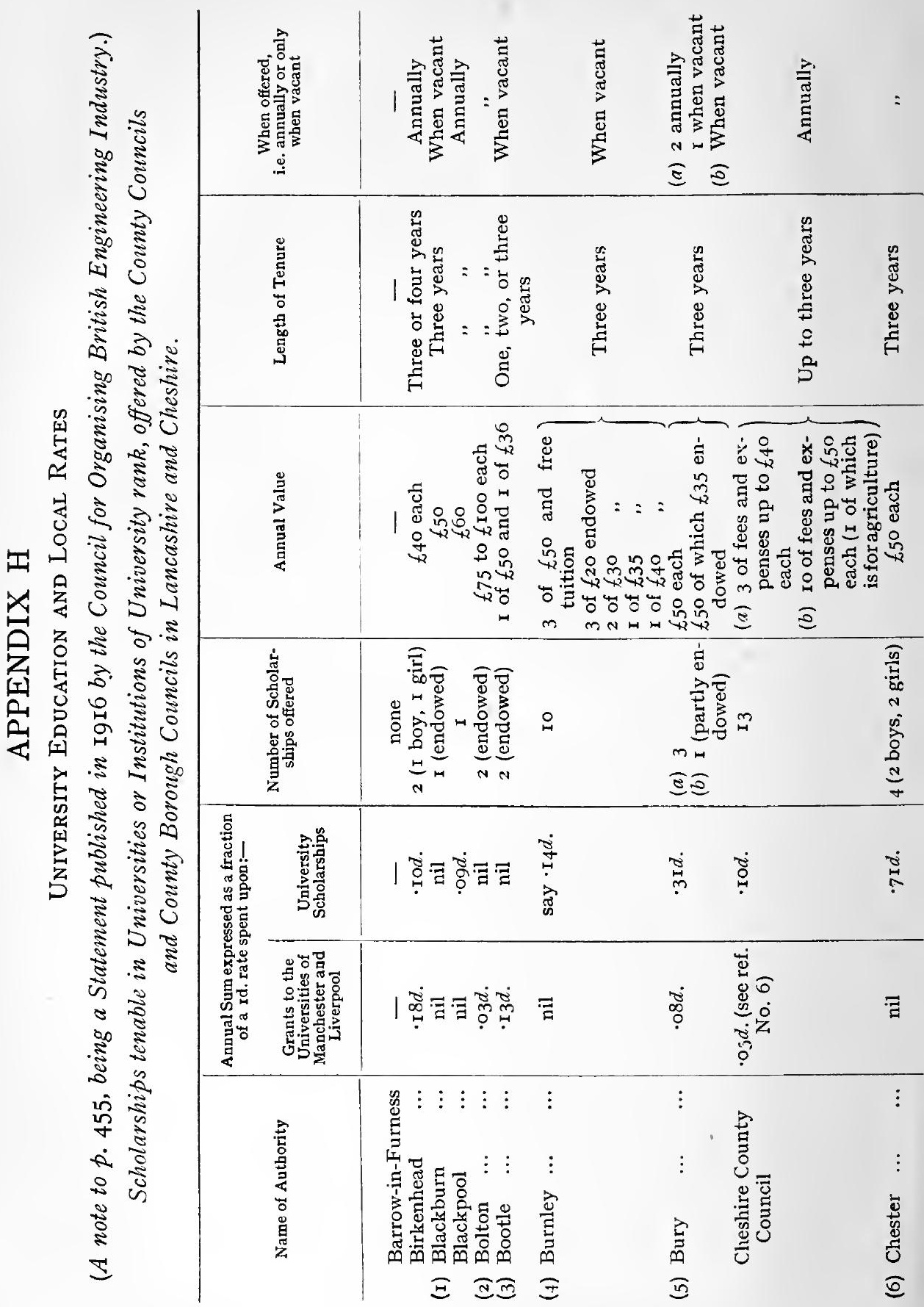




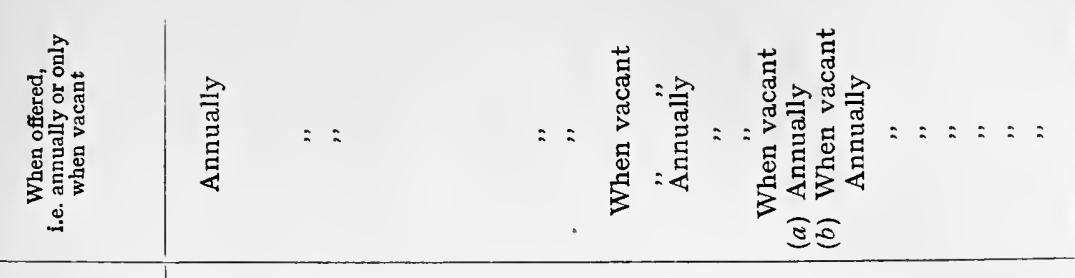

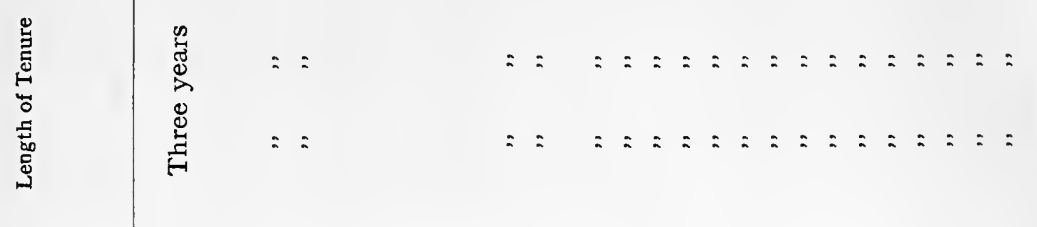
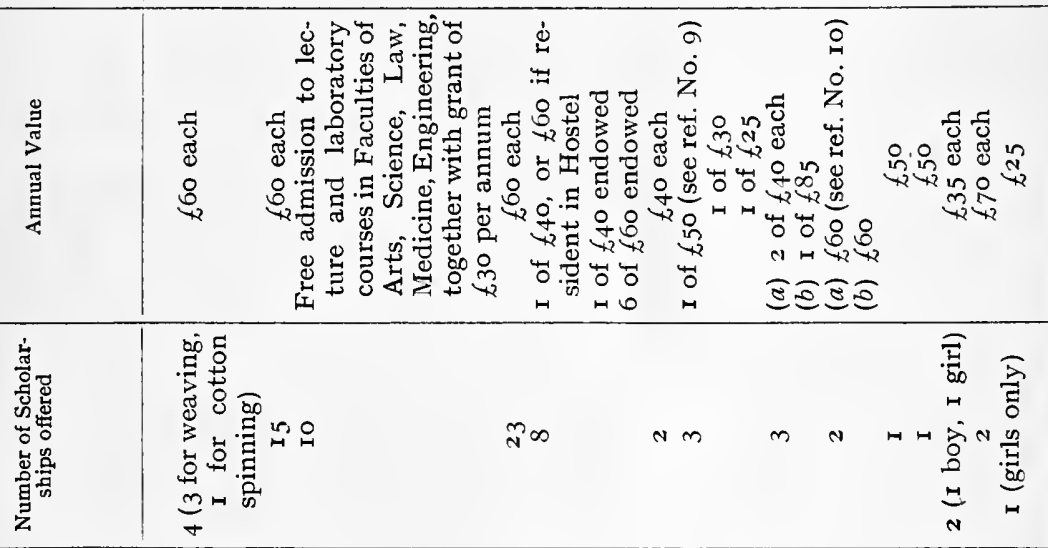

\begin{tabular}{|c|c|c|c|c|c|}
\hline 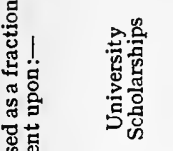 & : & 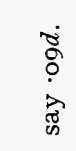 & 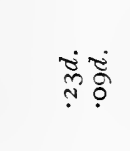 & ஜृष & 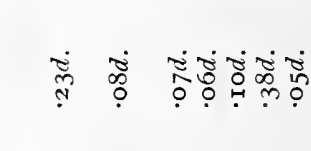 \\
\hline
\end{tabular}

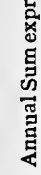

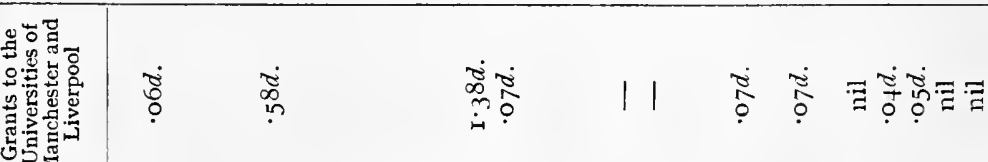

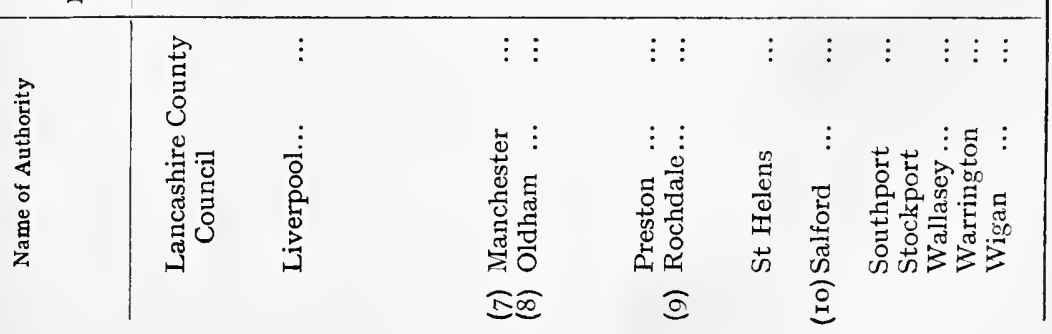




\section{REFERENCES.}

(I) One Endowed Scholarship, $£ 50$. Three years. Vaiue as fraction of $\mathrm{I} d$. rate $=$ $.023 d$.

(2) Two Endowed Scholarships (one boy, one girl) offered annually, $£ 75$ to $£$ I0o per annum each for three years. Value, as fraction of $\mathrm{I} d$. rate, is about $\cdot \mathbf{r} 8 d$.

(3) Two Endowed Scholarships, $£ 50$ each. One to three years. Value, expressed as fraction of $\mathrm{I} d$. rate, $=\cdot 044 d$. Grants by way of loan are made by this Education Committee to promising students who have matriculated and desire to enter Liverpool University.

(4) Seven Endowed Scholarships aggregating $£$ I95 per annum are offered. The total 'rate' value of all Scholarships offered is about $\cdot 26 d$., of which 11 I $6 d$. is endowed. These figures do not include a $£ 50$ Art Exhibition or two Scholarships for Student Teachers offered by this Authority.

(5) One Endowed Scholarship of $£ 35$ is increased to $£ 5^{\circ}$ by Local Authority if holder is son or ward of a resident in Bury. The total value of Scholarships offered, expressed as a fraction of $\mathrm{r} d$. rate, is $\cdot 34 d$., of which $\cdot 03 d$. is endowed.

(6) $£ 60$ set aside annually for Grants-in-aid, to assist deserving pupils at school, college, university, etc. The 'rate' value of this is 'o7d. (University Scholarship holders cannot claim this.)

(7) The figure given in first column includes contribution to maintenance of parttime as well as of University courses in the [College] of Technology.

(8) Seven Endowed Scholarships aggregating $£ 400$ per annum are offered. Total 'rate' value of all Scholarships offered is ' $29 d$., of which ' $20 d$. is endowed. Students may obtain payment of travelling expenses to [College] of Technology, Manchester, provided their attainments are such as will enable them to profit by instruction there, and their age is under twentyone years.

(9) The $£ 50$ Scholarship is a 'Local Science and Art Exhibition,' only half the cost of which is borne by the Rochdale Authority. The total 'rate' value of all Scholarships offered is $\cdot 17 d$., of which ${ }^{\circ} 0_{5} d$. is paid by the Board of Education.

(I0) Of the (a) Scholarship $£ 35$ is given by the Council of the Manchester University out of funds provided by the Salford Education Committee. The remaining $£^{25}$ is paid by the Salford Education Committee. The whole amount of both Scholarships is included in the figures given in the second column. 


\section{INDEX}

Aberystwyth, 497

Ability. See 'General ability'

Abnormal educational paths, 429, 430

Absolute truth, 196

Absorption, 272

Abstract, 187, I90, 193, 197, 198, 213 , $233,25 \mathrm{I}, 267,343,344,356-8,36 \mathrm{I}$, $362,377,382$

Action, 72, 1 46, I 53, 224, 225, 265, 273 et seq., 286, 289, 290

- freedom of, 225

Active education, 346, 347, 464

Activity, 28, 66, 74, 8I, 84, 248

Adams, Professor John, 5, 1 2-1 8, 21 , 22, I $02,290,292,3$ I $2,378,444$, 469-7 I, 473-5

Administration, industrial, 400, 4I 4 , 466

Administrator, 328

Adrenal glands, 133

Adrenin, I33

Advanced part-time studies, $368,4 \mathrm{I} 3$, $414,428,430,442$

- secondary studies, 349, 370, 373, 383-9o, 4II, 4I3, 4I6, 420, 44I, 44.3

Advisory committee, 426

Aesthetic satisfaction, 250-4, 274, 284 , 285

Affect, 54

Affective, 52, 54

Affective-conative, $54,89,238,298$

Afferent, 29

After-discharge, 84

ả ${ }^{\prime} \pi \eta, 259,302,308$

Age of transfer, 369, 445

Aim of education, 2I-3, 244, 312

Alexander, Professor S., $24 \mathrm{I}$

Alington, Rev. C. A., 338, $34^{\circ}$

Alternating reflexes, 76

Alternative Matriculation test, 395

Amateur, 357, 358

AMERICA, 303, 37I, 403, 404, 436

American Journal of Insanity, IOO, IOI, I 30,154

AMIEL, 299

Analysis, 198

Anger, 52, I66

Apprentice, college, $350,353,402,408$, 431

- Master, 350, 409, 4I 4, 420, 42I, 424,450

- special, 430, 431

- trade, 325,430

Apprenticeship, 136, 418, 424, 430, 449

Approved purpose, 144, 145

Arc, nervous, 29, 34

Archer, Professor R. L., 339, 397, 398, 402
ARChIMEDES, 82, 248, 274

Architect, 276

Aristotelian Society, 5I, 53, 54

ARISTOTLE, 6

ARNOLD, Matthew, 47I

Arrow, 374, 459, 460, 463. See also 'Scholarships'

Art, 373, 4I I

ArtaxerXes, 285

Association, 45

- areas, 33

- by similarity, 122

Association OF TECHNical InstituTIONS, 369,425

Athens, I4

'Atmosphere,' 432

Attention, 99, I I 8, 128, 272, 273

Aural instruction, 377

AUstralia, 205, 302, 394, 472

Authority, 304

Automatic, 377

Autonomic, 132

AvebuRy, Lord, 378

Bachelor of Arts, 399. See also 'Degree'

BACON, Francis (Lord Verulam), 433

BAIN, 69, 122

BALLARD, P. B., IOI, II3, I32

BANGOR, 497

Beauty, 249, 25I, 252, 433

Behaviour, consistency of, 224

- efficiency of, 224

Being, omniscient, 199, 208, 2 I 4, 236, 237

Bergson, Henri, 5, 7, 8

BERKELEY, I5I

Bertrand, A., 470

Bias, due to interest, 86

BineT, Alfred, 9, 98, I I I, I I 8

Biometry, 104

Bi-polar conflicts, 177

Birmingham, 393, 425, 437, 497

Blind-alley occupations, 425,426

BOARD OF EDUCATION, $212,446,447$, $449,45^{2}, 45^{8}, 47$ O, 5 OI

Books, school, 333, $38 \mathrm{I}$

BootLe, 4I8, 4I9

Boston (Massachusetts), 4II, 4I 7

Boundary essence, 214

Boy labour, 429

- Scouts, 422, 428

Brain and mind, 8-9

Branch endarchy, 208, 2I5, 24I, 242, 257

Brereton, Cloudesley, 439

BRISTOL, 497

British Engineers' Association, 325

BRItish ScIENCe Guild, 371, 435, 438, 439,457 
Brotherhood, 302

Brotherly love, 302, 347

BROUGHAM, Lord, 244

BRown, Dr William, IO3, I I 7

BRuCE, Hon. W. N., 359, 360, 4 I 7

BRYANT, C. L., $34^{8}$

BuRT, Cyril, I03, I05. I09, II0, II2, I 1 3-8, I66, 32 I, 367, 381, 460, $475-81$

CAMBRIDGE, I44, 349, 359, 374, 393, $403,404,438,456$

Campagnac, Professor E. T., 393, 4 I I

CANADA, 394

Canalise, 43, I I 5, I 54

Canals, semi-circular, I 75

CANNON, W. B., I33

CANTERBURY, 44I

CARDIFF, 497

CARLYLE, Thomas, 294

Carnivor, I97

CAstiglione, I 5

Censor, I 48 , I 5 I

Central Elementary School, 369, 423, $45 \mathrm{I}, 452$

- elements in personal endarchies, $230,237,239$

- essences, 31 I

- flow of excitement, 94, 163, 208, $224,225,244,281,282,286$

- purpose, 334, 342, 347

- teacher, 270

Cerebrum, 3I

Certificate, School, examination, 338 , $387,393,395,396,462$

- Higher School, examination, 387 , $390,392,393,396,397,498$

Chairs, professorial, 2 I 6

Character, 64, 94, I00, I 55, I 56, I 87 , $224,244,290$ et seq., 3 I 4

- strong, 299, 314

Chemists, 196, 237, 279, 290

Chesterfield, I6

Chesterton, G. K., 192, 296, 336

Childhood, 23, 339, 340

Christian, 24 I, 259, 303 et seq., 334, 335

- character, 308, 3I I, 3I5, 3I6

- Church, 309

- purpose, 309, 340

Christiania swing, I 75

Christianity, 305 et seq.

ChURCHILL, Winston, 322

Circuit, short-, 230, 237, 35 I, 354, 356

Circular nervous process, $53,60,82,92$, I $66,277,278$

Citizen, 301, 302, 305, 326, 327, 426--7

Civil Service, $324,472,473$

Classical side, $358,387,390,417,462$

Classics, $338,358,359,381,386,387$, 389

Classification of services, 326

Cleveland, 497

Cleverness, I I 8 et seq., I 29, I 55 , I 56 , 157,158, I 88,29 I, 294, 485

Cobalt glass, $184, \mathrm{I} 85$

Cognitive, 52
Coherence, 339, 356, 358, 359

Colet, John (Dean of St Paul's), 433

College apprentice, $350,353,402,408$, $43 \mathrm{I}$

College of TechNology (Manchester), $330,37 \mathrm{I}, 405,4 \mathrm{I} 4,438,44 \mathrm{I}$

Cologne, 436

Colour, 193, 2 I 8, 265, 372, 373

Common fund of energy, I I 8, 226

Commonwealth, 232, 241, 302, 345

Community, 227, 232, 240, 24I, 289, $293,296,299$

Complete endarchy, 197, 208, 213,221 , 248

- fact, 195, 443

Complex, 61, 62, 63, 64

Complication, 69

Comprehensiveness, $339,356,358,359$, 360,361

Conation, 52

Concentration (or reinforcement), 99, I 28, I $80,234,28$ I

Concept, I96, 2 I 8,358

Concomitant, 190, 198

Concrete (adj.), I93, I97, 214, 343, 344, $356,35^{8}$

- things, I9I, I92, I93

- (noun), 382

Conditions, correlation between columns, I ro-r 2,479

- for a hierarchy, Io4-Io, 475-8

- satisfied by endarchy of science, 203

Conduct, 226, 240,28I, 282, 290, 3 I 4 , 473

Conduction, law of forward, I83 (see also pp. 33-34)

Conflict, 64, 97, I 4I, I 42, I68, I 74, I 75, I $76,177,224,237,240,255,268$, $284,285,287,292,300,301,3$ I 3

- types of, 169

Conscience, 284,285

Conscious control, r68, I 75, 240

- purpose, I 44, I 45

- work, 256

Consistency, I6o, 224, 283, 285, 293 et seq.

Constructive instinct, 277,382

Consultative Commitee of the BoARD OF EdUCATION, I 5, 338, 34 I, $349,350,359,362,391,396,4 \mathrm{I} 2$, $442,443,454,459,463,469$

Consulting practice, University Teachers', 406, 407, 464

Continuity in education, $349-50,35 \mathrm{I}$, 360,366

- in thought, 67

Copernicus, 3

Corollary to third law, 89

Correlation, 104-27, I 56-60, 477 et seq.

- between columns, I 10-1 2, 479, 480

- partial, I59, 489

Correlatives, I 45

Cortex, 31, 32, 33

Cosine law, 484

Cost of education, $4,2 \mathrm{I}, 374,454,50 \mathrm{I}$ 
Cotton, $423,424,426$

COUNCIL FOR ORGANISING BRITISH ENGINEERING INDUSTRY, 325, 39I, $420,425,502$

Craftsman, 329

Creative thinking, 258

Credulity, 297

CREWE, Marquis of, 405

Cromer, Earl of, 473

Crusoe, Robinson, 227, 288

Culture, 6, 357, 360, 47 I, 472

Curiosity, 9I-94, 22I, 226, 236, 245-8, $256,259,263,300,3$ I 4

Curriculum, 337, 338, 348, 349, 359, $362,38 \mathrm{I}, 384,390,417,423,426$, $427,432,470$

Dalton, John, I9I

DARTMOUTH, 4 I 8

DARWIN, Charles, 72, 132, 219,246

David, Dr A. A., 338

Deduction, $263,264,265$

Deductive reasoning, $262,264-6,268$, 286

- syllogism, 26I, 262, 263

Defective, mentally, 278, 32 I, 362, 367

Definite fact, I9I, I94

Degree, honours, 392, 397, 402, 4 Io, $440,455,466$

- pass (or ordinary), 339, 392, 397, 399, 402, 4I 3, 466, 467

- research, 403, 4I 8, 464

- technical, 400, 409

DÉJÉRINE, 294

Delivigne, Sir Malcolm, 330

DENMARK, 372

DEPARTMENT OF SCIENTIFIC AND INDUSTRIAL RESEARCH, 404

Depth of neurogram, $44,88,89$

DERBY, 497

Derived essences, I19, 203, 209, 239, 260

Diagram, flow (facing page), 3 I9

Diagrammatic representation of facts, 213

Diagrams, list of. See 'Figures'

DiEsEl engine, 360

Differences. See 'Individual differences'

Diffusion, 69, I 80, 273, 282

Dignity of labour, 279

Dimensions, I 29, I 56, I 57, 277

Direct experience, facts of, 2 I 4

Discovery, 4I, I96, 248, 254, 256, 259 , 305

Disgust, 5 I

Disintegration, I68, I 74, I 82

Disorder, 264

Displacement, I68, I69, I 72, I 73, I 74, I 84,185

Disposition, 42

Distress, 5I, I67, 248

Division of labour, 3II. See also 'Individual differences'

Doctor of Philosophy, degree of, 403 , 464

Dogma, 297
Domestic occupations, 4 I I

- subjects, 386

Dotting apparatus, I I $3-14$

Double personality, I 70

Drainage of nervous impulses, 45, 75 , $79,89, \mathbf{1} 29,289$

Drawing, 386

DrIEsch, Hans, I 89

DURHAM, 456, 497

Economics, 400

Economy of effort, 2 I I, 223

- of thought, $223,250,288$

Educability of ' $g$,' 138,266

Educand, I 2, 356, 360, 470

Education (in Book II), 2 I2, 223, 243 , 244, 279, 292, 296, 3I I et seq.

- Act (I9I8), 323, 4I 5, 42 I, 423, 425, $447,448,449,453$

- - (I902), 455

- active, 347,464

- aim of, 244,312

- girls', 367, 386, 4I I , 4I 3

- passive, $346,465,470$

- private, 323

- types of. See 'Types'

Education Reform Council, 424, 456, 457

Educator, 23I, 243, 245, 269, 3I4, 400

- pupil his own chief, 27 r, 314, 400

Effective conduct, 224, 283, 293

Efferent, 29, 37, 57

Efficiency of thought organisation, 2 I I

Efficient reasoning, 223

Effort, I 4 I

- in organising thought, 2 I I

EGGAR, W. D., 348

Ego, I38, 258

Einstein, Professor, 192

Elegance, 252

Elementary education, 366, 374, 37783

- School, 366-9, 372, 375, 380-3, $423,45 \mathrm{I}, 452$

- - Central, 369, 423, 45I et seq.

Eliot, George, 6

Elliot Smith, Professor G., 33 I

EMERSON, $34^{\circ}$

Emotion, 5I, 53, 54, 55, 231, 242, 249 , $259,295,345,432,465$

Empirical neurographic connexions, 229-30

- thought contrasted with reasoned thought, I87

Emptying the mind, 148

Endarchy, I6I, I63, I 80, I 94-9, 20I-3, 206-I 3, 2I 5-I6, 220-23, 224, 225, $227-34,236,237,24 I-2,247,248$, $257,269,284,3$ I 3,335

- branch, 208, 21 5, 24I-2, 257

- complete. 197, 208, 213,221, $24^{8}$

- imperfect, 236

- incomplete, 199, 222, 269

- maximal, 180, 206 et seq., 222, 224, $225,227,247$ 
Endarchy, partial, 196, 197, 198, 202, 206, 208, 210,215

- personal, 196, I99, 219, 220, 23I, 232 et seq., 24I, 242, 269

- scientific, 228-34, 335 et seq.

- subject, 196, 208, 215, 236

Endarchy of science, 194-7, 199, 202, $208,215-7,227,237,346,361,379$, 416

Endowments, 438

Energy, common fund of, 118, 226

Engineer, 276, 353, 360, 390, 40I, 402, $419,424,430$

Engineering, $44 \mathrm{I}$

Engineering, 325, 326, 349, 350, 353, $354,359,36 \mathrm{I}, 390,39 \mathrm{I}, 403,408$, $409,412,420,425,426,429,430$

ENGLAND, 4, 10, 319, 363-6, 371, 374, $381,382,392,395,401,435,438$, $439,453,455,456,47^{2}$

English, 347, 348, 387, 388, 394, 396, $418,426,462$

Engram, 42

Epiphenomenon, 9, 95

ERAsmus, 433

Error, probable, I06-9, 475, 476

Essence, 180, I 87, I90, 19I, 193, 197 et seq., 203, 211, 213, 214, 215, 2I6-I 8, 229, 25I, 260, 3I I

- abstract, 187, I90, I91, 193, 197 et seq., $25 \mathrm{I}$

- boundary, 2I 4

- central, 3 II

- derived, II $9,203,209,239,260$

- first stage, 198, 199

- general, 229, 234, 251

- second stage, 199, 200

- third stage, 200

Ethical, 96, 97

Ethics, 240, 314, 427

ETON, 332,338

EUCLID, 193, 344, 365, 380

EUROPE, I 4, 15, 303

EwING, Sir J. A., 401

Examination, Higher School Certificate, $387,390,393,396,397,498$

- Matriculation, 392-6, 462, 471

- School Certificate, $338,386,387$. $393,395,396,462,47 \mathbf{I}$

- University Entrance, 393-6

Excitement, 29, 47, 66, 74, 81, 82, 172, I 73, I 75-84

EXETER, 497

Expenditure on Public Education, 4, $21,499-501,502-4$

Experience, direct, $2 \mathrm{I} 3$

- world of, 180,189

Expert, 232, 328, 352, 357, 358, 389

Explain, 265

Facilitate, 1 28, $218,266,268,285$

Fact, 187, 190, I01, 194-6, 199, 200, 21 3-19, 266, 267, 268

- abstract, 213-14

- complete, 195, 443
Fact, complex, 213, 2 I 5

- concrete, 187, 213, 214, 267

- definite, I9I, I94

- element, 195, 199, 213, 216, 217, 266

- general, 267

- important, 194, 195

- incomplete, 195

- of direct experience, 191, 213, 214, $215,216,218$

- real, I89, 190

- simple, 199, 213-16

Faculty, 99, IOI

FaIRGRIEVE, James, 333

Faith, 150, 297, 303, 307, 310

Fatigue, 273

Fear, 5I, 54, 57, 58

FEDERAL COUNCIL OF LANCASHIRE AND Cheshire Teachers' Associations, 457

Fees, 453, 499, 500

FÉRE, 78

Fiat, 140, 142

Figures:

$\begin{array}{lrr}1 \ldots 30 & 8 \ldots 121 & 14 \ldots 201 \\ 2 \ldots 53 & 9 \ldots 157 & 15 \ldots 201 \\ 3 \ldots 75 & 10 \ldots 178 & 16 \ldots 204 \\ 4 \ldots 76 & 11 \ldots 182 & 17 \ldots 235 \\ 5 \ldots 80 & 12 \ldots 200 & 18 \ldots 236 \\ 6 \ldots 87 & 13 \ldots 201 & 19 \ldots 493 \\ 7 \ldots 114 & & \end{array}$

(See also diagram)

Films, metallic, 264, 265

First stage essences, 198, 199

Fisher, The Right Hon. H. A. L., 323, 425,501

Flechsig, Professor, 34

Fleming, A. P. M., 325

Flow (of excitement), central, 94, 163 . $208,224,225,244,28 \mathrm{I}, 282,286$

Flow diagram (facing page) 319

FLUEGEL, J. C., I 42, I 48 , ' I 70 o, I 72, 175 , I $82,185,240$

Focus of consciousness, $47,66,67$

Foreign languages, $340,379,386,387$, $400,47 \mathrm{I}$

Foreman, 329

Formal training, 102

Form-master, 270, 338, 346-9, 387-9, 420,465

- sixth, 349, 350, 388, 389, 420, 465

France, 398

FRANCIS, M. E., 382

Freedom, 97, 225, 300, 302, 309, 363

French Revolution, 294, 303

Frequency distribution, 368, 475

FREUD, Professor Sigmund, 124, 154, I $68, \mathbf{1} 69$

Fringe of consciousness, 35, 67, 68

Froebel, 469

Fuegians, 72, 246

Functional system of nervous arcs, 37 , 277

Fund, common, of energy, 118,226

Future, 143, 297, 49I 
Future interest, I 52, I 53, I 54, I 55, I 59 , I $60,238,282,297,491$

Galton, Franc1s, 104

Games, 390, 41 8, 433

GARNETT, Dr William, 424

GAUCKLER, 294

General ability, 103, I20, 123, I25, 127 , I 29, 266, 278, 279, 292, 319, 333, $34 \mathrm{I}, 344,353,36 \mathrm{I}, 362,363,364$, $376,381,383,385,399,40 r, 460$, $46 I, 48 I$

- essence, 229, 234, $25 \mathrm{I}$

- fact, 267

- factor (g), ror et seq., 48r. See also 'General ability'

- intelligence, 102, 103,127

- laws, rgo

- sensory discrimination, 102

Generalisations, $384,385,428$

Genius, I25

Geography, 233, 348, 379, 386, 387, 396, $419,424,427$

GERMANY, 300, 332, 37 I, 398, 436, 44 I, 446,460

Girl Guides, 422, 428

Girls' education, $367,386,411,413$

Glycosuria, 264

Goal of scientific thought, r96, 287

GoEthe, 337, 470

Gold, r93, 2 I 4

Good purposes, I 72

GossE, Sir Edmund, I37, 333

Government grants, 2 I 2, $45^{8}$

Graduate, 37I

Graduate study and research, 402-6

'Greats,' 359

GREECE, 354, 389

Greek, 354, 364, 382, 387, 462

GREEN, J. R., 298

GREGORY, Sir Richard, 138

Group factor, I IO, I I2, I I9, 476, 478 , 480

- Cleverness, II9 et seq. See also 'Cleverness'

- Dr Webb's, IoI, I17, I19, I29, I 55 et seq., 476,490

Guiding of thought by neurograms and Will, I30

Habit, 42, 43, I I 5, I 52, 282, 288, 330

HALdANE, Viscount, 59,328

Hall, Sir Daniel, $34 \mathrm{I}$

HALI, Dr G. Stanley, 297, 3 10, $3^{84}$

Hamilton, Sir William R., 82

Handels Hochschule, 436

Handicraft, $379,380,382,386$

Handwork, 278, 361, 362, 422, 423, 428

Harmony, 1 42, 240, 244, 250, 284

- between interest-systems, 142

- of purpose, 240, 244, 299

HARRISON, Frederic, 295

HART, Dr Bernard, 7, 47, 59, 6r, 65, 79, $90,91,96,103,113,118,127,142$, I $63, \mathrm{I} 69, \mathrm{I} 7 \mathrm{I}, \mathrm{I} 73, \mathrm{I} 74, \mathrm{I} 75, \mathrm{I} 85$

Hartog, P. J., 473

G. E.
HEAD, Dr Henry, 3r, 32, 53, 248

Headlam, Professor W., 305

Headmasters, Incorporated AssoCIATION OF, 34I, 460

Hedonistic, 249

Hemispheres, cerebral, 34

HERBART, 63, 86, 90, 290, 292

Heuristic, 22 I, 224

HEY, Spurley, 369

HEYMANS, I6I

Hichens, W. L., IO3

Hierarchy, Io 4 et seq., I95, 477, $47^{8}$

High school (for girls), 4 I 3

Higher level arcs, 34, 36

- School Certificate Examination, $387,390,393,396,397,498$

- Secondary School, 366, 367, 369, $370,373,383,388,389,390,392$, $412,417,420,443-5,465$

HiLl, Dr Alex, I 3

Historian, 292, 346, 347

History, $347,378,379,386,387,394$, $396,424,427,433$

Hobby, 63, 90, 334, 495, 496

Holiday, 334, 382, 495, 496

Holmes, Dr Gordon, 31, 248

Homework, 380

Honours degree, 393, 397, 402, 410, 440, 455,466

- course, 397 et seq., 409, 466

- school, 386, 390, 392, 394, 396, 397 . 4OI, 402, 4I I, 444, 445

Hope, 298, 303, 308, 3 I 5

Hopkinson, Professor Bertram, 4I9

Hours of labour, 427

Housemaster, 347

Humanist, 338

Humanity, 301

Humberstone, T. L., 404

HUME, 249

Humour, sense of, I 20-3

HuXley, Professor, 8, 9, 12, 95, 399

Hydro-mechanical model, 77

Hypnotic suggestion, I44

Hypothesis, I $50,260,264,265,304$, 307,309

Idea, $45,46,47,147,233,235,276$

- of an object, 47

Identity theory, 183

Ideo-motor, 140 et seq.

Image, 38, 44 et seq., 69, I40, 225, 276

Imagination, $6,276,277$

Immortality, 241, 298, 299, 307

Importance of essences, 194-5, 199, 202, 238,304

Impulse, 27, 28, 29, 30

Incomplete endarchy, 199, 222, 269, 385

Individual differences, 225, 227, 228, $232,235,240,241,242,246,266$, 303, 3II, 313, 3I6, 319, 327, 344, $351,352,363$

Induction, 265, 304

Inductive syllogism, 262

Industrial administration, 400, 4I 4, 466 
Industrial bursaries, 408

- history, 4I9

- research, 404, 405, 406

- statesman, 326, 328

Industry, 325, 326-30, 406, 408, 409, 4 II, 466

- captain of, $323,326,328$

Infant school, 376

INGE, W. R., Dean of St Pauls, 2 I 7

Inhibition, 75, 76, 77, I 4 I, I 48, I 49, 259

Inspiration, 256, 257, 259, 260, 263, $264,265,266,274,297,304,400$, 464

Inspirational lectures, 400

Instinct, 5 I et seq., 1 38, 22 I, 245 et seq., $268,270,271,300,302$

- Dr McDougall's definition, 52

- neurograms, 53,82

Instrumental subjects, I 6

Insulation, 69, 80

Integral Education, 270, 470

Integrated mind, 244, 266, 269

Integration, I68, I 74, I 75

Intellective energy, i 8

Intellectual plane, I 2 I

- qualities, I 2 I et seq.

Intelligence, $102,103,127$

Intensity of neurograms, $45,88,89$

Interest, 62, 85

- single wide, 22, 23, 225, 243, 244, 268,28 I, 287, 3 I I, 3I 5

Interest-system, 62-4, 86, 88, 94, I 44, I62, I63, I64, I66-8, 243

- future-, I 44, I $46, I_{52}$, I 53, I 54 , I $59-6 \mathrm{I}, 2.38,49 \mathrm{I}$

Intermediate level, arcs of, 33

- part-time education, 373, 420-2, $428,429,430,44^{2}$

- secondary education, $369,370,373$, 383-90, 4I 3, 4I 7, 444

Interruption of trains of thought, $28 \mathrm{I}$, 282,285

Interview for scholarships, $46 \mathrm{I}$

Involuntary thought, Chapters 6, I3

JАСОві, C. G. J., I37, 333

JAMES, Professor William, 23, 33, 44, $46,47,51,54,66,69,74,77,78,79$, $82,83,89,90,96,97,99$, 1 О1, 124, I 25, I $28,130,132,138$, I 40, I 4 I, I 44, I $47, I_{4}, I_{49}, I_{50}, I_{5} I, I 6 I$, I 74, I 79, I 83, I86, I89, I90, I96, 197, 198, 205, 214, 217, 218, 229, $230,233,234,238,257,267,272$, $273,274,275,277,286,287,288$, $292,296,297,340,376$

JANET, Professor, 169

JOHN, St, 24I, 301

Joint Matriculation BoARD, 393, 395

JoWETT, Professor, 216, 303

JUDD, 427

JUNG, Dr, 6I, 9 I

Junior part-time education, 369, 424-9

- secondary education, 370,373 . $383-90,423,462$
Junior Technical School, 325, 369, 370, $373,417,418,419,421,425,430$ $445-8$

Justice, 300, 301, 302, 306

KaNT, 219

Kenyon, Sir Frederic G., 392

KEPLER, 195, 196, 238, 248

Kerschensteiner, Dr Georg, 279, 470 , 474

IIEYNES, J. Maynard, 303

Kinaesthetic, 30, 35, 59, I 39, 277

Kingdom of God, 306, 308, 311, 315 , 330

KIPLING's, Rudyard, Kim, 350

Knife and fork studies, 378,383

Knowledge, 62, 188, 189, I94, 2 I8, 235 , $237,239,244,256,308,309,335$ et seq., 357, 394, 473

- about, 358

- of, $35^{8}$

KRÜGER, 475

Labour, division of, 3I I

LANCASHIRE, 423,455

Language. See 'Foreign languages'

LAPLACE, 215,243

Latin, I5, 354, 380, 382, 387, 462, 471

Law, 7, 4I, 65-94, I29, 190, 198, I99, 3 I $3,353,384,400,428,475,484$

- cosine, 484

- general, 190

- normal probability, I I 2 et seq., 475

- of forward conduction, 183

- of nature, 65,191

- vector, I03, I57, 477

Laws of thought, 289

Ist: Psycho-neural parallelism, 65 2nd: Diffusion, 69

3rd: Inhibition 79 (corollary to 3 rd law, 89)

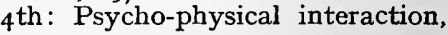
I 29

5th: Thought ends in action, 273

Leading hand, 326,329

LEAGUE OF NATIONS, International Labour Organisation of the, 330

Learn by doing, 59,279

Learned, 357, 394

Learning, 244

Leathes, Sir Stanley, I38, 296, 333, $337,338,340,347,357,380$

Lecturer, 400,464

LEEDS, 293, 332, 497

LEICESTER, 497

Liberty (see also 'Freedom'), 363

Light, 265

LIVERPOOL, 393, 455, 497

LOCKE, J., I6, 249

LOMBARD, 79

LONDON, 455, 497

- County Council, 367

- UnIVERsity (see also Royal Commission), 393, 395

LONGRIDGE, Michael, 436, 44 I

LOTZE, 286 
LouvaIN, I9, 332

Love, 259, 300, 302, 307, 308, 3I5, 347

Lower (or Ordinary) Secondary School, $324,369,370,375,382,383,413$, $4 I 7,443-6,452,461,462$

Lucas, Keith, 35

LUKE, St, 306

Lyttelton, Hon. and Rev. E. S., I9, 332

\section{$\mathrm{MACH}, 223$}

MACKenZIE, Compton, 433

Major premise, 26I

Manager, works, 329

MANCHESTER, 330, 369, 393, 4I3, 4 I 4, $4 I 5,42 I, 425,438,439,44 I, 449$, $45.5,497$

- See also 'Technology'

Manual workers, 232, 279

MARLBOROUGH, 4I 3

- Master of, 23

Marsupials, 205

Mass influence, 434

Mathematical Association, 348

Mathematical side, 358,390

Mathematician, 256, 276, 292, 347, 365, 433

Mathematics, $345,354,356,365,386$, $387,396,424,433$

Matriculation examination, 392-6, 462, $47 \mathrm{I}$

MatTHew, St, 306

Maximal endarchy, I80, 206 et seq., 222, $224,225,227,247$

MaXWell, James Clerk, I 34, 297, 433

McDougall, Dr William, 6, 9, 27-30, $33,34,35,36,38,39,41,42,45$, $47-51,52,53,54,55-8,60-3,65$, $69,70,74-80,81,82,84,91,92,95$, 96,99, I 00, I 1 3, I 22, I 23, I 28, I 30, I 32, I 46, I 54, I 66, I 83, 248, 249, $274,275,276,277,34 \mathrm{I}$

McKenNa, Stephen, 434

M'CoRMICK, Sir William, 396

Meaning, 45-6, 67, 68, 81, 91, 93, I 87

Medical studies, 32I, 400, 402, 408

Mellon Institute, 404,405

Memory, 38, 43, 44, I0o, I I6, I28, 279

Mentally defective children, 278,321 , 362,367

Mercier, Dr C. A., 20, I 24

Metallic films, 264, 265

Metallurgy, 400

Mill, John Stuart, I 2, 288, 401

Milton, John, I6, 337, 433, $47^{\circ}$

Mind, 8

Minimum path proposition, 209, 2 IO, 492

Minor premise, 26I

Miscellaneous part-time studies, 4 I 4 , $422,429,442$

MrtChell, 79

Modal age, 368,377

Modal child, 368, 377

Mode (of frequency distribution), 368
Modern humanities, $387,389,390$

— side, $358,359,390$

MonTAIGNE, I 6

Mood, 62, I60

Moral sense, I 72

Moseley, H. G. J., 196

Mot,, Dr John R., 297

Moulding the world, 2 I 8

Moulton, Lord, 328

Muir, Professor Ramsay, I 52

Multiple stimulation, 83, I34, I 79

MuMFord, Dr A., 34 I

Murray, Professor Gilbert, 59

Muscular sense, 30

Music, 373, 4I I

Names as essences, I98, I99, 356

NAPOLEON, 2 I 5

Natural science. See 'Science'

Natural selection, 275

Nature, 366, 456, 470, 472

Nautical Almanac, 196

Nebular hypothesis, 2 I 9

Negative purpose, 145-7

Negative self-feeling, $5 \mathrm{I}, 248$

' Neglect of Science,' 472, 473

NEHEMIAH, 285

Neighbour, 300-3, 309

Neural disposition, 42

Neurasthenic, 294

Neurin, 75,76

Neurogram, 27, 42 et seq.

—-element, I9I, 2 I2

- purpose, 143 et seq., 238 et seq.

Neurograms, system of, 42,44

Neurography, 63, I 32, 226 et seq., 291

Neurone, 28

Neurosis, 66

New science, 223

Newcastle, 497

Newman, Cardinal, 337, 435, 436, 437

NewToN, Sir Isaac, 93, 94, I 34, I92, I95, 433

Nomenclature, 2or

Normal Distribution, II 2 et seq., 32I, 475

- educational paths, $376,385,395$. 422,430

- probability law. See 'Normal Distribution

NoRwich, 497

Notation. See 'Symbols'

NotinghaM, 497

Nunn, Professor T. Percy, I04, 334, 348

Nursery education, 376,377

- school, 376

(8)-process, I 48, I 70, I 82

Obsession, I 7 I

Occupations, 319

Old age, 298, 299

Omniscient being, 199, 208, 214, 236, 237

Ordinary (or Lower) Secondary School, $324,369,370,375,382,383,413$, $4 I 7,443-6,452,461,462$ 
Ordinate, 367,369

Organisation of thought, 177

Organised thought, 335

Originality, $120-3$

OSBORNE, $4 \mathrm{I} 8,46 \mathrm{I}$

Ostwald, Professor, 290

OUNDLE, 4 I 3

OWens College, 432

OXFORD, I03, 349, 359, 374, 393, 403, $433,434,435,438$

Pain, 248

Pantheists, 24I

Parent, 347

Partial correlation, I 59, 489

- endarchy, 196, 197, 198, 202, 206, 208, 2 IO, 2 I 5

Partridge, G. E., I6I

Part-time education, $345,347,349,360$, $37^{2}, 373,406,4^{1} 3,420,424,44^{8}$

-- - miscellaneous, 4I3 et seq., 422, $429,431,442$

- Secondary School, 369, 402, 425-9, 448-5I

- secondary education, 424 et seq.

Pass courses, $397,398,402$

- degrees, 339, 392, 397, 399, 402, $413,466,467$

Passive education, $346,465,470$

Patellar reflex, 79

PAUl, St, 149, I 50, 284, 297, 300, 306, $307,320,363$

PAWLOW, 47,49

Pear, Professor T. H., 331

Pearson, Professor Karl, I04, 368

Perception, 38, 46, 69

Perry, Professor, 470, 472

Persistence of motives, I 55, 160, 490

Personal endarchy, 196, 199, 219,220 , 23I, 232 et seq., 24I, 242, 269

Personality, 292

Pestalozzi, I6, 21,469

Peter, St, 300

Philosopher, 232

Philosophy, 295, 296, 305, 306

- degree of Doctor of, 403, 464

Physical exercises, 386, 4 I 8

Physicists, 191, 196, 237, 256, 259, 279, 326,433

PitTsburg, 404, 405

Plato, 3, 303, 320, 335, 336

Pleasurable satisfaction, 248, 249

Plymouth, 497

Poincart, Henri, $7,8,85,90,92,190$, I93, I94, 195, 199, $217,219,223$, $249-58,260,264,268,274,356$

Polytechnics, 368, 412, 44I

Positive purpose, 145

- self-feeling, 5I, 248, 298

Potential, higher, ror

- lower, IOI

Poulton, Professor F. B., 382

Poulton, Ronald, 382

Practical instruction, 377

- man, 286

-. work, 278,279
Practice, consulting, 406, 407, 464

Pragmatism, I 89. 296

Prayer, 285

Preliminary one year undergraduate courses, $392,393,463$

Preparatory education, 373-82, 467

- School, 377-83

Primary emotions, 5I

Prince, Dr Morton, 40, 42, 43, 44, 47-9, $56,59-64,66,77,79,96$, го1, 244, $258,292,331$

Principles, 267, 287, 343, 344, 346, $35^{2}-7,384,428,465$

Private Schools, 323

- study, 380,432

Probability, 345

- law, I12 et seq., 475

Probable error, Io6-9, 475, 476

Professorial chairs, 216

Progress, 287, 303, 304, 313

Property, 229, 238

Propositions, universal, I90

Prosser, Dr C. A., 4I I, 4I 7, 420

Province, 319, 365, 366, 370, 439, 440, $45 \mathrm{I}, 456-8$

Provincial Education Authority. See 'Province'

Prussianism, 303

Psycho-analysis, 176

Psychodynamics, 65

Psychology, social, 222

Psycho-neural parallelism, 65

Psycho-physical interaction, 9, 95 et seq., 98, 132, 31 3

- parallelism, 9,65, 95

Psychosis, 66, 289

Psychostatics, 65

Public School, 323, 333, 381, 413, 434, 443,448

Purpose, 98, I 4I, I 43-6I, I 72, 238, $240,244,28 \mathrm{I}, 282-5,29 \mathrm{I}, 294-302$, 306, 309, 31 I, 314-16, 334, 335, $340-2,347,3^{8} 3,3^{86}, 387,420,433$, $46 \mathrm{I}, 490$

- and Will, 98, I 55, 288

- approved, I44, I45

- Christian, 309, 340

- good, 172

- harmony of, $240,244,299$

- negative, 145,147

- qualities, I 55-6I, 490

Purposefulness, I58 et seq., I6I, 294. 489-9I

Pyramidal cells, 32

- tract, $32,34,36,72,73,78$, I 39 , I $53,274,277$

Pythagoras' theorem, 344, 364

Quickness, I20-3

RAMSAY, Sir William, 20

Rationalisation, I73, I 74

READING, 497

Reading, 379

Real world, 62,372

Realisation, self-, 22 
Reason, I99, 297

Reasoned thought contrasted with empirical thought, 187

Reasoning, I 79, I86, 189, I9I, 222, 223, $239,255,257,262,265,266-8,313$

- deductive, 262, 264-6, 268, 286

- efficient, 223

- trains of, 223

Reciprocal innervation, 75

Reconstruction, Ministry OF, 355

Reflex, I 54, 245, 282, 29 I

REIMS, 332

Reinforcement or concentration, 99, I 28, I 80, 234, 28I

Relativity, 347

Religion, 294-9, 303, 334, 335, 347 , 488,490

Religious instruction, 296, 335, 347

Report on Engineering Education and Research, 325, 502-4

Report on Scholarships for Higher Education (Cd. 8291), 454, 463

Repression, I 48, I69, I 70, I 7 I, I 76, I79, I 82, I 84

Research, 257, 364, 40I-6, 435, 437, $438,463,464,465$

- association, 404,405

- degree, 403, 4I8, 464

- Department of Scientific and Industrial, 404

- industrial, 404-6

- scholarships, 463

Residence in universities, 374, 432, 436-8

Resistance, synaptic, 39-41, 50, 69, $80,8 \mathrm{I}$

RHODEs scholarships, 435

RICHMOND, Kenneth, 224, 258, 259 , 263,266

RICHTER, 299

RIVERS, Dr W. H. R., 34 I

Robertson, T. Brailsford, 39

Robinson, C. E., 333

Robinson CRUSOE, 227, 288

Rolandic area, 32-4, I 39, 278, 378

ROME, 354, 389

ROUSSEAU, I6, 22, 287, 288, 380

Rowntree, B. Seebohm, 4I 5

ROYAL COMMISSION ON UNIVERSITY EDUCATION IN LONDON, 396, 400, $401,407,4 I 4,435$

RugBY, 338

Rural districts, 423

Ruskin, John, 321, 322

Rutherford, Professor Sir Ernest, 196

SAdler, Sir Michael, 332, 372

ST ANDREWS, I 2, 40I

St Paul's School, 433

SALFORD, 425

Salvation, 307,308

SAMOA, 300

Sandwich system, 430

Satisfaction, $248-53,274,284,285,298$

- aesthetic, 250-4, 274, 284, 285

- pleasurable, 248,249
SCHILLER, I 24

Scholarships, $320,366,374,375,408$, 430, 439, 453 et seq.

Scholarships for Higher Education, Report on, 454,463

Scholasticism, 238

School, types of. See 'Types"

School Certificate examination, 338 , $386,387,393,395,396,462,471$

- - Higher, $387,390,393,397,498$

— of Art, 373

- of Commerce, 373

- of Domestic Subjects, 373

Schuster, Sir Arthur, 286

Science, applied, 395, 400, 464

- endarchy of, 194-7, 199, 202, 208, 2 I 5-I 7, 227, 237

- natural, I9I, 348, 388, 389, 394, 472,473

- new, 323

- side, 390

Scientific endarchy, 228-34, 335 et seq.

Scotland, 37r, 38r, 430, 438, 444

Scouts, Boy, 422, 428

SCRIPTURE, IOI

Secondary education, 4I5-20, 424-9. See also advanced, intermediate, senior, junior, and part-time secondary education

- Schools, 325, 346, 347, 362, 443-5, 448,450 . See also Higher Secondary Schools, Lower Secondary Schools, and Part-time Secondary Schools

Selection, 194, 227, 251, 319-25, 453

Self-denial, 308

Self-feeling, negative, 5 I, 248

- positive, 5I, 248, 298

Self government, 444

- realisation, 22

- -regarding sentiment, $146,152,285$, 299

Selfishness, $22,300,301$

Semi-circular canals, I 75

Senior elementary education, 364, 369, $373,422-4,429$

- part-time education, 373, 422, 424-9

- secondary education, $369,373,383$, 4I5-20, 462

- technical education, $370,373,3^{8} 3$, 409-1 4, 442

- Technical Schools, 325, 368, 373, $375,412-14,430,440-2,456,457$, 465

Sensation, $31,32,36,38$

- -reflex, 36, 37

Sensori-motor arc, 29, 30, 36

Sensory area, 32-34

Sentiment, $5^{8}$ et seq., 288

— -neurograms, 58

- self-regarding, I 46, I 52, 285, 299

Separate subjects, $212,215,216,223$, $269,336-8,346,356,384,393,470$, 474

Sermon on the Mount, 290 
Service, 227, 289, 302, 303, 308, 319, $322,323,324,325,326,345,357$, $360,363,372,416,422,427,460$

SHAND, A. F., 52, 56, 58, 96, 299

SHEFFIELD, 393, 403, 497

Shell-shock, I 7I, 33 I

Sherrington, Professor C. S., 3I, 35 , $79,83,84,245$

Shipbuilders, 430,431

ShOREDITCH, 419

Short-circuit, 230, 237, 351, 354, 356

SHREWSBURY, 338

Similarity, I 22

Simple essence, 229

Simple fact, I99, 2 I 3-16

Single general factor, ro3 et seq., rog et seq., 476 et seq. See also "General Ability'

Single wide interest, 22, 23, 225, 243 , $244,268,28$ I, 287, 3I I, 3 I 5

Sixth form, $349,390,444,465$

- - master, $349,388,465$

Skeletal system, 29, 30, 378

Skill in thinking, 327, 385, 399

Sleep, I 48, 273

Sleight, Dr W., 102

Smith, May, Ior

Smith, Professor G. Elliot, 33 I

Smithells, Professor W., 3

Social psychology, 222

Sociology, $37^{8}$

SOCRATES, I 4

Soddy, Professor, I96

Sodium, 264, 265

Soldiers, 82, I64, I68

Somnambulism, I69, I 70

Soul, 8, 9, I6, 17, 95-7, 99, I28, I38, I 4 I, I 43, I 49, I 50

South Pole, 300

SOUTHAMPTON, 497

Spearman, Professor W,, 96, IO2-5, I09, I I 2, I I 3, I I 7-20,427, 475-8 I

Special apprentices, 430,431

Specialist teacher, 270, 347

Specific education, I 3

- factor, I Io et seq., 477 et seq.

SPENCER, Herbert, I6, $47 \mathbf{I}$

Spinal cord, 30, 33, I3I

- reflexes, 35, I 38

'Spinal' dogs, 35,83

Standard deviation, 475

-- neurography, 228

Statesmen, 326, 328

Stevenson, R. L., 298, 340

Stories, 378,379

Stout, G. F., 56

STRACHAN, James, 34r, 365

Struggle for existence, 275

Strychnine, 77

Student-hours, 212

Study, contrasted types of, 35I-62

- private, 380,432

Sublimation, I68, I69, I 72

Subliminal, 258

Sub-conscious, 259

Sub-normal children, $278,279,362,367$
Subject, 62, 2 I 2

- endarchies, 196, 208, 2 I 5, 236

Subjects, separate, 212, 21 5, 2 I6, 223 , $269,336-8,340,356,357,470$

Summum bonum, 240, 284

SUNDERLAND, 430

Superconscious, 258,259

SWANSEA, 497

SYDENHAM, Lord, 332

Syllogism, 261, 262

Symbols, 28, 70, I46, I 79, I90, 207, 208, 233

Synapse, 28, 39-4I

System of neurograms, 42, 44

Teacher, 269-7I, 322, 33I, 346, 350, $363,402,420,433,456,457,464$ et seq.

- central, 270

- part-time, 466

- specialist, 270,347

- university, 464,465

Teachers' qualifications, 464 et seq.

Team work, 434

Technical education. See senior technical education, and advanced, miscellaneous, and intermediate parttime education

- School. See Technical School and Junior Technical School

Technische Hochschule, 436

TECHNOLOGY, COLlEGE OF (Manchester), 330, 37I, 405, 4I 4, 438, $44 \mathrm{I}, 504$

- Faculty of (Manchester), 392, 396

Teleological, 196, 234, 238, 240, 395

Telephone exchange, 204

Temperament, I6r

Temple, Dr William (Bishop of Manchester), 8, 103, 137, 296, 333, 347. 434

Temptation, I 79. 295

Tender emotion, 51, 302

Terminal type of education, 358-6r, $373,383,384,4$ I I, 4 I6-I 8,428

Thalamus, $32,36,50,53$

Theists, 241

Theologians, 232, 347

Things, I9I, I96

Things in themselves, I9I, 196

Thinking, voluntary, 138,256

Thomson, Professor G. H., 478

Thomson, Sir J. J., 338, 343, 348, 350, $358,389,392,395,396,401,404$, $406,417,420,433,446,462$

Thorndike, Professor W., I I I

Thornton, J. S., 372

Thought-activity, 47

- organisation of, 177

- organised, 335

Threshold, 86

Times Educational Supplement, 348

Tiredness, 273

Toy, 377

Trades, 279

Tradesman, 326, 329 
Trains of reasoning, 223

'Transfer,' 102, 320

Transfer, age of, 369,445

Transitional type of education, 356 , $35^{8-61}, 373,3^{83}, 385,390,4$ I6, 4I 7, 429

Trinity College, Cambridge, 433

Tripos, 386

TROTTER, W., I 74

Truth, I 89, 196, 260, 301, 306, 309

Tutorial superintendence, $374,432,436$

Types of education, $351-5,372,373$, 376 et seq.

I. Graduate study and research, 402-6

2. University part-time, 406-9

3. Undergraduate, 397-402

4. Advanced part-time, 4I3, 4I 4

5. Miscellaneous part-time, 4I 4

6. Advanced secondary, 383-90

7. Senior technical, 409-1 3

8. Intermediate part-time, 420-2

9. Senior part-time, 424-9

Io. Intermediate secondary, 383-90

I I. Senior secondary, 4I 5-20

I2. Junior part-time, 424-8

I3. Junior secondary, 383-90

I4. Senior elementary, 422-4

15. Preparatory, $377-83$

I6. Elementary, 377-83

I 7. Nursery, 376,377

Types of school, $365,373,374,432$ et seq.

I. University, 435-40

2. Senior Technical School, 440-2

3. Higher Secondary School, 443-5

4. Part-time Secondary School, $44^{8}-5$ I

5. Lower Secondary School, 443-5

6 . Junior Technical School, 445-8

7. Central Elementary School, 423, 45I, 452

8. Private Preparatory School, 380-3

9. Public Elementary School (or Primary School), 380-3, 423, $45 \mathrm{I}, 45^{2}$

Unconscious, $44,257,258$

- memory, 43

- work, 256, 258

Undergraduate, 349,350,37 I, 390, 397

- studies, 350, 389, 393, 394, 397-402

Unipolar conflicts, 176

Unique effect of psychical activity, IOI

United States (see also 'America'), 438

Universal propositions, I90

University, $349,350,359,366,370-2$, $386,388,406-9,413,435-40,462$, $463,497,502,503$

- Court, $44^{\circ}$

- entrance scholarships, 462-3

- entrahce tests, 393-6
University, finance, $437-9$

- part-time courses, 368, 372, 406-9, 4I 3

- teacher, $388,464,465$

Unpleasure, 248

Utility of thought organisation, 2 II, 212

UTOPIA, 298

Value I94, I99, 207, 2 I 5, 2 I 6, 25 I, 258, $342,35 \mathrm{I}-3,384$

Variety of curricula, $385-7,4$ I I, 4I 4 , $417,422,428$

Vector law, I 03, I 57, 477

Visceral, 29, 30, I 32

Visual instruction, 377,378

Visualisers, 276

Volition, 98

Voluntary attention, I I 5

- movements, I39, I40

- system, 30

- thinking, Chapter 7, especially pp. I 38 et seq., and Chapter 14

Wages, 427

$W^{\prime}$ ALES, $454,455,456$

WAlker, Professor Miles, 325

WALler, Mary, 226

WARD, H., 34 I

WEBв, Dr E., 98, го3, I I I, I I 2, 129 , I 35,138, I 55-6I, 333, 460, 483-91

Welfare workers, $42 \mathrm{I}$

Welton, Professor I., 19

WELIS, H. G., 292, 321, 336, 363

WHEWELL, William, 2 I 6

WHITEHAI.L, $45,46,67$

Whitehead, Dr A. N., I4, I77, I96, $220,243,268,269,357,390$

Whitley Councils, 457

Width (of interest-system), 89

WIERSMA, I 6 I

Will, 38, 98 et seq., 129 et seq., 135 et seq., 1 38 et seq., 255 et seq., 268 et seq., 287 et seq., $29 \mathrm{I}$ et seq., 36I, 482

- and purpose, 98, I 55, 288

Wilson, President, 303

WINCHESTER, 333

Wonder, 51, 92-4, 222, 236, 247, 248, $256,263,300,314$

Woon, Professor R. W., 264

Workers EDUCATIONAL Association, $372,408,424$

Workers, welfare, 42 I

Works schools, 449

World of experience, 180,189

Writing, 379

YoRk, 4I5

Young persons, 453

YULE, G. Udny, I04, I 59, 490

Zimmern, Professor A. E., 303

Zone, 206, 207, 213 
CAMBRIDGE : PRINTED BY J. B. PEACE, M.A., AT THE UNIVERSITY PRESS 



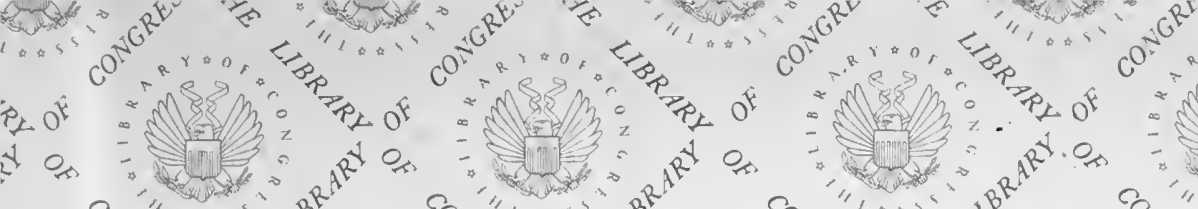
Ot" .

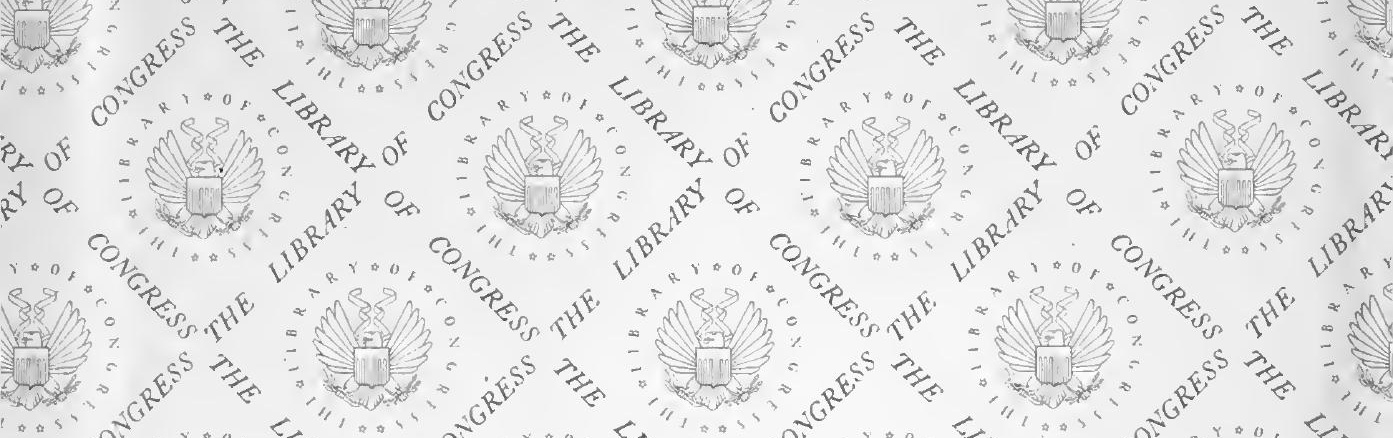

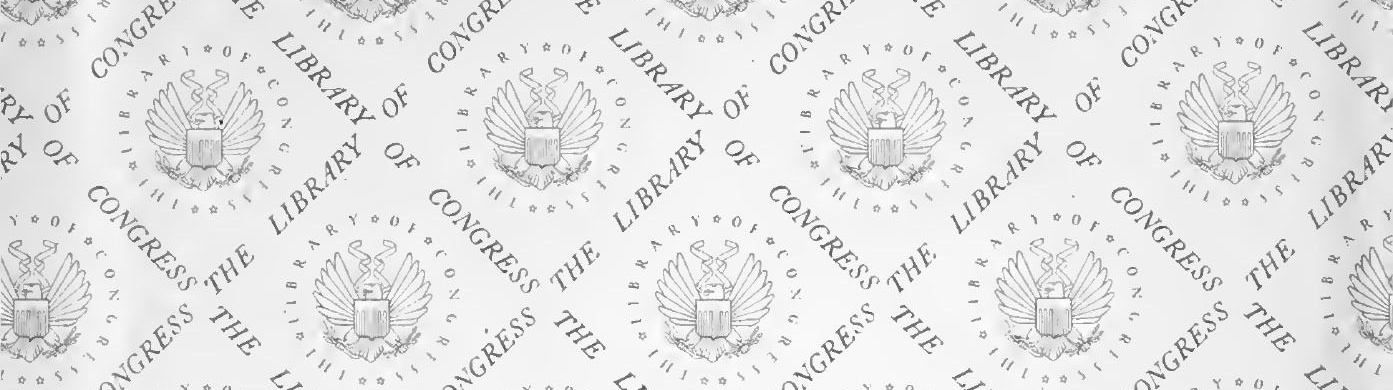
and

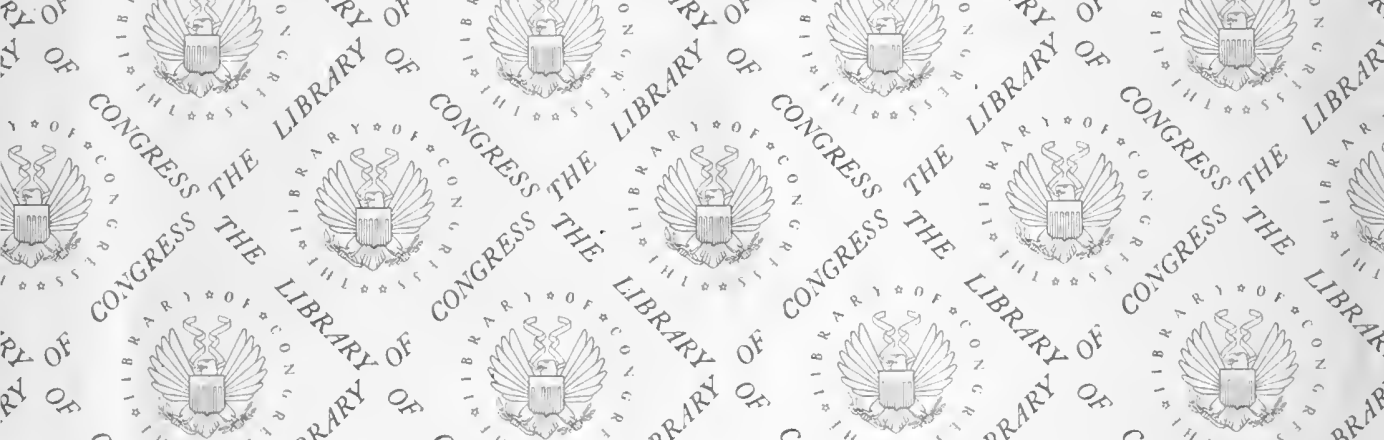

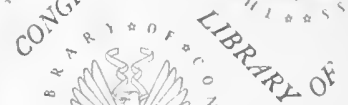
(स) as = $\hat{p}_{2}, 0^{5}$

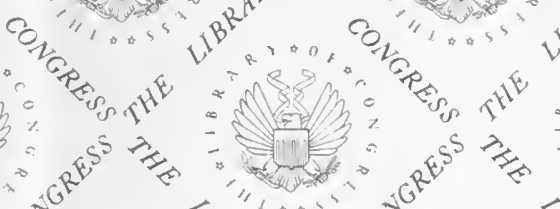

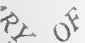
$p^{4} 0^{2}$

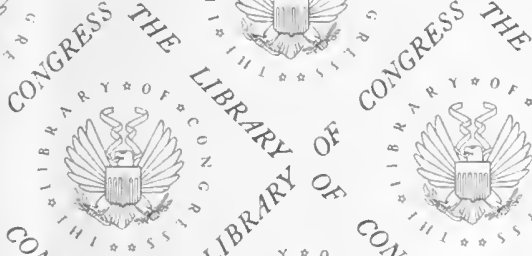

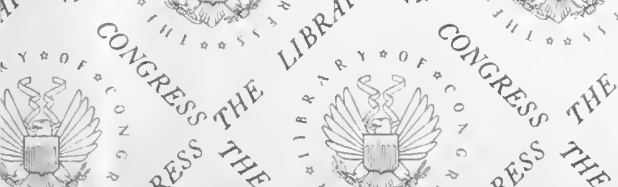

is os $P_{2} O^{4}$ (1) - .

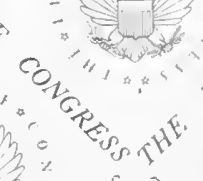

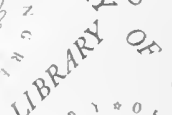

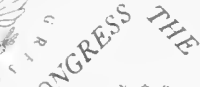
Pि

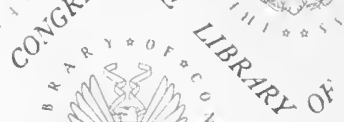
"If

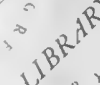
and (3)

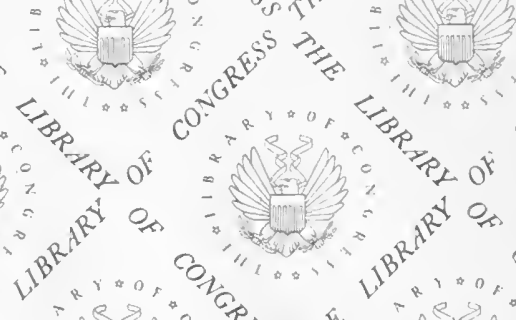
$\mathrm{CO}^{\mathrm{c}}$ $\log _{0}$ - UN " $=0$ $\sqrt{10}$, 
